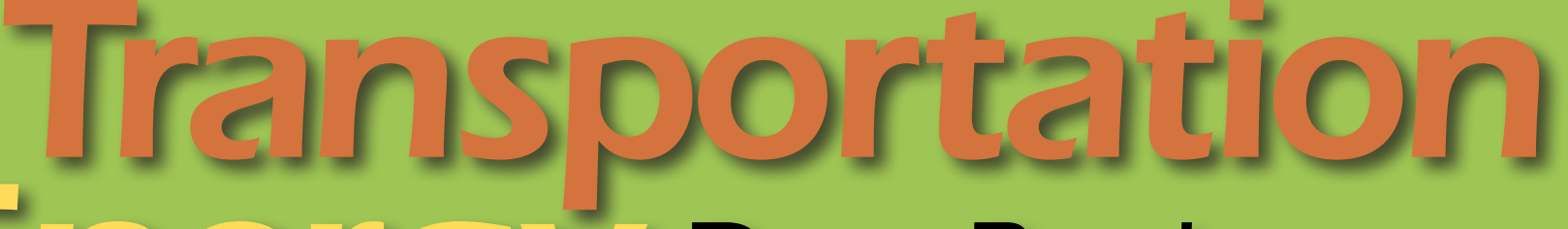 Es]esgy Data Book \\ Edition 36
}

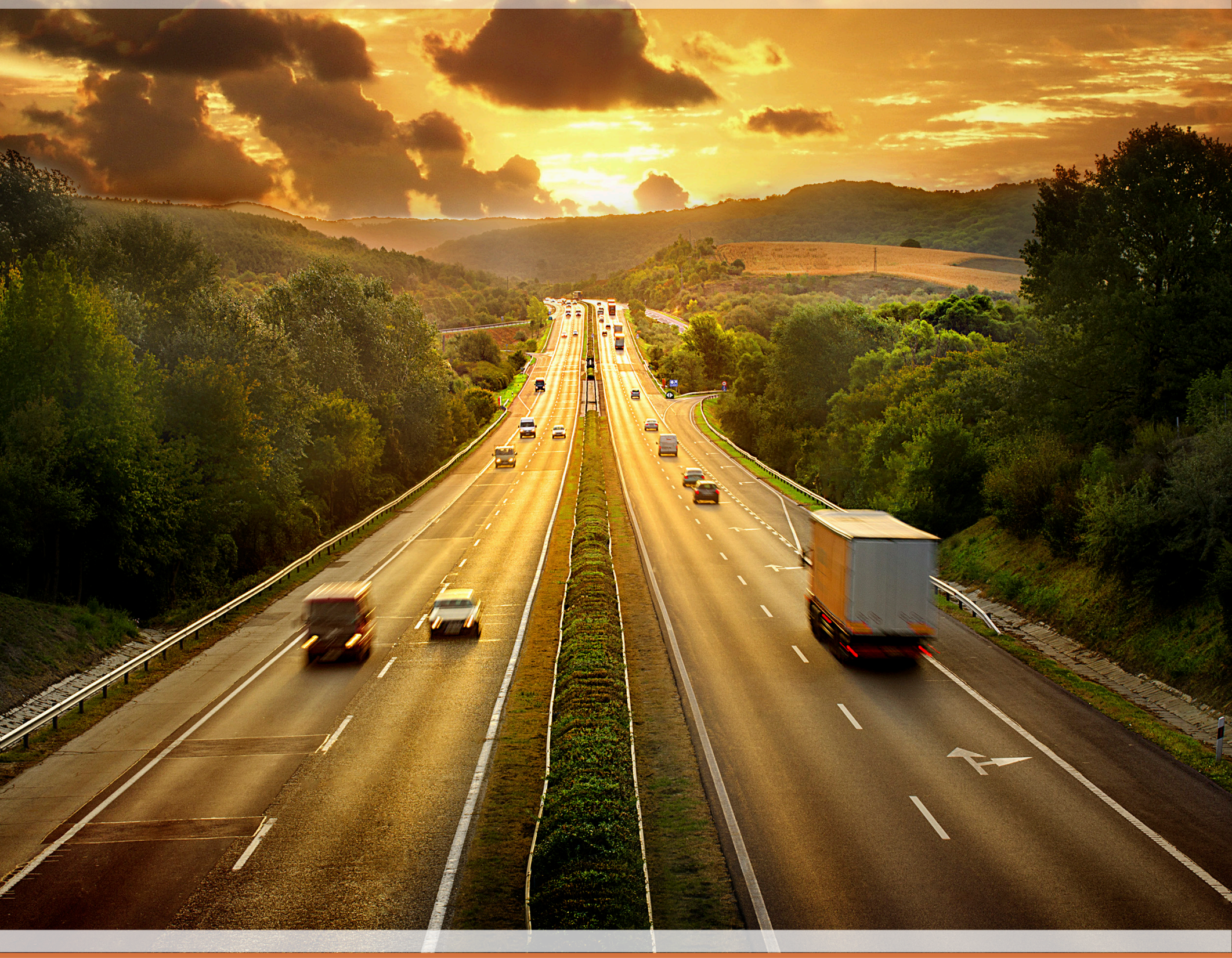

\section{OAK RIDGE NATIONAL LABORATORY}




\section{Transportation Energy Data Book Quick Facts}

\section{Petroleum}

- In 2016 the U.S. produced more than 12 million barrels of petroleum per day (mmbd), or $13.5 \%$ of the world's $91.3 \mathrm{mmbd}$.

- The U.S. consumed $19.5 \mathrm{mmbd}$, or $21 \%$ of the world's $95.4 \mathrm{mmbd}$ in 2015.

- Net imports of petroleum to the U.S. in 2016 were nearly $5 \mathrm{mmbd}$, which was $25 \%$ of U.S. petroleum consumption.

- U.S. transportation petroleum use was 70\% of total U.S. petroleum use in 2016.

- In 2016 U.S. transportation petroleum use was $111 \%$ of total U.S. petroleum production.

- Petroleum comprised 92\% of U.S. transportation energy use in 2016.

- Cars and light trucks accounted for 63\% of U.S. transportation petroleum use in 2015.

- Medium trucks (Class 3-6) accounted for 4\% of U.S. transportation petroleum use in 2015.

- Heavy trucks (Class 7-8) and buses accounted for 19\% of U.S. transportation petroleum use in 2015.

- Nonhighway modes accounted for the rest of U.S. transportation petroleum use in 2015 (14\%).

\section{Energy}

- In 2016 U.S. transportation energy use accounted for over $28 \%$ of total U.S. energy use.

- Cars and light trucks accounted for 59\% of U.S. transportation energy use in 2015.

- Medium trucks accounted for 5\% of U.S. transportation energy use in 2015.

- Heavy trucks and buses accounted for 19\% of U.S. transportation energy use in 2015.

- Nonhighway modes accounted for the rest of U.S. transportation energy use in 2015 (18\%).

\section{Light Vehicle Characteristics}

- In 2015 there were 113 million cars and 129 million light trucks in the U.S. (242 million total light vehicles).

- $\quad$ Light vehicles accounted for $90 \%$ of the 3.1 trillion vehicle miles driven in the U.S. in 2015.

- U.S. cars:

o $6,873,000$ cars were sold in 2016 which was $40 \%$ of new light vehicle sales.

o In 2016 the average age of a U.S. car was 11.6 years.

o In 2015 the average fuel economy for the U.S. car fleet (all cars on the road) was $26.2 \mathrm{mpg}$.

- U.S. light trucks:

o 10,296,000 light trucks were sold in 2016 which was $60 \%$ of new light vehicle sales.

o In 2016 the average age of a U.S. light truck was 11.6 years.

o In 2015 the average fuel economy for the U.S. light truck fleet (all light trucks on the road) was $18.8 \mathrm{mpg}$.

- The average U.S. household vehicle travels 11,300 miles per year (2009 NHTS).

\section{Heavy Truck Characteristics}

- $11,203,000$ heavy trucks were registered in the U.S. in 2015.

- Heavy trucks and buses accounted for $10 \%$ of the 3.1 trillion vehicle miles driven in 2015 .

- In 2002 (the last time a survey was conducted), heavy trucks accounted for $80 \%$ of medium and heavy truck fuel use. 
ORNL/TM-2017/513

(Edition 36 of ORNL-5198)

Center for Transportation Analysis

Energy and Transportation Science Division

\title{
TRANSPORTATION ENERGY DATA BOOK: EDITION 36
}

\author{
Stacy C. Davis \\ Susan E. Williams \\ Oak Ridge National Laboratory \\ Robert G. Boundy \\ Roltek, Inc.
}

December 2017

Transportation Energy Data Book: Edition 36 can be found online at: cta.ornl.gov/data

\author{
Prepared for the \\ Vehicle Technologies Office \\ Office of Energy Efficiency and Renewable Energy \\ U.S. Department of Energy
}

Prepared by the

Oak Ridge National Laboratory

Oak Ridge, Tennessee 37831-6073

Managed by

UT-BATTELLE, LLC

for the

U.S. DEPARTMENT OF ENERGY

under Contract No. DE-AC05-00OR22725

Approved for Public Release.

Distribution is Unlimited. 


DOCUMENT AVAILABILITY
Reports produced after January 1, 1996, are generally available free via US
Department of Energy (DOE) SciTech Connect.
Website http://www.osti.gov/scitech/
Reports produced before January 1, 1996, may be purchased by members of the
public from the following source:
National Technical Information Service
5285 Port Royal Road
Springfield, VA 22161
Telephone 703-605-6000 (1-800-553-6847)
TDD 703-487-4639
Fax 703-605-6900
E-mail info@ntis.gov
Website http://www.ntis.gov/help/ordermethods.aspx
Reports are available to DOE employees, DOE contractors, Energy Technology Data
Exchange representatives, and International Nuclear Information System
representatives from the following source:
Office of Scientific and Technical Information
PO Box 62 Oak Ridge, TN 37831
Telephone 865-576-8401
Fax 865-576-5728
E-mail reports@osti.gov
Website http://www.osti.gov/contact.html

This report was prepared as an account of work sponsored by an agency of the United States Government. Neither the United States Government nor any agency thereof, nor any of their employees, makes any warranty, express or implied, or assumes any legal liability or responsibility for the accuracy, completeness, or usefulness of any information, apparatus, product, or process disclosed, or represents that its use would not infringe privately owned rights. Reference herein to any specific commercial product, process, or service by trade name, trademark, manufacturer, or otherwise, does not necessarily constitute or imply its endorsement, recommendation, or favoring by the United States Government or any agency thereof. The views and opinions of authors expressed herein do not necessarily state or reflect those of the United States Government or any agency thereof. 
Users of the Transportation Energy Data Book are encouraged to comment on errors, omissions, emphases, and organization of this report to one of the persons listed below. Requests for additional complementary copies of this report, additional data, or information on an existing table should be referred to Ms. Stacy Davis, Oak Ridge National Laboratory.

\author{
Stacy C. Davis \\ Oak Ridge National Laboratory \\ National Transportation Research Center \\ 2360 Cherahala Boulevard \\ Knoxville, Tennessee 37932 \\ Telephone: (865) 946-1256 \\ FAX: (865) 946-1541 \\ E-mail: DAVISSC@ornl.gov \\ Website Location: cta.ornl.gov/data \\ Rachael Nealer \\ Vehicle Technologies Office \\ Energy Efficiency and Renewable Energy \\ Department of Energy, EE-3V \\ Forrestal Building \\ 1000 Independence Avenue, S.W. \\ Washington, D.C. 20585 \\ Telephone: (202) 586-3916 \\ FAX: (202) 586-1600 \\ E-mail: Rachael.Nealer@ee.doe.gov \\ Website Location: energy.gov/eere/vehicles
}

Spreadsheets of the tables in the

Transportation Energy Data Book include data for years not presented in the published document.

Spreadsheets can be found on the web at: cta.ornl.gov/data 
Find useful data and information in other U.S. Department of Energy, Office of Energy Efficiency and Renewable Energy products.

Vehicle Technologies Market Report:

cta.ornl.gov/vtmarketreport

Light Duty Electric Drive Vehicles:

www.anl.gov/energy-systems/project/light-duty-electricdrive-vehicles-monthly-sales-updates

Transportation-Related Consumer Preference Data www.nrel.gov/transportation/consumer-data.html

Subscribe to the Vehicle Technologies Fact of the Week:

energy.gov/eere/vehicles/transportation-fact-week 


\section{TABLE OF CONTENTS}

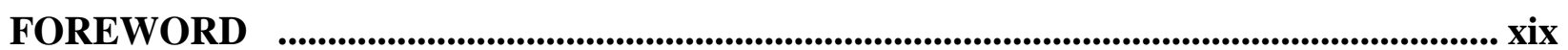

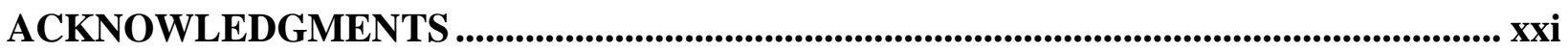

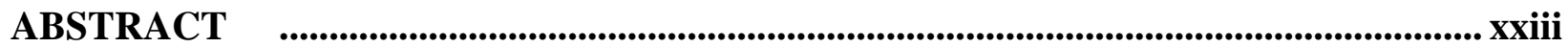

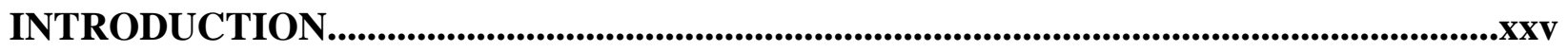

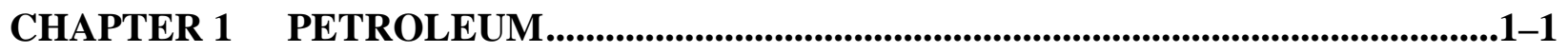

Table 1.1 Proved Reserves of Crude Oil and Natural Gas, 1980-2015.............................1-2

Table $1.2 \quad$ World Crude Oil Production, 1960-2016 .......................................................1-3

Table 1.3 World Petroleum Production, 1973-2016 ………............................................1-4

Table 1.4 World Petroleum Consumption, 1960-2015 …...............................................1-5

Figure 1.1 World Oil Reserves, Production, and Consumption, 1980...............................1-6

Figure 1.2 World Oil Reserves, Production, and Consumption, 1993...............................1-6

Figure 1.3 World Oil Reserves, Production, and Consumption, 2015..............................1-7

Table 1.5 World Oil Reserves, Production, and Consumption, 1980, 1993 and 2015 .....1-7

Table $1.6 \quad$ U.S. Petroleum Imports, 1960-2016..............................................................

Table $1.7 \quad$ Imported Crude Oil by Country of Origin, 1973-2016 ....................................1-9

Table $1.8 \quad$ Crude Oil Supplies, 1973-2016..........................................................1-10

Figure 1.4 Refinery Gross Output by World Region, 2006 and 2016 ..............................1-11

Table 1.9 U.S. Refinery Input of Crude Oil and Petroleum Products, 1987-2015..........1-12

Table 1.10 U.S. Refinery Yield of Petroleum Products from a Barrel of Crude Oil, 1978-2016............................................................................................1-13

Table 1.11 United States Petroleum Production, Imports, and Exports, 1950-2016 .......1-14

Table 1.12 Petroleum Production and Transportation Petroleum Consumption in

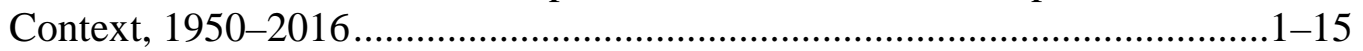


Figure 1.5 United States Petroleum Production and Consumption - All Sectors, 1973-2050. $1-16$

Figure 1.6 United States Petroleum Production and Transportation Consumption, 1970-2050. $1-17$

Table 1.13 Consumption of Petroleum by End-Use Sector, 1973-2016 $1-18$

Table 1.14 Highway Transportation Petroleum Consumption by Mode, 1970-2015 ......1-19

Table 1.15 Nonhighway Transportation Petroleum Consumption by Mode, 1970-2015. $1-20$

Table 1.16 Transportation Petroleum Use by Mode, 2014-2015 $1-21$

CHAPTER 2 ENERGY $.2-1$

Figure 2.1 World Consumption of Primary Energy, 2014 $2-2$

Table $2.1 \quad$ U.S. Consumption of Total Energy by End-Use Sector, 1973-2016 ..... $.2-3$

Table 2.2 Distribution of Energy Consumption by Source, 1973 and 2016. $.2-4$

Table 2.3 Distribution of Transportation Energy Consumption by Source, 1950-2016. $.2-5$

Figure 2.2 World Natural Gas Reserves, Production, and Consumption, 1980 ...............2-6

Figure 2.3 World Natural Gas Reserves, Production, and Consumption, 1997...............2-6

Figure 2.4 World Natural Gas Reserves, Production, and Consumption, 2015...............2-7

Table 2.4 World Natural Gas Reserves, Production, and Consumption, 1980, 1997, and 2015.

Figure 2.5 Natural Gas Production and Reserves for the Top Ten Natural Gas Producing Countries, 2015.........................................................................2-8

Table 2.5 Alternative Fuel and Oxygenate Consumption, 2005-2011...........................2-9

Table 2.6 Fuel Ethanol and Biodiesel Production, Net Imports, and Consumption, 1981-2016... $2-10$

Table 2.7 Domestic Consumption of Transportation Energy by Mode and Fuel Type, 2015. 
Figure 2.6 Domestic Consumption of Transportation Energy by Mode and Fuel Type, 2015 ...... $.2-12$

Table 2.8 Transportation Energy Use by Mode, 2014-2015 …..........................................2-13

Table 2.9 Highway Transportation Energy Consumption by Mode, 1970-2015............2-14

Table 2.10 Nonhighway Transportation Energy Consumption by Mode, 1970-2015.....2-15

Table 2.11 Off-Highway Transportation-Related Fuel Consumption from the Nonroad Model, 2015 ................................................................................2-16

Table 2.12 Fuel Consumption from Lawn and Garden Equipment, 2015 .........................2-17

Table 2.13 Highway Usage of Gasoline and Diesel, 1973-2015 ....................................2-18

Table 2.14 Passenger Travel and Energy Use, 2015........................................................2-19

Table 2.15 Energy Intensities of Highway Passenger Modes, 1970-2015........................2-20

Table 2.16 Energy Intensities of Nonhighway Passenger Modes, 1970-2015..................2-21

Figure 2.7 Energy Intensity of Light Rail Transit Systems, 2015 _...................................2-22

Figure 2.8 Energy Intensity of Heavy Rail Systems, 2015..............................................2-23

Figure 2.9 Energy Intensity of Commuter Rail Systems, 2015........................................2-23

Table 2.17 Energy Intensities of Freight Modes, 1970-2015.............................................2-24

CHAPTER 3 ALL HIGHWAY VEHICLES AND CHARACTERISTICS .......................3-1

Table $3.1 \quad$ World Production of Cars and Trucks, 2000 and 2015 .....................................

Figure $3.1 \quad$ World Car Production, 1983-2015 ………………………….........................3-3

Figure $3.2 \quad$ World Truck and Bus Production, 1983-2015 …………………….................3-3

Table 3.2 Car Registrations for Selected Countries, 1960-2015 .........................................

Table 3.3 Truck and Bus Registrations for Selected Countries, 1960-2015 …...................3-5

Table $3.4 \quad$ U.S. Cars and Trucks in Use, 1970-2015_.....................................................

Figure $3.3 \quad$ Vehicles per Thousand People: U.S. (Over Time) Compared to Other Countries (in 2005 and 2015) ........................................................................... 
Table $3.5 \quad$ Vehicles per Thousand People in Selected Countries/Regions, 2005 and 2015 ..... $.3-10$

Table 3.6 Vehicles per Thousand People in the United States, 1990-2015 $.3-11$

Table 3.7 Shares of Highway Vehicle-Miles Traveled by Vehicle Type, 1970-2015.

Table 3.8 Cars in Operation by Age, 1970, 2000, and 2013........................................13

Table $3.9 \quad$ Trucks in Operation by Age, 1970, 2000, and 2013 ...................................14

Table $3.10 \quad$ U.S. Average Vehicle Age, 1995-2016.......................................................15

Table $3.11 \quad$ New Retail Vehicle Sales, 1970-2016.........................................................16

Table 3.12 Survival Rates for Cars and Light Trucks by Vehicle Age ..........................3-17

Table 3.13 Annual Mileage for Cars and Light Trucks by Vehicle Age .........................3-18

Table 3.14 Heavy Truck Scrappage and Survival Rates, 1970, 1980, and 1990

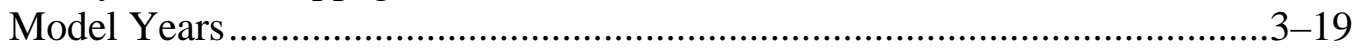

CHAPTER 4 LIGHT VEHICLES AND CHARACTERISTICS ................................4-1

Table 4.1 Summary Statistics for Cars, 1970-2015.................................................4-3

Table 4.2 Summary Statistics for Two-Axle, Four-Tire Trucks, 1970-2015.................4-4

Table 4.3 Summary Statistics for Light Vehicles, 1970-2014 _..................................4-5

Table 4.4 Summary Statistics on Class 1, Class 2a, and Class 2b Light Trucks .............4-6

Table 4.5 Sales Estimates of Class 1, Class 2a, and Class 2b Light Trucks,

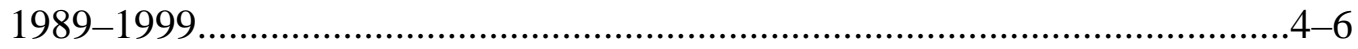

Table 4.6 New Retail Car Sales in the United States, 1970-2016................................4-7

Table 4.7 New Retail Sales of Trucks 10,000 Pounds GVW and Less in the United States, 1970-2016 .........................................................................

Table 4.8 Production, Production Shares, and Production-Weighted Fuel Economies of New Domestic and Import Cars, Model Years 1975-2016........................4-9

Table 4.9 Definition of Car Sport Utility Vehicles in Model Year 2016......................4-10

Table 4.10 Production, Production Shares, and Production-Weighted Fuel Economies of New Domestic and Import Light Trucks, Model Years 1975-2016 ..........4-11 
Table 4.11 Production and Production-Weighted Fuel Economies of New Domestic and Import Cars, Light Trucks and Light Vehicles, Model Years 1975-2016. $.4-12$

Table 4.12 Light Vehicle Production Shares, Model Years 1975-2016 $.4-13$

Figure 4.1 Light Vehicle Production Shares, Model Years 1975-2016. $.4-14$

Table 4.13 Production-Weighted Engine Size of New Domestic and Import Cars, Model Years 1975-2016.

Table 4.14 Production-Weighted Engine Size of New Domestic and Import Light Trucks, Model Years 1975-2016. $.4-16$

Table 4.15 Production-Weighted Loaded Vehicle Weight of New Domestic and Import Cars, Model Years 1975-2016 $.4-17$

Table 4.16 Production-Weighted Loaded Vehicle Weight of New Domestic and Import

Light Trucks, Model Years 1975-2016.... 4-18

Table 4.17 Average Material Consumption for a Domestic Light Vehicle, Model Years 1995, 2000, and 2015. $.4-19$

Table 4.18 New Light Vehicle Dealerships and Sales, 1970-2016. $.4-20$

Table 4.19 Conventional Refueling Stations, 1993-2013 . $.4-21$

Table 4.20 Fuel Economy and Carbon Dioxide Emissions Standards, MY 2012-2025 $.4-22$

Table 4.21 Fuel Economy and Carbon Dioxide Targets for Model Year 2025. $.4-23$

Table 4.22 Car Corporate Average Fuel Economy (CAFE) Standards versus Sales-Weighted Fuel Economy Estimates, 1978-2017

Table 4.23 Light Truck Corporate Average Fuel Economy (CAFE) Standards versus Sales-Weighted Fuel Economy Estimates, 1978-2017. $.4-25$

Table 4.24 Corporate Average Fuel Economy (CAFE) Fines Collected, 1983-2014......4-26

Table 4.25 The Gas Guzzler Tax on New Cars .........................................................4-27

Table 4.26 List of Model Year 2016 Cars with Gas Guzzler Taxes ..............................4-28

Table 4.27 Tax Receipts from the Sale of Gas Guzzlers, 1980-2015 ...........................4-30

Table 4.28 Fuel Economy by Speed, Autonomie Model Results .................................4-31

Table 4.29 Fuel Economy by Speed, 1973, 1984, 1997, and 2012 Studies....................4-32 
Figure 4.2 Fuel Economy by Speed, 1973, 1984, 1997, and 2012 Studies and Autonomie Model Results $.4-33$

Table 4.30 Driving Cycle Attributes. $.4-34$

Figure 4.3 City Driving Cycle $.4-35$

Figure 4.4 Highway Driving Cycle. $.4-35$

Figure 4.5 Air Conditioning (SCO3) Driving Cycle $4-36$

Figure 4.6 Cold Temperature (Cold FTP) Driving Cycle. $.4-36$

Figure 4.7 High Speed (US06) Driving Cycle. $.4-37$

Figure 4.8 New York City Driving Cycle. $.4-38$

Figure 4.9 Representative Number Five Driving Cycle $.4-38$

Table 4.31 Comparison of U.S., European, and Japanese Driving Cycles Attributes ......4-39

Table 4.32 Example of Differing Results Using the U.S., European, and Japanese Driving Cycles.....

\section{CHAPTER 5 HEAVY VEHICLES AND CHARACTERISTICS} $.5-1$

Table 5.1

Summary Statistics for Class 3-8 Single-Unit Trucks, 1970-2015 $.5-2$

Table 5.2 Summary Statistics for Class 7-8 Combination Trucks, 1970-2015. $.5-3$

Table 5.3 New Retail Truck Sales by Gross Vehicle Weight, 1970-2016 $.5-4$

Table 5.4 Truck Statistics by Gross Vehicle Weight Class, 2002 $.5-6$

Table 5.5 Truck Harmonic Mean Fuel Economy by Size Class, 1992, 1997, and 2002. $.5-6$

Table 5.6

Truck Statistics by Size, 2002 $.5-7$

Table 5.7 Percentage of Trucks by Size Ranked by Major Use, 2002 . $.5-8$

Table 5.8

Percentage of Trucks by Fleet Size and Primary Fueling Facility, 2002. $.5-9$

Table 5.9

Share of Trucks by Major Use and Primary Fueling Facility, 2002. $.5-10$

Figure 5.1

Distribution of Trucks over 26,000 lb by Vehicle-Miles Traveled. $.5-11$ 
Figure 5.2 Share of Heavy Trucks with Selected Electronic Features, 2002 $.5-12$

Table 5.10 Effect of Terrain on Class 8 Truck Fuel Economy $5-14$

Table 5.11 Fuel Economy for Class 8 Trucks as Function of Speed and

Tractor-Trailer Tire Combination $.5-15$

Figure 5.3 Class 8 Truck Fuel Economy as a Function of Speed and Tractor-Trailer Tire Combination and Percentage of Total Distance Traveled as a Function of Speed $.5-16$

Figure 5.4 Class 8 Truck Percent of Total Fuel Consumed as a Function of Speed and Tractor-Trailer Tire Combination. $.5-17$

Table 5.12 Class 8 Truck Weight by Component $.5-18$

Table 5.13 Gross Vehicle Weight vs. Empty Vehicle Weight..............................................5-19

Figure 5.5 Distribution of Class 8 Trucks by On-Road Vehicle Weight, 2008. $.5-20$

Table 5.14 Value of Goods Shipped in the United States: Comparison of the 1993, 1997, 2002, 2007, and 2012 Commodity Flow Surveys...... $.5-22$

Table 5.15 Tons of Freight in the United States: Comparison of the 1993, 1997, 2002, 2007, and 2012 Commodity Flow Surveys.

Table 5.16 Growth of Ton-Miles in the United States: Comparison of the 1993, 1997, 2002, 2007, and 2012 Commodity Flow Surveys.

Table 5.17 Average Miles per Shipment in the United States: Comparison of the 1993, 1997, 2002, 2007, and 2012 Commodity Flow Surveys. $.5-25$

\section{CHAPTER 6 ALTERNATIVE FUEL AND ADVANCED TECHNOLOGY} VEHICLES AND CHARACTERISTICS. $.6-1$

Table 6.1 Estimates of Alternative Fuel Highway Vehicles Made Available, 2004-2015. .6-3

Table 6.2 Hybrid and Plug-In Vehicle Sales, 1999-2016...............................................6-4

Table 6.3 Alternative Fuel Transit Vehicles, 2016 ...........................................................6-5

Table 6.4 E85 Flex-Fuel Vehicles Available by Manufacturer, Model Year 2017 ...........6-6

Table 6.5 B20, CNG, and LPG Vehicles Available by Manufacturer, Model Year 2017 $.6-7$ 
Table 6.6 Hybrid-Electric Vehicles Available by Manufacturer, Model Year 2017 6-8

Table 6.7 Electric-Drive Vehicles Available by Manufacturer, Model Year 2017 ..........6-9

Table 6.8 Number of Alternative Fuel Vehicle Models Available, 1991-2017 ............6-10

Table 6.9 Hybrid-Electric Medium/Heavy Trucks and Buses Available by Manufacturer, 2017 $.6-11$

Table 6.10 Electric-Drive Medium/Heavy Trucks and Buses Available by Manufacturer, 2017 $.6-12$

Table 6.11 Number of Alternative Refuel Sites by State and Fuel Type, 2017 $.6-13$

Table 6.12 Number of Alternative Refuel Stations, 1992-2016. $.6-14$

Figure 6.1

Clean Cities Coalitions $6-15$

Table 6.13 Properties of Conventional and Alternative Liquid Fuels $.6-16$

Table 6.14 Properties of Conventional and Alternative Gaseous Fuels $.6-17$

CHAPTER 7 FLEET VEHICLES AND CHARACTERISTICS. .7-1

Figure 7.1 Fleet Vehicles in Service as of January 1, 2016 7-2

Table 7.1 Fleet Vehicles in Service, 2006-2016 $.7-3$

Table 7.2 Average Length of Time Commercial Fleet Vehicles Are in Service, 2015 $.7-4$

Table 7.3 Average Annual Vehicle-Miles of Travel for Commercial Fleet Vehicles, 2015. $.7-4$

Figure 7.2 Average Miles per Domestic Federal Vehicle by Vehicle Type, 2015.............7-5

Table 7.4 Federal Government Vehicles, 2001-2015.................................................7-6

Table 7.5 Federal Fleet Vehicle Acquisitions by Fuel Type, FY 2002-2015 .................7-7

Table 7.6 Fuel Consumed by Federal Government Fleets, FY 2000-2015....................7-7

Table 7.7 Federal Government Vehicles by Agency, FY 2015 ...................................7-8

Table 7.8 Summary Statistics on Demand Response Vehicles, 1994-2014....................7-9

Table 7.9 Summary Statistics on Transit Buses and Trolleybuses, 1994-2015 ............7-10 
CHAPTER 8 HOUSEHOLD VEHICLES AND CHARACTERISTICS 8-1

Table 8.1 Population and Vehicle Profile, 1950-2015 $.8-2$

Table 8.2 Vehicles and Vehicle-Miles per Capita, 1950-2015 $.8-3$

Table 8.3 Household Vehicle Ownership, 1960-2015 Census..... $.8-4$

Table 8.4 Demographic Statistics from the 1969, 1977, 1983, 1990, 1995 NPTS and 2001, 2009 NHTS 8-5

Table $8.5 \quad$ Average Annual Vehicle-Miles, Vehicle Trips and Trip Length per Household 1969, 1977, 1983, 1990, 1995 NPTS and 2001, 2009 NHTS .......8-6

Table 8.6 Average Number of Vehicles and Vehicle Travel per Household, 1990 NPTS and 2001 and 2009 NHTS $8-7$

Table 8.7 Trip Statistics by Trip Purpose, 2001 and 2009 NHTS 8-8

Figure 8.1 Average Vehicle Occupancy by Vehicle Type, 1995 NPTS and 2009 NHTS.

Figure 8.2 Average Vehicle Occupancy by Trip Purpose, 1977 NPTS and 2009 NHTS. $.8-10$

Table 8.8 Average Annual Miles per Household Vehicle by Vehicle Age $.8-11$

Table 8.9 Self-Reported vs. Odometer Average Annual Miles, 1995 NPTS and 2001 NHTS $.8-12$

Figure 8.3 Share of Vehicle Trips by Trip Distance, 2009 NHTS. $.8-13$

Figure 8.4 Share of Vehicle Trips to Work by Trip Distance, 2009 NHTS $.8-13$

Table 8.10 Share of Vehicles by Annual Miles of Travel and Vehicle Age, 2009 NHTS $.8-14$

Table 8.11 Household Vehicle Trips, 2009 NHTS $.8-15$

Figure 8.5 Average Daily Miles Driven (per Driver), 2009 NHTS. $.8-15$

Table 8.12 Daily Vehicle Miles of Travel (per Vehicle) by Number of Vehicles in the Household, 2009 NHTS. $.8-16$

Table 8.13 Daily and Annual Vehicle Miles of Travel and Average Age for Each Vehicle in a Household, 2009 NHTS $.8-16$

Figure 8.6 Daily Vehicle Miles of Travel for Each Vehicle in a Household, 2009 NHTS. 
Figure 8.7 Annual Vehicle Miles of Travel for Each Vehicle in a Household, 2009 NHTS. $.8-17$

Table 8.14 Means of Transportation to Work, 1980, 1990, 2000 and 2015. $.8-18$

Table 8.15 Characteristics of U.S. Daily per Vehicle Driving vs. Dwelling Unit Type and Density $.8-19$

Table 8.16 Housing Unit Characteristics, 2015 $.8-19$

Table 8.17 Workers by Commute Time, 1990, 2000, and 2015. $.8-20$

Table 8.18 Bicycle Sales, 1981-2015 $.8-21$

Figure 8.8 Walk and Bike Trips by Trip Purpose, 2009 NHTS. $.8-22$

Table 8.19 Long-Distance Trip Characteristics, 2001 NHTS $.8-24$

CHAPTER 9 NONHIGHWAY MODES . $.9-1$

Table 9.1 Nonhighway Energy Use Shares, 1970-2015 . $.9-2$

Table 9.2 Summary Statistics for U.S. Domestic and International Certificated Route Air Carriers (Combined Totals), 1970-2016 $.9-3$

Table 9.3 Summary Statistics for General Aviation, 1970-2015. $.9-4$

Table 9.4 Tonnage Statistics for Domestic and International Waterborne Commerce, 1970-2015 $.9-5$

Table 9.5 Summary Statistics for Domestic Waterborne Commerce, 1970-2015 . $.9-6$

Table 9.6 Recreational Boat Energy Use, 1970-2015 $.9-7$

Table 9.7 Class I Railroad Freight Systems in the United States Ranked by Revenue Ton-Miles, 2015. $.9-8$

Table 9.8 Summary Statistics for Class I Freight Railroads, 1970-2015. $.9-9$

Table 9.9 Intermodal Rail Traffic, 1965-2015 $.9-10$ 
Table 9.10 Summary Statistics for the National Railroad Passenger Corporation (Amtrak), 1971-2015.............................................................................11

Table 9.11 Summary Statistics for Commuter Rail Operations, 1984-2015...................9-12

Table 9.12 Summary Statistics for Rail Transit Operations, 1970-2015 _......................9-13

CHAPTER 10 TRANSPORTATION AND THE ECONOMY ...........................................10-1

Figure 10.1 Transportation Services Index, January 1990-January 2017......................10-3

Table 10.1 Average Annual Expenditures of Households by Income, 2015...................10-4

Table 10.2 Annual Household Expenditures for Transportation, 1985-2015..................10-5

Table 10.3 Gasoline Prices for Selected Countries, 1990-2016..................................10-6

Table 10.4 Diesel Fuel Prices for Selected Countries, 1990-2016................................10-7

Figure 10.2 Gasoline Prices for Selected Countries, 1990 and 2016 ............................10-8

Figure 10.3 Diesel Prices for Selected Countries, 1990 and 2016 ...............................10-9

Table 10.5 Prices for a Barrel of Crude Oil and a Gallon of Gasoline, 1978-2016 .......10-10

Table 10.6 Retail Prices for Motor Fuel, 1978-2016 ...............................................10-11

Figure 10.4 Oil Price and Economic Growth, 1970-2016.........................................10-12

Figure 10.5 Costs of Oil Dependence to the U.S. Economy, 1970-2015 .......................10-13

Table 10.7 Refiner Sales Prices for Propane and No. 2 Diesel, 1978-2016..................10-14

Table 10.8 Refiner Sales Prices for Aviation Gasoline and Jet Fuel, 1978-2016 ..........10-15

Table $10.9 \quad$ Federal Excise Taxes on Motor Fuels, 2015..........................................10-16

Table $10.10 \quad$ Federal, State, and Local Alternative Fuel Incentives, 2017 ......................10-17

Table 10.11 Federal, State, and Local Advanced Technology Incentives, 2017 ..............10-18

Table 10.12 Average Price of a New Car, 1913-2016 ...............................................10-19

Table 10.13 Average Price of a New Car (Domestic and Import), 1970-2016...............10-20

Table 10.14 Car Operating Cost per Mile, 1985-2016...............................................10-21

Table 10.15 Fixed Car Operating Costs per Year, 1975-2016.....................................10-22 
Table 10.16 Personal Consumption Expenditures, 1970-2016 $.10-23$

Table 10.17 Consumer Price Indices, 1970-2016 $.10-23$

Table 10.18 Transportation-Related Employment, 2000 and 2016. $.10-24$

Table 10.19 U.S. Employment for Motor Vehicles and Motor Vehicle Parts Manufacturing, 1990-2016. $10-25$

CHAPTER 11 GREENHOUSE GAS EMISSIONS $.11-1$

Table 11.1 World Carbon Dioxide Emissions, 1990, 2005, and 2016 $.11-2$

Figure 11.1 World Carbon Dioxide Emissions, 1990-2016 $11-3$

Table 11.2 Numerical Estimates of Global Warming Potentials Compared with Carbon Dioxide. $.11-4$

Table 11.3 U.S. Emissions of Greenhouse Gases, Based on Global Warming Potential, 1990-2015 $11-5$

Table 11.4 Total U.S. Greenhouse Gas Emissions by End-Use Sector, 2015 $11-6$

Table 11.5 U.S. Carbon Emissions from Fossil Fuel Consumption by End-Use Sector, 1990-2015 $11-7$

Table 11.6 Transportation Sector Carbon Dioxide Emissions from Energy Consumption, 1973-2016. $11-8$

Table $11.7 \quad$ U.S. Carbon Emissions from Fossil Fuel Combustion in the Transportation End-Use Sector, 1990-2015 $.11-9$

Table 11.8 Transportation Carbon Dioxide Emissions by Mode, 1990-2015..................11-10

Figure 11.2 GREET Model .............................................................................11-11

Figure 11.3 GREET Model Feedstocks and Fuels ......................................................11-12

Figure 11.4 Well-to-Wheel Emissions for Various Fuels and Vehicle Technologies........11-13

Table $11.9 \quad$ Production-Weighted Annual Carbon Footprint of New Domestic and Import Cars, Model Years 1975-2016 $11-15$

Table 11.10 Production-Weighted Annual Carbon Footprint of New Domestic and Import Light Trucks, Model Years 1975-2016 $11-16$

Table 11.11 Average Annual Carbon Footprint of New Vehicles by Vehicle Classification, Model Years 1975 and 2016 $.11-17$ 
Table 11.12 Direct Carbon Dioxide Emissions from a Gallon of Fuel $.11-18$

Table 11.13 Carbon Content of Transportation Fuels $.11-19$

CHAPTER 12 CRITERIA AIR POLLUTANTS $.12-1$

Table 12.1 Total National Emissions of the Criteria Air Pollutants by Sector, 2016.......12-2

Table 12.2 Total National Emissions of Carbon Monoxide, 1970-2016..... $12-3$

Table 12.3 Emissions of Carbon Monoxide from Highway Vehicles, 1970-2014 ..........12-4

Table 12.4 Total National Emissions of Nitrogen Oxides, 1970-2016 .........................12-5

Table 12.5 Emissions of Nitrogen Oxides from Highway Vehicles, 1970-2014............12-6

Table 12.6 Total National Emissions of Volatile Organic Compounds, 1970-2016 .......12-7

Table 12.7 Emissions of Volatile Organic Compounds from Highway Vehicles, 1970-2014. $.12-8$

Table 12.8 Total National Emissions of Particulate Matter (PM-10), 1970-2016 $.12-9$

Table 12.9 Emissions of Particulate Matter (PM-10) from Highway Vehicles, 1970-2014. $12-10$

Table 12.10 Total National Emissions of Particulate Matter (PM-2.5), 1990-2016 $.12-11$

Table 12.11 Emissions of Particulate Matter (PM-2.5) from Highway Vehicles, 1990-2014. $12-12$

Table 12.12 Total National Emissions of Sulfur Dioxide, 1970-2016. $12-13$

Table 12.13 Tier 3 Non-Methane Organic Gases and Nitrogen Oxide Standards. $.12-15$

Table 12.14 Tier 3 Particulate Matter Emission Standards for Light Gasoline Vehicles, MY 2017 and Beyond. $12-16$

Table 12.15 Tier 3 Evaporative Emission Standards $.12-16$

Table 12.16 Light-Duty Vehicle, Light-Duty Truck, and Medium-Duty Passenger Vehicle - Tier 2 Exhaust Emission Standards. $12-17$

Table 12.17 Light-Duty Vehicle, Light-Duty Truck, and Medium-Duty Passenger Vehicle - Tier 2 Evaporative Emission Standards $.12-18$ 
Table 12.18 Heavy-Duty Highway Compression-Ignition Engines and Urban Buses Exhaust Emission Standards

Table 12.19 Heavy-Duty Highway Spark-Ignition Engines - Exhaust Emission Standards.

Table 12.20 Heavy-Duty Highway Compression-Ignition and Spark-Ignition Engines Evaporative Emission Standards.

Table 12.21 California New Car, Light Truck and Medium Truck Emission

Certification Standards, Model Year 2015-On

Table 12.22 Aircraft - Exhaust Emission Standards $12-25$

Table 12.23 Nonroad Compression-Ignition Engines - Exhaust Emission Standards .....12-26

Table 12.24 Nonroad Large Spark-Ignition Engines - Exhaust and Evaporative Emission Standards.

Table 12.25 Locomotives - Exhaust Emission Standards

Table 12.26 Marine Compression-Ignition (CI) Engines - Exhaust Emission Standards.

Table 12.27 Marine Spark-Ignition Engines and Vessels - Exhaust Emission Standards $12-35$

Table 12.28 Nonroad Recreational Engines and Vehicles - Exhaust Emission Standards $12-37$

Table 12.29 Gasoline Sulfur Standards. $.12-39$

Table 12.30 Highway, Nonroad, Locomotive, and Marine (NRLM) Diesel Fuel Sulfur Standards

APPENDIX A. SOURCES \& METHODOLOGIES .........................................................

APPENDIX B. CONVERSIONS.........................................................................................

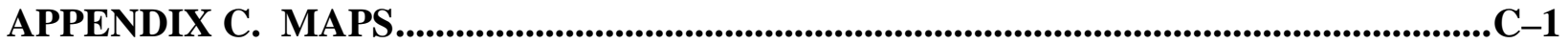

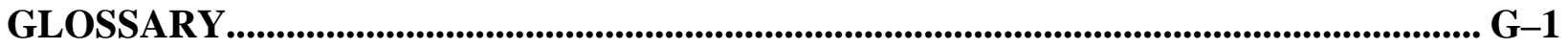




\section{FOREWORD}

Thank you for reading the 36th edition of the Transportation Energy Data Book. We hope you find the information you are looking for on transportation and energy. Beginning with this edition, the Data Book will only be posted on-line in both PDF and spreadsheet format at the website cta.ornl.gov/data. Please utilize the flexibility and convenience of PDF viewing, downloading, and searching. We will continue to update the Data Book as new source data are made available throughout the year, instead of waiting for a once-a-year update. We thank you for your support and flexibility as we make this transition and please do not hesitate to reach out if you have any questions or concerns. I have put together a few highlights of this year's Data Book:

- Before getting too far into the Transportation Energy Data Book check out Table 1.6 and Figure 1.5 which show some interesting trends on net imports of oil consumed for transportation.

- Energy use by transportation source can be found in Table 2.3 and broken down by consumption and Btu in Table 2.7 and Table 2.8, respectively. These tables and corresponding Figure 2.6, show how energy is being used across the transportation sector.

- You do not want to miss Table 2.15 on transportation energy consumption per mile; with our changing transportation environment, these data can inform the energy implications of our behavior and choices.

- Once you have gotten through the first couple chapters of petroleum consumption and energy use, there is still plenty of information on vehicles (light duty and heavy duty), alternative fuels, fleets, households, non-highway, the economy, and emissions.

- Note one change in the Data Book in this edition is the conversion factor for converting electricity usage into British thermal units (Btu). For this edition, only end-use energy was counted for electricity, and one kilowatt-hour (kWhr) of electricity is equal to 3,412 Btu. This change affected data in the energy-by-mode series going back to 1970 . The rail and pipeline modes were most affected because they use a greater share of electricity than other transportation modes.

- Check out some highlights of the GREET model (Figure 11.3 and 11.4), and data that helps inform household transportation needs in terms of economics of operating and purchasing vehicles (Table 10.10-10.13), mileage (Figure 8.5 and Table 8.14), and more.

Every figure and table compiled here is thanks to the hard work and diligence of our staff at Oak Ridge National Laboratory, specifically, Stacy Davis, Susan Williams, and Bob Boundy. We hope you enjoy this edition of the Transportation Energy Data Book. If you have any questions or suggestions for future improvements, please let us know.

Rachael Nealer

Vehicle Technologies Office

Office of Energy Efficiency and Renewable Energy

U.S. Department of Energy 


\section{ACKNOWLEDGMENTS}

The authors would like to express their gratitude to the many individuals who assisted in the preparation of this document. First, we would like to thank Rachael Nealer, Jacob Ward, David Gohlke, Kelly Fleming, and the Vehicle Technologies Office staff for their continued support of the Transportation Energy Data Book project. We would also like to thank Mark Robbins for the cover design. We are indebted to Debbie Bain, who has masterfully prepared the manuscript since 1998.

This book would not be possible without the leadership, guidance, and vision of Phil Patterson, who began this book in the 1970's. We hope to continue this report into the future with the same level of excellence. The authors and the transportation research community will be forever grateful for his efforts. 
xxii

TRANSPORTATION ENERGY DATA BOOK: EDITION 36-2017 


\begin{abstract}
The Transportation Energy Data Book: Edition 36 is a statistical compendium prepared and published by Oak Ridge National Laboratory (ORNL) under contract with the U.S. Department of Energy, Office of Energy Efficiency and Renewable Energy, Vehicle Technologies Office. Designed for use as a desk-top reference, the Data Book represents an assembly and display of statistics and information that characterize transportation activity, and presents data on other factors that influence transportation energy use. The purpose of this document is to present relevant statistical data in the form of tables and graphs. The latest edition of the Data Book is available via the Internet (cta.ornl.gov/data).

This edition of the Data Book has 12 chapters which focus on various aspects of the transportation industry. Chapter 1 focuses on petroleum; Chapter 2 - energy; Chapter 3 - highway vehicles; Chapter 4 - light vehicles; Chapter 5 - heavy vehicles; Chapter 6 - alternative fuel vehicles; Chapter 7 - fleet vehicles; Chapter 8 - household vehicles; Chapter 9 - nonhighway modes; Chapter 10 - transportation and the economy; Chapter 11 - greenhouse gas emissions; and Chapter 12 - criteria pollutant emissions. The sources used represent the latest available data. There are also three appendices which include detailed source information for some tables, measures of conversion, and the definition of Census divisions and regions. A glossary of terms is also included for the reader's convenience.
\end{abstract}


xxiv

TRANSPORTATION ENERGY DATA BOOK: EDITION 36-2017 


\section{INTRODUCTION}

In January 1976, the Transportation Energy Conservation (TEC) Division of the Energy Research and Development Administration contracted with Oak Ridge National Laboratory (ORNL) to prepare a Transportation Energy Conservation Data Book to be used by TEC staff in their evaluation of current and proposed conservation strategies. The major purposes of the Data Book were to draw together, under one cover, transportation data from diverse sources, to resolve data conflicts and inconsistencies, and to produce a comprehensive document. The first edition of the TEC Data Book was published in October 1976. With the passage of the Department of Energy (DOE) Organization Act, the work being conducted by the former Transportation Energy Conservation Division fell under the purview of the DOE's Office of Transportation Programs. This work continues today in the Vehicle Technologies Office.

Policymakers and analysts need to be well-informed about activity in the transportation sector. The organization and scope of the data book reflect the need for different kinds of information. For this reason, Edition 36 updates much of the same type of data that is found in previous editions.

In any attempt to compile a comprehensive set of statistics on transportation activity, numerous instances of inadequacies and inaccuracies in the basic data are encountered. Where such problems occur, estimates are developed by ORNL. To minimize the misuse of these statistics, an appendix (Appendix A) is included to document the estimation procedures. The attempt is to provide sufficient information for the conscientious user to evaluate the estimates and to form their own opinions as to their utility. Clearly, the accuracy of the estimates cannot exceed the accuracy of the primary data, an accuracy which in most instances is unknown. In cases where data accuracy is known or substantial errors are strongly suspected in the data, the reader is alerted. In all cases it should be recognized that the estimates are not precise.

The majority of the statistics contained in the data book are taken directly from published sources, although these data may be reformatted for presentation by ORNL. Consequently, neither ORNL nor DOE endorses the validity of these data. 
xxvi

TRANSPORTATION ENERGY DATA BOOK: EDITION 36-2017 


\section{Chapter 1 \\ Petroleum}

Summary Statistics from Tables/Figures in this Chapter

\section{Source}

Table 1.3 World Petroleum Production, 2016 (million barrels per day)

U.S. Production (million barrels per day)

U.S. Share

Table 1.4 World Petroleum Consumption, 2015 (million barrels per day)

U.S. Consumption (million barrels per day)

U.S. Share

$20.5 \%$

Figure 1.4 Average Refinery Yield, 2016

OECD $^{\mathbf{b}} \quad$ OECD $^{\mathbf{b}}$

Gasoline
Diesel oil
Residual fuel
Kerosene
Other
U.S. transportation petroleum use as a percent of U.S. petroleum
roduction, 2016

Europe

Americas

$20.1 \% \quad 41.5 \%$

$29.9 \% \quad 28.7 \%$

$9.2 \% \quad 4.2 \%$

$15.2 \% \quad 8.6 \%$

$10.3 \% \quad 14.2 \%$

Table 1.12 U.S. transportation petroleum use as a percent of U.S. petroleum

$110.9 \%$

Table 1.12 Net imports as a percentage of U.S. petroleum consumption, 2016

$24.8 \%$

Table 1.13 Transportation share of U.S. petroleum consumption, 2016

$69.8 \%$

Table 1.16 Highway share of transportation petroleum consumption, 2015

$86.2 \%$

Table 1.16 Light vehicle share of transportation petroleum consumption, 2015

In this document, petroleum is defined as crude oil (including lease condensate) and natural gas plant liquids.

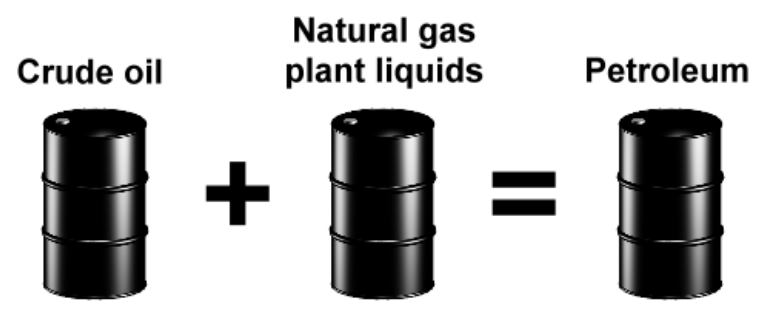

\footnotetext{
${ }^{a}$ Organization for Economic Co-operation and Development.
} 
As new technologies appear and new areas are explored, the amount of proved reserves of crude oil and natural gas has grown. Although the reserves of natural gas in the United States were 84\% higher in 2015 than it was in 1980, the U.S. share of World natural gas reserves is lower.

Table 1.1

Proved Reserves of Crude Oil and Natural Gas, 1980-2015

\begin{tabular}{|c|c|c|c|c|c|c|}
\hline \multirow[b]{2}{*}{ Year } & \multicolumn{2}{|c|}{$\begin{array}{l}\text { Crude Oil Reserves } \\
\text { (billion barrels) }\end{array}$} & \multirow{2}{*}{$\begin{array}{l}\text { U.S. Share of } \\
\text { Crude Oil } \\
\text { Reserves }\end{array}$} & \multicolumn{2}{|c|}{$\begin{array}{l}\text { Natural Gas Reserves } \\
\text { (trillion cubic feet) }\end{array}$} & \multirow{2}{*}{$\begin{array}{l}\text { U.S. Share of } \\
\text { Natural Gas } \\
\text { Reserves }\end{array}$} \\
\hline & World & United States & & World & United States & \\
\hline 1980 & 643.3 & 31.2 & $4.9 \%$ & $2,592.0$ & 201.0 & $7.8 \%$ \\
\hline 1981 & 650.7 & 31.3 & $4.8 \%$ & $2,653.9$ & 199.0 & $7.5 \%$ \\
\hline 1982 & 669.0 & 31.0 & $4.6 \%$ & $2,927.0$ & 201.7 & $6.9 \%$ \\
\hline 1983 & 667.2 & 29.5 & $4.4 \%$ & $3,038.4$ & 201.5 & $6.6 \%$ \\
\hline 1984 & 668.1 & 29.3 & $4.4 \%$ & $3,208.5$ & 200.2 & $6.2 \%$ \\
\hline 1985 & 699.3 & 30.0 & $4.3 \%$ & $3,407.2$ & 197.5 & $5.8 \%$ \\
\hline 1986 & 700.1 & 29.9 & $4.3 \%$ & $3,490.1$ & 193.4 & $5.5 \%$ \\
\hline 1987 & 699.4 & 28.3 & $4.1 \%$ & $3,648.7$ & 191.6 & $5.3 \%$ \\
\hline 1988 & 888.9 & 28.7 & $3.2 \%$ & 3,796.6 & 187.2 & $4.9 \%$ \\
\hline 1989 & 907.4 & 28.2 & $3.1 \%$ & $3,933.2$ & 168.0 & $4.3 \%$ \\
\hline 1990 & $1,001.9$ & 27.9 & $2.8 \%$ & $3,987.5$ & 167.1 & $4.2 \%$ \\
\hline 1991 & $1,000.0$ & 27.6 & $2.8 \%$ & $4,215.6$ & 169.3 & $4.0 \%$ \\
\hline 1992 & 990.7 & 25.9 & $2.6 \%$ & $4,376.7$ & 167.1 & $3.8 \%$ \\
\hline 1993 & 997.3 & 25.0 & $2.5 \%$ & $4,884.4$ & 165.0 & $3.4 \%$ \\
\hline 1994 & 999.5 & 24.1 & $2.4 \%$ & $5,013.8$ & 162.4 & $3.2 \%$ \\
\hline 1995 & $1,000.4$ & 23.6 & $2.4 \%$ & $4,981.9$ & 163.8 & $3.3 \%$ \\
\hline 1996 & $1,008.6$ & 23.5 & $2.3 \%$ & $4,935.3$ & 165.1 & $3.3 \%$ \\
\hline 1997 & $1,019.8$ & 23.3 & $2.3 \%$ & $4,947.0$ & 166.5 & $3.4 \%$ \\
\hline 1998 & $1,021.4$ & 23.9 & $2.3 \%$ & $5,087.6$ & 167.2 & $3.3 \%$ \\
\hline 1999 & $1,034.1$ & 22.4 & $2.2 \%$ & $5,141.9$ & 164.0 & $3.2 \%$ \\
\hline 2000 & $1,018.2$ & 23.2 & $2.3 \%$ & $5,150.0$ & 167.4 & $3.3 \%$ \\
\hline 2001 & 1,029.6 & 23.5 & $2.3 \%$ & $5,288.9$ & 177.4 & $3.4 \%$ \\
\hline 2002 & $1,033.4$ & 23.8 & $2.3 \%$ & $5,457.6$ & 183.5 & $3.4 \%$ \\
\hline 2003 & $1,214.5$ & 24.0 & $2.0 \%$ & $5,505.4$ & 186.9 & $3.4 \%$ \\
\hline 2004 & $1,266.2$ & 23.1 & $1.8 \%$ & $6,079.1$ & 189.0 & $3.1 \%$ \\
\hline 2005 & $1,278.5$ & 22.6 & $1.8 \%$ & $6,046.6$ & 192.5 & $3.2 \%$ \\
\hline 2006 & $1,289.2$ & 23.0 & $1.8 \%$ & $6,124.6$ & 204.4 & $3.3 \%$ \\
\hline 2007 & $1,319.9$ & 22.3 & $1.7 \%$ & $6,190.9$ & 211.1 & $3.4 \%$ \\
\hline 2008 & $1,328.5$ & 22.8 & $1.7 \%$ & $6,213.7$ & 237.7 & $3.8 \%$ \\
\hline 2009 & $1,336.4$ & 20.6 & $1.5 \%$ & $6,262.4$ & 244.7 & $3.9 \%$ \\
\hline 2010 & $1,356.7$ & 23.3 & $1.7 \%$ & $6,638.2$ & 272.5 & $4.1 \%$ \\
\hline 2011 & $1,475.0$ & 26.5 & $1.8 \%$ & $6,708.2$ & 304.6 & $4.5 \%$ \\
\hline 2012 & $1,523.4$ & 30.5 & $2.0 \%$ & $6,809.3$ & 334.1 & $4.9 \%$ \\
\hline 2013 & $1,643.9$ & 33.4 & $2.0 \%$ & $6,845.2$ & 308.0 & $4.5 \%$ \\
\hline 2014 & $1,650.6$ & 36.4 & $2.2 \%$ & $6,972.5$ & 338.3 & $4.9 \%$ \\
\hline 2015 & $1,658.0$ & 32.3 & $1.9 \%$ & $6,951.0$ & 369.0 & $5.3 \%$ \\
\hline \multicolumn{7}{|c|}{ Average Annual Percentage Change } \\
\hline 1970-2015 & $2.7 \%$ & $0.1 \%$ & & $2.9 \%$ & $1.8 \%$ & \\
\hline 2005-2015 & $2.6 \%$ & $4.9 \%$ & & $1.4 \%$ & $6.7 \%$ & \\
\hline
\end{tabular}

\section{Source:}

U.S. Department of Energy, Energy Information Administration, International Energy Statistics, August 2017.

(Additional resources: www.eia.doe.gov) 
In 2016, the Organization of Petroleum Exporting Countries (OPEC) accounted for 43.4\% of world oil production. World crude oil production reached an all-time high in 2016, while U.S. production was down slightly from 2015.

Table 1.2

World Crude Oil Production, 1960-2016 (million barrels per day)

\begin{tabular}{|c|c|c|c|c|c|c|}
\hline Year & $\begin{array}{l}\text { United } \\
\text { States }\end{array}$ & U.S. share & Total OPEC & OPEC share & $\begin{array}{c}\text { Total non- } \\
\text { OPEC }\end{array}$ & World \\
\hline 1960 & 7.04 & $33.5 \%$ & 8.70 & $41.4 \%$ & 12.29 & 20.99 \\
\hline 1965 & 7.80 & $25.7 \%$ & 14.35 & $47.3 \%$ & 15.98 & 30.33 \\
\hline 1970 & 9.64 & $21.0 \%$ & 23.30 & $50.8 \%$ & 22.59 & 45.89 \\
\hline 1975 & 8.38 & $15.9 \%$ & 26.01 & $49.2 \%$ & 26.82 & 52.83 \\
\hline 1980 & 8.60 & $14.4 \%$ & 25.56 & $42.9 \%$ & 34.00 & 59.56 \\
\hline 1985 & 8.97 & $16.6 \%$ & 15.54 & $28.8 \%$ & 38.43 & 53.97 \\
\hline 1986 & 8.68 & $15.4 \%$ & 17.75 & $31.5 \%$ & 38.57 & 56.33 \\
\hline 1987 & 8.35 & $14.7 \%$ & 17.89 & $31.6 \%$ & 38.76 & 56.65 \\
\hline 1988 & 8.14 & $13.9 \%$ & 19.90 & $33.9 \%$ & 38.80 & 58.70 \\
\hline 1989 & 7.61 & $12.7 \%$ & 21.61 & $36.1 \%$ & 38.19 & 59.80 \\
\hline 1990 & 7.36 & $12.2 \%$ & 22.77 & $37.6 \%$ & 37.73 & 60.50 \\
\hline 1991 & 7.42 & $12.3 \%$ & 22.72 & $37.8 \%$ & 37.41 & 60.13 \\
\hline 1992 & 7.17 & $11.9 \%$ & 24.03 & $40.0 \%$ & 36.08 & 60.10 \\
\hline 1993 & 6.85 & $11.4 \%$ & 24.78 & $41.2 \%$ & 35.40 & 60.17 \\
\hline 1994 & 6.66 & $10.9 \%$ & 25.20 & $41.2 \%$ & 35.97 & 61.17 \\
\hline 1995 & 6.56 & $10.5 \%$ & 25.86 & $41.4 \%$ & 36.57 & 62.43 \\
\hline 1996 & 6.46 & $10.1 \%$ & 26.37 & $41.3 \%$ & 37.45 & 63.82 \\
\hline 1997 & 6.45 & $9.8 \%$ & 27.64 & $42.0 \%$ & 38.16 & 65.81 \\
\hline 1998 & 6.25 & $9.3 \%$ & 28.70 & $42.8 \%$ & 38.33 & 67.03 \\
\hline 1999 & 5.88 & $8.9 \%$ & 27.53 & $41.7 \%$ & 38.44 & 65.97 \\
\hline 2000 & 5.82 & $8.5 \%$ & 29.26 & $42.7 \%$ & 39.27 & 68.53 \\
\hline 2001 & 5.80 & $8.5 \%$ & 28.40 & $41.7 \%$ & 39.73 & 68.13 \\
\hline 2002 & 5.74 & $8.5 \%$ & 26.72 & $39.7 \%$ & 40.57 & 67.29 \\
\hline 2003 & 5.65 & $8.1 \%$ & 28.22 & $40.6 \%$ & 41.24 & 69.46 \\
\hline 2004 & 5.44 & $7.5 \%$ & 30.67 & $42.2 \%$ & 41.92 & 72.60 \\
\hline 2005 & 5.18 & $7.0 \%$ & 32.16 & $43.5 \%$ & 41.70 & 73.87 \\
\hline 2006 & 5.09 & $6.9 \%$ & 31.84 & $43.3 \%$ & 41.63 & 73.48 \\
\hline 2007 & 5.08 & $6.9 \%$ & 31.60 & $43.2 \%$ & 41.58 & 73.18 \\
\hline 2008 & 5.00 & $6.8 \%$ & 32.97 & $44.5 \%$ & 41.08 & 74.05 \\
\hline 2009 & 5.35 & $7.3 \%$ & 31.29 & $42.9 \%$ & 41.58 & 72.87 \\
\hline 2010 & 5.48 & $7.4 \%$ & 32.20 & $43.2 \%$ & 42.34 & 74.54 \\
\hline 2011 & 5.65 & $7.6 \%$ & 32.39 & $43.4 \%$ & 42.23 & 74.62 \\
\hline 2012 & 6.49 & $8.5 \%$ & 33.57 & $44.2 \%$ & 42.46 & 76.03 \\
\hline 2013 & 7.47 & $9.8 \%$ & 32.62 & $42.8 \%$ & 43.56 & 76.18 \\
\hline 2014 & 8.76 & $11.2 \%$ & 32.68 & $41.9 \%$ & 45.38 & 78.06 \\
\hline 2015 & 9.42 & $11.7 \%$ & 33.94 & $42.2 \%$ & 46.50 & 80.44 \\
\hline 2016 & 8.88 & $11.0 \%$ & 34.94 & $43.4 \%$ & 45.63 & 80.57 \\
\hline & \multicolumn{6}{|c|}{ Average annual percentage change } \\
\hline 1960-2016 & $0.4 \%$ & & $2.5 \%$ & & $2.4 \%$ & $2.4 \%$ \\
\hline $1970-2016$ & $-0.2 \%$ & & $0.9 \%$ & & $1.5 \%$ & $1.2 \%$ \\
\hline 2006-2016 & $5.7 \%$ & & $0.9 \%$ & & $0.9 \%$ & $0.9 \%$ \\
\hline
\end{tabular}

\section{Source:}

U.S. Department of Energy, Energy Information Administration, International Energy Statistics website, August 2017. (Additional resources: www.eia.doe.gov)

a Includes lease condensate. Excludes natural gas plant liquids.

b See Glossary for membership. 
This table shows petroleum production, which includes both crude oil and natural gas plant liquids. Because other liquids and processing gain are not included, the world total is often smaller than world petroleum consumption (Table 1.4). The United States was responsible for 13.5\% of the world's petroleum production in 2016 and $11.0 \%$ of the world's crude oil production (Table 1.2).

Table 1.3

World Petroleum Production, 1973-2016 ${ }^{a}$

(million barrels per day)

\begin{tabular}{|c|c|c|c|c|c|c|c|}
\hline Year & $\begin{array}{l}\text { United } \\
\text { States }\end{array}$ & $\begin{array}{l}\text { U.S. } \\
\text { share }\end{array}$ & $\begin{array}{c}\text { Total } \\
\text { OPEC }^{\mathrm{b}}\end{array}$ & $\begin{array}{l}\text { OPEC } \\
\text { share }\end{array}$ & $\begin{array}{c}\text { Total } \\
\text { non- } \\
\text { OPEC }\end{array}$ & $\begin{array}{l}\text { Non- } \\
\text { OPEC } \\
\text { share }\end{array}$ & World \\
\hline 1973 & 10.95 & $18.7 \%$ & 29.99 & $51.3 \%$ & 28.48 & $48.7 \%$ & 58.47 \\
\hline 1975 & 10.01 & $18.0 \%$ & 26.16 & $47.0 \%$ & 28.48 & $51.2 \%$ & 55.62 \\
\hline 1980 & 10.17 & $16.1 \%$ & 26.05 & $41.3 \%$ & 35.77 & $56.8 \%$ & 63.00 \\
\hline 1985 & 10.58 & $18.3 \%$ & 16.20 & $28.0 \%$ & 40.90 & $70.6 \%$ & 57.90 \\
\hline 1990 & 8.91 & $13.7 \%$ & 23.71 & $36.4 \%$ & 40.80 & $62.6 \%$ & 65.14 \\
\hline 1991 & 9.08 & $14.0 \%$ & 23.65 & $36.4 \%$ & 40.53 & $62.4 \%$ & 64.95 \\
\hline 1992 & 8.87 & $13.7 \%$ & 25.02 & $38.5 \%$ & 39.37 & $60.6 \%$ & 64.95 \\
\hline 1993 & 8.58 & $13.2 \%$ & 25.83 & $39.6 \%$ & 38.82 & $59.5 \%$ & 65.23 \\
\hline 1994 & 8.39 & $12.6 \%$ & 26.52 & $39.8 \%$ & 39.21 & $58.9 \%$ & 66.55 \\
\hline 1995 & 8.32 & $12.2 \%$ & 27.19 & $40.0 \%$ & 40.21 & $59.1 \%$ & 68.01 \\
\hline 1996 & 8.29 & $11.9 \%$ & 27.70 & $39.8 \%$ & 41.26 & $59.3 \%$ & 69.52 \\
\hline 1997 & 8.27 & $11.5 \%$ & 29.05 & $40.5 \%$ & 42.05 & $58.7 \%$ & 71.65 \\
\hline 1998 & 8.01 & $11.0 \%$ & 30.21 & $41.4 \%$ & 42.33 & $58.0 \%$ & 73.04 \\
\hline 1999 & 7.73 & $10.7 \%$ & 29.13 & $40.4 \%$ & 43.02 & $59.6 \%$ & 72.15 \\
\hline 2000 & 7.73 & $10.3 \%$ & 32.78 & $43.8 \%$ & 42.12 & $56.2 \%$ & 74.90 \\
\hline 2001 & 7.67 & $10.3 \%$ & 32.05 & $42.8 \%$ & 42.78 & $57.2 \%$ & 74.83 \\
\hline 2002 & 7.62 & $10.3 \%$ & 30.37 & $41.0 \%$ & 43.73 & $59.0 \%$ & 74.10 \\
\hline 2003 & 7.37 & $9.6 \%$ & 31.91 & $41.7 \%$ & 44.60 & $58.3 \%$ & 76.52 \\
\hline 2004 & 7.25 & $9.0 \%$ & 34.75 & $43.4 \%$ & 45.38 & $56.6 \%$ & 80.12 \\
\hline 2005 & 6.90 & $8.4 \%$ & 36.50 & $44.7 \%$ & 45.23 & $55.3 \%$ & 81.73 \\
\hline 2006 & 6.82 & $8.4 \%$ & 36.16 & $44.3 \%$ & 45.44 & $55.7 \%$ & 81.59 \\
\hline 2007 & 6.86 & $8.4 \%$ & 35.98 & $44.1 \%$ & 45.51 & $55.9 \%$ & 81.49 \\
\hline 2008 & 6.78 & $8.2 \%$ & 37.53 & $45.5 \%$ & 44.97 & $54.5 \%$ & 82.50 \\
\hline 2009 & 7.26 & $8.9 \%$ & 35.84 & $44.0 \%$ & 45.62 & $56.0 \%$ & 81.46 \\
\hline 2010 & 7.55 & $9.0 \%$ & 36.85 & $44.1 \%$ & 46.65 & $55.9 \%$ & 83.49 \\
\hline 2011 & 7.86 & $9.4 \%$ & 37.03 & $44.2 \%$ & 46.76 & $55.8 \%$ & 83.79 \\
\hline 2012 & 8.89 & $10.4 \%$ & 38.32 & $44.8 \%$ & 47.27 & $55.2 \%$ & 85.59 \\
\hline 2013 & 10.07 & $11.7 \%$ & 37.23 & $43.4 \%$ & 48.61 & $56.6 \%$ & 85.84 \\
\hline 2014 & 11.78 & $13.4 \%$ & 37.20 & $42.2 \%$ & 50.98 & $57.8 \%$ & 88.18 \\
\hline 2015 & 12.76 & $14.0 \%$ & 38.55 & $42.4 \%$ & 52.47 & $57.6 \%$ & 91.01 \\
\hline 2016 & 12.35 & $13.5 \%$ & 39.62 & $43.4 \%$ & 51.70 & $56.6 \%$ & 91.32 \\
\hline & \multicolumn{7}{|c|}{ Average annual percentage change } \\
\hline 1973-2016 & $0.3 \%$ & & $0.6 \%$ & & $1.4 \%$ & & $1.0 \%$ \\
\hline $2006-2016$ & $6.1 \%$ & & $0.9 \%$ & & $1.3 \%$ & & $1.1 \%$ \\
\hline
\end{tabular}

Source:

U.S. Department of Energy, Energy Information Administration, International Energy Statistics website, August 2017. (Additional resources: www.eia.doe.gov)

${ }^{a}$ Includes natural gas plant liquids, crude oil and lease condensate. Does not account for all inputs or refinery processing gain.

${ }^{\mathrm{b}}$ Organization of Petroleum Exporting Countries. See Glossary for membership.

c Not comparable with previous data. Includes other inputs and refinery processing gains. 
During the 1980s and 1990s, the United States accounted for about one-quarter of the world's petroleum consumption, but from 2000 to 2012 that share had been decreasing. In 2015 the United States accounted for only 20.5\%. World petroleum consumption decreased in 2009 but has continued to increase beginning with 2010. NonOECD consumption has continued to increase.

Table 1.4

World Petroleum Consumption, 1960-2015 (million barrels per day)

\begin{tabular}{|c|c|c|c|c|c|}
\hline Year & United States & U.S. share & Total OECD ${ }^{\mathrm{a}}$ & Total non-OECD & World \\
\hline 1960 & 9.80 & $45.9 \%$ & 15.78 & 5.56 & 21.34 \\
\hline 1965 & 11.51 & $37.0 \%$ & 22.81 & 8.33 & 31.14 \\
\hline 1970 & 14.70 & $31.4 \%$ & 34.69 & 12.12 & 46.81 \\
\hline 1975 & 16.32 & $29.0 \%$ & 39.14 & 17.06 & 56.20 \\
\hline 1980 & 17.06 & $27.0 \%$ & 42.03 & 21.09 & 63.12 \\
\hline 1985 & 15.73 & $26.2 \%$ & 37.70 & 22.39 & 60.08 \\
\hline 1986 & 16.28 & $26.3 \%$ & 38.83 & 22.99 & 61.82 \\
\hline 1987 & 16.67 & $26.4 \%$ & 39.59 & 23.51 & 63.11 \\
\hline 1988 & 17.28 & $26.6 \%$ & 40.92 & 24.05 & 64.98 \\
\hline 1989 & 17.33 & $26.2 \%$ & 41.62 & 24.47 & 66.09 \\
\hline 1990 & 16.99 & $25.5 \%$ & 41.75 & 24.79 & 66.54 \\
\hline 1991 & 16.71 & $24.9 \%$ & 42.22 & 24.97 & 67.19 \\
\hline 1992 & 17.03 & $25.3 \%$ & 43.25 & 24.15 & 67.40 \\
\hline 1993 & 17.24 & $25.5 \%$ & 43.69 & 23.93 & 67.62 \\
\hline 1994 & 17.72 & $25.7 \%$ & 44.92 & 24.09 & 69.01 \\
\hline 1995 & 17.72 & $25.2 \%$ & 45.40 & 24.86 & 70.26 \\
\hline 1996 & 18.31 & $25.5 \%$ & 46.53 & 25.35 & 71.88 \\
\hline 1997 & 18.62 & $25.3 \%$ & 47.30 & 26.29 & 73.60 \\
\hline 1998 & 18.92 & $25.5 \%$ & 47.49 & 26.78 & 74.27 \\
\hline 1999 & 19.52 & $25.7 \%$ & 48.48 & 27.50 & 75.98 \\
\hline 2000 & 19.70 & $25.6 \%$ & 48.50 & 28.56 & 77.06 \\
\hline 2001 & 19.65 & $25.3 \%$ & 48.54 & 29.19 & 77.73 \\
\hline 2002 & 19.76 & $25.2 \%$ & 48.51 & 29.91 & 78.42 \\
\hline 2003 & 20.03 & $25.0 \%$ & 49.23 & 30.84 & 80.07 \\
\hline 2004 & 20.73 & $24.9 \%$ & 50.05 & 33.16 & 83.21 \\
\hline 2005 & 20.80 & $24.6 \%$ & 50.38 & 34.25 & 84.63 \\
\hline 2006 & 20.69 & $24.1 \%$ & 50.16 & 35.54 & 85.70 \\
\hline 2007 & 20.68 & $23.9 \%$ & 50.04 & 36.46 & 86.49 \\
\hline 2008 & 19.50 & $22.8 \%$ & 48.36 & 37.28 & 85.64 \\
\hline 2009 & 18.77 & $21.9 \%$ & 46.30 & 39.29 & 85.58 \\
\hline 2010 & 19.18 & $21.5 \%$ & 47.00 & 42.17 & 89.17 \\
\hline 2011 & 18.88 & $21.0 \%$ & 46.33 & 43.56 & 89.88 \\
\hline 2012 & 18.49 & $20.3 \%$ & 45.93 & 45.16 & 91.09 \\
\hline 2013 & 18.96 & $20.5 \%$ & 46.07 & 46.26 & 92.32 \\
\hline 2014 & 19.11 & $20.4 \%$ & 45.77 & 47.84 & 93.61 \\
\hline 2015 & 19.53 & $20.5 \%$ & 46.34 & 49.02 & 95.36 \\
\hline \multicolumn{6}{|c|}{ Average annual percentage change } \\
\hline 1960-2015 & $1.3 \%$ & & $2.0 \%$ & $4.0 \%$ & $2.8 \%$ \\
\hline 1970-2015 & $0.6 \%$ & & $0.6 \%$ & $3.2 \%$ & $1.6 \%$ \\
\hline 2005-2015 & $-0.6 \%$ & & $-0.8 \%$ & $3.3 \%$ & $1.1 \%$ \\
\hline
\end{tabular}

\section{Source:}

U.S. Department of Energy, Energy Information Administration, International Energy Statistics website, August 2017. (Additional resources: www.eia.doe.gov)

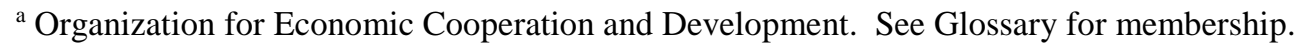


Figure 1.1. World Oil Reserves, Production, and Consumption, 1980

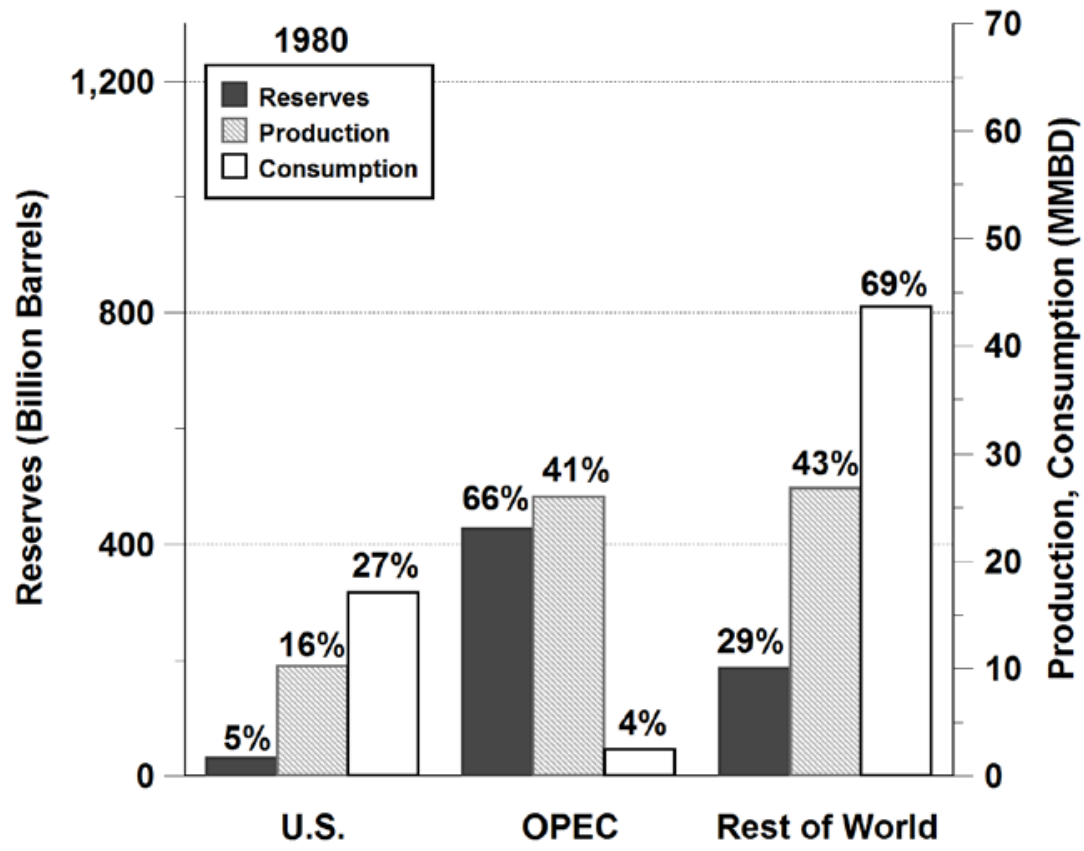

Source:

See Table 1.5.

Figure 1.2. World Oil Reserves, Production, and Consumption, 1993

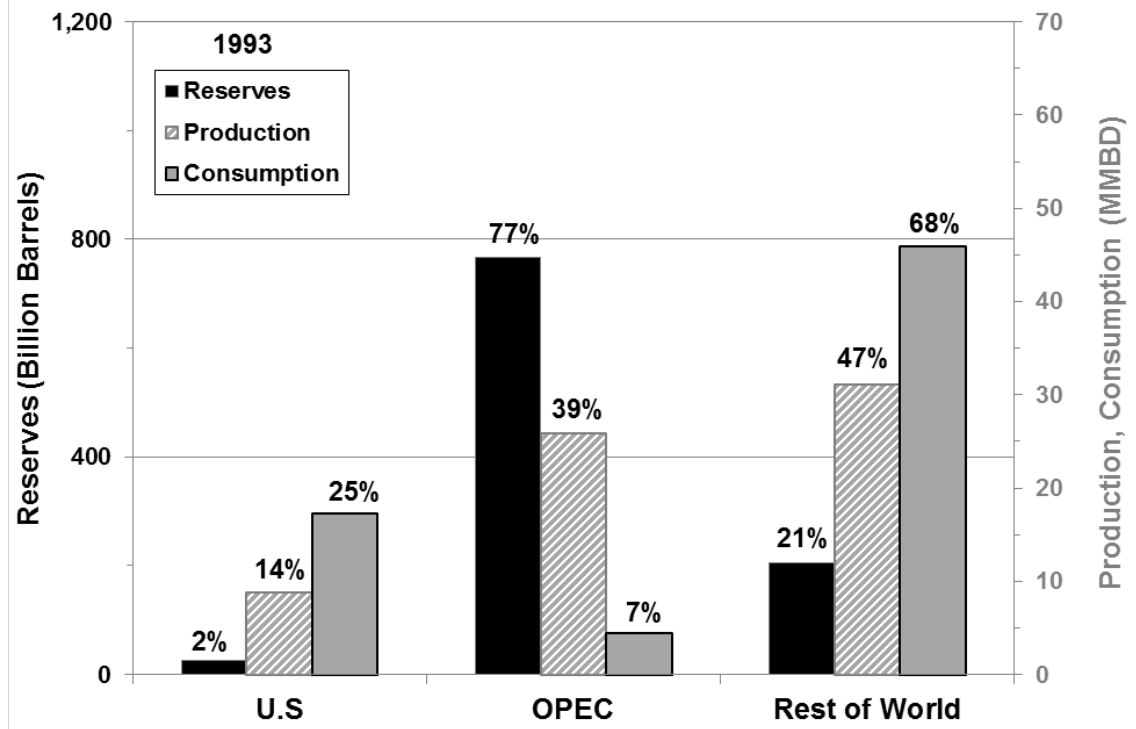

Source:

See Table 1.5. 
Figure 1.3. World Oil Reserves, Production, and Consumption, 2015

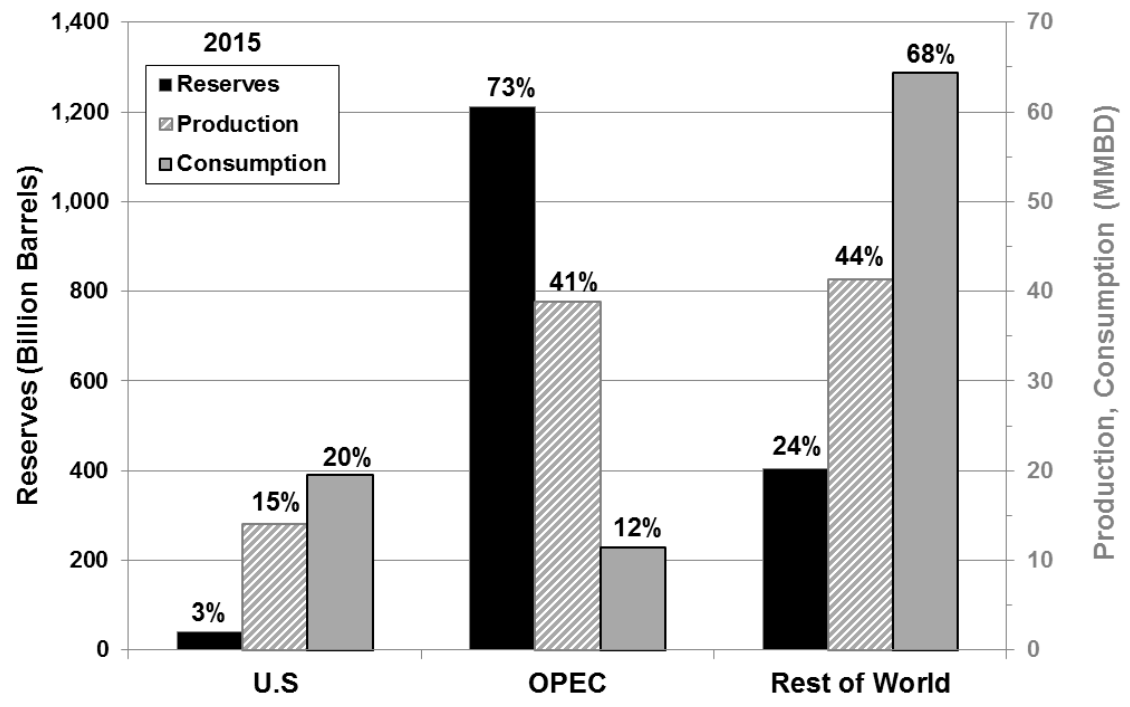

\section{Source:}

See Table 1.5.

Table 1.5

World Oil Reserves, Production, and Consumption, 1980, 1993 and 2015

\begin{tabular}{|c|c|c|c|c|c|c|}
\hline & $\begin{array}{l}\text { Crude oil } \\
\text { reserves } \\
\text { (billion } \\
\text { barrels) } \\
\end{array}$ & $\begin{array}{c}\text { Reserve } \\
\text { share }\end{array}$ & $\begin{array}{l}\text { Petroleum } \\
\text { production } \\
\text { (million } \\
\text { barrels per } \\
\text { day) } \\
\end{array}$ & $\begin{array}{c}\text { Production } \\
\text { share }\end{array}$ & $\begin{array}{c}\text { Petroleum } \\
\text { consumption } \\
\text { (million barrels } \\
\text { per day) }\end{array}$ & $\begin{array}{c}\text { Consumption } \\
\text { share }\end{array}$ \\
\hline & \multicolumn{6}{|c|}{1980} \\
\hline United States & 31.2 & $5 \%$ & 10.2 & $16 \%$ & 17.1 & $27 \%$ \\
\hline OPEC & 426.7 & $66 \%$ & 26.0 & $41 \%$ & 2.5 & $4 \%$ \\
\hline \multirow[t]{2}{*}{ Rest of world } & 185.4 & $29 \%$ & 26.8 & $43 \%$ & 43.6 & $69 \%$ \\
\hline & \multicolumn{6}{|c|}{1993} \\
\hline United States & 25.0 & $2 \%$ & 8.84 & $14 \%$ & 17.2 & $25 \%$ \\
\hline OPEC & 767.2 & $77 \%$ & 25.85 & $39 \%$ & 4.5 & $7 \%$ \\
\hline \multirow[t]{2}{*}{ Rest of world } & 205.2 & $21 \%$ & 31.18 & $47 \%$ & 45.9 & $68 \%$ \\
\hline & \multicolumn{6}{|c|}{2015} \\
\hline United States & 40.0 & $3 \%$ & 14.1 & $15 \%$ & 19.5 & $20 \%$ \\
\hline OPEC & $1,212.0$ & $73 \%$ & 38.8 & $41 \%$ & 11.5 & $12 \%$ \\
\hline Rest of world & 405.0 & $24 \%$ & 41.3 & $44 \%$ & 64.4 & $68 \%$ \\
\hline
\end{tabular}

Note: Total consumption is higher than total production due to refinery gains including alcohol and liquid products produced from coal and other sources. See Glossary for OPEC countries.

\section{Sources:}

Energy Information Administration, International Energy Statistics, August 2017. (Additional resources: www.eia.doe.gov) 
The share of petroleum imported to the United States can be calculated using total imports or net imports. Net imports, which are the preferred data, rose to over 50\% of U.S. petroleum consumption for the first time in 1998, while total imports reached $50 \%$ for the first time in 1993. OPEC share of net imports has been below $50 \%$ since 1993. Net imports as a share of consumption decreased to $24.1 \%$ in 2015 but rose slightly in 2016.

Table 1.6

U.S. Petroleum Imports, 1960-2016 (million barrels per day)

\begin{tabular}{|c|c|c|c|c|c|}
\hline Year & $\begin{array}{l}\text { Net OPEC } \\
\text { imports }\end{array}$ & $\begin{array}{l}\text { Net OPEC } \\
\text { share }\end{array}$ & Net imports & $\begin{array}{l}\text { Net imports as a share } \\
\text { of U.S. consumption }\end{array}$ & Total imports \\
\hline 1960 & 1.23 & $68.0 \%$ & 1.61 & $16.5 \%$ & 1.81 \\
\hline 1965 & 1.44 & $58.3 \%$ & 2.28 & $19.8 \%$ & 2.47 \\
\hline 1970 & 1.29 & $37.8 \%$ & 3.16 & $21.5 \%$ & 3.42 \\
\hline 1975 & 3.60 & $59.5 \%$ & 5.85 & $35.8 \%$ & 6.06 \\
\hline 1980 & 4.30 & $62.2 \%$ & 6.36 & $37.3 \%$ & 6.91 \\
\hline 1985 & 1.83 & $36.1 \%$ & 4.29 & $27.3 \%$ & 5.07 \\
\hline 1986 & 2.84 & $45.6 \%$ & 5.44 & $33.4 \%$ & 6.22 \\
\hline 1987 & 3.06 & $45.8 \%$ & 5.91 & $35.5 \%$ & 6.68 \\
\hline 1988 & 3.52 & $47.6 \%$ & 6.59 & $38.1 \%$ & 7.40 \\
\hline 1989 & 4.14 & $51.4 \%$ & 7.20 & $41.6 \%$ & 8.06 \\
\hline 1990 & 4.30 & $53.6 \%$ & 7.16 & $42.2 \%$ & 8.02 \\
\hline 1991 & 4.09 & $53.7 \%$ & 6.63 & $39.6 \%$ & 7.63 \\
\hline 1992 & 4.09 & $51.9 \%$ & 6.94 & $40.7 \%$ & 7.89 \\
\hline 1993 & 4.27 & $49.6 \%$ & 7.62 & $44.2 \%$ & 8.62 \\
\hline 1994 & 4.25 & $47.2 \%$ & 8.05 & $45.5 \%$ & 9.00 \\
\hline 1995 & 4.00 & $45.3 \%$ & 7.89 & $44.5 \%$ & 8.83 \\
\hline 1996 & 4.21 & $44.4 \%$ & 8.50 & $46.4 \%$ & 9.48 \\
\hline 1997 & 4.57 & $45.0 \%$ & 9.16 & $49.2 \%$ & 10.16 \\
\hline 1998 & 4.91 & $45.8 \%$ & 9.76 & $51.6 \%$ & 10.71 \\
\hline 1999 & 4.95 & $45.6 \%$ & 9.91 & $50.8 \%$ & 10.85 \\
\hline 2000 & 5.20 & $45.4 \%$ & 10.42 & $52.9 \%$ & 11.46 \\
\hline 2001 & 5.53 & $46.6 \%$ & 10.90 & $55.5 \%$ & 11.87 \\
\hline 2002 & 4.61 & $39.9 \%$ & 10.55 & $53.4 \%$ & 11.53 \\
\hline 2003 & 5.16 & $42.1 \%$ & 11.24 & $56.1 \%$ & 12.26 \\
\hline 2004 & 5.70 & $43.4 \%$ & 12.10 & $58.4 \%$ & 13.15 \\
\hline 2005 & 5.59 & $40.7 \%$ & 12.55 & $60.3 \%$ & 13.71 \\
\hline 2006 & 5.52 & $40.2 \%$ & 12.39 & $59.9 \%$ & 13.71 \\
\hline 2007 & 5.98 & $44.4 \%$ & 12.04 & $58.2 \%$ & 13.47 \\
\hline 2008 & 5.95 & $46.1 \%$ & 11.11 & $57.0 \%$ & 12.92 \\
\hline 2009 & 4.78 & $40.9 \%$ & 9.67 & $51.5 \%$ & 11.69 \\
\hline 2010 & 4.91 & $41.6 \%$ & 9.44 & $49.2 \%$ & 11.79 \\
\hline 2011 & 4.56 & $39.8 \%$ & 8.45 & $44.8 \%$ & 11.44 \\
\hline 2012 & 4.27 & $40.3 \%$ & 7.39 & $40.0 \%$ & 10.60 \\
\hline 2013 & 3.72 & $37.7 \%$ & 6.24 & $32.9 \%$ & 9.86 \\
\hline 2014 & 3.24 & $35.0 \%$ & 5.07 & $26.5 \%$ & 9.24 \\
\hline 2015 & 2.89 & $30.6 \%$ & 4.71 & $24.1 \%$ & 9.45 \\
\hline 2016 & 3.45 & $34.2 \%$ & 4.87 & $24.8 \%$ & 10.06 \\
\hline & \multicolumn{5}{|c|}{ Average annual percentage change } \\
\hline 1960-2016 & $1.9 \%$ & & $2.0 \%$ & & $3.1 \%$ \\
\hline $1970-2016$ & $2.2 \%$ & & $0.9 \%$ & & $2.4 \%$ \\
\hline 2006-2016 & $-4.6 \%$ & & $-8.9 \%$ & & $-3.0 \%$ \\
\hline
\end{tabular}

\section{Source:}

U.S. Department of Energy, Energy Information Administration, Monthly Energy Review, Washington, DC, August 2017, Table 3.3a. (Additional resources: www.eia.gov)

${ }^{\text {a }}$ Organization of Petroleum Exporting Countries. See Glossary for membership. 
More than half of the oil imported to the United States in 2016 was from the western hemisphere. Canada, Mexico, and Venezuela provided most of the oil from the western hemisphere, along with small amounts from Brazil, Columbia, Ecuador, and the U.S. Virgin Islands (these countries are not listed separately).

Table 1.7

Imported Crude Oil by Country of Origin, 1973-2016

(million barrels per day)

\begin{tabular}{|c|c|c|c|c|c|c|c|c|c|}
\hline Year & $\begin{array}{c}\text { Saudi } \\
\text { Arabia }\end{array}$ & Venezuela & Nigeria & $\begin{array}{c}\text { Other } \\
\text { OPEC }^{\mathrm{a}} \\
\text { countries }\end{array}$ & Canada & Mexico & Russia & $\begin{array}{c}\text { Other } \\
\text { non- } \\
\text { OPEC } \\
\text { countries } \\
\end{array}$ & $\begin{array}{c}\text { Total } \\
\text { imports }\end{array}$ \\
\hline 1973 & 0.49 & 1.13 & 0.46 & 0.91 & 1.32 & 0.02 & 0.03 & 1.90 & 6.26 \\
\hline 1975 & 0.71 & 0.70 & 0.76 & 1.42 & 0.85 & 0.07 & 0.01 & 1.52 & 6.06 \\
\hline 1980 & 1.26 & 0.48 & 0.86 & 1.70 & 0.45 & 0.53 & 0.00 & 1.62 & 6.91 \\
\hline 1985 & 0.17 & 0.60 & 0.29 & 0.76 & 0.77 & 0.82 & 0.01 & 1.64 & 5.07 \\
\hline 1986 & 0.68 & 0.79 & 0.44 & 0.92 & 0.81 & 0.70 & 0.02 & 1.86 & 6.22 \\
\hline 1987 & 0.75 & 0.80 & 0.53 & 0.97 & 0.85 & 0.65 & 0.01 & 2.10 & 6.68 \\
\hline 1988 & 1.07 & 0.79 & 0.62 & 1.03 & 1.00 & 0.75 & 0.03 & 2.11 & 7.40 \\
\hline 1989 & 1.22 & 0.87 & 0.82 & 1.23 & 0.93 & 0.77 & 0.05 & 2.17 & 8.06 \\
\hline 1990 & 1.34 & 1.02 & 0.80 & 1.13 & 0.93 & 0.76 & 0.04 & 1.99 & 8.02 \\
\hline 1991 & 1.80 & 1.03 & 0.70 & 0.55 & 1.03 & 0.81 & 0.03 & 1.67 & 7.63 \\
\hline 1992 & 1.72 & 1.17 & 0.68 & 0.52 & 1.07 & 0.83 & 0.02 & 1.88 & 7.89 \\
\hline 1993 & 1.41 & 1.30 & 0.74 & 0.82 & 1.18 & 0.92 & 0.05 & 2.19 & 8.62 \\
\hline 1994 & 1.40 & 1.33 & 0.64 & 0.87 & 1.27 & 0.98 & 0.03 & 2.46 & 9.00 \\
\hline 1995 & 1.34 & 1.48 & 0.63 & 0.55 & 1.33 & 1.07 & 0.02 & 2.41 & 8.83 \\
\hline 1996 & 1.36 & 1.68 & 0.62 & 0.56 & 1.42 & 1.24 & 0.03 & 2.57 & 9.48 \\
\hline 1997 & 1.41 & 1.77 & 0.70 & 0.69 & 1.56 & 1.39 & 0.01 & 2.63 & 10.16 \\
\hline 1998 & 1.49 & 1.72 & 0.70 & 1.00 & 1.60 & 1.35 & 0.02 & 2.83 & 10.71 \\
\hline 1999 & 1.48 & 1.49 & 0.66 & 1.33 & 1.54 & 1.32 & 0.09 & 2.95 & 10.85 \\
\hline 2000 & 1.57 & 1.55 & 0.90 & 1.19 & 1.81 & 1.37 & 0.07 & 3.00 & 11.46 \\
\hline 2001 & 1.66 & 1.55 & 0.89 & 1.43 & 1.83 & 1.44 & 0.09 & 2.98 & 11.87 \\
\hline 2002 & 1.55 & 1.40 & 0.62 & 1.03 & 1.97 & 1.55 & 0.21 & 3.20 & 11.53 \\
\hline 2003 & 1.77 & 1.38 & 0.87 & 1.14 & 2.07 & 1.62 & 0.25 & 3.15 & 12.26 \\
\hline 2004 & 1.56 & 1.55 & 1.14 & 1.45 & 2.14 & 1.66 & 0.30 & 3.34 & 13.15 \\
\hline 2005 & 1.54 & 1.53 & 1.17 & 1.36 & 2.18 & 1.66 & 0.41 & 3.87 & 13.71 \\
\hline 2006 & 1.46 & 1.42 & 1.11 & 1.52 & 2.35 & 1.71 & 0.37 & 3.76 & 13.71 \\
\hline 2007 & 1.48 & 1.36 & 1.13 & 2.00 & 2.45 & 1.53 & 0.41 & 3.09 & 13.47 \\
\hline 2008 & 1.53 & 1.19 & 0.99 & 2.25 & 2.49 & 1.30 & 0.47 & 2.70 & 12.92 \\
\hline 2009 & 1.00 & 1.06 & 0.81 & 1.90 & 2.48 & 1.21 & 0.56 & 2.66 & 11.69 \\
\hline 2010 & 1.10 & 0.99 & 1.02 & 1.80 & 2.54 & 1.28 & 0.61 & 2.46 & 11.79 \\
\hline 2011 & 1.19 & 0.95 & 0.82 & 1.59 & 2.73 & 1.21 & 0.62 & 2.32 & 11.44 \\
\hline 2012 & 1.37 & 0.96 & 0.44 & 1.51 & 2.95 & 1.03 & 0.48 & 1.87 & 10.60 \\
\hline 2013 & 1.33 & 0.81 & 0.28 & 1.30 & 3.14 & 0.92 & 0.46 & 1.62 & 9.86 \\
\hline 2014 & 1.17 & 0.79 & 0.09 & 1.19 & 3.39 & 0.84 & 0.33 & 1.44 & 9.24 \\
\hline 2015 & 1.06 & 0.83 & 0.08 & 0.93 & 3.76 & 0.76 & 0.37 & 1.66 & 9.45 \\
\hline 2016 & 1.10 & 0.80 & 0.24 & 1.31 & 3.80 & 0.67 & 0.43 & 1.71 & 10.06 \\
\hline
\end{tabular}

Sources:

U.S. Department of Energy, Energy Information Administration, Monthly Energy Review, Washington, DC, July 2017, Tables 3.3c and 3.3d. (Additional resources: www.eia.gov)

a Organization of Petroleum Exporting Countries. See Glossary for membership. 
The Strategic Petroleum Reserve (SPR) began in October 1977 as a result of the 1975 Energy Policy and Conservation Act. Its purpose is to provide protection against oil supply disruptions. The U.S. consumed 19.6 million barrels per day in 2016. At that rate of consumption, the SPR supply would last 35 days if used exclusively and continuously.

Table 1.8

Crude Oil Supplies, 1973-2016

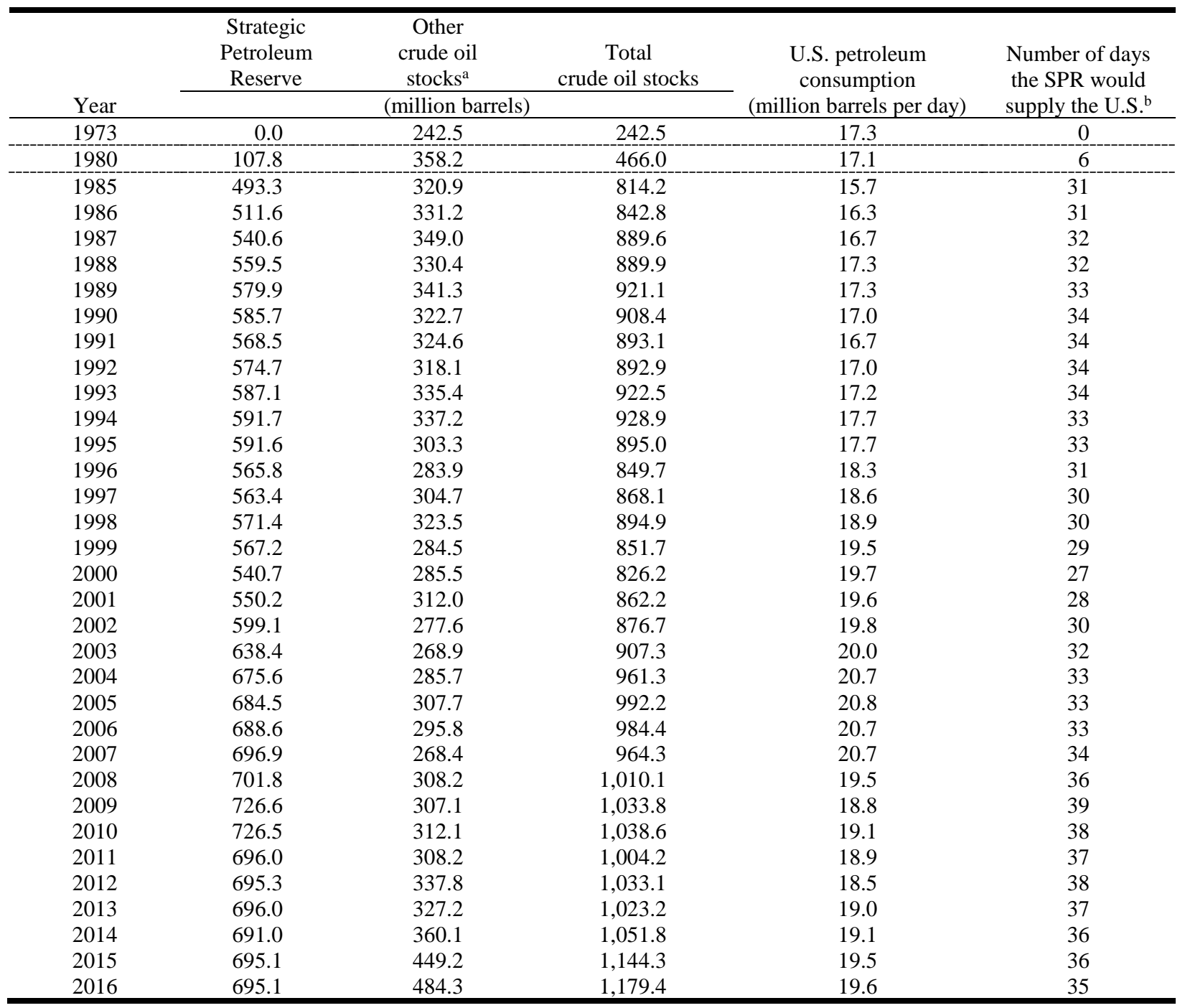

Sources:

U.S. Department of Energy, Energy Information Administration, Monthly Energy Review, Washington, DC, July 2017, Tables 3.1 and 3.4. (Additional resources: www.eia.gov) in transit.

a Other crude oil stocks include stocks held by petroleum companies, as well as stocks of Alaskan crude oil

${ }^{\mathrm{b}}$ Strategic Petroleum Reserves divided by U.S. consumption per day. This would only hold true if the SPR were the only oil used for that many days. 
Other parts of the world refine crude oil to produce more diesel fuel and less gasoline than does OECD Americas. The OECD Asia Oceania countries produce the lowest share of gasoline in 2016.

Figure 1.4. Refinery Gross Output by World Region, 2006 and 2016

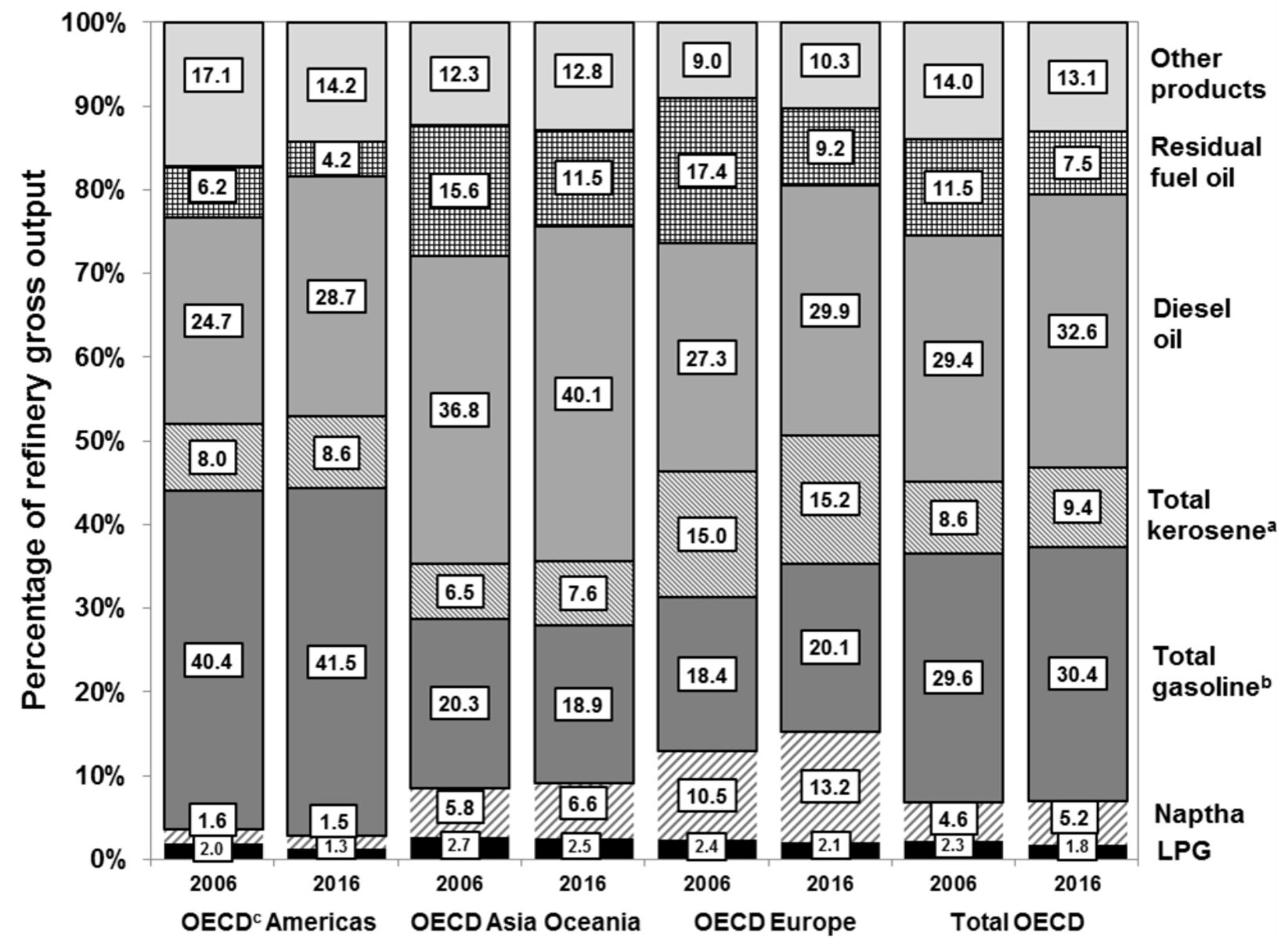

Source:

International Energy Agency, Monthly Oil Survey, May 2017 and Monthly Oil Statistics, February 2007. (Additional resources: www.iea.org)

${ }^{a}$ Includes jet kerosene and other kerosene.

${ }^{\mathrm{b}}$ Includes motor gasoline, jet gasoline, and aviation gasoline.

${ }^{\mathrm{c}}$ Organization for Economic Cooperation and Development. See Glossary for membership. 
Oxygenate refinery input increased significantly in 1995, most certainly due to the Clean Air Act Amendments of 1990 which mandated the sale of reformulated gasoline in certain areas beginning in January 1995. The use of MTBE has declined over the last ten years due to many states banning the additive. The other hydrocarbons and liquids category includes unfinished oils, motor gasoline blending components and aviation gasoline blending components.

Table 1.9

U.S. Refinery Input of Crude Oil and Petroleum Products, 1987-2015 (thousand barrels)

\begin{tabular}{|c|c|c|c|c|c|c|c|}
\hline \multirow[b]{2}{*}{ Year } & \multirow[b]{2}{*}{ Crude oil } & \multirow[b]{2}{*}{$\begin{array}{l}\text { Natural gas } \\
\text { liquids }\end{array}$} & \multicolumn{3}{|c|}{ Oxygenates } & \multirow{2}{*}{$\begin{array}{c}\text { Other } \\
\text { hydrocarbons } \\
\text { and liquids }\end{array}$} & \multirow[b]{2}{*}{$\begin{array}{l}\text { Total input to } \\
\text { refineries }\end{array}$} \\
\hline & & & $\begin{array}{c}\text { Fuel } \\
\text { ethanol }\end{array}$ & MTBE $^{a}$ & $\begin{array}{c}\text { Other } \\
\text { oxygenates }^{\mathrm{b}}\end{array}$ & & \\
\hline 1987 & $4,691,783$ & 280,889 & c & $\begin{array}{lc}\text { c } \\
\text {. }\end{array}$ & $\mathrm{d}$ & 132,720 & $5,105,392$ \\
\hline 1988 & $4,848,175$ & 304,566 & c & c & d & 105,645 & $5,258,386$ \\
\hline 1989 & $4,891,381$ & 182,109 & c & c & d & 223,797 & $5,297,287$ \\
\hline 1990 & 4,894,379 & 170,589 & c & c & d & 260,108 & $5,325,076$ \\
\hline 1991 & $4,855,016$ & 172,306 & c & c & d & 280,265 & $5,307,587$ \\
\hline 1992 & 4,908,603 & 171,701 & c & c & d & 272,676 & $5,352,980$ \\
\hline 1993 & $4,968,641$ & 179,213 & 3,351 & 49,393 & 1,866 & 280,074 & $5,482,538$ \\
\hline 1994 & $5,061,111$ & 169,868 & 3,620 & 52,937 & 1,918 & 193,808 & $5,483,262$ \\
\hline 1995 & $5,100,317$ & 172,026 & 9,055 & 79,396 & 4,122 & 190,411 & $5,555,327$ \\
\hline 1996 & $5,195,265$ & 164,552 & 11,156 & 79,407 & 3,570 & 214,282 & $5,668,232$ \\
\hline 1997 & $5,351,466$ & 151,769 & 11,803 & 86,240 & 4,246 & 201,268 & $5,806,792$ \\
\hline 1998 & $5,434,383$ & 146,921 & 11,722 & 89,362 & 4,038 & 206,135 & $5,892,561$ \\
\hline 1999 & $5,403,450$ & 135,756 & 13,735 & 94,784 & 4,147 & 225,779 & $5,877,651$ \\
\hline 2000 & $5,514,395$ & 138,921 & 15,268 & 90,288 & 4,005 & 201,135 & $5,964,012$ \\
\hline 2001 & $5,521,637$ & 156,479 & 16,929 & 87,116 & 4,544 & 192,632 & $5,979,337$ \\
\hline 2002 & $5,455,530$ & 155,429 & 26,320 & 90,291 & 2,338 & 224,567 & $5,955,475$ \\
\hline 2003 & $5,585,875$ & 152,763 & 55,626 & 67,592 & 1,937 & 163,459 & $6,027,252$ \\
\hline 2004 & $5,663,861$ & 154,356 & 74,095 & 47,600 & 940 & 194,203 & $6,135,055$ \\
\hline 2005 & $5,555,332$ & 161,037 & 84,088 & 39,751 & 612 & 295,064 & $6,135,884$ \\
\hline 2006 & $5,563,354$ & 182,924 & 117,198 & 11,580 & 57 & 322,989 & $6,198,102$ \\
\hline 2007 & $5,532,097$ & 184,383 & 136,603 & 1,610 & 0 & 349,807 & $6,204,500$ \\
\hline 2008 & $5,361,287$ & 177,559 & 190,084 & 480 & 0 & 548,843 & $6,277,893$ \\
\hline 2009 & $5,232,656$ & 177,194 & 240,955 & 90 & 0 & 518,998 & $6,169,893$ \\
\hline 2010 & $5,374,094$ & 161,479 & 285,883 & 901 & 0 & 523,015 & $6,345,372$ \\
\hline 2011 & $5,404,347$ & 178,884 & 297,266 & 1,154 & 0 & 541,059 & $6,422,710$ \\
\hline 2012 & $5,489,516$ & 186,270 & 304,155 & 806 & 0 & 425,946 & $6,406,693$ \\
\hline 2013 & $5,589,006$ & 181,112 & 310,568 & 915 & 0 & 495,476 & $6,577,077$ \\
\hline 2014 & $5,784,637$ & 186,601 & 317,171 & 719 & 1 & 490,213 & $6,779,342$ \\
\hline 2015 & $5,908,550$ & 188,722 & 325,858 & 830 & 0 & 446,744 & $6,870,704$ \\
\hline \multicolumn{8}{|c|}{ Average annual percentage change } \\
\hline 1987-2015 & $0.8 \%$ & $-1.4 \%$ & d & d & . & $4.4 \%$ & $1.1 \%$ \\
\hline 2005-2015 & $0.6 \%$ & $1.6 \%$ & $14.5 \%$ & $-32.1 \%$ & $-100.0 \%$ & $4.2 \%$ & $1.1 \%$ \\
\hline
\end{tabular}

\section{Source:}

U.S. Department of Energy, Energy Information Administration, Petroleum Supply Annual 2015, Vol. 1, September 2016, Table 16, and annual. (Additional resources: www.eia.doe.gov)

\footnotetext{
a Methyl tertiary butyl ether (MTBE).

b Includes methanol and other oxygenates.

c Reported in "Other hydrocarbons and liquids" category in this year.

${ }^{\mathrm{d}}$ Data are not available.
} 
When crude oil and other hydrocarbons are processed into products that are, on average, less dense than the input, a processing volume gain occurs. Due to this gain, the product yield from a barrel of crude oil is more than $100 \%$.

For the last 20 years, the processing volume gain has been about 5-7\%.

Table 1.10

\section{U.S. Refinery Yield of Petroleum Products from a Barrel of Crude Oil, 1978-2016 (percentage)}

\begin{tabular}{|c|c|c|c|c|c|c|}
\hline Year & $\begin{array}{c}\text { Motor } \\
\text { gasoline }\end{array}$ & $\begin{array}{c}\text { Distillate } \\
\text { fuel oil }\end{array}$ & Jet fuel & $\begin{array}{c}\text { Liquefied } \\
\text { petroleum gas }\end{array}$ & Other $^{\mathrm{a}}$ & Total ${ }^{\mathrm{b}}$ \\
\hline 1978 & 44.1 & 21.4 & 6.6 & 2.3 & 29.6 & 104.0 \\
\hline 1980 & 44.5 & 19.7 & 7.4 & 2.4 & 30.0 & 104.0 \\
\hline 1985 & 45.6 & 21.6 & 9.6 & 3.1 & 24.6 & 104.5 \\
\hline 1986 & 45.7 & 21.2 & 9.8 & 3.2 & 24.8 & 104.7 \\
\hline 1987 & 46.4 & 20.5 & 10.0 & 3.4 & 24.5 & 104.8 \\
\hline 1988 & 46.0 & 20.8 & 10.0 & 3.6 & 24.4 & 104.8 \\
\hline 1989 & 45.7 & 20.8 & 10.1 & 4.0 & 24.2 & 104.8 \\
\hline 1990 & 45.6 & 20.9 & 10.7 & 3.6 & 24.1 & 104.9 \\
\hline 1991 & 45.7 & 21.3 & 10.3 & 3.8 & 24.1 & 105.2 \\
\hline 1992 & 46.0 & 21.2 & 9.9 & 4.3 & 24.0 & 105.4 \\
\hline 1993 & 46.1 & 21.9 & 9.2 & 4.1 & 24.2 & 105.5 \\
\hline 1994 & 45.5 & 22.3 & 9.8 & 4.2 & 23.8 & 105.6 \\
\hline 1995 & 46.4 & 21.8 & 9.7 & 4.5 & 23.3 & 105.7 \\
\hline 1996 & 45.7 & 22.7 & 10.4 & 4.5 & 22.6 & 105.9 \\
\hline 1997 & 45.7 & 22.5 & 10.3 & 4.6 & 22.6 & 105.7 \\
\hline 1998 & 46.2 & 22.3 & 9.9 & 4.4 & 23.1 & 105.9 \\
\hline 1999 & 46.5 & 22.3 & 10.2 & 4.5 & 22.6 & 106.1 \\
\hline 2000 & 46.2 & 23.1 & 10.3 & 4.5 & 22.1 & 106.2 \\
\hline 2001 & 46.2 & 23.8 & 9.8 & 4.3 & 21.6 & 105.7 \\
\hline 2002 & 47.3 & 23.2 & 9.8 & 4.3 & 21.4 & 106.0 \\
\hline 2003 & 46.9 & 23.7 & 9.5 & 4.2 & 22.2 & 106.5 \\
\hline 2004 & 46.8 & 23.9 & 9.7 & 4.0 & 22.4 & 106.8 \\
\hline 2005 & 46.2 & 25.0 & 9.8 & 3.6 & 21.6 & 106.2 \\
\hline 2006 & 45.8 & 25.4 & 9.3 & 3.9 & 21.7 & 106.1 \\
\hline 2007 & 45.5 & 26.1 & 9.1 & 4.1 & 21.7 & 106.5 \\
\hline 2008 & 44.2 & 27.8 & 9.7 & 4.1 & 20.8 & 106.6 \\
\hline 2009 & 46.6 & 26.6 & 9.2 & 4.1 & 19.9 & 106.4 \\
\hline 2010 & 46.3 & 27.2 & 9.2 & 4.3 & 19.8 & 106.8 \\
\hline 2011 & 45.6 & 28.6 & 9.3 & 4.0 & 19.1 & 106.6 \\
\hline 2012 & 45.7 & 28.7 & 9.4 & 4.0 & 18.6 & 106.4 \\
\hline 2013 & 45.7 & 29.1 & 9.4 & 3.9 & 18.5 & 106.6 \\
\hline 2014 & 45.7 & 29.5 & 9.4 & 4.0 & 17.6 & 106.2 \\
\hline 2015 & 46.0 & 29.5 & 9.6 & 3.7 & 17.1 & 105.9 \\
\hline 2016 & 47.0 & 28.4 & 9.8 & 3.7 & 17.1 & 106.0 \\
\hline
\end{tabular}

Source:

Department of Energy, Energy Information Administration, Petroleum Supply Navigator, July 2017. (Additional resources: www.eia.doe.gov)

a Includes aviation gasoline $(0.1 \%)$, kerosene $(0.1 \%)$, residual fuel oil $(2.5 \%)$, naphtha and other oils for petrochemical feedstock use (1.1\%), other oils for petrochemical feedstock use $(0.6 \%)$, special naphthas $(0.2 \%)$, lubricants (0.6\%), petroleum coke (5.4\%) asphalt and road oil (1.9\%), still gas (4.1\%), and miscellaneous products $(0.5 \%)$

${ }^{\mathrm{b}}$ Products sum to greater than $100 \%$ due to processing gain. The processing gain for years 1978 to 1980 is assumed to be 4 percent. 
Domestic petroleum production increased in 2009 for the first time in 20 years and continued to increase through 2015. Most of the petroleum imported by the United States is in the form of crude oil. The United States does export small amounts of petroleum, mainly refined petroleum products which go to Canada and Mexico.

Table 1.11

United States Petroleum Production, Imports, and Exports, 1950-2016 (million barrels per day)

\begin{tabular}{|c|c|c|c|c|c|c|c|c|c|}
\hline & \multicolumn{3}{|c|}{ Domestic production } & \multicolumn{3}{|c|}{ Net imports } & \multicolumn{3}{|c|}{ Exports } \\
\hline & $\begin{array}{c}\text { Crude } \\
\text { oil }\end{array}$ & $\begin{array}{l}\text { Natural } \\
\text { gas } \\
\text { plant } \\
\text { liquids } \\
\end{array}$ & Total $^{\mathrm{a}}$ & $\begin{array}{c}\text { Crude } \\
\text { oil }\end{array}$ & $\begin{array}{c}\text { Petroleum } \\
\text { products }\end{array}$ & Total & $\begin{array}{c}\text { Crude } \\
\text { oil }\end{array}$ & $\begin{array}{l}\text { Petroleum } \\
\text { products }\end{array}$ & Total \\
\hline 1950 & 5.41 & 0.50 & 5.91 & 0.49 & 0.36 & 0.85 & 0.10 & 0.21 & 0.31 \\
\hline 1955 & 6.81 & 0.77 & 7.58 & 0.78 & 0.47 & 1.25 & 0.03 & 0.34 & 0.37 \\
\hline 1960 & 7.05 & 0.93 & 7.98 & 1.02 & 0.80 & 1.82 & 0.01 & 0.19 & 0.20 \\
\hline 1965 & 7.80 & 1.21 & 9.01 & 1.24 & 1.23 & 2.47 & 0.00 & 0.18 & 0.19 \\
\hline 1970 & 9.64 & 1.66 & 11.30 & 1.32 & 2.10 & 3.42 & 0.01 & 0.25 & 0.26 \\
\hline 1975 & 8.38 & 1.63 & 10.01 & 4.11 & 1.95 & 6.06 & 0.01 & 0.20 & 0.21 \\
\hline 1980 & 8.60 & 1.57 & 10.17 & 5.26 & 1.65 & 6.91 & 0.29 & 0.26 & 0.54 \\
\hline 1985 & 8.97 & 1.61 & 10.58 & 3.20 & 1.87 & 5.07 & 0.20 & 0.58 & 0.78 \\
\hline 1990 & 7.36 & 1.56 & 8.91 & 5.89 & 2.12 & 8.02 & 0.11 & 0.75 & 0.86 \\
\hline 1995 & 6.56 & 1.76 & 8.32 & 7.23 & 1.61 & 8.83 & 0.09 & 0.85 & 0.95 \\
\hline 1996 & 6.46 & 1.83 & 8.29 & 7.51 & 1.97 & 9.48 & 0.11 & 0.87 & 0.98 \\
\hline 1997 & 6.45 & 1.82 & 8.27 & 8.23 & 1.94 & 10.16 & 0.11 & 0.90 & 1.00 \\
\hline 1998 & 6.25 & 1.76 & 8.01 & 8.71 & 2.00 & 10.71 & 0.11 & 0.83 & 0.94 \\
\hline 1999 & 5.88 & 1.85 & 7.73 & 8.73 & 2.12 & 10.85 & 0.12 & 0.82 & 0.94 \\
\hline 2000 & 5.82 & 1.91 & 7.73 & 9.07 & 2.39 & 11.46 & 0.05 & 0.99 & 1.04 \\
\hline 2001 & 5.80 & 1.87 & 7.67 & 9.33 & 2.54 & 11.87 & 0.02 & 0.95 & 0.97 \\
\hline 2002 & 5.74 & 1.88 & 7.62 & 9.14 & 2.39 & 11.53 & 0.01 & 0.97 & 0.98 \\
\hline 2003 & 5.65 & 1.72 & 7.37 & 9.67 & 2.60 & 12.26 & 0.01 & 1.01 & 1.03 \\
\hline 2004 & 5.44 & 1.81 & 7.25 & 10.09 & 3.06 & 13.15 & 0.03 & 1.02 & 1.05 \\
\hline 2005 & 5.18 & 1.72 & 6.90 & 10.13 & 3.59 & 13.71 & 0.03 & 1.13 & 1.17 \\
\hline 2006 & 5.09 & 1.74 & 6.83 & 10.12 & 3.59 & 13.71 & 0.02 & 1.29 & 1.32 \\
\hline 2007 & 5.08 & 1.78 & 6.86 & 10.03 & 3.44 & 13.47 & 0.03 & 1.41 & 1.43 \\
\hline 2008 & 5.00 & 1.78 & 6.79 & 9.78 & 3.13 & 12.92 & 0.03 & 1.77 & 1.80 \\
\hline 2009 & 5.35 & 1.91 & 7.26 & 9.01 & 2.68 & 11.69 & 0.04 & 1.98 & 2.02 \\
\hline 2010 & 5.48 & 2.07 & 7.55 & 9.21 & 2.58 & 11.79 & 0.04 & 2.31 & 2.35 \\
\hline 2011 & 5.65 & 2.22 & 7.86 & 8.94 & 2.50 & 11.44 & 0.05 & 2.94 & 2.99 \\
\hline 2012 & 6.49 & 2.41 & 8.90 & 8.53 & 2.07 & 10.60 & 0.07 & 3.14 & 3.21 \\
\hline 2013 & 7.47 & 2.61 & 10.07 & 7.73 & 2.13 & 9.86 & 0.13 & 3.49 & 3.62 \\
\hline 2014 & 8.76 & 3.02 & 11.78 & 7.34 & 1.90 & 9.24 & 0.35 & 3.83 & 4.18 \\
\hline 2015 & 9.42 & 3.34 & 12.76 & 7.36 & 2.09 & 9.45 & 0.47 & 4.27 & 4.74 \\
\hline 2016 & 8.88 & 3.48 & 12.35 & 7.88 & 2.18 & 10.06 & 0.52 & 4.67 & 5.19 \\
\hline \multicolumn{10}{|c|}{ Average annual percentage change } \\
\hline 1950-2016 & $0.8 \%$ & $3.0 \%$ & $1.1 \%$ & $4.3 \%$ & $2.8 \%$ & $3.8 \%$ & $2.5 \%$ & $4.8 \%$ & $4.4 \%$ \\
\hline 1970-2016 & $-0.2 \%$ & $1.6 \%$ & $0.2 \%$ & $4.0 \%$ & $0.1 \%$ & $2.4 \%$ & $9.0 \%$ & $6.5 \%$ & $6.7 \%$ \\
\hline 2006-2016 & $5.7 \%$ & $7.2 \%$ & $6.1 \%$ & $-2.5 \%$ & $-4.9 \%$ & $-3.0 \%$ & $33.0 \%$ & $13.7 \%$ & $14.7 \%$ \\
\hline
\end{tabular}

\section{Source:}

U.S. Department of Energy, Energy Information Administration, Monthly Energy Review, Washington, DC, July 2017, Tables 3.1 and 3.3b. (Additional resources: www.eia.gov)

a Total domestic production includes crude oil, natural gas plant liquids and small amounts of other liquids. 
U.S. petroleum production has been increasing and petroleum imports decreasing from 2008 through 2015. Net imports of petroleum in 2015 were at the lowest level since 1985. In 2016, domestic production declined slightly.

Table 1.12

Petroleum Production and Transportation Petroleum Consumption in Context, 1950-2016

\begin{tabular}{|c|c|c|c|c|c|c|c|c|}
\hline & $\begin{array}{c}\text { Domestic } \\
\text { petroleum } \\
\text { production }^{\mathrm{a}}\end{array}$ & $\begin{array}{c}\text { Net } \\
\text { petroleum } \\
\text { imports } \\
\end{array}$ & $\begin{array}{c}\text { Transportation } \\
\text { petroleum } \\
\text { consumption } \\
\end{array}$ & $\begin{array}{c}\text { U.S. } \\
\text { petroleum } \\
\text { consumption }\end{array}$ & $\begin{array}{c}\text { World } \\
\text { petroleum } \\
\text { consumption }\end{array}$ & \multirow{2}{*}{$\begin{array}{l}\text { Net imports } \\
\text { as a share of } \\
\text { U.S. } \\
\text { consumption } \\
8.4 \%\end{array}$} & \multirow{3}{*}{$\begin{array}{l}\text { U.S. petroleum } \\
\text { consumption as } \\
\text { a share of world } \\
\text { consumption } \\
\text { b }\end{array}$} & \multirow{3}{*}{$\begin{array}{c}\text { Transportation } \\
\text { petroleum use as } \\
\text { a share of } \\
\text { domestic } \\
\text { production } \\
56.8 \%\end{array}$} \\
\hline & \multicolumn{5}{|c|}{ (million barrels per day) } & consumption & & \\
\hline 1950 & 5.91 & 0.55 & 3.36 & 6.46 & $\mathrm{~b}$ & & & \\
\hline 1955 & 7.58 & 0.88 & 4.46 & 8.46 & b & $10.4 \%$ & $\mathrm{~b}$ & $58.8 \%$ \\
\hline 1960 & 7.99 & 1.62 & 5.15 & 9.82 & 21.34 & $16.5 \%$ & $46.0 \%$ & $64.5 \%$ \\
\hline 1965 & 9.01 & 2.28 & 6.04 & 11.51 & 31.14 & $19.8 \%$ & $37.0 \%$ & $67.0 \%$ \\
\hline 1970 & 11.30 & 3.16 & 7.78 & 14.70 & 46.81 & $21.5 \%$ & $31.4 \%$ & $68.9 \%$ \\
\hline 1975 & 10.01 & 5.85 & 8.92 & 16.32 & 56.20 & $35.8 \%$ & $29.0 \%$ & $89.4 \%$ \\
\hline 1980 & 10.17 & 6.36 & 9.55 & 17.06 & 63.12 & $37.3 \%$ & $27.0 \%$ & $93.9 \%$ \\
\hline 1985 & 10.58 & 4.29 & 9.84 & 15.73 & 60.08 & $27.3 \%$ & $26.2 \%$ & $93.0 \%$ \\
\hline 1990 & 8.91 & 7.16 & 10.99 & 16.99 & 66.54 & $42.2 \%$ & $25.5 \%$ & $123.2 \%$ \\
\hline 1995 & 8.32 & 7.89 & 11.50 & 17.72 & 70.26 & $44.5 \%$ & $25.2 \%$ & $138.2 \%$ \\
\hline 1996 & 8.29 & 8.50 & 11.83 & 18.31 & 71.88 & $46.4 \%$ & $25.5 \%$ & $142.6 \%$ \\
\hline 1997 & 8.27 & 9.16 & 12.14 & 18.62 & 73.60 & $49.2 \%$ & $25.3 \%$ & $146.8 \%$ \\
\hline 1998 & 8.01 & 9.76 & 12.40 & 18.92 & 74.27 & $51.6 \%$ & $25.5 \%$ & $154.8 \%$ \\
\hline 1999 & 7.73 & 9.91 & 12.74 & 19.52 & 75.98 & $50.8 \%$ & $25.7 \%$ & $164.8 \%$ \\
\hline 2000 & 7.73 & 10.42 & 12.99 & 19.70 & 76.93 & $52.9 \%$ & $25.6 \%$ & $168.0 \%$ \\
\hline 2001 & 7.67 & 10.90 & 12.91 & 19.65 & 77.73 & $55.5 \%$ & $25.3 \%$ & $168.4 \%$ \\
\hline 2002 & 7.62 & 10.55 & 13.18 & 19.76 & 78.46 & $53.4 \%$ & $25.2 \%$ & $172.9 \%$ \\
\hline 2003 & 7.37 & 11.24 & 13.25 & 20.03 & 80.09 & $56.1 \%$ & $25.0 \%$ & $179.8 \%$ \\
\hline 2004 & 7.25 & 12.10 & 13.67 & 20.73 & 83.06 & $58.4 \%$ & $25.0 \%$ & $188.6 \%$ \\
\hline 2005 & 6.90 & 12.55 & 13.90 & 20.80 & 84.56 & $60.3 \%$ & $24.6 \%$ & $201.6 \%$ \\
\hline 2006 & 6.83 & 12.39 & 14.11 & 20.69 & 85.57 & $59.9 \%$ & $24.2 \%$ & $206.7 \%$ \\
\hline 2007 & 6.86 & 12.04 & 14.20 & 20.68 & 86.72 & $58.2 \%$ & $23.8 \%$ & $207.0 \%$ \\
\hline 2008 & 6.78 & 11.11 & 13.50 & 19.50 & 86.05 & $57.0 \%$ & $22.7 \%$ & $199.0 \%$ \\
\hline 2009 & 7.26 & 9.67 & 13.16 & 18.77 & 84.97 & $51.5 \%$ & $22.1 \%$ & $181.3 \%$ \\
\hline 2010 & 7.55 & 9.44 & 13.35 & 19.18 & 89.17 & $49.2 \%$ & $21.5 \%$ & $176.9 \%$ \\
\hline 2011 & 7.86 & 8.45 & 13.14 & 18.88 & 89.88 & $44.8 \%$ & $21.0 \%$ & $167.2 \%$ \\
\hline 2012 & 8.90 & 7.39 & 12.86 & 18.49 & 91.09 & $40.0 \%$ & $20.3 \%$ & $144.6 \%$ \\
\hline 2013 & 10.07 & 6.24 & 13.09 & 18.96 & 92.32 & $32.9 \%$ & $20.5 \%$ & $130.0 \%$ \\
\hline 2014 & 11.78 & 5.07 & 13.29 & 19.11 & 93.61 & $26.5 \%$ & $20.4 \%$ & $112.9 \%$ \\
\hline 2015 & 12.76 & 4.71 & 13.60 & 19.53 & 95.36 & $24.1 \%$ & $20.5 \%$ & $106.6 \%$ \\
\hline 2016 & 12.35 & 4.87 & 13.69 & 19.63 & $\mathrm{~b}$ & $24.8 \%$ & $\mathrm{~b}$ & $110.9 \%$ \\
\hline & & & Average ar & nual percent & change & & & \\
\hline 1950-2016 & $1.1 \%$ & $3.4 \%$ & $2.2 \%$ & $1.7 \%$ & $\mathrm{~b}$ & & & \\
\hline 1970-2016 & $0.2 \%$ & $0.9 \%$ & $1.2 \%$ & $0.6 \%$ & $\mathrm{~b}$ & & & \\
\hline 2006-2016 & $6.1 \%$ & $-8.9 \%$ & $-0.3 \%$ & $-0.5 \%$ & b & & & \\
\hline
\end{tabular}

Sources:

U.S. Department of Energy, Energy Information Administration, Monthly Energy Review, Washington, DC, July 2017, Tables 2.5, 3.1, and A3. (Pre-1973 data from the Annual Energy Review). World petroleum consumption - U.S. Department of Energy, Energy Information Administration, International Energy Statistics website, August 2017. (Additional resources: www.eia.doe.gov)

a Total domestic production includes crude oil, natural gas plant liquids and small amounts of other liquids.

${ }^{\mathrm{b}}$ Data are not available. 
Before 1989 the U.S. produced enough petroleum to meet the needs of the transportation sector, but was still short of meeting the petroleum needs of all the sectors, including industrial, residential and commercial, and electric utilities. In 1973 the gap between what the U.S. produced and what was consumed was 5.8 million barrels per day. By 2050, the gap is expected to be only about 4.8 million barrels per day if petroleum and other inputs are included or 6.1 million barrels per day if only conventional petroleum is used.

Figure 1.5. United States Petroleum Production and Consumption - All Sectors, 1973-2050

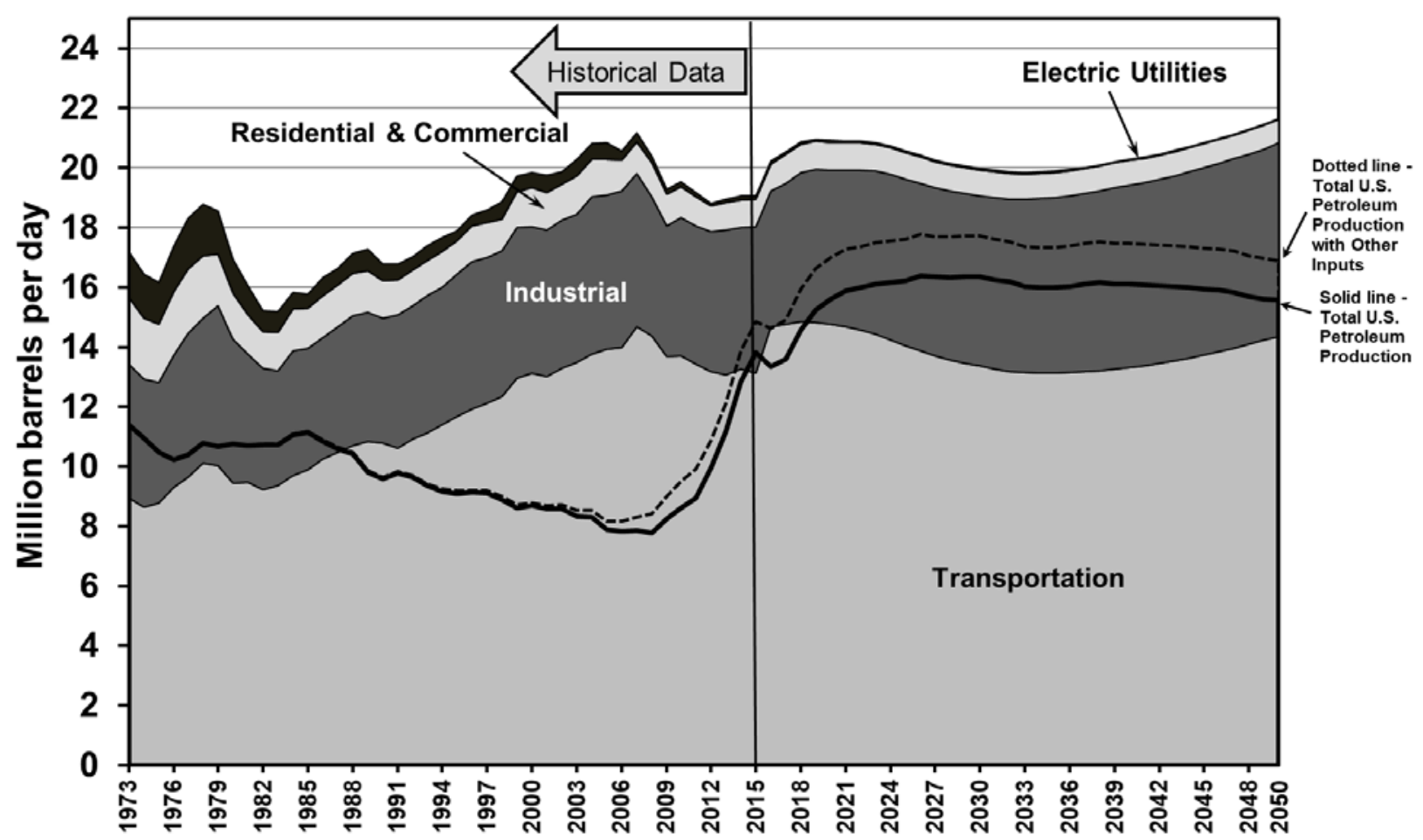

Notes: “Total U.S. Petroleum Production” includes crude oil, natural gas plant liquids, and refinery gains. It does not include dry natural gas.

"Total U.S. Petroleum Production" is for all uses.

"Total U.S. Petroleum Production with Other Inputs" also includes non-petroleum sources such as ethanol, biomass, liquids from coal, other blending components, other hydrocarbons, and ethers which were domestically produced.

The change from historical values to projected values is between 2015 and 2016.

The sharp increase in the value for heavy trucks between 2006 and 2007 is the result of the Federal Highway Administration's methodology change.

\section{Sources:}

Historical transportation petroleum use - See Tables 1.14 and 1.15. Historical petroleum use for other sectors - See Table 1.13. Historical U.S. petroleum production - Energy Information Administration, Monthly Energy Review August 2017, Table 3.1. Historical other inputs - Energy Information Administration, Monthly Energy Review August 2017, Tables 10.3 and 10.4. Forecasted petroleum use and petroleum production - Energy Information Administration, 2017 Annual Energy Outlook, January 2017, reference case tables 7, 11, and 36. 
In 1989, for the first time, the petroleum consumption for transportation surpassed total U.S. petroleum production, which was declining. These contrasting trends in production and consumption created a gap that was met with foreign imports of petroleum. In 2009, however, the U.S. production of petroleum (for all uses including, but not limited to, transportation) began to increase substantially because of new hydraulic fracturing and oil extraction technology. In 2015, total production exceeded all transportation sector petroleum consumption. With other inputs included, such as ethanol, domestic production has exceeded transportation consumption since 2014. Transportation accounts for about $70 \%$ of all U.S. petroleum consumption.

The Energy Information Administration expects petroleum production to be greater than transportation consumption through 2040. Including non-petroleum sources such as ethanol, the production will exceed transportation demand by about three million barrels per day in 2050.

Figure 1.6. United States Petroleum Production and Transportation Consumption, 1970-2050

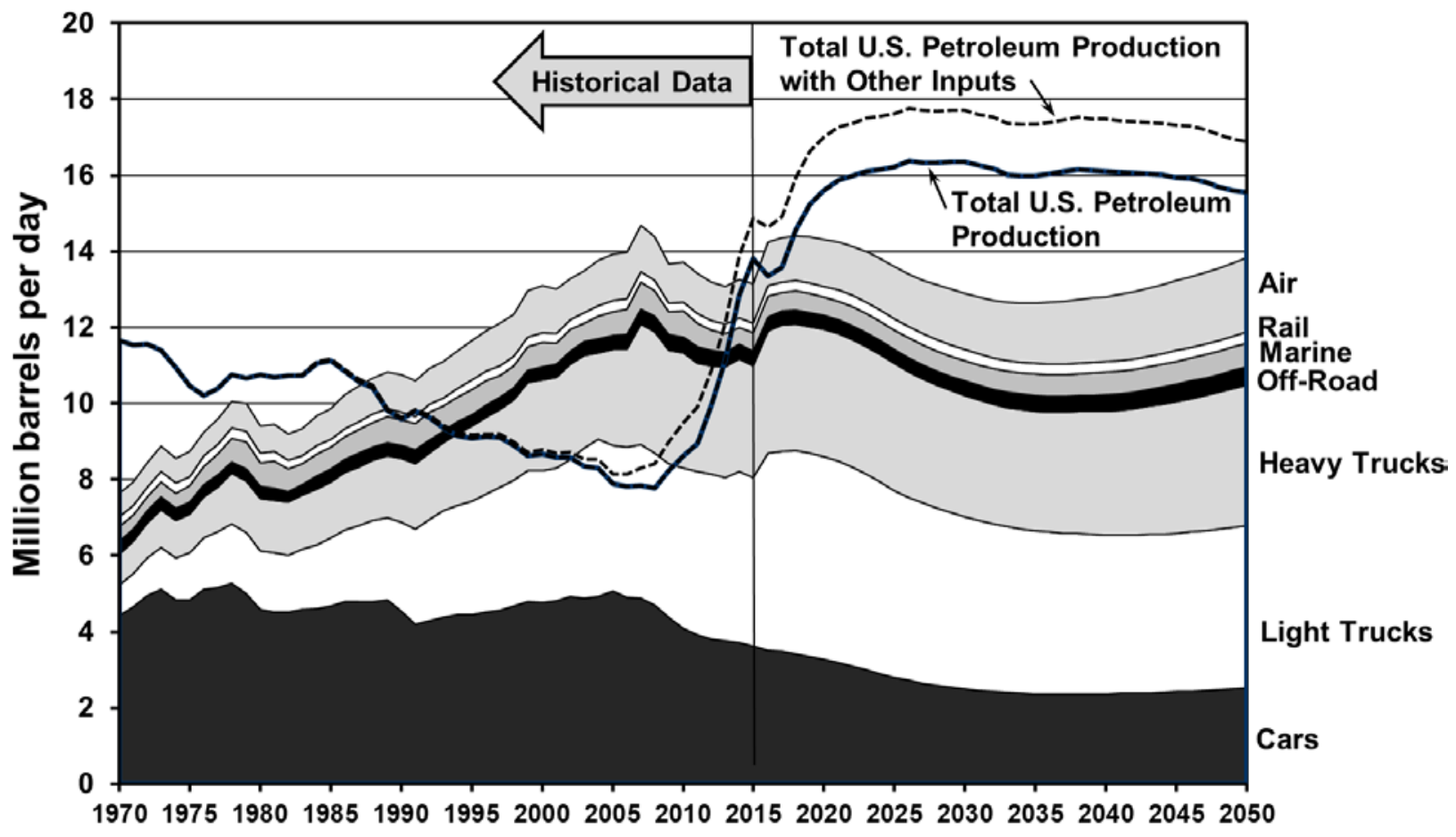

Notes: “Total U.S. Petroleum Production” includes crude oil, natural gas plant liquids, and refinery gains. It does not include dry natural gas.

"Total U.S. Petroleum Production” is for all uses, including but not limited to transportation.

"Total U.S. Petroleum Production with Other Inputs" also includes non-petroleum sources such as ethanol, biomass, liquids from coal, other blending components, other hydrocarbons, and ethers which were domestically produced.

The change from historical values to projected values is between 2015 and 2016.

The sharp increase in the value for heavy trucks between 2006 and 2007 is the result of the Federal Highway Administration's methodology change.

\section{Sources:}

Historical transportation petroleum use - See Tables 1.14 and 1.15. Historical U.S. petroleum production - Energy Information Administration, Monthly Energy Review August 2017, Table 3.1. Historical other inputs - Energy Information Administration, Monthly Energy Review August 2017, Tables 10.3 and 10.4. Forecasted petroleum use and petroleum production - Energy Information Administration, 2017 Annual Energy Outlook, January 2017, reference case tables 7, 11, and 36. 
Transportation accounted for about 70\% of the U.S. petroleum use from 2008 to 2016. Total petroleum consumption reached more than 20 million barrels per day from 2003 to 2007, but has been below that level from 2008 through present.

Table 1.13

Consumption of Petroleum by End-Use Sector, 1973-2016 (million barrels per day)

\begin{tabular}{|c|c|c|c|c|c|c|c|}
\hline Year & Transportation & Percentage & Residential & Commercial & Industrial & $\begin{array}{l}\text { Electric } \\
\text { utilities }\end{array}$ & Total \\
\hline 1973 & 9.05 & $52.3 \%$ & 1.46 & 0.77 & 4.48 & 1.54 & 17.31 \\
\hline 1975 & 8.95 & $54.8 \%$ & 1.29 & 0.65 & 4.04 & 1.39 & 16.32 \\
\hline 1980 & 9.55 & $56.0 \%$ & 0.89 & 0.63 & 4.84 & 1.15 & 17.06 \\
\hline 1981 & 9.49 & $59.1 \%$ & 0.79 & 0.54 & 4.27 & 0.96 & 16.06 \\
\hline 1982 & 9.31 & $60.8 \%$ & 0.75 & 0.50 & 4.06 & 0.69 & 15.30 \\
\hline 1983 & 9.41 & $61.8 \%$ & 0.72 & 0.57 & 3.85 & 0.68 & 15.23 \\
\hline 1984 & 9.59 & $61.0 \%$ & 0.79 & 0.60 & 4.19 & 0.56 & 15.73 \\
\hline 1985 & 9.84 & $62.6 \%$ & 0.82 & 0.53 & 4.07 & 0.48 & 15.73 \\
\hline 1986 & 10.19 & $62.6 \%$ & 0.80 & 0.57 & 4.09 & 0.64 & 16.28 \\
\hline 1987 & 10.51 & $63.0 \%$ & 0.85 & 0.55 & 4.21 & 0.55 & 16.67 \\
\hline 1988 & 10.84 & $62.7 \%$ & 0.87 & 0.54 & 4.35 & 0.68 & 17.28 \\
\hline 1989 & 10.89 & $62.8 \%$ & 0.87 & 0.51 & 4.31 & 0.74 & 17.33 \\
\hline 1990 & 10.99 & $64.7 \%$ & 0.75 & 0.50 & 4.19 & 0.57 & 16.99 \\
\hline 1991 & 10.56 & $63.2 \%$ & 0.73 & 0.46 & 4.45 & 0.52 & 16.71 \\
\hline 1992 & 10.94 & $64.2 \%$ & 0.76 & 0.45 & 4.46 & 0.44 & 17.03 \\
\hline 1993 & 10.98 & $63.7 \%$ & 0.77 & 0.40 & 4.60 & 0.49 & 17.24 \\
\hline 1994 & 11.46 & $64.7 \%$ & 0.76 & 0.41 & 4.61 & 0.47 & 17.72 \\
\hline 1995 & 11.50 & $64.9 \%$ & 0.74 & 0.38 & 4.78 & 0.33 & 17.72 \\
\hline 1996 & 11.83 & $64.6 \%$ & 0.81 & 0.40 & 4.92 & 0.36 & 18.31 \\
\hline 1997 & 12.14 & $65.2 \%$ & 0.79 & 0.38 & 4.90 & 0.41 & 18.62 \\
\hline 1998 & 12.40 & $65.5 \%$ & 0.72 & 0.36 & 4.86 & 0.58 & 18.92 \\
\hline 1999 & 12.74 & $65.3 \%$ & 0.82 & 0.37 & 5.05 & 0.54 & 19.52 \\
\hline 2000 & 12.99 & $65.9 \%$ & 0.87 & 0.42 & 4.92 & 0.51 & 19.70 \\
\hline 2001 & 12.91 & $65.7 \%$ & 0.85 & 0.41 & 4.91 & 0.57 & 19.65 \\
\hline 2002 & 13.18 & $66.7 \%$ & 0.82 & 0.38 & 4.95 & 0.43 & 19.76 \\
\hline 2003 & 13.25 & $66.1 \%$ & 0.87 & 0.44 & 4.95 & 0.54 & 20.03 \\
\hline 2004 & 13.67 & $65.9 \%$ & 0.84 & 0.42 & 5.26 & 0.54 & 20.73 \\
\hline 2005 & 13.90 & $66.8 \%$ & 0.82 & 0.39 & 5.14 & 0.55 & 20.80 \\
\hline 2006 & 14.11 & $68.2 \%$ & 0.69 & 0.35 & 5.25 & 0.29 & 20.69 \\
\hline 2007 & 14.20 & $68.7 \%$ & 0.72 & 0.34 & 5.12 & 0.30 & 20.68 \\
\hline 2008 & 13.50 & $69.2 \%$ & 0.78 & 0.36 & 4.65 & 0.21 & 19.50 \\
\hline 2009 & 13.16 & $70.1 \%$ & 0.70 & 0.36 & 4.38 & 0.18 & 18.77 \\
\hline 2010 & 13.35 & $69.6 \%$ & 0.68 & 0.35 & 4.62 & 0.18 & 19.18 \\
\hline 2011 & 13.14 & $69.6 \%$ & 0.62 & 0.34 & 4.63 & 0.14 & 18.88 \\
\hline 2012 & 12.86 & $69.6 \%$ & 0.53 & 0.31 & 4.68 & 0.10 & 18.49 \\
\hline 2013 & 13.09 & $69.1 \%$ & 0.59 & 0.31 & 4.84 & 0.12 & 18.96 \\
\hline 2014 & 13.29 & $69.6 \%$ & 0.61 & 0.32 & 4.74 & 0.14 & 19.11 \\
\hline 2015 & 13.60 & $69.6 \%$ & 0.59 & 0.32 & 4.89 & 0.13 & 19.53 \\
\hline 2016 & 13.69 & $69.8 \%$ & 0.58 & 0.48 & 4.76 & 0.12 & 19.63 \\
\hline \multicolumn{8}{|c|}{ Average annual percentage change } \\
\hline 1973-2016 & $1.0 \%$ & & $-2.1 \%$ & $-1.1 \%$ & $0.1 \%$ & $-5.8 \%$ & $0.3 \%$ \\
\hline 2006-2016 & $-0.3 \%$ & & $-1.7 \%$ & $3.2 \%$ & $-1.0 \%$ & $-8.4 \%$ & $-0.5 \%$ \\
\hline
\end{tabular}

Source:

U.S. Department of Energy, Energy Information Administration, Monthly Energy Review, Washington, DC, July 2017, Tables 2.2-2.6. Converted to million barrels per day using Table A3. (Additional resources: www.eia.doe.gov) 
Cars and light trucks use most of the petroleum in the transportation sector. Light trucks include pick-ups, minivans, sport-utility vehicles, and vans. See Table 2.9 for highway energy use in trillion Btu.

Table 1.14

Highway Transportation Petroleum Consumption by Mode, 1970-2015 ${ }^{\mathrm{a}}$ (thousand barrels per day)

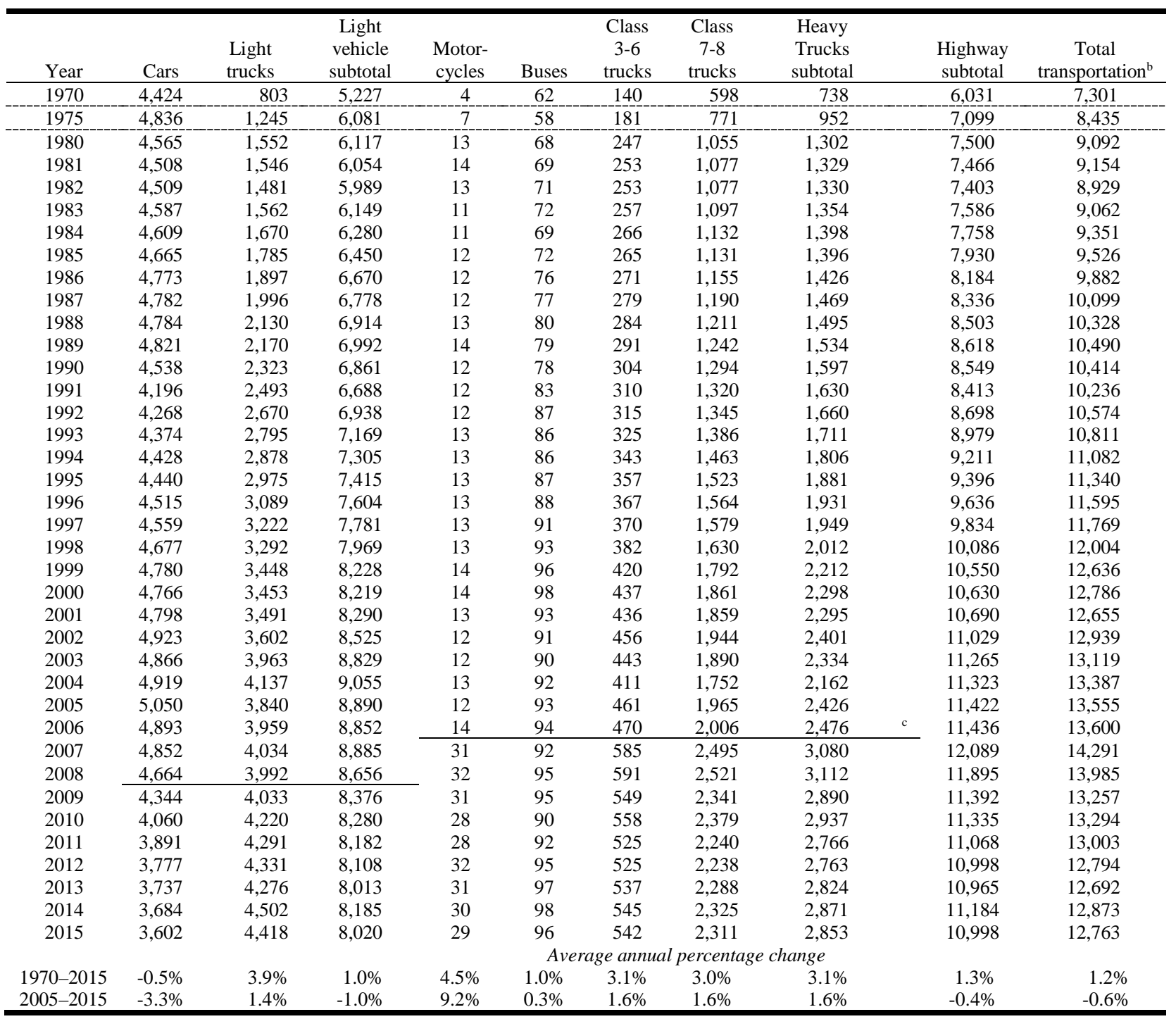

\section{Source:}

See Appendix A, Section 2.1 Highway Energy Use.

${ }^{\text {a }}$ Each gallon of petroleum product was assumed to equal one gallon of crude oil. The oil used to produce electricity is also estimated. See Appendix A, Section 2.4 for details.

b Total transportation figures do not include military and off-highway energy use and may not include all possible uses of fuel for transportation (e.g., snowmobiles).

${ }^{\mathrm{c}}$ Due to changes in the FHWA fuel use methodology, motorcycle, bus, and heavy truck data are not comparable with data before the year 2007. Car and light truck data changed after 2008; see Appendix A, Section 7, Car/Light Truck Shares. 
Although about $18 \%$ of transportation energy use is for nonhighway modes, only $14 \%$ of transportation petroleum use is for nonhighway. This is because some nonhighway modes, such as pipelines and transit rail, use electricity. An estimate for the petroleum used to make electricity is included in the data. See Table 2.10 for nonhighway transportation energy use in trillion Btu.

Table 1.15

Nonhighway Transportation Petroleum Consumption by Mode, 1970-2015 (thousand barrels per day)

\begin{tabular}{|c|c|c|c|c|c|c|}
\hline Year & Air & Water & Pipeline & Rail & $\begin{array}{l}\text { Nonhighway } \\
\text { subtotal }\end{array}$ & $\begin{array}{c}\text { Total } \\
\text { transportation }\end{array}$ \\
\hline 1970 & 625 & 381 & 14 & 250 & 1,270 & 7,301 \\
\hline 1975 & 651 & 423 & 16 & 246 & 1,336 & 8,435 \\
\hline 1980 & 697 & 625 & 11 & 259 & 1,592 & 9,092 \\
\hline 1985 & 814 & 564 & 4 & 214 & 1,596 & 9,526 \\
\hline 1986 & 884 & 601 & 6 & 207 & 1,698 & 9,882 \\
\hline 1987 & 920 & 626 & 5 & 211 & 1,763 & 10,099 \\
\hline 1988 & 958 & 644 & 6 & 217 & 1,825 & 10,328 \\
\hline 1989 & 960 & 688 & 6 & 218 & 1,872 & 10,490 \\
\hline 1990 & 991 & 655 & 5 & 214 & 1,865 & 10,414 \\
\hline 1991 & 928 & 690 & 4 & 201 & 1,823 & 10,236 \\
\hline 1992 & 942 & 724 & 3 & 207 & 1,876 & 10,574 \\
\hline 1993 & 961 & 653 & 4 & 213 & 1,831 & 10,811 \\
\hline 1994 & 1,004 & 635 & 4 & 229 & 1,871 & 11,082 \\
\hline 1995 & 1,036 & 668 & 2 & 238 & 1,944 & 11,340 \\
\hline 1996 & 1,068 & 644 & 3 & 244 & 1,959 & 11,595 \\
\hline 1997 & 1,113 & 574 & 3 & 245 & 1,935 & 11,769 \\
\hline 1998 & 1,102 & 566 & 4 & 246 & 1,918 & 12,004 \\
\hline 1999 & 1,202 & 625 & 4 & 255 & 2,086 & 12,636 \\
\hline 2000 & 1,236 & 662 & 3 & 254 & 2,156 & 12,786 \\
\hline 2001 & 1,161 & 546 & 4 & 255 & 1,966 & 12,655 \\
\hline 2002 & 1,079 & 572 & 3 & 256 & 1,910 & 12,939 \\
\hline 2003 & 1,094 & 496 & 3 & 262 & 1,855 & 13,119 \\
\hline 2004 & 1,188 & 596 & 3 & 276 & 2,064 & 13,387 \\
\hline 2005 & 1,226 & 625 & 3 & 279 & 2,133 & 13,555 \\
\hline 2006 & 1,216 & 661 & 2 & 285 & 2,163 & 13,600 \\
\hline 2007 & 1,215 & 709 & 2 & 276 & 2,202 & 14,291 \\
\hline 2008 & 1,160 & 664 & 1 & 265 & 2,091 & 13,985 \\
\hline 2009 & 1,029 & 613 & 1 & 221 & 1,864 & 13,257 \\
\hline 2010 & 1,040 & 677 & 1 & 240 & 1,959 & 13,294 \\
\hline 2011 & 1,044 & 638 & 1 & 253 & 1,935 & 13,003 \\
\hline 2012 & 1,006 & 543 & 1 & 247 & 1,797 & 12,794 \\
\hline 2013 & 987 & 486 & 1 & 253 & 1,727 & 12,692 \\
\hline 2014 & 997 & 426 & 1 & 265 & 1,689 & 12,873 \\
\hline 2015 & 1,025 & 486 & 1 & 253 & 1,765 & 12,763 \\
\hline \multicolumn{7}{|c|}{ Average annual percentage change } \\
\hline 1970-2015 & $1.1 \%$ & $0.5 \%$ & $-5.7 \%$ & $0.0 \%$ & $0.7 \%$ & $1.2 \%$ \\
\hline 2005-2015 & $-1.8 \%$ & $-2.5 \%$ & $-10.4 \%$ & $-1.0 \%$ & $-1.9 \%$ & $-0.6 \%$ \\
\hline
\end{tabular}

\section{Source:}

See Appendix A, Section 2.3. Nonhighway Energy Use.

${ }^{a}$ Each gallon of petroleum product was assumed to equal one gallon of crude oil. The oil used to produce electricity is also estimated. See Appendix A, Section 2.3 Nonhighway Energy Use for details.

b Total transportation figures do not include military and off-highway energy use and may not include all possible uses of fuel for transportation (e.g., snowmobiles). 
Highway vehicles were responsible for $86.2 \%$ of all transportation petroleum use in 2015. See Table 2.8 for transportation energy use in trillion Btu.

Table 1.16

Transportation Petroleum Use by Mode, 2014-2015

\begin{tabular}{|c|c|c|c|c|c|c|}
\hline & \multicolumn{2}{|c|}{$\begin{array}{c}\text { Thousand barrels } \\
\text { per day }\end{array}$} & \multicolumn{2}{|c|}{ Percentage of total ${ }^{\mathrm{b}}$} & \multicolumn{2}{|c|}{$\begin{array}{c}\text { Percentage of total U.S } \\
\text { petroleum } \\
\text { consumption }^{\mathrm{b}}\end{array}$} \\
\hline & 2014 & 2015 & 2014 & 2015 & 2014 & 2015 \\
\hline HIGHWAY & $11,184.1$ & $10,998.1$ & $86.9 \%$ & $86.2 \%$ & $58.5 \%$ & $56.3 \%$ \\
\hline Light vehicles & $8,215.3$ & $8,048.9$ & $63.8 \%$ & $63.1 \%$ & $43.0 \%$ & $41.2 \%$ \\
\hline Cars & 3,683.6 & 3,601.6 & $28.6 \%$ & $28.2 \%$ & $19.3 \%$ & $18.4 \%$ \\
\hline Light trucks ${ }^{c}$ & $4,501.8$ & $4,418.1$ & $35.0 \%$ & $34.6 \%$ & $23.6 \%$ & $22.6 \%$ \\
\hline Motorcycles & 29.9 & 29.2 & $0.2 \%$ & $0.2 \%$ & $0.2 \%$ & $0.1 \%$ \\
\hline Buses & 97.9 & 96.4 & $0.8 \%$ & $0.8 \%$ & $0.5 \%$ & $0.5 \%$ \\
\hline Transit & 41.2 & 38.9 & $0.3 \%$ & $0.3 \%$ & $0.2 \%$ & $0.2 \%$ \\
\hline Intercity & 16.3 & 16.5 & $0.1 \%$ & $0.1 \%$ & $0.1 \%$ & $0.1 \%$ \\
\hline School & 40.3 & 40.9 & $0.3 \%$ & $0.3 \%$ & $0.2 \%$ & $0.2 \%$ \\
\hline Medium/heavy trucks & $2,871.0$ & $2,852.8$ & $22.3 \%$ & $22.3 \%$ & $15.0 \%$ & $14.6 \%$ \\
\hline Class 3-6 & 545.5 & 542.0 & $4.2 \%$ & $4.2 \%$ & $2.9 \%$ & $2.8 \%$ \\
\hline Class 7-8 & 2,325.5 & $2,310.8$ & $18.1 \%$ & $18.1 \%$ & $12.2 \%$ & $11.8 \%$ \\
\hline NONHIGHWAY & $1,689.1$ & $1,764.8$ & $13.1 \%$ & $13.8 \%$ & $8.9 \%$ & $9.0 \%$ \\
\hline Air & 997.0 & $1,024.9$ & $7.7 \%$ & $8.0 \%$ & $5.2 \%$ & $5.2 \%$ \\
\hline General aviation & 108.6 & 102.6 & $0.8 \%$ & $0.8 \%$ & $0.6 \%$ & $0.5 \%$ \\
\hline Domestic air carriers & 682.2 & 712.9 & $5.3 \%$ & $5.6 \%$ & $3.6 \%$ & $3.7 \%$ \\
\hline International air carriers & 206.2 & 209.4 & $1.6 \%$ & $1.6 \%$ & $1.1 \%$ & $1.1 \%$ \\
\hline Water & 426.3 & 485.6 & $3.3 \%$ & $3.8 \%$ & $2.2 \%$ & $2.5 \%$ \\
\hline Freight & 301.0 & 360.0 & $2.3 \%$ & $2.8 \%$ & $1.6 \%$ & $1.8 \%$ \\
\hline Recreational & 125.4 & 125.6 & $1.0 \%$ & $1.0 \%$ & $0.7 \%$ & $0.6 \%$ \\
\hline Pipeline & 0.9 & 0.8 & $0.0 \%$ & $0.0 \%$ & $0.0 \%$ & $0.0 \%$ \\
\hline Rail & 264.9 & 253.5 & $2.1 \%$ & $2.0 \%$ & $1.4 \%$ & $1.3 \%$ \\
\hline Freight (Class I) & 254.2 & 242.9 & $2.0 \%$ & $1.9 \%$ & $1.3 \%$ & $1.2 \%$ \\
\hline Passenger & 10.7 & 10.6 & $0.1 \%$ & $0.1 \%$ & $0.1 \%$ & $0.1 \%$ \\
\hline Transit & 0.2 & 0.2 & $0.0 \%$ & $0.0 \%$ & $0.0 \%$ & $0.0 \%$ \\
\hline Commuter & 6.2 & 6.3 & $0.0 \%$ & $0.1 \%$ & $0.0 \%$ & $0.0 \%$ \\
\hline Intercity & 4.3 & 4.1 & $0.0 \%$ & $0.0 \%$ & $0.0 \%$ & $0.0 \%$ \\
\hline \multicolumn{7}{|l|}{ HWY \& NONHWY } \\
\hline Off-Highway & $1,095.3$ & $1,115.1$ & & & & \\
\hline
\end{tabular}

Source:

See Appendix A, Section 2. Energy Use Sources.

${ }^{\text {a }}$ Each gallon of petroleum product was assumed to equal one gallon of crude oil. The oil used to produce electricity is also estimated. See Appendix A, Section 2.4 for details.

${ }^{\mathrm{b}}$ Percentages may not sum to totals due to rounding.

c Two-axle, four-tire trucks.

d Civilian consumption only. Totals may not include all possible uses of fuels for transportation (e.g., snowmobiles). 
$1-22$ 


\section{Chapter 2 \\ Energy}

Summary Statistics from Tables in this Chapter

\begin{tabular}{clrc}
\hline Source & \multicolumn{2}{c}{} & \\
\hline Table 2.1 & Transportation share of U.S. energy & & \\
& consumption, 2016 & & \\
Table 2.2 & Petroleum share of transportation energy & & \\
& consumption, 2016 & \\
Table 2.6 & Fuel ethanol consumption (million gallons) & & $14,399.1$ \\
& Biodiesel consumption (million gallons) & & $2,059.6$ \\
Table 2.8 & Transportation energy use by mode, 2015 & (trillion Btu) & energy share) \\
& Cars & 6,797 & $26.3 \%$ \\
& Light trucks & 8,348 & $32.3 \%$ \\
& Medium/heavy trucks & 5,984 & $23.1 \%$ \\
& Buses & 203 & $0.8 \%$ \\
& Total Highway & 21,386 & $82.6 \%$ \\
& Air & 2,118 & $8.2 \%$ \\
& Water & 1,046 & $4.0 \%$ \\
& Pipeline & 767 & $3.0 \%$ \\
& Rail & 563 & $2.2 \%$ \\
\hline
\end{tabular}


Petroleum accounted for 34\% of the world's energy use in 2014. Although petroleum and natural gas are the dominant energy sources for OECD countries, the non-OECD countries rely on coal and petroleum. The U.S. shares of primary energy sources are similar to the OECD countries as a whole, but with a lesser reliance on renewables.

Figure 2.1. World Consumption of Primary Energy, 2014

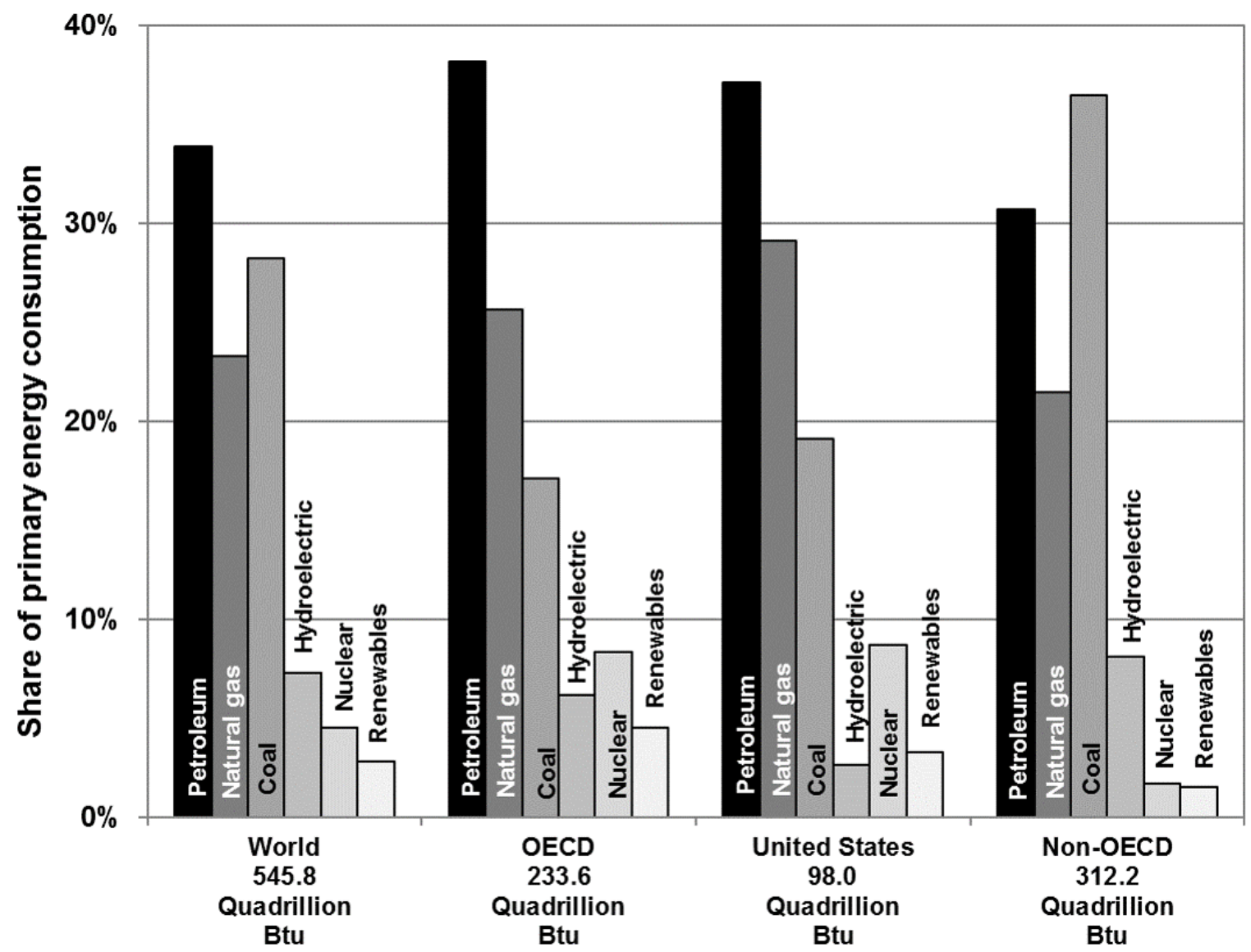

Note: The United States data are shown separately but are also included in the OECD data.

\section{Source:}

U.S. Department of Energy, Energy Information Administration, International Energy Statistics, August 2017. (Additional resources: www.eia.doe.gov) 
Total energy use was 97.4 quads in 2016 with transportation using 28.7\%. The Energy Information Administration includes renewable energy in each sector.

Table 2.1

\section{U. S. Consumption of Total Energy by End-Use Sector, 1973-2016 (quadrillion Btu)}

\begin{tabular}{|c|c|c|c|c|c|c|}
\hline Year & Transportation & $\begin{array}{c}\text { Percentage } \\
\text { transportation of } \\
\text { total }\end{array}$ & Industrial & Commercial & Residential & Total $^{\mathrm{a}}$ \\
\hline 1973 & 18.6 & $24.6 \%$ & 32.6 & 9.5 & 14.9 & 75.7 \\
\hline 1975 & 18.2 & $25.4 \%$ & 29.4 & 9.5 & 14.8 & 72.0 \\
\hline 1980 & 19.7 & $25.2 \%$ & 32.0 & 10.6 & 15.8 & 78.1 \\
\hline 1981 & 19.5 & $25.6 \%$ & 30.7 & 10.6 & 15.3 & 76.1 \\
\hline 1982 & 19.1 & $26.1 \%$ & 27.6 & 10.9 & 15.5 & 73.1 \\
\hline 1983 & 19.2 & $26.3 \%$ & 27.4 & 10.9 & 15.4 & 73.0 \\
\hline 1984 & 19.7 & $25.7 \%$ & 29.6 & 11.4 & 16.0 & 76.7 \\
\hline 1985 & 20.1 & $26.3 \%$ & 28.8 & 11.5 & 16.0 & 76.4 \\
\hline 1986 & 20.8 & $27.1 \%$ & 28.3 & 11.6 & 16.0 & 76.7 \\
\hline 1987 & 21.5 & $27.2 \%$ & 28.4 & 11.9 & 16.3 & 79.1 \\
\hline 1988 & 22.3 & $27.0 \%$ & 30.7 & 12.6 & 17.1 & 82.7 \\
\hline 1989 & 22.5 & $26.5 \%$ & 31.3 & 13.2 & 17.8 & 84.8 \\
\hline 1990 & 22.4 & $26.5 \%$ & 31.8 & 13.3 & 16.9 & 84.5 \\
\hline 1991 & 22.1 & $26.2 \%$ & 31.4 & 13.4 & 17.4 & 84.4 \\
\hline 1992 & 22.4 & $26.1 \%$ & 32.6 & 13.4 & 17.4 & 85.8 \\
\hline 1993 & 22.8 & $26.1 \%$ & 32.6 & 13.8 & 18.2 & 87.4 \\
\hline 1994 & 23.4 & $26.3 \%$ & 33.5 & 14.1 & 18.1 & 89.1 \\
\hline 1995 & 23.8 & $26.2 \%$ & 34.0 & 14.7 & 18.5 & 91.0 \\
\hline 1996 & 24.4 & $26.0 \%$ & 34.9 & 15.2 & 19.5 & 94.0 \\
\hline 1997 & 24.8 & $26.2 \%$ & 35.2 & 15.7 & 19.0 & 94.6 \\
\hline 1998 & 25.3 & $26.8 \%$ & 34.8 & 16.0 & 19.0 & 95.0 \\
\hline 1999 & 25.9 & $26.8 \%$ & 34.8 & 16.4 & 19.6 & 96.7 \\
\hline 2000 & 26.6 & $26.9 \%$ & 34.7 & 17.2 & 20.4 & 98.8 \\
\hline 2001 & 26.3 & $27.3 \%$ & 32.7 & 17.1 & 20.0 & 96.2 \\
\hline 2002 & 26.8 & $27.5 \%$ & 32.7 & 17.3 & 20.8 & 97.6 \\
\hline 2003 & 26.9 & $27.5 \%$ & 32.6 & 17.3 & 21.1 & 97.9 \\
\hline 2004 & 27.8 & $27.8 \%$ & 33.5 & 17.7 & 21.1 & 100.1 \\
\hline 2005 & 28.3 & $28.2 \%$ & 32.4 & 17.9 & 21.6 & 100.2 \\
\hline 2006 & 28.7 & $28.9 \%$ & 32.4 & 17.7 & 20.7 & 99.5 \\
\hline 2007 & 28.9 & $28.6 \%$ & 32.4 & 18.3 & 21.5 & 101.0 \\
\hline 2008 & 27.5 & $27.8 \%$ & 31.3 & 18.4 & 21.7 & 98.9 \\
\hline 2009 & 26.7 & $28.4 \%$ & 28.5 & 17.9 & 21.1 & 94.1 \\
\hline 2010 & 27.1 & $27.8 \%$ & 30.5 & 18.1 & 21.8 & 97.4 \\
\hline 2011 & 26.7 & $27.6 \%$ & 30.8 & 18.0 & 21.3 & 96.8 \\
\hline 2012 & 26.2 & $27.8 \%$ & 30.9 & 17.4 & 19.9 & 94.2 \\
\hline 2013 & 26.8 & $27.5 \%$ & 31.4 & 17.9 & 21.1 & 97.2 \\
\hline 2014 & 27.0 & $27.5 \%$ & 31.6 & 18.3 & 21.4 & 98.3 \\
\hline 2015 & 27.4 & $28.1 \%$ & 31.3 & 18.2 & 20.5 & 97.4 \\
\hline 2016 & 27.9 & $28.7 \%$ & 30.8 & 18.2 & 20.4 & 97.4 \\
\hline \multicolumn{7}{|c|}{ Average annual percentage change } \\
\hline 1973-2016 & $0.9 \%$ & & $-0.1 \%$ & $1.5 \%$ & $0.7 \%$ & $0.6 \%$ \\
\hline 2006-2016 & $-0.3 \%$ & & $-0.5 \%$ & $0.3 \%$ & $-0.1 \%$ & $-0.2 \%$ \\
\hline
\end{tabular}

Source:

U.S. Department of Energy, Energy Information Administration, Monthly Energy Review, July 2017, Washington, DC, Table 2.1. (Additional resources: www.eia.doe.gov)

${ }^{\text {a }}$ Electrical energy losses have been distributed among the sectors. 
In transportation, the alcohol fuels blended into gasoline to make gasohol (10\% ethanol or less) are counted under "renewables" and are not in with petroleum. The petroleum category, however, still contains other blending agents that are not actually petroleum, but are not broken out into a separate category.

Table 2.2

Distribution of Energy Consumption by Source, 1973 and 2016 (percentage)

\begin{tabular}{|c|c|c|c|c|c|c|}
\hline \multirow{2}{*}{$\begin{array}{l}\text { Energy } \\
\text { source }\end{array}$} & \multicolumn{2}{|c|}{ Transportation } & \multicolumn{2}{|c|}{ Residential } & \multicolumn{2}{|c|}{ Commercial } \\
\hline & 1973 & 2016 & 1973 & 2016 & 1973 & 2016 \\
\hline Petroleum $^{\mathrm{a}}$ & 95.8 & 91.9 & 18.8 & 4.7 & 16.8 & 4.7 \\
\hline Natural gas ${ }^{\mathrm{b}}$ & 4.0 & 2.7 & 33.4 & 22.3 & 27.8 & 17.8 \\
\hline Coal & 0.0 & 0.0 & 0.6 & 0.0 & 1.7 & 0.1 \\
\hline Renewable & 0.0 & 5.1 & 2.4 & 2.8 & 0.1 & 1.4 \\
\hline Nuclear & 0.0 & 0.0 & 0.0 & 0.0 & 0.0 & 0.0 \\
\hline Electricity ${ }^{\mathrm{c}}$ & 0.2 & 0.3 & 44.8 & 70.2 & 53.6 & 76.0 \\
\hline Total & 100.0 & 100.0 & 100.0 & 100.0 & 100.0 & 100.0 \\
\hline
\end{tabular}

\begin{tabular}{lrrrrr} 
Energy & \multicolumn{2}{c}{ Industrial } & & \multicolumn{2}{c}{ Electric utilities } \\
\cline { 2 - 3 } \cline { 5 - 6 } source & 1973 & 2016 & & 1973 & 2016 \\
\hline Petroleum $^{\mathrm{a}}$ & 27.9 & 26.6 & & 17.8 & 0.6 \\
Natural gas $^{\mathrm{b}}$ & 31.8 & 31.1 & & 19.0 & 27.3 \\
Coal & 12.4 & 3.9 & & 44.0 & 34.4 \\
Renewable & 3.7 & 7.5 & & 14.4 & 14.8 \\
Nuclear & 0.0 & 0.0 & & 4.6 & 22.3 \\
Electricity & 24.2 & 30.9 & & 0.2 & 0.6 \\
Total & 100.0 & 100.0 & & 100.0 & 100.0 \\
\hline
\end{tabular}

\section{Source:}

U.S. Department of Energy, Energy Information Administration, Monthly Energy Review, July 2017, Washington, DC, Tables 2.2, 2.3, 2.4, 2.5, and 2.6. (Additional resources: www.eia.doe.gov)

\footnotetext{
${ }^{a}$ In transportation, the petroleum category contains some blending agents which are not petroleum.

${ }^{\mathrm{b}}$ Includes supplemental gaseous fuels. Transportation sector includes pipeline fuel and natural gas vehicle use.

${ }^{\mathrm{c}}$ Includes electrical system energy losses.
} 
Total transportation energy consumption was 27.9 quads in 2016. Petroleum has accounted for more than $90 \%$ of transportation energy consumption since the mid-1950's. Renewables, including ethanol and biodiesel, were 5\% of the total in 2016.

Table 2.3. Distribution of Transportation Energy Consumption by Source, 1950-2016

\begin{tabular}{|c|c|c|c|c|c|c|}
\hline Year & Petroleum ${ }^{\mathrm{a}}$ & Natural gas & Coal & Renewables & Electricity $^{c}$ & $\begin{array}{c}\text { Total } \\
\text { (trillion Btu) }\end{array}$ \\
\hline 1950 & $78.8 \%$ & $1.5 \%$ & $18.4 \%$ & $0.0 \%$ & $1.3 \%$ & $8,492.5$ \\
\hline 1955 & $92.1 \%$ & $2.7 \%$ & $4.4 \%$ & $0.0 \%$ & $0.8 \%$ & $9,550.2$ \\
\hline 1960 & $95.6 \%$ & $3.4 \%$ & $0.7 \%$ & $0.0 \%$ & $0.3 \%$ & $10,595.9$ \\
\hline 1965 & $95.4 \%$ & $4.2 \%$ & $0.1 \%$ & $0.0 \%$ & $0.3 \%$ & $12,432.5$ \\
\hline 1970 & $95.1 \%$ & $4.6 \%$ & $0.0 \%$ & $0.0 \%$ & $0.2 \%$ & $16,098.2$ \\
\hline 1975 & $96.5 \%$ & $3.3 \%$ & $0.0 \%$ & $0.0 \%$ & $0.2 \%$ & $18,245.0$ \\
\hline 1980 & $96.5 \%$ & $3.3 \%$ & $0.0 \%$ & $0.0 \%$ & $0.2 \%$ & $19,696.7$ \\
\hline 1985 & $96.9 \%$ & $2.6 \%$ & $0.0 \%$ & $0.2 \%$ & $0.2 \%$ & $20,087.9$ \\
\hline 1986 & $97.1 \%$ & $2.4 \%$ & $0.0 \%$ & $0.3 \%$ & $0.2 \%$ & $20,788.8$ \\
\hline 1987 & $97.0 \%$ & $2.5 \%$ & $0.0 \%$ & $0.3 \%$ & $0.2 \%$ & $21,468.9$ \\
\hline 1988 & $96.6 \%$ & $2.8 \%$ & $0.0 \%$ & $0.3 \%$ & $0.2 \%$ & $22,317.7$ \\
\hline 1989 & $96.6 \%$ & $2.9 \%$ & $0.0 \%$ & $0.3 \%$ & $0.2 \%$ & $22,477.9$ \\
\hline 1990 & $96.5 \%$ & $3.0 \%$ & $0.0 \%$ & $0.3 \%$ & $0.2 \%$ & $22,419.6$ \\
\hline 1991 & $96.6 \%$ & $2.8 \%$ & $0.0 \%$ & $0.3 \%$ & $0.2 \%$ & $22,118.0$ \\
\hline 1992 & $96.7 \%$ & $2.7 \%$ & $0.0 \%$ & $0.4 \%$ & $0.2 \%$ & $22,415.1$ \\
\hline 1993 & $96.5 \%$ & $2.8 \%$ & $0.0 \%$ & $0.4 \%$ & $0.2 \%$ & $22,711.7$ \\
\hline 1994 & $96.3 \%$ & $3.0 \%$ & $0.0 \%$ & $0.4 \%$ & $0.2 \%$ & $23,365.1$ \\
\hline 1995 & $96.3 \%$ & $3.0 \%$ & $0.0 \%$ & $0.5 \%$ & $0.2 \%$ & $23,851.1$ \\
\hline 1996 & $96.4 \%$ & $3.0 \%$ & $0.0 \%$ & $0.3 \%$ & $0.2 \%$ & $24,439.2$ \\
\hline 1997 & $96.2 \%$ & $3.2 \%$ & $0.0 \%$ & $0.4 \%$ & $0.2 \%$ & $24,751.3$ \\
\hline 1998 & $96.7 \%$ & $2.6 \%$ & $0.0 \%$ & $0.4 \%$ & $0.2 \%$ & $25,260.1$ \\
\hline 1999 & $96.7 \%$ & $2.6 \%$ & $0.0 \%$ & $0.5 \%$ & $0.2 \%$ & $25,949.5$ \\
\hline 2000 & $96.7 \%$ & $2.5 \%$ & $0.0 \%$ & $0.5 \%$ & $0.2 \%$ & $26,555.3$ \\
\hline 2001 & $96.7 \%$ & $2.5 \%$ & $0.0 \%$ & $0.5 \%$ & $0.2 \%$ & $26,282.2$ \\
\hline 2002 & $96.5 \%$ & $2.6 \%$ & $0.0 \%$ & $0.6 \%$ & $0.2 \%$ & $26,845.7$ \\
\hline 2003 & $96.5 \%$ & $2.3 \%$ & $0.0 \%$ & $0.9 \%$ & $0.2 \%$ & $26,900.2$ \\
\hline 2004 & $96.5 \%$ & $2.2 \%$ & $0.0 \%$ & $1.0 \%$ & $0.3 \%$ & $27,842.8$ \\
\hline 2005 & $96.3 \%$ & $2.2 \%$ & $0.0 \%$ & $1.2 \%$ & $0.3 \%$ & $28,280.3$ \\
\hline 2006 & $95.9 \%$ & $2.2 \%$ & $0.0 \%$ & $1.7 \%$ & $0.3 \%$ & $28,716.7$ \\
\hline 2007 & $95.3 \%$ & $2.3 \%$ & $0.0 \%$ & $2.1 \%$ & $0.3 \%$ & $28,858.3$ \\
\hline 2008 & $94.2 \%$ & $2.5 \%$ & $0.0 \%$ & $3.0 \%$ & $0.3 \%$ & $27,486.3$ \\
\hline 2009 & $93.5 \%$ & $2.7 \%$ & $0.0 \%$ & $3.5 \%$ & $0.3 \%$ & $26,687.1$ \\
\hline 2010 & $93.1 \%$ & $2.7 \%$ & $0.0 \%$ & $4.0 \%$ & $0.3 \%$ & $27,059.1$ \\
\hline 2011 & $92.6 \%$ & $2.7 \%$ & $0.0 \%$ & $4.3 \%$ & $0.3 \%$ & $26,712.1$ \\
\hline 2012 & $92.3 \%$ & $3.0 \%$ & $0.0 \%$ & $4.4 \%$ & $0.3 \%$ & $26,219.3$ \\
\hline 2013 & $91.6 \%$ & $3.3 \%$ & $0.0 \%$ & $4.8 \%$ & $0.3 \%$ & $26,749.8$ \\
\hline 2014 & $92.1 \%$ & $2.8 \%$ & $0.0 \%$ & $4.8 \%$ & $0.3 \%$ & $26,996.5$ \\
\hline 2015 & $92.2 \%$ & $2.7 \%$ & $0.0 \%$ & $4.8 \%$ & $0.3 \%$ & $27,355.2$ \\
\hline 2016 & $91.9 \%$ & $2.6 \%$ & $0.0 \%$ & $5.1 \%$ & $0.3 \%$ & $27,925.3$ \\
\hline
\end{tabular}

\section{Source:}

U.S. Department of Energy, Energy Information Administration, Monthly Energy Review, July 2017, Washington, DC, Table 2.5. (Additional resources: www.eia.doe.gov)

\footnotetext{
${ }^{a}$ In transportation, the petroleum category contains some blending agents which are not petroleum.

${ }^{\mathrm{b}}$ Includes supplemental gaseous fuels. Transportation sector includes pipeline fuel and natural gas vehicle use.

${ }^{\mathrm{c}}$ Includes electrical system energy losses.
} 
Figure 2.2. World Natural Gas Reserves, Production, and Consumption, 1980

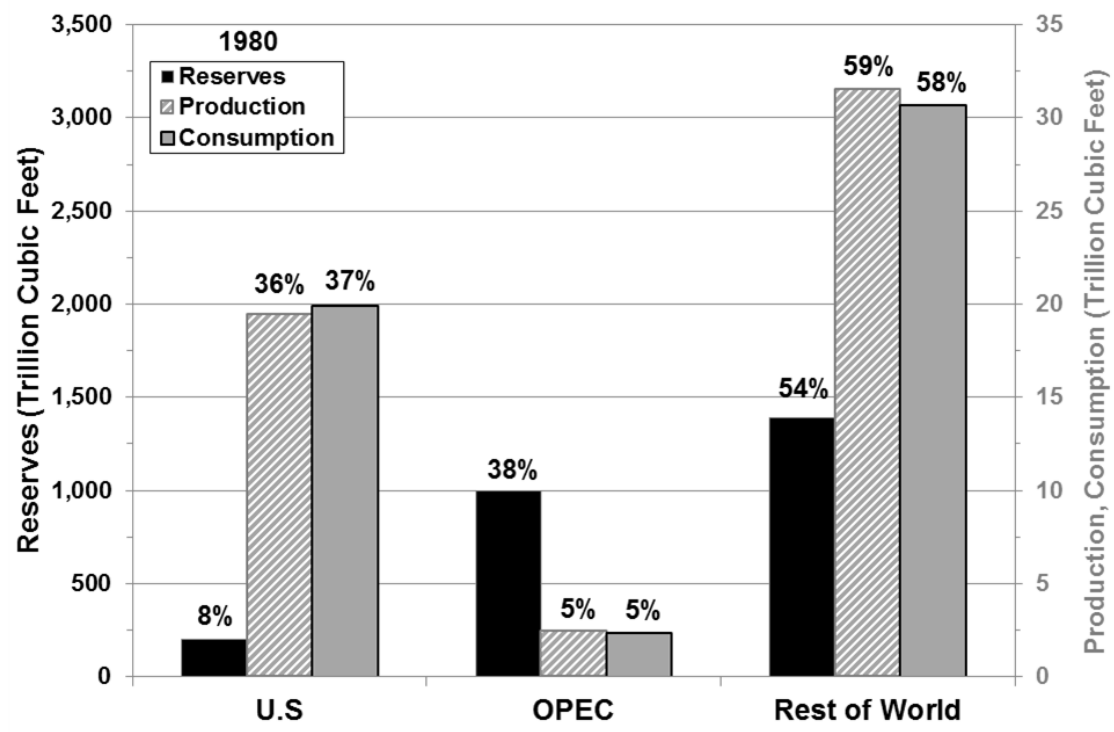

Source:

See Table 2.4 .

Figure 2.3. World Natural Gas Reserves, Production, and Consumption, 1997

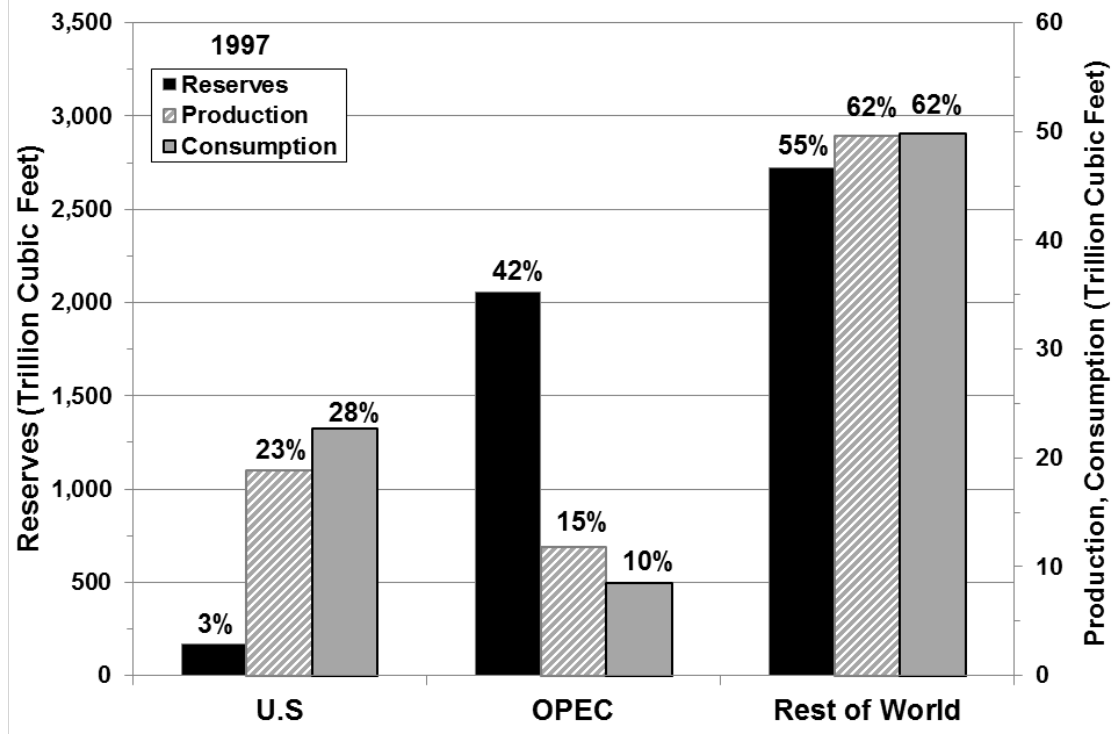

Source:

See Table 2.4. 
Figure 2.4. World Natural Gas Reserves, Production and Consumption, 2015

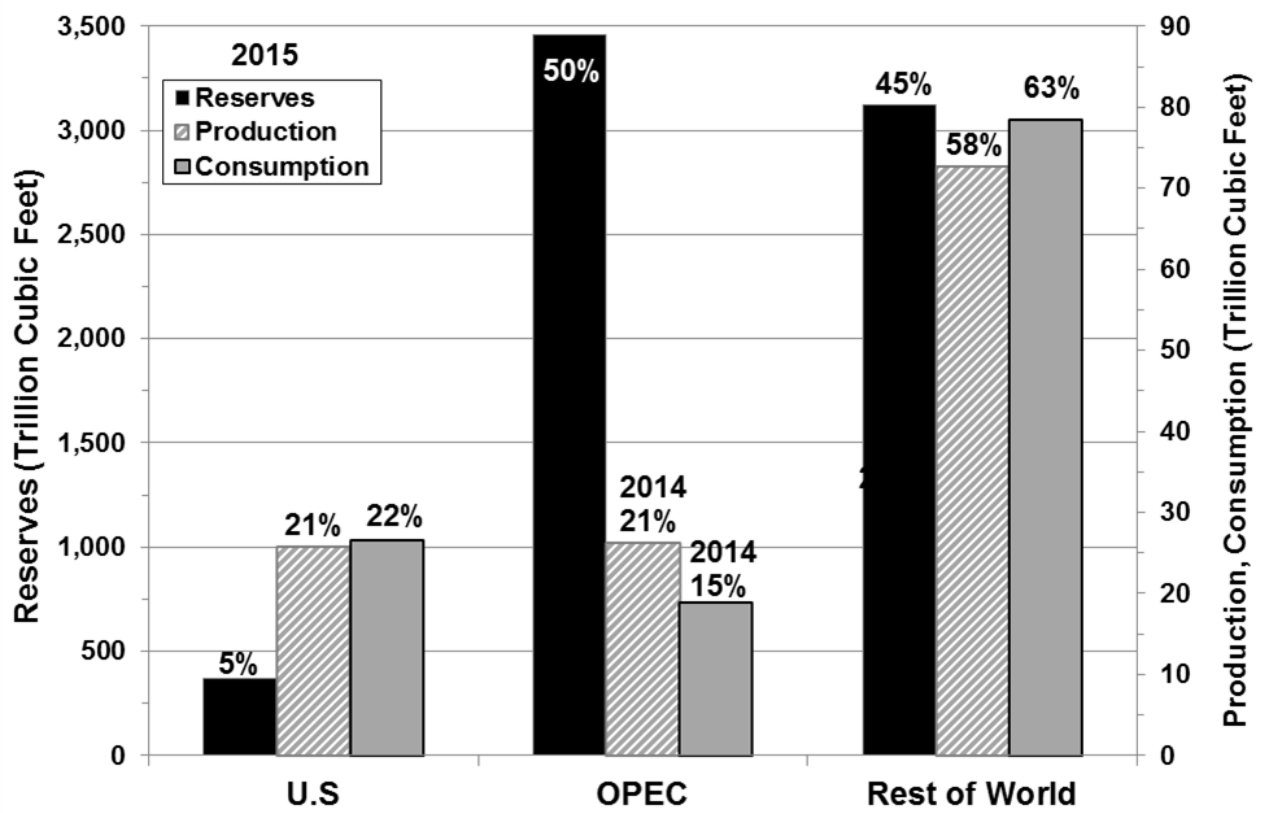

Source:

See Table 2.4.

Table 2.4

World Natural Gas Reserves, Production, and Consumption, 1980, 1997, and 2015

(trillion cubic feet)

\begin{tabular}{|c|c|c|c|c|c|c|}
\hline & $\begin{array}{c}\text { Natural } \\
\text { gas } \\
\text { reserves }\end{array}$ & $\begin{array}{c}\text { Reserve } \\
\text { share }\end{array}$ & $\begin{array}{l}\text { Natural gas } \\
\text { production }\end{array}$ & $\begin{array}{c}\text { Production } \\
\text { share }\end{array}$ & $\begin{array}{c}\text { Natural gas } \\
\text { consumption }\end{array}$ & $\begin{array}{c}\text { Consumption } \\
\text { share }\end{array}$ \\
\hline & \multicolumn{6}{|c|}{1980} \\
\hline United States & 201.0 & $8 \%$ & 19.4 & $36 \%$ & 19.9 & $37 \%$ \\
\hline OPEC & 997.1 & $38 \%$ & 2.4 & $5 \%$ & 2.4 & $5 \%$ \\
\hline \multirow[t]{2}{*}{ Rest of world } & $1,387.6$ & $54 \%$ & 31.5 & $59 \%$ & 30.7 & $58 \%$ \\
\hline & \multicolumn{6}{|c|}{1997} \\
\hline United States & 166.5 & $3 \%$ & 18.9 & $23 \%$ & 22.7 & $28 \%$ \\
\hline OPEC & $2,057.6$ & $42 \%$ & 11.9 & $15 \%$ & 8.5 & $10 \%$ \\
\hline \multirow[t]{2}{*}{ Rest of world } & $2,722.7$ & $55 \%$ & 49.7 & $62 \%$ & 49.9 & $62 \%$ \\
\hline & \multicolumn{6}{|c|}{2015} \\
\hline United States & 368.7 & $5 \%$ & 25.8 & $21 \%$ & 26.6 & $22 \%$ \\
\hline OPEC & $3,461.4$ & $50 \%$ & 26.2 & $21 \%$ & 18.9 & $15 \%$ \\
\hline Rest of world & $3,120.4$ & $45 \%$ & 72.6 & $58 \%$ & 78.4 & $63 \%$ \\
\hline
\end{tabular}

Note: Production data are dry gas production. OPEC production and consumption are 2014 data. See Glossary for OPEC countries.

\section{Source:}

Energy Information Administration, International Energy Statistics, and International Energy Outlook, August 2017.

(Additional resources: www.eia.doe.gov) 
In 2015, the United States and Russia were by far the top natural gas producing countries with more than triple that of any other country. Although the United States produced more than Russia, Russia has almost five times more reserves.

Figure 2.5. Natural Gas Production and Reserves for the Top Ten Natural Gas Producing Countries, 2015
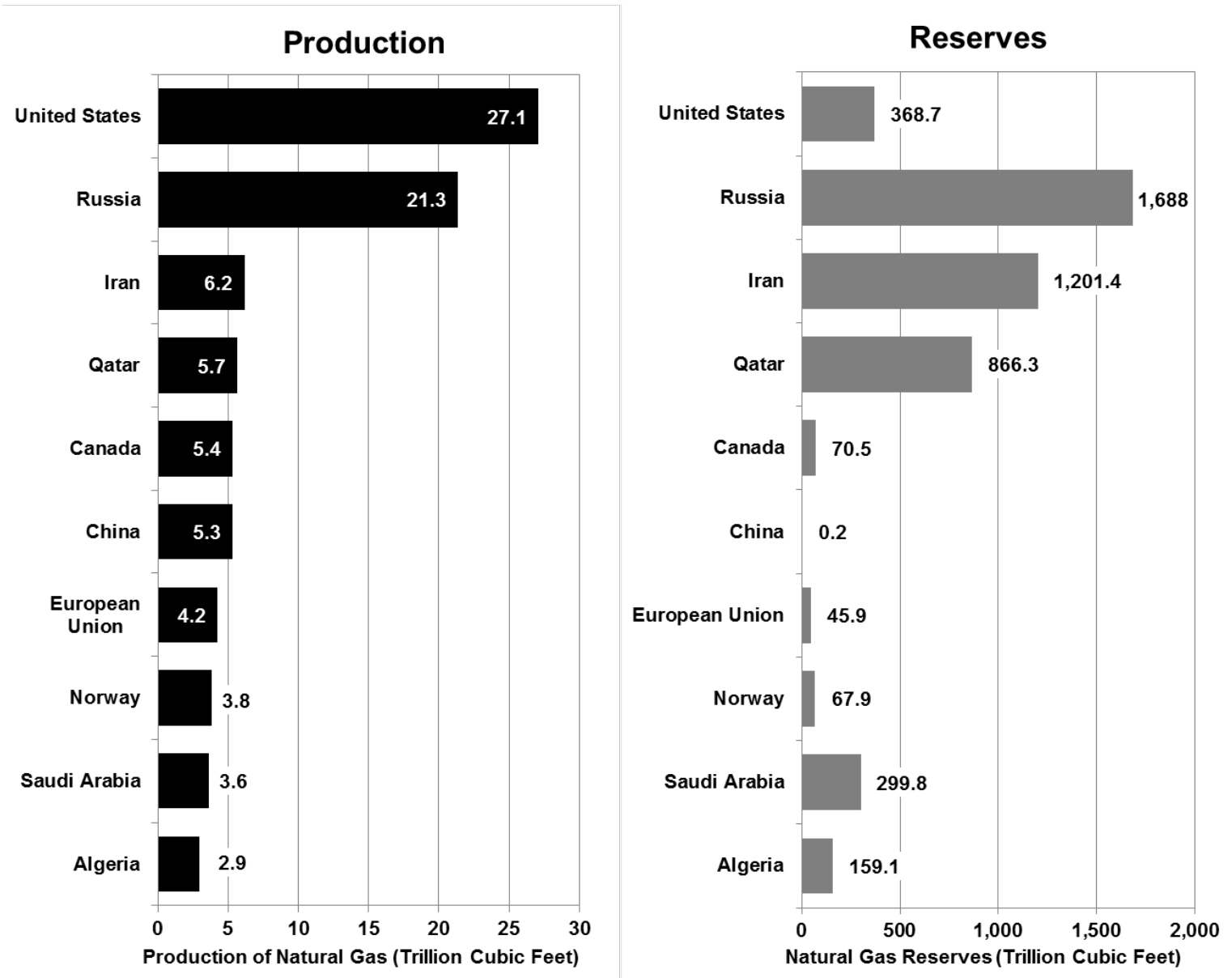

Note: The Energy Information Administration International Energy Statistics was formerly the source of this figure, but did not have 2015 production data available at the time of publication.

\section{Source:}

U.S. Central Intelligence Agency, The World Factbook, August 2017. (Additional resources: www.cia.gov/library/publications/the-world-factbook) 
The Energy Information Administration no longer publishes national data on alternative use. They do publish fuel use data for four types of alternative fuel vehicle fleets at www.eia.gov/renewable/afv.

Table 2.5

Alternative Fuel and Oxygenate Consumption, 2005-2011 (thousand gasoline-equivalent gallons)

\begin{tabular}{|c|c|c|c|c|c|c|c|}
\hline & 2005 & 2006 & 2007 & 2008 & 2009 & 2010 & 2011 \\
\hline \multicolumn{8}{|l|}{ Alternative fuel } \\
\hline Liquefied petroleum gas & 188,171 & 173,130 & 152,360 & 147,784 & 129,631 & 126,354 & 124,457 \\
\hline Compressed natural gas & 166,878 & 172,011 & 178,585 & 189,358 & 199,513 & 210,007 & 220,247 \\
\hline Liquefied natural gas & 22,409 & 23,474 & 24,594 & 25,554 & 25,652 & 26,072 & 26,242 \\
\hline $\mathrm{E}^{1} 5^{\mathrm{a}}$ & 38,074 & 44,041 & 54,091 & 62,464 & 71,213 & 90,323 & 137,165 \\
\hline Electricity ${ }^{\mathrm{b}}$ & 5,219 & 5,104 & 5,037 & 5,050 & 4,956 & 4,847 & 7,635 \\
\hline Hydrogen & 25 & 41 & 66 & 117 & 140 & 152 & 174 \\
\hline Biodiesel & 91,649 & 267,623 & 367,764 & 324,329 & 334,809 & 270,170 & 910,968 \\
\hline Other & 2 & 2 & 2 & 2 & 2 & 0 & 0 \\
\hline Subtotal & 512,427 & 685,426 & 782,479 & 754,658 & 756,916 & 727,925 & $1,426,888$ \\
\hline \multicolumn{8}{|l|}{ Oxygenates } \\
\hline $\mathrm{MTBE}^{\mathrm{c}}$ & $1,654,500$ & 435,000 & 0 & 0 & 0 & 0 & 0 \\
\hline Ethanol in gasohol & $2,756,663$ & $3,729,168$ & $4,694,304$ & $6,442,781$ & $7,343,133$ & $8,527,431$ & $8,563,841$ \\
\hline Total & $4,923,590$ & $4,849,594$ & $5,476,783$ & $7,197,439$ & $8,099,342$ & $9,255,356$ & $9,990,729$ \\
\hline
\end{tabular}

Note: These are the latest data available from the Energy Information Administration. See text box for additional information.

\section{Source:}

U.S. Department of Energy, Energy Information Administration, Alternative Fuel Vehicle Data website, May 2013, www.eia.doe.gov/renewable. (Additional resources: www.eia.doe.gov)

${ }^{\text {a }}$ Consumption includes gasoline portion of the mixture.

b Vehicle consumption only; does not include power plant inputs.

${ }^{c}$ Methyl Tertiary Butyl Ether. This category includes a very small amount of other ethers, primarily Tertiary Amyl Methyl Ether (TAME) and Ethyl Tertiary Butyl Ether (ETBE). 
Ethanol is an oxygenate blended with gasoline in amounts up to $10 \%$ to be used in conventional vehicles, and is blended in higher amounts up to $85 \%$ for use in flex-fuel vehicles. The production of ethanol grew to over 15 billion gallons in 2016, with consumption reaching over 14 billion gallons. Beginning in 2010, the United States began exporting more fuel ethanol than it imports. Biodiesel is a renewable fuel typically made from vegetable oils or animal fats. It can be burned in standard diesel engines and is often blended with petroleum diesel. In 2016, over 2.0 billion gallons of biodiesel were consumed.

Table 2.6

Fuel Ethanol and Biodiesel Production, Net Imports, and Consumption, 1981-2016 (million gallons)

\begin{tabular}{|c|c|c|c|c|c|c|}
\hline \multirow[b]{2}{*}{ Year } & \multicolumn{3}{|c|}{ Fuel ethanol } & \multicolumn{3}{|c|}{ Biodiesel } \\
\hline & Production & Net imports & Consumption & Production & Net imports & Consumption \\
\hline 1981 & 83.1 & $\mathrm{a}$ & 83.1 & $\mathrm{a}$ & $\mathrm{a}$ & $\mathrm{a}$ \\
\hline 1985 & 617.1 & $\mathrm{a}$ & 617.1 & a & a & a \\
\hline 1990 & 747.7 & a & 747.7 & a & a & a \\
\hline 1991 & 866.3 & a & 866.3 & a & $\mathrm{a}$ & a \\
\hline 1992 & 985.0 & a & 985.0 & $\mathrm{a}$ & $\mathrm{a}$ & a \\
\hline 1993 & $1,154.3$ & 10.2 & $1,151.0$ & a & $\mathrm{a}$ & a \\
\hline 1994 & $1,288.9$ & 11.7 & $1,288.9$ & a & a & $\mathrm{a}$ \\
\hline 1995 & $1,357.7$ & 16.3 & $1,382.6$ & a & a & a \\
\hline 1996 & 973.5 & 13.1 & 991.7 & a & $\mathrm{a}$ & a \\
\hline 1997 & $1,288.3$ & 3.6 & $1,255.8$ & $\mathrm{a}$ & $\mathrm{a}$ & $\mathrm{a}$ \\
\hline 1998 & $1,405.0$ & 2.8 & $1,387.6$ & a & a & a \\
\hline 1999 & $1,465.0$ & 3.7 & $1,442.7$ & a & a & a \\
\hline 2000 & $1,622.3$ & 4.9 & $1,653.4$ & a & $\mathrm{a}$ & $\mathrm{a}$ \\
\hline 2001 & $1,765.2$ & 13.2 & $1,740.7$ & 8.6 & 1.7 & 10.3 \\
\hline 2002 & $2,140.2$ & 12.9 & $2,073.1$ & 10.5 & 5.9 & 16.4 \\
\hline 2003 & $2,804.4$ & 12.3 & $2,826.0$ & 14.2 & $(0.7)$ & 13.5 \\
\hline 2004 & $3,404.4$ & 148.8 & $3,552.2$ & 28.0 & (1.1) & 26.8 \\
\hline 2005 & $3,904.4$ & 135.8 & 4,058.6 & 90.8 & 0.0 & 90.8 \\
\hline 2006 & $4,884.3$ & 731.1 & $5,481.2$ & 250.4 & 10.5 & 260.9 \\
\hline 2007 & $6,521.0$ & 439.2 & $6,885.7$ & 489.8 & $(136.1)$ & 353.7 \\
\hline 2008 & $9,308.8$ & 529.6 & $9,683.4$ & 678.1 & (374.6) & 303.6 \\
\hline 2009 & $10,937.8$ & 198.2 & $11,036.6$ & 515.8 & (194.9) & 321.8 \\
\hline 2010 & $13,297.9$ & (382.8) & $12,858.5$ & 343.4 & $(85.0)$ & 260.1 \\
\hline 2011 & $13,929.1$ & $(1,023.3)$ & $12,893.3$ & 967.5 & (38.1) & 886.2 \\
\hline 2012 & $13,218.0$ & (247.4) & $12,881.9$ & 990.7 & (92.5) & 899.0 \\
\hline 2013 & $13,292.7$ & (242.0) & $13,215.6$ & 1,359.5 & 146.0 & $1,428.8$ \\
\hline 2014 & $14,312.8$ & (771.6) & $13,444.0$ & $1,279.0$ & 109.4 & $1,416.9$ \\
\hline 2015 & $14,807.2$ & (740.5) & $13,946.7$ & 1,263.3 & 246.9 & $1,494.2$ \\
\hline \multirow[t]{2}{*}{2016} & $15,329.1$ & $(1,010.1)$ & $14,399.1$ & $1,555.5$ & 605.0 & $2,059.6$ \\
\hline & \multicolumn{6}{|c|}{ Average annual percentage change } \\
\hline $1981-2016$ & $16.1 \%$ & a & $15.9 \%$ & a & a & a \\
\hline 2006-2016 & $12.1 \%$ & a & $10.1 \%$ & $20.0 \%$ & $50.0 \%$ & $23.0 \%$ \\
\hline
\end{tabular}

Note: The Energy Information Administration no longer publishes the nationwide consumption of E-85. Net imports are total imports minus exports.

\section{Source:}

U.S. Department of Energy, Energy Information Administration, Monthly Energy Review, Washington, DC, July 2017, Table 10.3. (Additional resources: www.eia.doe.gov)

${ }^{\text {a }}$ Data are not available. 
As data about alternative fuel use become available, an attempt is made to incorporate them into this table. Sometimes assumptions must be made in order to use the data. Please see Appendix A for a description of the methodology used to develop these data. See Table 1.16 for transportation petroleum use in thousand barrels per day.

Table 2.7

Domestic Consumption of Transportation Energy by Mode and Fuel Type, 2015 (trillion Btu)

\begin{tabular}{|c|c|c|c|c|c|c|c|c|}
\hline & Gasoline & $\begin{array}{c}\text { Diesel } \\
\text { fuel }\end{array}$ & $\begin{array}{l}\text { Liquefied } \\
\text { petroleum } \\
\text { gas }\end{array}$ & Jet fuel & $\begin{array}{l}\text { Residual } \\
\text { fuel oil }\end{array}$ & $\begin{array}{l}\text { Natural } \\
\text { gas }\end{array}$ & Electricity $^{\mathrm{b}}$ & Total $^{\mathrm{c}}$ \\
\hline HIGHWAY & $15,343.4$ & $5,947.2$ & 70.1 & - & - & 21.9 & 3.7 & $21,386.3$ \\
\hline$\overline{\text { Light vehicles }}$ & $14,733.1$ & 414.1 & 49.5 & - & - & - & 3.4 & $15,200.1$ \\
\hline Cars & $6,754.8$ & 38.3 & & & & & 3.4 & $6,796.5$ \\
\hline Light trucks ${ }^{\mathrm{d}}$ & $7,922.3$ & 375.8 & 49.5 & & & & 0.0 & $8,347.6$ \\
\hline Motorcycles & 56.0 & & & & & & & 56.0 \\
\hline Buses & 8.9 & 170.9 & 0.7 & - & - & 21.9 & 0.3 & 202.7 \\
\hline Transit & 1.1 & 57.4 & 0.7 & & & 21.9 & 0.3 & 81.5 \\
\hline Intercity & & 35.1 & & & & & & 35.1 \\
\hline School & 7.8 & 78.3 & & & & & & 86.1 \\
\hline Medium/heavy trucks & 601.3 & $5,362.3$ & 20.0 & - & - & - & - & $5,983.6$ \\
\hline Class 3-6 trucks & 553.2 & 750.7 & 19.8 & & & & & $1,323.7$ \\
\hline Class 7-8 trucks & 48.1 & $4,611.5$ & 0.2 & & & & & $4,659.9$ \\
\hline NONHIGHWAY & 214.6 & 927.3 & - & $2,095.6$ & 464.6 & 686.9 & 104.2 & $4,493.2$ \\
\hline$\overline{\text { Air }}$ & 22.0 & - & - & $2,095.6$ & - & - & - & $2,117.7$ \\
\hline General aviation & 22.0 & & & 186.9 & & & & 208.9 \\
\hline Domestic air carriers & & & & $1,475.4$ & & & & $1,475.4$ \\
\hline International air carriers ${ }^{\mathrm{e}}$ & & & & 433.4 & & & & 433.4 \\
\hline Water & 192.6 & 388.4 & - & - & 464.6 & - & - & $1,045.6$ \\
\hline Freight & & 335.0 & & & 464.6 & & & 799.6 \\
\hline Recreational & 192.6 & 53.4 & & & & & & 246.0 \\
\hline Pipeline & - & - & - & - & - & 686.9 & 80.3 & 767.1 \\
\hline Rail & - & 538.8 & - & - & - & - & 24.0 & 562.8 \\
\hline Freight (Class I) & & 516.4 & & & & & & 516.4 \\
\hline Passenger & & 22.4 & & & & & 24.0 & 46.3 \\
\hline Transit & & & & & & & 16.1 & 16.1 \\
\hline Commuter & & 13.3 & & & & & 6.1 & 19.4 \\
\hline Intercity & & 9.1 & & & & & 1.8 & 10.9 \\
\hline $\begin{array}{l}\text { TOTAL HWY \& } \\
\text { NONHWYc }^{c}\end{array}$ & $15,558.0$ & $6,874.5$ & 70.1 & $2,095.6$ & 464.6 & 708.8 & 107.9 & $25,879.5$ \\
\hline
\end{tabular}

Source:

See Appendix A, Section 2. Energy Use Sources.

a Civilian consumption only. Totals may not include all possible uses of fuels for transportation (e.g., snowmobiles).

${ }^{\mathrm{b}}$ Only end-use energy was counted for electricity. Previous editions included primary energy use for electricity which included generation and distribution losses.

c Totals may not sum due to rounding.

d Two-axle, four-tire trucks.

e One half of fuel used by domestic carriers in international operation. 
The gasoline and diesel used in highway modes accounts for the majority of transportation energy use (81.6\%) and nearly all highway use.

Figure 2.6. Domestic Consumption of Transportation Energy Use by Mode and Fuel Type, 2015

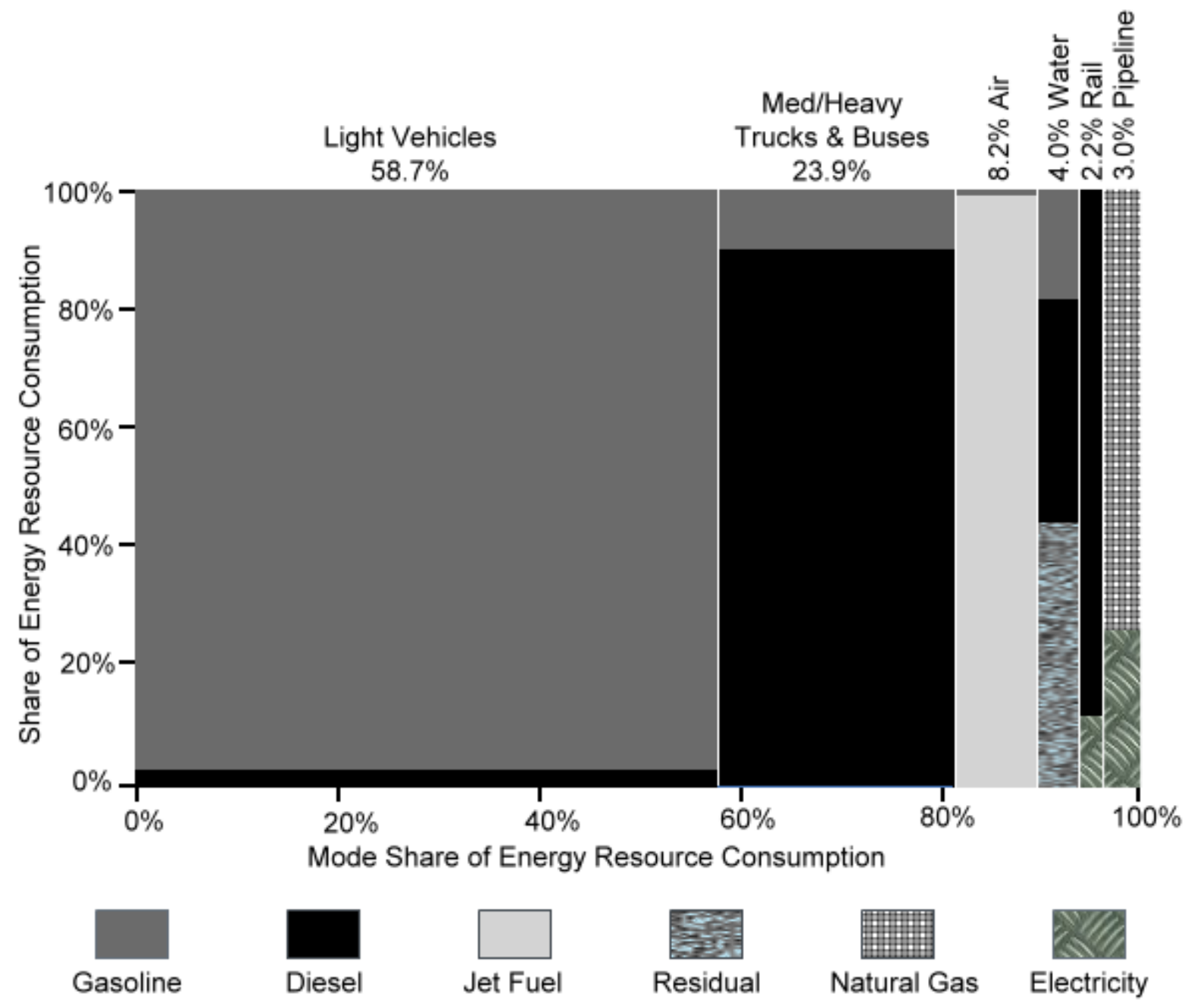

Note: Residual fuel oil is heavier oil which can be used in vessel bunkering.

\section{Source:}

See Table 2.7 or Appendix A, Section 2. Energy Use Sources.

a Civilian consumption only. Totals may not include all possible uses of fuels for transportation (e.g., snowmobiles). Only end-use energy was counted for electricity. Previous editions included primary energy use for electricity which included generation and distribution losses. 
Nonhighway modes were responsible for $18.0 \%$ of all transportation energy use in 2015. See Table 1.16 for transportation energy use in thousand barrels per day.

Table 2.8

Transportation Energy Use by Mode, 2014-2015

\begin{tabular}{|c|c|c|c|c|}
\hline & \multicolumn{2}{|c|}{ Trillion Btu } & \multicolumn{2}{|c|}{ Percentage of total based on Btus } \\
\hline & 2014 & 2015 & 2014 & 2015 \\
\hline HIGHWAY & $21,741.7$ & $21,386.3$ & $83.3 \%$ & $82.6 \%$ \\
\hline$\overline{\text { Light vehicles }}$ & $15,514.5$ & $15,200.1$ & $59.4 \%$ & $58.7 \%$ \\
\hline Cars & $6,951.2$ & $6,796.5$ & $26.6 \%$ & $26.3 \%$ \\
\hline Light trucks ${ }^{\mathrm{b}}$ & $8,505.9$ & $8,347.6$ & $32.6 \%$ & $32.3 \%$ \\
\hline Motorcycles & 57.3 & 56.0 & $0.2 \%$ & $0.2 \%$ \\
\hline Buses & 205.6 & 202.7 & $0.8 \%$ & $0.8 \%$ \\
\hline Transit & 86.2 & 81.5 & $0.3 \%$ & $0.3 \%$ \\
\hline Intercity & 34.6 & 35.1 & $0.1 \%$ & $0.1 \%$ \\
\hline School & 84.9 & 86.1 & $0.3 \%$ & $0.3 \%$ \\
\hline Medium/heavy trucks & $6,021.6$ & $5,983.6$ & $23.1 \%$ & $23.1 \%$ \\
\hline Class 3-6 trucks & $1,332.1$ & 1,323.7 & $5.1 \%$ & $5.1 \%$ \\
\hline Class 7-8 trucks & $4,689.5$ & $4,659.9$ & $18.0 \%$ & $18.0 \%$ \\
\hline NONHIGHWAY & $4,367.5$ & $4,493.2$ & $16.7 \%$ & $17.4 \%$ \\
\hline$\overline{\text { Air }}$ & $2,059.6$ & $2,117.7$ & $7.9 \%$ & $8.2 \%$ \\
\hline General aviation & 221.0 & 208.9 & $0.8 \%$ & $0.8 \%$ \\
\hline Domestic air carriers & $1,411.9$ & $1,475.4$ & $5.4 \%$ & $5.7 \%$ \\
\hline International air & 426.7 & 433.4 & $1.6 \%$ & $1.7 \%$ \\
\hline Water & 918.2 & $1,045.6$ & $3.5 \%$ & $4.0 \%$ \\
\hline Freight & 672.8 & 799.6 & $2.6 \%$ & $3.1 \%$ \\
\hline Recreational & 245.4 & 246.0 & $0.9 \%$ & $1.0 \%$ \\
\hline Pipeline & 802.6 & 767.1 & $3.1 \%$ & $3.0 \%$ \\
\hline Rail & 587.0 & 562.8 & $2.2 \%$ & $2.2 \%$ \\
\hline Freight (Class I) & 540.5 & 516.4 & $2.1 \%$ & $2.0 \%$ \\
\hline Passenger & 46.5 & 46.3 & $0.2 \%$ & $0.2 \%$ \\
\hline Transit & 16.4 & 16.1 & $0.1 \%$ & $0.1 \%$ \\
\hline Commuter & 19.2 & 19.4 & $0.1 \%$ & $0.1 \%$ \\
\hline Intercity & 11.0 & 10.9 & $0.0 \%$ & $0.0 \%$ \\
\hline HWY \& NONHWY TOTAL & $26,109.3$ & $25,879.5$ & $100.0 \%$ & $100.0 \%$ \\
\hline Off-highway & $2,194.3$ & $2,234.3$ & & \\
\hline
\end{tabular}

Source:

See Appendix A, Section 2. Energy Use Sources.

a Civilian consumption only. Totals may not include all possible uses of fuels for transportation (e.g., snowmobiles). Only end-use energy was counted for electricity. Previous editions included primary energy use for electricity which included generation and distribution losses.

b Two-axle, four-tire trucks. 
Light trucks include pick-ups, minivans, sport-utility vehicles, and vans. See Table 1.14 for highway petroleum use in thousand barrels per day.

Table 2.9

Highway Transportation Energy Consumption by Mode, 1970-2015 (trillion Btu)

\begin{tabular}{|c|c|c|c|c|c|c|c|c|c|c|c|}
\hline Year & Cars & $\begin{array}{l}\text { Light } \\
\text { trucks }\end{array}$ & $\begin{array}{c}\text { Light } \\
\text { vehicles } \\
\text { subtotal }\end{array}$ & $\begin{array}{l}\text { Motor- } \\
\text { cycles }\end{array}$ & Buses & $\begin{array}{c}\text { Class } \\
3-6 \\
\text { trucks } \\
\end{array}$ & $\begin{array}{c}\text { Class } \\
7-8 \\
\text { trucks }\end{array}$ & $\begin{array}{c}\text { Heavy } \\
\text { trucks } \\
\text { subtotal }\end{array}$ & & $\begin{array}{c}\text { Highway } \\
\text { subtotal }\end{array}$ & $\begin{array}{c}\text { Total } \\
\text { transportation }^{\mathrm{a}}\end{array}$ \\
\hline 1970 & 8,479 & 1,539 & 10,018 & 7 & 129 & 333 & 1,220 & 1,553 & & 11,707 & 15,192 \\
\hline 1975 & 9,298 & 2,384 & 11,682 & 14 & 124 & 430 & 1,574 & 2,003 & & 13,823 & 17,204 \\
\hline 1980 & 8,800 & 2,975 & 11,775 & 26 & 143 & 929 & 1,757 & 2,686 & & 14,630 & 18,760 \\
\hline 1981 & 8,693 & 2,963 & 11,656 & 27 & 145 & 1,065 & 1,659 & 2,724 & & 14,552 & 18,558 \\
\hline 1982 & 8,673 & 2,837 & 11,510 & 25 & 151 & 1,182 & 1,525 & 2,707 & & 14,393 & 18,055 \\
\hline 1983 & 8,802 & 2,990 & 11,792 & 22 & 152 & 1,121 & 1,649 & 2,770 & & 14,736 & 18,188 \\
\hline 1984 & 8,837 & 3,197 & 12,034 & 22 & 146 & 1,072 & 1,801 & 2,873 & & 15,075 & 18,773 \\
\hline 1985 & 8,932 & 3,413 & 12,345 & 23 & 153 & 986 & 1,897 & 2,883 & & 15,404 & 19,017 \\
\hline 1986 & 9,138 & 3,629 & 12,767 & 23 & 160 & 920 & 2,038 & 2,958 & & 15,908 & 20,086 \\
\hline 1987 & 9,157 & 3,819 & 12,976 & 24 & 164 & 858 & 2,203 & 3,061 & & 16,225 & 20,578 \\
\hline 1988 & 9,158 & 4,078 & 13,236 & 25 & 169 & 860 & 2,257 & 3,118 & & 16,548 & 21,131 \\
\hline 1989 & 9,232 & 4,156 & 13,388 & 26 & 169 & 869 & 2,330 & 3,199 & & 16,782 & 21,487 \\
\hline 1990 & 8,688 & 4,451 & 13,139 & 24 & 167 & 891 & 2,442 & 3,334 & & 16,664 & 21,383 \\
\hline 1991 & 8,029 & 4,774 & 12,803 & 23 & 177 & 895 & 2,507 & 3,402 & & 16,405 & 20,985 \\
\hline 1992 & 8,169 & 5,117 & 13,286 & 24 & 184 & 897 & 2,570 & 3,468 & & 16,962 & 21,646 \\
\hline 1993 & 8,368 & 5,356 & 13,724 & 25 & 183 & 906 & 2,671 & 3,577 & & 17,509 & 22,125 \\
\hline 1994 & 8,470 & 5,515 & 13,985 & 26 & 183 & 936 & 2,842 & 3,778 & & 17,972 & 22,729 \\
\hline 1995 & 8,489 & 5,695 & 14,184 & 25 & 184 & 954 & 2,983 & 3,937 & & 18,330 & 23,263 \\
\hline 1996 & 8,634 & 5,917 & 14,551 & 24 & 186 & 958 & 3,088 & 4,045 & & 18,806 & 23,773 \\
\hline 1997 & 8,710 & 6,169 & 14,879 & 25 & 192 & 945 & 3,141 & 4,086 & & 19,182 & 24,126 \\
\hline 1998 & 8,936 & 6,303 & 15,239 & 26 & 196 & 967 & 3,251 & 4,218 & & 19,679 & 24,461 \\
\hline 1999 & 9,134 & 6,602 & 15,736 & 26 & 203 & 1,054 & 3,584 & 4,638 & & 20,603 & 25,758 \\
\hline 2000 & 9,100 & 6,607 & 15,707 & 26 & 209 & 1,085 & 3,734 & 4,819 & & 20,761 & 26,069 \\
\hline 2001 & 9,161 & 6,678 & 15,839 & 24 & 196 & 1,074 & 3,738 & 4,813 & & 20,872 & 25,741 \\
\hline 2002 & 9,391 & 6,883 & 16,274 & 24 & 192 & 1,114 & 3,921 & 5,035 & & 21,525 & 26,331 \\
\hline 2003 & 9,255 & 7,551 & 16,806 & 24 & 190 & 1,083 & 3,812 & 4,895 & & 21,915 & 26,512 \\
\hline 2004 & 9,331 & 7,861 & 17,192 & 25 & 194 & 1,003 & 3,532 & 4,535 & & 21,946 & 26,970 \\
\hline 2005 & 9,579 & 7,296 & 16,875 & 24 & 196 & 1,126 & 3,963 & 5,088 & & 22,183 & 27,377 \\
\hline 2006 & 9,316 & 7,550 & 16,866 & 28 & 199 & 1,149 & 4,045 & 5,193 & $\mathrm{~b}$ & 22,286 & 27,554 \\
\hline 2007 & 9,221 & 7,679 & 16,900 & 59 & 195 & 1,429 & 5,031 & 6,460 & & 23,615 & 29,013 \\
\hline 2008 & 8,831 & 7,572 & 16,404 & 61 & 200 & 1,444 & 5,083 & 6,527 & & 23,192 & 28,381 \\
\hline 2009 & 8,209 & 7,635 & 15,843 & 60 & 200 & 1,341 & 4,720 & 6,061 & & 22,165 & 26,895 \\
\hline 2010 & 7,657 & 7,971 & 15,628 & 53 & 190 & 1,363 & 4,797 & 6,160 & & 22,032 & 26,974 \\
\hline 2011 & 7,336 & 8,104 & 15,440 & 53 & 195 & 1,283 & 4,517 & 5,801 & & 21,489 & 26,388 \\
\hline 2012 & 7,121 & 8,180 & 15,300 & 61 & 200 & 1,282 & 4,512 & 5,794 & & 21,356 & 26,001 \\
\hline 2013 & 7,047 & 8,077 & 15,124 & 58 & 204 & 1,310 & 4,613 & 5,924 & & 21,310 & 25,905 \\
\hline 2014 & 6,951 & 8,506 & 15,454 & 57 & 206 & 1,332 & 4,689 & 6,022 & & 21,742 & 26,109 \\
\hline 2015 & 6,802 & 8,348 & 15,141 & 56 & 203 & 1,324 & 4,660 & 5,984 & & 21,392 & 25,880 \\
\hline \multicolumn{12}{|c|}{ Average annual percentage change } \\
\hline $1970-2015$ & $-0.5 \%$ & $3.8 \%$ & $0.9 \%$ & $4.7 \%$ & $1.0 \%$ & $3.1 \%$ & $3.0 \%$ & $3.0 \%$ & & $1.3 \%$ & $1.2 \%$ \\
\hline $2005-2015$ & $-3.4 \%$ & $1.4 \%$ & $-1.1 \%$ & $8.8 \%$ & $0.4 \%$ & $1.6 \%$ & $1.6 \%$ & $1.6 \%$ & & $-0.4 \%$ & $-0.6 \%$ \\
\hline
\end{tabular}

Note: Totals may not add due to rounding.

\section{Source:}

See Appendix A, Section 2.1 Highway Energy Use.

a Total transportation figures do not include military and off-highway energy use and may not include all possible uses of fuel for transportation (e.g., snowmobiles). Only end-use energy was counted for electricity. Previous editions included primary energy use for electricity which included generation and distribution losses.

${ }^{\mathrm{b}}$ Due to changes in the FHWA fuel use methodology, motorcycle, bus, and heavy truck data are not comparable with data before the year 2007. Car and light truck data changed after 2008; see Appendix A for car/light truck shares. 
About $18 \%$ of transportation energy use is for nonhighway modes. Air travel accounts for almost $45 \%$ of nonhighway energy use. See Table 1.15 for nonhighway petroleum use in thousand barrels per day.

Table 2.10

Nonhighway Transportation Energy Consumption by Mode, 1970-2015 (trillion Btu)

\begin{tabular}{|c|c|c|c|c|c|c|}
\hline Year & Air & Water & Pipeline & Rail & $\begin{array}{c}\text { Nonhighway } \\
\text { subtotal }\end{array}$ & $\begin{array}{c}\text { Total } \\
\text { transportation }^{\mathrm{a}}\end{array}$ \\
\hline 1970 & 1,287 & 836 & 826 & 537 & 3,486 & 15,192 \\
\hline 1975 & 1,234 & 927 & 680 & 540 & 3,381 & 17,204 \\
\hline 1980 & 1,434 & 1,393 & 734 & 570 & 4,130 & 18,760 \\
\hline 1981 & 1,453 & 1,270 & 742 & 541 & 4,006 & 18,558 \\
\hline 1982 & 1,445 & 1,063 & 694 & 460 & 3,662 & 18,055 \\
\hline 1983 & 1,440 & 974 & 583 & 455 & 3,452 & 18,188 \\
\hline 1984 & 1,609 & 964 & 623 & 502 & 3,699 & 18,773 \\
\hline 1985 & 1,677 & 871 & 597 & 468 & 3,613 & 19,017 \\
\hline 1986 & 1,823 & 1,323 & 578 & 454 & 4,178 & 20,086 \\
\hline 1987 & 1,899 & 1,378 & 613 & 464 & 4,354 & 20,578 \\
\hline 1988 & 1,978 & 1,417 & 712 & 476 & 4,583 & 21,131 \\
\hline 1989 & 1,981 & 1,516 & 729 & 478 & 4,705 & 21,487 \\
\hline 1990 & 2,046 & 1,442 & 760 & 471 & 4,719 & 21,383 \\
\hline 1991 & 1,916 & 1,523 & 699 & 442 & 4,580 & 20,985 \\
\hline 1992 & 1,945 & 1,599 & 685 & 455 & 4,684 & 21,646 \\
\hline 1993 & 1,986 & 1,437 & 723 & 469 & 4,615 & 22,125 \\
\hline 1994 & 2,075 & 1,394 & 787 & 502 & 4,758 & 22,729 \\
\hline 1995 & 2,141 & 1,468 & 803 & 523 & 4,935 & 23,263 \\
\hline 1996 & 2,206 & 1,411 & 814 & 536 & 4,967 & 23,773 \\
\hline 1997 & 2,300 & 1,250 & 856 & 537 & 4,943 & 24,126 \\
\hline 1998 & 2,275 & 1,232 & 735 & 540 & 4,782 & 24,461 \\
\hline 1999 & 2,483 & 1,367 & 745 & 560 & 5,156 & 25,758 \\
\hline 2000 & 2,554 & 1,454 & 742 & 559 & 5,309 & 26,069 \\
\hline 2001 & 2,397 & 1,186 & 724 & 561 & 4,869 & 25,741 \\
\hline 2002 & 2,229 & 1,247 & 768 & 563 & 4,807 & 26,331 \\
\hline 2003 & 2,260 & 1,074 & 689 & 575 & 4,597 & 26,512 \\
\hline 2004 & 2,456 & 1,299 & 662 & 607 & 5,024 & 26,970 \\
\hline 2005 & 2,532 & 1,368 & 681 & 613 & 5,194 & 27,377 \\
\hline 2006 & 2,511 & 1,450 & 681 & 626 & 5,269 & 27,554 \\
\hline 2007 & 2,509 & 1,559 & 720 & 610 & 5,399 & 29,013 \\
\hline 2008 & 2,396 & 1,460 & 748 & 586 & 5,190 & 28,381 \\
\hline 2009 & 2,127 & 1,340 & 771 & 492 & 4,731 & 26,895 \\
\hline 2010 & 2,149 & 1,485 & 775 & 533 & 4,942 & 26,974 \\
\hline 2011 & 2,157 & 1,392 & 790 & 560 & 4,900 & 26,388 \\
\hline 2012 & 2,077 & 1,183 & 835 & 549 & 4,644 & 26,001 \\
\hline 2013 & 2,037 & 1,055 & 942 & 562 & 4,595 & 25,905 \\
\hline 2014 & 2,060 & 918 & 803 & 587 & 4,368 & 26,109 \\
\hline 2015 & 2,118 & 1,046 & 767 & 563 & 4,493 & 25,880 \\
\hline \multicolumn{7}{|c|}{ Average annual percentage change } \\
\hline 1970-2015 & $1.1 \%$ & $0.5 \%$ & $-0.2 \%$ & $0.1 \%$ & $0.6 \%$ & $1.2 \%$ \\
\hline $2005-2015$ & $-1.8 \%$ & $-2.6 \%$ & $1.2 \%$ & $-0.8 \%$ & $-1.4 \%$ & $-0.6 \%$ \\
\hline
\end{tabular}

Note: Totals may not add due to rounding.

Source:

See Appendix A, Section 2.3 Nonhighway Energy Use.

a Total transportation figures do not include military and off-highway energy use and may not include all possible uses of fuel for transportation (e.g., snowmobiles). Only end-use energy was counted for electricity. Previous editions included primary energy use for electricity which included generation and distribution losses. 
The Environmental Protection Agency's MOVES model estimates fuel use for different types of nonroad equipment and off-highway vehicles. Most of these vehicles/equipment use diesel fuel. Recreational equipment, such as offhighway motorcycles, snowmobiles, and all-terrain vehicles, are mainly fueled by gasoline.

Table 2.11

Off-Highway Transportation-Related Fuel Consumption from the Nonroad Model, 2015 (trillion Btu)

\begin{tabular}{|c|c|c|c|c|c|}
\hline & Gasoline & Diesel & LPG & CNG & Total \\
\hline $\begin{array}{l}\text { Agricultural equipment } \\
\text { Tractors, mowers, combines, balers, and other farm } \\
\text { equipment which has utility in its movement. }\end{array}$ & 8.6 & 610.6 & 0.0 & 0.0 & 619.3 \\
\hline Airport ground equipment & 0.3 & 16.6 & 0.3 & a & 17.2 \\
\hline $\begin{array}{l}\text { Construction and mining equipment } \\
\text { Pavers, rollers, drill rigs, graders, backhoes, } \\
\text { excavators, cranes, mining equipment }\end{array}$ & 11.4 & 987.4 & 1.9 & a & $1,000.7$ \\
\hline $\begin{array}{l}\text { Industrial equipment } \\
\quad \text { Forklifts, terminal tractors, sweeper/scrubbers }\end{array}$ & 8.2 & 140.7 & 210.9 & 19.1 & 378.9 \\
\hline $\begin{array}{l}\text { Logging equipment } \\
\text { Feller/buncher/skidder }\end{array}$ & 1.8 & 22.2 & $\mathrm{a}$ & a & 24.0 \\
\hline Railroad maintenance equipment & 0.2 & 3.9 & 0.0 & a & 4.0 \\
\hline $\begin{array}{l}\text { Recreational equipment } \\
\text { Off-road motorcycles, snowmobiles, all-terrain } \\
\text { vehicles, golf carts, specialty vehicles }\end{array}$ & 188.0 & 2.1 & 0.1 & a & 190.3 \\
\hline Total & 218.5 & $1,783.5$ & 213.2 & 19.1 & $2,234.3$ \\
\hline
\end{tabular}

Source:

Environmental Protection Agency, MOVES2014a model, www.epa.gov/otaq/models/moves.

a There is no equipment listed for this fuel type. 
Mowing equipment consumes nearly half of all the fuel used by lawn and garden equipment. The gasoline used in lawn and garden equipment is $2.0 \%$ of total gasoline use.

Table 2.12

Fuel Consumption from Lawn and Garden Equipment, 2015 (million gallons ${ }^{\mathrm{a}}$ )

\begin{tabular}{|c|c|c|c|c|c|}
\hline Equipment & Classification & Gasoline & Diesel & LPG & $\begin{array}{c}\text { Total fuel } \\
\text { consumption }\end{array}$ \\
\hline \multicolumn{6}{|l|}{ Mowing equipment } \\
\hline Front mowers & Commercial & 20.8 & 130.2 & 0.0 & 151 \\
\hline Lawn \& garden tractors & Commercial & 248.4 & 26.9 & 0.0 & 275.3 \\
\hline Lawn \& garden tractors & Residential & 581.7 & 0.0 & 0.0 & 581.7 \\
\hline Lawn mowers & Commercial & 166.2 & 0.0 & 0.0 & 166.2 \\
\hline Lawn mowers & Residential & 220.7 & 0.0 & 0.0 & 220.7 \\
\hline Rear engine riding mowers & Commercial & 18.3 & 0.0 & 0.0 & 18.3 \\
\hline Rear engine riding mowers & Residential & 43.4 & 0.0 & 0.0 & 43.4 \\
\hline Total & & $1,299.50$ & 157.1 & 0.0 & $1,456.60$ \\
\hline \multicolumn{6}{|l|}{ Soil and turf equipment } \\
\hline Commercial turf equipment ${ }^{\mathrm{b}}$ & Commercial & 801.0 & 20.9 & 0.0 & 821.9 \\
\hline Rotary tillers $<6 \mathrm{HP}$ & Commercial & 92.1 & 0.0 & 0.0 & 92.1 \\
\hline Rotary tillers $<6 \mathrm{HP}$ & Residential & 19.9 & 0.0 & 0.0 & 19.9 \\
\hline Total & & 913.0 & 20.9 & $\mathbf{0 . 0}$ & 933.9 \\
\hline \multicolumn{6}{|l|}{ Wood cutting equipment } \\
\hline Chain saws $<6 \mathrm{HP}$ & Commercial & 80.3 & 0.0 & 0.0 & 80.3 \\
\hline Chain saws $<6 \mathrm{HP}$ & Residential & 19.2 & 0.0 & 0.0 & 19.2 \\
\hline Chippers/stump grinders & Commercial & 41.3 & 177.2 & 19.8 & 238.3 \\
\hline Shredders $<6 \mathrm{HP}$ & Commercial & 9.9 & 0.0 & 0.0 & 9.9 \\
\hline Total & & 150.7 & 177.2 & 19.8 & 347.7 \\
\hline \multicolumn{6}{|l|}{ Blowers and vacuums } \\
\hline Leafblowers/vacuums & Commercial & 219.2 & 0.0 & 0.0 & 219.2 \\
\hline Leafblowers/vacuums & Residential & 19.5 & 0.0 & 0.0 & 19.5 \\
\hline Snowblowers & Commercial & 37.9 & 2.3 & 0.0 & 40.2 \\
\hline Snowblowers & Residential & 20.1 & 0.0 & 0.0 & 20.1 \\
\hline Total & & 296.7 & 2.3 & 0.0 & 299.0 \\
\hline \multicolumn{6}{|l|}{ Trimming equipment } \\
\hline Trimmers/edgers/brush cutter & Commercial & 67.8 & 0.0 & 0.0 & 67.8 \\
\hline Trimmers/edgers/brush cutter & Residential & 27.8 & 0.0 & 0.0 & 27.8 \\
\hline Other lawn \& garden equipment ${ }^{\mathrm{C}}$ & Commercial & 24.8 & 0.5 & 0.0 & 25.3 \\
\hline Other lawn \& garden equipment ${ }^{\mathrm{c}}$ & Residential & 20.8 & 0.0 & 0.0 & 20.8 \\
\hline Total & & 141.2 & 0.5 & $\mathbf{0 . 0}$ & 141.7 \\
\hline Total all equipment & & $2,801.1$ & 358.0 & 19.8 & $3,178.9$ \\
\hline
\end{tabular}

Source:

U.S. Environmental Protection Agency, MOVES2014a model, www.epa.gov/otaq/models/moves.

${ }^{a}$ Numbers may not sum due to rounding.

${ }^{\mathrm{b}}$ Includes equipment such as aerators, dethatchers, sod cutters, hydro-seeders, turf utility vehicles, golf course greens mowers, and sand trap groomers.

c Includes equipment not otherwise classified such as augers, sickle-bar mowers, and wood splitters. 
The Federal Highway Administration (FHWA) cautions that data from 1993 on may not be directly comparable to earlier years. Some states have improved reporting procedures in recent years, and the estimation procedures were revised in 1994. The FHWA no longer publishes separate estimates of gasohol or ethanol used in gasohol.

Table 2.13

Highway Usage of Gasoline and Diesel, 1973-2015

(billion gallons)

\begin{tabular}{|c|c|c|c|c|}
\hline Year & Total gasoline and gasohol & Diesel $^{\mathrm{a}}$ & Percent diesel & Total highway fuel use \\
\hline 1973 & 100.6 & 9.8 & $8.9 \%$ & 110.5 \\
\hline 1975 & 99.4 & 9.6 & $8.8 \%$ & 109.0 \\
\hline 1980 & 101.2 & 13.8 & $12.0 \%$ & 115.0 \\
\hline 1981 & 99.6 & 14.9 & $13.0 \%$ & 114.5 \\
\hline 1982 & 98.5 & 14.9 & $13.1 \%$ & 113.4 \\
\hline 1983 & 100.1 & 16.0 & $13.8 \%$ & 116.1 \\
\hline 1984 & 101.4 & 17.3 & $14.6 \%$ & 118.7 \\
\hline 1985 & 103.6 & 17.8 & $14.6 \%$ & 121.3 \\
\hline 1986 & 106.8 & 18.4 & $14.7 \%$ & 125.2 \\
\hline 1987 & 108.7 & 19.0 & $14.9 \%$ & 127.7 \\
\hline 1988 & 109.8 & 20.1 & $15.5 \%$ & 129.9 \\
\hline 1989 & 110.6 & 21.2 & $16.1 \%$ & 131.9 \\
\hline 1990 & 110.2 & 21.4 & $16.3 \%$ & 131.6 \\
\hline 1991 & 107.9 & 20.7 & $16.1 \%$ & 128.6 \\
\hline 1992 & 111.0 & 22.0 & $16.5 \%$ & 132.9 \\
\hline 1993 & 113.7 & 23.5 & $17.1 \%$ & 137.2 \\
\hline 1994 & 115.0 & 25.1 & $17.9 \%$ & 140.1 \\
\hline 1995 & 117.1 & 26.2 & $18.3 \%$ & 143.3 \\
\hline 1996 & 119.5 & 27.2 & $18.5 \%$ & 146.7 \\
\hline 1997 & 120.9 & 29.4 & $19.6 \%$ & 150.3 \\
\hline 1998 & 124.7 & 30.2 & $19.5 \%$ & 154.9 \\
\hline 1999 & 128.7 & 31.9 & $19.9 \%$ & 160.7 \\
\hline 2000 & 128.9 & 33.4 & $20.6 \%$ & 162.3 \\
\hline 2001 & 129.7 & 33.4 & $20.5 \%$ & 163.1 \\
\hline 2002 & 133.0 & 34.8 & $20.7 \%$ & 167.8 \\
\hline 2003 & 134.1 & 35.5 & $20.9 \%$ & 169.6 \\
\hline 2004 & 136.5 & 37.4 & $21.5 \%$ & 173.9 \\
\hline 2005 & 135.2 & 39.1 & $22.4 \%$ & 174.3 \\
\hline 2006 & 134.8 & 40.1 & $22.9 \%$ & 174.9 \\
\hline 2007 & 135.4 & 40.7 & $23.1 \%$ & 176.1 \\
\hline 2008 & 132.2 & 38.6 & $22.6 \%$ & 170.8 \\
\hline 2009 & 132.9 & 35.3 & $21.0 \%$ & 168.1 \\
\hline 2010 & 133.1 & 36.6 & $21.6 \%$ & 169.7 \\
\hline 2011 & 131.5 & 37.1 & $22.0 \%$ & 168.6 \\
\hline 2012 & 130.9 & 37.4 & $22.2 \%$ & 168.3 \\
\hline 2013 & 131.3 & 38.4 & $22.6 \%$ & 169.7 \\
\hline 2014 & 136.5 & 39.7 & $22.5 \%$ & 176.2 \\
\hline 2015 & 132.2 & 40.6 & $23.5 \%$ & 172.9 \\
\hline \multicolumn{5}{|c|}{ Average annual percentage change } \\
\hline 1973-2015 & $0.7 \%$ & $3.4 \%$ & & $1.1 \%$ \\
\hline 2005-2015 & $-0.2 \%$ & $0.4 \%$ & & $-0.1 \%$ \\
\hline
\end{tabular}

\section{Source:}

U.S. Department of Transportation, Federal Highway Administration, Highway Statistics 2015, Washington, DC, 2016, Table MF-21 and annual. (Additional resources: www.fhwa.dot.gov)

${ }^{\text {a }}$ Consists primarily of diesel fuel, with small quantities of other fuels, such as liquefied petroleum gas and E85. 
Great care should be taken when comparing modal energy intensity data among modes. Because of the inherent differences among the transportation modes in the nature of services, routes available, and many additional factors, it is not possible to obtain truly comparable national energy intensities among modes. These values are averages, and there is a great deal of variability even within a mode.

Table 2.14

Passenger Travel and Energy Use, 2015 ${ }^{\mathrm{a}}$

\begin{tabular}{|c|c|c|c|c|c|c|c|}
\hline & \multirow[b]{2}{*}{$\begin{array}{c}\text { Number of } \\
\text { vehicles } \\
\text { (thousands) }\end{array}$} & \multirow[b]{2}{*}{$\begin{array}{l}\text { Vehicle- } \\
\text { miles } \\
\text { (millions) }\end{array}$} & \multirow[b]{2}{*}{$\begin{array}{c}\text { Passenger- } \\
\text { miles } \\
\text { (millions) }\end{array}$} & \multirow[b]{2}{*}{$\begin{array}{c}\text { Load factor } \\
\text { (persons/ } \\
\text { vehicle) }\end{array}$} & \multicolumn{2}{|c|}{ Energy intensities } & \multirow[b]{2}{*}{$\begin{array}{c}\text { Energy use } \\
\text { (trillion Btu) }\end{array}$} \\
\hline & & & & & $\begin{array}{c}\text { (Btu per } \\
\text { vehicle- } \\
\text { mile) }\end{array}$ & $\begin{array}{l}\text { (Btu per } \\
\text { passenger- } \\
\text { mile) }\end{array}$ & \\
\hline Personal trucks & $113,054.6$ & $1,123,226$ & $2,066,736$ & 1.80 & 6,156 & 3,345 & $6,870.1$ \\
\hline Motorcycles & $8,601.0$ & 19,606 & 22,743 & 1.20 & 2,855 & 2,462 & 56.0 \\
\hline Demand response $^{b}$ & 71.4 & 1,595 & 2,267 & 1.4 & 20,047 & 14,106 & 32.0 \\
\hline Transit & 64.2 & 2,216 & 20,239 & 9.1 & 36,760 & 4,025 & 81.5 \\
\hline Intercity $^{\mathrm{d}}$ & c & c & c & c & c & c & 35.1 \\
\hline School $^{\mathrm{d}}$ & 628.1 & c & c & c & c & c & 86.1 \\
\hline Air & c & c & c & c & c & c & $1,684.3$ \\
\hline Certificated route ${ }^{\mathrm{e}}$ & c & 5,589 & 632,648 & 113.2 & 263,971 & 2,332 & $1,475.4$ \\
\hline General aviation & 210.0 & c & c & c & c & c & 208.9 \\
\hline Recreational boats & $13,915.6$ & c & c & c & c & c & 246.0 \\
\hline Rail & 20.5 & 1,496 & 39,050 & 26.1 & 30,972 & 1,187 & 46.3 \\
\hline Transit & 12.8 & 803 & 20,710 & 25.8 & 20.022 & 776 & 16.1 \\
\hline Commuter & 7.3 & 374 & 11,804 & 31.6 & 51,888 & 1,643 & 19.4 \\
\hline
\end{tabular}

\section{Source:}

See Appendix A, Section 3. Passenger Travel and Energy Use.

${ }^{a}$ Only end-use energy was counted for electricity. Previous editions included primary energy use for electricity which included generation and distribution losses.

${ }^{b}$ Demand response data are for 2014. Includes passenger cars, vans, and small buses operating in response to calls from passengers to the transit operator who dispatches the vehicles.

${ }^{\mathrm{c}}$ Data are not available.

${ }^{\mathrm{d}}$ Energy use is estimated.

e Only domestic service and domestic energy use are shown on this table. (Previous editions included half of international energy.) These energy intensities may be inflated because all energy use is attributed to passengerscargo energy use is not taken into account. 
Great care should be taken when comparing modal energy intensity data among modes. Because of the inherent differences among the transportation modes in the nature of services, routes available, and many additional factors, it is not possible to obtain truly comparable national energy intensities among modes. These values are averages, and there is a great deal of variability even within a mode.

Table 2.15

Energy Intensities of Highway Passenger Modes, 1970-2015

\begin{tabular}{|c|c|c|c|c|c|}
\hline \multirow[b]{2}{*}{ Year } & \multicolumn{2}{|c|}{ Cars } & \multirow{2}{*}{$\begin{array}{l}\text { Light truck }^{\mathrm{b}} \\
\text { (Btu per } \\
\text { vehicle-mile) }\end{array}$} & \multicolumn{2}{|c|}{ Transit Buses ${ }^{\mathrm{C}}$} \\
\hline & $\begin{array}{c}\text { (Btu per } \\
\text { vehicle-mile) }\end{array}$ & $\begin{array}{c}\text { (Btu per } \\
\text { passenger-mile) }\end{array}$ & & $\begin{array}{c}\text { (Btu per } \\
\text { vehicle-mile) }\end{array}$ & $\begin{array}{c}\text { (Btu per } \\
\text { passenger-mile) }\end{array}$ \\
\hline 1970 & 9,250 & 4,868 & 12,479 & 31,796 & 2,472 \\
\hline 1975 & 8,993 & 4,733 & 11,879 & 33,748 & 2,814 \\
\hline 1980 & 7,916 & 4,279 & 10,224 & 36,553 & 2,813 \\
\hline 1985 & 7,164 & 4,110 & 8,730 & 38,876 & 3,423 \\
\hline 1986 & 7,194 & 4,197 & 8,560 & 37,889 & 3,545 \\
\hline 1987 & 6,959 & 4,128 & 8,359 & 36,247 & 3,594 \\
\hline 1988 & 6,683 & 4,033 & 8,119 & 36,673 & 3,706 \\
\hline 1989 & 6,589 & 4,046 & 7,746 & 36,754 & 3,732 \\
\hline 1990 & 6,169 & 3,856 & 7,746 & 37,374 & 3,794 \\
\hline 1991 & 5,912 & 3,695 & 7,351 & 37,732 & 3,877 \\
\hline 1992 & 5,956 & 3,723 & 7,239 & 40,243 & 4,310 \\
\hline 1993 & 6,087 & 3,804 & 7,182 & 39,043 & 4,262 \\
\hline 1994 & 6,024 & 3,765 & 7,212 & 36,932 & 4,225 \\
\hline 1995 & 5,902 & 3,689 & 7,208 & 36,936 & 4,271 \\
\hline 1996 & 5,874 & 3,683 & 7,247 & 37,238 & 4,315 \\
\hline 1997 & 5,797 & 3,646 & 7,251 & 38,622 & 4,407 \\
\hline 1998 & 5,767 & 3,638 & 7,260 & 41,062 & 4,374 \\
\hline 1999 & 5,821 & 3,684 & 7,327 & 40,351 & 4,320 \\
\hline 2000 & 5,687 & 3,611 & 7,158 & 41,466 & 4,506 \\
\hline 2001 & 5,626 & 3,583 & 7,080 & 38,320 & 4,123 \\
\hline 2002 & 5,662 & 3,607 & 7,125 & 37,340 & 4,110 \\
\hline 2003 & 5,535 & 3,525 & 7,673 & 36,900 & 4,191 \\
\hline 2004 & 5,489 & 3,496 & 7,653 & 37,665 & 4,342 \\
\hline 2005 & 5,607 & 3,571 & 7,009 & 37,244 & 4,229 \\
\hline 2006 & 5,511 & 3,510 & 6,974 & 39,397 & 4,297 \\
\hline 2007 & 5,513 & 3,512 & 6,904 & 39,748 & 4,352 \\
\hline 2008 & 5,466 & 3,526 & 6,830 & 39,726 & 4,328 \\
\hline 2009 & 5,239 & 3,380 & 7,158 & 39,073 & 4,233 \\
\hline 2010 & 5,117 & 3,301 & 6,919 & 35,858 & 4,107 \\
\hline 2011 & 5,032 & 3,246 & 6,795 & 37,648 & 4,232 \\
\hline 2012 & 4,949 & 3,193 & 6,674 & 37,037 & 4,023 \\
\hline 2013 & 4,873 & 3,144 & 6,557 & 37,273 & 4,052 \\
\hline 2014 & 4,839 & 3,122 & 6,677 & 35,237 & 3,810 \\
\hline 2015 & 4,702 & 3,034 & 6,256 & 36,760 & 4,025 \\
\hline \multicolumn{6}{|c|}{ Average annual percentage change } \\
\hline 1970-2015 & $-1.5 \%$ & $-1.0 \%$ & $-1.5 \%$ & $0.3 \%$ & $1.1 \%$ \\
\hline $2005-2015$ & $-1.7 \%$ & $-1.6 \%$ & $-1.2 \%$ & $-0.1 \%$ & $-0.5 \%$ \\
\hline
\end{tabular}

\section{Source:}

See Appendix A, Section 4. Highway Passenger Mode Energy Intensities.

${ }^{a}$ Only end-use energy was counted for electricity. Previous editions included primary energy use for electricity which included generation and distribution losses.

${ }^{\mathrm{b}}$ All two-axle, four-tire trucks.

${ }^{c}$ Series not continuous between 1983 and 1984 because of a change in data source by the American Public Transportation Association (APTA). 
Great care should be taken when comparing modal energy intensity data among modes. Because of the inherent differences between the transportation modes in the nature of services, routes available, and many additional factors, it is not possible to obtain truly comparable national energy intensities among modes.

Table 2.16

Energy Intensities of Nonhighway Passenger Modes, 1970-2015

\begin{tabular}{|c|c|c|c|c|}
\hline \multirow[b]{2}{*}{ Year } & \multirow{2}{*}{$\begin{array}{c}\text { Air } \\
\text { Certificated air carriers }^{\mathrm{b}} \\
\text { (Btu per } \\
\text { passenger-mile) } \\
\end{array}$} & \multicolumn{3}{|c|}{ Rail } \\
\hline & & $\begin{array}{l}\text { Intercity Amtrak } \\
\text { (Btu per } \\
\text { passenger-mile) }\end{array}$ & $\begin{array}{c}\text { Rail transit } \\
\text { (Btu per } \\
\text { passenger-mile) }\end{array}$ & $\begin{array}{l}\text { Commuter rail } \\
\text { (Btu per } \\
\text { passenger-mile) }\end{array}$ \\
\hline 1970 & 10,115 & c & 712 & $c$ \\
\hline 1975 & 7,625 & 3,311 & 866 & c \\
\hline 1980 & 5,561 & 2,859 & 763 & c \\
\hline 1981 & 5,774 & 2,414 & 855 & c \\
\hline 1982 & 5,412 & 2,551 & 891 & c \\
\hline 1983 & 5,133 & 2,359 & 931 & c \\
\hline 1984 & 5,298 & 2,417 & 1,002 & 1,798 \\
\hline 1985 & 5,053 & 2,237 & 927 & 1,720 \\
\hline 1986 & 5,011 & 2,037 & 1,004 & 1,720 \\
\hline 1987 & 4,827 & 1,989 & 1,003 & 1,628 \\
\hline 1988 & 4,861 & 1,967 & 1,014 & 1,666 \\
\hline 1989 & 4,844 & 2,082 & 960 & 1,622 \\
\hline 1990 & 4,797 & 2,052 & 998 & 1,622 \\
\hline 1991 & 4,602 & 2,011 & 1,074 & 1,601 \\
\hline 1992 & 4,455 & 2,117 & 1,041 & 1,565 \\
\hline 1993 & 4,490 & 2,142 & 1,113 & 1,782 \\
\hline 1994 & 4,407 & 1,917 & 1,102 & 1,605 \\
\hline 1995 & 4,349 & 2,071 & 1,102 & 1,580 \\
\hline 1996 & 4,199 & 2,194 & 996 & 1,541 \\
\hline 1997 & 4,173 & 2,289 & 943 & 1,630 \\
\hline 1998 & 3,987 & 2,246 & 931 & 1,612 \\
\hline 1999 & 4,108 & 2,362 & 919 & 1,670 \\
\hline 2000 & 3,960 & 2,651 & 923 & 1,542 \\
\hline 2001 & 3,943 & 2,690 & 925 & 1,533 \\
\hline 2002 & 3,718 & 2,537 & 948 & 1,542 \\
\hline 2003 & 3,614 & 2,145 & 936 & 1,542 \\
\hline 2004 & 3,505 & 2,068 & 907 & 1,536 \\
\hline 2005 & 3,346 & 2,025 & 919 & 1,658 \\
\hline 2006 & 3,250 & 1,948 & 893 & 1,539 \\
\hline 2007 & 3,153 & 1,824 & 851 & 1,543 \\
\hline 2008 & 3,055 & 1,745 & 832 & 1,579 \\
\hline 2009 & 2,901 & 1,773 & 830 & 1,714 \\
\hline 2010 & 2,825 & 1,668 & 832 & 1,753 \\
\hline 2011 & 2,772 & 1,628 & 812 & 1,681 \\
\hline 2012 & 2,633 & 1,561 & 791 & 1,703 \\
\hline 2013 & 2,568 & 1,559 & 793 & 1,676 \\
\hline 2014 & 2,511 & 1,641 & 786 & 1,638 \\
\hline 2015 & 2,477 & 1,663 & 776 & 1,643 \\
\hline \multicolumn{5}{|c|}{ Average annual percentage change ${ }^{d}$} \\
\hline 1970-2015 & $-3.1 \%$ & $-1.7 \%$ & $0.2 \%$ & $-0.3 \%$ \\
\hline 2005-2015 & $-3.0 \%$ & $-2.0 \%$ & $-1.7 \%$ & $-0.1 \%$ \\
\hline
\end{tabular}

\section{Source:}

See Appendix A, Section 5. Nonhighway Passenger Mode Energy Intensities.

${ }^{\text {a }}$ Only end-use energy was counted for electricity. Previous editions included primary energy use for electricity which included generation and distribution losses.

$\mathrm{b}$ These data differ from the data on Table 2.14 because they include half of international services. These energy intensities may be inflated because all energy use is attributed to passengers-cargo energy use is not taken into account.

c Data are not available.

${ }^{\mathrm{d}}$ Average annual percentage calculated to earliest year possible. 
The energy intensity of light rail systems, measured in Btu per passenger-mile varies greatly. The weighted average of all light rail systems in 2015 is 3,823 Btu/passenger-mile.

Figure 2.7. Energy Intensity of Light Rail Transit Systemsa ${ }^{\mathrm{a}} 2015$

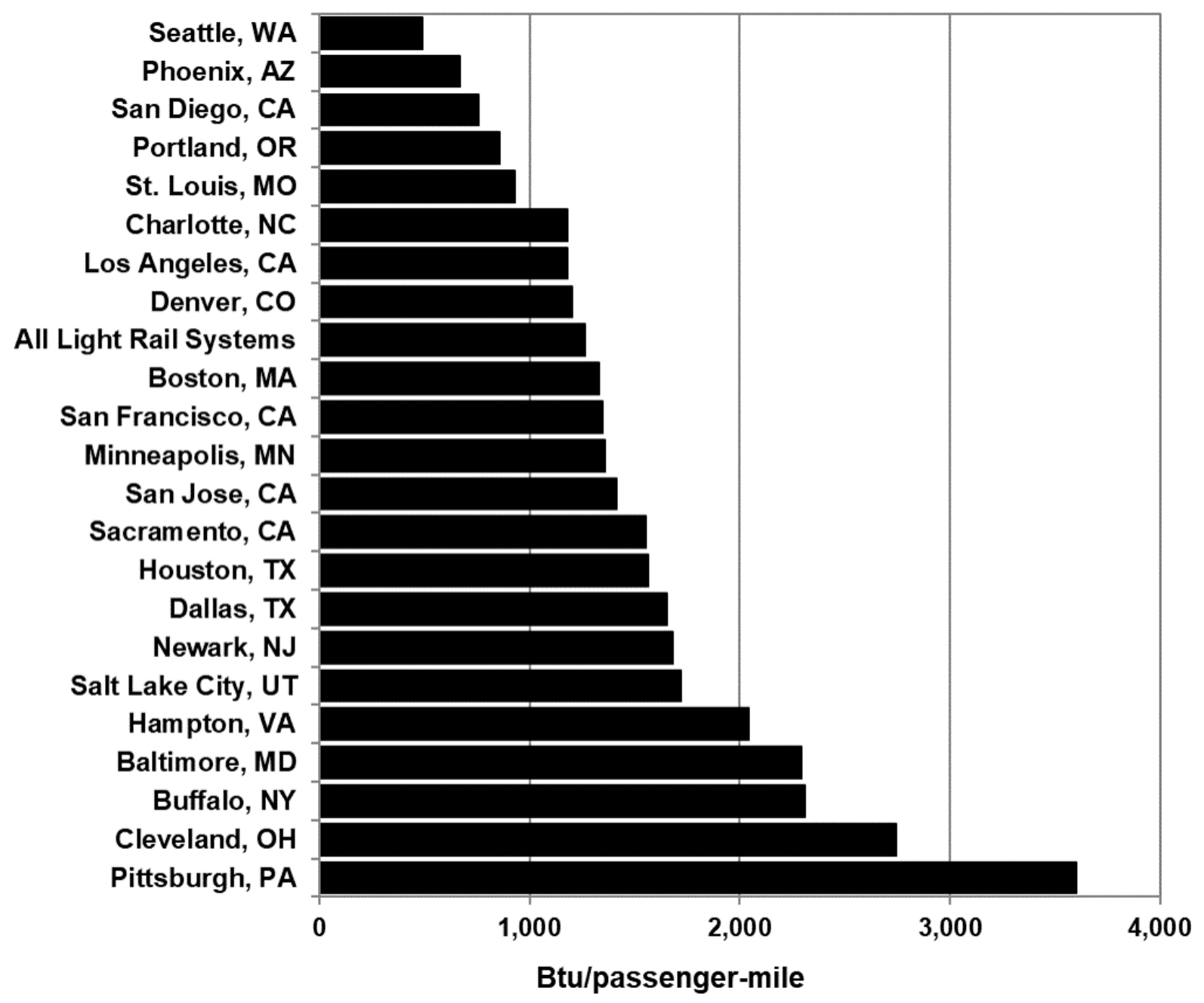

Source:

U.S. Department of Transportation, National Transit Database, August 2017. (Additional resources: www.transit.dot.gov/ntd)

a Typically an electric railway with a light volume traffic capacity with power drawn from an overhead electric line. Only end-use energy was counted for electricity. Previous editions included primary energy use for electricity which included generation and distribution losses. 
Figure 2.8. Energy Intensity of Heavy Rail Systems ${ }^{\mathrm{a}}, 2015$

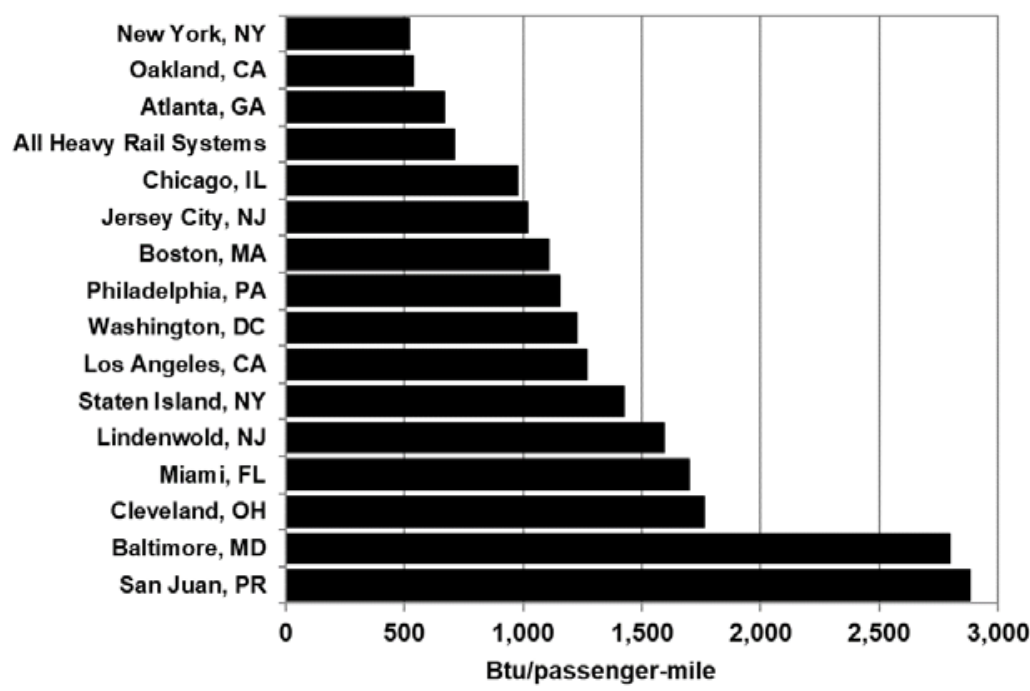

\section{Source:}

U.S. Department of Transportation, National Transit Database, August 2017. (Additional resources: www.transit.dot.gov/ntd)

Figure 2.9. Energy Intensity of Commuter Rail Systems ${ }^{b}, 2015$

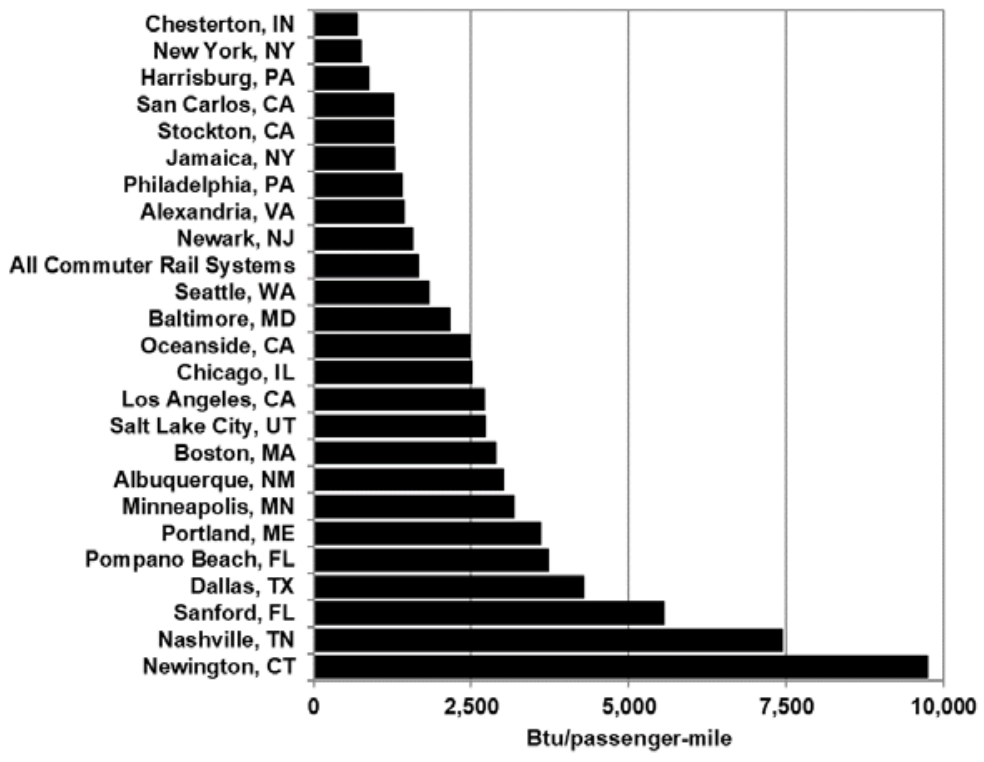

Note: Does not include systems classified as hybrid rail.

Source:

U.S. Department of Transportation, 2015 National Transit Database, August 2017. (Additional resources: www.transit.dot.gov/ntd)

a An electric railway with the capacity for a heavy volume of traffic. Only end-use energy was counted for electricity. Previous editions included primary energy use for electricity which included generation and distribution losses.

${ }^{\mathrm{b}}$ Electric car or diesel-propelled railway for urban passenger train service between a central city and adjacent suburbs. Only end-use energy was counted for electricity. Previous editions included primary energy use for electricity which included generation and distribution losses. 
Great care should be taken when comparing modal energy intensity data among modes. Because of the inherent differences between the transportation modes in the nature of services, routes available, and many additional factors, it is not possible to obtain truly comparable national energy intensities among modes.

Table 2.17

Energy Intensities of Freight Modes, 1970-2015

\begin{tabular}{|c|c|c|c|c|}
\hline \multirow[b]{2}{*}{ Year } & \multirow{2}{*}{$\begin{array}{l}\text { Heavy single-unit and } \\
\text { combination trucks } \\
\text { (Btu per vehicle-mile) }\end{array}$} & \multicolumn{2}{|c|}{ Class I freight railroad } & \multirow{2}{*}{$\begin{array}{c}\text { Waterborne commerce on } \\
\text { taxable waterways } \\
\text { (Btu per ton-mile) }\end{array}$} \\
\hline & & (Btu per freight car-mile) & (Btu per ton-mile) & \\
\hline 1970 & 24,960 & 17,669 & 691 & $\mathrm{a}$ \\
\hline 1975 & 24,631 & 18,739 & 687 & $\mathrm{a}$ \\
\hline 1980 & 24,758 & 18,742 & 597 & a \\
\hline 1981 & 25,059 & 18,629 & 572 & $\mathrm{a}$ \\
\hline 1982 & 24,297 & 18,404 & 553 & $\mathrm{a}$ \\
\hline 1983 & 23,853 & 17,864 & 525 & a \\
\hline 1984 & 23,585 & 17,795 & 510 & a \\
\hline 1985 & 23,343 & 17,500 & 497 & a \\
\hline 1986 & 23,352 & 17,265 & 486 & a \\
\hline 1987 & 22,923 & 16,790 & 456 & a \\
\hline 1988 & 22,596 & 16,758 & 443 & a \\
\hline 1989 & 22,411 & 16,894 & 437 & a \\
\hline 1990 & 22,795 & 16,619 & 420 & a \\
\hline 1991 & 22,749 & 15,835 & 391 & a \\
\hline 1992 & 22,609 & 16,043 & 393 & $\mathrm{a}$ \\
\hline 1993 & 22,373 & 16,056 & 389 & a \\
\hline 1994 & 22,193 & 16,340 & 388 & $\mathrm{a}$ \\
\hline 1995 & 22,097 & 15,992 & 372 & $\mathrm{a}$ \\
\hline 1996 & 22,109 & 15,747 & 368 & $\mathrm{a}$ \\
\hline 1997 & 21,340 & 15,784 & 370 & 266 \\
\hline 1998 & 21,516 & 15,372 & 365 & 256 \\
\hline 1999 & 22,884 & 15,363 & 363 & 266 \\
\hline 2000 & 23,449 & 14,917 & 352 & 270 \\
\hline 2001 & 23,024 & 15,108 & 346 & 253 \\
\hline 2002 & 23,462 & 15,003 & 345 & 253 \\
\hline 2003 & 22,461 & 15,016 & 344 & 251 \\
\hline 2004 & 20,540 & 15,274 & 341 & 241 \\
\hline 2005 & 22,866 & 15,152 & 337 & 241 \\
\hline 2006 & 23,340 & 14,990 & 330 & 235 \\
\hline 2007 & 21,238 & 14,846 & 320 & 225 \\
\hline 2008 & 21,008 & 14,573 & 305 & 252 \\
\hline 2009 & 21,024 & 13,907 & 291 & 225 \\
\hline 2010 & 21,499 & 13,733 & 289 & 217 \\
\hline 2011 & 21,677 & 14,043 & 298 & 211 \\
\hline 2012 & 21,524 & 13,800 & 294 & 211 \\
\hline 2013 & 21,540 & 14,607 & 296 & 233 \\
\hline 2014 & 21,573 & 14,533 & 292 & 214 \\
\hline 2015 & 21,382 & 14,401 & 296 & $\mathrm{a}$ \\
\hline & Ave & annual percentage change & & \\
\hline 1970-2015 & $-0.3 \%$ & $-0.4 \%$ & $-1.9 \%$ & a \\
\hline 2005-2015 & $-0.7 \%$ & $-0.5 \%$ & $-1.3 \%$ & a \\
\hline
\end{tabular}

Source:

See Appendix A, Section 6. Freight Mode Energy Intensities.

${ }^{\text {a }}$ Data are not available.

b Due to changes in the FHWA fuel use methodology, truck data are not comparable with data before the year 2007. 


\section{Chapter 3 \\ All Highway Vehicles and Characteristics}

Summary Statistics from Tables in this Chapter

\begin{tabular}{clr}
\hline Source & & \\
\hline Table 3.2 & U.S. share of world car registrations, 2015 & $13.6 \%$ \\
Table 3.3 & U.S. share of world truck \& bus registrations, 2015 & $43.3 \%$ \\
Table 3.4 & Number of U.S. cars, 2015 (thousands) & 112,864 \\
Table 3.4 & Number of U.S. trucks, 2015 (thousands) & 141,256 \\
Table 3.7 & Vehicle miles traveled, 2015 (million miles) & $3,104,179$ \\
& Cars & $46.6 \%$ \\
& Two-axle, four-tire trucks & $43.3 \%$ \\
& Combination trucks & $5.5 \%$ \\
& Other single-unit trucks & $3.5 \%$ \\
& Motorcycles & $0.6 \%$ \\
& Buses & $0.5 \%$ \\
& Average age of vehicles, 2016 & 11.6 \\
& Cars (years) & 11.6 \\
& Light trucks (years) & 11.6 \\
\hline
\end{tabular}


The top countries producing the world's cars and trucks have changed over the last ten years. In 2015, China was the largest producer of cars and trucks. In 2000, Japan produced the most cars and the United States produced the most trucks (includes light trucks).

Table 3.1

World Production of Cars and Trucks, 2000 and 2015 (thousands)

\begin{tabular}{lrrr}
\hline \multicolumn{1}{c}{ Cars } & & & Percent change \\
\hline China & 2000 & 2015 & $1,883 \%$ \\
Japan & 605 & 11,991 & $-6 \%$ \\
Germany & 8,363 & 7,831 & $11 \%$ \\
U.S. & 5,132 & 5,708 & $-25 \%$ \\
Brazil & 5,542 & 4,163 & $48 \%$ \\
India & 1,362 & 2,018 & $318 \%$ \\
Russia & 605 & 2,529 & $25 \%$ \\
Mexico & 969 & 1,216 & $74 \%$ \\
Spain & 1,130 & 1,968 & $-6 \%$ \\
UK & 2,366 & 2,219 & $-3 \%$ \\
France & 1,641 & 1,588 & $-46 \%$ \\
Czech Republic & 2,880 & 1,564 & $203 \%$ \\
All Other Countries & 428 & 1,297 & $14 \%$ \\
\hline Total World & 10,205 & 11,668 & $35 \%$ \\
\hline & 41,229 & 55,760 & Percent change \\
\multicolumn{1}{c}{ Trucks } & & & $2000-2015$ \\
\hline China & & 2015 & $754 \%$ \\
U.S. & 2000 & 12,512 & $9 \%$ \\
South Korea & 1,464 & 7,943 & $325 \%$ \\
India & 7,263 & 2,181 & $463 \%$ \\
Japan & 513 & 1,597 & $-19 \%$ \\
Canada & 283 & 1,448 & $-1 \%$ \\
Thailand & 1,781 & 1,397 & $266 \%$ \\
All Other Countries & 1,411 & 1,152 & $32 \%$ \\
\hline Total World & 315 & 6,172 & $94 \%$ \\
\hline
\end{tabular}

Source:

Ward's Communications, www.wardsauto.com

${ }^{a}$ Includes light trucks, heavy trucks, and buses. 
Figure 3.1. World Car Production, 1983-2015

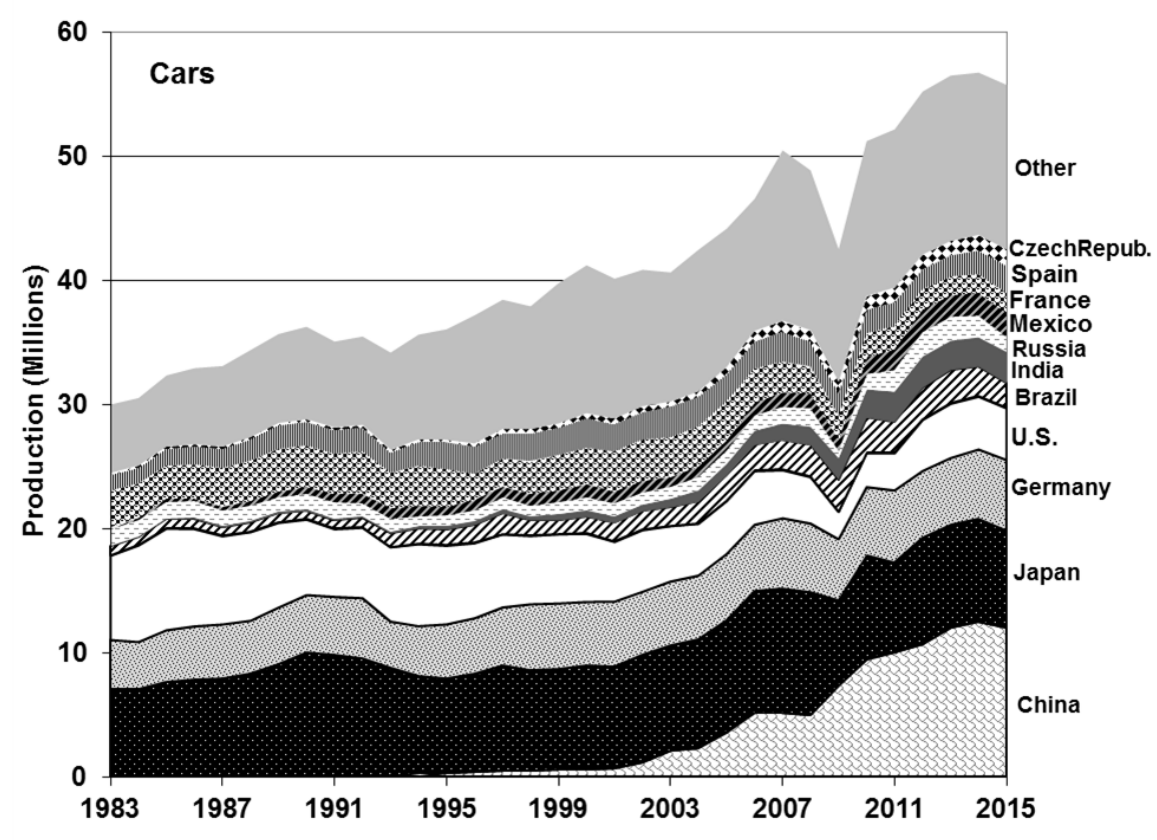

Source:

See Table 3.1.

Figure 3.2. World Truck and Bus Production, 1983-2015

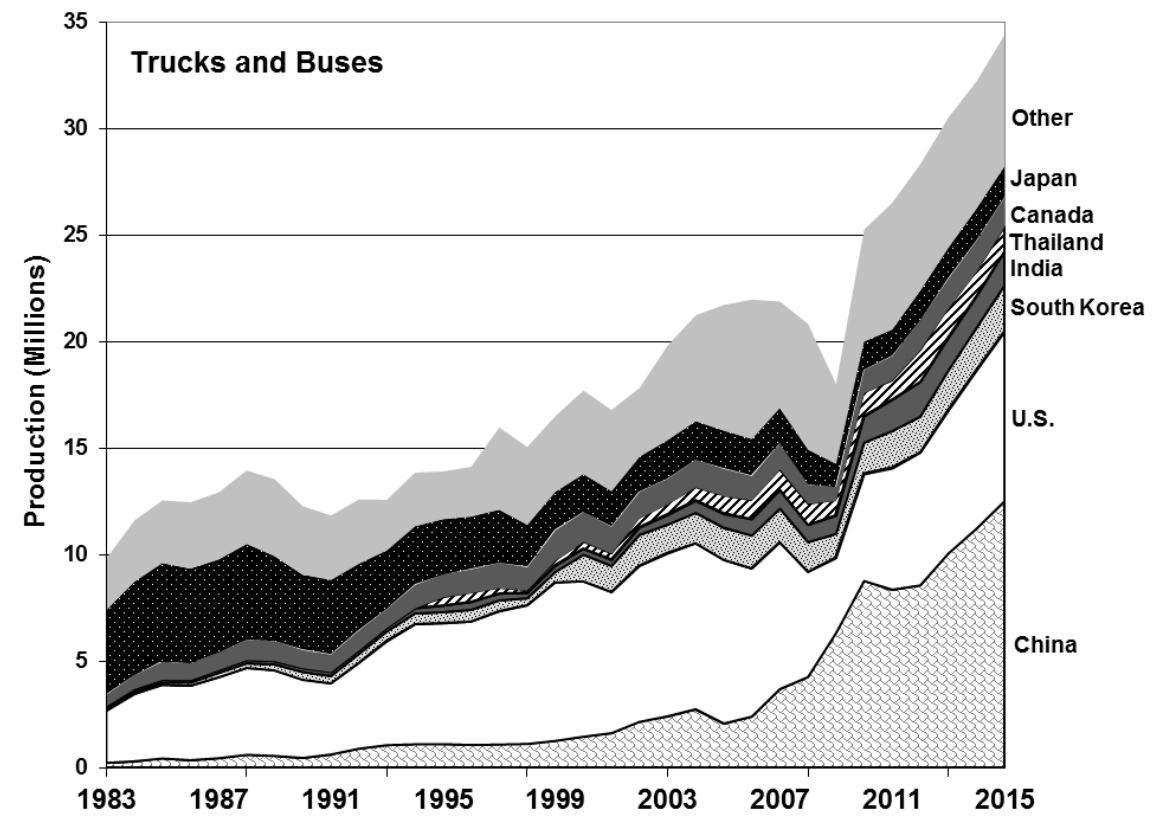

Source:

See Table 3.1.

${ }^{\text {a }}$ The sharp decrease in 2009 coincides with the recession. Note that the scales of the two figures differ. 
Use caution comparing historical data because of disconnects in data series. Also, the United States is unique in how many light trucks (SUVs, minivans, pickups) are used for personal travel. Those light trucks are not included on this table. The U.S. share of world cars continues to decline. The growth in the World total comes mainly from developing countries, like China, Indonesia, India, and South Korea.

Table 3.2

Car Registrations for Selected Countries, 1960-2015 (thousands)

\begin{tabular}{|c|c|c|c|c|c|c|c|c|c|c|}
\hline & & & & & & & & & & $\begin{array}{c}\text { Average } \\
\text { annual } \\
\text { percentage } \\
\text { change }\end{array}$ \\
\hline Country & 1960 & 1970 & 1980 & 1990 & 2000 & 2005 & 2010 & 2014 & 2015 & $1990-2015$ \\
\hline Argentina & 474 & 1,482 & 3,112 & 4,284 & 5,060 & 5,340 & 7,605 & 10,092 & 11,003 & $3.8 \%$ \\
\hline Brazil & a & a & a & 12,127 & 15,393 & 18,370 & 25,541 & 32,715 & 33,566 & $4.2 \%$ \\
\hline Canada ${ }^{\mathrm{b}}$ & 4,104 & 6,602 & 10,256 & 12,622 & 16,832 & 18,124 & 20,121 & 21,730 & 22,068 & $2.3 \%$ \\
\hline China & $\mathrm{a}$ & a & 351 & 1,897 & 3,750 & 8,900 & 34,430 & 120,724 & 135,119 & $18.6 \%$ \\
\hline France & 4,950 & 11,860 & 18,440 & 23,550 & 28,060 & 30,100 & 31,300 & 31,800 & 32,000 & $1.2 \%$ \\
\hline Germany ${ }^{c}$ & 4,856 & 14,376 & 23,236 & 35,512 & 43,772 & 46,090 & 42,302 & 44,403 & 45,071 & $1.0 \%$ \\
\hline India & a & a & a & 2,300 & 5,150 & 7,654 & 13,300 & 27,174 & 30,570 & $10.9 \%$ \\
\hline Indonesia & a & a & a & 1,200 & a & 3,850 & 8,891 & 12,595 & 13,846 & $10.3 \%$ \\
\hline Japan & 457 & 8,779 & 23,660 & 34,924 & 52,437 & 57,091 & 58,347 & 60,668 & 61,873 & $2.3 \%$ \\
\hline Malaysia & $\mathrm{a}$ & a & a & 1,811 & 4,213 & 6,402 & 9,115 & 11,149 & 11,279 & $7.6 \%$ \\
\hline Pakistan & $\mathrm{a}$ & $\mathrm{a}$ & a & 738 & 375 & 411 & 1,726 & 2,316 & 2,470 & $5.0 \%$ \\
\hline Russia & a & a & a & a & 20,353 & 25,285 & 34,350 & 40,850 & 41,000 & $4.8 \%{ }^{\mathrm{d}}$ \\
\hline $\begin{array}{l}\text { South Korea } \\
\text { United }\end{array}$ & a & a & a & 2,075 & 8,084 & 11,122 & 13,632 & 15,747 & 16,561 & $8.7 \%$ \\
\hline Kingdom & 5,650 & 11,802 & 15,438 & 22,528 & 27,185 & 30,652 & 31,258 & 32,613 & 33,542 & $1.6 \%$ \\
\hline $\begin{array}{l}\text { United States } \\
\text { U.S. percentage }\end{array}$ & 61,671 & 89,244 & 121,601 & 143,550 & 127,721 & 132,909 & 129,053 & 120,984 & 126,014 & $-0.5 \%$ \\
\hline of world & $62.7 \%$ & $46.1 \%$ & $38.0 \%$ & $32.3 \%$ & $23.3 \%$ & $21.5 \%$ & $17.8 \%$ & $13.7 \%$ & $13.6 \%$ & \\
\hline World total & 98,305 & 193,479 & 320,390 & 444,900 & 548,558 & 617,914 & 723,567 & 884,506 & 923,590 & $3.0 \%$ \\
\hline
\end{tabular}

Source:

Ward's Communications, www.wardsauto.com.

${ }^{a}$ Data are not available.

b Data from 2000 and later are not comparable to prior data. Canada reclassified autos and trucks prior to 2000.

c Data for 1990 and prior include West Germany only. Kraftwagen are included with automobiles.

${ }^{\mathrm{d}}$ Data for earliest year available. 
The United States totals include SUVs, minivans, and light trucks, many of which are used for personal travel. Thus, countries that only use trucks for freight movement will not be comparable to the United States.

Table 3.3

Truck and Bus Registrations for Selected Countries, 1960-2015 (thousands)

\begin{tabular}{|c|c|c|c|c|c|c|c|c|c|}
\hline Country & 1960 & 1970 & 1980 & 1990 & 2000 & 2005 & 2010 & 2015 & $\begin{array}{c}\text { Average } \\
\text { annual } \\
\text { percentage } \\
\text { change } \\
\text { 1990-2015 }\end{array}$ \\
\hline Argentina & 392 & 788 & 1,217 & 1,501 & 1,554 & 1,730 & 2,511 & 3,305 & $3.2 \%$ \\
\hline Brazil & $\mathrm{a}$ & a & a & 936 & 3,917 & 4,653 & 6,524 & 8,959 & $9.5 \%$ \\
\hline Canada $^{\mathrm{b}}$ & 1,056 & 1,481 & 2,955 & 3,931 & 739 & 786 & 933 & 1,147 & $-4.8 \%$ \\
\hline China & a & a & 1,480 & 4,314 & 9,650 & 21,750 & 43,590 & 23,191 & $7.0 \%$ \\
\hline France & 1,650 & 1,850 & 2,550 & 4,910 & 5,733 & 6,198 & 6,444 & 6,652 & $1.2 \%$ \\
\hline Germany $^{c}$ & 786 & 1,228 & 1,617 & 2,764 & 3,534 & 3,133 & 2,960 & 3,356 & $0.8 \%$ \\
\hline India & a & a & a & 2,050 & 2,390 & 4,145 & 9,500 & 11,230 & $7.0 \%$ \\
\hline Indonesia & a & a & a & 1,391 & 2,373 & 2,950 & 6,938 & 9,237 & $7.9 \%$ \\
\hline Japan & 896 & 8,803 & 14,197 & 22,773 & 20,211 & 16,734 & 15,512 & 14,981 & $-1.7 \%$ \\
\hline Malaysia & a & a & a & 616 & 1,030 & 1,323 & 1,138 & 1,335 & $3.1 \%$ \\
\hline Pakistan & $\mathrm{a}$ & a & a & 172 & 385 & 414 & 538 & 678 & $5.6 \%$ \\
\hline Russia & a & a & a & 7,200 & 5,041 & 5,705 & 6,304 & 8,000 & $0.4 \%$ \\
\hline South Korea & a & a & a & 1,320 & 3,956 & 4,275 & 4,310 & 4,428 & $5.0 \%$ \\
\hline United Kingdom & 1,534 & 1,769 & 1,920 & 3,774 & 3,361 & 3,943 & 4,220 & 4,677 & $0.9 \%$ \\
\hline United States & 12,186 & 19,175 & 34,195 & 45,106 & 85,579 & 104,788 & 119,179 & 146,154 & $4.8 \%$ \\
\hline $\begin{array}{l}\text { U.S. percentage } \\
\text { of world }\end{array}$ & $42.6 \%$ & $36.2 \%$ & $37.7 \%$ & $32.7 \%$ & $42.1 \%$ & $42.6 \%$ & $38.5 \%$ & $43.3 \%$ & \\
\hline World total & 28,583 & 52,899 & 90,592 & 138,082 & 203,272 & 245,798 & 309,395 & 337,250 & $3.6 \%$ \\
\hline
\end{tabular}

Source:

Ward's Communications, www.wardsauto.com.

\footnotetext{
a Data are not available.

b Data from 2000 and later are not comparable to prior data. Canada reclassified autos and trucks prior to 2000.

c Data for 1990 and prior include West Germany only. Kraftwagen are included with automobiles.
} 


\section{VEHICLES IN USE}

Both the Federal Highway Administration (FHWA) and IHS Automotive report figures on the car and truck population each year. The two estimates, however, differ by as much as $11.2 \%$ (1981). The differences can be attributed to several factors:

- The FHWA data include all vehicles which have been registered at any time throughout the calendar year. Therefore, the data include vehicles which were retired during the year and may double count vehicles which have been registered in different states or the same states to different owners. IHS Automotive data include only those vehicles which are registered on July 1 of the given year.

- The classification of mini-vans, station wagons on truck chassis, and utility vehicles as cars or trucks causes important differences in the two estimates. IHS Automotive data included passenger vans in the car count until 1980; since 1980 all vans have been counted as trucks.

- Starting in 1993, the FHWA reclassified some minivans and sport utility vehicles into the truck category which were previously included with cars. This change produced a dramatic change in the individual percentage differences of cars and trucks. The difference in total vehicles has been less than 5\% each year since 1990 and does not appear to be significantly affected by the FHWA reclassifications. Beginning with 2009, the FHWA discontinued the car/2-axle, 4-tire truck designations on Table VM-1. The data since 2009 come from Tables MV-1 and MV-9.

- The FHWA data include all non-military Federal vehicles, while IHS Automotive data include only those Federal vehicles which are registered within a state. Federal vehicles are not required to have State registrations, and, according to the General Services Administration, most Federal vehicles are not registered.

- In 2012 both IHS Automotive and FHWA changed their methodologies for the car/light truck split which created a significant decrease in the number of cars reported and a corresponding increase in the number of light trucks. 
In the early 1980's, researchers had to make a conscious choice of which data series to use, since they differed by as much as 11\%. In 2005 the two sources differed by less than 1\%. Both sources changed their methodologies for the car/light truck split causing significant decreases to the number of cars in 2012.

Table 3.4

U.S. Cars and Trucks in Use, 1970-2015 (thousands)

\begin{tabular}{|c|c|c|c|c|c|c|c|c|c|}
\hline \multirow[b]{2}{*}{ Year } & \multicolumn{3}{|c|}{ Cars } & \multicolumn{3}{|c|}{ Trucks } & \multicolumn{3}{|c|}{ Total } \\
\hline & FHWA & $\begin{array}{c}\text { IHS } \\
\text { Automotive } \\
\end{array}$ & $\begin{array}{c}\text { Percentage } \\
\text { difference }\end{array}$ & FHWA & $\begin{array}{c}\text { IHS } \\
\text { Automotive } \\
\end{array}$ & $\begin{array}{c}\text { Percentage } \\
\text { difference }\end{array}$ & FHWA & $\begin{array}{c}\text { IHS } \\
\text { Automotive } \\
\end{array}$ & $\begin{array}{l}\text { Percentage } \\
\text { difference }\end{array}$ \\
\hline 1970 & 89,243 & 80,448 & $10.9 \%$ & 18,797 & 17,688 & $6.3 \%$ & 108,040 & 98,136 & $10.1 \%$ \\
\hline 1975 & 106,706 & 95,241 & $12.0 \%$ & 25,781 & 24,813 & $3.9 \%$ & 132,487 & 120,054 & $10.4 \%$ \\
\hline 1980 & 121,601 & 104,564 & $16.3 \%$ & 33,667 & 35,268 & $-4.5 \%$ & 155,267 & 139,832 & $11.0 \%$ \\
\hline 1981 & 123,098 & 105,839 & $16.3 \%$ & 34,644 & 36,069 & $-4.0 \%$ & 157,743 & 141,908 & $11.2 \%$ \\
\hline 1982 & 123,702 & 106,867 & $15.8 \%$ & 35,382 & 36,987 & $-4.3 \%$ & 159,084 & 143,854 & $10.6 \%$ \\
\hline 1983 & 126,444 & 108,961 & $16.0 \%$ & 36,723 & 38,143 & $-3.7 \%$ & 163,166 & 147,104 & $10.9 \%$ \\
\hline 1984 & 128,158 & 112,019 & $14.4 \%$ & 37,507 & 40,143 & $-6.6 \%$ & 165,665 & 152,162 & $8.9 \%$ \\
\hline 1985 & 127,885 & 114,662 & $11.5 \%$ & 43,210 & 42,387 & $1.9 \%$ & 171,095 & 157,049 & $8.9 \%$ \\
\hline 1986 & 130,004 & 117,268 & $10.9 \%$ & 45,103 & 44,826 & $0.6 \%$ & 175,106 & 162,094 & $8.0 \%$ \\
\hline 1987 & 131,482 & 119,849 & $9.7 \%$ & 46,826 & 47,344 & $-1.1 \%$ & 178,308 & 167,193 & $6.6 \%$ \\
\hline 1988 & 133,836 & 121,519 & $10.1 \%$ & 49,941 & 50,221 & $-0.6 \%$ & 183,777 & 171,740 & $7.0 \%$ \\
\hline 1989 & 134,559 & 122,758 & $9.6 \%$ & 52,172 & 53,202 & $-1.9 \%$ & 186,731 & 175,960 & $6.1 \%$ \\
\hline 1990 & 133,700 & 123,276 & $8.5 \%$ & 54,470 & 56,023 & $-2.8 \%$ & 188,171 & 179,299 & $4.9 \%$ \\
\hline 1991 & 128,300 & 123,268 & $4.1 \%$ & 59,206 & 58,179 & $1.8 \%$ & 187,505 & 181,447 & $3.3 \%$ \\
\hline 1992 & 126,581 & 120,347 & $5.2 \%$ & 63,136 & 61,172 & $3.2 \%$ & 189,717 & 181,519 & $4.5 \%$ \\
\hline 1993 & 127,327 & 121,055 & $5.2 \%$ & 66,082 & 65,260 & $1.3 \%$ & 193,409 & 186,315 & $3.8 \%$ \\
\hline 1994 & 127,883 & 121,997 & $4.8 \%$ & 69,491 & 66,717 & $4.2 \%$ & 197,375 & 188,714 & $4.6 \%$ \\
\hline 1995 & 128,387 & 123,242 & $4.2 \%$ & 72,458 & 70,199 & $3.2 \%$ & 200,845 & 193,441 & $3.8 \%$ \\
\hline 1996 & 129,728 & 124,613 & $4.1 \%$ & 75,940 & 73,681 & $3.1 \%$ & 205,669 & 198,294 & $3.7 \%$ \\
\hline 1997 & 129,749 & 124,673 & $4.1 \%$ & 77,307 & 76,398 & $1.2 \%$ & 207,056 & 201,071 & $3.0 \%$ \\
\hline 1998 & 131,839 & 125,966 & $4.7 \%$ & 79,062 & 79,077 & $0.0 \%$ & 210,901 & 205,043 & $2.9 \%$ \\
\hline 1999 & 132,432 & 126,869 & $4.4 \%$ & 83,148 & 82,640 & $0.6 \%$ & 215,580 & 209,509 & $2.9 \%$ \\
\hline 2000 & 133,621 & 127,721 & $4.6 \%$ & 87,108 & 85,579 & $1.8 \%$ & 220,729 & 213,300 & $3.5 \%$ \\
\hline 2001 & 137,633 & 128,714 & $6.9 \%$ & 92,045 & 87,969 & $4.6 \%$ & 229,678 & 216,683 & $6.0 \%$ \\
\hline 2002 & 135,921 & 129,907 & $4.6 \%$ & 92,939 & 91,120 & $2.0 \%$ & 228,860 & 221,027 & $3.5 \%$ \\
\hline 2003 & 135,670 & 131,072 & $3.5 \%$ & 94,944 & 94,810 & $0.1 \%$ & 230,614 & 225,882 & $2.1 \%$ \\
\hline 2004 & 136,431 & 132,469 & $3.0 \%$ & 100,016 & 99,698 & $0.3 \%$ & 236,447 & 232,167 & $1.8 \%$ \\
\hline 2005 & 136,568 & 132,909 & $2.8 \%$ & 103,819 & 105,475 & $-1.6 \%$ & 240,387 & 238,384 & $0.8 \%$ \\
\hline 2006 & 135,400 & 135,047 & $0.3 \%$ & 107,944 & 109,596 & $-1.5 \%$ & 243,344 & 244,643 & $-0.5 \%$ \\
\hline 2007 & 135,933 & 135,222 & $0.5 \%$ & 110,498 & 113,479 & $-2.6 \%$ & 246,431 & 248,701 & $-0.9 \%$ \\
\hline 2008 & 137,080 & 135,882 & $0.9 \%$ & 110,242 & 113,931 & $-3.2 \%$ & 247,322 & 249,813 & $-1.0 \%$ \\
\hline 2009 & 134,880 & 132,500 & $1.8 \%$ & 110,561 & 116,472 & $-5.1 \%$ & 245,441 & 248,972 & $-1.4 \%$ \\
\hline 2010 & 130,892 & 129,053 & $1.4 \%$ & 110,322 & 119,179 & $-7.4 \%$ & 241,214 & 248,232 & $-2.8 \%$ \\
\hline 2011 & 125,657 & 127,577 & $-1.5 \%$ & 118,483 & 121,355 & $-2.4 \%$ & 244,140 & 248,932 & $-1.9 \%$ \\
\hline 2012 & 111,290 & 120,902 & $-8.0 \%$ & 133,130 & 130,595 & $1.9 \%$ & 244,420 & 251,497 & $-2.8 \%$ \\
\hline 2013 & 113,676 & 120,214 & $-5.4 \%$ & 132,931 & 132,501 & $0.3 \%$ & 246,607 & 252,715 & $-2.4 \%$ \\
\hline 2014 & 113,899 & 120,984 & $-5.9 \%$ & 137,531 & 137,043 & $0.4 \%$ & 251,430 & 258,027 & $-2.6 \%$ \\
\hline 2015 & 112,864 & 122,322 & $-7.7 \%$ & 141,256 & 141,872 & $-0.4 \%$ & 254,120 & 264,194 & $-3.8 \%$ \\
\hline
\end{tabular}

\section{Source:}

FHWA - U.S. Department of Transportation, Federal Highway Administration, 1970-2008, Highway Statistics 2008 and earlier, Washington, DC, 2009, Table VM-1 and annual. 2009-2015 data from Tables MV-1 and MV-9, Highway Statistics 2015. (Additional resources: www.fhwa.dot.gov)

IHS Automotive - IHS Automotive, Detroit, Michigan. FURTHER REPRODUCTION PROHIBITED.

(Additional resources: https://www.ihs.com/industry/automotive.html) 
The graphs below show the number of motor vehicles per thousand people for various countries. The data for the

United States are displayed in the line which goes from 1900 to 2015. The points labeled on that line show data for

the other countries/regions around the world and how their vehicles per thousand people compare to the United

States at two different points in time, 2005 and 2015. For instance, the graph shows that in 2005, Eastern Europe's

vehicles per thousand people was about where the United States was in 1942, but by 2015 it is about where the

United States was in 1953. The lower part of the graph (1900-1930) is shown enlarged on the facing page.

Figure 3.3. Vehicles per Thousand People: U.S. (Over Time) Compared to Other Countries (in 2005 and 2015)

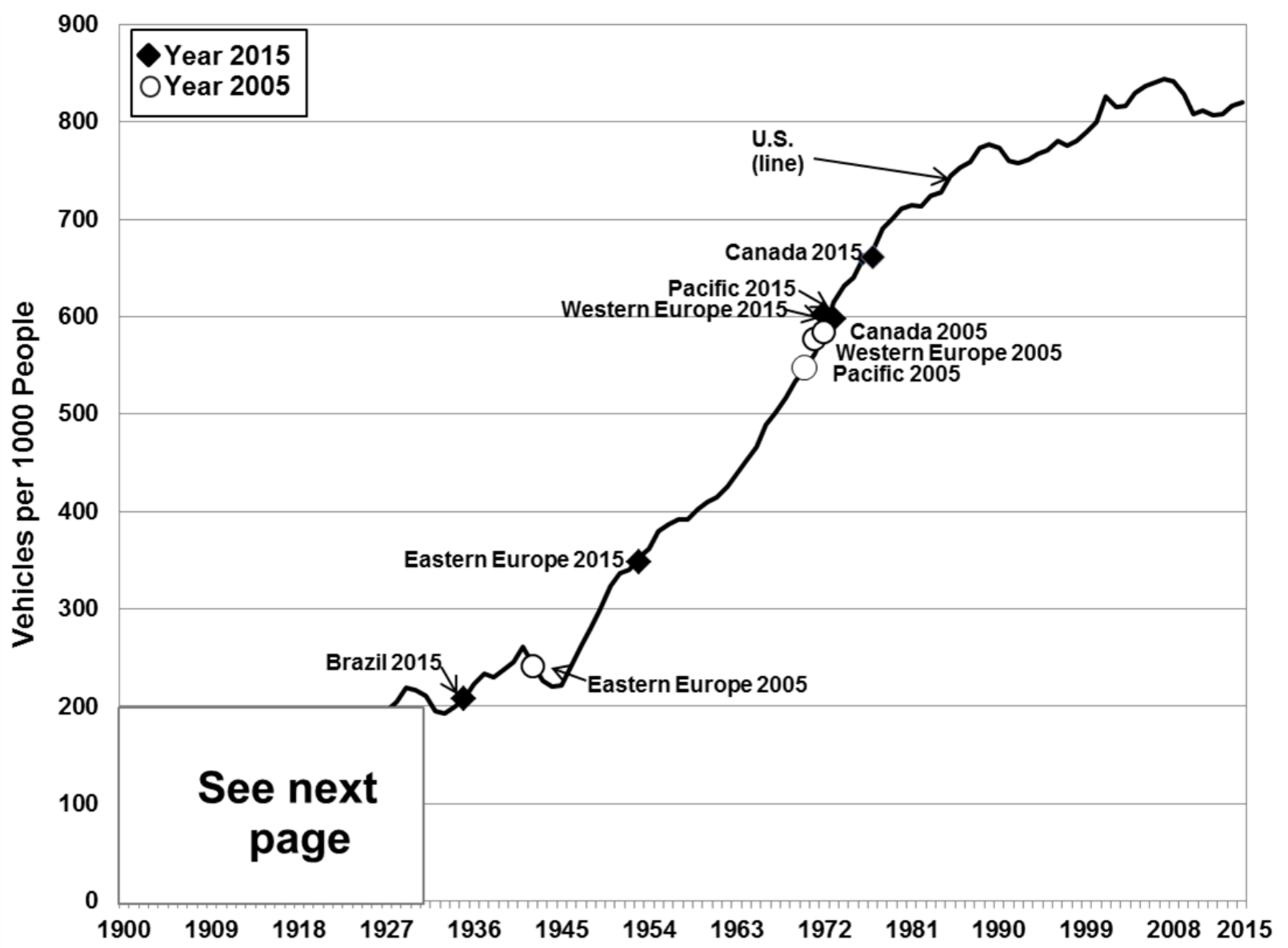




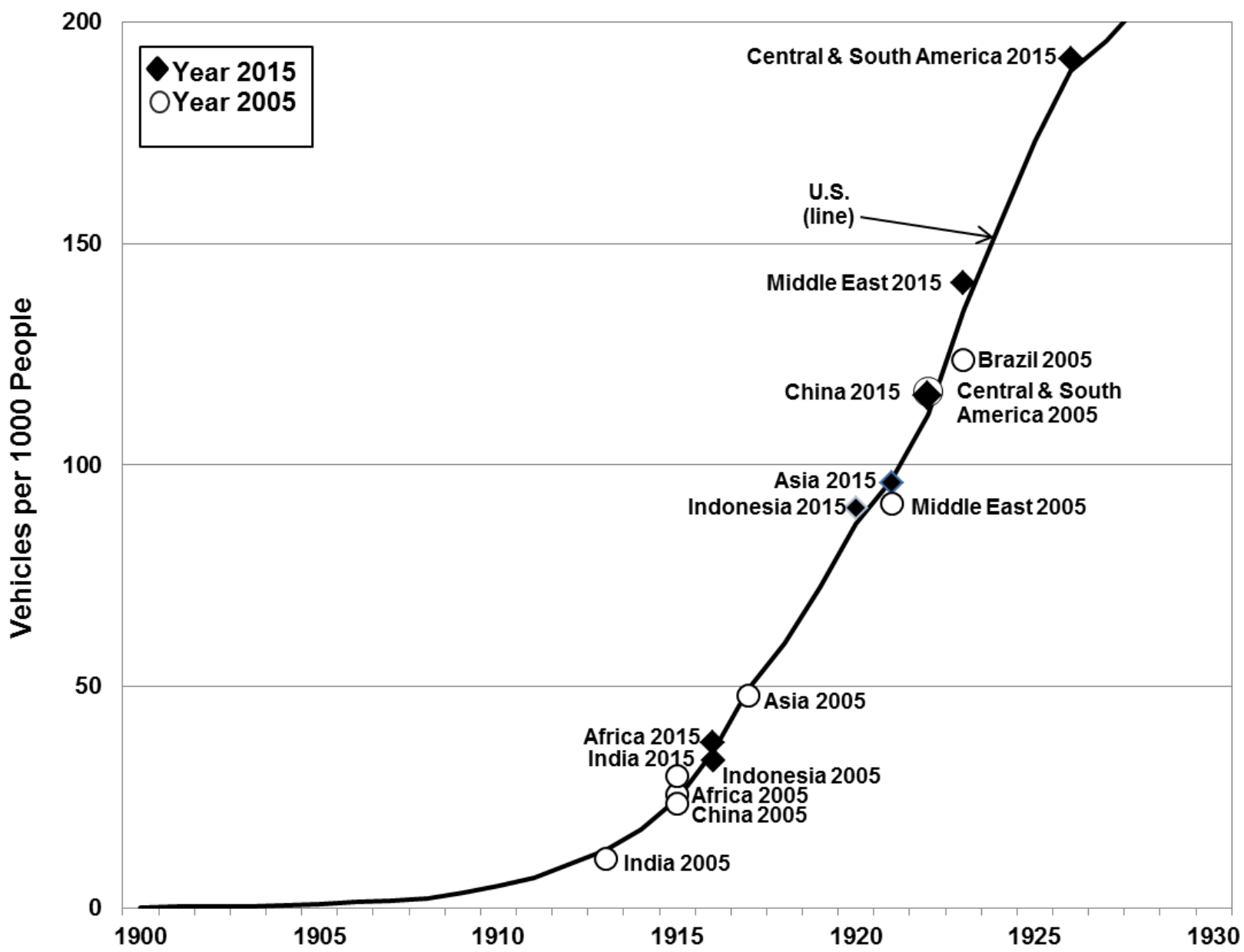

Source:

See Tables 3.5 and 3.6. 
Though some countries are listed separately in this table, those countries are also included in the regional total.

For instance, China is listed separately, but is also included in the Asia, Far East region.

Table 3.5

Vehicles per Thousand People in Selected Countries/Regions, 2005 and 2015

\begin{tabular}{lcc}
\hline & \multicolumn{2}{c}{ Vehicles per 1,000 people } \\
\cline { 2 - 3 } Country/Region & 2005 & 2015 \\
\hline Africa & 25.5 & 37.4 \\
Asia, Far East & 47.9 & 95.9 \\
Asia, Middle East & 91.2 & 141.2 \\
Brazil & 123.8 & 208.2 \\
Canada & 583.9 & 661.4 \\
Central \& South America & 116.5 & 191.7 \\
China & 23.5 & 115.8 \\
Europe, East & 241.3 & 348.0 \\
Europe, West & 577.8 & 597.6 \\
India & 11.1 & 33.4 \\
Indonesia & 29.7 & 90.2 \\
Pacific & 548.2 & 602.4 \\
United States & 837.2 & 820.1 \\
\hline
\end{tabular}

\section{Sources:}

Population - U.S. Census Bureau, Population Division, International Data Base (IDB) World, July 21, 2017. (Additional resources: www.census.gov/population/international)

Vehicles - United States: See Table 3.6. All other countries: Ward's Communications, www.wardsauto.com. 
The number of vehicles per thousand people in the United States has grown tremendously since 1900. After a peak in 2007 at 844.5, the number has declined and in 2015 was 820.2.

Table 3.6

Vehicles per Thousand People in the United States, 1990-2015

\begin{tabular}{|c|c|c|c|c|c|c|c|c|c|}
\hline Year & $\begin{array}{c}\text { U.S. } \\
\text { vehicles } \\
\text { per } 1,000 \\
\text { people }\end{array}$ & Year & $\begin{array}{c}\text { U.S. } \\
\text { vehicles } \\
\text { per } 1,000 \\
\text { people }\end{array}$ & Year & $\begin{array}{c}\text { U.S. } \\
\text { vehicles } \\
\text { per } 1,000 \\
\text { people }\end{array}$ & Year & $\begin{array}{c}\text { U.S. } \\
\text { vehicles } \\
\text { per } 1,000 \\
\text { people }\end{array}$ & Year & $\begin{array}{c}\text { U.S. } \\
\text { vehicles } \\
\text { per } 1,000 \\
\text { people }\end{array}$ \\
\hline 1900 & 0.1 & 1924 & 154.4 & 1948 & 280.2 & 1972 & 585.6 & 1996 & 781.2 \\
\hline 1901 & 0.2 & 1925 & 173.3 & 1949 & 299.6 & 1973 & 615.2 & 1997 & 776.0 \\
\hline 1902 & 0.3 & 1926 & 189.1 & 1950 & 323.7 & 1974 & 632.3 & 1998 & 781.2 \\
\hline 1903 & 0.4 & 1927 & 195.8 & 1951 & 337.1 & 1975 & 640.1 & 1999 & 790.1 \\
\hline 1904 & 0.7 & 1928 & 204.9 & 1952 & 340.6 & 1976 & 659.5 & 2000 & 800.3 \\
\hline 1905 & 0.9 & 1929 & 219.3 & 1953 & 353.7 & 1977 & 669.0 & 2001 & 825.8 \\
\hline 1906 & 1.3 & 1930 & 217.3 & 1954 & 361.4 & 1978 & 690.2 & 2002 & 815.7 \\
\hline 1907 & 1.7 & 1931 & 210.4 & 1955 & 379.8 & 1979 & 700.4 & 2003 & 816.1 \\
\hline 1908 & 2.2 & 1932 & 195.4 & 1956 & 387.6 & 1980 & 710.7 & 2004 & 829.9 \\
\hline 1909 & 3.5 & 1933 & 192.4 & 1957 & 392.1 & 1981 & 715.2 & 2005 & 837.3 \\
\hline 1910 & 5.1 & 1934 & 199.9 & 1958 & 392.2 & 1982 & 714.0 & 2006 & 840.7 \\
\hline 1911 & 6.8 & 1935 & 208.6 & 1959 & 402.8 & 1983 & 724.3 & 2007 & 844.5 \\
\hline 1912 & 9.9 & 1936 & 222.6 & 1960 & 410.4 & 1984 & 728.2 & 2008 & 841.6 \\
\hline 1913 & 12.9 & 1937 & 233.3 & 1961 & 415.1 & 1985 & 744.5 & 2009 & 828.7 \\
\hline 1914 & 17.8 & 1938 & 229.7 & 1962 & 426.1 & 1986 & 753.3 & 2010 & 808.4 \\
\hline 1915 & 24.8 & 1939 & 236.9 & 1963 & 438.8 & 1987 & 758.6 & 2011 & 812.7 \\
\hline 1916 & 35.5 & 1940 & 245.6 & 1964 & 451.6 & 1988 & 772.9 & 2012 & 808.0 \\
\hline 1917 & 49.6 & 1941 & 261.6 & 1965 & 466.9 & 1989 & 777.0 & 2013 & 808.6 \\
\hline 1918 & 59.7 & 1942 & 244.7 & 1966 & 489.3 & 1990 & 773.4 & 2014 & 816.4 \\
\hline 1919 & 72.5 & 1943 & 225.9 & 1967 & 500.7 & 1991 & 760.2 & 2015 & 820.2 \\
\hline 1920 & 86.8 & 1944 & 220.2 & 1968 & 516.5 & 1992 & 758.0 & & \\
\hline 1921 & 96.7 & 1945 & 221.8 & 1969 & 533.4 & 1993 & 761.9 & & \\
\hline 1922 & 111.5 & 1946 & 243.1 & 1970 & 545.4 & 1994 & 766.9 & & \\
\hline 1923 & 134.9 & 1947 & 262.6 & 1971 & 562.5 & 1995 & 771.0 & & \\
\hline
\end{tabular}

Sources:

Population - U.S. Census Bureau, Population Division, International Data Base (IDB) World, July 19, 2017. (Additional resources: www.census.gov/ipc/www/idb/)

Vehicles - U.S. Department of Transportation, Federal Highway Administration, Highway Statistics 2015, Washington, DC, 2016. 
Total vehicle-miles traveled increased each year from 2011 to 2015. The trend of using two-axle, four-tire trucks, such as pickups, vans, and sport-utility vehicles, for personal travel is evident in these data; two-axle, four-tire trucks account for 32.0\% more travel in 2015 than in 1970, and cars account for 35.9\% less travel in that time period.

Table 3.7

Shares of Highway Vehicle-Miles Traveled by Vehicle Type, 1970-2015

\begin{tabular}{|c|c|c|c|c|c|c|c|}
\hline Year & Cars & Motorcycles & $\begin{array}{c}\text { Two-axle, } \\
\text { four-tire } \\
\text { trucks }\end{array}$ & $\begin{array}{l}\text { Other } \\
\text { single-unit } \\
\text { trucks }\end{array}$ & $\begin{array}{c}\text { Combination } \\
\text { trucks }\end{array}$ & Buses & $\begin{array}{c}\text { Total } \\
\text { vehicle-miles } \\
\text { traveled } \\
\text { (million miles) }\end{array}$ \\
\hline 1970 & $82.6 \%$ & $0.3 \%$ & $11.1 \%$ & $2.4 \%$ & $3.2 \%$ & $0.4 \%$ & $1,109,724$ \\
\hline 1975 & $77.9 \%$ & $0.4 \%$ & $15.1 \%$ & $2.6 \%$ & $3.5 \%$ & $0.5 \%$ & $1,327,664$ \\
\hline 1980 & $72.8 \%$ & $0.7 \%$ & $19.0 \%$ & $2.6 \%$ & $4.5 \%$ & $0.4 \%$ & $1,527,295$ \\
\hline 1985 & $70.2 \%$ & $0.5 \%$ & $22.0 \%$ & $2.6 \%$ & $4.4 \%$ & $0.3 \%$ & $1,774,826$ \\
\hline 1986 & $69.2 \%$ & $0.5 \%$ & $23.1 \%$ & $2.5 \%$ & $4.4 \%$ & $0.3 \%$ & $1,834,872$ \\
\hline 1987 & $68.5 \%$ & $0.5 \%$ & $23.8 \%$ & $2.5 \%$ & $4.5 \%$ & $0.3 \%$ & $1,921,204$ \\
\hline 1988 & $67.6 \%$ & $0.5 \%$ & $24.8 \%$ & $2.4 \%$ & $4.4 \%$ & $0.3 \%$ & $2,025,962$ \\
\hline 1989 & $66.8 \%$ & $0.5 \%$ & $25.6 \%$ & $2.4 \%$ & $4.4 \%$ & $0.3 \%$ & $2,096,487$ \\
\hline 1990 & $65.7 \%$ & $0.4 \%$ & $26.8 \%$ & $2.4 \%$ & $4.4 \%$ & $0.3 \%$ & $2,144,362$ \\
\hline 1991 & $62.5 \%$ & $0.4 \%$ & $29.9 \%$ & $2.4 \%$ & $4.4 \%$ & $0.3 \%$ & $2,172,050$ \\
\hline 1992 & $61.0 \%$ & $0.4 \%$ & $31.5 \%$ & $2.4 \%$ & $4.4 \%$ & $0.3 \%$ & $2,247,151$ \\
\hline 1993 & $59.9 \%$ & $0.4 \%$ & $32.5 \%$ & $2.5 \%$ & $4.5 \%$ & $0.3 \%$ & $2,296,378$ \\
\hline 1994 & $59.6 \%$ & $0.4 \%$ & $32.4 \%$ & $2.6 \%$ & $4.6 \%$ & $0.3 \%$ & $2,357,588$ \\
\hline 1995 & $59.4 \%$ & $0.4 \%$ & $32.6 \%$ & $2.6 \%$ & $4.8 \%$ & $0.3 \%$ & $2,422,696$ \\
\hline 1996 & $59.1 \%$ & $0.4 \%$ & $32.8 \%$ & $2.6 \%$ & $4.8 \%$ & $0.3 \%$ & $2,485,848$ \\
\hline 1997 & $58.7 \%$ & $0.4 \%$ & $33.2 \%$ & $2.6 \%$ & $4.9 \%$ & $0.3 \%$ & $2,561,695$ \\
\hline 1998 & $58.9 \%$ & $0.4 \%$ & $33.0 \%$ & $2.6 \%$ & $4.9 \%$ & $0.3 \%$ & $2,631,522$ \\
\hline 1999 & $58.3 \%$ & $0.4 \%$ & $33.5 \%$ & $2.6 \%$ & $4.9 \%$ & $0.3 \%$ & $2,691,056$ \\
\hline 2000 & $58.3 \%$ & $0.4 \%$ & $33.6 \%$ & $2.6 \%$ & $4.9 \%$ & $0.3 \%$ & $2,746,925$ \\
\hline 2001 & $58.2 \%$ & $0.3 \%$ & $33.7 \%$ & $2.6 \%$ & $4.9 \%$ & $0.3 \%$ & $2,797,287$ \\
\hline 2002 & $58.1 \%$ & $0.3 \%$ & $33.8 \%$ & $2.7 \%$ & $4.9 \%$ & $0.2 \%$ & $2,855,508$ \\
\hline 2003 & $57.8 \%$ & $0.3 \%$ & $34.0 \%$ & $2.7 \%$ & $4.8 \%$ & $0.2 \%$ & $2,890,412$ \\
\hline 2004 & $57.3 \%$ & $0.3 \%$ & $34.6 \%$ & $2.6 \%$ & $4.8 \%$ & $0.2 \%$ & $2,964,788$ \\
\hline 2005 & $57.1 \%$ & $0.3 \%$ & $34.8 \%$ & $2.6 \%$ & $4.8 \%$ & $0.2 \%$ & $2,989,430$ \\
\hline 2006 & $56.1 \%$ & $0.4 \%$ & $35.9 \%$ & $2.7 \%$ & $4.7 \%$ & $0.2 \%$ & $3,014,369$ а \\
\hline 2007 & $55.2 \%$ & $0.4 \%$ & $36.7 \%$ & $2.7 \%$ & $4.8 \%$ & $0.2 \%$ & $3,032,399$ \\
\hline 2008 & $54.3 \%$ & $0.5 \%$ & $37.3 \%$ & $2.8 \%$ & $4.8 \%$ & $0.2 \%$ & $2,973,509$ \\
\hline 2009 & $53.0 \%$ & $0.7 \%$ & $36.1 \%$ & $4.1 \%$ & $5.7 \%$ & $0.5 \%$ & $2,956,764$ \\
\hline 2010 & $50.4 \%$ & $0.6 \%$ & $38.8 \%$ & $3.7 \%$ & $5.9 \%$ & $0.5 \%$ & $2,967,266$ \\
\hline 2011 & $49.4 \%$ & $0.6 \%$ & $40.4 \%$ & $3.5 \%$ & $5.6 \%$ & $0.5 \%$ & $2,950,401$ \\
\hline 2012 & $48.4 \%$ & $0.7 \%$ & $41.3 \%$ & $3.6 \%$ & $5.5 \%$ & $0.5 \%$ & $2,969,433$ \\
\hline 2013 & $48.4 \%$ & $0.7 \%$ & $41.2 \%$ & $3.6 \%$ & $5.6 \%$ & $0.5 \%$ & $2,988,281$ \\
\hline 2014 & $47.5 \%$ & $0.7 \%$ & $42.1 \%$ & $3.6 \%$ & $5.6 \%$ & $0.5 \%$ & $3,025,656$ \\
\hline 2015 & $46.7 \%$ & $0.6 \%$ & $43.1 \%$ & $3.5 \%$ & $5.5 \%$ & $0.5 \%$ & $3,095,372$ \\
\hline \multicolumn{8}{|c|}{ Average annual percentage change } \\
\hline 1970-2015 & & & & & & & $2.3 \%$ \\
\hline $2005-2015$ & & & & & & & $0.3 \%$ \\
\hline
\end{tabular}

\section{Source:}

U.S. Department of Transportation, Federal Highway Administration, Highway Statistics 2015, Washington, DC, 2016, Table VM-1 and annual. (Additional resources: www.fhwa.dot.gov). 2009-2015 cars and 2-axle 4-tire trucks - see Section 7 in Appendix A.

a Due to FHWA methodology changes, data from 2007-on are not comparable with previous data. 
In 1970 only $2.9 \%$ of the car population was 15 years old or older; by 2013 that number rose to nearly 20\%.

Table 3.8

Cars in Operation by Age, 1970, 2000, and 2013

\begin{tabular}{|c|c|c|c|c|c|c|c|c|c|}
\hline \multirow[b]{2}{*}{ Age (years) } & \multicolumn{3}{|c|}{1970} & \multicolumn{3}{|c|}{2000} & \multicolumn{3}{|c|}{2013} \\
\hline & $\begin{array}{l}\text { Vehicles } \\
\text { (thousands) }\end{array}$ & Percentage $^{\mathrm{a}}$ & $\begin{array}{l}\text { Cumulative } \\
\text { percentage }^{\text {a }}\end{array}$ & $\begin{array}{c}\text { Vehicles } \\
\text { (thousands) }\end{array}$ & Percentage $^{\mathrm{a}}$ & $\begin{array}{l}\text { Cumulative } \\
\text { percentage }^{\mathrm{a}}\end{array}$ & $\begin{array}{c}\text { Vehicles } \\
\text { (thousands) }\end{array}$ & Percentage $^{\mathrm{a}}$ & $\begin{array}{l}\text { Cumulative } \\
\text { percentage }^{\mathrm{a}}\end{array}$ \\
\hline Under $1^{\mathrm{b}}$ & 6,288 & $7.8 \%$ & $7.8 \%$ & 6,665 & $5.2 \%$ & $5.2 \%$ & 9,287 & $7.1 \%$ & $7.1 \%$ \\
\hline 1 & 9,299 & $11.6 \%$ & $19.4 \%$ & 8,177 & $6.4 \%$ & $11.6 \%$ & 7,700 & $5.9 \%$ & $13.1 \%$ \\
\hline 2 & 8,816 & $11.0 \%$ & $30.3 \%$ & 7,655 & $6.0 \%$ & $17.6 \%$ & 5,957 & $4.6 \%$ & $17.6 \%$ \\
\hline 3 & 7,878 & $9.8 \%$ & $40.1 \%$ & 7,906 & $6.2 \%$ & $23.8 \%$ & 6,159 & $4.7 \%$ & $22.4 \%$ \\
\hline 4 & 8,538 & $10.6 \%$ & $50.8 \%$ & 7,413 & $5.8 \%$ & $29.6 \%$ & 5,484 & $4.2 \%$ & $26.6 \%$ \\
\hline 5 & 8,506 & $10.6 \%$ & $61.3 \%$ & 8,675 & $6.8 \%$ & $36.4 \%$ & 7,226 & $5.6 \%$ & $32.1 \%$ \\
\hline 6 & 7,116 & $8.8 \%$ & $70.2 \%$ & 7,628 & $6.0 \%$ & $42.4 \%$ & 7,896 & $6.1 \%$ & $38.2 \%$ \\
\hline 7 & 6,268 & $7.8 \%$ & $78.0 \%$ & 7,650 & $6.0 \%$ & $48.4 \%$ & 7,706 & $5.9 \%$ & $44.1 \%$ \\
\hline 8 & 5,058 & $6.3 \%$ & $84.3 \%$ & 7,021 & $5.5 \%$ & $53.9 \%$ & 7,843 & $6.0 \%$ & $50.2 \%$ \\
\hline 9 & 3,267 & $4.1 \%$ & $88.3 \%$ & 7,109 & $5.6 \%$ & $59.4 \%$ & 6,924 & $5.3 \%$ & $55.5 \%$ \\
\hline 10 & 2,776 & $3.5 \%$ & $91.8 \%$ & 7,071 & $5.5 \%$ & $65.0 \%$ & 7,237 & $5.6 \%$ & $61.1 \%$ \\
\hline 11 & 1,692 & $2.1 \%$ & $93.9 \%$ & 7,338 & $5.7 \%$ & $70.7 \%$ & 7,167 & $5.5 \%$ & $66.6 \%$ \\
\hline 12 & 799 & $1.0 \%$ & $94.9 \%$ & 6,876 & $5.4 \%$ & $76.1 \%$ & 6,660 & $5.1 \%$ & $71.7 \%$ \\
\hline 13 & 996 & $1.2 \%$ & $96.1 \%$ & 6,084 & $4.8 \%$ & $80.9 \%$ & 6,889 & $5.3 \%$ & $77.0 \%$ \\
\hline 14 & 794 & $1.0 \%$ & $97.1 \%$ & 5,334 & $4.2 \%$ & $85.0 \%$ & 5,487 & $4.2 \%$ & $81.2 \%$ \\
\hline 15 and older & 2,336 & $2.9 \%$ & $100.0 \%$ & 19,119 & $15.0 \%$ & $100.0 \%$ & 24,457 & $18.8 \%$ & $100.0 \%$ \\
\hline Subtotal & 80,427 & $100.0 \%$ & & 127,721 & $100.0 \%$ & & 130,078 & $100.0 \%$ & \\
\hline Age not given & 22 & & & $\mathbf{0}$ & & & $\mathbf{0}$ & & \\
\hline Total & 80,449 & & & 121,721 & & & 130,078 & & \\
\hline
\end{tabular}

Source:

IHS Automotive, Detroit, MI. FURTHER REPRODUCTION PROHIBITED.

a Percentages may not sum to totals due to rounding.

${ }^{\mathrm{b}}$ Includes cars which were sold prior to July 1, 1970, and similarly, sold prior to July 1, 2000. For 2013, cars sold prior to December 31, 2013 were included. 
The number of trucks in the United States has grown significantly since 1970, some of it due to the use of light trucks (pickups, vans, sport utility vehicles) as personal passenger vehicles. Those light trucks, as well as medium and heavy trucks, are included in the data. In 1970 about 15\% of trucks were age 15 or older; by 2013, that increased to $20.8 \%$.

Table 3.9

Trucks in Operation by Age, 1970, 2000, and 2013

\begin{tabular}{|c|c|c|c|c|c|c|c|c|c|}
\hline \multirow[b]{2}{*}{ Age (years) } & \multicolumn{3}{|c|}{1970} & \multicolumn{3}{|c|}{2000} & \multicolumn{3}{|c|}{2013} \\
\hline & $\begin{array}{c}\text { Vehicles } \\
\text { (thousands) }\end{array}$ & Percentage $^{\mathrm{a}}$ & $\begin{array}{l}\text { Cumulative } \\
\text { percentage }^{\text {a }}\end{array}$ & $\begin{array}{c}\text { Vehicles } \\
\text { (thousands) }\end{array}$ & Percentage $^{\mathrm{a}}$ & $\begin{array}{l}\text { Cumulative } \\
\text { percentage }^{\text {a }}\end{array}$ & $\begin{array}{c}\text { Vehicles } \\
\text { (thousands) }\end{array}$ & Percentage $^{\mathrm{a}}$ & $\begin{array}{l}\text { Cumulative } \\
\text { percentage }^{\text {a }}\end{array}$ \\
\hline Under $1^{\mathrm{b}}$ & 1,262 & $7.1 \%$ & $7.1 \%$ & 6,439 & $7.5 \%$ & $7.5 \%$ & 8,097 & $6.5 \%$ & $6.5 \%$ \\
\hline 1 & 1,881 & $10.6 \%$ & $17.8 \%$ & 7,726 & $9.0 \%$ & $16.6 \%$ & 6,391 & $5.1 \%$ & $11.6 \%$ \\
\hline 2 & 1,536 & $8.7 \%$ & $26.5 \%$ & 6,630 & $7.7 \%$ & $24.3 \%$ & 6,417 & $5.2 \%$ & $16.8 \%$ \\
\hline 3 & 1,428 & $8.1 \%$ & $34.6 \%$ & 6,313 & $7.4 \%$ & $31.7 \%$ & 4,972 & $4.0 \%$ & $20.8 \%$ \\
\hline 4 & 1,483 & $8.4 \%$ & $43.0 \%$ & 5,300 & $6.2 \%$ & $37.9 \%$ & 3,991 & $3.2 \%$ & $24.0 \%$ \\
\hline 5 & 1,339 & $7.6 \%$ & $50.5 \%$ & 5,818 & $6.8 \%$ & $44.7 \%$ & 6,927 & $5.6 \%$ & $29.5 \%$ \\
\hline 6 & 1,154 & $6.5 \%$ & $57.1 \%$ & 5,206 & $6.1 \%$ & $50.8 \%$ & 7,587 & $6.1 \%$ & $35.6 \%$ \\
\hline 7 & 975 & $5.5 \%$ & $62.6 \%$ & 4,335 & $5.1 \%$ & $55.8 \%$ & 7,580 & $6.1 \%$ & $41.7 \%$ \\
\hline 8 & 826 & $4.7 \%$ & $67.3 \%$ & 3,547 & $4.1 \%$ & $60.0 \%$ & 7,585 & $6.1 \%$ & $47.8 \%$ \\
\hline 9 & 621 & $3.5 \%$ & $70.8 \%$ & 3,411 & $4.0 \%$ & $63.9 \%$ & 7,978 & $6.4 \%$ & $54.2 \%$ \\
\hline 10 & 658 & $3.7 \%$ & $74.5 \%$ & 3,258 & $3.8 \%$ & $67.8 \%$ & 7,201 & $5.8 \%$ & $60.0 \%$ \\
\hline 11 & 583 & $3.3 \%$ & $77.8 \%$ & 3,665 & $4.3 \%$ & $72.0 \%$ & 6,850 & $5.5 \%$ & $65.5 \%$ \\
\hline 12 & 383 & $2.2 \%$ & $80.0 \%$ & 3,421 & $4.0 \%$ & $76.0 \%$ & 6,163 & $4.9 \%$ & $70.4 \%$ \\
\hline 13 & 417 & $2.4 \%$ & $82.3 \%$ & 2,860 & $3.3 \%$ & $79.4 \%$ & 5,673 & $4.6 \%$ & $75.0 \%$ \\
\hline 14 & 414 & $2.3 \%$ & $84.7 \%$ & 2,812 & $3.3 \%$ & $82.7 \%$ & 5,217 & $4.2 \%$ & $79.2 \%$ \\
\hline 15 and older & 2,710 & $15.3 \%$ & $100.0 \%$ & 14,838 & $17.3 \%$ & $100.0 \%$ & 25,917 & $20.8 \%$ & $100.0 \%$ \\
\hline Subtotal & 17,670 & $100.0 \%$ & & 85,579 & $100.0 \%$ & & 124,545 & $100.0 \%$ & \\
\hline Age note given & 15 & & & $\mathbf{0}$ & & & $\mathbf{0}$ & & \\
\hline Total & 17,685 & & & 85,579 & & & 124,545 & & \\
\hline
\end{tabular}

Source:

IHS Automotive, Detroit, MI. FURTHER REPRODUCTION PROHIBITED.

${ }^{\text {a }}$ Percentages may not sum to totals due to rounding.

b Includes trucks which were sold prior to July 1, 1970, and similarly, sold prior to July 1, 2000. For 2013, trucks sold prior to December 31, 2013 were included. 
The average age of cars and light trucks has grown to a record level in 2016-11.6 years. Light trucks, which include pickups, vans, and sport utility vehicles, previously had a lower average age than cars. In 2014 through 2016, however, there is no difference in their ages.

Table 3.10

U.S. Average Vehicle Age, 1995-2016

\begin{tabular}{cccc}
\hline Calendar Year & Passenger cars & Light trucks & All light vehicles \\
\hline 1995 & 8.4 & 8.3 & 8.4 \\
1996 & 8.5 & 8.3 & 8.5 \\
1997 & 8.7 & 8.5 & 8.6 \\
1998 & 8.9 & 8.5 & 8.8 \\
1999 & 9.1 & 8.5 & 8.8 \\
2000 & 9.1 & 8.4 & 8.9 \\
2001 & 9.3 & 8.4 & 8.9 \\
2002 & 9.8 & 9.4 & 9.6 \\
2003 & 9.9 & 9.0 & 9.7 \\
2004 & 10.0 & 9.5 & 9.8 \\
2005 & 10.1 & 9.5 & 9.8 \\
2006 & 10.2 & 9.5 & 9.9 \\
2007 & 10.3 & 9.6 & 10.0 \\
2008 & 10.4 & 9.8 & 10.1 \\
2009 & 10.5 & 10.1 & 10.3 \\
2010 & 10.8 & 10.5 & 10.6 \\
2011 & 11.1 & 10.8 & 10.9 \\
2012 & 11.3 & 11.1 & 11.2 \\
2013 & 11.4 & 11.3 & 11.4 \\
2014 & 11.4 & 11.4 & 11.4 \\
2015 & 11.5 & 11.5 & 11.5 \\
2016 & 11.6 & 11.6 & 11.6 \\
\hline
\end{tabular}

Source:

IHS Automotive, Detroit, MI. FURTHER REPRODUCTION PROHIBITED. (Additional resources: https://www.ihs.com/industry/automotive.html)

${ }^{a}$ In 2013, IHS Automotive published a data series showing vehicle age from 2002-2013. These data did not match the previous data published by IHS Automotive. The data prior to 2002 came from earlier IHS Automotive news releases and is not comparable to the revised data. 
Table 3.11

New Retail Vehicle Sales, 1970-2016

(thousands)

\begin{tabular}{|c|c|c|c|c|c|}
\hline $\begin{array}{c}\text { Calendar } \\
\text { Year }\end{array}$ & Cars & $\begin{array}{c}\text { Light } \\
\text { Trucks }^{\mathrm{a}}\end{array}$ & $\begin{array}{c}\text { Subtotal } \\
\text { Light Vehicles }\end{array}$ & $\begin{array}{l}\text { Heavy } \\
\text { Trucks }\end{array}$ & $\begin{array}{c}\text { Total } \\
\text { Vehicle } \\
\text { Sales }\end{array}$ \\
\hline 1970 & 8,399 & 1,457 & 9,856 & 334 & 10,190 \\
\hline 1975 & 8,624 & 2,053 & 10,677 & 298 & 10,975 \\
\hline 1976 & 10,110 & 2,719 & 12,829 & 324 & 13,153 \\
\hline 1977 & 11,183 & 3,109 & 14,292 & 376 & 14,668 \\
\hline 1978 & 11,314 & 3,474 & 14,788 & 441 & 15,229 \\
\hline 1979 & 10,673 & 2,845 & 13,518 & 391 & 13,909 \\
\hline 1980 & 8,949 & 1,960 & 10,909 & 271 & 11,180 \\
\hline 1981 & 8,489 & 1,746 & 10,235 & 226 & 10,461 \\
\hline 1982 & 7,956 & 2,063 & 10,019 & 184 & 10,203 \\
\hline 1983 & 9,148 & 2,521 & 11,669 & 189 & 11,858 \\
\hline 1984 & 10,324 & 3,255 & 13,579 & 282 & 13,861 \\
\hline 1985 & 10,979 & 3,688 & 14,667 & 295 & 14,962 \\
\hline 1986 & 11,404 & 4,594 & 15,998 & 277 & 16,275 \\
\hline 1987 & 10,192 & 4,610 & 14,802 & 302 & 15,104 \\
\hline 1988 & 10,547 & 4,800 & 15,347 & 348 & 15,695 \\
\hline 1989 & 9,779 & 4,610 & 14,389 & 330 & 14,719 \\
\hline 1990 & 9,303 & 4,548 & 13,851 & 297 & 14,148 \\
\hline 1991 & 8,185 & 4,122 & 12,307 & 242 & 12,549 \\
\hline 1992 & 8,213 & 4,629 & 12,842 & 276 & 13,118 \\
\hline 1993 & 8,518 & 5,351 & 13,869 & 330 & 14,199 \\
\hline 1994 & 8,991 & 6,033 & 15,024 & 387 & 15,411 \\
\hline 1995 & 8,620 & 6,053 & 14,673 & 428 & 15,101 \\
\hline 1996 & 8,479 & 6,519 & 14,998 & 411 & 15,409 \\
\hline 1997 & 8,217 & 6,797 & 15,014 & 430 & 15,444 \\
\hline 1998 & 8,085 & 7,299 & 15,384 & 526 & 15,910 \\
\hline 1999 & 8,638 & 8,073 & 16,711 & 641 & 17,352 \\
\hline 2000 & 8,778 & 8,386 & 17,164 & 579 & 17,743 \\
\hline 2001 & 8,352 & 8,598 & 16,950 & 452 & 17,402 \\
\hline 2002 & 8,042 & 8,633 & 16,675 & 402 & 17,077 \\
\hline 2003 & 7,556 & 8,938 & 16,494 & 420 & 16,914 \\
\hline 2004 & 7,483 & 9,254 & 16,737 & 538 & 17,275 \\
\hline 2005 & 7,660 & 9,114 & 16,774 & 664 & 17,438 \\
\hline 2006 & 7,762 & 8,574 & 16,336 & 694 & 17,030 \\
\hline 2007 & 7,562 & 8,305 & 15,867 & 537 & 16,404 \\
\hline 2008 & 6,769 & 6,246 & 13,015 & 432 & 13,447 \\
\hline 2009 & 5,402 & 4,834 & 10,236 & 312 & 10,548 \\
\hline 2010 & 5,636 & 5,758 & 11,394 & 378 & 11,772 \\
\hline 2011 & 6,093 & 6,449 & 12,542 & 500 & 13,042 \\
\hline 2012 & 7,245 & 6,975 & 14,220 & 569 & 14,789 \\
\hline 2013 & 7,586 & 7,693 & 15,279 & 606 & 15,884 \\
\hline 2014 & 7,708 & 8,484 & 16,192 & 671 & 16,862 \\
\hline 2015 & 7,517 & 9,578 & 17,095 & 732 & 17,827 \\
\hline 2016 & 6,873 & 10,296 & 17,169 & 697 & 17,866 \\
\hline \multicolumn{6}{|c|}{ Average annual percentage change } \\
\hline 1970-2016 & $-0.4 \%$ & $4.3 \%$ & $1.2 \%$ & $1.6 \%$ & $1.2 \%$ \\
\hline 2006-2016 & $-1.2 \%$ & $1.8 \%$ & $0.5 \%$ & $0.0 \%$ & $0.5 \%$ \\
\hline
\end{tabular}

Source:

Ward's Communications, www.wardsauto.com.

${ }^{a}$ Includes light trucks of 10,000 lb. gross vehicle weight and less. 
The Environmental Protection Agency estimated the survival rates for cars and light trucks for the mid-term evaluation of the Light Vehicle Greenhouse Gas Emissions Standards and Corporate Average Fuel Economy Standards.

Table 3.12

Survival Rates for Cars and Light Trucks by Vehicle Age

\begin{tabular}{|c|c|c|}
\hline $\begin{array}{l}\text { Vehicle age } \\
\text { (years) }\end{array}$ & $\begin{array}{l}\text { Estimated } \\
\text { survival rate } \\
\text { for cars }\end{array}$ & $\begin{array}{c}\text { Estimated } \\
\text { survival rate } \\
\text { for light trucks }\end{array}$ \\
\hline 0 & 1.000 & 1.000 \\
\hline 1 & 0.997 & 0.991 \\
\hline 2 & 0.994 & 0.982 \\
\hline 3 & 0.991 & 0.973 \\
\hline 4 & 0.984 & 0.960 \\
\hline 5 & 0.974 & 0.941 \\
\hline 6 & 0.961 & 0.919 \\
\hline 7 & 0.942 & 0.891 \\
\hline 8 & 0.920 & 0.859 \\
\hline 9 & 0.893 & 0.823 \\
\hline 10 & 0.862 & 0.784 \\
\hline 11 & 0.826 & 0.741 \\
\hline 12 & 0.788 & 0.697 \\
\hline 13 & 0.718 & 0.651 \\
\hline 14 & 0.613 & 0.605 \\
\hline 15 & 0.510 & 0.553 \\
\hline 16 & 0.415 & 0.502 \\
\hline 17 & 0.332 & 0.453 \\
\hline 18 & 0.261 & 0.407 \\
\hline 19 & 0.203 & 0.364 \\
\hline 20 & 0.157 & 0.324 \\
\hline 21 & 0.120 & 0.288 \\
\hline 22 & 0.092 & 0.255 \\
\hline 23 & 0.070 & 0.225 \\
\hline 24 & 0.053 & 0.198 \\
\hline 25 & 0.040 & 0.174 \\
\hline 26 & 0.030 & 0.153 \\
\hline 27 & 0.023 & 0.133 \\
\hline 28 & 0.013 & 0.117 \\
\hline 29 & 0.010 & 0.102 \\
\hline 30 & 0.007 & 0.089 \\
\hline 31 & 0.002 & 0.027 \\
\hline
\end{tabular}

\section{Source:}

U.S. Environmental Protection Agency, Draft Technical Assessment Report: Midterm Evaluation of Light-Duty Vehicle Greenhouse Gas Emission Standards and Corporate Average Fuel Economy Standards for Model Years 2022-2025, EPA-420-D-16-900, July 2016. (Additional resources: https://www.epa.gov/regulations-emissionsvehicles-and-engines/midterm-evaluation-light-duty-vehicle-greenhouse-gas-ghg\#TAR) 
The Environmental Protection Agency estimated the annual vehicle miles of travel for cars and light trucks up to 30 years old for the mid-term evaluation of the Light Vehicle Greenhouse Gas Emissions Standards and Corporate Average Fuel Economy Standards. The "Total" row represents the number of miles a car or light truck would travel if it is in operation for 30 years. Typical lifetime miles from a 2006 study by the National Highway Traffic Safety Administration (NHTSA) are shown below the total.

Table 3.13

Annual Mileage for Cars and Light Trucks by Vehicle Age

\begin{tabular}{|c|c|c|}
\hline $\begin{array}{l}\text { Vehicle age } \\
\text { (years) }\end{array}$ & $\begin{array}{c}\text { Estimated annual } \\
\text { vehicle miles of } \\
\text { travel for cars }\end{array}$ & $\begin{array}{c}\text { Estimated annual } \\
\text { vehicle miles of travel } \\
\text { for light trucks }\end{array}$ \\
\hline 0 & 13,843 & 15,962 \\
\hline 1 & 13,580 & 15,670 \\
\hline 2 & 13,296 & 15,320 \\
\hline 3 & 12,992 & 15,098 \\
\hline 4 & 12,672 & 14,528 \\
\hline 5 & 12,337 & 14,081 \\
\hline 6 & 11,989 & 13,548 \\
\hline 7 & 11,630 & 13,112 \\
\hline 8 & 11,262 & 12,544 \\
\hline 9 & 10,887 & 12,078 \\
\hline 10 & 10,509 & 11,595 \\
\hline 11 & 10,129 & 11,131 \\
\hline 12 & 9,748 & 10,641 \\
\hline 13 & 9,370 & 10,153 \\
\hline 14 & 8,997 & 9,691 \\
\hline 15 & 8,629 & 9,239 \\
\hline 16 & 8,270 & 8,797 \\
\hline 17 & 7,922 & 8,383 \\
\hline 18 & 7,586 & 8,009 \\
\hline 19 & 7,265 & 7,666 \\
\hline 20 & 6,962 & 7,358 \\
\hline 21 & 6,679 & 7,089 \\
\hline 22 & 6,416 & 6,862 \\
\hline 23 & 6,177 & 6,684 \\
\hline 24 & 5,963 & 6,556 \\
\hline 25 & 5,778 & 6,481 \\
\hline 26 & 5,623 & 6,466 \\
\hline 27 & 5,499 & 6,466 \\
\hline 28 & 5,410 & 6,466 \\
\hline 29 & 5,358 & 6,466 \\
\hline 30 & 5,358 & 6,466 \\
\hline Total & 278,134 & 310,610 \\
\hline $\begin{array}{l}\text { NHTSA } 2006 \text { study - } \\
\text { typical lifetime miles }\end{array}$ & 152,137 & 179,954 \\
\hline
\end{tabular}

Sources:

U.S. Environmental Protection Agency, Draft Technical Assessment Report: Midterm Evaluation of Light-Duty Vehicle Greenhouse Gas Emission Standards and Corporate Average Fuel Economy Standards for Model Years 2022-2025, EPA-420-D-16-900, July 2016. (Additional resources: https://www.epa.gov/regulations-emissionsvehicles-and-engines/midterm-evaluation-light-duty-vehicle-greenhouse-gas-ghg\#TAR)

U.S. Department of Transportation, National Highway Traffic Safety Administration, Vehicle Survivability and Travel Mileage Schedules, January 2006. 
Using current registration data and a scrappage model by Greenspan and Cohen [1996 paper:

www.federalreserve.gov/pubs/feds/1996/199640/199640pap.pdf], ORNL calculated heavy truck (trucks over 26,000 lb gross vehicle weight) scrappage rates. The expected median lifetime for a 1990 model year heavy truck is 29 years. These data are fitted model values which assume constant economic conditions.

Table 3.14

Heavy Truck ${ }^{a}$ Scrappage and Survival Rates 1970, 1980, and 1990 Model Years

\begin{tabular}{|c|c|c|c|c|c|c|}
\hline \multirow{2}{*}{$\begin{array}{c}\text { Vehicle } \\
\text { age }^{\mathrm{b}} \\
\text { (years) }\end{array}$} & \multicolumn{2}{|c|}{1970 model year } & \multicolumn{2}{|c|}{1980 model year } & \multicolumn{2}{|c|}{1990 model year } \\
\hline & $\begin{array}{l}\text { Survival } \\
\text { rate }^{\mathrm{c}}\end{array}$ & $\begin{array}{l}\text { Scrappage } \\
\text { rate }^{\mathrm{d}}\end{array}$ & $\begin{array}{c}\text { Survival } \\
\text { rate }^{\mathrm{c}}\end{array}$ & $\begin{array}{l}\text { Scrappage } \\
\text { rate }^{\mathrm{d}}\end{array}$ & $\begin{array}{l}\text { Survival } \\
\text { rate }^{\mathrm{c}}\end{array}$ & $\begin{array}{l}\text { Scrappage } \\
\text { rate }^{\mathrm{d}}\end{array}$ \\
\hline 4 & 98.8 & 1.2 & 98.5 & 1.5 & 99.4 & 0.6 \\
\hline 5 & 97.2 & 1.6 & 96.7 & 1.9 & 98.6 & 0.8 \\
\hline 6 & 95.3 & 1.9 & 94.5 & 2.3 & 97.6 & 1.0 \\
\hline 7 & 93.2 & 2.3 & 92.0 & 2.7 & 96.5 & 1.2 \\
\hline 8 & 90.7 & 2.6 & 89.1 & 3.1 & 95.2 & 1.3 \\
\hline 9 & 88.1 & 3.0 & 86.0 & 3.5 & 93.8 & 1.5 \\
\hline 10 & 85.2 & 3.3 & 82.7 & 3.9 & 92.2 & 1.7 \\
\hline 11 & 82.1 & 3.6 & 79.1 & 4.3 & 90.5 & 1.9 \\
\hline 12 & 78.8 & 4.0 & 75.4 & 4.7 & 88.6 & 2.0 \\
\hline 13 & 75.4 & 4.3 & 71.6 & 5.1 & 86.7 & 2.2 \\
\hline 14 & 71.9 & 4.7 & 67.7 & 5.5 & 84.6 & 2.4 \\
\hline 15 & 68.3 & 5.0 & 63.7 & 5.9 & 82.4 & 2.6 \\
\hline 16 & 64.6 & 5.3 & 59.7 & 6.3 & 80.2 & 2.7 \\
\hline 17 & 61.0 & 5.7 & 55.7 & 6.7 & 77.9 & 2.9 \\
\hline 18 & 57.3 & 6.0 & 51.8 & 7.1 & 75.5 & 3.1 \\
\hline 19 & 53.7 & 6.3 & 47.9 & 7.4 & 73.0 & 3.3 \\
\hline 20 & 50.1 & 6.7 & 44.2 & 7.8 & 70.5 & 3.4 \\
\hline 21 & 46.6 & 7.0 & 40.6 & 8.2 & 68.0 & 3.6 \\
\hline 22 & 43.2 & 7.3 & 37.1 & 8.6 & 65.4 & 3.8 \\
\hline 23 & 39.9 & 7.6 & 33.7 & 9.0 & 62.8 & 3.9 \\
\hline 24 & 36.7 & 8.0 & 30.6 & 9.4 & 60.3 & 4.1 \\
\hline 25 & 33.7 & 8.3 & 27.6 & 9.7 & 57.7 & 4.3 \\
\hline 26 & 30.8 & 8.6 & 24.8 & 10.1 & 55.1 & 4.5 \\
\hline 27 & 28.0 & 8.9 & 22.2 & 10.5 & 52.6 & 4.6 \\
\hline 28 & 25.4 & 9.3 & 19.8 & 10.9 & 50.0 & 4.8 \\
\hline 29 & 23.0 & 9.6 & 17.6 & 11.2 & 47.6 & 5.0 \\
\hline 30 & 20.7 & 9.9 & 15.5 & 11.6 & 45.1 & 5.1 \\
\hline $\begin{array}{l}\text { Median } \\
\text { lifetime }\end{array}$ & \multicolumn{2}{|c|}{20.0 years } & \multicolumn{2}{|c|}{18.5 years } & \multicolumn{2}{|c|}{28.0 years } \\
\hline
\end{tabular}

Source:

Schmoyer, Richard L., unpublished study on scrappage rates, Oak Ridge National Laboratory, Oak Ridge, TN, 2001.

${ }^{a}$ Heavy trucks are trucks over 26,000 lb gross vehicle weight.

${ }^{\mathrm{b}}$ It was assumed that scrappage for vehicles less than 4 years old is 0 .

${ }^{c}$ The percentage of heavy trucks which will be in use at the end of the year.

${ }^{d}$ The percentage of heavy trucks which will be retired from use during the year. 


\section{Chapter 4 \\ Light Vehicles and Characteristics}

Summary Statistics from Tables in this Chapter

Source

Table $4.1 \quad$ Cars, 2015

Registrations (thousands)

112,864

Vehicle miles (billion miles)

$1,445.4$

Fleet average fuel economy (miles per gallon)

26.2

Table 4.2 Two-axle, four-tire trucks, 2015

Registrations (thousands)

128,553

Vehicle miles (billion miles)

$1,343.1$

Fleet average fuel economy (miles per gallon)

18.8

Table 4.7 Light truck share of total light vehicle sales

1970 calendar year

$14.8 \%$

2016 calendar year

$60.0 \%$

Table 4.8 Cars, 2015 model year

Production (thousands)

7,899

New car fuel economy (miles per gallon) 29.4

Table 4.8 Car SUVs, 2015 model year

Production (thousands) 1,701

New car SUV fuel economy (miles per gallon) 25.3

Table 4.8 Truck SUVs, 2015 model year

Production (thousands) 4,697

New truck SUV fuel economy (miles per gallon) 22.6

Table 4.8 Pickups, 2015 model year

Production (thousands) $\quad 1,786$

New pickup fuel economy (miles per gallon) $\quad 18.8$

Table 4.8 Vans, 2015 model year

Production (thousands) 655

New van fuel economy (miles per gallon) 21.9

Table $4.29 \quad$ Average fuel economy loss from 50 to $70 \mathrm{mph} \quad 24.5 \%$

The definition of light truck can change from table to table in this document due to differing definitions among federal government regulations and public nomenclature. See page 4-2 for additional information. 


\section{Definition of Light Truck}

Often for regulatory purposes, agencies within the federal government have differing definitions for the term "light truck." Private data collectors, such as Ward's Communications or IHS Automotive/Polk, have their own definitions as well. The paragraphs below are intended as a guide to the different definitions which are used in this document.

The data on Table 4.2 are from the Federal Highway Administration (FHWA). From 1970 to 2008 the FHWA defined light trucks as two-axle, four-tire trucks, including pickups, vans, SUVs, and other two-axle, four-tire trucks under 10,000 lb gross vehicle weight rating (GVWR). In 2009, the FHWA changed methodologies and no longer publishes vehicle miles, fuel use, and fuel economy of light trucks separately from cars. They continue to publish vehicle registrations for pickups, vans, SUVs and other two-axle, four tire trucks under 10,000 lb. The methodology used by Oak Ridge National Laboratory (ORNL) to continue the data series on Table 4.2 after 2008 is based on the FHWA data for all light vehicles, thus uses the same definition of light trucks. See Section 7.2 in Appendix A for the methodology of light truck data on Table 4.2 after 2008. Data on energy use in Tables 2.7 through 2.9 also use the FHWA definition of light truck.

Tables 3.11, 4.4, 4.5, and 4.7 are light truck sales based on Ward's Communications data. Ward's definition of light trucks includes pickups, vans, SUVs, and specialty purpose vehicles up to $14,000 \mathrm{lb}$ GVWR. However, in most cases, data are available by individual GVWR and ORNL summarized only light trucks that were 10,000 lb GVWR or less and did not include the heavier trucks. Thus, the definition on these tables is nearly identical to the FHWA definition.

The Environmental Protection Agency (EPA) and the Department of Transportation, National Highway Traffic Safety Administration (NHTSA), issued joint rulemaking to establish Corporate Average Fuel Economy (CAFE) standards and greenhouse gas emissions standards beginning with model year 2012. The rulemaking established new definitions of cars and light trucks. Before the rule, CAFE standards applied to cars and light trucks (pickups, vans, SUVs, and other trucks) less than 8,500 lb GVWR. After the rule, some two-wheel drive SUVs are considered cars instead of light trucks, and personal passenger vehicles (vans and SUVs) up to 10,000 lb GVWR are considered light trucks. Thus, data are now categorized as cars, car SUVs, truck SUVs, pickups and vans. Table 4.9 gives a listing of which SUVs are considered car SUVs for model year 2016. The EPA revised their data series back to 1975, so the definitions are consistent historically. Data on tables 4.8 through 4.15 are based on EPA data and thus use this definition of cars and light trucks. The CAFE data on Table 4.21 apply to cars only through 2011 and cars plus car SUVs after that. The CAFE data on Table 4.22 are for trucks up to 8,500 lb GVWR through 2011 and after that are for truck SUVs and vans up to 10,000 lb GVWR, and pickup trucks up to 8,500 lb GVWR.

Because of these different definitions, caution is advised when comparing light truck data from different sources. 
The data in this table from 1985-on DO NOT include minivans, pickups, or sport utility vehicles. Much of the data for 2009-on were estimated; the FHWA no longer publishes travel and fuel data for cars. A methodology change for the number of cars registered affected the series in 2012.

Table 4.1

Summary Statistics for Cars, 1970-2015

\begin{tabular}{|c|c|c|c|c|c|c|}
\hline Year & $\begin{array}{c}\text { Registrations }^{\mathrm{a}} \\
\text { (thousands) }\end{array}$ & $\begin{array}{l}\text { Vehicle travel } \\
\text { (billion miles) }\end{array}$ & $\begin{array}{c}\text { Miles } \\
\text { (per vehicle) }\end{array}$ & $\begin{array}{c}\text { Fuel use } \\
\text { (million gallons) }\end{array}$ & $\begin{array}{l}\text { Fuel economy } \\
\text { (miles per gallon) }\end{array}$ & \\
\hline 1970 & 89,244 & 916.7 & 10,272 & 67,820 & 13.5 & \\
\hline 1975 & 106,706 & $1,034.0$ & 9,690 & 74,140 & 13.9 & \\
\hline 1980 & 121,601 & $1,111.6$ & 9,141 & 69,981 & 15.9 & \\
\hline $1985^{\mathrm{c}}$ & 127,885 & $1,246.8$ & 9,749 & 71,518 & 17.4 & \\
\hline 1986 & 130,004 & $1,270.2$ & 9,770 & 73,174 & 17.4 & \\
\hline 1987 & 131,482 & $1,316.0$ & 10,009 & 73,308 & 18.0 & \\
\hline 1988 & 133,836 & $1,370.3$ & 10,238 & 73,345 & 18.7 & \\
\hline 1989 & 134,559 & $1,401.2$ & 10,413 & 73,913 & 19.0 & \\
\hline 1990 & 133,700 & $1,408.3$ & 10,533 & 69,568 & 20.2 & \\
\hline 1991 & 128,300 & 1,358.2 & 10,586 & 64,318 & 21.1 & \\
\hline 1992 & 126,581 & $1,371.6$ & 10,836 & 65,436 & 21.0 & \\
\hline 1993 & 127,327 & $1,374.7$ & 10,797 & 67,047 & 20.5 & \\
\hline 1994 & 127,883 & $1,406.1$ & 10,995 & 67,874 & 20.7 & \\
\hline 1995 & 128,387 & $1,438.3$ & 11,203 & 68,072 & 21.1 & \\
\hline 1996 & 129,728 & 1,469.9 & 11,330 & 69,221 & 21.2 & \\
\hline 1997 & 129,749 & 1,502.6 & 11,580 & 69,892 & 21.5 & \\
\hline 1998 & 131,839 & 1,549.6 & 11,754 & 71,695 & 21.6 & \\
\hline 1999 & 132,432 & 1,569.1 & 11,848 & 73,283 & 21.4 & \\
\hline 2000 & 133,621 & $1,600.3$ & 11,976 & 73,065 & 21.9 & \\
\hline 2001 & 137,633 & $1,628.3$ & 11,831 & 73,559 & 22.1 & \\
\hline 2002 & 135,921 & $1,658.5$ & 12,202 & 75,471 & 22.0 & \\
\hline 2003 & 135,670 & $1,672.1$ & 12,325 & 74,590 & 22.4 & \\
\hline 2004 & 136,431 & 1,699.9 & 12,460 & 75,402 & 22.5 & \\
\hline 2005 & 136,568 & $1,708.4$ & 12,510 & 77,418 & 22.1 & \\
\hline 2006 & 135,400 & $1,690.5$ & 12,485 & 75,009 & 22.5 & \\
\hline 2007 & 135,933 & $1,672.5$ & 12,304 & 74,377 & 22.5 & \\
\hline 2008 & 137,080 & $1,615.9$ & 11,788 & 71,497 & 22.6 & d \\
\hline 2009 & 134,880 & $1,566.8$ & 11,616 & 66,587 & 23.5 & \\
\hline 2010 & 130,892 & $1,496.4$ & 11,432 & 62,245 & 24.0 & \\
\hline 2011 & 125,657 & $1,457.8$ & 11,601 & 59,646 & 24.4 & \\
\hline 2012 & 111,290 & 1,438.6 & 12,928 & 57,899 & 24.9 & \\
\hline 2013 & 113,676 & $1,446.0$ & 12,720 & 57,290 & 25.2 & \\
\hline 2014 & 113,899 & 1,436.6 & 12,613 & 56,470 & 25.4 & \\
\hline 2015 & 112,864 & $1,445.4$ & 12,807 & 55,212 & 26.2 & \\
\hline & \multicolumn{6}{|c|}{ Average annual percentage change } \\
\hline 1970-2015 & $0.5 \%$ & $1.0 \%$ & $0.5 \%$ & $-0.5 \%$ & $1.5 \%$ & \\
\hline 2005-2015 & $-1.9 \%$ & $-1.7 \%$ & $0.2 \%$ & $-3.3 \%$ & $1.7 \%$ & \\
\hline
\end{tabular}

\section{Source:}

1970-2008: U.S. Department of Transportation, Federal Highway Administration, Highway Statistics 2009, Washington, DC, 2011, Table VM-1 and annual. 2009-on: See Section 7.1 in Appendix A. (Additional resources: www.fhwa.dot.gov)

a This number differs from IHS Automotive’s estimates of "number of cars in use.” See Table 3.4.

${ }^{\mathrm{b}}$ Fuel economy for car population.

c Beginning in this year the data were revised to exclude minivans, pickups and sport utility vehicles which may have been previously included.

${ }^{\mathrm{d}}$ Due to FHWA methodology changes, data from 2009-on are not comparable with previous data. 
Much of the data for 2009-on were estimated; the FHWA no longer publishes travel and fuel use data for two-axle, four-tire trucks. A methodology change for the number of registrations affected the data series in 2012.

Table 4.2

Summary Statistics for Two-Axle, Four-Tire Trucks, 1970-2015

\begin{tabular}{|c|c|c|c|c|c|c|}
\hline Year & $\begin{array}{l}\text { Registrations } \\
\text { (thousands) }\end{array}$ & $\begin{array}{l}\text { Vehicle travel } \\
\text { (billion miles) }\end{array}$ & $\begin{array}{c}\text { Miles } \\
\text { (per vehicle) }\end{array}$ & $\begin{array}{c}\text { Fuel use } \\
\text { (million gallons) }\end{array}$ & $\begin{array}{c}\text { Fuel economy } \\
\text { (miles per gallon) }\end{array}$ & \\
\hline 1970 & 14,211 & 123.3 & 8,675 & 12,313 & 10.0 & \\
\hline 1975 & 20,418 & 200.7 & 9,830 & 19,081 & 10.5 & \\
\hline 1980 & 27,876 & 290.9 & 10,437 & 23,796 & 12.2 & \\
\hline $1985^{a}$ & 37,214 & 391.0 & 10,506 & 27,363 & 14.3 & \\
\hline 1986 & 39,382 & 423.9 & 10,764 & 29,074 & 14.6 & \\
\hline 1987 & 41,107 & 456.9 & 11,114 & 30,598 & 14.9 & \\
\hline 1988 & 43,805 & 502.2 & 11,465 & 32,653 & 15.4 & \\
\hline 1989 & 45,945 & 536.5 & 11,676 & 33,271 & 16.1 & \\
\hline 1990 & 48,275 & 574.6 & 11,902 & 35,611 & 16.1 & \\
\hline 1991 & 53,033 & 649.4 & 12,245 & 38,217 & 17.0 & \\
\hline 1992 & 57,091 & 706.9 & 12,381 & 40,929 & 17.3 & \\
\hline 1993 & 59,994 & 745.8 & 12,430 & 42,851 & 17.4 & \\
\hline 1994 & 62,904 & 764.6 & 12,156 & 44,112 & 17.3 & \\
\hline 1995 & 65,738 & 790.0 & 12,018 & 45,605 & 17.3 & \\
\hline 1996 & 69,134 & 816.5 & 11,811 & 47,354 & 17.2 & \\
\hline 1997 & 70,224 & 850.7 & 12,115 & 49,389 & 17.2 & \\
\hline 1998 & 71,330 & 868.3 & 12,173 & 50,462 & 17.2 & \\
\hline 1999 & 75,356 & 901.0 & 11,957 & 52,859 & 17.0 & \\
\hline 2000 & 79,085 & 923.1 & 11,672 & 52,939 & 17.4 & \\
\hline 2001 & 84,188 & 943.2 & 11,204 & 53,522 & 17.6 & \\
\hline 2002 & 85,011 & 966.0 & 11,364 & 55,220 & 17.5 & \\
\hline 2003 & 87,187 & 984.1 & 11,287 & 60,758 & 16.2 & \\
\hline 2004 & 91,845 & $1,027.2$ & 11,184 & 63,417 & 16.2 & \\
\hline 2005 & 95,337 & $1,041.1$ & 10,920 & 58,869 & 17.7 & \\
\hline 2006 & 99,125 & $1,082.5$ & 10,920 & 60,685 & 17.8 & \\
\hline 2007 & 101,470 & $1,112.3$ & 10,962 & 61,836 & 18.0 & \\
\hline 2008 & 101,235 & $1,108.6$ & 10,951 & 61,199 & 18.1 & b \\
\hline 2009 & 100,154 & $1,066.5$ & 10,649 & 61,824 & 17.3 & \\
\hline 2010 & 102,702 & $1,152.1$ & 11,218 & 64,687 & 17.8 & \\
\hline 2011 & 105,571 & $1,192.7$ & 11,298 & 65,786 & 18.1 & \\
\hline 2012 & 120,847 & $1,225.5$ & 10,142 & 66,395 & 18.5 & \\
\hline 2013 & 120,523 & $1,231.8$ & 10,220 & 65,555 & 18.8 & \\
\hline 2014 & 124,681 & $1,274.0$ & 10,218 & 69,012 & 18.5 & \\
\hline 2015 & 128,553 & $1,334.3$ & 10,448 & 67,730 & 18.8 & \\
\hline & \multicolumn{6}{|c|}{ Average annual percentage change } \\
\hline 1970-2015 & $5.0 \%$ & $5.5 \%$ & $0.4 \%$ & $3.9 \%$ & $1.4 \%$ & \\
\hline 2005-2015 & $3.0 \%$ & $2.6 \%$ & $-0.4 \%$ & $1.4 \%$ & $0.6 \%$ & \\
\hline
\end{tabular}

\section{Source:}

1970-2008: U.S. Department of Transportation, Federal Highway Administration, Highway Statistics 2009, Washington, DC, 2011, Table MV-9. Previous years Table VM-1. 2009-on: See Section 7.2 in Appendix A. (Additional resources: www.fhwa.dot.gov) vehicles.

${ }^{a}$ Beginning in this year the data were revised to include all vans (including mini-vans), pickups and sport utility

${ }^{\mathrm{b}}$ Due to FHWA methodology changes, data from 2009-on are not comparable with previous data. 
These data are the combination of the car and two-axle, four-tire truck data from Tables 4.1 and 4.2 thus the data may not match exactly with the FHWA VM-1 table's light-duty vehicle data. The methodology change after 2008 affects these data as well.

Table 4.3

Summary Statistics for Light Vehicles, 1970-2015

\begin{tabular}{|c|c|c|c|c|c|c|}
\hline Year & $\begin{array}{l}\text { Registrations } \\
\text { (thousands) }\end{array}$ & $\begin{array}{l}\text { Vehicle travel } \\
\text { (billion miles) }\end{array}$ & $\begin{array}{c}\text { Miles } \\
\text { (per vehicle) }\end{array}$ & $\begin{array}{c}\text { Fuel use } \\
\text { (million gallons) }\end{array}$ & $\begin{array}{c}\text { Fuel economy } \\
\text { (miles per gallon) }\end{array}$ & \\
\hline 1970 & 103,455 & 1,040 & 10,053 & 80,133 & 13.0 & \\
\hline 1975 & 127,124 & 1,235 & 9,712 & 93,221 & 13.2 & \\
\hline 1980 & 149,477 & 1,403 & 9,383 & 93,777 & 15.0 & \\
\hline 1981 & 152,026 & 1,430 & 9,404 & 92,809 & 15.4 & \\
\hline 1982 & 153,494 & 1,468 & 9,563 & 91,818 & 16.0 & \\
\hline 1983 & 157,658 & 1,523 & 9,658 & 94,267 & 16.2 & \\
\hline 1984 & 160,264 & 1,585 & 9,890 & 96,267 & 16.5 & \\
\hline $1985^{a}$ & 165,099 & 1,638 & 9,920 & 98,881 & 16.6 & \\
\hline 1986 & 169,386 & 1,694 & 10,001 & 102,248 & 16.6 & \\
\hline 1987 & 172,589 & 1,773 & 10,272 & 103,906 & 17.1 & \\
\hline 1988 & 177,641 & 1,872 & 10,541 & 105,998 & 17.7 & \\
\hline 1989 & 180,504 & 1,938 & 10,735 & 107,184 & 18.1 & \\
\hline 1990 & 181,975 & 1,983 & 10,896 & 105,179 & 18.9 & \\
\hline 1991 & 181,333 & 2,008 & 11,071 & 102,535 & 19.6 & \\
\hline 1992 & 183,672 & 2,078 & 11,316 & 106,365 & 19.5 & \\
\hline 1993 & 187,321 & 2,120 & 11,320 & 109,898 & 19.3 & \\
\hline 1994 & 190,787 & 2,171 & 11,378 & 111,986 & 19.4 & \\
\hline 1995 & 194,125 & 2,228 & 11,479 & 113,677 & 19.6 & \\
\hline 1996 & 198,862 & 2,286 & 11,497 & 116,575 & 19.6 & \\
\hline 1997 & 199,973 & 2,353 & 11,768 & 119,281 & 19.7 & \\
\hline 1998 & 203,169 & 2,418 & 11,901 & 122,157 & 19.8 & \\
\hline 1999 & 207,788 & 2,470 & 11,888 & 126,142 & 19.6 & \\
\hline 2000 & 212,706 & 2,523 & 11,863 & 126,004 & 20.0 & \\
\hline 2001 & 221,821 & 2,572 & 11,593 & 127,081 & 20.2 & \\
\hline 2002 & 220,932 & 2,625 & 11,879 & 130,691 & 20.1 & \\
\hline 2003 & 222,857 & 2,656 & 11,919 & 135,348 & 19.6 & \\
\hline 2004 & 228,276 & 2,727 & 11,946 & 138,819 & 19.6 & \\
\hline 2005 & 231,905 & 2,749 & 11,856 & 136,287 & 20.2 & \\
\hline 2006 & 234,525 & 2,773 & 11,824 & 135,694 & 20.4 & \\
\hline 2007 & 237,403 & 2,785 & 11,730 & 136,213 & 20.4 & \\
\hline 2008 & 238,315 & 2,724 & 11,432 & 132,696 & 20.5 & b \\
\hline 2009 & 235,034 & 2,633 & 11,204 & 128,411 & 20.5 & \\
\hline 2010 & 233,594 & 2,648 & 11,338 & 126,932 & 20.9 & \\
\hline 2011 & 231,228 & 2,650 & 11,463 & 125,432 & 21.1 & \\
\hline 2012 & 232,137 & 2,664 & 11,476 & 124,294 & 21.4 & \\
\hline 2013 & 234,199 & 2,678 & 11,434 & 122,845 & 21.8 & \\
\hline 2014 & 238,580 & 2,711 & 11,361 & 125,482 & 21.6 & \\
\hline 2015 & 241,417 & 2,780 & 11,514 & 122,940 & 22.6 & \\
\hline & \multicolumn{6}{|c|}{ Average annual percentage change } \\
\hline 1970-2015 & $1.9 \%$ & $2.2 \%$ & $0.3 \%$ & $1.0 \%$ & $1.2 \%$ & \\
\hline 2005-2015 & $0.4 \%$ & $0.1 \%$ & $-0.3 \%$ & $-1.0 \%$ & $1.1 \%$ & \\
\hline
\end{tabular}

\section{Sources:}

Tables 4.1 and 4.2 . vehicles.

a Beginning in this year the data were revised to include all vans (including mini-vans), pickups and sport utility

b Due to FHWA methodology changes, data from 2009-on are not comparable with previous data. 
Because data on Class $2 b$ trucks are scarce, the U.S. DOE funded a study to investigate available sources of data. In the final report, four methodologies are described to estimate the sales of Class $2 b$ trucks. Until another study is funded, the 1999 data are the latest available.

Table 4.4

Summary Statistics on Class 1, Class 2a, and Class 2b Light Trucks

\begin{tabular}{lccccrrc}
\hline & $\begin{array}{c}\text { CY 1999 } \\
\text { truck sales } \\
\text { (millions) }\end{array}$ & $\begin{array}{c}\text { 2000 truck } \\
\text { population } \\
\text { (millions) }\end{array}$ & $\begin{array}{c}\text { Percent } \\
\text { diesel trucks } \\
\text { in } \\
\text { population }\end{array}$ & $\begin{array}{c}\text { Average } \\
\text { age } \\
\text { (years) }\end{array}$ & $\begin{array}{c}\text { Estimated } \\
\text { annual } \\
\text { miles }^{\text {a }} \\
\text { (billions) }\end{array}$ & $\begin{array}{c}\text { Estimated } \\
\text { fuel use } \\
\text { (billion }^{\text {a }} \\
\text { gallons) }\end{array}$ & $\begin{array}{c}\text { Estimated fuel } \\
\text { economy } \\
\text { (miles per } \\
\text { gallon) }\end{array}$ \\
\hline Class 1 & 5.7 & 49.7 & $0.3 \%$ & 7.3 & 672.7 & 37.4 & 18.0 \\
Class 2a & 1.8 & 19.2 & $2.5 \%$ & 7.4 & 251.9 & 18.0 & 14.0 \\
Class 2b & 0.5 & 5.8 & $24.0 \%$ & 8.6 & 76.7 & 5.5 & 13.9 \\
\hline
\end{tabular}

Note: CY - calendar year.

Source:

Davis, S.C. and L.F. Truett, Investigation of Class $2 b$ Trucks (Vehicles of 8,500 to 10,000 lbs GVWR), ORNL/TM2002/49, March 2002, Table 16.

Table 4.5

Sales Estimates of Class 1, Class 2a, and Class 2b Light Trucks, 1989-1999

\begin{tabular}{ccccc}
\hline & \multicolumn{4}{c}{ Sales estimates (thousands) } \\
\cline { 2 - 5 } Calendar year & Class 1 & $\begin{array}{c}\text { Class 2a } \\
(6,001-8,500 \mathrm{lb})\end{array}$ & $\begin{array}{c}\text { Class 2b } \\
(8,501-10,000 \mathrm{lb})\end{array}$ & Total \\
\hline 1989 & $3,000 \mathrm{lb}$ and under $)$ & 918 & 379 & 4,610 \\
1990 & 3,313 & 829 & 268 & 4,548 \\
1991 & 3,451 & 670 & 206 & 4,122 \\
1992 & 3,246 & 827 & 194 & 4,629 \\
1993 & 3,608 & 975 & 257 & 5,351 \\
1994 & 4,119 & 1,241 & 265 & 6,033 \\
1995 & 4,527 & 1,304 & 327 & 6,053 \\
1996 & 4,422 & 1,356 & 334 & 6,519 \\
1997 & 4,829 & 1,315 & 397 & 6,797 \\
1998 & 5,085 & 1,694 & 342 & 7,299 \\
1999 & 5,263 & 1,845 & 521 & 8,073 \\
$1989-1999$ & 5,707 & & $37.5 \%$ & $75.1 \%$ \\
\hline
\end{tabular}

Note: These data were calculated using Methodology 4 from the report.

\section{Source:}

Davis, S.C. and L.F. Truett, Investigation of Class $2 b$ Trucks (Vehicles of 8,500 to 10,000 lbs GVWR), ORNL/TM2002/49, March 2002, Table 1.

${ }^{a}$ Estimates derived using 2000 population data and 1997 usage data. See source for details. 
Car sales in 2009 and 2010 were below 6 million but increased to more than 7.7 million by 2014 before declining in both 2015 and 2016. In 1980, Chrysler/FCA, Ford and General Motors held 73.8\% of the market; by 2016, that had dropped to $27.9 \%$.

Table 4.6

New Retail Car Sales in the United States, 1970-2016

\begin{tabular}{|c|c|c|c|c|c|c|}
\hline $\begin{array}{c}\text { Calendar } \\
\text { year }\end{array}$ & Domestic $^{\mathrm{a}}$ & $\frac{\text { Import }^{\mathrm{b}}}{\text { (thousands) }^{2}}$ & Total $^{\mathrm{c}}$ & $\begin{array}{l}\text { Percentage } \\
\text { imports }\end{array}$ & $\begin{array}{c}\text { Percentage } \\
\text { FCA/Ford/GM } \\
\text { sales }^{\mathrm{d}}\end{array}$ & $\begin{array}{c}\text { Percentage } \\
\text { diesel }\end{array}$ \\
\hline 1970 & 7,119 & 1,280 & 8,399 & $15.2 \%$ & e & $0.07 \%$ \\
\hline 1975 & 7,053 & 1,571 & 8,624 & $18.2 \%$ & e & $0.31 \%$ \\
\hline 1980 & 6,580 & 2,369 & 8,949 & $26.5 \%$ & $73.8 \%$ & $4.32 \%$ \\
\hline 1985 & 8,205 & 2,775 & 10,979 & $25.3 \%$ & $72.9 \%$ & $0.83 \%$ \\
\hline 1990 & 6,919 & 2,384 & 9,303 & $25.6 \%$ & $65.7 \%$ & $0.08 \%$ \\
\hline 1991 & 6,162 & 2,023 & 8,185 & $24.7 \%$ & $64.2 \%$ & $0.10 \%$ \\
\hline 1992 & 6,286 & 1,927 & 8,213 & $23.5 \%$ & $65.8 \%$ & $0.06 \%$ \\
\hline 1993 & 6,742 & 1,776 & 8,518 & $20.9 \%$ & $67.3 \%$ & $0.04 \%$ \\
\hline 1994 & 7,255 & 1,735 & 8,991 & $19.3 \%$ & $65.9 \%$ & $0.04 \%$ \\
\hline 1995 & 7,114 & 1,506 & 8,620 & $17.5 \%$ & $65.3 \%$ & $0.03 \%$ \\
\hline 1996 & 7,206 & 1,272 & 8,479 & $15.0 \%$ & $64.1 \%$ & $0.09 \%$ \\
\hline 1997 & 6,862 & 1,355 & 8,217 & $16.5 \%$ & $62.2 \%$ & $0.09 \%$ \\
\hline 1998 & 6,705 & 1,380 & 8,085 & $17.1 \%$ & $59.7 \%$ & $0.14 \%$ \\
\hline 1999 & 6,919 & 1,719 & 8,638 & $19.9 \%$ & $58.3 \%$ & $0.16 \%$ \\
\hline 2000 & 6,762 & 2,016 & 8,778 & $23.0 \%$ & $55.0 \%$ & $0.26 \%$ \\
\hline 2001 & 6,254 & 2,098 & 8,352 & $25.1 \%$ & $51.4 \%$ & $0.18 \%$ \\
\hline 2002 & 5,817 & 2,226 & 8,042 & $27.7 \%$ & $48.4 \%$ & $0.39 \%$ \\
\hline 2003 & 5,473 & 2,083 & 7,556 & $27.6 \%$ & $47.1 \%$ & $0.52 \%$ \\
\hline 2004 & 5,333 & 2,149 & 7,483 & $28.7 \%$ & $44.9 \%$ & $0.40 \%$ \\
\hline 2005 & 5,473 & 2,187 & 7,660 & $28.5 \%$ & $43.1 \%$ & $0.63 \%$ \\
\hline 2006 & 5,417 & 2,345 & 7,762 & $30.2 \%$ & $40.5 \%$ & $0.82 \%$ \\
\hline 2007 & 5,197 & 2,365 & 7,562 & $31.3 \%$ & $36.9 \%$ & $0.10 \%$ \\
\hline 2008 & 4,491 & 2,278 & 6,769 & $33.7 \%$ & $34.2 \%$ & $0.11 \%$ \\
\hline 2009 & 3,558 & 1,843 & 5,402 & $34.1 \%$ & $31.3 \%$ & $2.93 \%$ \\
\hline 2010 & 3,791 & 1,844 & 5,636 & $32.7 \%$ & $31.7 \%$ & $2.69 \%$ \\
\hline 2011 & 4,146 & 1,947 & 6,093 & $32.0 \%$ & $33.3 \%$ & $1.47 \%$ \\
\hline 2012 & 5,120 & 2,125 & 7,245 & $29.3 \%$ & $31.6 \%$ & $2.69 \%$ \\
\hline 2013 & 5,433 & 2,153 & 7,586 & $28.4 \%$ & $32.4 \%$ & $2.45 \%$ \\
\hline 2014 & 5,610 & 2,098 & 7,708 & $27.2 \%$ & $31.2 \%$ & $2.41 \%$ \\
\hline 2015 & 5,595 & 1,922 & 7,517 & $25.6 \%$ & $29.7 \%$ & $1.14 \%$ \\
\hline 2016 & 5,169 & 1,703 & 6,873 & $24.8 \%$ & $27.9 \%$ & $0.12 \%$ \\
\hline \multicolumn{7}{|c|}{ Average annual percentage change } \\
\hline 1970-2016 & $-0.7 \%$ & $0.6 \%$ & $-0.4 \%$ & & & \\
\hline 2006-2016 & $-0.5 \%$ & $-3.1 \%$ & $-1.2 \%$ & & & \\
\hline
\end{tabular}

\section{Source:}

Domestic and import data - 1970-97: American Automobile Manufacturers Association, Motor Vehicle Facts and Figures 1998, Detroit, MI, 1998, p. 15, and annual. 1997 data from Economic Indicators, 4th Quarter 1997. 1998-2016: Ward's Communication, www.wardsauto.com.

Diesel data - Ward's Communications, www.wardsauto.com.

${ }^{a}$ Any vehicle built in North America regardless of manufacturer. deliveries.

${ }^{b}$ Any vehicle built outside of North America regardless of manufacturer. Does not include import tourist

c Sums may not add to totals due to rounding.

${ }^{\mathrm{d}}$ Big 3 includes Ford, General Motors, and Fiat-Chrysler (and predecessor entities).

e Data are not available. 
Light trucks, which include pick-ups, minivans, sport-utility vehicles, and other trucks less than 10,000 pounds gross vehicle weight (GVW), accounted for more than half of light vehicle sales from 2001 to 2007 and again in 2010, 2011, and from 2013 to 2016.

Table 4.7

New Retail Sales of Trucks 10,000 Pounds GVW and Less in the United States, 1970-2016

\begin{tabular}{|c|c|c|c|c|c|c|}
\hline \multirow[b]{2}{*}{$\begin{array}{l}\text { Calendar } \\
\text { year }\end{array}$} & \multirow[b]{2}{*}{$\begin{array}{l}\text { Light truck } \\
\quad \text { sales }^{\mathrm{a}} \\
\text { (thousands) }\end{array}$} & \multicolumn{5}{|c|}{ Percentages } \\
\hline & & Import $^{\mathrm{b}}$ & $\begin{array}{c}\text { FCA/Ford/GM } \\
\text { sales }^{\mathrm{c}}\end{array}$ & Diesel $^{\mathrm{d}}$ & $\begin{array}{l}\text { Light trucks of } \\
\text { light-duty } \\
\text { vehicle sales }\end{array}$ & $\begin{array}{c}\text { Light trucks } \\
\text { of total } \\
\text { truck sales } \\
\end{array}$ \\
\hline 1970 & 1,457 & $4.5 \%$ & Not available & $\mathrm{f}$ & $14.8 \%$ & $80.5 \%$ \\
\hline 1975 & 2,053 & $10.0 \%$ & Not available & f & $20.9 \%$ & $82.8 \%$ \\
\hline 1980 & 1,960 & $24.4 \%$ & Not available & $4.0 \%$ & $17.5 \%$ & $78.6 \%$ \\
\hline 1985 & 3,688 & $22.6 \%$ & $78.2 \%$ & $4.0 \%$ & $25.1 \%$ & $77.7 \%$ \\
\hline 1990 & 4,548 & $13.5 \%$ & $80.9 \%$ & $2.3 \%$ & $32.8 \%$ & $93.8 \%$ \\
\hline 1991 & 4,122 & $13.1 \%$ & $79.4 \%$ & $3.2 \%$ & $33.5 \%$ & $94.4 \%$ \\
\hline 1992 & 4,629 & $8.8 \%$ & $83.1 \%$ & $2.5 \%$ & $36.0 \%$ & $94.4 \%$ \\
\hline 1993 & 5,351 & $7.1 \%$ & $83.4 \%$ & $2.3 \%$ & $38.6 \%$ & $94.2 \%$ \\
\hline 1994 & 6,033 & $6.8 \%$ & $82.9 \%$ & $2.5 \%$ & $40.2 \%$ & $94.0 \%$ \\
\hline 1995 & 6,053 & $6.6 \%$ & $83.4 \%$ & $3.8 \%$ & $41.3 \%$ & $93.2 \%$ \\
\hline 1996 & 6,519 & $6.7 \%$ & $83.8 \%$ & $3.1 \%$ & $43.5 \%$ & $93.4 \%$ \\
\hline 1997 & 6,797 & $8.5 \%$ & $81.9 \%$ & $2.7 \%$ & $45.3 \%$ & $93.4 \%$ \\
\hline 1998 & 7,299 & $9.0 \%$ & $80.5 \%$ & $2.6 \%$ & $47.4 \%$ & $92.6 \%$ \\
\hline 1999 & 8,073 & $9.6 \%$ & $78.0 \%$ & $2.9 \%$ & $48.3 \%$ & $92.0 \%$ \\
\hline 2000 & 8,386 & $10.2 \%$ & $76.1 \%$ & $3.4 \%$ & $48.9 \%$ & $92.8 \%$ \\
\hline 2001 & 8,598 & $11.4 \%$ & $75.3 \%$ & $2.9 \%$ & $50.7 \%$ & $94.3 \%$ \\
\hline 2002 & 8,633 & $12.4 \%$ & $74.7 \%$ & $2.7 \%$ & $51.8 \%$ & $94.9 \%$ \\
\hline 2003 & 8,938 & $13.7 \%$ & $72.4 \%$ & $2.9 \%$ & $54.2 \%$ & $95.0 \%$ \\
\hline 2004 & 9,254 & $13.5 \%$ & $70.1 \%$ & $2.8 \%$ & $55.3 \%$ & $94.3 \%$ \\
\hline 2005 & 9,114 & $13.3 \%$ & $68.2 \%$ & $2.7 \%$ & $54.3 \%$ & $93.1 \%$ \\
\hline 2006 & 8,574 & $15.7 \%$ & $63.9 \%$ & $2.8 \%$ & $52.5 \%$ & $92.3 \%$ \\
\hline 2007 & 8,305 & $16.7 \%$ & $61.9 \%$ & $3.2 \%$ & $52.3 \%$ & $93.3 \%$ \\
\hline 2008 & 6,246 & $17.6 \%$ & $61.2 \%$ & $3.4 \%$ & $48.0 \%$ & $92.9 \%$ \\
\hline 2009 & 4,834 & $18.3 \%$ & $57.8 \%$ & $4.2 \%$ & $47.2 \%$ & $93.0 \%$ \\
\hline 2010 & 5,758 & $15.6 \%$ & $57.6 \%$ & $4.9 \%$ & $50.5 \%$ & $93.8 \%$ \\
\hline 2011 & 6,449 & $15.2 \%$ & $59.4 \%$ & $5.4 \%$ & $51.4 \%$ & $92.7 \%$ \\
\hline 2012 & 6,975 & $15.2 \%$ & $57.7 \%$ & $5.5 \%$ & $49.0 \%$ & $92.6 \%$ \\
\hline 2013 & 7,693 & $16.1 \%$ & $57.3 \%$ & $5.3 \%$ & $50.3 \%$ & $92.7 \%$ \\
\hline 2014 & 8,484 & $16.0 \%$ & $57.6 \%$ & $5.4 \%$ & $52.4 \%$ & $92.7 \%$ \\
\hline 2015 & 9,578 & $18.6 \%$ & $57.0 \%$ & $5.5 \%$ & $56.0 \%$ & $92.7 \%$ \\
\hline 2016 & 10,296 & $20.7 \%$ & $55.6 \%$ & g & $60.0 \%$ & $93.7 \%$ \\
\hline \multicolumn{7}{|c|}{ Average annual percentage change } \\
\hline 1970-2016 & $4.3 \%$ & & & & & \\
\hline $2006-2016$ & $1.8 \%$ & & & & & \\
\hline
\end{tabular}

Source:

Ward's Communications, www.wardsauto.com.

${ }^{\text {a }}$ Includes all trucks of 10,000 pounds gross vehicle weight and less sold in the United States.

${ }^{\mathrm{b}}$ Excluding transplants.

c Includes Ford, General Motors, and Fiat-Chrysler (and predecessor entities).

${ }^{\mathrm{d}}$ Based on model year factory installations.

e Light-duty vehicles include cars and light trucks.

${ }^{\mathrm{f}}$ Indicates less than 1 percent.

g Not available. 
The production-weighted fuel economy of cars increased dramatically from 1975 (13.5 mpg) to 1985 (23.0 mpg), but rose only $0.5 \mathrm{mpg}$ from 1985 to 2005. Since 2005, fuel economy rose $5.8 \mathrm{mpg}$-from $23.5 \mathrm{mpg}$ in 2005 to 29.8 mpg in 2016. The fuel economy values have been adjusted to provide the best estimate of real world performance.

Table 4.8

Production, Production Shares, and Production-Weighted Fuel Economies of New Domestic and Import Cars, Model Years 1975-2016

\begin{tabular}{|c|c|c|c|c|c|c|}
\hline \multirow[b]{2}{*}{ Model year } & \multicolumn{3}{|c|}{ Car } & \multicolumn{3}{|c|}{ Car SUV } \\
\hline & $\begin{array}{l}\text { Production } \\
\text { (thousands) }\end{array}$ & $\begin{array}{l}\text { Production } \\
\text { share }(\%)^{\mathrm{b}}\end{array}$ & $\begin{array}{c}\text { Fuel economy } \\
\text { (mpg) }\end{array}$ & $\begin{array}{l}\text { Production } \\
\text { (thousands) }\end{array}$ & $\begin{array}{l}\text { Production } \\
\text { share }(\%)^{b}\end{array}$ & $\begin{array}{c}\text { Fuel } \\
\text { economy } \\
\text { (mpg) }\end{array}$ \\
\hline 1975 & 8,237 & $99.9 \%$ & 13.5 & 10 & $0.1 \%$ & 11.1 \\
\hline 1980 & 9,443 & $100.0 \%$ & 20.0 & 0 & $0.0 \%$ & 14.6 \\
\hline 1990 & 8,810 & $99.3 \%$ & 23.3 & 65 & $0.7 \%$ & 18.8 \\
\hline 1991 & 8,524 & $97.4 \%$ & 23.4 & 224 & $2.6 \%$ & 18.2 \\
\hline 1992 & 8,108 & $97.1 \%$ & 23.1 & 243 & $2.9 \%$ & 17.8 \\
\hline 1993 & 8,456 & $94.7 \%$ & 23.5 & 473 & $5.3 \%$ & 17.0 \\
\hline 1994 & 8,415 & $96.2 \%$ & 23.3 & 332 & $3.8 \%$ & 18.0 \\
\hline 1995 & 9,396 & $97.7 \%$ & 23.4 & 220 & $2.3 \%$ & 17.8 \\
\hline 1996 & 7,890 & $96.5 \%$ & 23.3 & 287 & $3.5 \%$ & 18.4 \\
\hline 1997 & 8,334 & $95.8 \%$ & 23.4 & 361 & $4.2 \%$ & 19.2 \\
\hline 1998 & 7,971 & $94.6 \%$ & 23.4 & 454 & $5.4 \%$ & 18.2 \\
\hline 1999 & 8,376 & $94.5 \%$ & 23.0 & 488 & $5.5 \%$ & 18.5 \\
\hline 2000 & 9,125 & $93.7 \%$ & 22.9 & 617 & $6.3 \%$ & 17.9 \\
\hline 2001 & 8,405 & $91.9 \%$ & 23.0 & 743 & $8.1 \%$ & 18.8 \\
\hline 2002 & 8,301 & $93.2 \%$ & 23.1 & 603 & $6.8 \%$ & 19.3 \\
\hline 2003 & 7,921 & $93.2 \%$ & 23.3 & 575 & $6.8 \%$ & 19.9 \\
\hline 2004 & 7,537 & $92.2 \%$ & 23.1 & 639 & $7.8 \%$ & 20.0 \\
\hline 2005 & 8,027 & $90.8 \%$ & 23.5 & 813 & $9.2 \%$ & 20.2 \\
\hline 2006 & 7,993 & $91.4 \%$ & 23.3 & 751 & $8.6 \%$ & 20.5 \\
\hline 2007 & 8,082 & $89.8 \%$ & 24.1 & 919 & $10.2 \%$ & 20.6 \\
\hline 2008 & 7,319 & $88.8 \%$ & 24.3 & 924 & $11.2 \%$ & 21.2 \\
\hline 2009 & 5,636 & $90.3 \%$ & 25.3 & 608 & $9.7 \%$ & 22.0 \\
\hline 2010 & 6,061 & $86.9 \%$ & 26.2 & 915 & $13.1 \%$ & 23.0 \\
\hline 2011 & 5,743 & $82.6 \%$ & 26.1 & 1,207 & $17.4 \%$ & 23.7 \\
\hline 2012 & 7,392 & $85.4 \%$ & 27.9 & 1,265 & $14.6 \%$ & 23.4 \\
\hline 2013 & 8,226 & $84.5 \%$ & 28.6 & 1,514 & $15.5 \%$ & 24.5 \\
\hline 2014 & 7,639 & $83.0 \%$ & 28.7 & 1,566 & $17.0 \%$ & 24.6 \\
\hline 2015 & 7,899 & $82.3 \%$ & 29.4 & 1,701 & $17.7 \%$ & 25.3 \\
\hline 2016 & c & $82.8 \%$ & 29.8 & c & $17.2 \%$ & 25.6 \\
\hline
\end{tabular}

Note: See Table 4.11 for all cars (car + car SUV). See Table 4.9 for car SUV listing.

\section{Source:}

U.S. Environmental Protection Agency, Light-Duty Automotive Technology, Carbon Dioxide Emissions, and Fuel Economy Trends: 1975 Through 2016, November 2016. (Additional resources: www.epa.gov/fueleconomy/lightduty-automotive-technology-carbon-dioxide-emissions-and-fuel-economy-trends-1975-0)

a The fuel economy data on this table are adjusted to provide the best estimate of real world performance. See section 10 of the source document for details on adjustment methodology. These data are not directly comparable to Corporate Average Fuel Economy data.

${ }^{\mathrm{b}}$ Production share is based on total of cars plus car SUVs. Percentages may not sum to totals due to rounding.

${ }^{\mathrm{c}}$ Data are not available. 
A vehicle classification was created to match the Corporate Average Fuel Economy (CAFE) methodology. Under CAFE, small, two-wheel drive sport utility vehicles will be held to the same standards as cars. The Environmental

Protection Agency has defined these vehicles as "car SUVs." The vehicles below make up this category.

Table 4.9

Definition of Car Sport Utility Vehicles in Model Year 2016

\begin{tabular}{ll}
\hline Acura RDX 2WD & Jeep Cherokee FWD \\
BMW X3 sDrive 28i & Jeep Compass FWD \\
Buick Encore & Jeep Patriot FWD \\
Cadillac SRX & Jeep Renegade 4x2 \\
Cadillac SRX AWD & Kia Sorento AWD \\
Chevrolet Equinox AWD & Kia Sorento FWD \\
Chevrolet Equinox FWD & Kia Sportage FWD \\
Chevrolet Trax & Kia Sportage SX FWD \\
Dodge Journey & Lexus NX 200t \\
Fiat 500X & Lexus NX 200t AWD F Sport \\
Ford Edge FWD & Lexus NX 300h \\
Ford Escape FWD & Lexus RX 350 \\
GMC Terrain AWD & Lexus RX 450h \\
GMC Terrain FWD & Lincoln MKC FWD \\
Honda CR-V 2WD & Lincoln MKX FWD \\
Honda HR-V 2WD & Mazda CX-5 2WD \\
Honda HR-V 4WD & Mercedes GLC 300 \\
Hyundai Santa Fe Sport AWD & Mitsubishi Outlander Sport 2WD \\
Hyundai Santa Fe Sport FWD & Nissan Rogue FWD \\
Hyundai Santa Fe Sport Ultimate FWD & Tesla Model X AWD \\
Hyundai Tucson AWD & Toyota RAV4 \\
Hyundai Tucson Eco AWD & Toyota RAV4 LE/XLE \\
Hyundai Tucson Eco FWD & Toyota RAV4 Limited AWD/SE AWD \\
Hyundai Tucson Fuel Cell & Volvo XC60 FWD \\
Hyundai Tucson FWD & Volvo XC70 FWD \\
Infiniti QX70 RWD & VW Tiguan \\
\hline
\end{tabular}

Note: $2 \mathrm{WD}=$ Two-wheel drive. $4 \mathrm{WD}=$ Four-wheel drive. $\mathrm{AWD}=$ All-wheel drive. $\mathrm{FWD}=$ Front-wheel drive.

\section{Source:}

U.S. Environmental Protection Agency, Light-Duty Automotive Technology, Carbon Dioxide Emissions, and Fuel Economy Trends: 1975 Through 2016, November 2016. (Additional resources: www.epa.gov/fueleconomy/lightduty-automotive-technology-carbon-dioxide-emissions-and-fuel-economy-trends-1975-0)

${ }^{a}$ Alternative fuel vehicle. 
Production of sport utility vehicles (SUVs) has grown substantially since 1975. The production-weighted fuel economy of SUVs was more than 22 mpg in 2016. Almost 62\% of all light trucks produced in 2016 were SUVs.

Table 4.10

Production, Production Shares, and Production-Weighted Fuel Economies of New Domestic and Import Light Trucks, Model Years 1975-2016 ${ }^{a}$

\begin{tabular}{|c|c|c|c|c|c|c|c|c|c|}
\hline \multirow[b]{2}{*}{$\begin{array}{c}\text { Model } \\
\text { Year }\end{array}$} & \multicolumn{3}{|c|}{ Pickup } & \multicolumn{3}{|c|}{ Van } & \multicolumn{3}{|c|}{ Truck SUV } \\
\hline & $\begin{array}{l}\text { Production } \\
\text { (Thousands) }\end{array}$ & $\begin{array}{l}\text { Share } \\
(\%)^{\mathrm{b}}\end{array}$ & $\begin{array}{c}\text { Fuel } \\
\text { Economy } \\
\text { (mpg) }\end{array}$ & $\begin{array}{l}\text { Production } \\
\text { (Thousands) }\end{array}$ & $\begin{array}{l}\text { Share } \\
(\%)^{\mathrm{b}}\end{array}$ & $\begin{array}{c}\text { Fuel } \\
\text { Economy } \\
\text { (mpg) }\end{array}$ & $\begin{array}{l}\text { Production } \\
\text { (Thousands) }\end{array}$ & $\begin{array}{l}\text { Share } \\
(\%)^{\mathrm{b}}\end{array}$ & $\begin{array}{c}\text { Fuel } \\
\text { Economy } \\
(\mathrm{mpg})\end{array}$ \\
\hline 1975 & 1,343 & $67.9 \%$ & 11.9 & 457 & $23.1 \%$ & 11.1 & 177 & $9.0 \%$ & 11.0 \\
\hline 1980 & 1,437 & $77.1 \%$ & 16.5 & 242 & $13.0 \%$ & 14.1 & 184 & $9.9 \%$ & 13.2 \\
\hline 1985 & 2,078 & $58.0 \%$ & 18.2 & 855 & $23.9 \%$ & 16.5 & 648 & $18.1 \%$ & 16.5 \\
\hline 1986 & 2,532 & $59.0 \%$ & 18.9 & 1,044 & $24.3 \%$ & 17.5 & 714 & $16.6 \%$ & 17.0 \\
\hline 1987 & 2,147 & $53.2 \%$ & 19.0 & 1,114 & $27.6 \%$ & 17.7 & 779 & $19.3 \%$ & 17.3 \\
\hline 1988 & 2,459 & $55.3 \%$ & 18.1 & 1,133 & $25.5 \%$ & 17.9 & 859 & $19.3 \%$ & 17.0 \\
\hline 1989 & 2,232 & $51.6 \%$ & 17.8 & 1,278 & $29.5 \%$ & 17.8 & 818 & $18.9 \%$ & 16.6 \\
\hline 1990 & 1,835 & $49.1 \%$ & 17.4 & 1,262 & $33.7 \%$ & 17.8 & 643 & $17.2 \%$ & 16.4 \\
\hline 1991 & 1,920 & $50.2 \%$ & 18.2 & 1,034 & $27.0 \%$ & 17.9 & 871 & $22.8 \%$ & 16.7 \\
\hline 1992 & 1,840 & $48.1 \%$ & 17.5 & 1,221 & $31.9 \%$ & 17.9 & 761 & $19.9 \%$ & 16.2 \\
\hline 1993 & 2,002 & $46.8 \%$ & 17.6 & 1,441 & $33.7 \%$ & 18.2 & 838 & $19.6 \%$ & 16.3 \\
\hline 1994 & 2,669 & $49.6 \%$ & 17.4 & 1,418 & $26.4 \%$ & 17.8 & 1,291 & $24.0 \%$ & 16.0 \\
\hline 1995 & 2,271 & $41.1 \%$ & 16.9 & 1,662 & $30.1 \%$ & 18.1 & 1,596 & $28.9 \%$ & 16.0 \\
\hline 1996 & 1,955 & $39.4 \%$ & 17.1 & 1,409 & $28.4 \%$ & 18.3 & 1,603 & $32.3 \%$ & 16.2 \\
\hline 1997 & 2,408 & $41.8 \%$ & 16.8 & 1,265 & $22.0 \%$ & 18.2 & 2,089 & $36.3 \%$ & 16.1 \\
\hline 1998 & 2,415 & $40.0 \%$ & 17.0 & 1,489 & $24.7 \%$ & 18.7 & 2,127 & $35.3 \%$ & 16.2 \\
\hline 1999 & 2,544 & $40.1 \%$ & 16.3 & 1,463 & $23.0 \%$ & 18.3 & 2,342 & $36.9 \%$ & 16.1 \\
\hline 2000 & 2,612 & $38.2 \%$ & 16.7 & 1,691 & $24.8 \%$ & 18.6 & 2,526 & $37.0 \%$ & 16.0 \\
\hline 2001 & 2,519 & $39.0 \%$ & 16.0 & 1,232 & $19.1 \%$ & 18.0 & 2,707 & $41.9 \%$ & 16.4 \\
\hline 2002 & 2,380 & $33.0 \%$ & 15.8 & 1,243 & $17.2 \%$ & 18.7 & 3,588 & $49.8 \%$ & 16.3 \\
\hline 2003 & 2,474 & $34.0 \%$ & 16.1 & 1,232 & $16.9 \%$ & 19.0 & 3,571 & $49.1 \%$ & 16.4 \\
\hline 2004 & 2,505 & $33.3 \%$ & 15.7 & 953 & $12.7 \%$ & 19.2 & 4,075 & $54.1 \%$ & 16.5 \\
\hline 2005 & 2,300 & $32.6 \%$ & 15.8 & 1,481 & $21.0 \%$ & 19.3 & 3,272 & $46.4 \%$ & 16.7 \\
\hline 2006 & 2,188 & $34.4 \%$ & 16.1 & 1,166 & $18.3 \%$ & 19.5 & 3,006 & $47.3 \%$ & 17.2 \\
\hline 2007 & 2,113 & $33.7 \%$ & 16.2 & 847 & $13.5 \%$ & 19.5 & 3,314 & $52.8 \%$ & 17.7 \\
\hline 2008 & 1,794 & $31.7 \%$ & 16.5 & 790 & $14.0 \%$ & 19.8 & 3,072 & $54.3 \%$ & 18.2 \\
\hline 2009 & 989 & $32.2 \%$ & 16.9 & 368 & $12.0 \%$ & 20.1 & 1,714 & $55.8 \%$ & 19.3 \\
\hline 2010 & 1,276 & $30.8 \%$ & 16.9 & 559 & $13.5 \%$ & 20.1 & 2,305 & $55.7 \%$ & 19.7 \\
\hline 2011 & 1,479 & $29.2 \%$ & 17.2 & 521 & $10.3 \%$ & 21.0 & 3,069 & $60.5 \%$ & 19.8 \\
\hline 2012 & 1,357 & $28.3 \%$ & 17.2 & 661 & $13.8 \%$ & 21.3 & 2,771 & $57.8 \%$ & 20.0 \\
\hline 2013 & 1,577 & $28.9 \%$ & 17.4 & 571 & $10.5 \%$ & 21.1 & 3,310 & $60.6 \%$ & 20.9 \\
\hline 2014 & 1,929 & $30.6 \%$ & 18.0 & 672 & $10.7 \%$ & 21.3 & 3,706 & $58.8 \%$ & 21.7 \\
\hline 2015 & 1,786 & $25.0 \%$ & 18.8 & 655 & $9.2 \%$ & 21.9 & 4,697 & $65.8 \%$ & 22.6 \\
\hline 2016 & c & $28.5 \%$ & 19.0 & c & $9.5 \%$ & 21.9 & c & $61.7 \%$ & 22.6 \\
\hline
\end{tabular}

Note: Includes light trucks of 8,500 lb or less. See Table 4.11 for all light trucks (pickup + van + truck SUV).

\section{Source:}

U.S. Environmental Protection Agency, Light-Duty Automotive Technology, Carbon Dioxide Emissions, and Fuel Economy Trends: 1975 Through 2016, November 2016. (Additional resources: www.epa.gov/fueleconomy/lightduty-automotive-technology-carbon-dioxide-emissions-and-fuel-economy-trends-1975-0)

a The fuel economy data on this table are adjusted to provide the best estimate of real world performance. See section 10 of the source document for details on adjustment methodology. These data are not directly comparable to Corporate Average Fuel Economy data.

${ }^{\mathrm{b}}$ Production share is based on the total of pickups, plus vans and truck SUVs. Percentages may not sum to totals due to rounding.

\footnotetext{
${ }^{\mathrm{c}}$ Data are not available.
} 
The average fuel economy of cars more than doubled from 1975 to 2016 while the average fuel economy of light trucks grew by $84 \%$ in that same time period. This was not steady annual growth, but growth in the 1970's and early 1980's followed by a long period with little improvement. Growth resumed around 2008-2009.

Table 4.11

Production and Production-Weighted Fuel Economies of New Domestic and Import Cars, Light Trucks and Light Vehicles, Model Years 1975-2016

\begin{tabular}{|c|c|c|c|c|c|c|}
\hline \multirow[b]{2}{*}{$\begin{array}{c}\text { Model } \\
\text { Year }\end{array}$} & \multicolumn{2}{|c|}{ All Cars } & \multicolumn{2}{|c|}{ All Light Trucks } & \multicolumn{2}{|c|}{ All Light Vehicles } \\
\hline & $\begin{array}{l}\text { Production } \\
\text { (Thousands) }\end{array}$ & $\begin{array}{c}\text { Fuel } \\
\text { Economy (mpg) }\end{array}$ & $\begin{array}{c}\text { Production } \\
\text { (Thousands) }\end{array}$ & $\begin{array}{c}\text { Fuel } \\
\text { Economy (mpg) }\end{array}$ & $\begin{array}{l}\text { Production } \\
\text { (Thousands) }\end{array}$ & $\begin{array}{c}\text { Fuel } \\
\text { Economy (mpg) }\end{array}$ \\
\hline 1975 & 8,247 & 13.5 & 1,977 & 11.6 & 10,224 & 13.1 \\
\hline 1980 & 9,444 & 20.0 & 1,863 & 15.8 & 11,307 & 19.2 \\
\hline 1985 & 10,879 & 23.0 & 3,581 & 17.5 & 14,460 & 21.3 \\
\hline 1986 & 11,074 & 23.7 & 4,291 & 18.2 & 15,365 & 21.8 \\
\hline 1987 & 10,826 & 23.8 & 4,039 & 18.3 & 14,865 & 22.0 \\
\hline 1988 & 10,845 & 24.1 & 4,450 & 17.8 & 15,295 & 21.9 \\
\hline 1989 & 10,126 & 23.6 & 4,327 & 17.6 & 14,453 & 21.4 \\
\hline 1990 & 8,875 & 23.3 & 3,740 & 17.4 & 12,615 & 21.2 \\
\hline 1991 & 8,748 & 23.3 & 3,825 & 17.8 & 12,573 & 21.3 \\
\hline 1992 & 8,350 & 22.9 & 3,822 & 17.3 & 12,172 & 20.8 \\
\hline 1993 & 8,929 & 23.0 & 4,281 & 17.5 & 13,210 & 20.9 \\
\hline 1994 & 8,747 & 23.0 & 5,378 & 17.2 & 14,125 & 20.4 \\
\hline 1995 & 9,616 & 23.3 & 5,529 & 17.0 & 15,145 & 20.5 \\
\hline 1996 & 8,177 & 23.1 & 4,967 & 17.2 & 13,144 & 20.4 \\
\hline 1997 & 8,695 & 23.2 & 5,762 & 16.8 & 14,457 & 20.2 \\
\hline 1998 & 8,425 & 23.0 & 6,030 & 17.1 & 14,455 & 20.1 \\
\hline 1999 & 8,865 & 22.7 & 6,350 & 16.6 & 15,215 & 19.7 \\
\hline 2000 & 9,742 & 22.5 & 6,829 & 16.8 & 16,571 & 19.8 \\
\hline 2001 & 9,148 & 22.6 & 6,458 & 16.5 & 15,606 & 19.6 \\
\hline 2002 & 8,904 & 22.8 & 7,211 & 16.5 & 16,115 & 19.5 \\
\hline 2003 & 8,496 & 23.0 & 7,277 & 16.7 & 15,773 & 19.6 \\
\hline 2004 & 8,176 & 22.9 & 7,533 & 16.5 & 15,709 & 19.3 \\
\hline 2005 & 8,839 & 23.1 & 7,053 & 16.9 & 15,892 & 19.9 \\
\hline 2006 & 8,744 & 23.0 & 6,360 & 17.2 & 15,104 & 20.1 \\
\hline 2007 & 9,001 & 23.7 & 6,275 & 17.4 & 15,276 & 20.6 \\
\hline 2008 & 8,243 & 23.9 & 5,656 & 17.8 & 13,898 & 21.0 \\
\hline 2009 & 6,244 & 25.0 & 3,071 & 18.5 & 9,316 & 22.4 \\
\hline 2010 & 6,976 & 25.7 & 4,141 & 18.8 & 11,116 & 22.6 \\
\hline 2011 & 6,949 & 25.6 & 5,069 & 19.1 & 12,018 & 22.4 \\
\hline 2012 & 8,658 & 27.1 & 4,790 & 19.3 & 13,448 & 23.7 \\
\hline 2013 & 9,740 & 27.9 & 5,458 & 19.8 & 15,198 & 24.3 \\
\hline 2014 & 9,205 & 27.9 & 6,307 & 20.4 & 15,512 & 24.3 \\
\hline 2015 & 9,601 & 28.6 & 7,138 & 21.1 & 16,739 & 24.8 \\
\hline 2016 & b & 29.0 & $\mathrm{~b}$ & 21.4 & $\mathrm{~b}$ & 25.6 \\
\hline
\end{tabular}

Source:

U.S. Environmental Protection Agency, Light-Duty Automotive Technology, Carbon Dioxide Emissions, and Fuel Economy Trends: 1975 Through 2016, November 2016. (Additional resources: www.epa.gov/fueleconomy/lightduty-automotive-technology-carbon-dioxide-emissions-and-fuel-economy-trends-1975-0)

a The fuel economy data on this table are adjusted to provide the best estimate of real world performance. See section 10 of the source document for details on adjustment methodology. These data are not directly comparable to Corporate Average Fuel Economy data.

${ }^{\mathrm{b}}$ Data are not available, but $62.1 \%$ of all light vehicles were cars and 37.9\% were light trucks in 2016. 
Back in 1975 only 19.3\% of new light vehicles produced were light trucks. Because of the boom in production of minivans, sport utility vehicles, and pick-up trucks, that number rose to over $40 \%$ in 1998. The car SUV category was almost $11 \%$ of production in 2016 and the truck SUVs were $23 \%$.

Table 4.12

Light Vehicle Production Shares ${ }^{\mathrm{a}}$, Model Years 1975-2016

\begin{tabular}{|c|c|c|c|c|c|c|c|c|}
\hline \multirow[b]{2}{*}{$\begin{array}{c}\text { Model } \\
\text { Year }\end{array}$} & \multirow[b]{2}{*}{ Car } & \multirow[b]{2}{*}{$\begin{array}{c}\text { Car } \\
\text { SUV }\end{array}$} & \multirow[b]{2}{*}{ Pickup } & \multirow[b]{2}{*}{ Van } & \multirow[b]{2}{*}{$\begin{array}{l}\text { Truck } \\
\text { SUV }\end{array}$} & \multirow{2}{*}{$\begin{array}{l}\text { Total Light } \\
\text { Vehicles } \\
\text { Produced } \\
\text { (thousands) }\end{array}$} & \multicolumn{2}{|c|}{ Production Share } \\
\hline & & & & & & & Cars $^{\mathrm{b}}$ & $\begin{array}{c}\text { Light } \\
\text { Trucks }\end{array}$ \\
\hline 1975 & $80.6 \%$ & $0.1 \%$ & $13.1 \%$ & $4.5 \%$ & $1.7 \%$ & 10,224 & $80.7 \%$ & $19.3 \%$ \\
\hline 1980 & $83.5 \%$ & $0.0 \%$ & $12.7 \%$ & $2.1 \%$ & $1.6 \%$ & 11,306 & $83.5 \%$ & $16.5 \%$ \\
\hline 1985 & $74.6 \%$ & $0.6 \%$ & $14.4 \%$ & $5.9 \%$ & $4.5 \%$ & 14,460 & $75.2 \%$ & $24.8 \%$ \\
\hline 1986 & $71.7 \%$ & $0.4 \%$ & $16.5 \%$ & $6.8 \%$ & $4.6 \%$ & 15,365 & $72.1 \%$ & $27.9 \%$ \\
\hline 1987 & $72.2 \%$ & $0.6 \%$ & $14.4 \%$ & $7.5 \%$ & $5.2 \%$ & 14,865 & $72.8 \%$ & $27.2 \%$ \\
\hline 1988 & $70.2 \%$ & $0.7 \%$ & $16.1 \%$ & $7.4 \%$ & $5.6 \%$ & 15,295 & $70.9 \%$ & $29.1 \%$ \\
\hline 1989 & $69.3 \%$ & $0.7 \%$ & $15.4 \%$ & $8.8 \%$ & $5.7 \%$ & 14,453 & $70.1 \%$ & $29.9 \%$ \\
\hline 1990 & $69.8 \%$ & $0.5 \%$ & $14.5 \%$ & $10.0 \%$ & $5.1 \%$ & 12,615 & $70.4 \%$ & $29.6 \%$ \\
\hline 1991 & $67.8 \%$ & $1.8 \%$ & $15.3 \%$ & $8.2 \%$ & $6.9 \%$ & 12,573 & $69.6 \%$ & $30.4 \%$ \\
\hline 1992 & $66.6 \%$ & $2.0 \%$ & $15.1 \%$ & $10.0 \%$ & $6.2 \%$ & 12,172 & $68.6 \%$ & $31.4 \%$ \\
\hline 1993 & $64.0 \%$ & $3.6 \%$ & $15.2 \%$ & $10.9 \%$ & $6.3 \%$ & 13,211 & $67.6 \%$ & $32.4 \%$ \\
\hline 1994 & $59.6 \%$ & $2.3 \%$ & $18.9 \%$ & $10.0 \%$ & $9.1 \%$ & 14,125 & $61.9 \%$ & $38.1 \%$ \\
\hline 1995 & $62.0 \%$ & $1.5 \%$ & $15.0 \%$ & $11.0 \%$ & $10.5 \%$ & 15,145 & $63.5 \%$ & $36.5 \%$ \\
\hline 1996 & $60.0 \%$ & $2.2 \%$ & $14.9 \%$ & $10.7 \%$ & $12.2 \%$ & 13,144 & $62.2 \%$ & $37.8 \%$ \\
\hline 1997 & $57.6 \%$ & $2.5 \%$ & $16.7 \%$ & $8.8 \%$ & $14.5 \%$ & 14,458 & $60.1 \%$ & $39.9 \%$ \\
\hline 1998 & $55.1 \%$ & $3.1 \%$ & $16.7 \%$ & $10.3 \%$ & $14.7 \%$ & 14,456 & $58.3 \%$ & $41.7 \%$ \\
\hline 1999 & $55.1 \%$ & $3.2 \%$ & $16.7 \%$ & $9.6 \%$ & $15.4 \%$ & 15,215 & $58.3 \%$ & $41.7 \%$ \\
\hline 2000 & $55.1 \%$ & $3.7 \%$ & $15.8 \%$ & $10.2 \%$ & $15.2 \%$ & 16,571 & $58.8 \%$ & $41.2 \%$ \\
\hline 2001 & $53.9 \%$ & $4.8 \%$ & $16.1 \%$ & $7.9 \%$ & $17.3 \%$ & 15,605 & $58.6 \%$ & $41.4 \%$ \\
\hline 2002 & $51.5 \%$ & $3.7 \%$ & $14.8 \%$ & $7.7 \%$ & $22.3 \%$ & 16,115 & $55.3 \%$ & $44.7 \%$ \\
\hline 2003 & $50.2 \%$ & $3.6 \%$ & $15.7 \%$ & $7.8 \%$ & $22.6 \%$ & 15,773 & $53.9 \%$ & $46.1 \%$ \\
\hline 2004 & $48.0 \%$ & $4.1 \%$ & $15.9 \%$ & $6.1 \%$ & $25.9 \%$ & 15,709 & $52.0 \%$ & $48.0 \%$ \\
\hline 2005 & $50.5 \%$ & $5.1 \%$ & $14.5 \%$ & $9.3 \%$ & $20.6 \%$ & 15,892 & $55.6 \%$ & $44.4 \%$ \\
\hline 2006 & $52.9 \%$ & $5.0 \%$ & $14.5 \%$ & $7.7 \%$ & $19.9 \%$ & 15,104 & $57.9 \%$ & $42.1 \%$ \\
\hline 2007 & $52.9 \%$ & $6.0 \%$ & $13.8 \%$ & $5.5 \%$ & $21.7 \%$ & 15,276 & $58.9 \%$ & $41.1 \%$ \\
\hline 2008 & $52.7 \%$ & $6.6 \%$ & $12.9 \%$ & $5.7 \%$ & $22.1 \%$ & 13,898 & $59.3 \%$ & $40.7 \%$ \\
\hline 2009 & $60.5 \%$ & $6.5 \%$ & $10.6 \%$ & $4.0 \%$ & $18.4 \%$ & 9,316 & $67.0 \%$ & $33.0 \%$ \\
\hline 2010 & $54.5 \%$ & $8.2 \%$ & $11.5 \%$ & $5.0 \%$ & $20.7 \%$ & 11,116 & $62.8 \%$ & $37.3 \%$ \\
\hline 2011 & $47.8 \%$ & $10.0 \%$ & $12.3 \%$ & $4.3 \%$ & $25.5 \%$ & 12,018 & $57.8 \%$ & $42.2 \%$ \\
\hline 2012 & $55.0 \%$ & $9.4 \%$ & $10.1 \%$ & $4.9 \%$ & $20.6 \%$ & 13,448 & $64.4 \%$ & $35.6 \%$ \\
\hline 2013 & $54.1 \%$ & $10.0 \%$ & $10.4 \%$ & $3.8 \%$ & $21.8 \%$ & 15,198 & $64.1 \%$ & $35.9 \%$ \\
\hline 2014 & $49.2 \%$ & $10.1 \%$ & $12.4 \%$ & $4.3 \%$ & $23.9 \%$ & 15,512 & $59.3 \%$ & $40.7 \%$ \\
\hline 2015 & $47.2 \%$ & $10.2 \%$ & $10.7 \%$ & $3.9 \%$ & $28.1 \%$ & 16,739 & $57.4 \%$ & $42.6 \%$ \\
\hline 2016 & $51.4 \%$ & $10.7 \%$ & $10.8 \%$ & $3.7 \%$ & $23.4 \%$ & c & $62.1 \%$ & $37.9 \%$ \\
\hline
\end{tabular}

Note: Includes light trucks of $8,500 \mathrm{lb}$ or less.

\section{Source:}

U.S. Environmental Protection Agency, Light-Duty Automotive Technology, Carbon Dioxide Emissions, and Fuel Economy Trends: 1975 Through 2016, November 2016. (Additional resources: www.epa.gov/fueleconomy/lightduty-automotive-technology-carbon-dioxide-emissions-and-fuel-economy-trends-1975-0)

a Percentages may not sum to totals due to rounding.

b Cars include both car and car SUV categories.

c Data are not available. 
The effects of the Japanese earthquake/tsunami in 2011 are apparent in the large decline in car production for that year. Light trucks were gaining market share from the early 1980s until 2004, mainly due to increases in the market share of sport utility vehicles (SUVs) and pickup trucks. Car SUVs are two-wheel drive SUVs that are counted as cars in the Corporate Average Fuel Economy Standards for model years 2011-on. A listing of the makes/models of car SUVs is in Table 4.9.

Figure 4.1. Light Vehicle Production Shares, Model Years 1975-2016

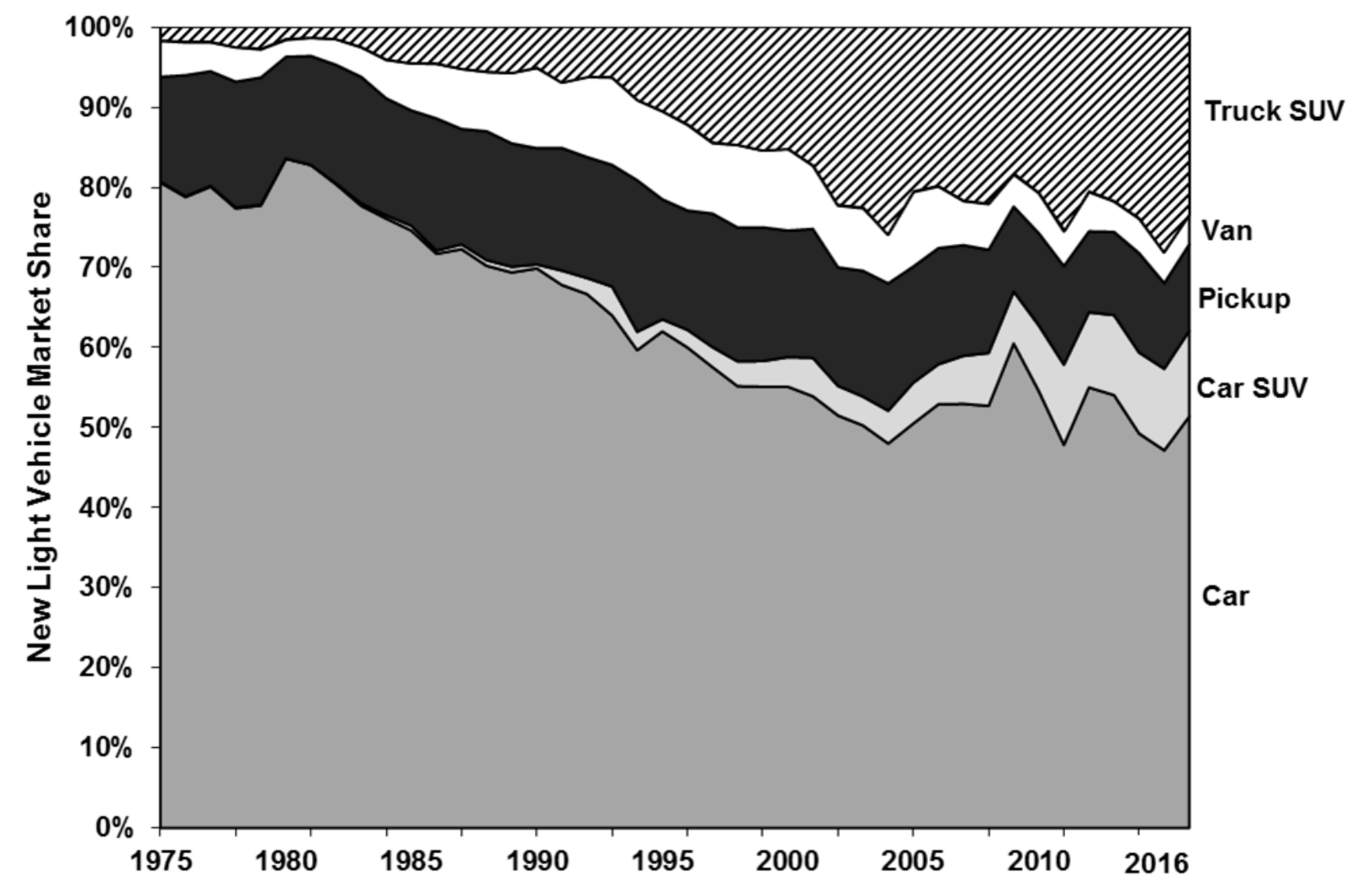

Source:

See Table 4.12. 
The production-weighted average engine displacement of cars in 1975 was 4.72 liters, but had declined to 2.34 liters by 2016. Car SUVs also experienced a decline in engine displacement. For a list of car SUVs, see

Table 4.9.

Table 4.13

Production-Weighted Engine Size of New Domestic and Import Cars

Model Years 1975-2016

$\left(\right.$ liters $\left.^{\mathrm{a}}\right)$

\begin{tabular}{|c|c|c|}
\hline Model Year & Car & Car SUV \\
\hline 1975 & 4.72 & 4.29 \\
\hline 1980 & 3.08 & 4.59 \\
\hline 1985 & 2.90 & 2.80 \\
\hline 1986 & 2.74 & 2.79 \\
\hline 1987 & 2.65 & 2.93 \\
\hline 1988 & 2.62 & 3.26 \\
\hline 1989 & 2.67 & 3.70 \\
\hline 1990 & 2.67 & 3.43 \\
\hline 1991 & 2.67 & 3.52 \\
\hline 1992 & 2.79 & 3.44 \\
\hline 1993 & 2.72 & 3.90 \\
\hline 1994 & 2.75 & 3.43 \\
\hline 1995 & 2.74 & 3.51 \\
\hline 1996 & 2.70 & 3.52 \\
\hline 1997 & 2.69 & 3.11 \\
\hline 1998 & 2.69 & 3.57 \\
\hline 1999 & 2.72 & 3.46 \\
\hline 2000 & 2.70 & 3.46 \\
\hline 2001 & 2.70 & 3.18 \\
\hline 2002 & 2.72 & 3.00 \\
\hline 2003 & 2.70 & 2.97 \\
\hline 2004 & 2.75 & 3.13 \\
\hline 2005 & 2.72 & 3.05 \\
\hline 2006 & 2.82 & 3.02 \\
\hline 2007 & 2.70 & 3.03 \\
\hline 2008 & 2.70 & 2.93 \\
\hline 2009 & 2.54 & 2.87 \\
\hline 2010 & 2.56 & 2.82 \\
\hline 2011 & 2.61 & 2.72 \\
\hline 2012 & 2.43 & 2.74 \\
\hline 2013 & 2.38 & 2.64 \\
\hline 2014 & 2.39 & 2.52 \\
\hline 2015 & 2.38 & 2.51 \\
\hline 2016 & 2.34 & 2.43 \\
\hline \multicolumn{3}{|c|}{ Annual average percentage change } \\
\hline 1975-2016 & $-1.7 \%$ & $-1.4 \%$ \\
\hline 2006-2016 & $-1.8 \%$ & $-2.2 \%$ \\
\hline
\end{tabular}

\section{Source:}

U.S. Environmental Protection Agency, Light-Duty Automotive Technology, Carbon Dioxide Emissions, and Fuel Economy Trends: 1975 Through 2016, November 2016. (Additional resources: www.epa.gov/fueleconomy/lightduty-automotive-technology-carbon-dioxide-emissions-and-fuel-economy-trends-1975-0)

a 1 liter $=61.02$ cubic inches. 
The production-weighted engine size of truck sport utility vehicles (SUVs) declined an average of $2.3 \%$ per year from 2006 to 2016, while the engine size of pickups in 2016 decreased by only 0.5\%.

Table 4.14

Production-Weighted Engine Size of New Domestic and Import Light Trucks, Model Years 1975-2016 $\left(\right.$ (liters $\left.^{a}\right)$

\begin{tabular}{|c|c|c|c|}
\hline Model Year & Pickup & Van & Truck SUV \\
\hline 1975 & 5.01 & 5.21 & 5.44 \\
\hline 1980 & 3.87 & 4.72 & 4.83 \\
\hline 1985 & 3.62 & 3.87 & 3.64 \\
\hline 1986 & 3.38 & 3.74 & 3.44 \\
\hline 1987 & 3.31 & 3.70 & 3.47 \\
\hline 1988 & 3.75 & 3.75 & 3.64 \\
\hline 1989 & 3.87 & 3.69 & 4.00 \\
\hline 1990 & 4.05 & 3.69 & 3.85 \\
\hline 1991 & 3.80 & 3.61 & 3.82 \\
\hline 1992 & 4.02 & 3.64 & 3.85 \\
\hline 1993 & 4.00 & 3.57 & 4.00 \\
\hline 1994 & 4.06 & 3.70 & 4.02 \\
\hline 1995 & 4.21 & 3.79 & 4.02 \\
\hline 1996 & 4.11 & 3.61 & 4.24 \\
\hline 1997 & 4.33 & 3.61 & 4.18 \\
\hline 1998 & 4.13 & 3.56 & 4.13 \\
\hline 1999 & 4.38 & 3.65 & 4.15 \\
\hline 2000 & 4.18 & 3.56 & 4.15 \\
\hline 2001 & 4.41 & 3.75 & 3.92 \\
\hline 2002 & 4.44 & 3.57 & 4.02 \\
\hline 2003 & 4.33 & 3.59 & 4.05 \\
\hline 2004 & 4.61 & 3.59 & 4.13 \\
\hline 2005 & 4.65 & 3.54 & 4.00 \\
\hline 2006 & 4.56 & 3.54 & 3.87 \\
\hline 2007 & 4.69 & 3.59 & 3.93 \\
\hline 2008 & 4.69 & 3.61 & 3.77 \\
\hline 2009 & 4.70 & 3.52 & 3.46 \\
\hline 2010 & 4.80 & 3.51 & 3.47 \\
\hline 2011 & 4.62 & 3.47 & 3.56 \\
\hline 2012 & 4.69 & 3.44 & 3.52 \\
\hline 2013 & 4.62 & 3.43 & 3.36 \\
\hline 2014 & 4.80 & 3.49 & 3.21 \\
\hline 2015 & 4.54 & 3.33 & 3.24 \\
\hline 2016 & 4.34 & 3.33 & 3.08 \\
\hline \multicolumn{4}{|c|}{ Annual average percentage change } \\
\hline $1975-2016$ & $-0.3 \%$ & $-1.1 \%$ & $-1.4 \%$ \\
\hline 2006-2016 & $-0.5 \%$ & $-0.6 \%$ & $-2.3 \%$ \\
\hline
\end{tabular}

Note: Includes light trucks of $8,500 \mathrm{lb}$ or less.

\section{Source:}

U.S. Environmental Protection Agency, Light-Duty Automotive Technology, Carbon Dioxide Emissions, and Fuel Economy Trends: 1975 Through 2016, November 2016. (Additional resources: www.epa.gov/fueleconomy/lightduty-automotive-technology-carbon-dioxide-emissions-and-fuel-economy-trends-1975-0)

a 1 liter $=61.02$ cubic inches. 
The production-weighted loaded vehicle weight of cars declined almost $550 \mathrm{lb}$ from 1975 to 2016, while car SUVs declined by $151 \mathrm{lb}$.

Table 4.15

Production-Weighted Loaded Vehicle Weight ${ }^{\mathrm{a}}$ of New Domestic and Import Cars, Model Years 1975-2016 (pounds)

\begin{tabular}{|c|c|c|}
\hline Model Year & Car & Car SUV \\
\hline 1975 & 4,058 & 4,000 \\
\hline 1980 & 3,101 & 4,000 \\
\hline 1981 & 3,076 & 4,000 \\
\hline 1982 & 3,054 & 2,630 \\
\hline 1983 & 3,112 & 3,124 \\
\hline 1984 & 3,099 & 3,487 \\
\hline 1985 & 3,093 & 3,469 \\
\hline 1986 & 3,041 & 3,479 \\
\hline 1987 & 3,031 & 3,492 \\
\hline 1988 & 3,047 & 3,495 \\
\hline 1989 & 3,099 & 3,497 \\
\hline 1990 & 3,176 & 3,518 \\
\hline 1991 & 3,154 & 3,733 \\
\hline 1992 & 3,240 & 3,713 \\
\hline 1993 & 3,207 & 3,848 \\
\hline 1994 & 3,250 & 3,735 \\
\hline 1995 & 3,263 & 3,763 \\
\hline 1996 & 3,282 & 3,710 \\
\hline 1997 & 3,274 & 3,549 \\
\hline 1998 & 3,306 & 3,824 \\
\hline 1999 & 3,365 & 3,831 \\
\hline 2000 & 3,369 & 3,870 \\
\hline 2001 & 3,380 & 3,765 \\
\hline 2002 & 3,391 & 3,747 \\
\hline 2003 & 3,417 & 3,716 \\
\hline 2004 & 3,462 & 3,854 \\
\hline 2005 & 3,463 & 3,848 \\
\hline 2006 & 3,534 & 3,876 \\
\hline 2007 & 3,507 & 3,935 \\
\hline 2008 & 3,527 & 3,902 \\
\hline 2009 & 3,464 & 3,846 \\
\hline 2010 & 3,474 & 3,949 \\
\hline 2011 & 3,559 & 3,890 \\
\hline 2012 & 3,452 & 3,915 \\
\hline 2013 & 3,465 & 3,966 \\
\hline 2014 & 3,497 & 3,865 \\
\hline 2015 & 3,489 & 3,868 \\
\hline 2016 & 3,509 & 3,849 \\
\hline \multicolumn{3}{|c|}{ Annual average percentage change } \\
\hline 1975-2016 & $-0.4 \%$ & $-0.1 \%$ \\
\hline 2006-2016 & $-0.1 \%$ & $-0.1 \%$ \\
\hline
\end{tabular}

\section{Source:}

U.S. Environmental Protection Agency, Light-Duty Automotive Technology, Carbon Dioxide Emissions, and Fuel Economy Trends: 1975 Through 2016, November 2016. (Additional resources: www.epa.gov/fueleconomy/lightduty-automotive-technology-carbon-dioxide-emissions-and-fuel-economy-trends-1975-0)

${ }^{\text {a }}$ Loaded vehicle weight is equal to the vehicle's curb weight plus 300 pounds. 
The production-weighted loaded vehicle weight of pickups, vans, and truck SUVs increased from 1975 to 2016.

Pickups gained almost 1,200 lb while vans gained $210 \mathrm{lb}$ and truck SUVs gained $261 \mathrm{lb}$.

Table 4.16

Production-Weighted Loaded Vehicle Weight of New Domestic and Import Light Trucks, Model Years 1975-2016

(pounds)

\begin{tabular}{|c|c|c|c|}
\hline Model Year & Pickup & Van & Truck SUV \\
\hline 1975 & 4,012 & 4,196 & 4,214 \\
\hline 1980 & 3,740 & 4,353 & 4,237 \\
\hline 1981 & 3,679 & 4,324 & 4,208 \\
\hline 1982 & 3,629 & 4,342 & 4,494 \\
\hline 1983 & 3,544 & 4,414 & 4,270 \\
\hline 1984 & 3,619 & 4,075 & 4,049 \\
\hline 1985 & 3,642 & 3,975 & 4,092 \\
\hline 1986 & 3,574 & 3,998 & 3,958 \\
\hline 1987 & 3,526 & 3,972 & 3,882 \\
\hline 1988 & 3,737 & 4,053 & 3,906 \\
\hline 1989 & 3,803 & 4,057 & 4,086 \\
\hline 1990 & 3,928 & 4,095 & 4,098 \\
\hline 1991 & 3,779 & 4,133 & 4,157 \\
\hline 1992 & 3,976 & 4,151 & 4,204 \\
\hline 1993 & 3,996 & 4,105 & 4,331 \\
\hline 1994 & 4,056 & 4,156 & 4,331 \\
\hline 1995 & 4,182 & 4,110 & 4,323 \\
\hline 1996 & 4,190 & 4,195 & 4,386 \\
\hline 1997 & 4,415 & 4,240 & 4,463 \\
\hline 1998 & 4,282 & 4,183 & 4,450 \\
\hline 1999 & 4,486 & 4,306 & 4,518 \\
\hline 2000 & 4,340 & 4,276 & 4,602 \\
\hline 2001 & 4,551 & 4,518 & 4,546 \\
\hline 2002 & 4,690 & 4,394 & 4,636 \\
\hline 2003 & 4,642 & 4,393 & 4,754 \\
\hline 2004 & 4,939 & 4,487 & 4,756 \\
\hline 2005 & 4,988 & 4,430 & 4,756 \\
\hline 2006 & 4,968 & 4,475 & 4,715 \\
\hline 2007 & 5,144 & 4,479 & 4,797 \\
\hline 2008 & 5,161 & 4,527 & 4,727 \\
\hline 2009 & 5,176 & 4,572 & 4,548 \\
\hline 2010 & 5,309 & 4,533 & 4,555 \\
\hline 2011 & 5,268 & 4,502 & 4,665 \\
\hline 2012 & 5,335 & 4,442 & 4,640 \\
\hline 2013 & 5,429 & 4,543 & 4,584 \\
\hline 2014 & 5,485 & 4,489 & 4,483 \\
\hline 2015 & 5,165 & 4,416 & 4,533 \\
\hline 2016 & 5,177 & 4,406 & 4,475 \\
\hline \multicolumn{4}{|c|}{ Annual average percentage change } \\
\hline 1975-2016 & $0.6 \%$ & $0.1 \%$ & $0.1 \%$ \\
\hline 2006-2016 & $0.4 \%$ & $-0.2 \%$ & $-0.5 \%$ \\
\hline
\end{tabular}

\section{Source:}

U.S. Environmental Protection Agency, Light-Duty Automotive Technology, Carbon Dioxide Emissions, and Fuel Economy Trends: 1975 Through 2016, November 2016. (Additional resources: www.epa.gov/fueleconomy/lightduty-automotive-technology-carbon-dioxide-emissions-and-fuel-economy-trends-1975-0)

${ }^{\text {a }}$ Loaded vehicle weight is equal to the vehicle's curb weight plus 300 pounds. 
The average light vehicle in 2015 contained more than 2,000 pounds of steel, most of it conventional steel. High and medium strength steel, however, almost 18\% of the vehicle. The use of aluminum grew from 1995 to 2015, while the use of iron castings declined.

Table 4.17

Average Material Consumption for a Domestic Light Vehiclea Model Years 1995, 2000, and 2015

\begin{tabular}{|c|c|c|c|c|c|c|}
\hline \multirow[b]{2}{*}{ Material } & \multicolumn{2}{|c|}{1995} & \multicolumn{2}{|c|}{2000} & \multicolumn{2}{|c|}{2015} \\
\hline & Pounds & Percentage & Pounds & Percentage & Pounds & Percentage \\
\hline Regular steel & 1,630 & $44.1 \%$ & 1,655 & $42.4 \%$ & 1,330 & $33.3 \%$ \\
\hline High and medium strength steel & 324 & $8.8 \%$ & 408 & $10.5 \%$ & 701 & $17.6 \%$ \\
\hline Stainless steel & 51 & $1.4 \%$ & 62 & $1.6 \%$ & 75 & $1.9 \%$ \\
\hline Other steels & 46 & $1.2 \%$ & 26 & $0.7 \%$ & 32 & $0.8 \%$ \\
\hline Iron castings & 466 & $12.6 \%$ & 432 & $11.1 \%$ & 268 & $6.7 \%$ \\
\hline Aluminum & 231 & $6.3 \%$ & 268 & $6.9 \%$ & 395 & $9.9 \%$ \\
\hline Magnesium castings & 4 & $0.1 \%$ & 8 & $0.2 \%$ & 10 & $0.2 \%$ \\
\hline Copper and brass & 50 & $1.4 \%$ & 52 & $1.3 \%$ & 66 & $1.7 \%$ \\
\hline Lead & 33 & $0.9 \%$ & 36 & $0.9 \%$ & 35 & $0.9 \%$ \\
\hline Zinc castings & 19 & $0.5 \%$ & 13 & $0.3 \%$ & 8 & $0.2 \%$ \\
\hline Powder metal parts & 29 & $0.8 \%$ & 36 & $0.9 \%$ & 45 & $1.1 \%$ \\
\hline Other metals & 4 & $0.1 \%$ & 4 & $0.1 \%$ & 5 & $0.1 \%$ \\
\hline Plastics and plastic composites & 240 & $6.5 \%$ & 286 & $7.3 \%$ & 334 & $8.4 \%$ \\
\hline Rubber & 149 & $4.0 \%$ & 166 & $4.3 \%$ & 198 & $5.0 \%$ \\
\hline Coatings & 23 & $0.6 \%$ & 25 & $0.6 \%$ & 29 & $0.7 \%$ \\
\hline Textiles & 42 & $1.1 \%$ & 44 & $1.1 \%$ & 45 & $1.1 \%$ \\
\hline Fluids and lubricants & 192 & $5.2 \%$ & 207 & $5.3 \%$ & 225 & $5.6 \%$ \\
\hline Glass & 97 & $2.6 \%$ & 103 & $2.6 \%$ & 95 & $2.4 \%$ \\
\hline Other materials & 64 & $1.7 \%$ & 71 & $1.8 \%$ & 95 & $2.4 \%$ \\
\hline Total & 3,694 & $100.0 \%$ & 3,902 & $100.0 \%$ & 3,991 & $100.0 \%$ \\
\hline
\end{tabular}

Source:

Ward's Communications, www.wardsauto.com.

a Percentages may not sum to totals due to rounding. 
The number of franchised dealerships which sell new light-duty vehicles (cars and light trucks) has declined 46\% since 1970. This decline, along with increasing light vehicle sales, caused the average number of vehicles sold to be 1,028 vehicles per dealer.

Table 4.18

New Light Vehicle Dealerships and Sales, 1970-2016

\begin{tabular}{|c|c|c|c|}
\hline Calendar year & $\begin{array}{l}\text { Number of franchised new } \\
\text { light vehicle dealerships }\end{array}$ & $\begin{array}{c}\text { New light vehicle sales } \\
\text { (thousands) }\end{array}$ & $\begin{array}{c}\text { Light vehicle sales per } \\
\text { dealer }\end{array}$ \\
\hline 1970 & 30,800 & 9,856 & 320 \\
\hline 1975 & 29,600 & 10,677 & 361 \\
\hline 1980 & 27,900 & 10,909 & 391 \\
\hline 1981 & 26,350 & 10,235 & 388 \\
\hline 1982 & 25,700 & 10,019 & 390 \\
\hline 1983 & 24,725 & 11,669 & 472 \\
\hline 1984 & 24,725 & 13,579 & 549 \\
\hline 1985 & 24,725 & 14,667 & 593 \\
\hline 1986 & 24,825 & 15,998 & 644 \\
\hline 1987 & 25,150 & 14,802 & 589 \\
\hline 1988 & 25,025 & 15,347 & 613 \\
\hline 1989 & 25,000 & 14,389 & 576 \\
\hline 1990 & 24,825 & 13,851 & 558 \\
\hline 1991 & 24,200 & 12,307 & 509 \\
\hline 1992 & 23,500 & 12,842 & 546 \\
\hline 1993 & 22,950 & 13,869 & 604 \\
\hline 1994 & 22,850 & 15,024 & 657 \\
\hline 1995 & 22,800 & 14,673 & 644 \\
\hline 1996 & 22,750 & 14,998 & 659 \\
\hline 1997 & 22,700 & 15,014 & 661 \\
\hline 1998 & 22,600 & 15,384 & 681 \\
\hline 1999 & 22,400 & 16,711 & 746 \\
\hline 2000 & 22,250 & 17,164 & 771 \\
\hline 2001 & 22,150 & 16,950 & 765 \\
\hline 2002 & 21,800 & 16,675 & 765 \\
\hline 2003 & 21,725 & 16,494 & 759 \\
\hline 2004 & 21,650 & 16,737 & 773 \\
\hline 2005 & 21,640 & 16,774 & 775 \\
\hline 2006 & 21,495 & 16,336 & 760 \\
\hline 2007 & 21,200 & 15,867 & 748 \\
\hline 2008 & 20,770 & 13,015 & 627 \\
\hline 2009 & 20,010 & 10,236 & 512 \\
\hline 2010 & 18,460 & 11,394 & 617 \\
\hline 2011 & 17,700 & 12,542 & 709 \\
\hline 2012 & 17,540 & 14,220 & 811 \\
\hline 2013 & 17,665 & 15,279 & 865 \\
\hline 2014 & 16,396 & 16,192 & 988 \\
\hline 2015 & 16,545 & 17,095 & 1,033 \\
\hline 2016 & 16,708 & 17,169 & 1,028 \\
\hline \multicolumn{4}{|c|}{ Average annual percentage change } \\
\hline 1970-2016 & $-1.3 \%$ & $1.2 \%$ & $2.6 \%$ \\
\hline $2006-2016$ & $-2.5 \%$ & $0.5 \%$ & $3.1 \%$ \\
\hline
\end{tabular}

\section{Source:}

Number of dealers - National Automobile Dealers Association website, www.nada.org. (Additional resources: www.nada.org/2016NADAdataHighlights/). Light-duty vehicle sales - See tables 4.5 and 4.6.

a As of the beginning of the year. 
In 2010 the number of conventional refueling stations fell below 160,000 for the first time in the series history and continued to decline through 2013. The number of vehicles fueling at those stations fell in 2009 for the first time in several years but began rising slowly in 2011. In 2013, there were 0.61 fueling stations per thousand vehicles or 1.65 thousand vehicles per station.

Table 4.19

Conventional Refueling Stations, 1993-2013

\begin{tabular}{ccccc}
\hline & $\begin{array}{c}\text { Number of retail } \\
\text { outlets }\end{array}$ & $\begin{array}{c}\text { Vehicles in } \\
\text { operation } \\
\text { (thousands) }\end{array}$ & $\begin{array}{c}\text { Thousand } \\
\text { Stations per } \\
\text { thousand vehicles }\end{array}$ & $\begin{array}{c}\text { vehicles per } \\
\text { station }\end{array}$ \\
\cline { 2 - 5 } Year & \multicolumn{4}{c}{ Conventional fuels } \\
1993 & 207,416 & 186,315 & 1.11 & 0.90 \\
1994 & 202,878 & 188,714 & 1.08 & 0.93 \\
1995 & 195,455 & 193,441 & 1.01 & 0.99 \\
1996 & 190,246 & 198,294 & 0.96 & 1.04 \\
1997 & 187,892 & 201,071 & 0.93 & 1.07 \\
1998 & 182,596 & 205,043 & 0.89 & 1.16 \\
1999 & 180,567 & 209,509 & 0.86 & 1.21 \\
2000 & 175,941 & 213,299 & 0.82 & 1.26 \\
2001 & 172,169 & 216,683 & 0.79 & 1.30 \\
2002 & 170,018 & 221,027 & 0.77 & 1.35 \\
2003 & 167,571 & 225,882 & 0.74 & 1.41 \\
2004 & 167,346 & 232,167 & 0.72 & 1.46 \\
2005 & 168,987 & 238,384 & 0.71 & 1.51 \\
2006 & 167,476 & 244,643 & 0.69 & 1.55 \\
2007 & 164,292 & 248,701 & 0.66 & 1.53 \\
2008 & 161,068 & 249,813 & 0.64 & 1.56 \\
2009 & 162,350 & 248,972 & 0.65 & 1.58 \\
2010 & 159,006 & 248,231 & 0.64 & 1.61 \\
2011 & 157,393 & 248,932 & 0.63 & 1.65 \\
2012 & 156,065 & 251,497 & 0.62 & \\
2013 & 152,995 & 252,715 & 0.61 & \\
\hline
\end{tabular}

Notes: This data series was discontinued after 2013. The County Business Patterns (CBP) data published by the Bureau of the Census tells the number of establishments by North American Industry Classification System (NAICS). NAICS is an industry classification system that groups establishments into industries based on the activities in which they are primarily engaged. NAICS 447 represents gasoline stations. However, the CBP gasoline station data differ from the National Petroleum News Survey data by as much as 30\% (117,189 stations in 2005); the CBP may not include every gasoline retail outlet due to the classification of the primary activity of the business.

Alternative Fuel Refueling Stations are listed in Chapter 6.

\section{Sources:}

Conventional refueling stations: National Petroleum News Survey, 2013. (Source discontinued after 2013.)

Conventional vehicles: IHS Automotive, Detroit, MI. FURTHER REPRODUCTION PROHIBITED. 
The National Highway Traffic Safety Administration and the Environmental Protection Agency issued joint rulemaking to establish a new National Program to regulate fuel economy and greenhouse gas emissions for model year (MY) 2012-2025 cars and light trucks. The standards for model years 2021-2025 are currently under review.

Table 4.20

Fuel Economy and Carbon Dioxide Emissions Standards, MY 2012-2025

\begin{tabular}{|c|c|c|c|c|}
\hline Year & Cars & Light trucks & Combined cars and light trucks & \\
\hline \multicolumn{5}{|c|}{$\begin{array}{l}\text { Average required fuel economy } \\
\text { (miles per gallon) }\end{array}$} \\
\hline 2012 & 33.3 & 25.4 & 29.7 & \\
\hline 2013 & 34.2 & 26.0 & 30.5 & \\
\hline 2014 & 34.9 & 26.6 & 31.3 & \\
\hline 2015 & 36.2 & 27.5 & 32.6 & \\
\hline 2016 & 37.8 & 28.8 & 34.1 & \\
\hline 2017 & 40.1 & 29.4 & 35.4 & \\
\hline 2018 & 41.6 & 30.0 & 36.5 & \\
\hline 2019 & 43.1 & 30.6 & 37.7 & \\
\hline 2020 & 44.8 & 31.2 & 38.9 & \\
\hline 2021 & 46.8 & 33.3 & 41.0 & Standards \\
\hline 2022 & 49.0 & 34.9 & 43.0 & under review \\
\hline 2023 & 51.2 & 36.6 & 45.1 & \\
\hline 2024 & 53.6 & 38.5 & 47.4 & \\
\hline 2025 & 56.2 & 40.3 & 49.7 & \\
\hline \multicolumn{5}{|c|}{$\begin{array}{l}\text { Average projected emissions compliance levels under } \\
\text { the footprint-based carbon dioxide standards } \\
\text { (grams per mile) }\end{array}$} \\
\hline 2012 & 263 & 346 & 295 & \\
\hline 2013 & 256 & 337 & 286 & \\
\hline 2014 & 247 & 326 & 276 & \\
\hline 2015 & 236 & 312 & 263 & \\
\hline 2016 & 225 & 298 & 250 & \\
\hline 2017 & 212 & 295 & 243 & \\
\hline 2018 & 202 & 285 & 232 & \\
\hline 2019 & 191 & 277 & 222 & \\
\hline 2020 & 182 & 269 & 213 & \\
\hline 2021 & 172 & 249 & 199 & Standards \\
\hline 2022 & 164 & 237 & 190 & under review \\
\hline 2023 & 157 & 225 & 180 & \\
\hline 2024 & 150 & 214 & 171 & \\
\hline 2025 & 143 & 203 & 163 & \\
\hline
\end{tabular}

Note: The required fuel economy, along with projections of $\mathrm{CO}_{2}$ emissions, shown here use a model year 2008 baseline. The presented rates of increase in stringency for NHTSA CAFE standards are lower than the Environmental Protection Agency (EPA) rates of increase in stringency for greenhouse gas (GHG) standards. One major difference is that NHTSA's standards, unlike EPA's, do not reflect the inclusion of air conditioning system refrigerant and leakage improvements, but EPA's standards would allow consideration of such improvements which reduce GHGs but generally do not affect fuel economy. The 2025 EPA GHG standard of 163 grams/mile would be equivalent to $54.5 \mathrm{mpg}$, if the vehicles were to meet this level all through fuel economy improvements. The agencies expect, however, that a portion of these improvements will be made through reductions in air conditioning leakage, which would not contribute to fuel economy.

\section{Source:}

Federal Register, Vol. 77, No. 199, October 15, 2012. (Additional resources: www.nhtsa.gov/fuel-economy) 
The target levels for the fuel economy and carbon dioxide emission standards for vehicles manufactured in model years 2012-on are assigned based on a vehicle's "footprint." Each footprint has a different target. The vehicle footprint is calculated as:

$$
\begin{gathered}
\text { footprint }=\text { track width } \times \text { wheelbase, } \\
\text { where }
\end{gathered}
$$

track width = lateral distance between the centerlines of the base tires at ground, and wheelbase = longitudinal distance between the front and rear wheel centerlines.

Table 4.21

Fuel Economy and Carbon Dioxide Targets for Model Year 2025

\begin{tabular}{llccc}
\hline Vehicle type & Example models & $\begin{array}{c}\text { Example } \\
\text { model footprint } \\
\text { (square feet) }\end{array}$ & $\begin{array}{c}\mathrm{CO}_{2} \text { emissions } \\
\text { target } \\
\text { (grams per mile) }\end{array}$ & $\begin{array}{c}\text { Fuel economy target } \\
\text { (miles per gallon) }\end{array}$ \\
\hline Compact car & Honda Fit & Example Passenger Cars & \\
Midsize car & Ford Fusion & 40 & 131 & 61.1 \\
Fullsize car & Chrysler 300 & 46 & 147 & 54.9 \\
\hline \multicolumn{5}{c}{ Example Light-Duty Trucks } \\
\hline Small SUV & 4WD Ford Escape & 44 & 170 & 48.0 \\
Midsize crossover & Nissan Murano & 49 & 170 & 47.5 \\
Minivan & Toyota Sienna & 55 & 188 & 43.4 \\
Large pickup truck & Chevy Silverado & 67 & 209 & 39.2 \\
\hline
\end{tabular}

Notes: The model year 2025 targets are currently under review. Examples in table use model year 2012 vehicle specifications. The fuel economy from this table will not match the fuel economy listed on the window sticker of a new vehicle. Window sticker fuel economy is calculated by a different methodology than the Corporate Average Fuel Economy.

\section{Source:}

Federal Register, Vol. 77, No. 199, October 15, 2012. (Additional resources: www.nhtsa.gov/fuel-economy) 
The Corporate Average Fuel Economy standards were first established by the U.S. Energy Policy and Conservation Act of 1975 (PL94-163). These standards must be met at the manufacturer level. Some manufacturers fall short of meeting the standards while others exceed them. Legislation passed in December 2007 changed the CAFE standards beginning in the 2011 model year (MY). Some two-wheel drive sport utility vehicles are classified as cars under the final standards for MY 2011-2021.

Table 4.22

Car Corporate Average Fuel Economy (CAFE) Standards versus Sales-Weighted Fuel Economy Estimates, 1978-2017 ${ }^{\text {a }}$ (miles per gallon)

\begin{tabular}{|c|c|c|c|c|c|}
\hline \multirow{3}{*}{$\begin{array}{l}\text { Model } \\
\text { yearb }^{\text {b }}\end{array}$} & \multicolumn{4}{|c|}{ Cars } & \multirow{3}{*}{$\begin{array}{l}\text { CAFE estimates } \\
\text { Cars and light } \\
\text { trucks combined }\end{array}$} \\
\hline & \multicolumn{2}{|c|}{ CAFE standards } & \multicolumn{2}{|c|}{ CAFE estimates ${ }^{c}$} & \\
\hline & Domestic & Import & Domestic & Import & \\
\hline 1978 & 18.0 & 18.0 & 18.7 & 27.3 & 19.9 \\
\hline 1980 & 20.0 & 20.0 & 22.6 & 29.6 & 23.1 \\
\hline 1985 & 27.5 & 27.5 & 26.3 & 31.5 & 25.4 \\
\hline 1990 & 27.5 & 27.5 & 26.9 & 29.9 & 25.4 \\
\hline 1991 & 27.5 & 27.5 & 27.3 & 30.1 & 25.6 \\
\hline 1992 & 27.5 & 27.5 & 27.0 & 29.2 & 25.1 \\
\hline 1993 & 27.5 & 27.5 & 27.8 & 29.6 & 25.2 \\
\hline 1994 & 27.5 & 27.5 & 27.5 & 29.6 & 24.7 \\
\hline 1995 & 27.5 & 27.5 & 27.7 & 30.3 & 24.9 \\
\hline 1996 & 27.5 & 27.5 & 28.1 & 29.6 & 24.9 \\
\hline 1997 & 27.5 & 27.5 & 27.8 & 30.1 & 24.6 \\
\hline 1998 & 27.5 & 27.5 & 28.6 & 29.2 & 24.7 \\
\hline 1999 & 27.5 & 27.5 & 28.0 & 29.0 & 24.5 \\
\hline 2000 & 27.5 & 27.5 & 28.7 & 28.3 & 24.8 \\
\hline 2001 & 27.5 & 27.5 & 28.7 & 29.0 & 24.5 \\
\hline 2002 & 27.5 & 27.5 & 29.1 & 28.8 & 24.7 \\
\hline 2003 & 27.5 & 27.5 & 29.1 & 29.9 & 25.1 \\
\hline 2004 & 27.5 & 27.5 & 29.9 & 28.7 & 24.6 \\
\hline 2005 & 27.5 & 27.5 & 30.5 & 29.9 & 25.4 \\
\hline 2006 & 27.5 & 27.5 & 30.3 & 29.7 & 25.8 \\
\hline 2007 & 27.5 & 27.5 & 30.6 & 32.2 & 26.6 \\
\hline 2008 & $27.5^{d}$ & 27.5 & 31.2 & 31.8 & 27.1 \\
\hline 2009 & $27.5^{\mathrm{d}}$ & 27.5 & 32.1 & 33.8 & 29.0 \\
\hline 2010 & $27.5^{d}$ & 27.5 & 33.1 & 35.2 & 29.3 \\
\hline 2011 & 30.0 & 30.4 & 32.7 & 33.7 & 29.0 \\
\hline 2012 & 32.7 & 33.4 & 34.8 & 36.0 & 30.8 \\
\hline 2013 & 33.2 & 33.9 & 36.1 & 36.8 & 31.6 \\
\hline 2014 & 34.0 & 34.6 & 36.3 & 36.9 & 31.7 \\
\hline 2015 & 35.2 & 35.8 & 37.2 & 37.3 & 32.2 \\
\hline 2016 & $36.5^{e}$ & $37.3^{e}$ & $37.2^{\mathrm{e}}$ & $37.6^{\mathrm{e}}$ & $32.1^{\mathrm{e}}$ \\
\hline 2017 & $38.5^{\mathrm{e}}$ & $34.5^{\mathrm{e}}$ & $38.5^{e}$ & $32.8^{\mathrm{e}}$ & $31.8^{\mathrm{e}}$ \\
\hline
\end{tabular}

\section{Source:}

U.S. Department of Transportation, NHTSA, "Summary of Fuel Economy Performance," Washington, DC, December 2014 and updates 2017. (Additional resources: www.nhtsa.gov)

${ }^{a}$ Only vehicles with at least 75 percent domestic content can be counted in the average domestic fuel economy for a manufacturer.

${ }^{\mathrm{b}}$ Model year as determined by the manufacturer on a vehicle by vehicle basis.

${ }^{\mathrm{c}}$ All CAFE calculations are sales-weighted.

${ }^{\mathrm{d}}$ Unreformed standards, which were an option from 2008-2010. See Table 4.20 for reformed standards.

${ }^{\text {e }}$ Projected required average fuel economy standards value based on pre-model year reports. 
The Corporate Average Fuel Economy standards for light trucks are lower than the car standards. Light trucks include pickups, minivans, sport utility vehicles and vans. New legislation passed in December 2007 changed the CAFE standards beginning in the 2011 model year (MY). Some two-wheel drive sport utility vehicles are classified

as cars under the final standards for MY 2011-2021.

Table 4.23

Light Truck Corporate Average Fuel Economy (CAFE) Standards versus Sales-Weighted Fuel Economy Estimates, 1978-2017 ${ }^{\mathrm{a}}$ (miles per gallon)

\begin{tabular}{|c|c|c|c|c|c|}
\hline \multirow{3}{*}{$\begin{array}{c}\text { Model } \\
\text { year }^{c}\end{array}$} & \multicolumn{4}{|c|}{ Light trucks $^{\mathrm{b}}$} & \multirow{3}{*}{$\begin{array}{c}\text { CAFE estimates } \\
\text { Cars and light } \\
\text { trucks combined }\end{array}$} \\
\hline & \multirow{2}{*}{$\begin{array}{c}\text { CAFE } \\
\text { standards }\end{array}$} & \multicolumn{3}{|c|}{ CAFE estimates $^{\mathrm{d}}$} & \\
\hline & & Domestic & Import & Combined & \\
\hline 1978 & e & f & $\mathrm{f}$ & f & 19.9 \\
\hline 1980 & $\mathrm{e}$ & 16.8 & 24.3 & 18.5 & 23.1 \\
\hline 1985 & 19.5 & 19.6 & 26.5 & 20.7 & 25.4 \\
\hline 1990 & 20.0 & 20.3 & 23.0 & 20.8 & 25.4 \\
\hline 1991 & 20.2 & 20.9 & 23.0 & 21.3 & 25.6 \\
\hline 1992 & 20.2 & 20.5 & 22.7 & 20.8 & 25.1 \\
\hline 1993 & 20.4 & 20.7 & 22.8 & 21.0 & 25.2 \\
\hline 1994 & 20.5 & 20.5 & 22.1 & 20.8 & 24.7 \\
\hline 1995 & 20.6 & 20.3 & 21.5 & 20.5 & 24.9 \\
\hline 1996 & 20.7 & 20.5 & 22.2 & 20.8 & 24.9 \\
\hline 1997 & 20.7 & 20.1 & 22.1 & 20.6 & 24.6 \\
\hline 1998 & 20.7 & 20.5 & 23.0 & 21.0 & 24.7 \\
\hline 1999 & 20.7 & 20.4 & 22.5 & 20.9 & 24.5 \\
\hline 2000 & 20.7 & 21.1 & 19.7 & 21.3 & 24.8 \\
\hline 2001 & 20.7 & 20.6 & 21.8 & 20.9 & 24.5 \\
\hline 2002 & 20.7 & 20.6 & 21.9 & 21.4 & 24.7 \\
\hline 2003 & 20.7 & 21.8 & 22.4 & 21.8 & 25.1 \\
\hline 2004 & 20.7 & 20.7 & 22.3 & 21.5 & 24.6 \\
\hline 2005 & 21.0 & f & f & 22.1 & 25.4 \\
\hline 2006 & 21.6 & f & f & 22.5 & 25.8 \\
\hline 2007 & 22.2 & f & f & 23.1 & 26.6 \\
\hline 2008 & $22.4^{\mathrm{g}}$ & f & f & 23.6 & 27.1 \\
\hline 2009 & $23.0^{\mathrm{g}}$ & f & f & 24.8 & 29.0 \\
\hline 2010 & $23.4^{\mathrm{g}}$ & $\mathrm{f}$ & $\mathrm{f}$ & 25.2 & 29.3 \\
\hline 2011 & 24.3 & f & $\mathrm{f}$ & 24.7 & 29.0 \\
\hline 2012 & 25.3 & $\mathrm{f}$ & $\mathrm{f}$ & 25.0 & 30.8 \\
\hline 2013 & 25.9 & $\mathrm{f}$ & $\mathrm{f}$ & 25.7 & 31.6 \\
\hline 2014 & 26.3 & f & f & 26.5 & 31.7 \\
\hline 2015 & 27.6 & f & $\mathrm{f}$ & 27.3 & 32.2 \\
\hline 2016 & $28.8^{\mathrm{h}}$ & f & $\mathrm{f}$ & $27.2^{\mathrm{h}}$ & $32.1^{\mathrm{h}}$ \\
\hline 2017 & $29.3^{\mathrm{h}}$ & f & f & $28.0^{\mathrm{h}}$ & $31.8^{\mathrm{h}}$ \\
\hline
\end{tabular}

\section{Source:}

U.S. Department of Transportation, NHTSA, "Summary of Fuel Economy Performance," Washington, DC, December 2014 and updates 2017. (Additional resources: www.nhtsa.gov)

${ }^{a}$ Only vehicles with at least $75 \%$ domestic content can be counted in the average domestic fuel economy for a manufacturer.

${ }^{\mathrm{b}}$ Represents two- and four-wheel drive trucks combined. Gross vehicle weight of 0-6,000 pounds for model year 1978-1979 and 0-8,500 pounds for subsequent years.

c Model year as determined by the manufacturer on a vehicle by vehicle basis.

${ }^{\mathrm{d}}$ All CAFE calculations are sales-weighted.

e Standards were set for two-wheel drive and four-wheel drive light trucks, but no combined standard was set in this year.

${ }^{\mathrm{f}}$ Data are not available.

g Unreformed standards, which were an option from 2008-2010. See Table 4.20 for reformed standards.

${ }^{\text {h }}$ Projected required average fuel economy standards value based on pre-model year reports. 
Manufacturers of cars and light trucks whose vehicles do not meet the CAFE standards are fined. Data from the National Highway Traffic Safety Administration show the CAFE fine in the year in which the money was collected, which may not be the same year in which it was assessed. A manufacturer can also use CAFE credits to offset fines.

Fines for recent model years are still being collected.

Table 4.24

Corporate Average Fuel Economy (CAFE) Fines Collected, 1983-2014 ${ }^{\mathrm{a}}$

\begin{tabular}{|c|c|c|}
\hline Model year & $\begin{array}{l}\text { Current } \\
\text { dollars }\end{array}$ & $\begin{array}{l}2014 \text { constant } \\
\text { dollars }^{\mathrm{b}}\end{array}$ \\
\hline 1983 & $\$ 57,970$ & $\$ 137,795$ \\
\hline 1984 & $\$ 5,958,020$ & $\$ 13,572,370$ \\
\hline 1985 & $\$ 15,564,540$ & $\$ 34,241,988$ \\
\hline 1986 & $\$ 29,871,815$ & $\$ 64,523,120$ \\
\hline 1987 & $\$ 31,260,530$ & $\$ 65,146,945$ \\
\hline 1988 & $\$ 43,470,545$ & $\$ 86,984,561$ \\
\hline 1989 & $\$ 48,549,420$ & $\$ 92,680,843$ \\
\hline 1990 & $\$ 48,308,615$ & $\$ 87,486,902$ \\
\hline 1991 & $\$ 42,243,030$ & $\$ 73,418,386$ \\
\hline 1992 & $\$ 38,286,565$ & $\$ 64,589,435$ \\
\hline 1993 & $\$ 28,688,380$ & $\$ 46,991,566$ \\
\hline 1994 & $\$ 31,498,570$ & $\$ 50,303,216$ \\
\hline 1995 & $\$ 40,787,498$ & $\$ 63,342,984$ \\
\hline 1996 & $\$ 19,301,930$ & $\$ 29,126,612$ \\
\hline 1997 & $\$ 36,211,850$ & $\$ 53,412,479$ \\
\hline 1998 & $\$ 21,739,774$ & $\$ 31,566,151$ \\
\hline 1999 & $\$ 27,516,451$ & $\$ 39,100,876$ \\
\hline 2000 & $\$ 51,067,038$ & $\$ 70,217,177$ \\
\hline 2001 & $\$ 35,507,412$ & $\$ 47,473,409$ \\
\hline 2002 & $\$ 20,041,533$ & $\$ 26,374,657$ \\
\hline 2003 & $\$ 15,225,419$ & $\$ 19,595,114$ \\
\hline 2004 & $\$ 30,411,986$ & $\$ 38,106,218$ \\
\hline 2005 & $\$ 25,057,126$ & $\$ 30,369,236$ \\
\hline 2006 & $\$ 40,933,954$ & $\$ 48,056,461$ \\
\hline 2007 & $\$ 37,385,941$ & $\$ 42,694,745$ \\
\hline 2008 & $\$ 11,619,696$ & $\$ 12,781,666$ \\
\hline 2009 & $\$ 9,148,425$ & $\$ 10,090,713$ \\
\hline 2010 & $\$ 23,803,412$ & $\$ 25,850,505$ \\
\hline 2011 & $\$ 40,013,270$ & $\$ 42,093,960$ \\
\hline 2012 & $\$ 14,962,382$ & $\$ 15,426,216$ \\
\hline 2013 & $\$ 19,036,963$ & $\$ 19,341,554$ \\
\hline 2014 & $\$ 2,289,788$ & $\$ 2,289,788$ \\
\hline
\end{tabular}

\section{Source:}

U.S. Department of Transportation, National Highway Traffic Safety Administration, Office of Vehicle Safety Compliance, Washington, DC, December 2014 and updates, 2017. Data accessed July 18, 2017. (Additional resources: www.nhtsa.gov)

${ }^{a}$ These are fines which are actually collected. Fines which are assessed in certain year may not have been collected in that year.

${ }^{\mathrm{b}}$ Adjusted using the Consumer Price Inflation Index. 
Consumers must pay the Gas Guzzler Tax when purchasing a car that has an Environmental Protection Agency (EPA) fuel economy rating (combined city and highway) less than that stipulated in the table below. The Gas Guzzler Tax doubled in 1991 after remaining constant from 1986 to 1990. The tax has not changed since 1991.

This tax does not apply to light trucks such as pickups, minivans, sport utility vehicles, and vans.

Table 4.25

The Gas Guzzler Tax on New Cars (dollars per vehicle)

\begin{tabular}{crrrrrrrr}
\hline $\begin{array}{c}\text { Vehicle fuel } \\
\text { economy (mpg) }\end{array}$ & 1980 & 1981 & 1982 & 1983 & 1984 & 1985 & $1986-90$ & 1991 - on \\
\hline Over 22.5 & 0 & 0 & 0 & 0 & 0 & 0 & 0 & 0 \\
$22.0-22.5$ & 0 & 0 & 0 & 0 & 0 & 0 & 500 & 1,000 \\
$21.5-22.0$ & 0 & 0 & 0 & 0 & 0 & 0 & 500 & 1,000 \\
$21.0-21.5$ & 0 & 0 & 0 & 0 & 0 & 0 & 650 & 1,300 \\
$20.5-21.0$ & 0 & 0 & 0 & 0 & 0 & 500 & 650 & 1,300 \\
$20.0-20.5$ & 0 & 0 & 0 & 0 & 0 & 500 & 850 & 1,700 \\
$19.5-20.0$ & 0 & 0 & 0 & 0 & 0 & 600 & 850 & 1,700 \\
$19.0-19.5$ & 0 & 0 & 0 & 0 & 450 & 600 & 1,050 & 2,100 \\
$18.5-19.0$ & 0 & 0 & 0 & 350 & 450 & 800 & 1,050 & 2,100 \\
$18.0-18.5$ & 0 & 0 & 200 & 350 & 600 & 800 & 1,300 & 2,600 \\
$17.5-18.0$ & 0 & 0 & 200 & 500 & 600 & 1,000 & 1,300 & 2,600 \\
$17.0-17.5$ & 0 & 0 & 350 & 500 & 750 & 1,000 & 1,500 & 3,000 \\
$16.5-17.0$ & 0 & 200 & 350 & 650 & 750 & 1,200 & 1,500 & 3,000 \\
$16.0-16.5$ & 0 & 200 & 450 & 650 & 950 & 1,200 & 1,850 & 3,700 \\
$15.5-16.0$ & 0 & 350 & 450 & 800 & 950 & 1,500 & 1,850 & 3,700 \\
$15.0-15.5$ & 0 & 350 & 600 & 800 & 1,150 & 1,500 & 2,250 & 4,500 \\
$14.5-15.0$ & 200 & 450 & 600 & 1,000 & 1,150 & 1,800 & 2,250 & 4,500 \\
$14.0-14.5$ & 200 & 450 & 750 & 1,000 & 1,450 & 1,800 & 2,700 & 5,400 \\
$13.5-14.0$ & 300 & 550 & 750 & 1,250 & 1,450 & 2,200 & 2,700 & 5,400 \\
$13.0-13.5$ & 300 & 550 & 950 & 1,250 & 1,750 & 2,200 & 3,200 & 6,400 \\
$12.5-13.0$ & 550 & 650 & 950 & 1,550 & 1,750 & 2,650 & 3,200 & 6,400 \\
Under 12.5 & 550 & 650 & 1,200 & 1,550 & 2,150 & 2,650 & 3,850 & 7,700 \\
\hline
\end{tabular}

Source:

Internal Revenue Service, Form 6197, (Rev. 10-05), "Gas Guzzler Tax." (Additional resources: www.irs.ustreas.gov) 
Table 4.26

List of Model Year 2016 Cars with Gas Guzzler Taxes ${ }^{a}$

\begin{tabular}{|c|c|c|c|c|}
\hline Manufacturer & Model(s) & Size class & $\begin{array}{l}\text { Unadjusted } \\
\text { combined } \\
\text { city/highway } \\
\text { fuel } \\
\text { economy }\end{array}$ & $\begin{array}{c}\text { Adjusted } \\
\text { combined } \\
\text { city/highway } \\
\text { fuel } \\
\text { economy }\end{array}$ \\
\hline Aston Martin & DB9 & Minicompact Cars & 19 & 15 \\
\hline Aston Martin & Rapide S & Subcompact Cars & 21 & 17 \\
\hline Aston Martin & V12 Vantage S & Two Seaters & 18 & 14 \\
\hline Aston Martin & V8 Vantage & Two Seaters & 19 & 15 \\
\hline Aston Martin & V8 Vantage & Two Seaters & 21 & 16 \\
\hline Aston Martin & V8 Vantage S & Two Seaters & 19 & 15 \\
\hline Aston Martin & V8 Vantage S & Two Seaters & 21 & 16 \\
\hline Aston Martin & Vanquish & Minicompact Cars & 20 & 16 \\
\hline Aston Martin & Vantage GT & Two Seaters & 19 & 15 \\
\hline Aston Martin & Vantage GT & Two Seaters & 21 & 16 \\
\hline Audi & A8L & Large Cars & 21 & 17 \\
\hline Bentley & Continental GT & Compact Cars & 19 & 15 \\
\hline Bentley & Continental GT Convertible & Subcompact Cars & 18 & 15 \\
\hline Bentley & Continental GT Convertible & Subcompact Cars & 22 & 17 \\
\hline Bentley & Flying Spur & Midsize Cars & 18 & 15 \\
\hline Bentley & Flying Spur & Midsize Cars & 22 & 17 \\
\hline Bentley & Mulsanne & Midsize Cars & 17 & 13 \\
\hline BMW & M5 & Midsize Cars & 21 & 16 \\
\hline BMW & M5 & Midsize Cars & 22 & 17 \\
\hline BMW & M6 Convertible & Subcompact Cars & 21 & 16 \\
\hline BMW & M6 Convertible & Subcompact Cars & 22 & 17 \\
\hline BMW & M6 Coupe & Subcompact Cars & 21 & 16 \\
\hline BMW & M6 Coupe & Subcompact Cars & 22 & 17 \\
\hline BMW & M6 Gran Coupe & Compact Cars & 21 & 16 \\
\hline BMW & M6 Gran Coupe & Compact Cars & 22 & 17 \\
\hline FCA & Challenger & Midsize Cars & 22 & 17 \\
\hline FCA & Challenger SRT8 & Midsize Cars & 20 & 16 \\
\hline FCA & Challenger SRT8 & Midsize Cars & 22 & 17 \\
\hline FCA & Charger SRT8 & Large Cars & 20 & 16 \\
\hline FCA & Viper SRT & Two Seaters & 19 & 15 \\
\hline Ferrari & F12 & Two Seaters & 17 & 13 \\
\hline Ferrari & $\mathrm{F} 12 \mathrm{tdf}$ & Two Seaters & 16 & 12 \\
\hline Ferrari & $\mathrm{F} 12 \mathrm{tdf}$ & Two Seaters & 17 & 13 \\
\hline Ferrari & F60 America & Two Seaters & 16 & 13 \\
\hline Ferrari & F60 America & Two Seaters & 17 & 13 \\
\hline Ferrari & FF & Midsize Cars & 16 & 13 \\
\hline Ferrari & FF & Midsize Cars & 17 & 13 \\
\hline Ford & Shelby GT350 Mustang & Subcompact Cars & 21 & 16 \\
\hline GM & Corvette & Two Seaters & 20 & 16 \\
\hline GM & CTS-V & Midsize Cars & 21 & 17 \\
\hline GM & SS & Large Cars & 20 & 16 \\
\hline GM & SS & Large Cars & 21 & 17 \\
\hline GM & XTS Limo & Special Purpose Vehicle 2WD & 22 & 17 \\
\hline
\end{tabular}


Table 4.26 (Continued)

List of Model Year 2016 Cars with Gas Guzzler Taxes ${ }^{a}$

\begin{tabular}{|c|c|c|c|c|}
\hline Make & Model(s) & Size class & $\begin{array}{c}\text { Unadjusted } \\
\text { combined } \\
\text { city/highway } \\
\text { fuel } \\
\text { economy } \\
\end{array}$ & $\begin{array}{c}\text { Adjusted } \\
\text { combined } \\
\text { city/highway } \\
\text { fuel } \\
\text { economy } \\
\end{array}$ \\
\hline Lamborghini & Aventador Coupe & Two Seaters & 16 & 13 \\
\hline Lamborghini & Aventador Roadster & Two Seaters & 15 & 12 \\
\hline Lamborghini & Huracan & Two Seaters & 21 & 17 \\
\hline Lamborghini & Huracan Spyder & Two Seaters & 20 & 16 \\
\hline Maserati & Granturismo & Subcompact Cars & 20 & 16 \\
\hline Maserati & Granturismo Convertible & Subcompact Cars & 20 & 15 \\
\hline Maserati & Quattroporte GTS & Large Cars & 22 & 17 \\
\hline Mercedes-Benz & AMG S 65 & Large Cars & 19 & 15 \\
\hline Mercedes-Benz & AMG S 65 (coupe) & Compact Cars & 19 & 15 \\
\hline Mercedes-Benz & AMG SL 65 & Two Seaters & 21 & 17 \\
\hline Mercedes-Benz & Maybach S 600 & Large Cars & 20 & 15 \\
\hline Mercedes-Benz & S 600 & Large Cars & 21 & 16 \\
\hline Pagani & Нuayra Coupe & Two Seaters & 15 & 13 \\
\hline Porsche & 911 GT3 & Two Seaters & 22 & 17 \\
\hline Porsche & 911 GT3RS & Two Seaters & 21 & 16 \\
\hline Porsche & $911 \mathrm{R}$ & Minicompact Cars & 21 & 16 \\
\hline Rolls-Royce & Dawn & Compact Cars & 18 & 14 \\
\hline Rolls-Royce & Ghost & Large Cars & 19 & 15 \\
\hline Rolls-Royce & Ghost EWB & Large Cars & 19 & 15 \\
\hline Rolls-Royce & Phantom & Large Cars & 17 & 14 \\
\hline Rolls-Royce & Phantom Coupe & Compact Cars & 17 & 14 \\
\hline Rolls-Royce & Phantom Drophead Coupe & Compact Cars & 17 & 14 \\
\hline Rolls-Royce & Phantom EWB & Large Cars & 17 & 14 \\
\hline Rolls-Royce & Wraith & Midsize Cars & 19 & 15 \\
\hline Roush & Roush Stage 3 Mustang & Subcompact Cars & 20 & 16 \\
\hline Roush & Roush Stage 3 Mustang & Subcompact Cars & 21 & 16 \\
\hline
\end{tabular}

Source:

U.S. Department of Energy and U.S. Environmental Protection Agency, Fuel Economy Guide database, www.fueleconomy.gov stickers.

a Tax is based on unadjusted combined fuel economy; adjusted combined fuel economy is used on window 
The IRS collected \$58.7 million in 2015 from those buying cars with combined city/highway fuel economy less than 22.5 miles per gallon. This tax does not apply to light trucks such as pickups, minivans, sport utility vehicles, and vans. It is worthy to note that total revenue from fines paid by consumers to purchase gas-guzzling vehicles greatly exceeds the overall fines paid by manufacturers whose vehicles fail to meet CAFE standards (see Table 4.22).

Table 4.27

Tax Receipts from the Sale of Gas Guzzlers, 1980-2015

(thousands)

\begin{tabular}{|c|c|c|}
\hline Model year & Current dollars & $\begin{array}{c}2015 \\
\text { constant dollars }^{\mathrm{a}}\end{array}$ \\
\hline 1980 & 740 & 2,129 \\
\hline 1981 & 780 & 2,034 \\
\hline 1982 & 1,720 & 4,225 \\
\hline 1983 & 4,020 & 9,566 \\
\hline 1984 & 8,820 & 20,120 \\
\hline 1985 & 39,790 & 87,648 \\
\hline 1986 & 147,660 & 319,324 \\
\hline 1987 & 145,900 & 304,408 \\
\hline 1988 & 116,780 & 233,972 \\
\hline 1989 & 109,640 & 209,569 \\
\hline 1990 & 103,200 & 187,147 \\
\hline 1991 & 118,400 & 206,041 \\
\hline 1992 & 144,200 & 243,605 \\
\hline 1993 & 111,600 & 183,053 \\
\hline 1994 & 64,100 & 102,515 \\
\hline 1995 & 73,500 & 114,309 \\
\hline 1996 & 52,600 & 79,459 \\
\hline 1997 & 48,200 & 71,179 \\
\hline 1998 & 47,700 & 69,360 \\
\hline 1999 & 68,300 & 97,168 \\
\hline 2000 & 70,800 & 97,449 \\
\hline 2001 & 78,200 & 104,657 \\
\hline 2002 & 79,700 & 105,004 \\
\hline 2003 & 126,700 & 163,207 \\
\hline 2004 & 140,800 & 176,665 \\
\hline 2005 & 163,800 & 198,788 \\
\hline 2006 & 201,700 & 237,135 \\
\hline 2007 & 178,700 & 204,276 \\
\hline 2008 & 172,428 & 189,818 \\
\hline 2009 & 99,300 & 109,705 \\
\hline 2010 & 85,226 & 92,637 \\
\hline 2011 & 68,905 & 72,605 \\
\hline 2012 & 73,500 & 75,876 \\
\hline 2013 & 61,300 & 62,368 \\
\hline 2014 & 48,200 & 48,257 \\
\hline 2015 & 58,700 & 58,700 \\
\hline
\end{tabular}

\section{Source:}

Ward's Communications, Detroit, MI, 2017. Original data source: Internal Revenue Service. (Additional resources: www.epa.gov/fueleconomy/guzzler)

${ }^{a}$ Adjusted using the Consumer Price Inflation Index. 
Autonomie is a system simulation tool for vehicle energy consumption and performance analysis. It is used to evaluate the energy consumption and cost of multiple advanced powertrain technologies. Autonomie was used to develop data on the relationship between steady-state vehicle speed and fuel economy.

Table 4.28

Fuel Economy by Speed, Autonomie Model Results

\begin{tabular}{|c|c|c|c|c|c|c|c|}
\hline \multirow[b]{2}{*}{ Speed (mph) } & \multicolumn{3}{|c|}{ Gasoline conventional } & \multicolumn{3}{|c|}{ Diesel conventional } & \multirow{2}{*}{$\begin{array}{c}\text { Hybrid } \\
\text { vehicle } \\
\text { Midsize } \\
\text { Car }\end{array}$} \\
\hline & $\begin{array}{c}\text { Midsize } \\
\text { car }\end{array}$ & $\begin{array}{l}\text { Small } \\
\text { SUV }\end{array}$ & $\begin{array}{l}\text { Large } \\
\text { SUV }\end{array}$ & $\begin{array}{c}\text { Midsize } \\
\text { car }\end{array}$ & $\begin{array}{l}\text { Small } \\
\text { SUV }\end{array}$ & $\begin{array}{l}\text { Large } \\
\text { SUV }\end{array}$ & \\
\hline \multicolumn{8}{|c|}{ (miles per gallon) } \\
\hline 45 & 43 & 37 & 35 & 57 & 48 & 48 & 55 \\
\hline 55 & 45 & 36 & 31 & 55 & 45 & 40 & 46 \\
\hline 65 & 38 & 30 & 29 & 45 & 36 & 35 & 38 \\
\hline 75 & 32 & 26 & 25 & 37 & 30 & 29 & 33 \\
\hline \multicolumn{8}{|c|}{ Fuel economy loss } \\
\hline $55-65 \mathrm{mph}$ & $15 \%$ & $16 \%$ & $7 \%$ & $18 \%$ & $19 \%$ & $13 \%$ & $18 \%$ \\
\hline 65 - $75 \mathrm{mph}$ & $15 \%$ & $16 \%$ & $15 \%$ & $18 \%$ & $18 \%$ & $17 \%$ & $12 \%$ \\
\hline $55-75 \mathrm{mph}$ & $28 \%$ & $29 \%$ & $21 \%$ & $33 \%$ & $34 \%$ & $27 \%$ & $28 \%$ \\
\hline
\end{tabular}

Source:

Argonne National Laboratory, Autonomie model, August 2016, www.autonomie.net. (Additional resources: www.anl.gov/energy/transportation) 
The latest study of vehicle fuel economy by speed indicated higher fuel economy around 40 miles per hour, as did the 1973 and 1984 studies. Engineers at Oak Ridge National Laboratory believe that the lowest speed in the vehicle's highest gear is where the best fuel economy is typically obtained. That speed will be different for individual vehicles.

Table 4.29

Fuel Economy by Speed, 1973, 1984, 1997, and 2012 Studies (miles per gallon)

\begin{tabular}{ccccc}
\hline $\begin{array}{c}\text { Speed } \\
\text { (miles per hour) }\end{array}$ & $\begin{array}{c}1973^{\mathrm{a}} \\
(13 \text { vehicles })\end{array}$ & $\begin{array}{c}1984^{\mathrm{b}} \\
(15 \text { vehicles })\end{array}$ & $\begin{array}{c}1997^{\mathrm{c}} \\
(9 \text { vehicles })\end{array}$ & $\begin{array}{c}2012^{\mathrm{d}} \\
(74 \text { vehicles })\end{array}$ \\
\hline 15 & $\mathrm{e}$ & 21.1 & 24.4 & $\mathrm{e}$ \\
20 & $\mathrm{e}$ & 25.5 & 27.9 & $\mathrm{e}$ \\
25 & $\mathrm{e}$ & 30.0 & 30.5 & $\mathrm{e}$ \\
30 & 21.1 & 31.8 & 31.7 & $\mathrm{e}$ \\
35 & 21.1 & 33.6 & 31.2 & $\mathrm{e}$ \\
40 & 21.1 & 33.6 & 31.0 & 33.2 \\
45 & 20.3 & 33.5 & 31.6 & $\mathrm{e}$ \\
50 & 19.5 & 31.9 & 32.4 & 31.9 \\
55 & 18.5 & 30.3 & 32.4 & $\mathrm{e}$ \\
60 & 17.5 & 27.6 & 31.4 & 27.9 \\
65 & 16.2 & 24.9 & 29.2 & $\mathrm{e}$ \\
70 & 14.9 & 22.5 & 26.8 & 24.1 \\
75 & $\mathrm{e}$ & 20.0 & 24.8 & $\mathrm{e}$ \\
80 & $\mathrm{e}$ & $\mathrm{e}$ & $\mathrm{e}$ & 20.5 \\
& & Fuel economy loss & \\
$50-60 \mathrm{mph}$ & $10.3 \%$ & $13.5 \%$ & $3.1 \%$ & $12.5 \%$ \\
$60-70 \mathrm{mph}$ & $14.9 \%$ & $18.5 \%$ & $14.6 \%$ & $13.6 \%$ \\
$50-70 \mathrm{mph}$ & $23.6 \%$ & $29.5 \%$ & $17.3 \%$ & $24.5 \%$ \\
\hline
\end{tabular}

\section{Sources:}

1973- U.S. Department of Transportation, Federal Highway Administration, Office of Highway Planning, The Effect of Speed on Automobile Gasoline Consumption Rates, Washington, DC, October 1973.

1984 - U.S. Department of Transportation, Federal Highway Administration, Fuel Consumption and Emission Values for Traffic Models, Washington, DC, May 1985.

1997 - West, B.H., R.N. McGill, J.W. Hodgson, S.S. Sluder, and D.E. Smith, Development and Verification of LightDuty Modal Emissions and Fuel Consumption Values for Traffic Models, FHWA-RD-99-068, U.S. Department of Transportation, Federal Highway Administration, Washington, DC, March 1999.

2012 - U.S. Department of Energy and U.S. Environmental Protection Agency, Fuel Economy Guide website: www.fueleconomy.gov. The Green Car Congress, "ORNL researchers quantify the effect of increasing highway speed on fuel economy." February 8, 2013.

${ }^{a}$ Model years 1970 and earlier cars.

${ }^{\mathrm{b}}$ Model years 1981-84 cars and light trucks.

${ }^{\text {c }}$ Model years 1988-97 cars and light trucks.

${ }^{\mathrm{d}}$ Model years 2003-2012 cars and light trucks.

${ }^{\text {e }}$ Data are not available. 
Figure 4.2. Fuel Economy by Speed, 1973, 1984, 1997, and 2012 Studies and Autonomie Model Results

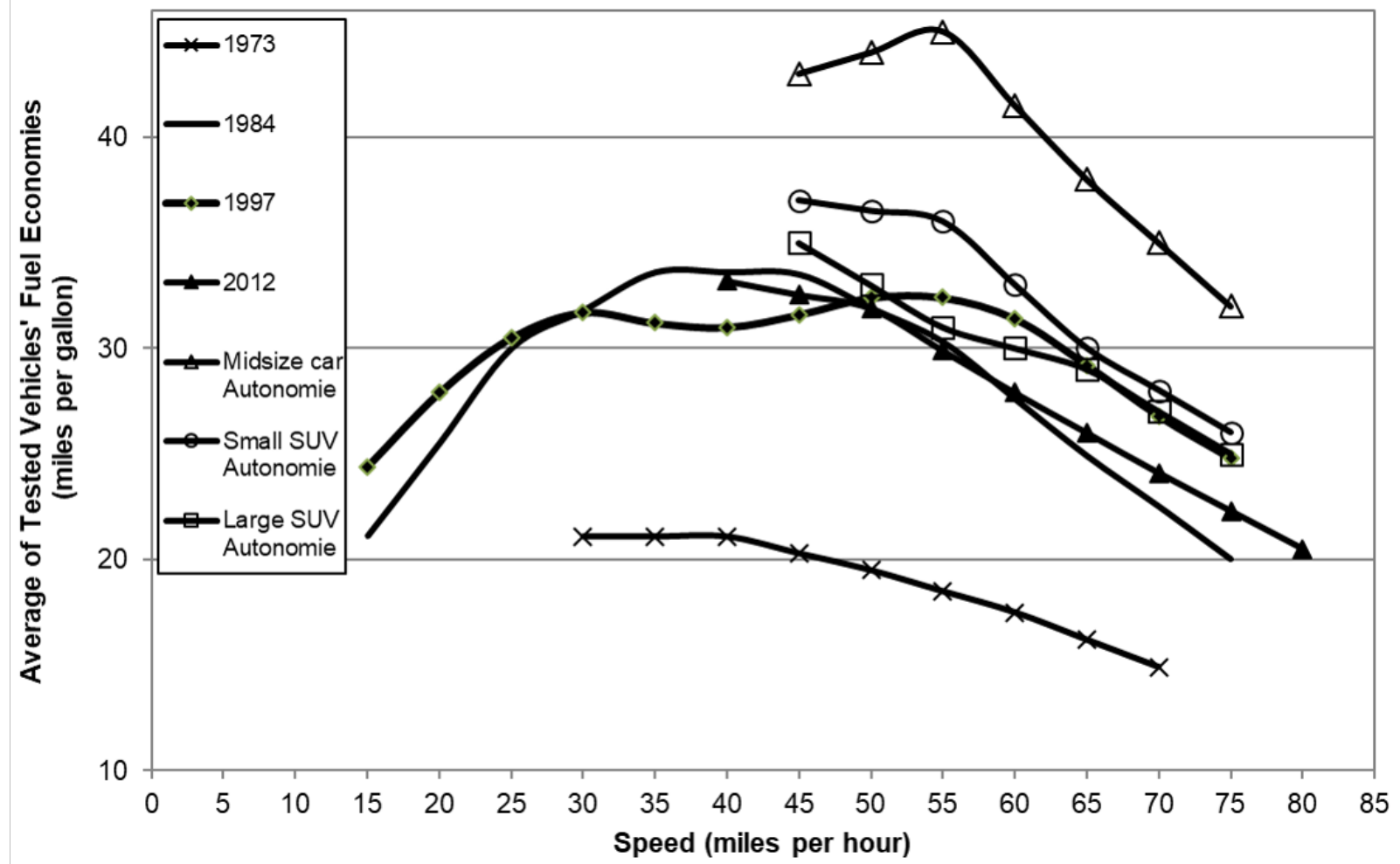

Sources:

See Tables 4.28 and 4.29. 
This table shows the driving cycles for the new methodology that the Environmental Protection Agency (EPA) used to determine fuel economy ratings for new vehicles beginning in model year 2008. In addition to the Urban Driving Cycle and the Highway Driving cycle, the EPA will also use three additional tests to adjust fuel economy ratings to account for higher speeds, air conditioner use, and colder temperatures. Though the EPA uses a complex combination of these five cycles to determine the fuel economy that will be posted on a new vehicle window sticker, the manufacturer's Corporate Average Fuel Economy is still calculated using only the city and highway driving cycles. To know more about new vehicle fuel economy ratings, visit www.fueleconomy.gov.

\section{Table 4.30}

Driving Cycle Attributes

\begin{tabular}{|c|c|c|c|c|c|}
\hline & \multicolumn{5}{|c|}{ Test schedule } \\
\hline & City & Highway & High speed & $\begin{array}{l}\text { Air conditioner } \\
\text { (AC) }\end{array}$ & Cold temp \\
\hline Trip type & $\begin{array}{l}\text { Low speeds in } \\
\text { stop-and-go } \\
\text { urban traffic } \\
\end{array}$ & $\begin{array}{c}\text { Free-flow traffic } \\
\text { at highway } \\
\text { speeds } \\
\end{array}$ & $\begin{array}{c}\text { Higher speeds; } \\
\text { harder acceleration } \\
\text { \& braking } \\
\end{array}$ & $\begin{array}{c}\text { AC use under hot } \\
\text { ambient } \\
\text { conditions } \\
\end{array}$ & $\begin{array}{c}\text { City test } \\
\text { w/colder outside } \\
\text { temperature } \\
\end{array}$ \\
\hline Top speed & $56 \mathrm{mph}$ & $60 \mathrm{mph}$ & $80 \mathrm{mph}$ & $54.8 \mathrm{mph}$ & $56 \mathrm{mph}$ \\
\hline Average speed & $21 \mathrm{mph}$ & $48 \mathrm{mph}$ & $48 \mathrm{mph}$ & $21 \mathrm{mph}$ & $21 \mathrm{mph}$ \\
\hline Max. acceleration & $3.3 \mathrm{mph} / \mathrm{sec}$ & $3.2 \mathrm{mph} / \mathrm{sec}$ & $8.46 \mathrm{mph} / \mathrm{sec}$ & $5.1 \mathrm{mph} / \mathrm{sec}$ & $3.3 \mathrm{mph} / \mathrm{sec}$ \\
\hline Simulated distance & $11 \mathrm{mi}$. & $10.3 \mathrm{mi}$. & $8 \mathrm{mi}$. & $3.6 \mathrm{mi}$. & $11 \mathrm{mi}$. \\
\hline Time & $31.2 \mathrm{~min}$. & $12.6 \mathrm{~min}$. & $9.9 \mathrm{~min}$. & $9.9 \mathrm{~min}$. & $31.2 \mathrm{~min}$. \\
\hline Stops & 23 & None & 4 & 5 & 23 \\
\hline Idling time & $18 \%$ of time & None & $7 \%$ of time & $19 \%$ of time & $18 \%$ of time \\
\hline Engine startup ${ }^{\mathrm{a}}$ & Cold & Warm & Warm & Warm & Cold \\
\hline Lab temperature & $68-86^{\circ} \mathrm{F}$ & $68-86^{\circ} \mathrm{F}$ & $68-86^{\circ} \mathrm{F}$ & $95^{\circ} \mathrm{F}$ & $20^{\circ} \mathrm{F}$ \\
\hline Vehicle air conditioning & Off & Off & Off & On & Off \\
\hline
\end{tabular}

Source:

U.S. Department of Energy and U.S. Environmental Protection Agency, Fuel Economy website, www.fueleconomy.gov.

a A vehicle’s engine doesn't reach maximum fuel efficiency until it is warm. 
These driving cycles simulate the performance of an engine while driving in the city and on the highway. Once the city cycle is completed, the engine is stopped, and then started again for the 8.5-minute hot start cycle. Three additional cycles also influence new vehicle fuel economy ratings beginning with the 2008 model year.

Figure 4.3. City Driving Cycle

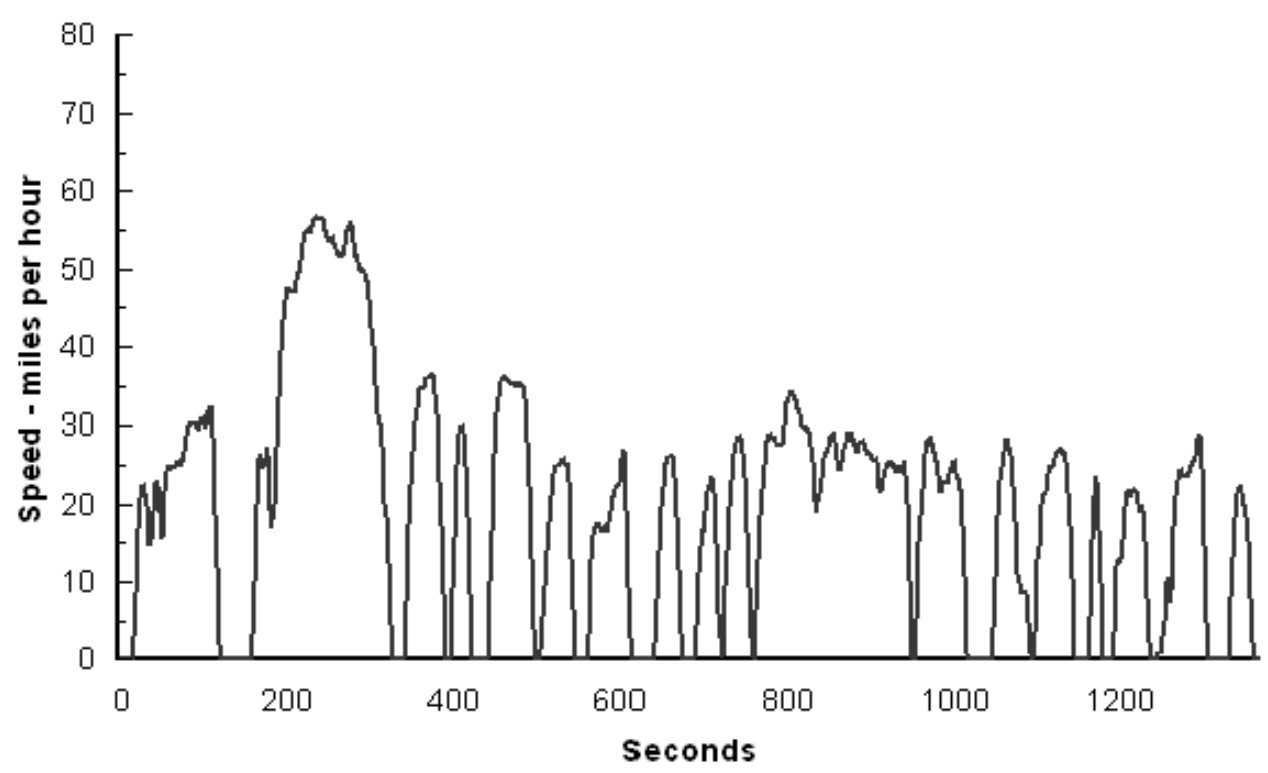

Figure 4.4. Highway Driving Cycle

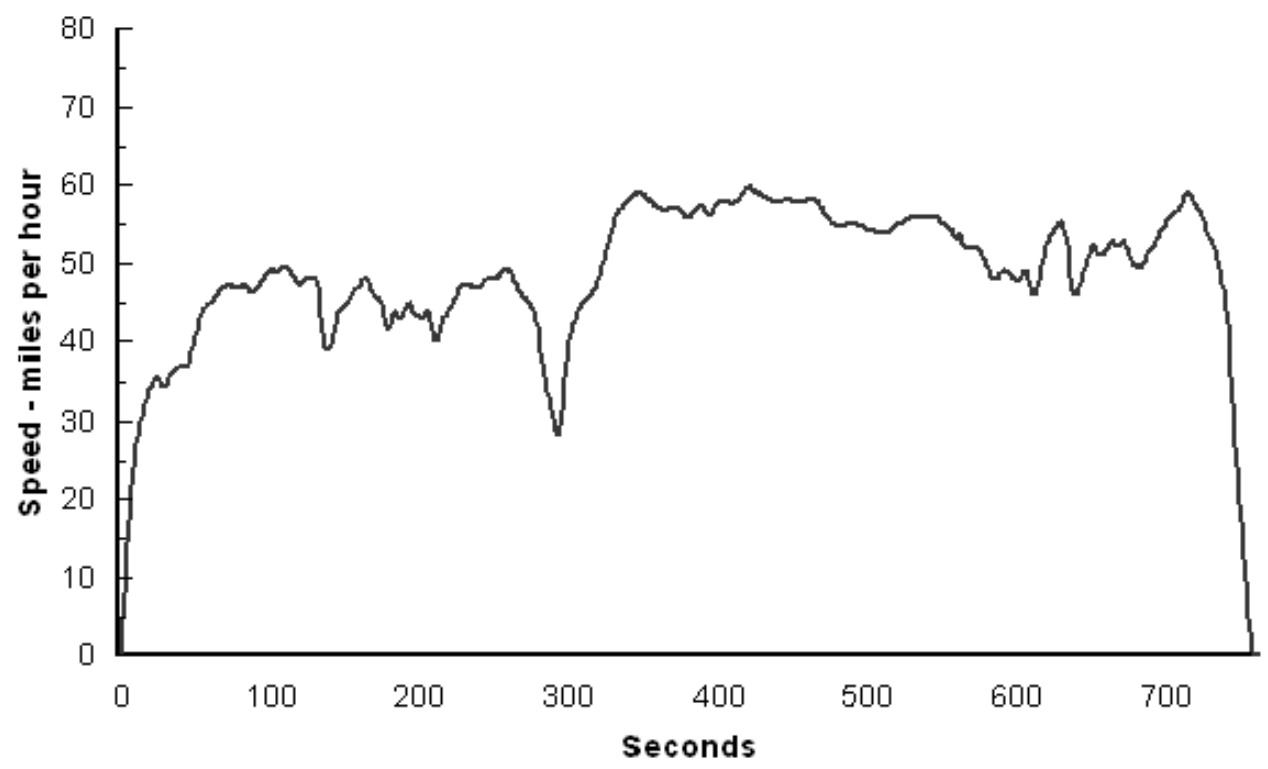

\section{Source:}

Code of Federal Regulations, 40CFR, "Subpart B - Fuel Economy Regulations for 1978 and Later Model Year Automobiles - Test Procedures," July 1, 1988 edition, p. 676. 
Figure 4.5. Air Conditioning (SC03) Driving Cycle

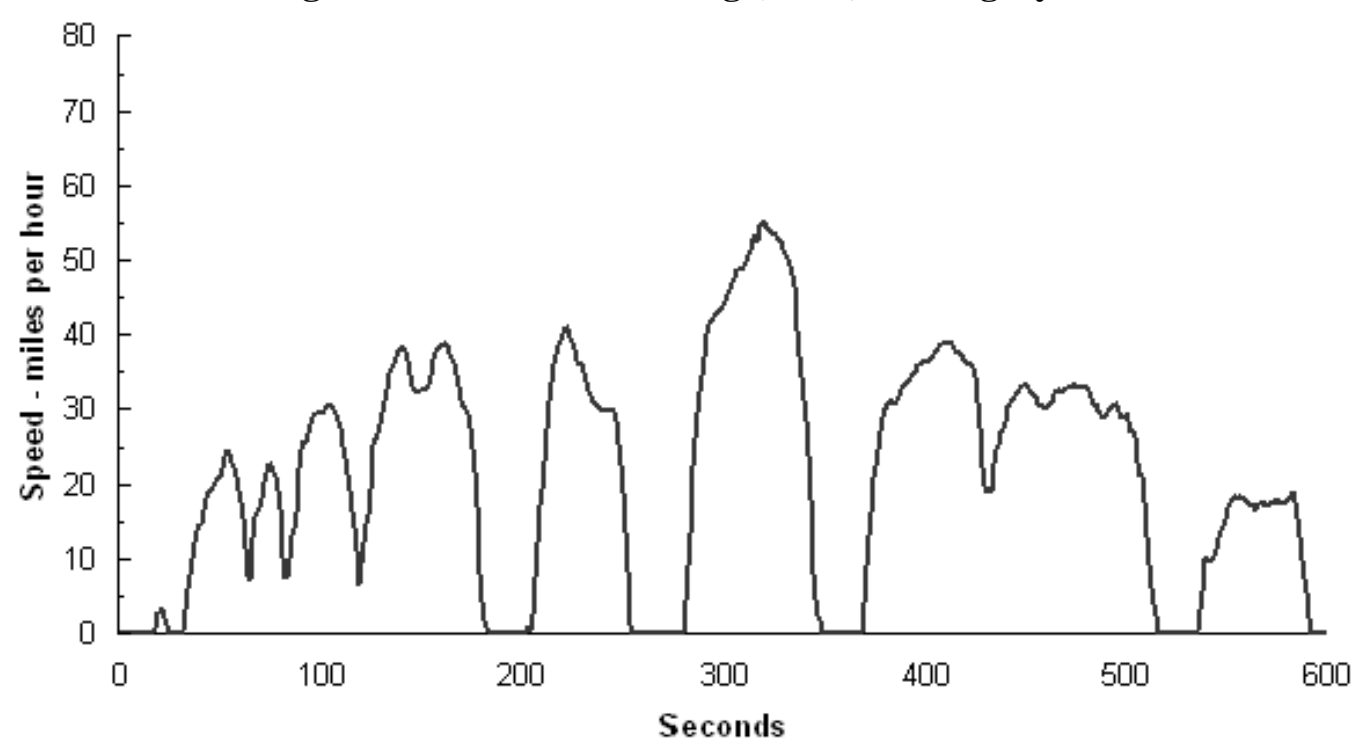

Source:

U.S. Department of Energy and Environmental Protection Agency, Fuel Economy website, www.fueleconomy.gov.

Figure 4.6. Cold Temperature (Cold FTP) Driving Cycle

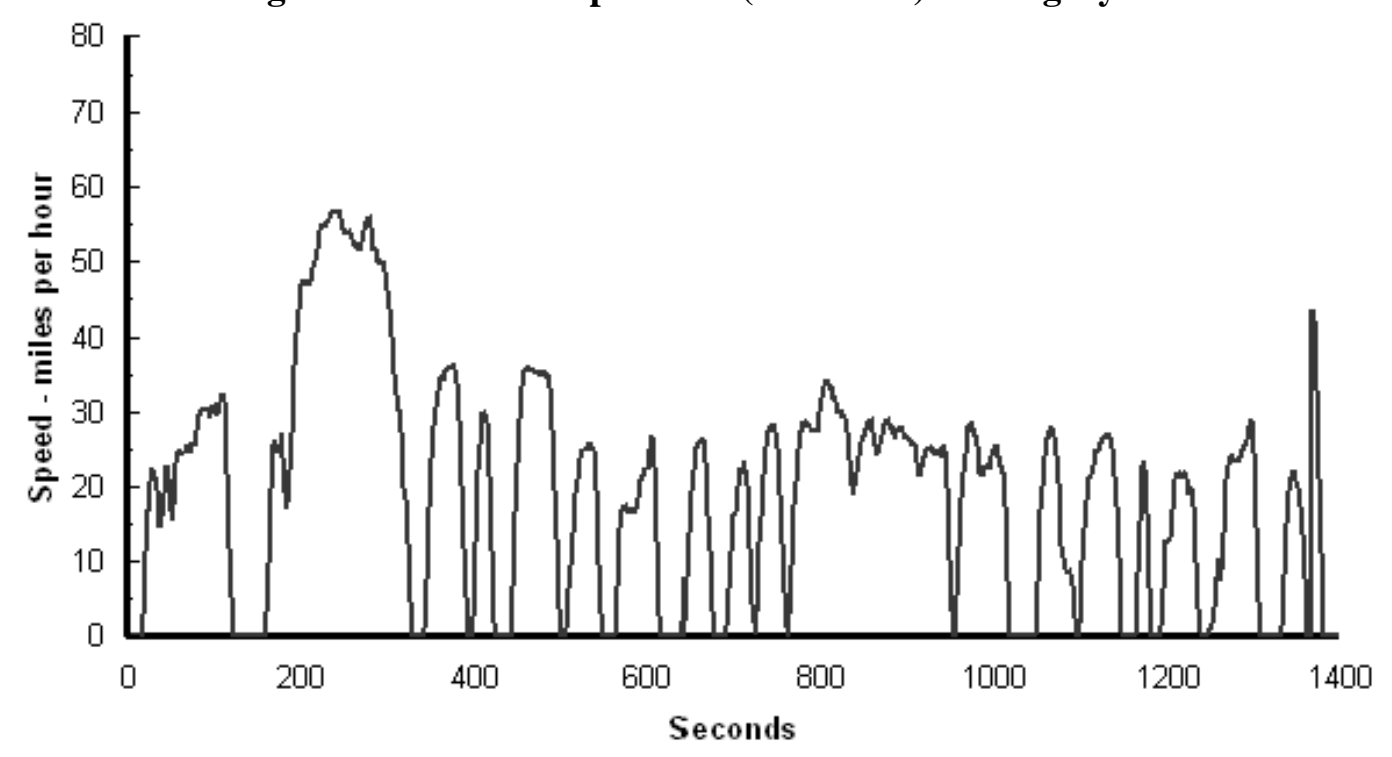

Source:

U.S. Department of Energy and Environmental Protection Agency, Fuel Economy website, www.fueleconomy.gov. 
Beginning with the 2008 model year, this cycle influences the new vehicle fuel economy ratings. The US06 driving cycle was originally developed as a supplement to the Federal Test Procedure. It is a short-duration cycle (600 seconds) which represents hard-acceleration driving.

Figure 4.7. High-Speed (US06) Driving Cycle

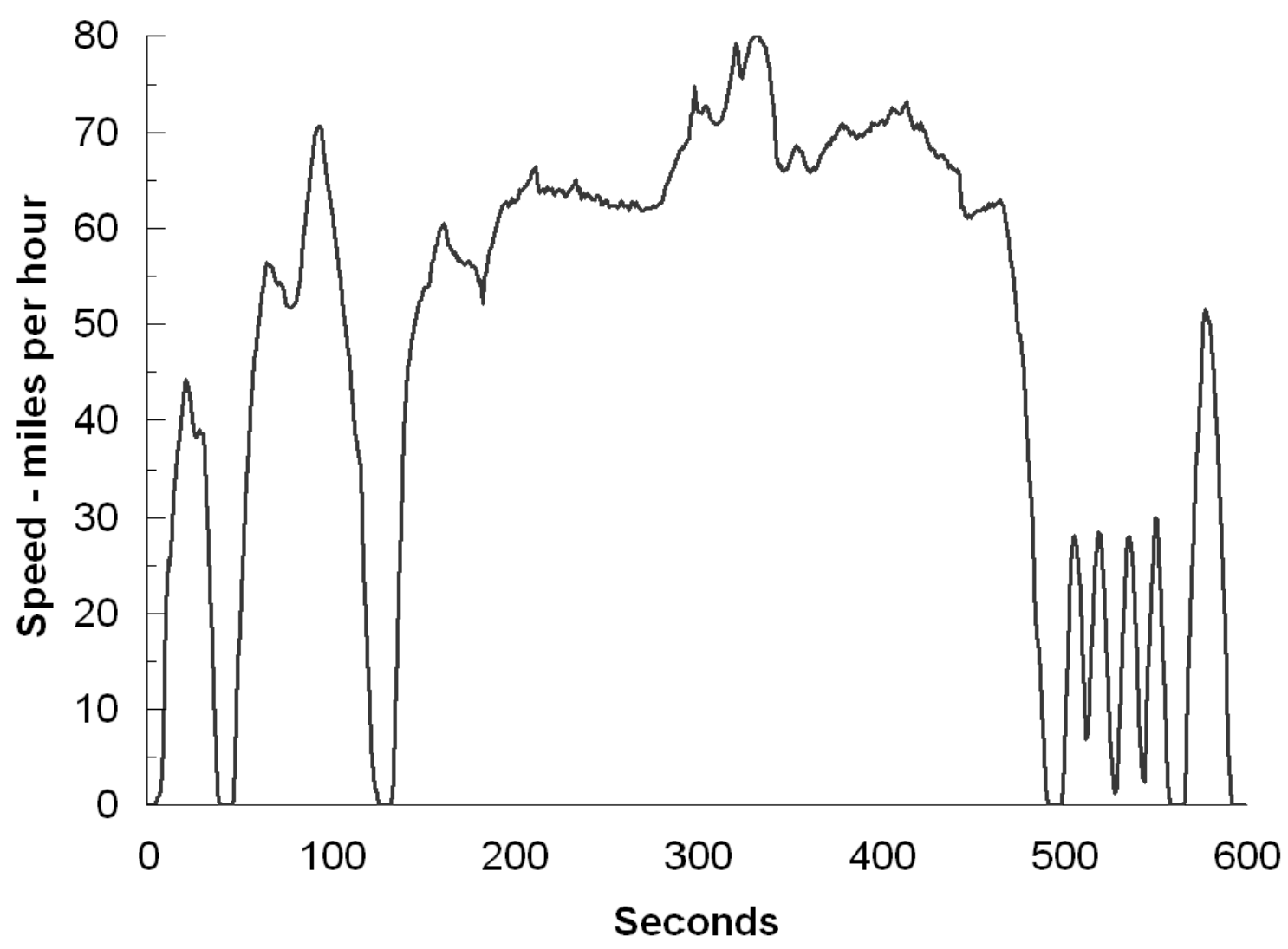

Source:

U.S. Department of Energy and Environmental Protection Agency, Fuel Economy website, www.fueleconomy.gov. 
The Environmental Protection Agency also uses other driving cycles to test new vehicles (although these do not affect the fuel economy ratings). The New York Test Cycle was developed in the 1970's in order to simulate driving in downtown congested areas. The Representative Number Five Test Cycle was developed in the 1990's to better represent actual on-road driving by combining modern city and freeway driving.

Figure 4.8. New York City Driving Cycle

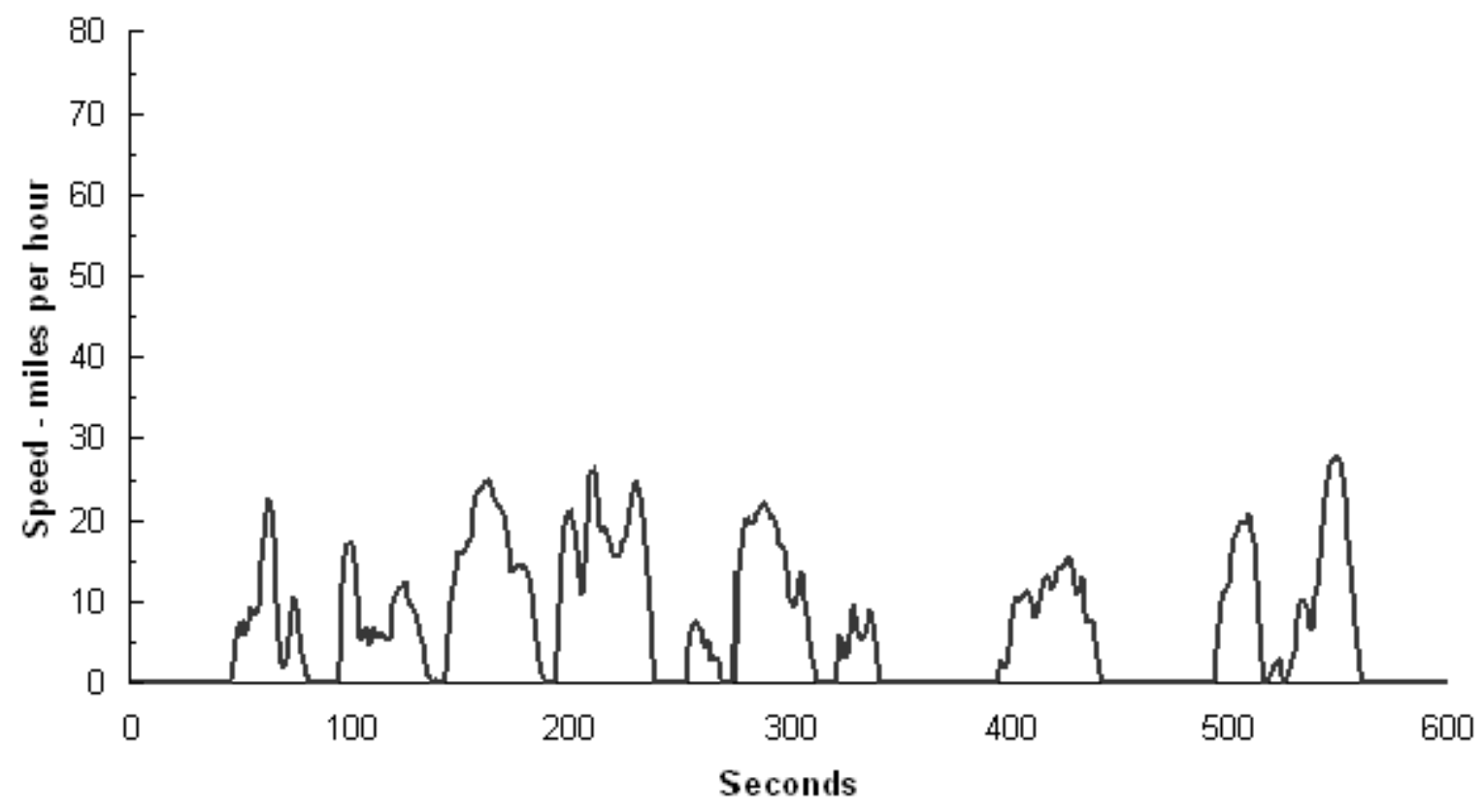

Figure 4.9. Representative Number Five Driving Cycle

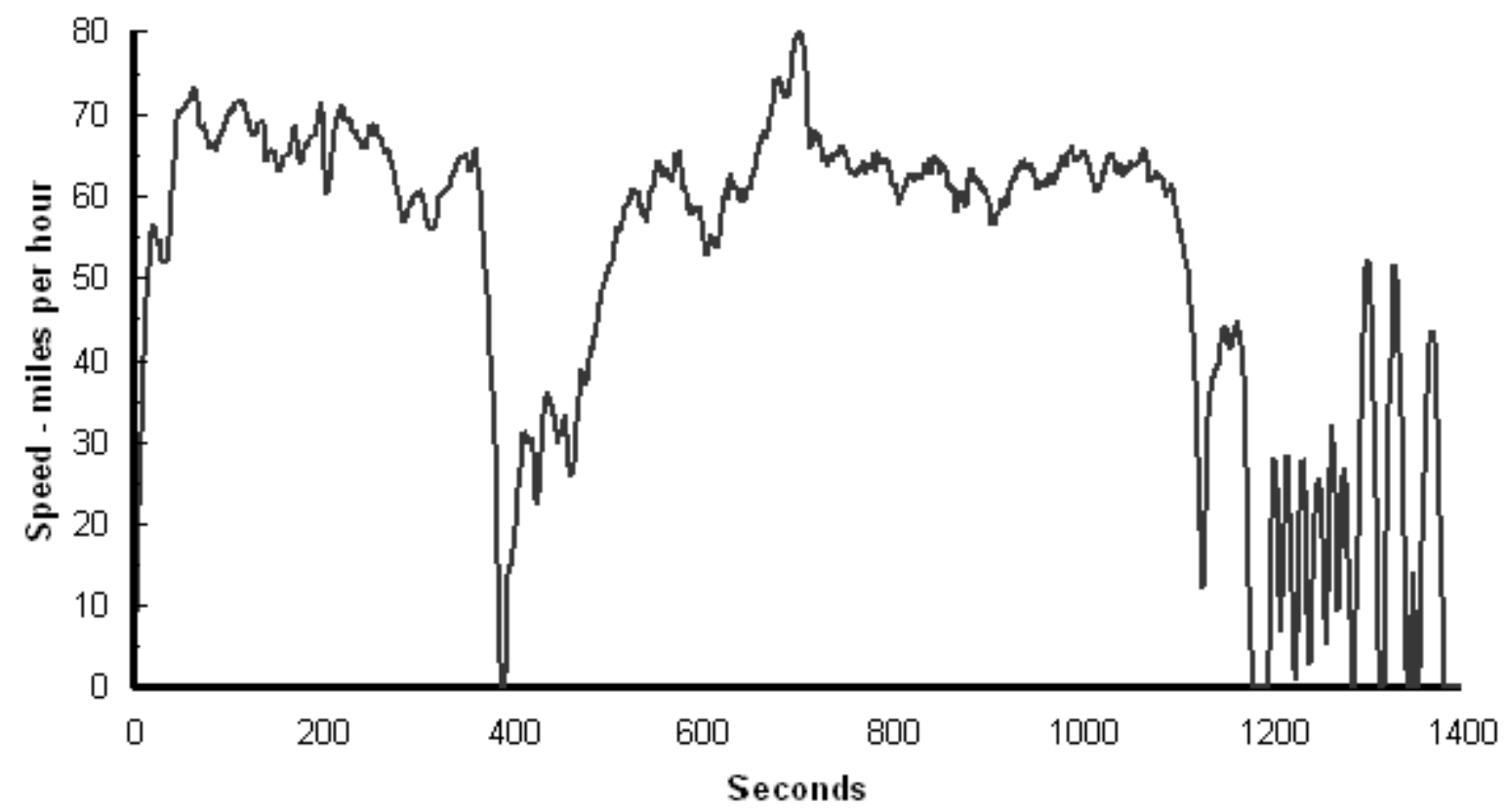

Source:

Data obtained from Michael Wang, Argonne National Laboratory, Argonne, IL, 1997. 
Testing cycles to determine vehicle fuel economy and emissions vary by country. The United States currently uses five different drive cycles to determine vehicle fuel economy. In Europe, the NEDC cycle is being replaced by the WLTC, but the NEDC continues to be used in China. The ARTEMIS cycles are not used in vehicle certification, but are used to represent real world driving in Europe.

Table 4.31

Comparison of U.S., European, and Japanese Driving Cycles Attributes

\begin{tabular}{|c|c|c|c|c|c|}
\hline Cycle & $\begin{array}{c}\text { Time } \\
\text { (seconds) }\end{array}$ & $\begin{array}{c}\text { Distance } \\
\text { (miles) }\end{array}$ & $\begin{array}{l}\text { Average } \\
\text { Speed } \\
\text { (mph) }\end{array}$ & $\begin{array}{l}\text { Maximum } \\
\text { Speed } \\
\text { (mph) }\end{array}$ & $\begin{array}{l}\text { Maximum } \\
\text { Acceleration } \\
(\mathrm{mph} / \mathrm{s})\end{array}$ \\
\hline \multicolumn{6}{|c|}{ United States } \\
\hline City & 1,872 & 11.0 & 21.2 & 56.0 & 3.3 \\
\hline Highway & 765 & 10.3 & 48.3 & 60.0 & 3.2 \\
\hline High-Speed & 594 & 8.0 & 48.4 & 80.0 & 8.5 \\
\hline Air Conditioner Use & 594 & 3.6 & 21.2 & 54.8 & 5.1 \\
\hline Cold Temperatures & 1,872 & 11.0 & 21.2 & 56.0 & 3.3 \\
\hline \multicolumn{6}{|c|}{ World Light Vehicle Test Cycle (WLTC) } \\
\hline Low & 589 & 1.9 & 11.7 & 35.1 & 3.6 \\
\hline Medium & 433 & 3.0 & 24.5 & 47.6 & 3.6 \\
\hline High & 455 & 4.4 & 35.1 & 60.5 & 3.7 \\
\hline Extra High & 323 & 5.1 & 57.0 & 81.6 & 2.3 \\
\hline Total WLTC & 1,800 & 14.5 & 28.9 & 81.6 & 3.7 \\
\hline \multicolumn{6}{|c|}{ Japan } \\
\hline JC08 & 1,204 & 5.1 & 15.2 & 50.7 & 3.8 \\
\hline \multicolumn{6}{|c|}{ New European Driving Cycle (NEDC) } \\
\hline Urban Driving Cycle (UDC) & 780 & 2.5 & 11.8 & 31.1 & 2.3 \\
\hline Extra Urban Driving Cycle (EUDC) & 400 & 4.3 & 38.9 & 74.6 & 1.9 \\
\hline Total NEDC & 1,180 & 6.8 & 20.9 & 74.6 & 2.3 \\
\hline \multicolumn{6}{|c|}{ ARTEMIS } \\
\hline Urban & 993 & 3.0 & 11.0 & 35.9 & 6.4 \\
\hline Rural Road & 1,082 & 10.7 & 35.7 & 69.3 & 5.3 \\
\hline Motorway & 1,068 & 17.9 & 60.1 & 81.9 & 4.3 \\
\hline Total ARTEMIS & 3,143 & 31.6 & 36.2 & 81.9 & 6.4 \\
\hline
\end{tabular}

\section{Source:}

United States - U.S. Department of Energy, Fuel Economy Guide website, www.fueleconomy.gov/feg/fe_test_schedules.shtml

All other - Compiled from public sources by Aymeric Rousseau, Argonne National Laboratory, September 2016. 
Testing cycles to determine vehicle fuel economy and emissions vary by country and therefore it is difficult to make a direct comparison. Simulation results show up to a $28 \%$ difference in the test cycles for each vehicle type. Note that the differences in these cycle results also vary with each individual vehicle tested.

Table 4.32

Example of Differing Results Using the U.S., European, and Japanese Driving Cycles

\begin{tabular}{|c|c|c|c|c|c|}
\hline \multirow[b]{2}{*}{ Vehicle type } & \multicolumn{3}{|c|}{ Miles per gallon } & \multicolumn{2}{|c|}{ Percentage difference from } \\
\hline & $\begin{array}{l}\text { U.S. Corporate } \\
\text { Average Fuel } \\
\text { Economy } \\
\text { (CAFE) cycle }\end{array}$ & $\begin{array}{c}\text { New European } \\
\text { Driving Cycle } \\
\text { (NEDC) }\end{array}$ & $\begin{array}{c}\text { Japan JC08 } \\
\text { cycle }\end{array}$ & $\begin{array}{c}\text { CAFE to } \\
\text { NEDC }\end{array}$ & $\begin{array}{c}\text { CAFE to } \\
\text { JC08 }\end{array}$ \\
\hline Small car & 34.8 & 32.4 & 27.6 & $-7 \%$ & $-21 \%$ \\
\hline Large car & 26.6 & 24.7 & 21.5 & $-7 \%$ & $-19 \%$ \\
\hline Minivan & 23.9 & 20.5 & 17.2 & $-14 \%$ & $-28 \%$ \\
\hline Sport-utility vehicle & 20.2 & 17.6 & 14.6 & $-13 \%$ & $-28 \%$ \\
\hline Pickup & 18.8 & 15.9 & 13.5 & $-15 \%$ & $-28 \%$ \\
\hline
\end{tabular}

Note: Simulation results for identical gasoline vehicles (i.e., results for the same small car on each of the three cycles).

\section{Source:}

The International Council on Clean Transportation, Passenger Vehicle Greenhouse Gas and Fuel Economy Standards: A Global Update, July 2009. 


\section{Chapter 5 \\ Heavy Vehicles and Characteristics}

Summary Statistics from Tables in this Chapter

\begin{tabular}{ccr}
\hline Source & & \\
\hline Table 5.1 & Class 3-8 single-unit trucks, 2015 & \\
& Registration (thousands) & Vehicle miles (millions) \\
& Fuel economy (miles per gallon) & 109,597 \\
Table 5.2 & Class 7-8 combination trucks, 2015 & 7.4 \\
& Registration (thousands) & 2,747 \\
Table 5.14 & Vehicle miles (millions) & 170,246 \\
Table 5.14 & Fuel economy (miles per gallon) & 5.9 \\
Table 5.15 & Shipments, 2012 Commodity Flow Survey & 13,852 \\
Table 5.16 & Tons (millions) & 11,299 \\
\hline
\end{tabular}


Class 3-8 single-unit trucks include trucks over 10,000 lb gross vehicle weight with the cab/engine and cargo space together as one unit. Most of these trucks would be used for business or for individuals with heavy hauling or towing needs. Very heavy single-units, such as concrete mixers and dump trucks, are also in this category. The data series was changed by the FHWA back to 2007.

Table 5.1

Summary Statistics for Class 3-8 Single-Unit Trucks, 1970-2015

\begin{tabular}{|c|c|c|c|c|c|}
\hline Year & $\begin{array}{c}\text { Registrations } \\
\text { (thousands) }\end{array}$ & $\begin{array}{l}\text { Vehicle travel } \\
\text { (million miles) }\end{array}$ & $\begin{array}{c}\text { Average annual } \\
\text { miles per vehicle }\end{array}$ & $\begin{array}{c}\text { Fuel use } \\
\text { (million gallons) }\end{array}$ & $\begin{array}{c}\text { Fuel economy } \\
\text { (miles per gallon) }\end{array}$ \\
\hline 1970 & 3,681 & 27,081 & 7,357 & 3,968 & 6.8 \\
\hline 1975 & 4,232 & 34,606 & 8,177 & 5,420 & 6.4 \\
\hline 1980 & 4,374 & 39,813 & 9,102 & 6,923 & 5.8 \\
\hline 1981 & 4,455 & 39,568 & 8,882 & 6,867 & 5.8 \\
\hline 1982 & 4,325 & 40,658 & 9,401 & 6,803 & 6.0 \\
\hline 1983 & 4,204 & 42,546 & 10,120 & 6,965 & 6.1 \\
\hline 1984 & 4,061 & 44,419 & 10,938 & 7,240 & 6.1 \\
\hline 1985 & 4,593 & 45,441 & 9,894 & 7,399 & 6.1 \\
\hline 1986 & 4,313 & 45,637 & 10,581 & 7,386 & 6.2 \\
\hline 1987 & 4,188 & 48,022 & 11,467 & 7,523 & 6.4 \\
\hline 1988 & 4,470 & 49,434 & 11,059 & 7,701 & 6.4 \\
\hline 1989 & 4,519 & 50,870 & 11,257 & 7,779 & 6.5 \\
\hline 1990 & 4,487 & 51,901 & 11,567 & 8,357 & 6.2 \\
\hline 1991 & 4,481 & 52,898 & 11,805 & 8,172 & 6.5 \\
\hline 1992 & 4,370 & 53,874 & 12,328 & 8,237 & 6.5 \\
\hline 1993 & 4,408 & 56,772 & 12,879 & 8,488 & 6.7 \\
\hline 1994 & 4,906 & 61,284 & 12,492 & 9,032 & 6.8 \\
\hline 1995 & 5,024 & 62,705 & 12,481 & 9,216 & 6.8 \\
\hline 1996 & 5,266 & 64,072 & 12,167 & 9,409 & 6.8 \\
\hline 1997 & 5,293 & 66,893 & 12,638 & 9,576 & 7.0 \\
\hline 1998 & 5,414 & 67,894 & 12,540 & 9,741 & 7.0 \\
\hline 1999 & 5,763 & 70,304 & 12,199 & 9,372 & 7.5 \\
\hline 2000 & 5,926 & 70,500 & 11,897 & 9,563 & 7.4 \\
\hline 2001 & 5,704 & 72,448 & 12,701 & 9,667 & 7.5 \\
\hline 2002 & 5,651 & 75,866 & 13,425 & 10,321 & 7.4 \\
\hline 2003 & 5,849 & 77,757 & 13,294 & 8,881 & 8.8 \\
\hline 2004 & 6,161 & 78,441 & 12,732 & 8,959 & 8.8 \\
\hline 2005 & 6,395 & 78,496 & 12,275 & 9,501 & 8.3 \\
\hline 2006 & 6,649 & 80,344 & 12,084 & 9,852 & 8.2 \\
\hline 2007 & 8,117 & 119,979 & 14,781 & 16,314 & 7.3 \\
\hline 2008 & 8,228 & 126,855 & 15,417 & 17,144 & 7.4 \\
\hline 2009 & 8,356 & 120,207 & 14,386 & 16,253 & 7.4 \\
\hline 2010 & 8,217 & 110,738 & 13,477 & 15,097 & 7.3 \\
\hline 2011 & 7,819 & 103,803 & 13,276 & 14,214 & 7.3 \\
\hline 2012 & 8,190 & 105,605 & 12,894 & 14,376 & 7.3 \\
\hline 2013 & 8,126 & 106,582 & 13,116 & 14,502 & 7.3 \\
\hline 2014 & 8,329 & 109,301 & 13,123 & 14,894 & 7.3 \\
\hline \multirow[t]{2}{*}{2015} & 8,456 & 109,597 & 12,961 & 14,850 & 7.4 \\
\hline & \multicolumn{5}{|c|}{ Average annual percentage change } \\
\hline 1970-2015 & $1.9 \%$ & $3.2 \%$ & $1.3 \%$ & $3.0 \%$ & $0.2 \%$ \\
\hline 2005-2015 & $2.8 \%$ & $3.4 \%$ & $0.5 \%$ & $4.6 \%$ & $-1.1 \%$ \\
\hline
\end{tabular}

\section{Source:}

U. S. Department of Transportation, Federal Highway Administration, Highway Statistics 2015, Washington, DC, 2016, Table VM-1 and annual. (Additional resources: www.fhwa.dot.gov)

${ }^{a}$ Due to FHWA methodology changes, data from 2007-on are not comparable with previous data. 
Class 7-8 combination trucks include all trucks designed to be used in combination with one or more trailers with a gross vehicle weight rating over 26,000 lb. The average vehicle travel of these trucks (on a per truck basis) far surpasses the travel of other trucks due to long-haul freight movement. The data series was changed by the FHWA back to 2007.

Table 5.2

Summary Statistics for Class 7-8 Combination Trucks, 1970-2015

\begin{tabular}{|c|c|c|c|c|c|c|}
\hline Year & $\begin{array}{l}\text { Registrations } \\
\text { (thousands) }\end{array}$ & $\begin{array}{l}\text { Vehicle travel }^{\mathrm{a}} \\
\text { (million miles) }\end{array}$ & $\begin{array}{l}\text { Average annual } \\
\text { miles per vehicle }\end{array}$ & $\begin{array}{c}\text { Fuel use } \\
\text { (million gallons) }\end{array}$ & $\begin{array}{c}\text { Fuel economy } \\
\text { (miles per gallon) }\end{array}$ & \\
\hline 1970 & 905 & 35,134 & 38,822 & 7,348 & 4.8 & \\
\hline 1975 & 1,131 & 46,724 & 41,312 & 9,177 & 5.1 & \\
\hline 1980 & 1,417 & 68,678 & 48,467 & 13,037 & 5.3 & \\
\hline 1981 & 1,261 & 69,134 & 54,825 & 13,509 & 5.1 & \\
\hline 1982 & 1,265 & 70,765 & 55,941 & 13,583 & 5.2 & \\
\hline 1983 & 1,304 & 73,586 & 56,431 & 13,796 & 5.3 & \\
\hline 1984 & 1,340 & 77,377 & 57,744 & 14,188 & 5.5 & \\
\hline 1985 & 1,403 & 78,063 & 55,640 & 14,005 & 5.6 & \\
\hline 1986 & 1,408 & 81,038 & 57,555 & 14,475 & 5.6 & \\
\hline 1987 & 1,530 & 85,495 & 55,879 & 14,990 & 5.7 & \\
\hline 1988 & 1,667 & 88,551 & 53,120 & 15,224 & 5.8 & \\
\hline 1989 & 1,707 & 91,879 & 53,825 & 15,733 & 5.8 & \\
\hline 1990 & 1,709 & 94,341 & 55,202 & 16,133 & 5.8 & \\
\hline 1991 & 1,691 & 96,645 & 57,153 & 16,809 & 5.7 & \\
\hline 1992 & 1,675 & 99,510 & 59,409 & 17,216 & 5.8 & \\
\hline 1993 & 1,680 & 103,116 & 61,379 & 17,748 & 5.8 & \\
\hline 1994 & 1,681 & 108,932 & 64,802 & 18,653 & 5.8 & \\
\hline 1995 & 1,696 & 115,451 & 68,073 & 19,777 & 5.8 & \\
\hline 1996 & 1,747 & 118,899 & 68,059 & 20,192 & 5.9 & \\
\hline 1997 & 1,790 & 124,584 & 69,600 & 20,302 & 6.1 & \\
\hline 1998 & 1,831 & 128,159 & 69,994 & 21,100 & 6.1 & \\
\hline 1999 & 2,029 & 132,384 & 65,246 & 24,537 & 5.4 & \\
\hline 2000 & 2,097 & 135,020 & 64,387 & 25,666 & 5.3 & \\
\hline 2001 & 2,154 & 136,584 & 63,409 & 25,512 & 5.4 & \\
\hline 2002 & 2,277 & 138,737 & 60,930 & 26,480 & 5.2 & \\
\hline 2003 & 1,908 & 140,160 & 73,459 & 23,815 & 5.9 & \\
\hline 2004 & 2,010 & 142,370 & 70,831 & 24,191 & 5.9 & \\
\hline 2005 & 2,087 & 144,028 & 69,012 & 27,689 & 5.2 & \\
\hline 2006 & 2,170 & 142,169 & 65,516 & 28,107 & 5.1 & b \\
\hline 2007 & 2,635 & 184,199 & 69,905 & 30,904 & 6.0 & \\
\hline 2008 & 2,585 & 183,826 & 71,113 & 30,561 & 6.0 & \\
\hline 2009 & 2,617 & 168,100 & 64,234 & 28,050 & 6.0 & \\
\hline 2010 & 2,553 & 175,789 & 68,856 & 29,927 & 5.9 & \\
\hline 2011 & 2,452 & 163,791 & 66,809 & 28,181 & 5.8 & \\
\hline 2012 & 2,469 & 163,602 & 66,262 & 27,975 & 5.8 & \\
\hline 2013 & 2,471 & 168,436 & 68,155 & 28,795 & 5.8 & \\
\hline 2014 & 2,577 & 169,830 & 65,897 & 29,118 & 5.8 & \\
\hline 2015 & 2,747 & 170,246 & 61,978 & 28,884 & 5.9 & \\
\hline & \multicolumn{6}{|c|}{ Average annual percentage change } \\
\hline 1970-2015 & $2.5 \%$ & $3.6 \%$ & $1.0 \%$ & $3.1 \%$ & $0.5 \%$ & \\
\hline $2005-2015$ & $2.8 \%$ & $1.7 \%$ & $-1.1 \%$ & $0.4 \%$ & $1.3 \%$ & \\
\hline
\end{tabular}

\section{Source:}

U. S. Department of Transportation, Federal Highway Administration, Highway Statistics 2015, Washington, DC, 2016, Table VM-1 and annual. (Additional resources: www.fhwa.dot.gov)

a The Federal Highway Administration changed the combination truck travel methodology in 1993.

${ }^{\mathrm{b}}$ Due to FHWA methodology changes, data from 2007-on are not comparable with previous data. 
Truck sales rose in 2010 for the first time since the sales peak in 2004 and have thus far continued to rise. Trucks under 10,000 lb continue to dominate truck sales.

Table 5.3

New Retail Truck Sales by Gross Vehicle Weight, 1970-2016 ${ }^{\mathrm{a}}$ (thousands)

\begin{tabular}{|c|c|c|c|c|c|c|c|c|c|}
\hline $\begin{array}{c}\text { Calendar } \\
\text { year }\end{array}$ & $\begin{array}{c}\text { Class } 1 \\
6,000 \mathrm{lb} \\
\text { or less }\end{array}$ & $\begin{array}{c}\text { Class } 2 \\
6,001- \\
10,000 \mathrm{lb} \\
\end{array}$ & $\begin{array}{c}\text { Class } 3 \\
10,001- \\
14,000 \mathrm{lb}\end{array}$ & $\begin{array}{c}\text { Class } 4 \\
14,001- \\
16,000 \mathrm{lb} \\
\end{array}$ & $\begin{array}{c}\text { Class } 5 \\
16,001- \\
19,500 \mathrm{lb} \\
\end{array}$ & $\begin{array}{c}\text { Class } 6 \\
19,501- \\
26,000 \mathrm{lb} \\
\end{array}$ & $\begin{array}{c}\text { Class } 7 \\
26,001- \\
33,000 \mathrm{lb} \\
\end{array}$ & $\begin{array}{c}\text { Class } 8 \\
33,001 \mathrm{lb} \\
\text { and over }\end{array}$ & Total \\
\hline \multicolumn{10}{|c|}{ Domestic sales (import data are not available) } \\
\hline $1970^{\mathrm{b}}$ & 1,049 & 408 & 6 & 12 & 58 & 133 & 36 & 89 & 1,791 \\
\hline 1975 & 1,101 & 952 & 23 & 1 & 9 & 159 & 23 & 83 & 2,351 \\
\hline 1980 & 985 & 975 & 4 & c & 2 & 90 & 58 & 117 & 2,231 \\
\hline 1981 & 896 & 850 & 1 & c & 2 & 72 & 51 & 100 & 1,972 \\
\hline 1983 & 1,314 & 1,207 & c & c & 1 & 47 & 59 & 82 & 2,710 \\
\hline 1984 & 2,031 & 1,224 & 6 & c & 5 & 55 & 78 & 138 & 3,538 \\
\hline 1985 & 2,408 & 1,280 & 11 & c & 5 & 48 & 97 & 134 & 3,983 \\
\hline \multicolumn{10}{|c|}{ Domestic and import sales } \\
\hline 1986 & 3,380 & 1,214 & 12 & c & 6 & 45 & 101 & 113 & 4,870 \\
\hline 1987 & 3,435 & 1,175 & 14 & 2 & 8 & 44 & 103 & 131 & 4,912 \\
\hline 1988 & 3,467 & 1,333 & 14 & 21 & 8 & 54 & 103 & 148 & 5,149 \\
\hline 1989 & 3,313 & 1,297 & 19 & 27 & 7 & 39 & 93 & 145 & 4,942 \\
\hline 1991 & 3,246 & 876 & 21 & 24 & 3 & 22 & 73 & 99 & 4,365 \\
\hline 1992 & 3,608 & 1,021 & 26 & 26 & 4 & 28 & 73 & 119 & 4,903 \\
\hline 1993 & 4,119 & 1,232 & 27 & 33 & 4 & 27 & 81 & 158 & 5,681 \\
\hline 1994 & 4,527 & 1,506 & 35 & 44 & 4 & 20 & 98 & 186 & 6,421 \\
\hline 1995 & 4,422 & 1,631 & 40 & 53 & 4 & 23 & 107 & 201 & 6,481 \\
\hline 1996 & 4,829 & 1,690 & 52 & 59 & 7 & 19 & 104 & 170 & 6,930 \\
\hline 1997 & 5,085 & 1,712 & 53 & 57 & 9 & 18 & 114 & 179 & 7,226 \\
\hline 1998 & 5,263 & 2,036 & 102 & 43 & 25 & 32 & 115 & 209 & 7,826 \\
\hline 1999 & 5,707 & 2,366 & 122 & 49 & 30 & 48 & 130 & 262 & 8,716 \\
\hline 2000 & 5,965 & 2,421 & 117 & 47 & 29 & 51 & 123 & 212 & 8,965 \\
\hline 2001 & 6,073 & 2,525 & 102 & 52 & 24 & 42 & 92 & 140 & 9,050 \\
\hline 2002 & 6,068 & 2,565 & 80 & 38 & 24 & 45 & 69 & 146 & 9,035 \\
\hline 2003 & 6,267 & 2,671 & 91 & 40 & 29 & 51 & 67 & 142 & 9,357 \\
\hline 2004 & 6,458 & 2,796 & 107 & 47 & 36 & 70 & 75 & 203 & 9,793 \\
\hline 2005 & 6,586 & 2,528 & 167 & 49 & 46 & 60 & 89 & 253 & 9,777 \\
\hline 2006 & 6,136 & 2,438 & 150 & 50 & 49 & 70 & 91 & 284 & 9,268 \\
\hline 2009 & 3,528 & 1,306 & 112 & 20 & 24 & 22 & 39 & 95 & 5,145 \\
\hline 2010 & 4,245 & 1,513 & 161 & 12 & 31 & 29 & 38 & 107 & 6,137 \\
\hline 2011 & 4,714 & 1,735 & 195 & 10 & 42 & 41 & 41 & 171 & 6,951 \\
\hline 2012 & 5,164 & 1,811 & 223 & 9 & 55 & 40 & 47 & 195 & 7,544 \\
\hline 2013 & 5,615 & 2,077 & 254 & 12 & 60 & 47 & 48 & 185 & 8,298 \\
\hline 2014 & 6,209 & 2,275 & 264 & 13 & 67 & 52 & 54 & 220 & 9,154 \\
\hline 2015 & 7,161 & 2,417 & 283 & 14 & 72 & 55 & 59 & 249 & 10,310 \\
\hline 2016 & 7,724 & 2,572 & 296 & 14 & 72 & 62 & 60 & 193 & 10,993 \\
\hline \multicolumn{10}{|c|}{ Average annual percentage change } \\
\hline 1970-1985 & $5.7 \%$ & $7.9 \%$ & $4.1 \%$ & c & $-15.1 \%$ & $-6.6 \%$ & $6.8 \%$ & $2.8 \%$ & $5.5 \%$ \\
\hline 1986-2016 & $2.8 \%$ & $2.5 \%$ & $11.3 \%$ & $6.9 \%{ }^{\mathrm{d}}$ & $8.6 \%$ & $1.1 \%$ & $-1.7 \%$ & $1.8 \%$ & $2.8 \%$ \\
\hline 2006-2016 & $2.3 \%$ & $0.5 \%$ & $7.0 \%$ & $-12.0 \%$ & $3.9 \%$ & $-1.2 \%$ & $-4.1 \%$ & $-3.8 \%$ & $1.7 \%$ \\
\hline
\end{tabular}

Source:

Ward's Communications, www.wardsauto.com. (Additional resources: www.wardsauto.com)

a Sales include domestic-sponsored imports.

${ }^{\mathrm{b}}$ Data for 1970 is based on new truck registrations.

${ }^{\mathrm{c}}$ Data are not available.

d $1987-2016$. 
The Vehicle Inventory and Use Survey (VIUS) was discontinued, thus the 2002 VIUS data remain the latest available.

The United States Department of Transportation, the United States Department of Energy, and the United States Department of Agriculture are funding a planning study to design and scope a new VIUS, possibly utilizing new forms of survey technologies.

There is an opportunity to provide input on how VIUS data have been used; which survey elements are essential to your future data and policy needs; and, elements which should be added or deleted. To review the questions from the 2002 survey prior to providing input, go to www.census.gov/svsd/www/vius/questionnaires.html.

To give input towards a new VIUS data collection, email VIUS@dot.gov.

\section{Vehicle Inventory and Use Survey}

The Vehicle Inventory and Use Survey (VIUS), which was formerly the Truck Inventory and Use Survey (TIUS), provides data on the physical and operational characteristics of the Nation's truck population. It is based on a probability sample of private and commercial trucks registered (or licensed) in each state. In 1997, the survey was changed to the Vehicle Inventory and Use Survey due to future possibilities of including additional vehicle types. The 2002 VIUS, however, only includes trucks. Copies of the 2002 VIUS report or CD may be obtained by contacting the U.S. Bureau of the Census, Transportation Characteristics Surveys Branch (301) 457-2797. Internet site:

\section{www.census.gov/svsd/www/tiusview.html}

Since 1987, the survey has included minivans, vans, station wagons on truck chassis, and sport utility vehicles in addition to the bigger trucks. The 1977 and 1982 surveys did not include those vehicle types. The estimated number of trucks that were within the scope of the 2002 VIUS and registered in the United States as of July 1, 2002 was 85.2 million. These trucks were estimated to have been driven a total of 1,115 billion miles during 2002, an increase of $6.8 \%$ from 1997. The average annual miles traveled per truck was estimated at 13,100 miles.

In 2016, the California Department of Transportation is conducting a survey to collect data on the physical and operational characteristics of the State's commercial vehicle population called the California Vehicle Inventory Use and Survey. Internet site:

www.dot.ca.gov/hq/tpp/offices/omsp/statewide_modeling/cal_vehicle_survey.html 
Table 5.4

Truck Statistics by Gross Vehicle Weight Class, 2002

\begin{tabular}{|c|c|c|c|c|c|}
\hline $\begin{array}{l}\text { Manufacturer's gross vehicle } \\
\text { weight class }\end{array}$ & $\begin{array}{l}\text { Number of } \\
\text { trucks }\end{array}$ & $\begin{array}{l}\text { Percentage } \\
\text { of trucks }\end{array}$ & $\begin{array}{c}\text { Average } \\
\text { annual miles } \\
\text { per truck }\end{array}$ & $\begin{array}{l}\text { Harmonic } \\
\text { mean fuel } \\
\text { economy }\end{array}$ & $\begin{array}{l}\text { Percentage } \\
\text { of fuel use }\end{array}$ \\
\hline 1) 6,000 lb and less & $51,941,389$ & $61.0 \%$ & 11,882 & 17.6 & $42.7 \%$ \\
\hline 2) $6,001-10,000 \mathrm{lb}$ & $28,041,234$ & $32.9 \%$ & 12,684 & 14.3 & $30.5 \%$ \\
\hline 3) $10,001-14,000 \mathrm{lb}$ & 691,342 & $0.8 \%$ & 14,094 & 10.5 & $1.1 \%$ \\
\hline 4) $14,001-16,000 \mathrm{lb}$ & 290,980 & $0.3 \%$ & 15,441 & 8.5 & $0.5 \%$ \\
\hline 5) $16,001-19,500 \mathrm{lb}$ & 166,472 & $0.2 \%$ & 11,645 & 7.9 & $0.3 \%$ \\
\hline 6) $19,501-26,000 \mathrm{lb}$ & $1,709,574$ & $2.0 \%$ & 12,671 & 7.0 & $3.2 \%$ \\
\hline Medium truck subtotal & $2,858,368$ & $3.4 \%$ & 13,237 & 8.0 & $5.2 \%$ \\
\hline 7) $26,001-33,000 \mathrm{lb}$ & 179,790 & $0.2 \%$ & 30,708 & 6.4 & $0.9 \%$ \\
\hline 8) 33,001 lb and up & $2,153,996$ & $2.5 \%$ & 45,739 & 5.7 & $20.7 \%$ \\
\hline Total & $85,174,777$ & $100.0 \%$ & 13,088 & 13.5 & $100.0 \%$ \\
\hline Light truck subtotal & $79,982,623$ & $93.9 \%$ & 12,163 & 16.2 & $73.2 \%$ \\
\hline Medium truck subtotal & $2,858,368$ & $3.4 \%$ & 13,237 & 8.0 & $5.2 \%$ \\
\hline Heavy truck subtotal & $2,333,786$ & $2.7 \%$ & 44,581 & 5.8 & $21.6 \%$ \\
\hline
\end{tabular}

Source:

U.S. Department of Commerce, Bureau of the Census, 2002 Vehicle Inventory and Use Survey, Microdata File on CD, 2005. (Additional resources: www.census.gov/svsd/www.tiusview.html)

Table 5.5

Truck Harmonic Mean Fuel Economy by Size Class, 1992, 1997, and 2002 (miles per gallon)

\begin{tabular}{lrrr}
\hline $\begin{array}{c}\text { Manufacturer's gross vehicle } \\
\text { weight class }\end{array}$ & $\begin{array}{r}1992 \\
\text { TIUS }\end{array}$ & 1997 & VIUS \\
\hline 1) $6,000 \mathrm{lb}$ and less & 17.2 & 17.1 & VIUS \\
2) $6,001-10,000 \mathrm{lb}$ & 13.0 & 13.6 & 17.6 \\
3) $10,000-14,000 \mathrm{lb}$ & 8.8 & 9.4 & 14.3 \\
4) $14,001-16,000 \mathrm{lb}$ & 8.8 & 9.3 & 10.5 \\
5) $16,001-19,500 \mathrm{lb}$ & 7.4 & 8.7 & 8.5 \\
6) 19,501-26,000 lb & 6.9 & 7.3 & 7.9 \\
7) 26,001-33,000 lb & 6.5 & 6.4 & 7.0 \\
8) 33,001 lb and over & 5.5 & 5.7 & 6.4 \\
\hline Light truck subtotal & 15.7 & 15.8 & 5.7 \\
Medium truck subtotal & 7.3 & 8.6 & 8.0 \\
Large truck subtotal & 5.6 & 6.1 & 5.8 \\
\hline
\end{tabular}

Note: Based on average fuel economy as reported by respondent.

\section{Sources:}

Estimates are based on data provided on the following public use files: U.S. Department of Commerce, Bureau of the Census, Census of Transportation, Washington, DC, 1992 Truck Inventory and Use Survey, 1995; 1997 Vehicle Inventory and Use Survey, 2000, and 2002 Vehicle Inventory and Use Survey, 2005. (Additional resources: www.census.gov/svsd/www/tiusview.html) 
As expected, most light trucks travel within 50 miles of their home base and refuel at public stations. About sixty percent of heavy trucks travel over 50 miles from their home base and 36\% of them refuel at central companyowned refueling stations.

Table 5.6

Truck Statistics by Size, 2002

\begin{tabular}{|c|c|c|c|c|}
\hline & \multicolumn{3}{|c|}{ Manufacturer's gross vehicle weight class } & \multirow[b]{2}{*}{ Total } \\
\hline & $\begin{array}{c}\text { Light } \\
(<10,000 \mathrm{lb})\end{array}$ & $\begin{array}{l}\text { Medium } \\
(10,001- \\
26,000 \mathrm{lb})\end{array}$ & $\begin{array}{c}\text { Heavy } \\
(>26,000 \mathrm{lb})\end{array}$ & \\
\hline & \multicolumn{4}{|c|}{ Typical trip miles or range of operation ${ }^{\mathrm{a}}$} \\
\hline Under 50 miles & $69.2 \%$ & $61.5 \%$ & $40.7 \%$ & $68.2 \%$ \\
\hline 51-100 miles & $8.5 \%$ & $11.7 \%$ & $13.5 \%$ & $8.7 \%$ \\
\hline 101-200 miles & $2.4 \%$ & $3.2 \%$ & $6.7 \%$ & $2.5 \%$ \\
\hline 201-500 miles & $1.1 \%$ & $1.8 \%$ & $7.6 \%$ & $1.3 \%$ \\
\hline 501 miles or more & $1.4 \%$ & $2.2 \%$ & $10.4 \%$ & $1.7 \%$ \\
\hline Off-road & $1.1 \%$ & $3.5 \%$ & $3.2 \%$ & $1.2 \%$ \\
\hline Vehicle not in use & $2.2 \%$ & $4.4 \%$ & $3.2 \%$ & $2.3 \%$ \\
\hline Not reported & $14.1 \%$ & $11.7 \%$ & $14.7 \%$ & $14.1 \%$ \\
\hline \multirow[t]{2}{*}{ Total $^{\mathbf{b}}$} & $100.0 \%$ & $100.0 \%$ & $100.0 \%$ & $100.0 \%$ \\
\hline & \multicolumn{4}{|c|}{ Primary refueling facility } \\
\hline Gas station & $96.9 \%$ & $62.4 \%$ & $28.4 \%$ & $93.9 \%$ \\
\hline Truck stop & $0.7 \%$ & $7.7 \%$ & $31.9 \%$ & $1.8 \%$ \\
\hline Own facility & $2.0 \%$ & $27.3 \%$ & $36.2 \%$ & $3.7 \%$ \\
\hline Other nonpublic facility & $0.3 \%$ & $2.6 \%$ & $3.5 \%$ & $0.5 \%$ \\
\hline Other & $0.0 \%$ & $0.0 \%$ & $0.0 \%$ & $0.0 \%$ \\
\hline Total $^{\text {b }}$ & $100.0 \%$ & $100.0 \%$ & $100.0 \%$ & $100.0 \%$ \\
\hline
\end{tabular}

Source:

U.S. Department of Commerce, Bureau of the Census, 2002 Vehicle Inventory and Use Survey, Microdata. File on CD, 2005. (Additional resources: www.census.gov/svsd/www/tiusview.html)

a The respondent was asked to choose the category which best described the trips made by the vehicle.

${ }^{\mathrm{b}}$ Percentages may not sum to totals due to rounding. 
More medium truck owners listed construction as the truck's major use than any other major use category.

Construction was the second highest major use for light trucks and heavy trucks.

Table 5.7

Percentage of Trucks by Size Ranked by Major Use, 2002

\begin{tabular}{|c|c|c|c|}
\hline Rank & $\begin{array}{c}\text { Light } \\
(<10,000 \mathrm{lb} \\
\text { average weight })\end{array}$ & $\begin{array}{c}\text { Medium } \\
(10,001-26,000 \mathrm{lb} \\
\text { average weight) }\end{array}$ & $\begin{array}{c}\text { Heavy } \\
\text { (> 26,000 lb average } \\
\text { weight) }\end{array}$ \\
\hline \multirow[t]{2}{*}{1} & Personal & Construction & For hire \\
\hline & $81.5 \%$ & $18.4 \%$ & $30.1 \%$ \\
\hline \multirow[t]{2}{*}{2} & Construction & Agriculture & Construction \\
\hline & $4.6 \%$ & $16.2 \%$ & $15.9 \%$ \\
\hline \multirow[t]{2}{*}{3} & Other services a & For hire & Agriculture \\
\hline & $2.5 \%$ & $9.6 \%$ & $12.2 \%$ \\
\hline \multirow[t]{2}{*}{4} & Not in use & Retail & Retail \\
\hline & $2.2 \%$ & $7.1 \%$ & $5.4 \%$ \\
\hline \multirow[t]{2}{*}{5} & Agriculture & Not in use & Not in use \\
\hline & $1.9 \%$ & $6.4 \%$ & $5.1 \%$ \\
\hline \multirow[t]{2}{*}{6} & Retail & Leasing & Waste management \\
\hline & $1.5 \%$ & $6.2 \%$ & $5.0 \%$ \\
\hline \multirow[t]{2}{*}{7} & Unknown & Wholesale & Manufacturing \\
\hline & $1.3 \%$ & $5.5 \%$ & $4.9 \%$ \\
\hline \multirow[t]{2}{*}{8} & Leasing & Waste management & Wholesale \\
\hline & $0.7 \%$ & $5.4 \%$ & $4.8 \%$ \\
\hline \multirow[t]{2}{*}{9} & Manufacturing & Utilities & Leasing \\
\hline & $0.7 \%$ & $5.0 \%$ & $4.6 \%$ \\
\hline \multirow[t]{2}{*}{10} & Utilities & Personal & Unknown \\
\hline & $0.6 \%$ & $4.8 \%$ & $3.2 \%$ \\
\hline \multirow[t]{2}{*}{11} & Waste management & Unknown & Personal \\
\hline & $0.6 \%$ & $4.4 \%$ & $2.5 \%$ \\
\hline \multirow[t]{2}{*}{12} & Wholesale & Manufacturing & Mining \\
\hline & $0.6 \%$ & $3.3 \%$ & $2.4 \%$ \\
\hline \multirow[t]{2}{*}{13} & Information services & Other services $^{a}$ & Other services $^{\mathrm{a}}$ \\
\hline & $0.4 \%$ & $3.2 \%$ & $1.3 \%$ \\
\hline \multirow[t]{2}{*}{14} & For hire & Food services & Utilities \\
\hline & $0.4 \%$ & $1.6 \%$ & $1.1 \%$ \\
\hline \multirow[t]{2}{*}{15} & Food services & Information services & Food services \\
\hline & $0.3 \%$ & $1.3 \%$ & $1.1 \%$ \\
\hline \multirow[t]{2}{*}{16} & Arts & Mining & Arts \\
\hline & $0.2 \%$ & $1.1 \%$ & $0.3 \%$ \\
\hline \multirow[t]{2}{*}{17} & Mining & Arts & Information services \\
\hline & $0.1 \%$ & $0.5 \%$ & $0.1 \%$ \\
\hline
\end{tabular}

Source:

U.S. Department of Commerce, Bureau of the Census, 2002 Vehicle Inventory and Use Survey, Micro data File on CD, 2005. (Additional resources: www.census.gov/svsd/www/tiusview.html)

${ }^{a}$ Business and personal services. 
Nearly half of trucks in fleets of 11-20 and 21-50 vehicles use company-owned facilities. Most trucks in smaller fleets use public gas stations for fueling.

Table 5.8

Percentage of Trucks by Fleet Size and Primary Fueling Facility, 2002

\begin{tabular}{|c|c|c|c|c|c|}
\hline \multirow[b]{2}{*}{ Truck fleet size } & \multicolumn{4}{|c|}{ Primary refueling facility } & \multirow[b]{2}{*}{ Total $^{\mathrm{a}}$} \\
\hline & Gas station & Truck stop & Own facility & Other's facility & \\
\hline $1-5$ & $73.8 \%$ & $6.1 \%$ & $18.2 \%$ & $1.9 \%$ & $100.0 \%$ \\
\hline $6-10$ & $55.3 \%$ & $5.7 \%$ & $35.5 \%$ & $3.4 \%$ & $100.0 \%$ \\
\hline $11-20$ & $41.1 \%$ & $5.1 \%$ & $48.9 \%$ & $4.9 \%$ & $100.0 \%$ \\
\hline $21-50$ & $42.9 \%$ & $3.7 \%$ & $49.8 \%$ & $3.6 \%$ & $100.0 \%$ \\
\hline 51 or more & $48.3 \%$ & $6.3 \%$ & $44.4 \%$ & $1.0 \%$ & $100.0 \%$ \\
\hline $\begin{array}{l}\text { Fleets of } 6 \text { or more } \\
\text { vehicles }\end{array}$ & $47.6 \%$ & $5.2 \%$ & $43.9 \%$ & $3.4 \%$ & $100.0 \%$ \\
\hline No fleet & $96.4 \%$ & $1.6 \%$ & $1.7 \%$ & $0.3 \%$ & $100.0 \%$ \\
\hline
\end{tabular}

Source:

U.S. Department of Commerce, Bureau of the Census, 2002 Vehicle Inventory and Use Survey, Microdata File on CD, 2005. (Additional resources: www.census.gov/svsd/www/tiusview.html)

a Percentages may not sum to totals due to rounding. 
Most trucks are fueled at gas stations but for-hire or warehousing trucks are more often fueled at truck stops. Mining trucks and vehicle leasing or rental trucks fuel at the companies' own facility more than $30 \%$ of the time.

Table 5.9

Share of Trucks by Major Use and Primary Fueling Facility, 2002

\begin{tabular}{|c|c|c|c|c|c|c|}
\hline Major use & $\begin{array}{c}\text { Gas } \\
\text { station }\end{array}$ & $\begin{array}{l}\text { Truck } \\
\text { stop }\end{array}$ & $\begin{array}{c}\text { Own } \\
\text { facility }\end{array}$ & $\begin{array}{l}\text { Others } \\
\text { facility }\end{array}$ & Other & $\mathrm{All}^{\mathrm{a}}$ \\
\hline Personal & $98.6 \%$ & $0.6 \%$ & $0.7 \%$ & $0.1 \%$ & $0.1 \%$ & $100.0 \%$ \\
\hline Other services & $96.0 \%$ & $1.4 \%$ & $1.6 \%$ & $0.9 \%$ & $0.1 \%$ & $100.0 \%$ \\
\hline All & $93.9 \%$ & $1.8 \%$ & $3.7 \%$ & $0.5 \%$ & $0.0 \%$ & $100.0 \%$ \\
\hline Information services & $92.3 \%$ & $0.4 \%$ & $7.2 \%$ & $0.1 \%$ & $0.0 \%$ & $100.0 \%$ \\
\hline Retail trade & $86.6 \%$ & $3.5 \%$ & $8.6 \%$ & $1.2 \%$ & $0.0 \%$ & $100.0 \%$ \\
\hline Construction & $84.7 \%$ & $3.3 \%$ & $9.8 \%$ & $2.2 \%$ & $0.0 \%$ & $100.0 \%$ \\
\hline Accommodation or food services & $82.4 \%$ & $7.5 \%$ & $8.8 \%$ & $1.3 \%$ & $0.0 \%$ & $100.0 \%$ \\
\hline Manufacturing & $81.5 \%$ & $5.1 \%$ & $11.9 \%$ & $1.5 \%$ & $0.0 \%$ & $100.0 \%$ \\
\hline Arts, entertainment, recreation services & $81.1 \%$ & $4.3 \%$ & $14.2 \%$ & $0.3 \%$ & $0.0 \%$ & $100.0 \%$ \\
\hline Waste mgmt, landscaping, admin/support services & $78.2 \%$ & $3.0 \%$ & $17.1 \%$ & $1.6 \%$ & $0.0 \%$ & $100.0 \%$ \\
\hline Wholesale trade & $76.2 \%$ & $6.6 \%$ & $12.0 \%$ & $5.1 \%$ & $0.0 \%$ & $100.0 \%$ \\
\hline Utilities & $72.6 \%$ & $1.8 \%$ & $24.3 \%$ & $1.3 \%$ & $0.0 \%$ & $100.0 \%$ \\
\hline Agriculture, forestry, fishing, hunting & $62.7 \%$ & $6.7 \%$ & $29.4 \%$ & $1.0 \%$ & $0.1 \%$ & $100.0 \%$ \\
\hline Vehicle leasing or rental & $60.2 \%$ & $1.3 \%$ & $31.8 \%$ & $6.8 \%$ & $0.0 \%$ & $100.0 \%$ \\
\hline Mining & $48.7 \%$ & $8.5 \%$ & $34.3 \%$ & $8.5 \%$ & $0.0 \%$ & $100.0 \%$ \\
\hline For-hire or warehousing & $33.3 \%$ & $38.7 \%$ & $25.8 \%$ & $2.3 \%$ & $0.0 \%$ & $100.0 \%$ \\
\hline Overall & $93.9 \%$ & $1.8 \%$ & $3.7 \%$ & $0.5 \%$ & $0.0 \%$ & $100.0 \%$ \\
\hline
\end{tabular}

Source:

U.S. Department of Commerce, Bureau of the Census, 2002 Vehicle Inventory and Use Survey, Microdata File on CD, 2005. (Additional resources: www.census.gov/svsd/www/tiusview.html)

a Percentages may not sum to totals due to rounding. 
The figure below shows the distribution of annual travel the two types of Class 7 and 8 vehicles-combination units (separate tractor and trailer) and single units (tractor and trailer on a single chassis). This information is for all trucks and trucks two years old or less. Combination trucks, dominated by box-type trailers, display the greatest amount of annual travel of all heavy vehicle types, as is evidenced both by the range of annual use. Most of the single-unit trucks in the survey travel 40,000 miles per year or less.

Figure 5.1. Distribution of Trucks over 26,000 lb by Vehicle-Miles Traveled

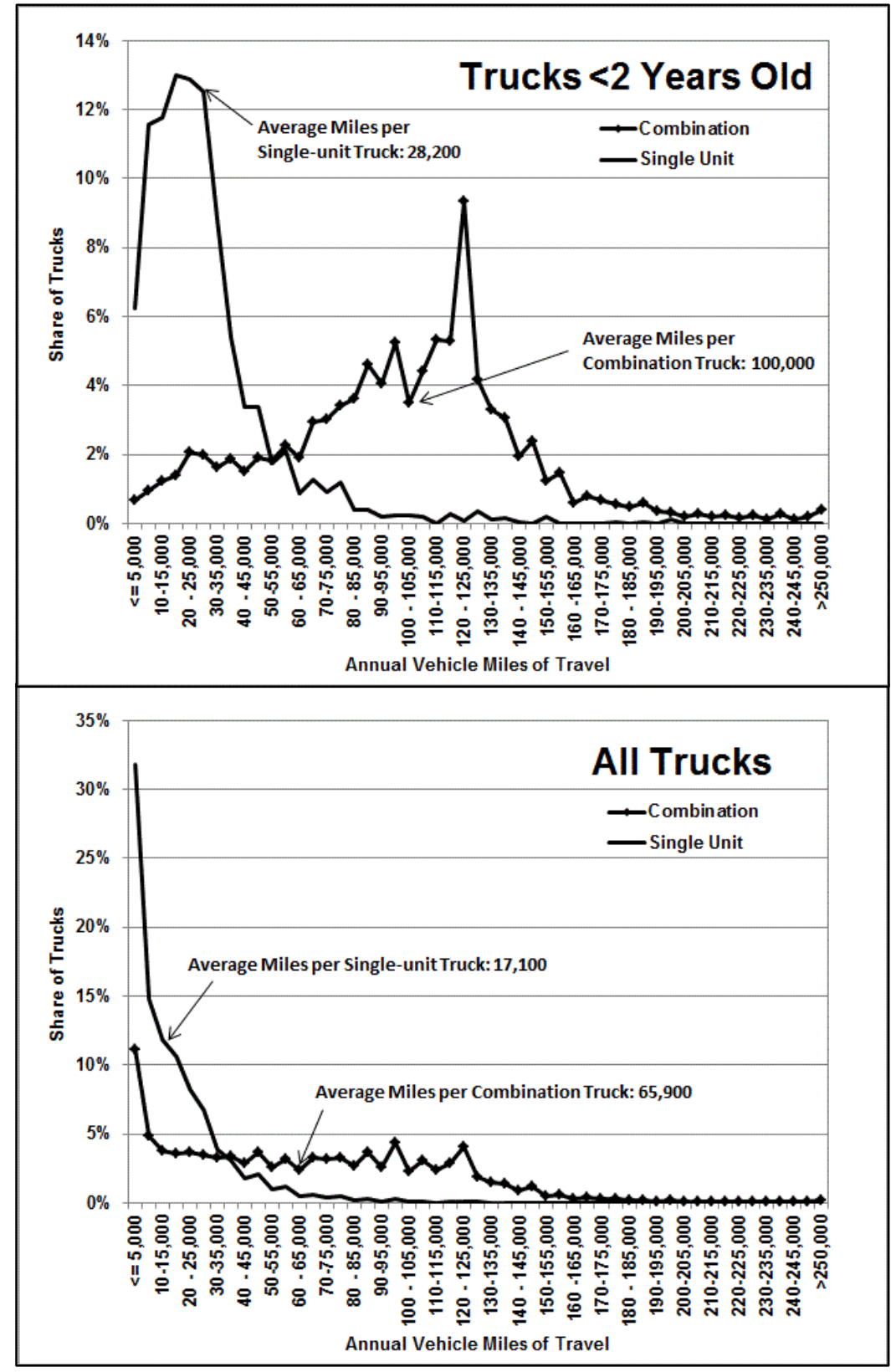

Note: Heavy trucks (class $7 \&$ 8) are greater than 26,000 pounds gross vehicle weight based on the manufacturer's rating.

\section{Source:}

U.S. Department of Commerce, Bureau of the Census, 2002 Vehicle Inventory and Use Survey, Microdata File on CD, 2005. (Additional resources: www.census.gov/svsd/www/tiusview.html) 
The latest Vehicle Inventory and Use Survey asked truck owners if the truck had certain features as permanent equipment on the truck. Some of the features asked about were onboard computers, idle-reduction devices, navigational systems, and Internet access. Of the 2.3 million heavy trucks (class 7 \& 8) in the United States, nearly $10 \%$ were equipped with onboard computers that had communication capabilities and another $5 \%$ had onboard computers without communication capabilities. Six percent of heavy trucks were equipped with idle-reducing technology. Navigational systems and Internet access were available in less than one percent of heavy trucks.

Figure 5.2. Share of Heavy Trucks with Selected Electronic Features, 2002

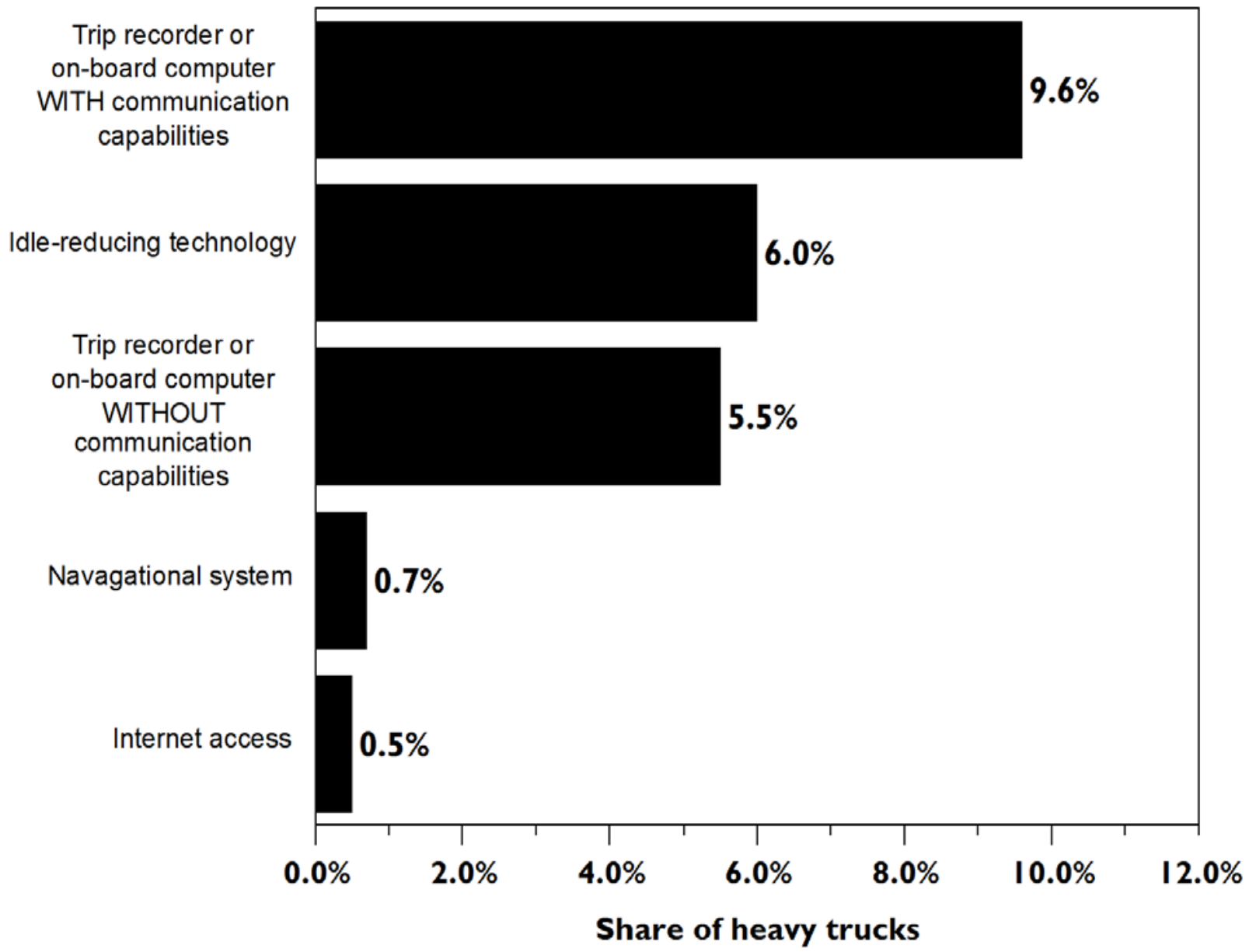

Note: Heavy trucks (class $7 \&$ 8) are greater than 26,000 pounds gross vehicle weight based on the manufacturer's rating.

\section{Source:}

U.S. Department of Commerce, Bureau of the Census, 2002 Vehicle Inventory and User Survey, Microdata File on CD, 2005. 


\section{Fuel Economy Study for Class 8 Trucks}

As part of a study sponsored by the U.S. Department of Energy (DOE) Vehicle Technologies Office (VTO), the Oak Ridge National Laboratory (ORNL) in conjunction with several industry partners has collected data and information related to heavy-truck operation in real-world highway environments. The primary objective of the project was to collect real-world performance and spatial data for long-haul operations of Class 8 tractor-trailers from a fleet engaged in normal freight operations. Six model-year 2005 Class 8 trucks from the selected fleet, which operates within a large area of the country extending from the east coast to Mountain Time Zone and from Canada to the US-Mexican border, were instrumented and 60 channels of data were collected for over a year at a rate of $5 \mathrm{~Hz}$ (or 5 readings per second). Those channels included information such as instantaneous fuel rate, engine speed, gear ratio, vehicle speed, and other information read from the vehicle's databus; weather information (wind speed, precipitation, air temperature, etc.) gathered from an on-board weather station; spatial information (latitude, longitude, altitude) acquired from a GPS (Global Positioning System) device; and instantaneous tractor and trailer weight obtained from devices mounted on the six participating tractors and ten trailers. Three of the six instrumented tractors and five of the ten instrumented trailers were mounted with New Generation Single Wide-Based Tires and the others with regular dual tires. Over the duration of this phase of the project (just over a year) the six tractors traveled nearly 700,000 miles.

To find out more about this project, contact Oscar Franzese, franzeseo@ornl.gov, 865-9461304. The final report on this project is available on-line at: cta.ornl.gov/cta/Publications/Reports/ORNL_TM_2008-122.pdf. 
The type of terrain a truck is traveling on can cause significant differences in fuel efficiency. This study (see page 5-13 for project description) shows fuel economy on severe upslopes is less than half that on flat terrain. On severe downslopes, the fuel economy was two times higher than on flat terrain.

Table 5.10

Effect of Terrain on Class 8 Truck Fuel Economy

\begin{tabular}{|c|c|c|c|c|c|}
\hline \multirow[b]{2}{*}{ Type of terrain } & \multirow[b]{2}{*}{$\begin{array}{c}\text { Share of data } \\
\text { records }\end{array}$} & \multicolumn{4}{|c|}{ Average fuel efficiency (mpg) } \\
\hline & & $\begin{array}{c}\text { All } \\
\text { trucks }\end{array}$ & $\begin{array}{c}\text { Tractors } \\
\text { with dual } \\
\text { tires }\end{array}$ & $\begin{array}{c}\text { Tractors } \\
\text { with single } \\
\text { (wide) tires }\end{array}$ & $\begin{array}{c}\text { Difference } \\
\text { between dual } \\
\text { and single } \\
\text { tires (percent) }\end{array}$ \\
\hline Severe upslope (>4\%) & $0.7 \%$ & 2.90 & 2.86 & 2.94 & $2.91 \%$ \\
\hline Mild upslope (1\% to $4 \%$ ) & $13.2 \%$ & 4.35 & 4.25 & 4.44 & $4.35 \%$ \\
\hline Flat terrain (1\% to $1 \%)$ & $72.4 \%$ & 7.33 & 7.08 & 7.58 & $7.13 \%$ \\
\hline Mild downslope (-4\% to $-1 \%)$ & $12.6 \%$ & 15.11 & 14.64 & 15.57 & $6.36 \%$ \\
\hline Severe downslope $(<-4 \%)$ & $1.1 \%$ & 23.5 & 21.82 & 25.3 & $15.97 \%$ \\
\hline
\end{tabular}

\section{Source:}

Capps, Gary, Oscar Franzese, Bill Knee, M.B. Lascurain, and Pedro Otaduy. Class-8 Heavy Truck Duty Cycle Project Final Report, ORNL/TM-2008/122, Oak Ridge National Laboratory, Oak Ridge, TN, December 2008. (Additional resources: cta.ornl.gov/cta/Publications/Reports/ORNL_TM_2008-122.pdf) 
This table presents a distribution of distance traveled, fuel consumed, and fuel economy by speed and by type of tires for the vehicles participating in the project (see page 5-13 for project description). The speed bins are divided into 5-mile intervals, going from 0+ mph (i.e., speed > $0.00 \mathrm{mph}$ ) to $85 \mathrm{mph}$, while the four main columns of the table are organized by the type of tires that were mounted on the tractor and trailers. The first row of the table contains information about fuel consumed while the vehicle was idling (i.e., the vehicle was static with the engine on) with the following rows presenting information about the distance traveled, fuel consumed, and fuel economy for each one of the speed intervals. The next-to-the-last row shows the totals for both traveled distances and fuel consumed as well as the overall fuel economy for each tire-combination category. The latter are then used to compute the percentage difference in terms of fuel economy from dual tire tractors and trailers, which is the most common tire setup for large trucks at the present time.

Table 5.11

Fuel Economy for Class 8 Trucks as Function of Speed and Tractor-Trailer Tire Combination

\begin{tabular}{|c|c|c|c|c|c|c|c|c|c|c|c|c|}
\hline \multirow[b]{2}{*}{$\begin{array}{l}\text { Speed } \\
(\mathrm{mph})\end{array}$} & \multicolumn{3}{|c|}{$\begin{array}{l}\text { Dual tire tractor - } \\
\text { dual tire trailer }\end{array}$} & \multicolumn{3}{|c|}{$\begin{array}{c}\text { Dual tire tractor - } \\
\text { single (wide) tire trailer }\end{array}$} & \multicolumn{3}{|c|}{$\begin{array}{c}\text { Single (wide) tire tractor - } \\
\text { dual tire trailer }\end{array}$} & \multicolumn{3}{|c|}{$\begin{array}{l}\text { Single (wide) tire tractor - } \\
\text { single (wide) tire trailer }\end{array}$} \\
\hline & $\begin{array}{c}\text { Distance } \\
\text { traveled } \\
\text { (miles) }\end{array}$ & $\begin{array}{l}\text { Fuel } \\
\text { cons. } \\
\text { (gal) }\end{array}$ & $\begin{array}{c}\text { Fuel } \\
\text { econ. } \\
\text { (MPG) }\end{array}$ & $\begin{array}{c}\text { Distance } \\
\text { traveled } \\
\text { (miles) }\end{array}$ & $\begin{array}{l}\text { Fuel } \\
\text { cons. } \\
\text { (gal) }\end{array}$ & $\begin{array}{c}\text { Fuel } \\
\text { econ. } \\
\text { (MPG) }\end{array}$ & $\begin{array}{c}\text { Distance } \\
\text { traveled } \\
\text { (miles) }\end{array}$ & $\begin{array}{l}\text { Fuel } \\
\text { cons. } \\
\text { (gal) }\end{array}$ & $\begin{array}{c}\text { Fuel } \\
\text { econ. } \\
\text { (MPG) }\end{array}$ & $\begin{array}{c}\text { Distance } \\
\text { traveled } \\
\text { (miles) }\end{array}$ & $\begin{array}{l}\text { Fuel } \\
\text { cons. } \\
\text { (gal) }\end{array}$ & $\begin{array}{c}\text { Fuel } \\
\text { econ. } \\
\text { (MPG) }\end{array}$ \\
\hline Idling & N/A & $1,858.5$ & N/A & N/A & 967.9 & N/A & N/A & $1,676.4$ & N/A & N/A & 706.0 & N/A \\
\hline $0+$ to 5 & 281 & 101.8 & 2.76 & 148 & 50.4 & 2.93 & 368.0 & 124.2 & 3.0 & 156 & 52.8 & 2.96 \\
\hline $5+$ to 10 & 674 & 198.8 & 3.39 & 368 & 103.2 & 3.56 & 808.0 & 245.4 & 3.3 & 331 & 98.8 & 3.35 \\
\hline $10+$ to 15 & 723 & 192.0 & 3.77 & 396 & 98.3 & 4.03 & 848.0 & 216.5 & 3.9 & 343 & 87.0 & 3.95 \\
\hline $15+$ to 20 & 744 & 199.1 & 3.73 & 404 & 100.9 & 4.00 & 882.0 & 221.6 & 4.0 & 361 & 90.5 & 3.98 \\
\hline $20+$ to 25 & 938 & 228.4 & 4.11 & 489 & 113.6 & 4.31 & $1,111.0$ & 244.2 & 4.6 & 462 & 101.1 & 4.57 \\
\hline $25+$ to 30 & 1,178 & 266.9 & 4.41 & 609 & 131.5 & 4.63 & $1,420.0$ & 286.9 & 5.0 & 580 & 117.6 & 4.93 \\
\hline $30+$ to 35 & 1,481 & 336.8 & 4.40 & 753 & 154.2 & 4.88 & $1,774.0$ & 341.1 & 5.2 & 708 & 141.1 & 5.02 \\
\hline $35+$ to 40 & 1,917 & 403.5 & 4.75 & 1,000 & 193.6 & 5.17 & $2,284.0$ & 433.6 & 5.3 & 941 & 184.3 & 5.10 \\
\hline $40+$ to 45 & 2,955 & 584.1 & 5.06 & 1,543 & 285.9 & 5.40 & $3,380.0$ & 603.6 & 5.6 & 1,350 & 254.4 & 5.31 \\
\hline $45+$ to 50 & 4,935 & 907.9 & 5.43 & 2,573 & 447.7 & 5.75 & $5,410.0$ & 872.8 & 6.2 & 2,177 & 360.4 & 6.04 \\
\hline $50+$ to 55 & 9,397 & $1,629.8$ & 5.77 & 4,962 & 811.5 & 6.11 & $10,046.0$ & $1,622.7$ & 6.2 & 3,877 & 625.5 & 6.20 \\
\hline $55+$ to 60 & 20,656 & $3,297.2$ & 6.26 & 11,707 & $1,721.9$ & 6.80 & $22,373.0$ & $3,257.8$ & 6.9 & 8,710 & $1,246.9$ & 6.99 \\
\hline $60+$ to 65 & 38,964 & $5,879.6$ & 6.63 & 21,472 & $2,980.8$ & 7.20 & $34,517.0$ & $4,840.0$ & 7.1 & 14,944 & $2,049.4$ & 7.29 \\
\hline \multicolumn{13}{|c|}{ NOT ADJUSTED FOR TERRAIN: See note below. } \\
\hline $65+$ to 70 & 58,304 & $8,313.2$ & 7.01 & 27,931 & $3,652.2$ & 7.65 & $65,063.0$ & $9,256.4$ & 7.0 & 27,144 & $3,880.1$ & 7.00 \\
\hline $70+$ to 75 & 56,378 & $7,483.2$ & 7.53 & 21,751 & $2,745.5$ & 7.92 & $66,882.0$ & $8,435.6$ & 7.9 & 32,887 & $4,056.1$ & 8.11 \\
\hline $75+$ to 85 & 7,849 & 808.2 & 9.71 & 3,610 & 403.2 & 8.95 & $11,513.0$ & 911.1 & 12.6 & 6,817 & 512.2 & 13.31 \\
\hline Total $^{\mathrm{a}}$ & 207,374 & $30,831.0$ & 6.73 & 99,714 & $13,994.0$ & 7.13 & $228,680.0$ & $31,913.0$ & 7.2 & 101,790 & $13,858.0$ & 7.35 \\
\hline $\begin{array}{l}\text { Percent } \\
\text { increase in } \\
\text { fuel } \\
\text { economy } \\
\text { from dual } \\
\text { tire } \\
\text { trac/trail }\end{array}$ & & & $0.00 \%$ & & & $5.93 \%$ & & & $6.53 \%$ & & & $9.20 \%$ \\
\hline
\end{tabular}

Note: These data were not adjusted to account for the effects of terrain. The increase in fuel economy for speeds above $70 \mathrm{mph}$ is likely due to the vehicle achieving high speeds while traveling down slope. Therefore, this increase in fuel economy is not expected to be characteristic of all travel at these higher speeds.

\section{Source:}

Capps, Gary, Oscar Franzese, Bill Knee, M.B. Lascurain, and Pedro Otaduy. Class-8 Heavy Truck Duty Cycle Project Final Report, ORNL/TM-2008/122, Oak Ridge National Laboratory, Oak Ridge, TN, December 2008. (Additional resources: cta.ornl.gov/cta/Publications/Reports/ORNL_TM_2008-122.pdf)

a Total fuel consumed does not include fuel consumed while idling. 
The fuel economy information presented in Table 5.11 is on the upper limits of today's large-truck fleets and is mostly a result of driver training and the extensive vehicle maintenance (including constant tire pressure) to which the fleet company participating in this project adheres. Nevertheless, the results of this extensive test indicate that there are substantial gains in terms of fuel economy for large trucks when single (wide) tires are used in combination with dual tires or alone (best case). Figure 5.3 shows the information from Table 5.11 in a graphical form (bars) and also displays for each speed bin the percentage of the total distance that is traveled at that speed (line). It is possible to observe that above $80 \%$ of the distance traveled by long-haul Class 8 trucks is done at speeds above $55 \mathrm{mph}$. Therefore, any gains in fuel economies at these speeds derived from a given tire combination would have a very large impact on the overall fuel economy of these types of trucks. Figure 5.3 shows that, except for the D-S combination within the $65+$ to $70 \mathrm{mph}$, the combinations with all single (wide) tires perform better and, therefore, obtain the largest overall fuel economy.

Figure 5.3. Class 8 Truck Fuel Economy as a Function of Speed and Tractor-Trailer Tire Combination and Percentage of Total Distance Traveled as a Function of Speed

NOT ADJUSTED FOR TERRAIN: See note below.

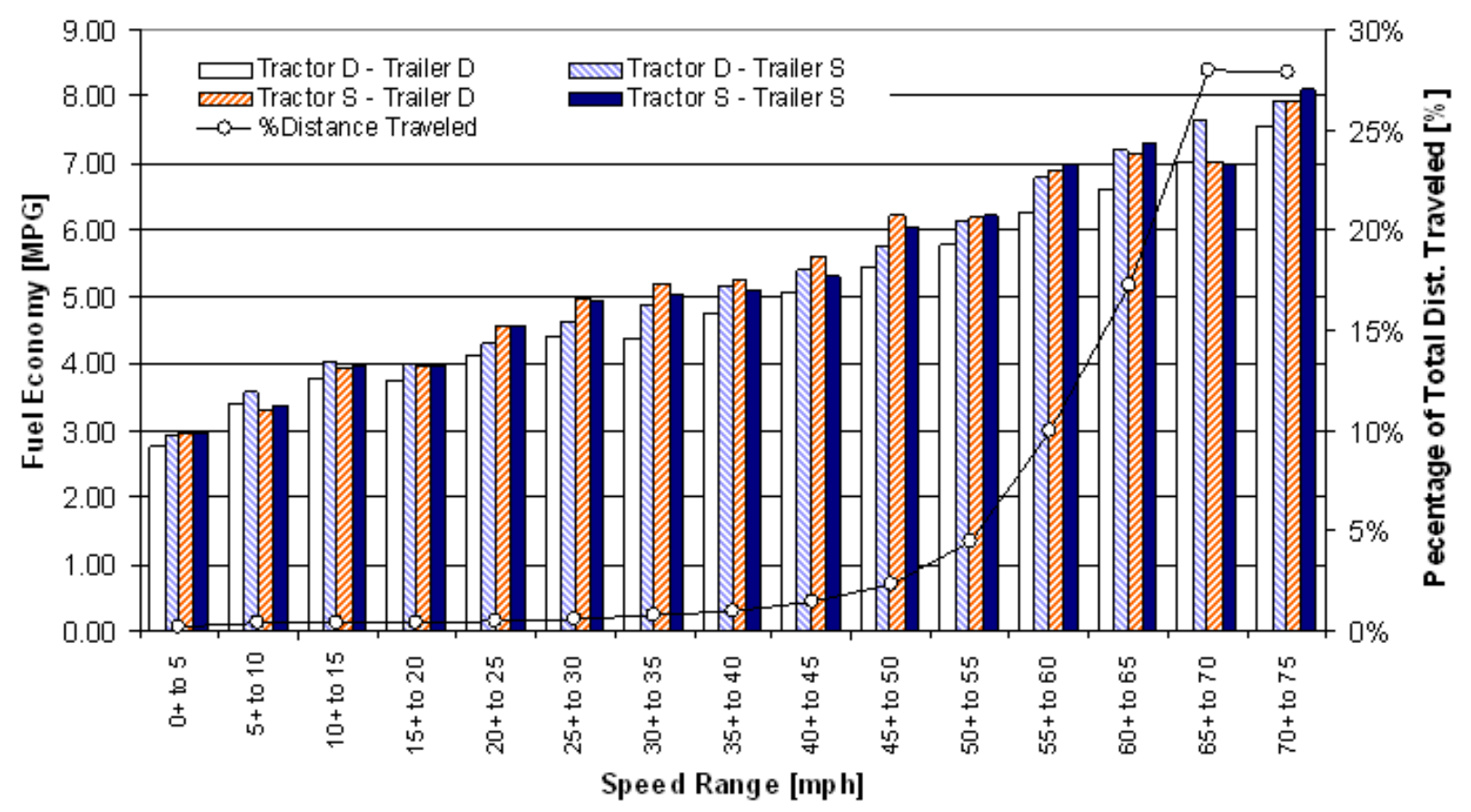

Note: $\mathrm{D}=$ Dual tire. $\mathrm{S}=$ Single (wide) tire.

These data were not adjusted to account for the effects of terrain. The increase in fuel economy for speeds above 70 mph is likely due to the vehicle achieving high speeds while traveling down slope. Therefore, this increase in fuel economy is not expected to be characteristic of all travel at these higher speeds.

\section{Source:}

Capps, Gary, Oscar Franzese, Bill Knee, M.B. Lascurain, and Pedro Otaduy. Class-8 Heavy Truck Duty Cycle Project Final Report, ORNL/TM-2008/122, Oak Ridge National Laboratory, Oak Ridge, TN, December 2008. (Additional resources: cta.ornl.gov/cta/Publications/Reports/ORNL_TM_2008-122.pdf) 
This graph presents for each one of the four tire-combination categories the percent of total fuel that is consumed when traveling at different speeds (bars) as well as the average percent of fuel consumed for each speed bin (line). As opposed to Table 5.11, the total fuel consumed on this graph includes the fuel consumed while idling.

Figure 5.4. Class 8 Truck Percent of Total Fuel Consumed as a Function of Speed and Tractor-Trailer Tire Combination

\section{NOT ADJUSTED FOR TERRAIN: See note below}

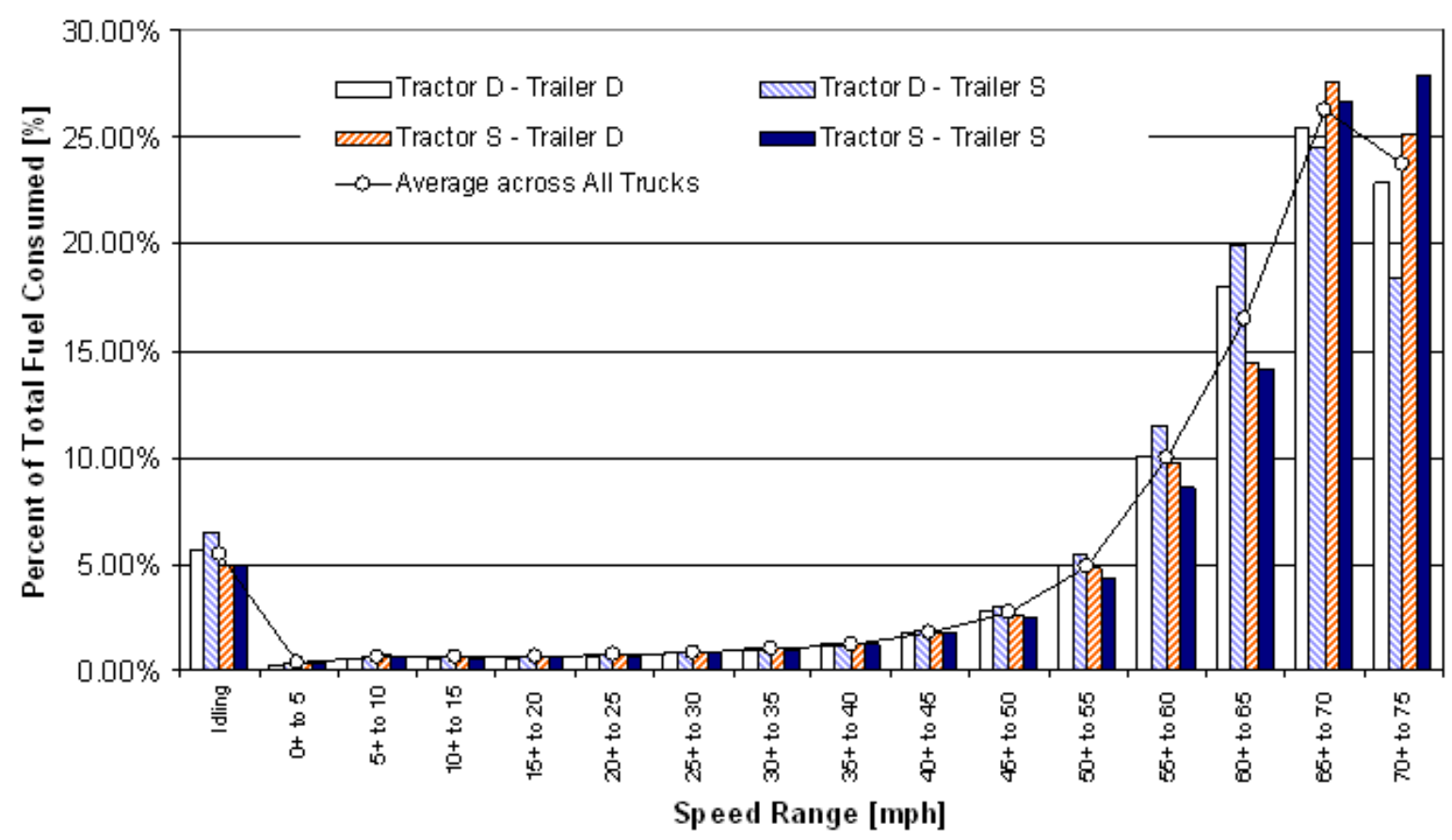

Note: $\mathrm{D}=$ Dual tire. $\mathrm{S}=$ Single (wide) tire.

These data were not adjusted to account for the effects of terrain. The increase in fuel economy for speeds above 70 $\mathrm{mph}$ is likely due to the vehicle achieving high speeds while traveling down slope. Therefore, this increase in fuel economy is not expected to be characteristic of all travel at these higher speeds.

\section{Source:}

Capps, Gary, Oscar Franzese, Bill Knee, M.B. Lascurain, and Pedro Otaduy. Class-8 Heavy Truck Duty Cycle Project Final Report, ORNL/TM-2008/122, Oak Ridge National Laboratory, Oak Ridge, TN, December 2008. (Additional resources: cta.ornl.gov/cta/Publications/Reports/ORNL_TM_2008-122.pdf) 
A typical class 8 truck tractor weighs about 17,000 lb. The powertrain is nearly a quarter of the weight (24\%) while the truck body structure is $19 \%$.

Table 5.12

Class 8 Truck Weight by Component

\begin{tabular}{lrc}
\hline & Pounds & Share of total \\
\hline Wheels and tires & 1,700 & $10 \%$ \\
Chassis/frame & 2,040 & $12 \%$ \\
Drivetrain and suspension & 2,890 & $17 \%$ \\
Misc. accessories/systems & 3,060 & $18 \%$ \\
Truck body structure & 3,230 & $19 \%$ \\
Powertrain & 4,080 & $24 \%$ \\
\hline Total & 17,000 & $100 \%$ \\
\hline
\end{tabular}

Notes:

- Powertrain includes engine and cooling system, transmission and accessories.

- Truck body structure includes cab-in-white, sleeper unit, hood and fairings, interior and glass.

- Miscellaneous accessories/systems include batteries, fuel system, and exhaust hardware.

- Drivetrain and suspension includes drive axles, steer axle, and suspension system.

- Chassis/frame includes frame rails and crossmembers, fifth wheel and brackets. Wheels and tires include a set of 10 aluminum wheels, plus tires.

\section{Source:}

National Academy of Sciences, Technologies and Approaches to Reducing the Fuel Consumption of Medium and Heavy-Duty Vehicles, prepublication copy, March 2010, p. 5-42. 
The gross weight of a vehicle (GVW) is the weight of the empty vehicle plus the weight of the maximum payload that the vehicle was designed to carry. In cars and small light trucks, the difference between the empty weight of the vehicle and the GVW is not significantly different (1,000 to 1,500 lb). The largest trucks and tractor-trailers, however, have a payload capacity share of $200 \%$, which means they can carry $200 \%$ of their empty weight. The medium-sized trucks (truck classes 3-6) have payload capacity shares between 50\% and 100\%.

Table 5.13

Gross Vehicle Weight vs. Empty Vehicle Weight

\begin{tabular}{|c|c|c|c|c|c|}
\hline Vehicle description & Truck class & $\begin{array}{l}\text { Gross vehicle } \\
\text { weight range } \\
\text { (pounds) }\end{array}$ & $\begin{array}{l}\text { Empty vehicle } \\
\text { weight range } \\
\text { (pounds) }\end{array}$ & $\begin{array}{l}\text { Maximum } \\
\text { payload } \\
\text { capacity } \\
\text { (pounds) }\end{array}$ & $\begin{array}{l}\text { Payload } \\
\text { capacity share } \\
\text { (percent of } \\
\text { empty weight) }\end{array}$ \\
\hline Cars & & $3,200-6,000$ & $2,400-5,000$ & 1,000 & $20 \%$ \\
\hline $\begin{array}{l}\text { Minivans, small SUVs, } \\
\text { small pick-ups }\end{array}$ & 1 & $4,000-2,400$ & $3,200-4,500$ & 1,500 & $33 \%$ \\
\hline $\begin{array}{l}\text { Large SUVs, standard pick- } \\
\text { ups }\end{array}$ & $2 a$ & $6,001-8,500$ & $4,500-6,000$ & 2,500 & $40 \%$ \\
\hline $\begin{array}{l}\text { Large SUVs, standard pick- } \\
\text { ups }\end{array}$ & $2 b$ & 8,501-10,000 & $5,000-6,300$ & 3,700 & $60 \%$ \\
\hline $\begin{array}{l}\text { Utility van, multi- purpose, } \\
\text { mini-bus, step van }\end{array}$ & 3 & $10,001-14,000$ & $7,650-8,750$ & 5,250 & $60 \%$ \\
\hline $\begin{array}{l}\text { City delivery, parcel } \\
\text { delivery, large walk-in, } \\
\text { bucket, landscaping }\end{array}$ & 4 & $14,001-16,000$ & 7,650-8,750 & 7,250 & $80 \%$ \\
\hline $\begin{array}{l}\text { City delivery, parcel } \\
\text { delivery, large walk-in, } \\
\text { bucket }\end{array}$ & 5 & $16,001-19,500$ & $9,500-10,000$ & 8,700 & $80 \%$ \\
\hline $\begin{array}{l}\text { City delivery, school bus, } \\
\text { large walk-in, bucket }\end{array}$ & 6 & $19,501-26,000$ & $11,500-14,500$ & 11,500 & $80 \%$ \\
\hline $\begin{array}{l}\text { City bus, furniture, } \\
\text { refrigerated, refuse, fuel } \\
\text { tanker, dump, tow, concrete, } \\
\text { fire engine, tractor-trailer }\end{array}$ & 7 & $26,001-33,000$ & $11,500-14,500$ & 18,500 & $125 \%$ \\
\hline $\begin{array}{l}\text { Refuse, concrete, furniture, } \\
\text { city bus, tow, fire engine } \\
\text { (straight trucks) }\end{array}$ & $8 a$ & $33,001-80,000$ & $20,000-26,000$ & 54,000 & $200 \%$ \\
\hline $\begin{array}{l}\text { Tractor-trailer: van, } \\
\text { refrigerated, bulk tanker, flat } \\
\text { bed (combination trucks) }\end{array}$ & $8 b$ & $33,001-80,000$ & $20,000-26,000$ & 54,000 & $200 \%$ \\
\hline
\end{tabular}

\section{Source:}

National Academy of Sciences, Technologies and Approaches to Reducing the Fuel Consumption of Medium and Heavy-Duty Vehicles, prepublication copy, March 2010, pp. 2-2 and 5-42. 
According to weigh-in-motion data collected by fifteen states, the majority of 5-axle tractor-trailers on the road weigh between 33,000 and 73,000 lb. Eleven percent of the tractor-trailers had weight recorded around 72,800 $\mathrm{lb}$ and $10 \%$ around $68,300 \mathrm{lb}$. Another $10 \%$ of tractor-trailers were on the lighter end of the scale - around 37,500

$\mathrm{lb}$. These data show that only a small percent of trucks on the road are near the maximum roadway gross vehicle weight of 80,000 lb. Thus, most trucks are filling the trailer space to capacity (cubing-out) before they reach the maximum weight limit (weighing-out).

Figure 5.5. Distribution of Class 8 Trucks by On-Road Vehicle Weight, 2008a

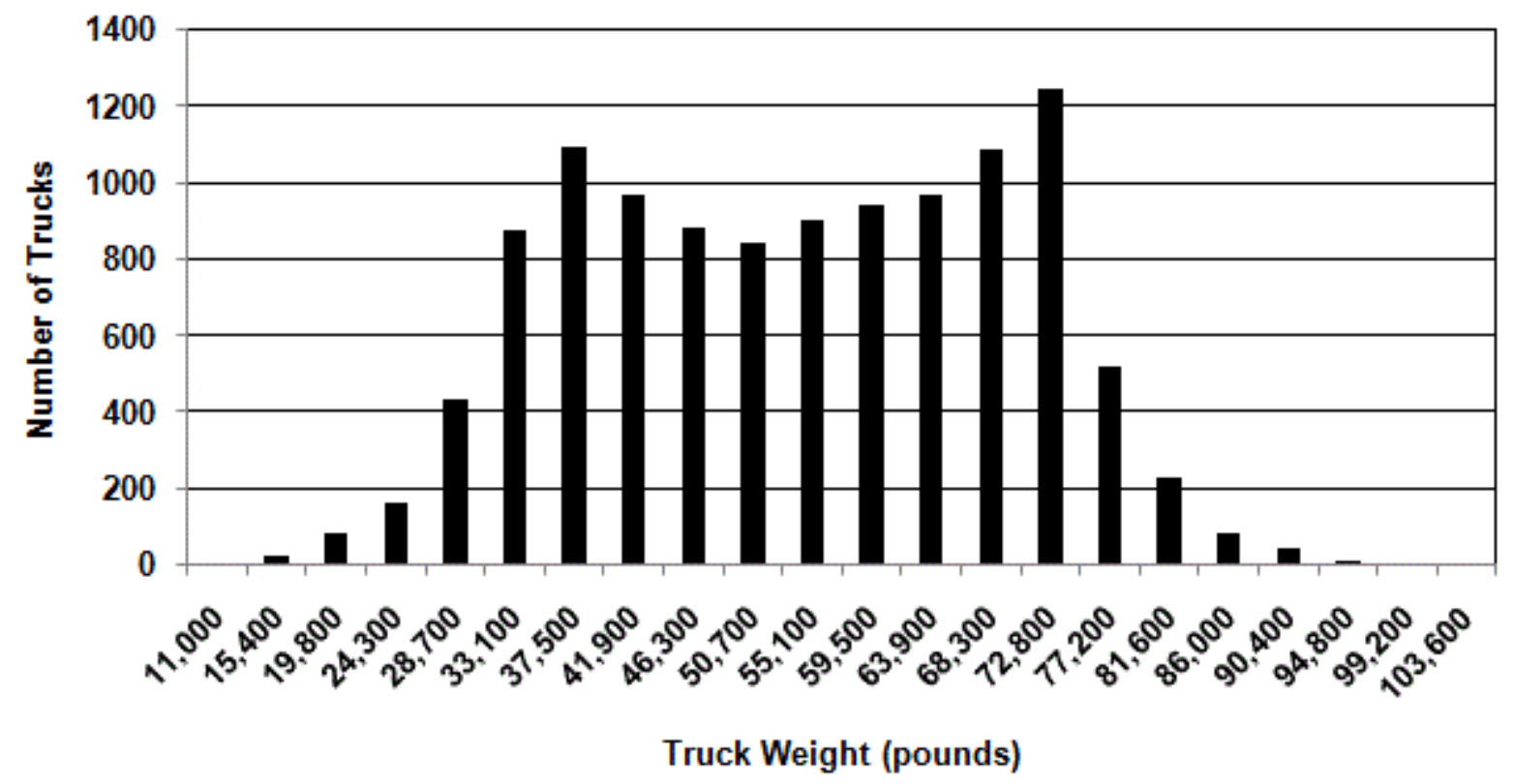

Note: Data are from these 15 States: California, Connecticut, Florida, Georgia, Hawaii, Iowa, Minnesota, Missouri, Montana, North Carolina, Oregon, Pennsylvania, South Dakota, Texas, and Washington.

\section{Source:}

National Academy of Sciences, Technologies and Approaches to Reducing the Fuel Consumption of Medium and Heavy-Duty Vehicles, prepublication copy, March 2010, p. 5-45. Original source: Federal Highway Administration, Vehicle Travel Information System, 2008.

a Study reported data on 5-axle tractor-trailers which are class 8 trucks. Single-unit class 8 trucks were not considered in the study. 


\section{Commodity Flow Survey}

The Commodity Flow Survey (CFS) designed to provide data on the flow of goods and materials by mode of transport. The survey was first conducted in various years from 1963 to 1977, and was again conducted in 1993, 1997, 2002, 2007, and 2012 with improvements in methodology, sample size, and scope. Data collection for the 2017 survey began in late 2016. It is a shipper-based survey which covers business establishments from these industries:

- Mining

- Manufacturing

- Wholesale trade

- Select Retail and Services

Industries not covered by CFS include transportation, construction, most retail and services industries, farms, fisheries, foreign establishments, and most government-owned establishments. Before 1993 data were collected only on the principal mode of travel, but after that time all modes of a shipment were captured in the data.

The CFS is a joint effort of the Bureau of Transportation Statistics and the U.S. Census Bureau. Additional information on the survey can be found at: www.rita.dot.gov/bts/sites/rita.dot.gov.bts/files/publications/commodity_flow_survey/index.html or www.census.gov/econ/cfs/ 
Industries covered by the 2012 Commodity Flow Survey (CFS) shipped goods worth over \$13 trillion. Compared to the $1993 \mathrm{CFS}$, the value of shipments is up $1.7 \%$ per year and tons shipped are up $0.8 \%$ per year. By value, multiple mode shipments increased 2.8\% per year from 1993 to 2012.

Table 5.14

Value of Goods Shipped in the United States: Comparison of the 1993, 1997, 2002, 2007 and 2012 Commodity Flow Surveys ${ }^{a}$

\begin{tabular}{|c|c|c|c|c|c|c|}
\hline & $\begin{array}{c}1993 \\
\text { (billion } \\
2012 \text { dollars) }\end{array}$ & $\begin{array}{c}1997 \\
\text { (billion } \\
2012 \text { dollars) }\end{array}$ & $\begin{array}{c}2002 \\
\text { (billion } \\
2012 \text { dollars) }\end{array}$ & $\begin{array}{c}2007 \\
\text { (billion } \\
2012 \text { dollars) }\end{array}$ & $\begin{array}{c}2012 \\
\text { (billion } \\
\text { dollars) }\end{array}$ & $\begin{array}{c}\text { Average } \\
\text { annual } \\
\text { percent } \\
\text { change } \\
(1993-2012)\end{array}$ \\
\hline All modes & $10,106.6$ & $9,933.3$ & $10,716.8$ & $12,938.9$ & $13,852.1$ & $1.7 \%$ \\
\hline Single modes & $8,542.3$ & $8,181.8$ & $8,996.6$ & $10,562.8$ & $11,900.4$ & $1.8 \%$ \\
\hline Truck $^{b}$ & $7,612.4$ & $7,126.0$ & 7,957.3 & $9,230.4$ & 10,132.2 & $1.5 \%$ \\
\hline For-hire truck & $4,538.0$ & $4,150.4$ & $4,794.9$ & $5,487.5$ & $6,504.6$ & $1.9 \%$ \\
\hline Private truck & $3,035.4$ & 2,913.2 & $3,120.8$ & $3,742.8$ & $3,627.6$ & $0.9 \%$ \\
\hline Rail & 427.7 & 457.2 & 396.8 & 483.3 & 473.1 & $0.5 \%$ \\
\hline Water & 106.6 & 108.5 & 114.0 & 127.2 & 301.6 & $5.6 \%$ \\
\hline Inland water & 70.4 & 77.1 & 73.3 & 100.8 & 218.9 & $6.2 \%$ \\
\hline Great Lakes & c & 2.2 & 1.1 & c & 0.4 & c \\
\hline Deep sea & 34.2 & 29.2 & 39.6 & 25.5 & 59.9 & $3.0 \%$ \\
\hline Multiple waterways & c & d & d & d & 22.3 & c \\
\hline Air (includes truck and air) & 240.4 & 327.7 & 338.1 & 279.4 & 450.6 & $3.4 \%$ \\
\hline Pipeline $^{\mathrm{e}}$ & 155.4 & 162.4 & 190.4 & 442.5 & 542.9 & $6.8 \%$ \\
\hline Multiple modes & $1,145.4$ & $1,353.1$ & $1,377.3$ & $2,067.1$ & $1,950.8$ & $2.8 \%$ \\
\hline Parcel, U.S.P.S. or courier & 973.7 & $1,224.4$ & $1,260.6$ & $1,729.5$ & $1,688.2$ & $2.9 \%$ \\
\hline Truck and rail & 143.6 & 108.3 & 89.2 & 207.3 & 224.8 & $2.4 \%$ \\
\hline Truck and water & 16.2 & 11.8 & 18.3 & 64.7 & 29.0 & $3.1 \%$ \\
\hline Rail and water & 6.4 & 2.5 & 4.2 & 15.4 & 8.0 & $1.2 \%$ \\
\hline Other multiple modes & 5.6 & 6.1 & 4.9 & 50.2 & 0.7 & $-10.3 \%$ \\
\hline $\begin{array}{l}\text { Other and unknown } \\
\text { modes }\end{array}$ & 418.8 & 398.5 & 342.8 & 309.1 & 1.0 & $-27.2 \%$ \\
\hline
\end{tabular}

Source:

U.S. Department of Transportation, Bureau of Transportation Statistics and U.S. Department of Commerce, Bureau of the Census, 1993, 1997, 2002, 2007, and 2012 Commodity Flow Surveys, Table 1a. (Additional resources: http://www.census.gov/econ/cfs/historical_data.html)

a Detail may not add to total because of rounding.

b "Truck" as a single mode includes shipments which went by private truck only, for-hire truck only, or a combination of private truck and for-hire truck.

${ }^{c}$ Denotes data do not meet publication standards because of high sampling variability or poor response quality.

d Data are not available.

e CFS data for pipeline exclude most shipments of crude oil. 
Industries covered by the 2012 Commodity Flow Survey (CFS) shipped over 11 billion tons of goods nationwide. Nearly three-quarters of the freight tonnage was shipped by truck.

Table 5.15

Tons of Freight in the United States: Comparison of the 1993, 1997, 2002, 2007 and 2012 Commodity Flow Surveys ${ }^{\mathrm{a}}$

\begin{tabular}{|c|c|c|c|c|c|c|}
\hline & $\begin{array}{c}1993 \\
\text { (millions) }\end{array}$ & $\begin{array}{c}1997 \\
\text { (millions) }\end{array}$ & $\begin{array}{c}2002 \\
\text { (millions) }\end{array}$ & $\begin{array}{c}2007 \\
\text { (millions) }\end{array}$ & $\begin{array}{c}2012 \\
\text { (millions) }\end{array}$ & $\begin{array}{c}\text { Average } \\
\text { annual } \\
\text { percent } \\
\text { change } \\
(1993-2012) \\
\end{array}$ \\
\hline All modes & $9,688.50$ & $11,089.7$ & $11,667.9$ & $12,543.4$ & $11,299.4$ & $0.8 \%$ \\
\hline Single modes & $8,922.30$ & $10,436.5$ & $11,086.7$ & $11,698.1$ & $10,905.5$ & $1.1 \%$ \\
\hline Truck $^{\mathrm{b}}$ & $6,385.9$ & $7,700.7$ & $7,842.8$ & $8,778.7$ & $8,060.2$ & $1.2 \%$ \\
\hline For-hire truck & $2,808.3$ & $3,402.6$ & $3,657.3$ & $4,075.1$ & $4,298.7$ & $2.3 \%$ \\
\hline Private truck & $3,543.5$ & $4,137.3$ & $4,149.7$ & $4,703.6$ & $3,761.3$ & $0.3 \%$ \\
\hline Rail & $1,544.10$ & $1,549.8$ & 1,873.9 & $1,861.3$ & $1,628.5$ & $0.3 \%$ \\
\hline Water & 505.4 & 563.4 & 681.2 & 403.6 & 576.0 & $0.7 \%$ \\
\hline Inland water & 362.5 & 414.8 & 458.6 & 343.3 & 424.5 & $0.8 \%$ \\
\hline Great Lakes & 33 & 38.4 & 38.0 & 17.8 & 31.4 & $-0.3 \%$ \\
\hline Deep sea & 109.9 & 110.2 & 184.6 & 42.5 & 73.0 & $-2.1 \%$ \\
\hline Multiple waterways & c & c & c & c & 47.1 & $c$ \\
\hline Air (includes truck and air) & 3.1 & 4.5 & 3.8 & 3.6 & 4.8 & $2.3 \%$ \\
\hline Pipeline $^{\mathrm{d}}$ & 483.6 & 618.2 & 685.0 & 650.9 & 636.0 & $1.5 \%$ \\
\hline Multiple modes & 225.7 & 216.7 & 216.7 & 573.7 & 357.0 & $2.4 \%$ \\
\hline Parcel, U.S.P.S. or courier & 18.9 & 23.7 & 25.5 & 33.9 & 28.5 & $2.2 \%$ \\
\hline Truck and rail & 40.6 & 54.2 & 43.0 & 225.6 & 213.8 & $9.1 \%$ \\
\hline Truck and water & 68 & 33.2 & 23.3 & 145.5 & 56.7 & $-1.0 \%$ \\
\hline Rail and water & 79.2 & 79.3 & 105.1 & 54.9 & 55.6 & $-1.8 \%$ \\
\hline Other multiple modes & 18.9 & 26.2 & 19.8 & 113.8 & 2.5 & $-10.1 \%$ \\
\hline Other and unknown modes & 540.5 & 436.5 & 364.6 & 271.6 & 36.8 & $-13.2 \%$ \\
\hline
\end{tabular}

\section{Source:}

U.S. Department of Transportation, Bureau of Transportation Statistics and U.S. Department of Commerce, Bureau of the Census, 1993, 1997, 2002, 2007, and 2012 Commodity Flow Survey, Table 1a. (Additional resources: http://www.census.gov/econ/cfs/historical_data.html)

a Detail may not add to total because of rounding.

b "Truck" as a single mode includes shipments which went by private truck only, for-hire truck only, or a combination of private truck and for-hire truck.

c Data are not available.

${ }^{\mathrm{d}}$ CFS data for pipeline exclude most shipments of crude oil. 
Industries covered by the 2012 Commodity Flow Survey (CFS) accounted for 2.9 trillion ton-miles on the nation's highways, railways, waterways, pipelines, and aviation system. Ton-miles increased an average of $1.1 \%$ per year from 1993 to 2012.

Table 5.16

Growth of Ton-Miles in the United States: Comparison of the 1993, 1997, 2002, 2007 and 2012 Commodity Flow Surveys ${ }^{a}$

\begin{tabular}{|c|c|c|c|c|c|c|}
\hline & $\begin{array}{c}1993 \\
\text { (billions) }\end{array}$ & $\begin{array}{c}1997 \\
\text { (billions) }\end{array}$ & $\begin{array}{c}2002 \\
\text { (billions) }\end{array}$ & $\begin{array}{c}2007 \\
\text { (billions) }\end{array}$ & $\begin{array}{c}2012 \\
\text { (billions) }\end{array}$ & $\begin{array}{c}\text { Average } \\
\text { annual } \\
\text { percent } \\
\text { change } \\
\text { (1993-2012) }\end{array}$ \\
\hline All modes & $2,420.90$ & $2,661.4$ & $3,137.9$ & $3,344.7$ & $2,969.5$ & $1.1 \%$ \\
\hline Single modes & $2,136.90$ & $2,383.5$ & $2,867.9$ & $2,894.3$ & $2,697.4$ & $1.2 \%$ \\
\hline Truck $^{\mathrm{b}}$ & 869.5 & $1,023.5$ & $1,255.9$ & $1,342.1$ & $1,247.7$ & $1.9 \%$ \\
\hline For-hire truck & 629 & 741.1 & 959.6 & $1,055.6$ & $1,050.9$ & $2.7 \%$ \\
\hline Private truck & 235.9 & 268.6 & 291.1 & 286.5 & 196.8 & $-0.9 \%$ \\
\hline Rail & 942.6 & $1,022.5$ & $1,261.6$ & $1,344.0$ & $1,211.5$ & $1.3 \%$ \\
\hline Water & 272 & 261.7 & 282.7 & 157.3 & 192.9 & $-1.8 \%$ \\
\hline Inland water & 164.4 & 189.3 & 211.5 & 117.5 & 118.7 & $-1.7 \%$ \\
\hline Great Lakes & 12.4 & 13.4 & 13.8 & 6.9 & 11.0 & $-0.6 \%$ \\
\hline Deep sea & 95.2 & 59.0 & 57.4 & 33.0 & 22.1 & $-7.4 \%$ \\
\hline Multiple waterways & c & c & c & c & 41.0 & c \\
\hline Air (includes truck and air) & 4 & 6.2 & 5.8 & 4.5 & 5.8 & $2.0 \%$ \\
\hline Pipeline $^{\mathrm{d}}$ & c & e & e & e & e & c \\
\hline Multiple modes & 191.5 & 204.5 & 225.7 & 416.6 & 271.8 & $1.9 \%$ \\
\hline Parcel, U.S.P.S. or courier & 13.2 & 18.0 & 19.0 & 28.0 & 22.7 & $2.9 \%$ \\
\hline Truck and rail & 37.7 & 55.6 & 45.5 & 196.8 & 169.5 & $8.2 \%$ \\
\hline Truck and water & 40.6 & 34.8 & 32.4 & 98.4 & 48.6 & $1.0 \%$ \\
\hline Rail and water & 70.2 & 77.6 & 115.0 & 47.1 & 29.2 & $-4.5 \%$ \\
\hline Other multiple modes & c & 18.6 & 13.8 & 46.4 & 1.9 & c \\
\hline Other and unknown modes & 92.6 & 73.4 & 44.2 & 33.8 & 0.3 & $-26.0 \%$ \\
\hline
\end{tabular}

\section{Source:}

U.S. Department of Transportation, Bureau of Transportation Statistics and U.S. Department of Commerce, Bureau of the Census, 1993, 1997, 2002, 2007 and 2012 Commodity Flow Surveys, Table 1a. (Additional resources: http://www.census.gov/econ/cfs/historical_data.html)

a Detail may not add to total because of rounding.

b "Truck" as a single mode includes shipments which went by private truck only, for-hire truck only, or a combination of private truck and for-hire truck.

c Data are not available.

${ }^{\mathrm{d}}$ CFS data for pipeline exclude most shipments of crude oil.

e Denotes data do not meet publication standards because of high sampling variability or poor response quality. 
Industries covered by the 2012 Commodity Flow Survey (CFS) had an average shipment length of 630 miles, a 49\% increase from the 1993 survey. For single mode shipments, air had the highest shipment length in 2012; for multiple modes, truck and water had the highest length.

Table 5.17

Average Miles per Shipment in the United States: Comparison of the 1993, 1997, 2002, 2007 and 2012 Commodity Flow Surveys ${ }^{\text {a }}$

\begin{tabular}{|c|c|c|c|c|c|c|}
\hline & $\begin{array}{r}1993 \\
\text { (miles) } \\
\end{array}$ & $\begin{array}{r}1997 \\
\text { (miles) } \\
\end{array}$ & $\begin{array}{r}2002 \\
\text { (miles) } \\
\end{array}$ & $\begin{array}{r}2007 \\
\text { (miles) } \\
\end{array}$ & $\begin{array}{r}2012 \\
\text { (miles) } \\
\end{array}$ & $\begin{array}{r}\text { Average } \\
\text { annual } \\
\text { percent } \\
\text { change } \\
\text { (1997-2012) }\end{array}$ \\
\hline All modes & 424 & 472 & 546 & 619 & 630 & $2.1 \%$ \\
\hline Single modes & 197 & 184 & 240 & 234 & 262 & $1.5 \%$ \\
\hline Truck $^{\mathrm{b}}$ & 144 & 144 & 173 & 206 & 227 & $2.4 \%$ \\
\hline For-hire truck & 472 & 485 & 523 & 599 & 508 & $0.4 \%$ \\
\hline Private truck & 52 & 53 & 64 & 57 & 58 & $0.6 \%$ \\
\hline Rail & 766 & 769 & 807 & 728 & 805 & $0.3 \%$ \\
\hline Water & c & 482 & 568 & 520 & 908 & c \\
\hline Inland water & c & 177 & 450 & 144 & 275 & c \\
\hline Great Lakes & 534 & 204 & 339 & 657 & 347 & $-2.2 \%$ \\
\hline Deep sea & 1,861 & 1,024 & 664 & 923 & 1,157 & $-2.5 \%$ \\
\hline Multiple waterways & c & c & c & c & 1,034 & c \\
\hline Air (includes truck and air) & 1,415 & 1,380 & 1,919 & 1,304 & 1,295 & $-0.5 \%$ \\
\hline Pipeline $^{\mathrm{d}}$ & c & e & e & e & e & c \\
\hline Multiple modes & 736 & 813 & 895 & 975 & 922 & $1.2 \%$ \\
\hline Parcel, U.S.P.S. or courier & 734 & 813 & 894 & 975 & 922 & $1.2 \%$ \\
\hline Truck and rail & 1,403 & 1,347 & 1,413 & 1,007 & 988 & $-1.8 \%$ \\
\hline Truck and water & 1,417 & 1,265 & 1,950 & 1,429 & 1,562 & $0.5 \%$ \\
\hline Rail and water & 627 & 1,092 & 957 & 1,928 & 1,073 & $2.9 \%$ \\
\hline Other multiple modes & 1,082 & e & e & 1,182 & e & c \\
\hline Other and unknown modes & 229 & 122 & 130 & 116 & 2 & $-22.1 \%$ \\
\hline
\end{tabular}

Source:

U.S. Department of Transportation, Bureau of Transportation Statistics and U.S. Department of Commerce, Bureau of the Census, 1993, 1997, 2002, 2007 and 2012 Commodity Flow Surveys, Table 1a. (Additional resources: http://www.census.gov/econ/cfs/historical_data.html)

${ }^{a}$ Detail may not add to total because of rounding.

b "Truck" as a single mode includes shipments which went by private truck only, for-hire truck only, or a combination of private truck and for-hire truck.

${ }^{\mathrm{c}}$ Data are not available.

${ }^{\mathrm{d}}$ CFS data for pipeline exclude most shipments of crude oil.

${ }^{\mathrm{e}}$ Denotes data do not meet publication standards because of high sampling variability or poor response quality. 


\section{Chapter 6 \\ Alternative Fuel and Advanced Technology Vehicles and Characteristics}

Summary Statistics from Tables in this Chapter

Source

Table 6.1 Alternative fuel vehicles made available, 2015

$2,011,061$

E85

$1,881,500$

$L P G$

2,248

CNG

8,744

Electric

118,560

LNG

Hydrogen

Table 6.11 Number of alternative fuel refuel sites, 2017

59,926

Electric outlets

50,481

$L P G$

3,541

CNG

1,697

Biodiesel

710

Hydrogen

Fuel type abbreviations are used throughout this chapter.

B20 = $20 \%$ biodiesel, $80 \%$ petroleum diesel

CNG $=$ compressed natural gas

E85 = 85\% ethanol, 15\% gasoline

E95 = 95\% ethanol, $5 \%$ gasoline

$\mathrm{H}_{2}=$ hydrogen

$L N G=$ liquefied natural gas

$L P G=$ liquefied petroleum gas 


\section{Alternative Fuels}

The Energy Policy Act of 1992 defines alternative fuels and allows the U.S. Department of Energy (DOE) to add to the list of alternative fuels if the fuel is substantially nonpetroleum, yields substantial energy security benefits, and offers substantial environmental benefits. DOE currently recognizes the following as alternative fuels:

- methanol, ethanol, and other alcohols,

- blends of $85 \%$ or more of alcohol with gasoline,

- natural gas and liquid fuels domestically produced from natural gas,

- liquefied petroleum gas (propane),

- coal-derived liquid fuels,

- hydrogen,

- electricity,

- biodiesel (B100),

- fuels (other than alcohol) derived from biological materials,

- P-series.

\section{Alternative Fuels Data Center}

DOE established the Alternative Fuels Data Center (AFDC) in 1991 to support its work aimed at fulfilling the Alternative Motor Fuels Act directives. Since then, the AFDC has expanded its focus to include all advanced transportation fuels, vehicles, and technologies. The AFDC is operated and managed by the National Renewable Energy Laboratory (NREL) in Golden, Colorado.

The purposes of the AFDC are:

- to gather and analyze information on the fuel consumption, emissions, operation, and durability of alternative fuel vehicles, and

- to provide unbiased, accurate information on alternative fuels and alternative fuel vehicles to government agencies, private industry, research institutions, and other interested organizations.

Much of the AFDC data can be obtained through their website: www.afdc.energy.gov. Several tables and graphs in this chapter contain statistics which were generated by the AFDC. Below are some links to specific areas of the AFDC website.

Alternative \& Advanced Fuels - www.afdc.energy.gov

Alternative Fueling Station Locator - www.afdc.energy.gov/afdc/locator/stations

Alternative \& Advanced Vehicles - www.afdc.energy.gov/fuels

State \& Federal Incentives \& Laws - www.afdc.energy.gov/afdc/laws

Data Analysis \& Trends - www.afdc.energy.gov/data

Tools - www.afdc.energy.gov/tools 
The Energy Information Administration (EIA) is no longer publishing estimates of the number of alternative vehicles in use in the United States. EIA does publish the number of alternative fuel vehicles "made available" each year, beginning in 2004. The alternative fuel vehicles "made available" are estimates from vehicle manufacturer production and companies performing vehicle conversions. The data are more of a proxy for alternative fuel vehicle sales than for vehicle population, but EIA cautions that the data are not actual sales data.

Table 6.1

Estimates of Alternative Fuel Highway Vehicles Made Available, 2004-2015

\begin{tabular}{rrrrrrr}
\hline Year & CNG & Electricity & E85 & Hydrogen & LNG & \multicolumn{1}{c}{ LPG } \\
\hline 2004 & 7,752 & 2,200 & 674,678 & 31 & 136 & 2,150 \\
2005 & 3,304 & 2,281 & 743,948 & 74 & 68 & 700 \\
2006 & 3,128 & 2,715 & $1,011,399$ & 40 & 92 & 473 \\
2007 & 2,487 & 3,152 & $1,115,069$ & 63 & 26 & 356 \\
2008 & 4,440 & 2,802 & $1,175,345$ & 63 & 384 & 695 \\
2009 & 3,770 & 2,255 & 805,777 & 26 & 126 & 861 \\
2010 & 4,973 & 2,229 & $1,484,945$ & 64 & 231 & 747 \\
2011 & 5,674 & 25,382 & $2,116,273$ & 107 & 137 & 1,054 \\
2012 & 7,672 & 46,624 & $2,446,966$ & 56 & 101 & 1,134 \\
2013 & 9,454 & 130,323 & $2,665,470$ & 10 & 344 & 2,700 \\
2014 & 6,662 & 92,594 & $2,433,113$ & 3 & 535 & 1,708 \\
2015 & 8,744 & 118,560 & $1,881,500$ & 2 & 7 & 2,248 \\
\hline
\end{tabular}

Note: "Made available" refers to the supply of warrantied alternative fuel vehicles by manufacturers and aftermarket conversion companies. These do not represent sales.

\section{Source:}

U. S. Department of Energy, Energy Information Administration website, "Alternative Fuel Vehicle Data," www.eia.gov/renewable/afv, August 2017. (Additional resources: www.eia.gov) 
The hybrid share of all light vehicles fell to $2.0 \%$ of all light vehicle sales in 2016. Plug-in vehicles certified for highway use began selling in 2010 and were 0.9\% of the light vehicle market in 2016.

Table 6.2

Hybrid and Plug-In Vehicle Sales, 1999-2016

\begin{tabular}{cccccc}
\hline $\begin{array}{c}\text { Calendar } \\
\text { year }\end{array}$ & $\begin{array}{c}\text { Hybrid vehicle } \\
\text { sales } \\
\text { (thousands) }\end{array}$ & $\begin{array}{c}\text { Plug-in vehicle } \\
\text { sales }^{\mathrm{a}} \\
\text { thousands) }^{\text {thourid }}\end{array}$ & $\begin{array}{c}\text { All light } \\
\text { vehicle sales } \\
\text { (thousands) }\end{array}$ & $\begin{array}{c}\text { Hybrid share } \\
\text { of all light } \\
\text { vehicles }\end{array}$ & $\begin{array}{c}\text { Plug-in share } \\
\text { of all light } \\
\text { vehicles }\end{array}$ \\
\hline 1999 & 0.0 & 0.0 & 16,711 & $0.0 \%$ & $0.0 \%$ \\
2000 & 9.4 & 0.0 & 17,164 & $0.1 \%$ & $0.0 \%$ \\
2001 & 20.3 & 0.0 & 16,950 & $0.1 \%$ & $0.0 \%$ \\
2002 & 36.0 & 0.0 & 16,675 & $0.2 \%$ & $0.0 \%$ \\
2003 & 47.6 & 0.0 & 16,494 & $0.3 \%$ & $0.0 \%$ \\
2004 & 84.2 & 0.0 & 16,737 & $0.5 \%$ & $0.0 \%$ \\
2005 & 205.9 & 0.0 & 16,774 & $1.2 \%$ & $0.0 \%$ \\
2006 & 251.9 & 0.0 & 16,336 & $1.5 \%$ & $0.0 \%$ \\
2007 & 351.1 & 0.0 & 15,867 & $2.2 \%$ & $0.0 \%$ \\
2008 & 315.8 & 0.0 & 13,015 & $2.4 \%$ & $0.0 \%$ \\
2009 & 290.3 & 0.0 & 10,236 & $2.8 \%$ & $0.0 \%$ \\
2010 & 274.6 & 0.3 & 11,394 & $2.4 \%$ & $0.0 \%$ \\
2011 & 266.5 & 17.8 & 12,542 & $2.1 \%$ & $0.1 \%$ \\
2012 & 434.6 & 53.2 & 14,220 & $3.1 \%$ & $0.4 \%$ \\
2013 & 495.5 & 97.1 & 15,279 & $3.2 \%$ & $0.6 \%$ \\
2014 & 452.2 & 118.9 & 16,192 & $2.8 \%$ & $0.7 \%$ \\
2015 & 384.4 & 115.3 & 17,095 & $2.2 \%$ & $0.7 \%$ \\
2016 & 346.9 & 159.6 & 17,169 & $2.0 \%$ & $0.9 \%$ \\
\hline
\end{tabular}

Note: Plug-in vehicle sales include only those vehicles certified for highway use. Small electric carts and neighborhood electric vehicles are excluded.

\section{Sources:}

Hybrid and Electric Vehicle Sales - Compiled by the Transportation Research Center at Argonne National Laboratory, 2017. (Additional resources: www.anl.gov/energy-systems/project/light-duty-electric-drivevehicles-monthly-sales-updates)

All Light Vehicle Sales - Table 3.11.

\footnotetext{
${ }^{a}$ Includes plug-in hybrid-electric vehicles and all-electric vehicles.
} 
Trollybus, heavy rail, and light rail use nearly all alternative fuels. However, the $41.4 \%$ of buses using alternative fuels replace a lot of traditional fuel use. Heavy rail units have the highest average age.

Table 6.3

Alternative Fuel Transit Vehicles, 2016

\begin{tabular}{|c|c|c|c|}
\hline Mode & $\begin{array}{c}\text { Average } \\
\text { age }\end{array}$ & $\begin{array}{c}\text { Percent } \\
\text { powered by } \\
\text { alternative fuels }\end{array}$ & $\begin{array}{c}\text { Number } \\
\text { of } \\
\text { vehicles }\end{array}$ \\
\hline Bus $^{\mathrm{a}}$ & 7.3 & $41.4 \%$ & 63,573 \\
\hline Commuter rail locomotive ${ }^{\mathrm{b}}$ & 21.7 & $9.2 \%$ & 6,278 \\
\hline Commuter rail self-propelled car & 20.7 & $90.6 \%$ & c \\
\hline Ferry Boat & 23.6 & c & 119 \\
\hline Heavy Rail ${ }^{\mathrm{d}}$ & 26.2 & $100.0 \%$ & 10,737 \\
\hline Light Rail ${ }^{\mathrm{e}}$ & 18.4 & $100.0 \%$ & 2,083 \\
\hline Demand Response & 5.8 & $2.3 \%$ & c \\
\hline Trolleybus & 10.3 & $100.0 \%$ & 611 \\
\hline Vanpool & 3.7 & $7.5 \%$ & 13,129 \\
\hline
\end{tabular}

Note: See Glossary for definition of modes, such as paratransit and vanpool. The number of vehicles is a 2015 number.

\section{Source:}

U.S. Department of Transportation, Federal Transit Administration, National Transit Database. (Additional resources: www.transit.dot.gov/ntd)

${ }^{a}$ Includes bus rapid transit and commuter bus vehicles. suburbs.

${ }^{\mathrm{b}}$ Electric car or diesel-propelled railway for urban passenger train service between a central city and adjacent

${ }^{\mathrm{c}}$ Data are not available.

${ }^{\mathrm{d}}$ An electric railway with the capacity for a heavy volume of traffic.

e Typically an electric railway with a light volume traffic capacity with power drawn from an overhead electric line. 
Table 6.4

E85 Flex-Fuel Vehicles Available by Manufacturer, Model Year 2017

\begin{tabular}{|c|c|c|}
\hline Model & Type & Emission class \\
\hline Audi A5 Cabriolet quattro & Sedan/Wagon & LEV II ULEV, Tier 3 Bin 125 \\
\hline Audi A5 quattro & Sedan/Wagon & LEV II ULEV \\
\hline Audi Q5 AWD & SUV & LEV II ULEV, Tier 2 Bin 5 \\
\hline Chevrolet Equinox AWD & SUV & TBD \\
\hline Chevrolet Equinox FWD & SUV & TBD \\
\hline Chevrolet Impala & Sedan/Wagon & TBD \\
\hline Chevrolet Silverado C15 2WD 4.3L & Truck & TBD \\
\hline Chevrolet Silverado C15 2WD 5.3L & Truck & TBD \\
\hline Chevrolet Silverado K15 4WD 4.3L & Truck & TBD \\
\hline Chevrolet Silverado K15 4WD 5.3L & Truck & TBD \\
\hline Chevrolet Suburban C1500 2WD & SUV & TBD \\
\hline Chevrolet Suburban K1500 4WD & SUV & TBD \\
\hline Chevrolet Tahoe C1500 2WD & SUV & TBD \\
\hline Chevrolet Tahoe K1500 4WD & SUV & TBD \\
\hline Chrysler 200 3.6L & Sedan/Wagon & TBD \\
\hline Chrysler 200 2.4L & Sedan/Wagon & TBD \\
\hline Chrysler 200 AWD & Sedan/Wagon & TBD \\
\hline Dodge Grand Caravan & Van & TBD \\
\hline Dodge Journey & SUV & TBD \\
\hline Ford Escape FWD FFV & SUV & TBD \\
\hline Ford Explorer 2WD FFV & SUV & TBD \\
\hline Ford Explorer AWD FFV & SUV & TBD \\
\hline Ford Transit Connect Van FFV & Van & TBD \\
\hline Ford Transit Connect Wagon FFV & Sedan/Wagon & TBD \\
\hline Ford Transit Connect Wagon LWB FFV & Sedan/Wagon & TBD \\
\hline Ford Transit T150 Wagon FFV & Van & TBD \\
\hline GMC Sierra C15 2WD & Truck & TBD \\
\hline GMC Sierra C15 2WD & Truck & TBD \\
\hline GMC Sierra K15 4WD & Truck & TBD \\
\hline GMC Sierra K15 4WD & Truck & TBD \\
\hline GMC Terrain FWD & SUV & TBD \\
\hline GMC Yukon C1500 2WD & SUV & TBD \\
\hline GMC Yukon C1500 XL 2WD & SUV & TBD \\
\hline GMC Yukon K1500 4WD & SUV & TBD \\
\hline GMC Yukon K1500 XL 4WD & SUV & TBD \\
\hline Jeep Cherokee 4WD & SUV & TBD \\
\hline Jeep Cherokee FWD & SUV & TBD \\
\hline Jeep Renegade 2WD & SUV & TBD \\
\hline Mercedes-Benz CLA250 4matic & Sedan/Wagon & TBD \\
\hline Mercedes-Benz GLA250 4matic & SUV & TBD \\
\hline Mercedes-Benz GLE350 4matic & SUV & TBD \\
\hline Ram 1500 2WD & Truck & TBD \\
\hline Ram 1500 4WD & Truck & TBD \\
\hline Toyota Tundra 2WD FFV & Truck & TBD \\
\hline Toyota Tundra 4WD FFV & Truck & TBD \\
\hline
\end{tabular}

Note: LEV = low emission vehicle. ILEV = inherently low emission vehicle. ULEV = ultra low emission vehicle. $\mathrm{ZEV}=$ zero emission vehicle. TLEV = transitional low emission vehicle. SULEV = super ultra low emission vehicle. See Chapter 12 for details on emissions. TBD = to be determined.

Source:

U.S. Department of Energy, Alternative Fuels Data Center website, “Alternative Fuel and Advanced Vehicle Search,” www.afdc.energy.gov/vehicles/search, August 2017. (Additional resources: www.afdc.energy.gov) 
Table 6.5

B20, CNG, and LPG Vehicles Available by Manufacturer, Model Year 2017

\begin{tabular}{|c|c|c|c|}
\hline Model & Fuel & Vehicle Type & Emission Class \\
\hline Chevrolet Colorado 2WD & B20 & Truck & Tier 3 Bin 125, LEV III ULEV125 \\
\hline Chevrolet Colorado 4WD & B20 & Truck & Tier 3 Bin 125, LEV III ULEV125 \\
\hline Chevrolet Express 2500 & B20 & Van & TBD \\
\hline Chevrolet Silverado 2500 HD 2WD/4WD & B20 & Truck & TBD \\
\hline Ford Super Duty F-250 & B20 & Truck & TBD \\
\hline Ford Transit T150 Wagon & B20 & Van & TBD \\
\hline GMC Canyon 2WD & B20 & Truck & Tier 3 Bin 125, LEV III ULEV125 \\
\hline GMC Canyon 4WD & B20 & Truck & Tier 3 Bin 125, LEV III ULEV125 \\
\hline GMC Savanna 2500 & B20 & Van & TBD \\
\hline GMC Sierra 2500 Denali HD 2WD/4WD & B20 & Truck & TBD \\
\hline GMC Sierra 2500 HD 2WD/4WD & B20 & Truck & TBD \\
\hline Jaguar F-Pace & B20 & SUV & Tier 3 Bin 160, LEV III LEV160 \\
\hline Jaguar XE & B20 & Sedan/Wagon & Tier 3 Bin 160, LEV III LEV160 \\
\hline Jaguar XE AWD & B20 & Sedan/Wagon & Tier 3 Bin 160, LEV III LEV160 \\
\hline Jaguar XF AWD & B20 & Sedan/Wagon & Tier 3 Bin 160, LEV III LEV160 \\
\hline Jeep Grand Cherokee 2WD/4WD & B20 & SUV & TBD \\
\hline Land Rover Range Rover Sport & B20 & SUV & TBD \\
\hline Nissan Titan HD 2WD/4WD & B20 & Truck & TBD \\
\hline Ram 1500 HD & B20 & Truck & TBD \\
\hline Ram 2500 HD & B20 & Truck & TBD \\
\hline Ram Promaster 1500/2500 & $\mathrm{B} 20$ & Van & TBD \\
\hline Chevrolet Express 2500 & CNG & Van & TBD \\
\hline Chevrolet Silverado 2500 HD 2WD/4WD & CNG & Truck & TBD \\
\hline Ford F-150 & CNG & Truck & TBD \\
\hline Ford Transit 150/250 & CNG & Van & TBD \\
\hline Ford Transit Connect & CNG & Van & TBD \\
\hline GMC Savanna 2500 & CNG & Van & TBD \\
\hline GMC Sierra 2500 HD 2WD/4WD & CNG & Truck & TBD \\
\hline Ram $2500 \mathrm{HD}$ & CNG & Truck & TBD \\
\hline Chevrolet Express 2500 & LPG & Van & TBD \\
\hline Chevrolet Silverado 2500 HD 2WD/4WD & LPG & Truck & TBD \\
\hline Ford F-150 & LPG & Truck & TBD \\
\hline Ford Super Duty F-250 & LPG & Truck & TBD \\
\hline Ford Transit 150/250 & LPG & Van & TBD \\
\hline Ford Transit Connect & LPG & Van & TBD \\
\hline GMC Savanna 2500 & LPG & Van & TBD \\
\hline GMC Sierra 2500 HD 2WD/4WD & LPG & Truck & TBD \\
\hline Ram 2500 HD & LPG & Truck & TBD \\
\hline
\end{tabular}

Note: $\mathrm{LEV}=$ low emission vehicle. ILEV = inherently low emission vehicle. ULEV = ultra low emission vehicle. $\mathrm{ZEV}=$ zero emission vehicle. TLEV = transitional low emission vehicle. SULEV = super ultra low emission vehicle. See Chapter 12 for details on emissions. TBD = to be determined.

\section{Source:}

U.S. Department of Energy, Alternative Fuels Data Center website, "Alternative Fuel and Advanced Vehicle Search,” www.afdc.energy.gov/vehicles/search, August 2017. (Additional resources: www.afdc.energy.gov) 
Table 6.6

Hybrid-Electric Vehicles Available by Manufacturer, Model Year 2017

\begin{tabular}{|c|c|c|}
\hline Model & Vehicle Type & Emission Class \\
\hline Acura MDX Hybrid & SUV & TBD \\
\hline Acura NSX Hybrid & Two-Seater & LEV III ULEV125, Tier 3 Bin 125 \\
\hline Acura RLX Hybrid & Sedan/Wagon & Tier 3 Bin 30, LEV-III SULEV30 \\
\hline BMW I8 & Two-Seater & Tier 3 Bin 125, LEV-II ULEV \\
\hline Chevrolet Malibu Hybrid & Sedan/Wagon & Tier 3 Transitional Bin 110 \\
\hline Chevrolet Silverado 15 Hybrid 2WD & Truck & LEV III ULEV125, Bin 5 \\
\hline Chevrolet Silverado 15 Hybrid 4WD & Truck & Bin 5, LEV III ULEV125 \\
\hline Ferrari LaFerrari Aperta & Two-Seater & Tier 3 Bin 125 \\
\hline Ford C-MAX Hybrid & Sedan/Wagon & TBD \\
\hline Ford Fusion Hybrid FWD & Sedan/Wagon & LEV-III SULEV30, Tier 3 Bin 30 \\
\hline GMC Sierra 15 Hybrid 2WD & Truck & LEV III ULEV125, Bin 5 \\
\hline GMC Sierra 15 Hybrid 4WD & Truck & LEV III ULEV125, Bin 5 \\
\hline Honda Accord Hybrid & Sedan/Wagon & LEV-III SULEV30/PZEV, Tier 3 \\
\hline Hyundai Ioniq & Sedan/Wagon & TBD \\
\hline Hyundai Ioniq Blue & Sedan/Wagon & TBD \\
\hline Hyundai Sonata Hybrid & Sedan/Wagon & LEV III ULEV125, Tier 3 Bin 125 \\
\hline Hyundai Sonata Hybrid SE & Sedan/Wagon & Tier 3 Bin 125, LEV III ULEV125 \\
\hline Infiniti Q50 Hybrid & Sedan/Wagon & Tier 3 Bin 125, LEV II ULEV \\
\hline Infiniti Q50 Hybrid AWD & Sedan/Wagon & Tier 3 Bin 125, LEV II ULEV \\
\hline Infiniti Q60 Hybrid AWD & SUV & LEV II ULEV, Tier 3 Bin 125 \\
\hline Infiniti Q60 Hybrid FWD & SUV & Tier 3 Bin 125, LEV II ULEV \\
\hline Infiniti Q70 Hybrid & Sedan/Wagon & Tier 3 Bin 125, LEV II ULEV \\
\hline Kia Niro & Sedan/Wagon & TBD \\
\hline Kia Niro FE & Sedan/Wagon & TBD \\
\hline Kia Niro Touring & Sedan/Wagon & TBD \\
\hline Kia Optima Hybrid & Sedan/Wagon & LEV III ULEV125, Tier 3 Bin 125 \\
\hline Lexus CT 200h & Sedan/Wagon & Tier 3 Bin 30, LEV-III SULEV30 \\
\hline Lexus ES 300h & Sedan/Wagon & TBD \\
\hline Lexus GS 450h & Sedan/Wagon & TBD \\
\hline Lexus NX 300h & SUV & Tier 3 Bin 30, LEV-III SULEV30 \\
\hline Lexus RX 450h & SUV & TBD \\
\hline Lincoln MKZ Hybrid FWD & Sedan/Wagon & TBD \\
\hline Nissan Rogue AWD Hybrid & SUV & TBD \\
\hline Nissan Rogue FWD Hybrid & SUV & TBD \\
\hline Nissan Rogue SV FWD Hybrid & SUV & TBD \\
\hline Toyota Avalon Hybrid & Sedan/Wagon & Tier 3 Bin 30, LEV-III \\
\hline Toyota Camry Hyrid LE & Sedan/Wagon & TBD \\
\hline Toyota Camry Hyrid XLE/SE & Sedan/Wagon & TBD \\
\hline Toyota Highlander Hybrid 4WD & SUV & TBD \\
\hline Toyota Highlander Hybrid 4WD LE Plus & SUV & TBD \\
\hline Toyota Prius & Sedan/Wagon & TBD \\
\hline Toyota Prius c & Sedan/Wagon & TBD \\
\hline Toyota Prius Eco & Sedan/Wagon & TBD \\
\hline Toyota Prius v & Sedan/Wagon & TBD \\
\hline Toyota RAV4 Hybrid AWD & SUV & TBD \\
\hline
\end{tabular}

Note: LEV = low emission vehicle. ILEV = inherently low emission vehicle. ULEV = ultra-low emission vehicle. $\mathrm{ZEV}=$ zero emission vehicle. TLEV $=$ transitional low emission vehicle. Does not include plug-in hybrid electric vehicles. See Chapter 12 for details on emissions.

Source:

U.S. Department of Energy, Alternative Fuels Data Center website, “Alternative Fuel and Advanced Vehicle Search,” www.afdc.energy.gov/vehicles/search, August 2017. (Additional resources: www.afdc.energy.gov) 
Table 6.7

Electric-Drive Vehicles Available by Manufacturer, Model Year 2017

\begin{tabular}{|c|c|c|c|}
\hline Model & Drive Type & Vehicle Type & Emission Class \\
\hline BMW i3 BEV & $\mathrm{EV}$ & Sedan/Wagon & TBD \\
\hline BMW i3 BEV & $\mathrm{EV}$ & Sedan/Wagon & TBD \\
\hline BYD Motors e6 & $\mathrm{EV}$ & Sedan/Wagon & TBD \\
\hline Chevrolet Bolt & $\mathrm{EV}$ & Sedan/Wagon & ZEV, Tier III Bin 0 \\
\hline Fiat $500 \mathrm{e}$ & $\mathrm{EV}$ & Sedan/Wagon & ZEV, Tier III Bin 0 \\
\hline Ford Focus Electric & $\mathrm{EV}$ & Sedan/Wagon & TBD \\
\hline Honda Clarity & $\mathrm{EV}$ & Sedan/Wagon & TBD \\
\hline Hyundai Ioniq & $\mathrm{EV}$ & Sedan/Wagon & TBD \\
\hline Kia Soul & $\mathrm{EV}$ & Sedan/Wagon & ZEV, Tier III Bin 0 \\
\hline Mercedes-Benz B250e & $\mathrm{EV}$ & Sedan/Wagon & ZEV, Tier III Bin 0 \\
\hline Mitsubishi i-MiEV & $\mathrm{EV}$ & Sedan/Wagon & ZEV, Tier III Bin 0 \\
\hline Nissan Leaf & $\mathrm{EV}$ & Sedan/Wagon & ZEV, Tier III Bin 0 \\
\hline Smart ForTwo & $\mathrm{EV}$ & Two-Seater & TBD \\
\hline Tesla Model S AWD - 60D & $\mathrm{EV}$ & Sedan/Wagon & TBD \\
\hline Tesla Model S AWD - 75D & $\mathrm{EV}$ & Sedan/Wagon & TBD \\
\hline Tesla Model S AWD - P100D & $\mathrm{EV}$ & Sedan/Wagon & TBD \\
\hline Tesla Model S AWD - P90D & $\mathrm{EV}$ & Sedan/Wagon & TBD \\
\hline Tesla Model S RWD $60 \mathrm{kWh}$ & $\mathrm{EV}$ & Sedan/Wagon & TBD \\
\hline Tesla Model S RWD 75 kWh & $\mathrm{EV}$ & Sedan/Wagon & TBD \\
\hline Tesla Model X AWD - 60D & $\mathrm{EV}$ & SUV & TBD \\
\hline Tesla Model X AWD - 75D & $\mathrm{EV}$ & SUV & TBD \\
\hline Tesla Model X AWD - 90D & $\mathrm{EV}$ & SUV & TBD \\
\hline Tesla Model X AWD - P100D & $\mathrm{EV}$ & SUV & TBD \\
\hline Tesla Model X AWD - P90D & $\mathrm{EV}$ & SUV & TBD \\
\hline Volkswagen e-Golf & $\mathrm{EV}$ & Sedan/Wagon & TBD \\
\hline Audi A3 e-tron & PHEV & Sedan/Wagon & LEV-III SULEV30/PZEV, Tier 3 \\
\hline BMW 330e & PHEV & Sedan/Wagon & Tier 3 Bin 125, LEV III ULEV125 \\
\hline BMW 740e xDrive & PHEV & Sedan/Wagon & Tier 3 Bin 125, LEV III ULEV125 \\
\hline BMW I3 REX & PHEV & Sedan/Wagon & TBD \\
\hline BMW X5 xDrive40e & PHEV & SUV & LEV-II ULEV, Tier 2 Bin 5 \\
\hline Cadillac CT6 PHEV & PHEV & Sedan/Wagon & TBD \\
\hline Chevrolet Volt & PHEV & Sedan/Wagon & Tier 3 Bin 125, LEV-III SULEV30 \\
\hline Chrysler Pacifica PHEV & PHEV & Sedan/Wagon & TBD \\
\hline Ford C-Max Energi & PHEV & Sedan/Wagon & TBD \\
\hline Ford Fusion Energi Plug in Hybrid & PHEV & Sedan/Wagon & Tier 3 Bin 30, LEV-III SULEV30 \\
\hline Hyundai Ioniq & PHEV & Sedan/Wagon & TBD \\
\hline Hyundai Sonata Plug-in Hybrid & PHEV & Sedan/Wagon & LEV-III SULEV30/PZEV, Tier 3 \\
\hline Kia Optima Plug-In Hybrid & PHEV & Sedan/Wagon & Tier 3 Bin 125, LEV III ULEV125 \\
\hline Mercedes-Benz C350e & PHEV & Sedan/Wagon & TBD \\
\hline Mercedes-Benz GLE550e & PHEV & SUV & TBD \\
\hline Mercedes-Benz S550e & PHEV & Sedan/Wagon & TBD \\
\hline Porsche 918 Spyder & PHEV & Two-Seater & TBD \\
\hline Porsche Cayenne S e-Hybrid & PHEV & SUV & LEV-II ULEV, Bin 5 \\
\hline Porsche Panamera 4 e-Hybrid & PHEV & Sedan/Wagon & TBD \\
\hline Toyota Prius Prime & PHEV & Sedan/Wagon & TBD \\
\hline Volvo XC90 T8 Plug-in Hybrid & PHEV & SUV & LEV-III SULEV30/PZEV, Tier 3 Bin 30 \\
\hline Honda Clarity Fuel Cell & FCEV & Sedan/Wagon & TBD \\
\hline Hyundai Tucson & FCEV & SUV & Tier III Bin 0, ZEV \\
\hline Toyota Mirai & FCEV & Sedan/Wagon & ZEV, Tier III Bin 0 \\
\hline
\end{tabular}

Note: EV = electric vehicle; PHEV = plug-in hybrid-electric vehicle; FCEV = hydrogen fuel cell vehicle; LEV = low emission vehicle; ILEV = inherently low emission vehicle; ULEV = ultra-low emission vehicle; ZEV = zero emission vehicle; TLEV = transitional low emission vehicle; SULEV = super ultra-low emission vehicle; AT-PZEV = advanced technology - partial zero emissions vehicle. See Chapter 12 for details on emissions.

\section{Source:}

U.S. Department of Energy, Alternative Fuels Data Center website, “Alternative Fuel and Advanced Vehicle Search,” www.afdc.energy.gov/vehicles/search, August 2017. (Additional resources: www.afdc.energy.gov) 
In 1991 there were only two alternative fuel vehicle (AFV) models on the market which were fueled by M85. In 2017 there were 111 different models of AFV on the market, with 41\% of those fueled by E85. Another $41 \%$ of the models available in 2017 are electric vehicles, which include plug-in hybrid-electric vehicles.

Table 6.8.

Number of Alternative Fuel Vehicle Models Available, 1991-2017 (number of models available)

\begin{tabular}{|c|c|c|c|c|c|c|c|}
\hline Year & Propane $^{\mathrm{a}}$ & $\mathrm{CNG}^{\mathrm{a}}$ & $\begin{array}{c}\text { Ethanol } \\
\text { (E85) }\end{array}$ & $\begin{array}{l}\text { Methanol } \\
\text { (M85) }\end{array}$ & $\begin{array}{l}\text { Electric } \\
\text { vehicle }^{\mathrm{b}}\end{array}$ & Hydrogen & Total \\
\hline 1991 & 0 & 0 & 0 & 2 & 0 & 0 & 2 \\
\hline 1992 & 0 & 2 & 1 & 2 & 0 & 0 & 5 \\
\hline 1993 & 0 & 2 & 1 & 4 & 0 & 0 & 7 \\
\hline 1994 & 0 & 2 & 1 & 2 & 0 & 0 & 5 \\
\hline 1995 & 0 & 10 & 0 & 2 & 1 & 0 & 13 \\
\hline 1996 & 0 & 10 & 1 & 1 & 0 & 0 & 12 \\
\hline 1997 & 3 & 9 & 1 & 1 & 3 & 0 & 17 \\
\hline 1998 & 3 & 12 & 2 & 0 & 8 & 0 & 25 \\
\hline 1999 & 5 & 16 & 6 & 0 & 16 & 0 & 43 \\
\hline 2000 & 2 & 15 & 8 & 0 & 12 & 0 & 37 \\
\hline 2001 & 5 & 16 & 11 & 0 & 10 & 0 & 42 \\
\hline 2002 & 5 & 18 & 16 & 0 & 6 & 0 & 45 \\
\hline 2003 & 1 & 16 & 22 & 0 & 5 & 0 & 44 \\
\hline 2004 & 1 & 16 & 19 & 0 & 1 & 0 & 37 \\
\hline 2005 & 0 & 5 & 24 & 0 & 0 & 0 & 29 \\
\hline 2006 & 0 & 5 & 22 & 0 & 0 & 0 & 27 \\
\hline 2007 & 0 & 1 & 31 & 0 & 0 & 0 & 32 \\
\hline 2008 & 1 & 1 & 31 & 0 & 1 & 0 & 34 \\
\hline 2009 & 1 & 1 & 36 & 0 & 1 & 0 & 39 \\
\hline 2010 & 0 & 1 & 34 & 0 & 1 & 0 & 36 \\
\hline 2011 & 0 & 1 & 72 & 0 & 2 & 0 & 75 \\
\hline 2012 & 1 & 6 & 62 & 0 & 6 & 1 & 76 \\
\hline 2013 & 6 & 11 & 84 & 0 & 15 & 1 & 117 \\
\hline 2014 & 14 & 19 & 90 & 0 & 16 & 2 & 141 \\
\hline 2015 & 10 & 17 & 84 & 0 & 27 & 3 & 141 \\
\hline 2016 & 5 & 12 & 66 & 0 & 29 & 3 & 115 \\
\hline 2017 & 9 & 8 & 45 & 0 & 46 & 3 & 111 \\
\hline \multicolumn{8}{|c|}{ Average annual percentage change } \\
\hline 1991-2017 & c & c & c & $-100.0 \%$ & c & c & $16.7 \%$ \\
\hline $2007-2017$ & c & $23.1 \%$ & $3.8 \%$ & c & c & c & $13.2 \%$ \\
\hline
\end{tabular}

Source:

U.S. Department of Energy, Alternative Fuels Data Center website, "Light-Duty AFC, HEV, and Diesel Model Offerings, By Fuel Type,” www.afdc.energy.gov/data/10303, September 2016 and estimates for 2017. (Additional resources: www.afdc.energy.gov)

${ }^{a}$ Dedicated and bi-fuel vehicles.

${ }^{\mathrm{b}}$ Electric vehicles include plug-in hybrid-electric vehicles but do not include neighborhood electric vehicles, low-speed electric vehicles, or two-wheeled electric vehicles.

${ }^{\mathrm{c}}$ Average annual percentage change cannot be calculated from zero. 
Table 6.9

Hybrid-Electric Medium/Heavy Trucks and Buses Available by Manufacturer, 2017

\begin{tabular}{|c|c|c|}
\hline Manufacturer - Model & Drive type & Truck type \\
\hline Daimler Buses North America Orion VII Hybrid Low-Floor & Hybrid Electric & Transit Bus \\
\hline DesignLine Corp. EcoSaver IV & Hybrid Electric & Transit Bus \\
\hline Foton America FCB 30-foot; FCB 35-foot; FCB 40-foot & Hybrid Electric & Transit Bus \\
\hline Glaval Bus Universal & Hybrid Electric & Shuttle Bus \\
\hline International DuraStar Hybrid & Hybrid Electric & Vocational/Cab Chassis \\
\hline Navistar HC300 Hybrid & Hybrid Electric & School Bus \\
\hline North American Bus Industries 31LFW / 35LFW / 40LFW & Hybrid Electric & Transit Bus \\
\hline North American Bus Industries CompoBus & Hybrid Electric & Transit Bus \\
\hline North American Bus Industries 42BRT & Hybrid Electric & Transit Bus \\
\hline North American Bus Industries 60BRT & Hybrid Electric & Transit Bus \\
\hline Turtle Top Odyssey XLT & Hybrid Electric & Shuttle Bus \\
\hline XL Hybrid - Chevrolet Express 2500/3500 HD & Hybrid Electric & Van \\
\hline XL Hybrid - Ford Transit & Hybrid Electric & Van \\
\hline XL Hybrid - General Motors Savana 2500/3500 HD & Hybrid Electric & Van \\
\hline XL Hybrid - Isuzu Reach & Hybrid Electric & Van \\
\hline XL Hybrid - Ford E 350/450 & Hybrid Electric & Vocational/Cab Chassis \\
\hline XL Hybrid - Ford F-59 Super Duty & Hybrid Electric & Vocational/Cab Chassis \\
\hline XL Hybrid - General Motors 3500/4500 HD & Hybrid Electric & Vocational/Cab Chassis \\
\hline Autocar E3-Hybrid Drive & Hybrid - Diesel & Refuse \\
\hline ElDorado National E-Z Rider II & Hybrid - Diesel & Transit Bus \\
\hline ENC E-Z RIDER II & Hybrid - Diesel & Transit Bus \\
\hline Gillig Corp. Trolley & Hybrid - Diesel & Transit Bus \\
\hline Gillig Corp. Standard, BRT, BRTPlus, Commuter & Hybrid - Diesel & Transit Bus \\
\hline Hino 195h Hybrid COE, 195h-DC Hybrid COE & Hybrid - Diesel & Vocational/Cab Chassis \\
\hline Motor Coach Industries D4500 CT Hybrid Commuter Coach & Hybrid - Diesel & Transit Bus \\
\hline New Flyer Xcelsior & Hybrid - Diesel & Transit Bus \\
\hline Nova Bus LFS Artic HEV & Hybrid - Diesel & Transit Bus \\
\hline Nova Bus LFS HEV & Hybrid - Diesel & Transit Bus \\
\hline DesignLine Corp. EcoSaver IV & Hybrid - CNG & Transit Bus \\
\hline
\end{tabular}

Source:

U.S. Department of Energy, Alternative Fuels Data Center website, www.afdc.energy.gov/vehicles/search, August 2017. (Additional resources: www.afdc.energy.gov) 
Table 6.10

Electric-Drive Medium/Heavy Trucks and Buses Available by Manufacturer, 2017

\begin{tabular}{|c|c|c|}
\hline Manufacturer - Model & Drive type & Truck type \\
\hline Via Motors VTRUX & Plug-in Hybrid Electric & Van \\
\hline BYD (Build Your Dream) C10 45ft Coach & Electric & Transit Bus \\
\hline BYD (Build Your Dream) C6 23ft Coach & Electric & Transit Bus \\
\hline BYD (Build Your Dream) C9 40ft Coach & Electric & Transit Bus \\
\hline BYD (Build Your Dream) K11 60ft Transit Bus & Electric & Transit Bus \\
\hline BYD (Build Your Dream) K7 30ft Transit Bus & Electric & Transit Bus \\
\hline BYD (Build Your Dream) K9 40ft Transit Bus & Electric & Transit Bus \\
\hline BYD (Build Your Dream) K9S 35ft Transit Bus & Electric & Transit Bus \\
\hline BYD (Build Your Dream) Q1M & Electric & Truck \\
\hline BYD (Build Your Dream) Step Van & Electric & Truck \\
\hline BYD (Build Your Dream) T9 & Electric & Truck \\
\hline BYD (Build Your Dream) T5 & Electric & Vocational/Cab Chassis \\
\hline BYD (Build Your Dream) T7 & Electric & Vocational/Cab Chassis \\
\hline Capacity Trucks HETT & Electric & Tractor \\
\hline DesignLine Corp. Eco-Smart 1 & Electric & Transit Bus \\
\hline Ebus 40 Foot CompositeEbus & Electric & Transit Bus \\
\hline Ebus EBUS22 & Electric & Transit Bus \\
\hline First Priority GreenFleet - Lion Bus Type C & Electric & School Bus \\
\hline First Priority GreenFleet - Trans Tech Type A & Electric & School Bus \\
\hline First Priority GreenFleet Medium Duty Truck & Electric & Truck \\
\hline First Priority GreenFleet Walk-In Van & Electric & Van \\
\hline GGT Electric Electric & Electric & Vocational/Cab Chassis \\
\hline Navistar-Modec EV Alliance eStar & Electric & Step Van \\
\hline New Flyer Xcelsior & Electric & Transit Bus \\
\hline Nova Bus LFSE & Electric & Transit Bus \\
\hline Orange EV Multiple & Electric & Tractor \\
\hline Phoenix Motorcars Shuttle Bus & Electric & Shuttle Bus \\
\hline Phoenix Motorcars Flatbed & Electric & Vocational/Cab Chassis \\
\hline Phoenix Motorcars Utility Vehicle & Electric & Vocational/Cab Chassis \\
\hline Proterra Catalyst & Electric & Transit Bus \\
\hline Trans Tech ETrans & Electric & School Bus \\
\hline Workhorse Custom Chassis E-Gen & Electric & Vocational/Cab Chassis \\
\hline Zenith Motors 250/350 & Electric & Van \\
\hline Zenith Motors Shuttle Van & Electric & Van \\
\hline ZeroTruck ZeroTruck & Electric & Vocational/Cab Chassis \\
\hline Capacity Trucks ZETT & Hydrogen Fuel Cell & Tractor \\
\hline Van Hool A300L Fuel Cel & Hydrogen Fuel Cell & Transit Bus \\
\hline Vision Motor Corp. Tyrano & Hydrogen Fuel Cell & Tractor \\
\hline Vision Motor Corp. ZETT Zero Emission Terminal Tractor & Hydrogen Fuel Cell & Tractor \\
\hline
\end{tabular}

Source:

U.S. Department of Energy, Alternative Fuels Data Center website, www.afdc.energy.gov/vehicles/search, August 2017. (Additional resources: www.afdc.energy.gov) 
This list includes public and private refuel sites; therefore, not all of these sites are available to the public.

Table 6.11

Number of Alternative Refuel Sites by State and Fuel Type, 2017

\begin{tabular}{|c|c|c|c|c|c|c|c|c|c|}
\hline State & $\begin{array}{l}\mathrm{B} 20 \\
\text { sites }\end{array}$ & $\begin{array}{l}\mathrm{CNG} \\
\text { sites }\end{array}$ & $\begin{array}{l}\mathrm{E} 85 \\
\text { sites }\end{array}$ & $\begin{array}{l}\text { Electric } \\
\text { stations }\end{array}$ & $\begin{array}{c}\text { Electric charging } \\
\text { outlets }\end{array}$ & $\begin{array}{l}\text { Hydrogen } \\
\text { sites }\end{array}$ & $\begin{array}{l}\text { LNG } \\
\text { sites }\end{array}$ & $\begin{array}{l}\text { LPG } \\
\text { sites }\end{array}$ & $\begin{array}{c}\text { Totals by } \\
\text { State }^{\mathrm{a}}\end{array}$ \\
\hline Alabama & 5 & 32 & 41 & 126 & 252 & 0 & 2 & 97 & 429 \\
\hline Alaska & 0 & 1 & 0 & 6 & 9 & 0 & 0 & 7 & 17 \\
\hline Arizona & 76 & 34 & 26 & 426 & 1,055 & 0 & 8 & 93 & 1,292 \\
\hline Arkansas & 5 & 16 & 44 & 56 & 85 & 0 & 1 & 40 & 191 \\
\hline California & 36 & 326 & 137 & 4,442 & 16,052 & 42 & 45 & 294 & 16,932 \\
\hline Colorado & 16 & 44 & 92 & 503 & 1,204 & 1 & 1 & 59 & 1,417 \\
\hline Connecticut & 2 & 21 & 4 & 347 & 777 & 2 & 1 & 21 & 828 \\
\hline Delaware & 1 & 1 & 1 & 37 & 97 & 1 & 0 & 9 & 110 \\
\hline Dist. of Columbia & 7 & 2 & 3 & 100 & 268 & 1 & 0 & 0 & 281 \\
\hline Florida & 14 & 57 & 79 & 1,035 & 2,301 & 0 & 3 & 142 & 2,596 \\
\hline Georgia & 23 & 48 & 55 & 678 & 1,905 & 0 & 4 & 101 & 2,136 \\
\hline Hawaii & 8 & 1 & 2 & 271 & 623 & 4 & 0 & 5 & 643 \\
\hline Idaho & 2 & 11 & 6 & 68 & 151 & 0 & 2 & 31 & 203 \\
\hline Illinois & 14 & 48 & 268 & 520 & 1,141 & 1 & 2 & 123 & 1,597 \\
\hline Indiana & 6 & 35 & 202 & 190 & 384 & 0 & 2 & 186 & 815 \\
\hline Iowa & 9 & 9 & 242 & 109 & 222 & 0 & 0 & 33 & 515 \\
\hline Kansas & 4 & 20 & 21 & 195 & 740 & 0 & 1 & 41 & 827 \\
\hline Kentucky & 4 & 12 & 73 & 78 & 163 & 0 & 1 & 36 & 289 \\
\hline Louisiana & 2 & 23 & 11 & 81 & 180 & 0 & 1 & 47 & 264 \\
\hline Maine & 3 & 2 & 0 & 116 & 199 & 0 & 0 & 14 & 218 \\
\hline Maryland & 13 & 16 & 36 & 518 & 1,255 & 0 & 1 & 34 & 1,355 \\
\hline Massachusetts & 11 & 16 & 7 & 545 & 1,404 & 2 & 1 & 34 & 1,475 \\
\hline Michigan & 9 & 23 & 252 & 538 & 1,159 & 2 & 0 & 103 & 1,548 \\
\hline Minnesota & 7 & 25 & 355 & 298 & 741 & 0 & 0 & 48 & 1,176 \\
\hline Mississippi & 4 & 7 & 3 & 45 & 69 & 0 & 2 & 91 & 176 \\
\hline Missouri & 3 & 22 & 96 & 356 & 1,476 & 0 & 1 & 69 & 1,667 \\
\hline Montana & 7 & 1 & 2 & 30 & 79 & 0 & 0 & 48 & 137 \\
\hline Nebraska & 2 & 11 & 86 & 60 & 142 & 0 & 1 & 27 & 269 \\
\hline Nevada & 4 & 5 & 19 & 193 & 553 & 0 & 1 & 34 & 616 \\
\hline New Hampshire & 4 & 3 & 0 & 98 & 192 & 0 & 0 & 25 & 224 \\
\hline New Jersey & 5 & 28 & 5 & 255 & 566 & 0 & 0 & 19 & 623 \\
\hline New Mexico & 5 & 14 & 12 & 58 & 151 & 0 & 1 & 59 & 242 \\
\hline New York & 34 & 94 & 69 & 845 & 1,724 & 1 & 0 & 71 & 1,993 \\
\hline North Carolina & 116 & 42 & 77 & 543 & 1,270 & 0 & 1 & 93 & 1,599 \\
\hline North Dakota & 3 & 1 & 41 & 9 & 13 & 0 & 0 & 24 & 82 \\
\hline Ohio & 14 & 61 & 159 & 346 & 691 & 3 & 6 & 91 & 1,025 \\
\hline Oklahoma & 5 & 122 & 31 & 54 & 117 & 0 & 1 & 140 & 416 \\
\hline Oregon & 70 & 16 & 10 & 554 & 1,343 & 0 & 2 & 56 & 1,497 \\
\hline Pennsylvania & 6 & 73 & 56 & 371 & 738 & 0 & 3 & 104 & 980 \\
\hline Rhode Island & 5 & 4 & 0 & 88 & 224 & 0 & 0 & 7 & 240 \\
\hline South Carolina & 24 & 12 & 64 & 221 & 446 & 2 & 1 & 48 & 597 \\
\hline South Dakota & 2 & 1 & 73 & 23 & 40 & 0 & 0 & 27 & 143 \\
\hline Tennessee & 29 & 22 & 79 & 445 & 1,039 & 0 & 5 & 88 & 1,262 \\
\hline Texas & 18 & 125 & 215 & 1,020 & 2,661 & 1 & 19 & 476 & 3,515 \\
\hline Utah & 1 & 86 & 1 & 138 & 345 & 0 & 6 & 48 & 487 \\
\hline Vermont & 2 & 3 & 0 & 164 & 413 & 0 & 0 & 2 & 420 \\
\hline Virginia & 11 & 20 & 29 & 448 & 1,053 & 0 & 2 & 92 & 1,207 \\
\hline Washington & 39 & 26 & 20 & 779 & 2,066 & 0 & 2 & 88 & 2,241 \\
\hline West Virginia & 2 & 4 & 33 & 58 & 151 & 0 & 0 & 13 & 203 \\
\hline Wisconsin & 5 & 58 & 153 & 295 & 487 & 0 & 1 & 75 & 779 \\
\hline Wyoming & 13 & 13 & 13 & 30 & 65 & 0 & 0 & 28 & 132 \\
\hline Totals by Fuel & 710 & 1,697 & 3,303 & 18,809 & 50,481 & 63 & 31 & 3,541 & 59,926 \\
\hline
\end{tabular}

Source:

U.S. Department of Energy, Alternative Fuels Data Center website,

www.afdc.energy.gov/afdc/fuels/stations_counts.html, August 2017. (Additional resources: www.afdc.energy.gov)

${ }^{\text {a }}$ Totals by State is the total number of fuel types available at stations. Stations are counted once for each type of fuel available. For electric, the number of charging outlets was used. 
There were just over 3,000 propane stations in the United States in 1992 making up 89\% of all alternative refueling stations. Electric vehicle stations, which after 2011 are counted by the number of plugs rather than by the geographic location, have the largest number of stations in 2016.

Table 6.12

Number of Alternative Refuel Stations, 1992-2016 (number of stations)

\begin{tabular}{|c|c|c|c|c|c|c|c|c|c|}
\hline Year & Propane & $\mathrm{CNG}$ & LNG & Biodiesel $^{\mathrm{a}}$ & $\begin{array}{c}\text { Ethanol } \\
\text { (E85) }\end{array}$ & $\begin{array}{c}\text { Methanol } \\
\text { (M85) }\end{array}$ & $\begin{array}{c}\text { Electric } \\
\text { vehicle }^{\mathrm{b}}\end{array}$ & Hydrogen & Total \\
\hline 1992 & 3,297 & 349 & c & 0 & 2 & 43 & c & c & 3,691 \\
\hline 1993 & 3,297 & 497 & c & 0 & 7 & 50 & c & c & 3,851 \\
\hline 1994 & 3,299 & 1,042 & c & 0 & 32 & 82 & c & c & 4,455 \\
\hline 1995 & 3,299 & 1,065 & c & 0 & 37 & 88 & 188 & c & 4,677 \\
\hline 1996 & 4,252 & 1,419 & 72 & 0 & 68 & 95 & 194 & c & 6,100 \\
\hline 1997 & 4,255 & 1,426 & 71 & 0 & 71 & 106 & 310 & c & 6,239 \\
\hline 1998 & 5,318 & 1,268 & 66 & 0 & 40 & 91 & 486 & c & 7,269 \\
\hline 1999 & 4,153 & 1,267 & 46 & 0 & 49 & 51 & 490 & c & 6,056 \\
\hline 2000 & 3,268 & 1,217 & 44 & 2 & 113 & 3 & 558 & c & 5,205 \\
\hline 2001 & 3,403 & 1,232 & 44 & 16 & 154 & 0 & 693 & c & 5,542 \\
\hline 2002 & 3,431 & 1,166 & 36 & 79 & 149 & 0 & 873 & 7 & 5,741 \\
\hline 2003 & 3,966 & 1,035 & 62 & 142 & 188 & 0 & 830 & 7 & 6,230 \\
\hline 2004 & 3,689 & 917 & 58 & 176 & 200 & 0 & 671 & 9 & 5,720 \\
\hline 2005 & 2,995 & 787 & 40 & 304 & 436 & 0 & 588 & 14 & 5,164 \\
\hline 2006 & 2,619 & 732 & 37 & 459 & 762 & 0 & 465 & 17 & 5,091 \\
\hline 2007 & 2,371 & 721 & 35 & 742 & 1,208 & 0 & 442 & 32 & 5,551 \\
\hline 2008 & 2,175 & 778 & 38 & 645 & 1,644 & 0 & 430 & 46 & 5,756 \\
\hline 2009 & 2,468 & 772 & 36 & 679 & 1,928 & 0 & 465 & 63 & 6,411 \\
\hline 2010 & 2,647 & 841 & 39 & 644 & 2,142 & 0 & 541 & 58 & 6,912 \\
\hline 2011 & 2,597 & 910 & 45 & 627 & 2,442 & 0 & 3,394 & 56 & 10,071 \\
\hline 2012 & 2,654 & 1,107 & 59 & 675 & 2,553 & 0 & 13,392 & 58 & 20,498 \\
\hline 2013 & 2,956 & 1,263 & 81 & 757 & 2,639 & 0 & 19,410 & 53 & 27,159 \\
\hline 2014 & 2,931 & 1,495 & 103 & 783 & 2,840 & 0 & 25,602 & 51 & 33,805 \\
\hline 2015 & 3,594 & 1,563 & 111 & 721 & 2,990 & 0 & 30,945 & 39 & 39,963 \\
\hline 2016 & 3,665 & 1,725 & 140 & 697 & 3,090 & 0 & 42,011 & 54 & 51,382 \\
\hline & \multicolumn{9}{|c|}{ Average annual percentage change } \\
\hline $1992-2016$ & $0.4 \%$ & $6.9 \%$ & c & c & $35.8 \%$ & $-100.0 \%$ & c & c & $11.6 \%$ \\
\hline 2006-2016 & $3.4 \%$ & $9.0 \%$ & $14.2 \%$ & $4.3 \%$ & $15.0 \%$ & c & d & $12.3 \%$ & $26.0 \%$ \\
\hline
\end{tabular}

\section{Source:}

U.S. Department of Energy, Alternative Fuels Data Center website, “U.S. Alternative Fueling Stations by Fuel Type,” www.afdc.energy.gov/data/10332. (Additional resources: www.afdc.energy.gov)

${ }^{a}$ Stations selling biodiesel blends less than B20 are included in the station count for years 2005-2007 only.

b Starting in 2011, electric stations are counted by the plug rather than by the geographical location. This is different from the other fuels, which count only the geographical location regardless of how many dispensers or nozzles are on site.

${ }^{\mathrm{c}}$ Data are not available.

${ }^{\mathrm{d}}$ Because data are not comparable from 2006 to 2016, an average annual percentage change is not provided. 
Clean Cities is a locally-based government/industry partnership, coordinated by the U.S. Department of Energy to expand the use of alternatives to gasoline and diesel fuel. By combining the decision-making with voluntary action by partners, the "grass-roots" approach of Clean Cities departs from traditional "top-down" Federal programs.

\section{Figure 6.1. Clean Cities Coalitions}

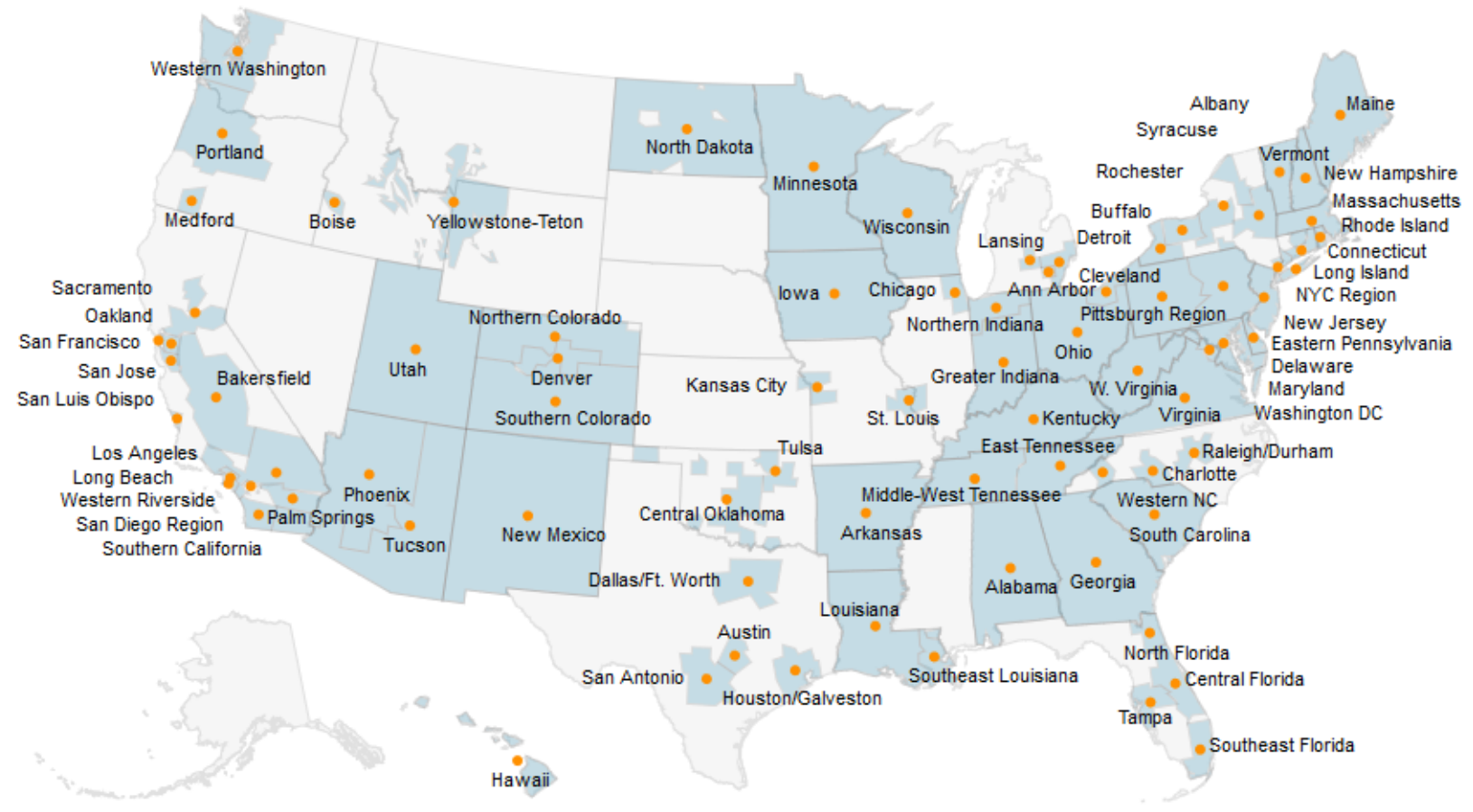

\section{Source:}

U.S. Department of Energy, Clean Cities website, "Clean Cities Coalition Locations," cleancities.energy.gov/coalitions/locations, August 2017. (Additional resources: cleancities.energy.gov) 
Table 6.13

Properties of Conventional and Alternative Liquid Fuels

\begin{tabular}{|c|c|c|c|c|}
\hline \multirow[b]{2}{*}{ Property } & \multicolumn{4}{|c|}{ Liquid Fuels } \\
\hline & Gasoline & Low-sulfur diesel & Methanol & Ethanol (E100) \\
\hline Standard chemical formula & $\mathrm{C}_{4}$ to $\mathrm{C}_{12}$ & $\mathrm{C}_{8}$ to $\mathrm{C}_{25}$ & $\mathrm{CH}_{3} \mathrm{OH}$ & $\mathrm{CH}_{3} \mathrm{CH}_{2} \mathrm{OH}$ \\
\hline Physical state & Liquid & Liquid & Liquid & Liquid \\
\hline Molecular weight & $100-105$ & $\sim 200$ & 32.04 & 46.07 \\
\hline \multicolumn{5}{|l|}{ Composition (weight \%) } \\
\hline Carbon & $85-88$ & 87 & 37.5 & 52.2 \\
\hline Hydrogen & $12-15$ & 13 & 12.6 & 13.1 \\
\hline Oxygen & 0 & 0 & 49.9 & 34.7 \\
\hline Main fuel source(s) & Crude oil & Crude oil & $\begin{array}{l}\text { Natural gas, coal, or } \\
\text { woody biomass }\end{array}$ & $\begin{array}{l}\text { Corn, grains, or } \\
\text { agricultural waste }\end{array}$ \\
\hline $\begin{array}{l}\text { Gasoline gallon equivalent (GGE) } \\
\text { (Fuel unit measured/GGE) }\end{array}$ & $\begin{array}{c}1.0 \\
\text { (E0 gasoline) }\end{array}$ & $\begin{array}{l}0.889 \text { (Diesel } \\
\text { gal/GGE) }\end{array}$ & $\begin{array}{l}\text { 2.04 Methanol } \\
\text { gal/GGE) }\end{array}$ & $\begin{array}{c}1.20-1.37\left(\mathrm{E} 85^{\mathrm{b}}\right. \\
\text { gal/GGE) } \\
1.03(\mathrm{E} 10 \mathrm{gal} / \mathrm{GGE})\end{array}$ \\
\hline Specific gravity $\left(60^{\circ} \mathrm{F} / 60^{\circ} \mathrm{F}\right)$ & $0.72-0.78$ & 0.85 & 0.796 & 0.794 \\
\hline Density (lb./gal @ 60F) & $6.0-6.5$ & 7.079 & 6.63 & 6.61 \\
\hline Boiling temperature $\left(\mathrm{F}^{\circ}\right)$ & $80-437$ & $356-644$ & 149 & 172 \\
\hline Freezing point $\left(\mathrm{F}^{\circ}\right)$ & -40 & $-40-30$ & -143.5 & -173.2 \\
\hline Autoignition temperature $\left(\mathrm{F}^{\circ}\right)$ & 495 & $\sim 600$ & 897 & 793 \\
\hline Reid vapor pressure (psi) & $8-15$ & $<0.2$ & 4.6 & 2.3 \\
\hline
\end{tabular}

Source:

U.S. Department of Energy, Alternative Fuels Data Center website, "Fuel Properties Comparison," www.afdc.energy.gov/fuels/fuel_comparison_chart.pdf, July 2015, and communication with George Mitchell, National Renewable Energy Laboratory, July 2015.

${ }^{\text {a }}$ Standard Chemical Formulas represent idealized fuels. Some table values are expressed in ranges to represent typical fuel variations that are encountered in the field.

b 1 gallon of E85 has 73\% to $83 \%$ of the energy of one gallon of gasoline (variation due to ethanol content in E85). 
Table 6.14

Properties of Conventional and Alternative Gaseous Fuels

\begin{tabular}{|c|c|c|c|}
\hline \multirow[b]{2}{*}{ Property } & \multicolumn{3}{|c|}{ Gaseous Fuels } \\
\hline & Propane (LPG) & CNG & Hydrogen \\
\hline Standard chemical formula ${ }^{\mathrm{a}}$ & $\mathrm{C}_{3} \mathrm{H}_{8}$ & $\mathrm{CH}_{4}$ & $\mathrm{H}_{2}$ \\
\hline Physical state & Pressurized liquid & Compressed gas & Compressed gas or liquid \\
\hline Molecular weight & 44.1 & 16.04 & 2.02 \\
\hline \multicolumn{4}{|l|}{ Composition (weight \%) } \\
\hline Carbon & 82 & 75 & 0 \\
\hline Hydrogen & 18 & 25 & 100 \\
\hline Oxygen & $\mathrm{n} / \mathrm{a}$ & $\mathrm{n} / \mathrm{a}$ & 0 \\
\hline Main fuel source(s) & Underground reserves & $\begin{array}{l}\text { Underground reserves } \\
\text { and renewable Bio-gas }\end{array}$ & $\begin{array}{l}\text { Natural gas, methanol, } \\
\text { electrolysis, and other energy } \\
\text { sources }\end{array}$ \\
\hline $\begin{array}{l}\text { Gasoline gallon equivalent (GGE) } \\
\text { (Fuel unit measured/GGE) }\end{array}$ & 1.34-1.38 (LPG gal/GGE) & $\begin{array}{l}\text { 5.56-5.71 (lb. } \\
\text { mass/GGE) }\end{array}$ & 0.991-1.017 (kg mass/GGE) \\
\hline $\begin{array}{l}\text { Diesel gallon equivalent (DGE) } \\
\text { (Fuel unit measured/DGE) }\end{array}$ & 1.54 (LPG gal/DGE) & 6.38 (lb. mass/DGE) & $\mathrm{n} / \mathrm{a}$ \\
\hline Specific Gravity $\left(60^{\circ} \mathrm{F} / 60^{\circ} \mathrm{F}\right)$ & 1.55 & 0.60 & 0.069 \\
\hline Density (lb./cu ft @60F) & 0.124 & 0.0458 & 0.0056 \\
\hline Freezing point $\left(\mathrm{F}^{\circ}\right)$ & -305.8 & -296 & -435 \\
\hline Boiling Point $\left({ }^{\circ} \mathrm{F}\right)$ & -44 & -260 & -423 \\
\hline Autoignition temperature $\left(\mathrm{F}^{\circ}\right)$ & $850-950$ & 1,004 & $1,050-1,080$ \\
\hline Reid vapor pressure (psi) & 208 & $\mathrm{n} / \mathrm{a}$ & $\mathrm{n} / \mathrm{a}$ \\
\hline
\end{tabular}

Note: $\mathrm{n} / \mathrm{a}=$ not applicable

\section{Source:}

U.S. Department of Energy, Alternative Fuels Data Center website, "Fuel Properties Comparison," www.afdc.energy.gov/fuels/fuel_comparison_chart.pdf, July 2015, and communication with George Mitchell, National Renewable Energy Laboratory, July 2015.

a Standard Chemical Formulas represent idealized fuels.

${ }^{\mathrm{b}}$ CNG: 1 Gasoline Gallon Equivalent $=5.66 \mathrm{lb}$. (as referenced by NIST Special Publication 854; Report of the 78th NCWM (1993); p. 326; NG data derived from field sampling of pipeline natural gas by IGT/GRI). 
$6-18$

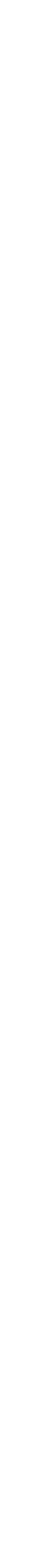

TRANSPORTATION ENERGy DATA BOOK: EDITION 36-2017 


\section{Chapter 7 \\ Fleet Vehicles and Characteristics}

Summary Statistics from Tables in this Chapter

\begin{tabular}{|c|c|c|}
\hline Source & & \\
\hline Figure 7.1 & Fleet cars, 2016 & $4,756,800$ \\
\hline Figure 7.1 & Fleet trucks $\leq 19,500 \mathrm{lbs} . \mathrm{GVW}, 2016$ & $4,809,400$ \\
\hline \multirow[t]{4}{*}{ Table 7.3} & Average annual miles per business fleet vehicle & \\
\hline & SUVS & 26,352 \\
\hline & Intermediate cars & 24,540 \\
\hline & Pickup trucks & 25,500 \\
\hline \multirow[t]{8}{*}{ Figure 7.2} & $\begin{array}{l}\text { Average annual miles per Federal Government } \\
\text { fleet vehicle, } 2015\end{array}$ & \\
\hline & Sedans & 9,855 \\
\hline & SUVS & 9,508 \\
\hline & Buses & 8,256 \\
\hline & Heavy trucks & 6,730 \\
\hline & Medium trucks & 6,600 \\
\hline & Light trucks & 6,245 \\
\hline & Ambulances & 5,403 \\
\hline \multirow[t]{6}{*}{ Table 7.4} & Federal government vehicles, FY 2015 & 640,304 \\
\hline & Light trucks $(<8,500$ lbs. GVW) & 281,993 \\
\hline & Cars and other passenger vehicles & 235,319 \\
\hline & Medium trucks $(8,500-26,000$ lbs. GVW) & 79,421 \\
\hline & Heavy trucks (>26,000 lbs. GVW) & 34,049 \\
\hline & Buses and ambulances & 9,522 \\
\hline
\end{tabular}


Vehicles in fleets of 15 or more are counted as fleet vehicles, as well as vehicles in fleets where five or more vehicles are purchased annually. There are more trucks in fleets than cars in 2016.

Figure 7.1. Fleet Vehicles in Service as of January 1, 2016

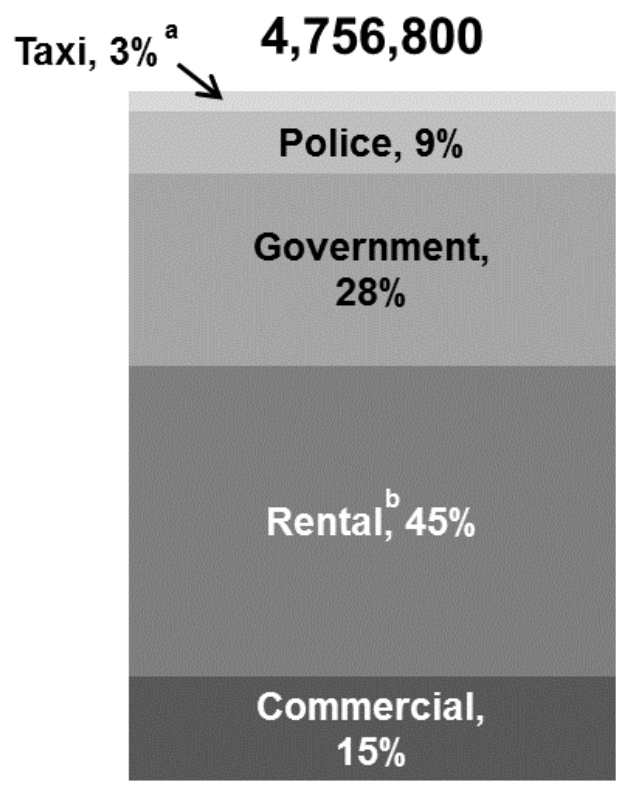

Cars $^{c}$

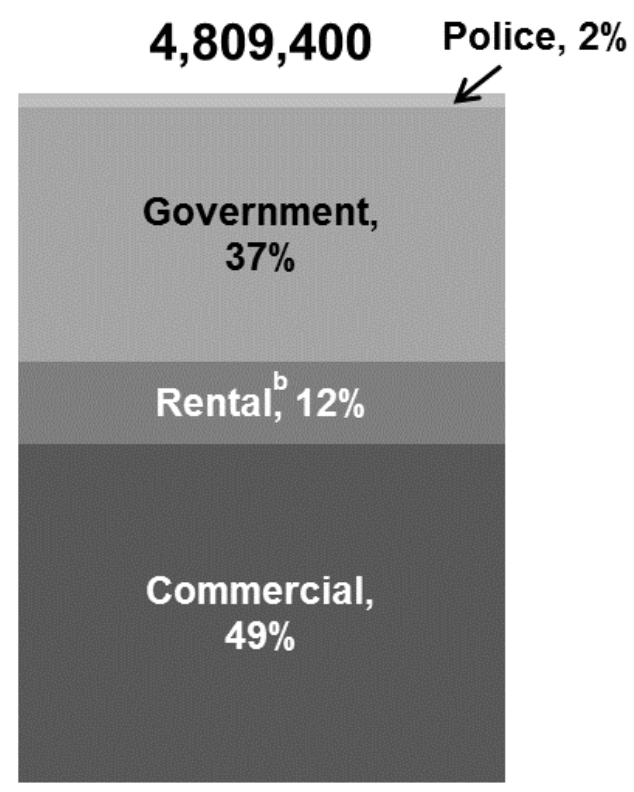

\section{Trucks $^{c}<19,501$ lbs gross vehicle weight}

Source:

Bobit Publishing Company, Automotive Fleet Research Department, Automotive Fleet Factbook 2016, Redondo Beach, CA, 2017.

a Taxi category includes taxis that are vans in addition to cars.

${ }^{\mathrm{b}}$ Rental category includes vans and sports utility vehicles under cars, not trucks.

${ }^{\mathrm{c}}$ Fleets of 15 or more in operation or 5 or more fleet vehicles purchased annually. 
Data for fleet vehicles (cars and trucks less than 19,501 pounds) show that rental fleets are the largest share of cars and commercial fleets are the largest share of trucks. Government fleets are the second largest share for both cars and trucks.

Table 7.1

Fleet Vehicles in Service, 2006-2016 (thousands of vehicles)

\begin{tabular}{|c|c|c|c|c|c|}
\hline Year & Commercial & Rental $^{\mathrm{a}}$ & Government & Police \& Taxi ${ }^{\mathrm{b}}$ & Total \\
\hline \multicolumn{6}{|c|}{ Cars $^{\mathrm{c}}$} \\
\hline 2006 & 896.9 & $1,623.0$ & $1,195.9$ & 555.2 & $4,271.0$ \\
\hline 2007 & 911.8 & $1,650.0$ & $1,215.8$ & 564.5 & $4,342.0$ \\
\hline 2008 & 879.1 & $1,465.1$ & $1,255.8$ & 586.0 & $4,186.0$ \\
\hline 2009 & 791.0 & $1,289.0$ & $1,299.0$ & 607.0 & $3,986.0$ \\
\hline 2010 & 741.2 & $1,175.0$ & $1,352.0$ & 575.7 & $3,843.8$ \\
\hline 2011 & 803.9 & $1,553.2$ & $1,330.0$ & 578.6 & $4,265.7$ \\
\hline 2012 & 834.7 & $1,745.0$ & $1,240.0$ & 556.6 & $4,376.3$ \\
\hline 2013 & 727.7 & $1,850.0$ & $1,290.0$ & 570.6 & $4,438.3$ \\
\hline 2014 & 688.5 & $1,920.0$ & $1,245.2$ & 582.4 & $4,443.2$ \\
\hline 2015 & 659.2 & $2,040.0$ & $1,325.0$ & 595.8 & $4,620.0$ \\
\hline 2016 & 685.0 & $2,156.0$ & $1,340.0$ & 575.8 & $4,756.8$ \\
\hline \multicolumn{6}{|c|}{ Trucks $^{\mathrm{c}}<19,501$ lbs. } \\
\hline 2006 & $2,362.4$ & 499.7 & $1,635.5$ & 45.4 & $4,543.0$ \\
\hline 2007 & $2,383.2$ & 560.8 & $1,682.3$ & 46.7 & $4,673.0$ \\
\hline 2008 & $2,318.5$ & 500.1 & $1,682.0$ & 45.5 & $4,546.0$ \\
\hline 2009 & $2,224.0$ & 381.0 & $1,701.0$ & 59.0 & $4,365.0$ \\
\hline 2010 & $1,999.5$ & 380.0 & $1,751.0$ & 55.4 & $4,185.8$ \\
\hline 2011 & $2,136.3$ & 391.0 & $1,684.0$ & 58.4 & $4,269.7$ \\
\hline 2012 & $2,236.8$ & 417.0 & $1,512.0$ & 62.0 & $4,227.8$ \\
\hline 2013 & $2,186.9$ & 465.0 & $1,560.0$ & 66.5 & $4,278.4$ \\
\hline 2014 & $2,136.4$ & 480.0 & $1,631.5$ & 74.9 & $4,322.8$ \\
\hline 2015 & $2,231.8$ & 535.0 & $1,727.4$ & 77.4 & $4,571.6$ \\
\hline 2016 & $2,340.0$ & 582.0 & $1,810.0$ & 77.4 & $4,809.0$ \\
\hline
\end{tabular}

\section{Source:}

Bobit Publishing Company, Automotive Fleet Research Department, Automotive Fleet Factbook 2016, and annual, Redondo Beach, CA. (Additional resources: www.fleet-central.com)

\footnotetext{
${ }^{a}$ Rental category includes vans and sports utility vehicles under cars, not trucks.

b Taxi category includes vans.

${ }^{\mathrm{c}}$ Fleets of 15 or more in operation or 5 or more fleet vehicles purchased annually.
} 
In commercial fleets, full size vans stay in service the longest—an average of 63 months. Most commercial fleet vehicles drive about 25,000 miles in a year.

Table 7.2

Average Length of Time Commercial Fleet Vehicles Are in Service, 2015

\begin{tabular}{lc}
\hline Vehicle type & $\begin{array}{c}\text { Average months } \\
\text { in service }\end{array}$ \\
\hline Compact cars & 35 \\
Intermediate cars & 33 \\
Pickup trucks & 56 \\
Minivans & 42 \\
Sport utility vehicles & 35 \\
Full-size vans & 63 \\
\hline
\end{tabular}

Note: Based on data collected from four leading Fleet Management companies.

\section{Source:}

Bobit Publishing Company, Automotive Fleet Factbook 2016, www.automotive-fleet.com. (Additional resources: www.fleet-central.com)

Table 7.3

Average Annual Vehicle-Miles of Travel

for Commercial Fleet Vehicles, 2015

\begin{tabular}{lc}
\hline & Average annual miles of \\
Business fleet vehicles & 24,408 \\
\hline Compact cars & 24,540 \\
Intermediate cars & 25,500 \\
Pickup trucks & 26,616 \\
Minivans & 26,352 \\
Sport utility vehicles & 25,152 \\
Full-size vans & \\
\hline
\end{tabular}

Source:

Bobit Publishing Company, Automotive Fleet Factbook 2016, www.automotive-fleet.com. (Additional resources: www.fleet-central.com) 
These data, which apply to domestic Federal fleet vehicles, indicate that sedans now have the highest average annual miles per vehicle, followed closely by sport utility vehicles (SUVs).

Figure 7.2. Average Miles per Domestic Federal Vehicle by Vehicle Type, 2015

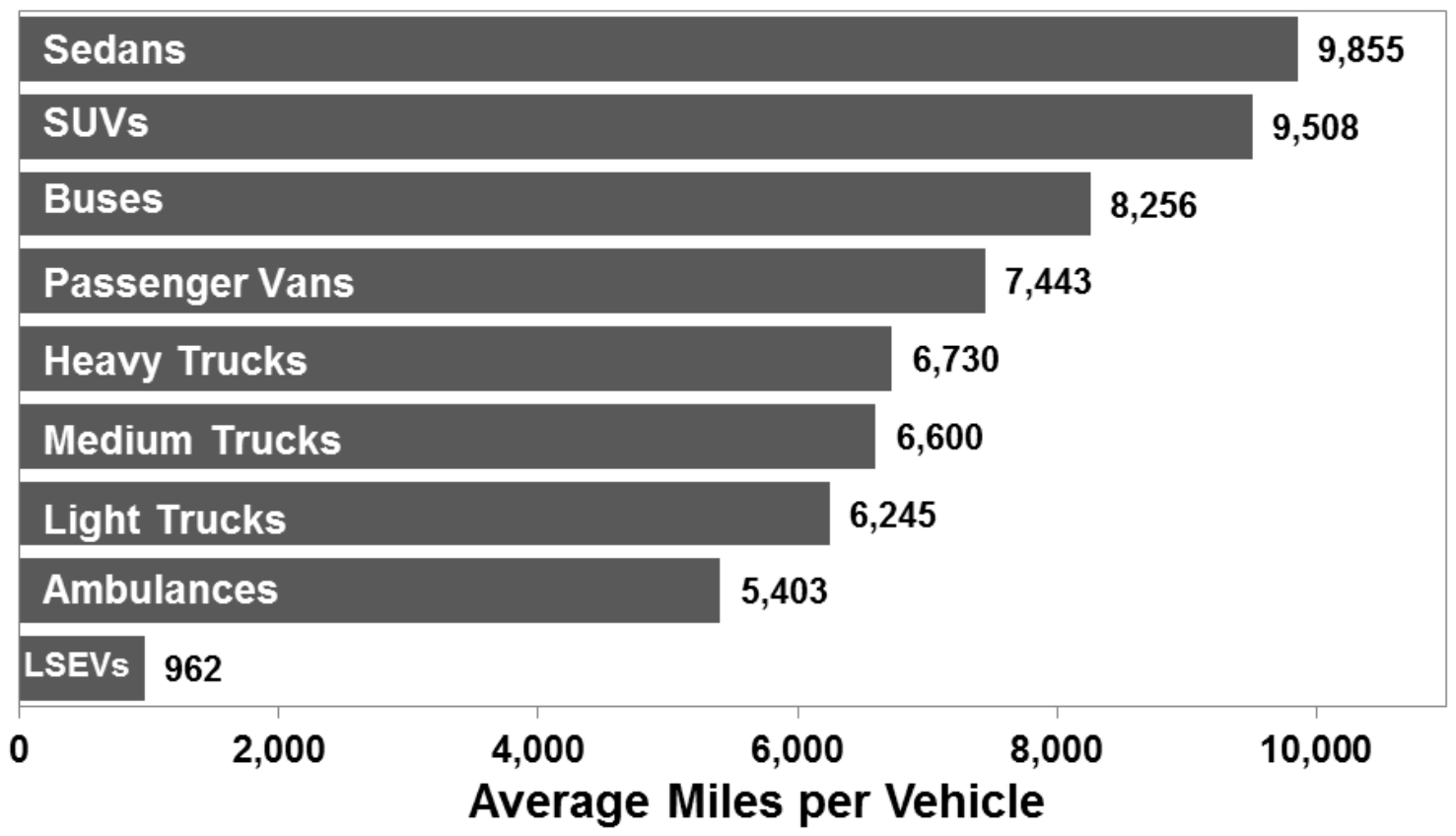

Note: Light trucks $=$ less than 8,500 pounds gross vehicle weight ratio (GVWR).

Medium trucks $=8,501-23,999$ pounds GVWR.

Heavy trucks $=24,000$ pounds GVWR or more.

LSEVs = low-speed electric vehicles.

\section{Source:}

U.S. General Services Administrations, Federal Vehicle Policy Division, FY 2015 Federal Fleet Report, Washington, DC, 2016, Table 4-2. (Additional resources: www.gsa.gov) 
The Federal Government vehicle inventory includes 20\% more light trucks than passenger vehicles.

Table 7.4

Federal Government Vehicles, 2001-2015

\begin{tabular}{lrrrrrrrr}
\hline Vehicle Type & $\mathbf{2 0 0 1}$ & $\mathbf{2 0 0 5}$ & $\mathbf{2 0 1 0}$ & $\mathbf{2 0 1 1}$ & $\mathbf{2 0 1 2}$ & $\mathbf{2 0 1 3}$ & $\mathbf{2 0 1 4}$ & $\mathbf{2 0 1 5}$ \\
\hline Passenger vehicles & & & & & & & & \\
Low-speed electric vehicle & 0 & 0 & 3,029 & 3,869 & 3,893 & 3,729 & 3,830 & 3,686 \\
Subcompact & 5,462 & 2,401 & 6,797 & 10,658 & 13,867 & 20,368 & 25,076 & 27,356 \\
Compact & 60,938 & 58,284 & 46,489 & 49,657 & 47,999 & 41,564 & 39,079 & 38,766 \\
Midsize & 36,921 & 36,656 & 48,242 & 38,057 & 33,321 & 30,659 & 28,271 & 24,775 \\
Large & 11,107 & 15,966 & 10,063 & 9,146 & 8,571 & 6,753 & 5,760 & 7,150 \\
Limousines & 116 & 191 & 412 & 158 & 130 & 123 & 126 & 83 \\
Light duty passenger vans & 56,563 & 42,109 & 41,676 & 40,964 & 39,518 & 38,409 & 37,439 & 37,448 \\
Medium duty passenger vans & 727 & 13,252 & 15,218 & 16,633 & 15,740 & 14,115 & 14,515 & 14,617 \\
Light duty SUVs & 40,842 & 50,445 & 66,316 & 68,807 & 73,356 & 70,371 & 70,235 & 73,203 \\
Medium duty SUVs & 0 & 6,096 & 11,117 & 11,448 & 9,405 & 9,533 & 9,978 & 8,235 \\
\hline Total passenger vehicles & $\mathbf{2 1 2 , 6 7 6}$ & $\mathbf{2 2 5 , 4 0 0}$ & $\mathbf{2 4 9 , 3 5 9}$ & $\mathbf{2 4 9 , 3 9 7}$ & $\mathbf{2 4 5 , 8 0 0}$ & $\mathbf{2 3 5 , 6 2 4}$ & $\mathbf{2 3 4 , 3 0 9}$ & $\mathbf{2 3 5 , 3 1 9}$ \\
\hline Trucks and other vehicles & & & & & & & & \\
Light trucks 4x2 & 227,937 & 243,477 & 241,011 & 238,261 & 233,629 & 231,886 & 231,215 & 232,914 \\
Light trucks 4x4 & 29,975 & 35,417 & 40,105 & 47,035 & 48,690 & 46,830 & 46,528 & 49,079 \\
Medium trucks & 88,993 & 83,747 & 89,253 & 81,791 & 78,630 & 77,325 & 78,372 & 79,421 \\
Heavy trucks & 27,988 & 35,230 & 32,760 & 33,951 & 33,642 & 34,624 & 33,996 & 34,049 \\
Ambulances & 1,819 & 1,580 & 1,480 & 1,445 & 1,401 & 1,363 & 1,231 & 1,349 \\
Buses & 6,726 & 7,837 & 8,186 & 7,978 & 8,269 & 8,186 & 8,200 & 8,173 \\
\hline Total trucks and other & $\mathbf{3 8 3 , 4 3 8}$ & $\mathbf{4 0 7 , 2 8 8}$ & $\mathbf{4 1 2 , 7 9 5}$ & $\mathbf{4 1 0 , 4 6 1}$ & $\mathbf{4 0 4 , 2 6 1}$ & $\mathbf{4 0 0 , 1 2 4}$ & $\mathbf{3 9 9 , 5 4 2}$ & $\mathbf{4 0 4 , 9 8 5}$ \\
vehicles & $\mathbf{5 9 6 , 1 1 4}$ & $\mathbf{6 3 2 , 6 8 8}$ & $\mathbf{6 6 2 , 1 5 4}$ & $\mathbf{6 5 9 , 8 5 8}$ & $\mathbf{6 5 0 , 0 6 1}$ & $\mathbf{6 3 5 , 7 4 8}$ & $\mathbf{6 3 3 , 8 5 1}$ & $\mathbf{6 4 0 , 3 0 4}$ \\
\hline GRAND TOTAL ALL & & & & & & &
\end{tabular}

Note: Light trucks $=$ less than 8,500 pounds gross vehicle weight rating (GVWR).

Medium trucks $=8,501-23,999$ pounds GVWR.

Heavy trucks $=24,000$ pounds GVWR or more.

\section{Source:}

U.S. General Services Administration, Federal Supply Service, FY 2015 Federal Fleet Report, Washington, DC, 2016,

Tables 2-5 and 2-6. (Additional resources: www.gsa.gov) 
Table 7.5

Federal Fleet Vehicle Acquisitions by Fuel Type, FY 2002-2015

\begin{tabular}{lrrrrrrrr}
\hline & \multicolumn{1}{c}{ Acquisitions by year } \\
\cline { 2 - 9 } Fuel type & 2002 & \multicolumn{1}{c}{2005} & 2007 & \multicolumn{1}{c}{2010} & 2012 & 2013 & 2014 & 2015 \\
\hline Gasoline & 44,850 & 41,247 & 32,089 & 26,547 & 15,660 & 15,994 & 18,412 & 17,080 \\
Gasoline hybrid & $\mathrm{a}$ & 222 & 458 & 4,853 & 1,254 & 1,364 & 4,124 & 2,500 \\
Gasoline LGHG $^{\mathrm{b}}$ & 0 & 0 & 0 & 0 & 745 & 369 & 562 & 224 \\
Gasoline plug-in hybrid $^{\text {Diesel }}$ & 0 & 0 & 0 & 0 & 144 & 258 & 283 & 263 \\
Diesel hybrid & 8,107 & 6,049 & 5,809 & 4,136 & 4,383 & 4,625 & 5,974 & 6,215 \\
Diesel LGHG & $\mathrm{c}$ & 1 & 4 & 27 & 36 & 51 & 8 & 7 \\
CNG & 0 & 0 & 0 & 0 & 10 & 0 & 1 & 0 \\
E-85 & 1,267 & 188 & 129 & 60 & 106 & 123 & 87 & 241 \\
Electric & 8,054 & 16,892 & 26,581 & 26,789 & 24,214 & 21,644 & 26,267 & 24,651 \\
LNG & 7 & 13 & 7 & 1,376 & 258 & 284 & 349 & 231 \\
LPG & 3 & 0 & 0 & 0 & 0 & 0 & 0 & 0 \\
M-85 & 59 & 1 & 4 & 2 & 15 & 23 & 7 & 6 \\
Hydrogen & 25 & 0 & 0 & 0 & 0 & 0 & 0 & 0 \\
\hline Grand total & 0 & 0 & 0 & 4 & 0 & 2 & 5 & 0 \\
\hline
\end{tabular}

Source:

U.S. General Services Administrations, Federal Vehicle Policy Division, FY 2015 Federal Fleet Report, Washington, DC, 2016, Table 5-4. (Additional resources: www.gsa.gov)

Table 7.6

Fuel Consumed by Federal Government Fleets, FY 2000-2015 (thousand gasoline equivalent gallons)

\begin{tabular}{lrrrrrrrrr}
\hline & FY00 & FY05 & FY07 & FY09 & FY10 & FY12 & FY13 & FY14 & FY15 \\
\hline Gasoline & 284,480 & 300,261 & 293,848 & 301,437 & 322,023 & 302,089 & 295,076 & 296,083 & 310,416 \\
Diesel & 70,181 & 53,363 & 74,806 & 76,456 & 75,329 & 73,228 & 67,332 & 65,168 & 66,736 \\
CNG & 865 & 1,245 & 889 & 499 & 504 & 421 & 369 & 333 & 400 \\
Electricity & 1 & 6 & 5 & 4 & 36 & 85 & 88 & 142 & 197 \\
Biodiesel (B20) & 569 & 8,052 & 9,515 & 7,393 & 8,258 & 6,353 & 5,619 & 5,329 & 4,722 \\
Biodiesel (B100) & 0 & 0 & 0 & 5 & 0 & 344 & 358 & 17 & 11 \\
Methanol/M-85 & 14 & 0 & 0 & 0 & 0 & 0 & 0 & 0 & 0 \\
LPG & 34 & 231 & 322 & 208 & 195 & 211 & 257 & 231 & 150 \\
Ethanol/E-85 & 347 & 3,060 & 3,854 & 7,923 & 8,201 & 12,261 & 14,158 & 12,911 & 13,512 \\
LNG & 0 & 102 & 95 & 35 & 0 & 0 & 0 & 3 & 7 \\
Hydrogen & 0 & 0 & 0 & 0 & 1 & 1 & 0 & 0 \\
\hline Total & 356,491 & 366,320 & 383,334 & 393,961 & 414,548 & 394,994 & 383,257 & 380,217 & 396,152 \\
\hline
\end{tabular}

Source:

U.S. General Services Administrations, Federal Vehicle Policy Division, FY 2015 Federal Fleet Report, Washington, DC, 2016, Table 5-1. (Additional resources: www.gsa.gov)

${ }^{\text {a }}$ Combined with gasoline.

b Low greenhouse gas emissions.

${ }^{c}$ Combined with diesel.

${ }^{\mathrm{d}}$ B100 cannot be separated from B20 from 2000-2007. 
In FY 2005, the General Services Administration owned 143,948 vehicles which they leased to other agencies. In FY 2015, they owned 967 vehicles. The U.S. Postal Service owned 67.5\% of all federal light trucks.

Table 7.7

Federal Government Vehicles by Agency, FY 2015

\begin{tabular}{|c|c|c|c|c|c|c|}
\hline Department or agency & Cars & $\begin{array}{l}\text { Light } \\
\text { trucks }\end{array}$ & $\begin{array}{c}\text { Medium } \\
\text { trucks }\end{array}$ & $\begin{array}{l}\text { Heavy } \\
\text { trucks }\end{array}$ & Other* & Total \\
\hline \multicolumn{7}{|l|}{ CIVILIAN } \\
\hline American Battle Monuments Commission & 27 & 8 & 6 & 0 & 0 & 41 \\
\hline Broadcasting Board of Governors & 76 & 39 & 24 & 18 & 7 & 164 \\
\hline Consumer Product Safety Commission & 91 & 1 & 3 & 0 & 0 & 95 \\
\hline Court Services and Offender Supervision Agency & 75 & 1 & 0 & 0 & 0 & 76 \\
\hline Department of Agriculture & 13,805 & 16,210 & 8,087 & 2,388 & 85 & 40,575 \\
\hline Department of Commerce & 1,157 & 442 & 352 & 70 & 9 & 2,030 \\
\hline Department of Education & 91 & 0 & 0 & 0 & 1 & 92 \\
\hline Department of Energy & 4,761 & 3,432 & 3,785 & 2,078 & 241 & 14,297 \\
\hline Department of Health and Human Services & 3,824 & 559 & 295 & 105 & 78 & 4,861 \\
\hline Department of Homeland Security & 37,440 & 10,056 & 3,404 & 838 & 451 & 52,189 \\
\hline Department of Housing and Urban Development & 348 & 1 & 0 & 0 & 0 & 349 \\
\hline Department of Justice & 36,188 & 4,614 & 1,583 & 957 & 199 & 43,541 \\
\hline Department of Labor & 3,220 & 247 & 205 & 21 & 298 & 3,991 \\
\hline Department of State & 10,156 & 1,605 & 1,367 & 666 & 166 & 13,960 \\
\hline Department of the Interior & 11,333 & 9,256 & 9,100 & 3,144 & 675 & 33,508 \\
\hline Department of Transportation & 4,145 & 730 & 1,023 & 144 & 5 & 6,047 \\
\hline Department of the Treasury & 3,147 & 121 & 45 & 9 & 0 & 3,322 \\
\hline Department of Veterans Affairs & 14,736 & 1,942 & 989 & 728 & 1,127 & 19,522 \\
\hline Environmental Protection Agency & 755 & 72 & 127 & 25 & 7 & 986 \\
\hline Equal Employment Opportunity Commission & 81 & 1 & 0 & 0 & 0 & 82 \\
\hline Federal Communications Commission & 93 & 0 & 0 & 0 & 0 & 93 \\
\hline Federal Housing Finance Agency & 7 & 0 & 0 & 0 & 0 & 7 \\
\hline Federal Maritime Commission & 9 & 0 & 0 & 0 & 0 & 9 \\
\hline General Services Administration & 893 & 46 & 25 & 1 & 2 & 967 \\
\hline Government Printing Office & 12 & 19 & 6 & 5 & 0 & 42 \\
\hline Library of Congress & 9 & 0 & 1 & 4 & 1 & 15 \\
\hline National Aeronautics and Space Administration & 1,385 & 665 & 600 & 318 & 59 & 3,027 \\
\hline National Archives \& Records Administration & 36 & 4 & 10 & 7 & 0 & 57 \\
\hline National Gallery of Art & 6 & 2 & 2 & 1 & 0 & 11 \\
\hline National Labor Relations Board & 34 & 0 & 1 & 0 & 0 & 35 \\
\hline National Science Foundation & 165 & 91 & 183 & 90 & 17 & 546 \\
\hline National Transportation Safety Board & 5 & 0 & 0 & 0 & 0 & 5 \\
\hline Nuclear Regulatory Commission & 14 & 1 & 0 & 2 & 0 & 17 \\
\hline Office of Personnel Management & 1,538 & 2 & 2 & 0 & 0 & 1,542 \\
\hline Peace Corps & 590 & 67 & 0 & 0 & 14 & 671 \\
\hline Pretrial Services Agency for the Dist of Columbia & 3 & 0 & 0 & 0 & 0 & 3 \\
\hline Small Business Administration & 157 & 2 & 1 & 0 & 0 & 160 \\
\hline Smithsonian Institution & 209 & 149 & 57 & 39 & 10 & 464 \\
\hline Social Security Administration & 422 & 5 & 6 & 22 & 5 & 460 \\
\hline Tennessee Valley Authority & 969 & 541 & 992 & 58 & 1 & 2,561 \\
\hline US International Trade Commission & 1 & 1 & 0 & 0 & 0 & 2 \\
\hline TOTAL CIVILIAN AGENCIES & 152,013 & 50,932 & 32,281 & 11,738 & 3,458 & 250,422 \\
\hline \multicolumn{7}{|l|}{ MILITARY } \\
\hline Corps of Engineers, Civil Works & 2,162 & 2,739 & 1,791 & 658 & 2 & 7,352 \\
\hline Defense Agencies & 4,625 & 611 & 572 & 596 & 370 & 6,774 \\
\hline Department of Air Force & 11,199 & 13,399 & 15,049 & 6,116 & 1,794 & 47,557 \\
\hline Department of Army & 31,873 & 11,682 & 12,046 & 5,366 & 2,520 & 63,487 \\
\hline Department of Navy & 17,240 & 10,113 & 7,660 & 3,385 & 851 & 39,249 \\
\hline United States Marine Corps & 7,189 & 2,032 & 2,064 & 1,293 & 525 & 13,103 \\
\hline TOTAL MILITARY AGENCIES & 74,288 & 40,576 & 39,182 & 17,414 & 6,062 & 177,522 \\
\hline \multirow[t]{2}{*}{ U. S. POSTAL SERVICE } & 9,018 & 190,485 & 7,958 & 4,897 & 2 & 212,360 \\
\hline & 235,319 & 281,993 & 79,421 & 34,049 & 9,522 & 640,304 \\
\hline
\end{tabular}

* Other $=$ Buses and ambulances.

Note: Light trucks - Less than 8,500 pounds gross vehicle weight ratio (GVWR) (Includes ambulances). Medium trucks 8,501-23,999 pounds GVWR. Heavy trucks - 24,000 pounds GVWR or more. Does not include low-speed vehicles.

Source:

U.S. General Services Administration, Federal Supply Service, FY 2015 Federal Fleet Report, Washington, DC, 2016 , Table 2-1.

(Additional resources: www.gsa.gov) 
Demand response vehicles (also called paratransit or dial-a-ride) are widely used by transit agencies. The vehicles do not operate over a fixed route or on a fixed schedule. The vehicle may be dispatched to pick up several passengers at different pick-up points before taking them to their respective destinations and may even be interrupted en route to these destinations to pick up other passengers. Demand response service is provided primarily by vans. In 2007, the data changed substantially due to improved estimation methodologies. Unfortunately, those data are no longer comparable to the rest of the historical series.

Table 7.8

Summary Statistics on Demand Response Vehicles, 1994-2014

\begin{tabular}{cccccccc}
\hline & $\begin{array}{c}\text { Number of } \\
\text { agencies }\end{array}$ & $\begin{array}{c}\text { Number of } \\
\text { active vehicles }\end{array}$ & $\begin{array}{c}\text { Vehicle-miles } \\
\text { (millions) }\end{array}$ & $\begin{array}{c}\text { Average } \\
\text { miles per } \\
\text { vehicle }\end{array}$ & $\begin{array}{c}\text { Passenger- } \\
\text { miles } \\
\text { (millions) }\end{array}$ & $\begin{array}{c}\text { Average } \\
\text { load } \\
\text { factor }\end{array}$ & $\begin{array}{c}\text { Energy use } \\
\text { (trillion Btu) }\end{array}$ \\
\hline 1994 & 5,214 & 28,729 & 463.7 & 16.14 & 577 & a & 9.5 \\
1995 & 5,214 & 29,352 & 506.5 & 17.26 & 607 & 1.41 & 9.2 \\
1996 & 5,214 & 30,804 & 548.3 & 17.80 & 656 & 1.21 & 9.9 \\
1997 & 5,214 & 32,509 & 585.3 & 18.00 & 754 & 1.36 & 9.8 \\
1998 & 5,214 & 29,646 & 670.9 & 22.63 & 735 & 1.21 & 10.4 \\
1999 & 5,252 & 31,884 & 718.4 & 22.53 & 813 & 1.34 & 10.6 \\
2000 & 5,252 & 33,080 & 758.9 & 22.94 & 839 & 1.30 & 10.8 \\
2001 & 5,251 & 34,661 & 789.3 & 22.77 & 855 & 1.28 & 11.3 \\
2002 & 5,251 & 34,699 & 802.6 & 23.13 & 853 & 1.24 & 11.6 \\
2003 & 5,346 & 35,954 & 864.0 & 24.03 & 930 & 1.27 & 12.9 \\
2004 & 5,960 & 37,078 & 889.5 & 23.99 & 962 & 1.25 & 13.3 \\
2005 & 5,960 & 41,958 & 978.3 & 23.32 & 1,058 & 1.25 & 14.8 \\
2006 & 5,960 & 43,509 & $1,013.0$ & 23.28 & 1,078 & 1.24 & 15.5 \\
\hline 2007 & 7,300 & 64,865 & $1,471.4$ & 22.68 & 1,502 & 1.18 & $\mathrm{~b}$ \\
2008 & 7,200 & 65,799 & $1,495.2$ & 22.72 & 1,412 & 1.09 & 24.7 \\
2009 & 6,700 & 68,957 & $1,529.2$ & 22.18 & 1,477 & 1.12 & 24.7 \\
2010 & 6,741 & 68,621 & $1,693.6$ & 24.68 & 1,494 & 1.03 & 23.1 \\
2011 & 6,600 & 65,336 & $1,611.8$ & 24.67 & 1,580 & 1.13 & 22.8 \\
2012 & 6,511 & 68,632 & $1,618.1$ & 23.58 & 1,756 & 1.24 & 24.1 \\
2013 & 6,270 & 68,559 & $1,565.1$ & 22.83 & 2,171 & 1.59 & 24.8 \\
\end{tabular}

Note: See Glossary for a detailed definition of demand response.

\section{Source:}

American Public Transportation Association, 2016 Public Transportation Fact Book, Washington, DC, April 2016. (Additional resources: www.apta.com)

a Data are not available.

b Data are not continuous between 2006 and 2007 due to changes in estimation methodology. See source document for details. 
In 2007, the data changed substantially due to improved estimation methodologies. Unfortunately, those data are no longer comparable to the rest of the historical series.

Table 7.9

Summary Statistics on Transit Buses and Trolleybuses, 1994-2015

\begin{tabular}{cccccc}
\hline Year & $\begin{array}{c}\text { Number of } \\
\text { active buses }\end{array}$ & $\begin{array}{c}\text { Vehicle-miles } \\
\text { (millions) }\end{array}$ & $\begin{array}{c}\text { Passenger- } \\
\text { miles } \\
\text { (millions) }\end{array}$ & $\begin{array}{c}\text { Btu/passenger- } \\
\text { mile }\end{array}$ & $\begin{array}{c}\text { Energy use } \\
\text { (trillion Btu) }\end{array}$ \\
\hline 1994 & 68,766 & 2,176 & 19,019 & 4,262 & 81.1 \\
1995 & 67,802 & 2,198 & 19,005 & 4,307 & 81.9 \\
1996 & 72,353 & 2,234 & 19,280 & 4,340 & 83.7 \\
1997 & 73,425 & 2,259 & 19,793 & 4,434 & 87.8 \\
1998 & 72,788 & 2,188 & 20,542 & 4,399 & 90.4 \\
1999 & 74,885 & 2,290 & 21,391 & 4,344 & 92.9 \\
2000 & 75,665 & 2,329 & 21,433 & 4,531 & 97.1 \\
2001 & 76,675 & 2,389 & 22,209 & 4,146 & 92.1 \\
2002 & 76,806 & 2,425 & 22,029 & 4,133 & 91.1 \\
2003 & 78,000 & 2,435 & 21,438 & 4,213 & 90.3 \\
2004 & 81,630 & 2,484 & 21,550 & 4,364 & 94.0 \\
2005 & 82,642 & 2,498 & 21,998 & 4,250 & 93.5 \\
2006 & 83,689 & 2,507 & 22,985 & 4,316 & 99.2 \\
\hline 2007 & 65,808 & 2,314 & 21,132 & 4,372 & 92.4 \\
2008 & 67,096 & 2,388 & 21,918 & 4,348 & 95.3 \\
2009 & 65,363 & 2,345 & 21,645 & 4,242 & 91.8 \\
2010 & 66,810 & 2,425 & 21,172 & 4,118 & 87.2 \\
2011 & 69,654 & 2,425 & 21,574 & 4,240 & 91.5 \\
2012 & 70,757 & 2,417 & 21,251 & 4,030 & 89.7 \\
2013 & 71,699 & 2,425 & 22,306 & 4,071 & 90.8 \\
2014 & 71,603 & 2,445 & 22,614 & 3,829 & 86.6 \\
2015 & 64,184 & 2,205 & 20,239 & 4,051 & 82.0 \\
\hline
\end{tabular}

\section{Source:}

American Public Transportation Association, 2016 Public Transportation Fact Book, Washington, DC, April 2016, Appendix A. Data for 2015 are from the U.S. Department of Transportation, Federal Transit Administration, Federal Transit Database. (Additional resources: www.apta.com)

a Data are not continuous between 2006 and 2007 due to changes in estimation methodology. See source document for details. 


\section{Chapter 8 \\ Household Vehicles and Characteristics}

Summary Statistics from Tables/Figures in this Chapter

\begin{tabular}{|c|c|c|}
\hline Source & & \\
\hline \multirow[t]{3}{*}{ Table 8.2} & Vehicles per capita, 2015 & 0.823 \\
\hline & Vehicles per licensed driver, 2015 & 1.21 \\
\hline & Vehicles per household, 2015 & 2.10 \\
\hline \multirow[t]{8}{*}{ Table 8.3} & Share of households owning 3 or more vehicles & \\
\hline & 1960 & $2.5 \%$ \\
\hline & 1970 & $5.5 \%$ \\
\hline & 1980 & $17.5 \%$ \\
\hline & 1990 & $17.3 \%$ \\
\hline & 2000 & $18.3 \%$ \\
\hline & 2010 & $19.5 \%$ \\
\hline & 2015 & $20.3 \%$ \\
\hline \multirow[t]{5}{*}{ Figure 8.1} & Average occupancy rates by vehicle type, 2009 & \\
\hline & Van & 2.35 \\
\hline & Sport Utility Vehicle & 1.90 \\
\hline & Car & 1.55 \\
\hline & Pickup & 1.49 \\
\hline Table 8.8 & Average annual miles per household vehicle, 2009 & 11,300 \\
\hline Table 8.14 & Share of workers who car pooled, 2015 & $9.0 \%$ \\
\hline \multirow[t]{3}{*}{ Table 8.19} & Long-distance trips in the United States, 2001 & \\
\hline & Person-trips & 2,554 million \\
\hline & Person-miles & 1,138 billion \\
\hline
\end{tabular}


The number of vehicles in the United States is growing faster than the population. The growth in vehicle-miles has slowed to $0.3 \%$ from 2005-2015. See Table 8.2 for vehicles per capita and vehicle-miles per capita.

Table 8.1

Population and Vehicle Profile, 1950-2015

\begin{tabular}{|c|c|c|c|c|c|c|}
\hline Year & $\begin{array}{c}\text { Resident } \\
\text { population } \\
\text { (thousands) }\end{array}$ & $\begin{array}{c}\text { Total } \\
\text { households } \\
\text { (thousands) }\end{array}$ & $\begin{array}{l}\text { Number of } \\
\text { vehicles in } \\
\text { operation } \\
\text { (thousands) }\end{array}$ & $\begin{array}{c}\text { Total vehicle- } \\
\text { miles } \\
\text { (millions) }\end{array}$ & $\begin{array}{l}\text { Number of } \\
\text { licensed drivers } \\
\text { (thousands) }\end{array}$ & $\begin{array}{l}\text { Number of } \\
\text { civilian } \\
\text { employed } \\
\text { persons } \\
\text { (thousands) }\end{array}$ \\
\hline 1950 & 151,868 & 43,554 & 43,501 & 458,246 & 62,194 & 58,920 \\
\hline 1955 & 165,069 & 47,874 & 56,540 & 605,646 & 74,686 & 62,171 \\
\hline 1960 & 179,979 & 52,799 & 67,906 & 718,762 & 87,253 & 65,778 \\
\hline 1965 & 193,526 & 57,436 & 82,066 & 887,812 & 98,502 & 71,088 \\
\hline 1970 & 205,052 & 63,401 & 98,136 & $1,109,724$ & 111,543 & 78,628 \\
\hline 1975 & 215,973 & 71,120 & 120,054 & $1,327,664$ & 129,791 & 85,846 \\
\hline 1980 & 227,226 & 80,776 & 139,831 & $1,527,295$ & 145,295 & 99,303 \\
\hline 1985 & 238,466 & 86,789 & 157,048 & $1,774,826$ & 156,868 & 107,150 \\
\hline 1990 & 250,132 & 93,347 & 179,299 & $2,144,362$ & 167,015 & 118,793 \\
\hline 1991 & 253,493 & 94,312 & 181,438 & $2,172,050$ & 168,995 & 117,718 \\
\hline 1992 & 256,894 & 95,669 & 181,519 & $2,247,151$ & 173,125 & 118,492 \\
\hline 1993 & 260,255 & 96,391 & 186,315 & $2,296,378$ & 173,149 & 120,259 \\
\hline 1994 & 263,436 & 97,107 & 188,714 & $2,357,588$ & 175,403 & 123,060 \\
\hline 1995 & 266,557 & 98,990 & 193,441 & $2,422,696$ & 176,628 & 124,900 \\
\hline 1996 & 269,667 & 99,627 & 198,294 & $2,485,848$ & 179,539 & 126,708 \\
\hline 1997 & 272,912 & 101,018 & 201,071 & $2,561,695$ & 182,709 & 129,558 \\
\hline 1998 & 276,115 & 102,528 & 205,043 & $2,631,522$ & 184,980 & 131,463 \\
\hline 1999 & 279,295 & 103,874 & 209,509 & $2,691,056$ & 187,170 & 133,488 \\
\hline 2000 & 282,385 & 104,705 & 213,300 & $2,746,925$ & 190,625 & 136,891 \\
\hline 2001 & 285,309 & 108,209 & 216,683 & $2,797,287$ & 191,276 & 136,933 \\
\hline 2002 & 288,105 & 109,297 & 221,027 & $2,855,508$ & 194,296 & 136,485 \\
\hline 2003 & 290,820 & 111,278 & 225,882 & $2,890,450$ & 196,166 & 137,736 \\
\hline 2004 & 293,463 & 112,000 & 232,167 & $2,964,788$ & 198,889 & 139,252 \\
\hline 2005 & 296,186 & 113,343 & 238,384 & $2,989,430$ & 200,549 & 141,730 \\
\hline 2006 & 298,996 & 114,384 & 244,643 & $3,014,371$ & 202,810 & 144,427 \\
\hline 2007 & 302,004 & 116,011 & 248,701 & $3,031,124$ & 205,742 & 146,047 \\
\hline 2008 & 304,798 & 116,783 & 249,813 & $2,976,528$ & 208,321 & 145,362 \\
\hline 2009 & 307,439 & 117,181 & 248,972 & $2,956,764$ & 209,618 & 139,877 \\
\hline 2010 & 309,347 & 117,538 & 248,231 & $2,967,266$ & 210,115 & 139,064 \\
\hline 2011 & 311,719 & 118,682 & 248,932 & $2,950,402$ & 211,875 & 139,869 \\
\hline 2012 & 314,103 & 121,084 & 251,497 & $2,969,433$ & 211,815 & 142,469 \\
\hline 2013 & 316,427 & 122,459 & 252,715 & $2,988,280$ & 212,160 & 143,929 \\
\hline 2014 & 318,907 & 123,027 & 258,027 & $3,025,656$ & 214,092 & 146,305 \\
\hline 2015 & 320,897 & 125,819 & 264,194 & $3,095,373$ & 218,084 & 148,834 \\
\hline \multicolumn{7}{|c|}{ Average annual percentage change } \\
\hline 1950-2015 & $1.2 \%$ & $1.6 \%$ & $2.8 \%$ & $3.0 \%$ & $1.9 \%$ & $1.4 \%$ \\
\hline 2005-2015 & $0.8 \%$ & $1.0 \%$ & $1.0 \%$ & $0.3 \%$ & $0.8 \%$ & $0.5 \%$ \\
\hline
\end{tabular}

Sources:

Resident population and civilian employed persons - U.S. Department of Commerce, Bureau of the Census, Online Data Retrieval, Washington, DC, 2017. (Additional resources: www.census.gov)

Vehicles in operation - IHS Automotive. FURTHER REPRODUCTION PROHIBITED. (Additional resources: https://www.ihs.com/industry/automotive.html)

Licensed drivers and vehicle-miles - U.S. Department of Transportation, Federal Highway Administration, Highway Statistics 2015, Tables DL-20 and VM-1, and annual. (Additional resources: www.fhwa.dot.gov)

a Estimates as of July 1. Includes Armed Forces in the United States. 
Vehicle-miles per capita were over 10,000 miles from 2004 to 2007 but were 9,646 miles in 2015. There were 1.78 vehicles for every employed civilian in the United States in 2015.

Table 8.2

Vehicles and Vehicle-Miles per Capita, 1950-2015

\begin{tabular}{|c|c|c|c|c|c|c|}
\hline Year & $\begin{array}{l}\text { Vehicles } \\
\text { per capita }\end{array}$ & $\begin{array}{l}\text { Vehicle-miles } \\
\text { per capita }\end{array}$ & $\begin{array}{l}\text { Licensed } \\
\text { drivers per } \\
\text { household }\end{array}$ & $\begin{array}{l}\text { Vehicles per } \\
\text { household }\end{array}$ & $\begin{array}{c}\text { Vehicles } \\
\text { per licensed } \\
\text { driver }\end{array}$ & $\begin{array}{c}\text { Vehicles per } \\
\text { civilian } \\
\text { employed } \\
\text { persons }\end{array}$ \\
\hline 1950 & 0.286 & 3,017 & 1.43 & 1.00 & 0.70 & 0.74 \\
\hline 1955 & 0.343 & 3,669 & 1.56 & 1.18 & 0.76 & 0.91 \\
\hline 1960 & 0.377 & 3,994 & 1.65 & 1.29 & 0.78 & 1.03 \\
\hline 1965 & 0.424 & 4,588 & 1.71 & 1.43 & 0.83 & 1.15 \\
\hline 1970 & 0.479 & 5,412 & 1.76 & 1.55 & 0.88 & 1.25 \\
\hline 1975 & 0.556 & 6,147 & 1.82 & 1.69 & 0.92 & 1.40 \\
\hline 1980 & 0.614 & 6,707 & 1.80 & 1.73 & 0.96 & 1.41 \\
\hline 1985 & 0.659 & 7,443 & 1.81 & 1.81 & 1.00 & 1.47 \\
\hline 1990 & 0.717 & 8,573 & 1.79 & 1.92 & 1.07 & 1.51 \\
\hline 1991 & 0.716 & 8,568 & 1.79 & 1.92 & 1.07 & 1.54 \\
\hline 1992 & 0.707 & 8,747 & 1.81 & 1.90 & 1.05 & 1.53 \\
\hline 1993 & 0.716 & 8,824 & 1.80 & 1.93 & 1.08 & 1.55 \\
\hline 1994 & 0.716 & 8,949 & 1.81 & 1.94 & 1.08 & 1.53 \\
\hline 1995 & 0.726 & 9,089 & 1.78 & 1.95 & 1.10 & 1.55 \\
\hline 1996 & 0.735 & 9,218 & 1.80 & 1.99 & 1.10 & 1.56 \\
\hline 1997 & 0.737 & 9,387 & 1.81 & 1.99 & 1.10 & 1.55 \\
\hline 1998 & 0.743 & 9,531 & 1.80 & 2.00 & 1.11 & 1.56 \\
\hline 1999 & 0.750 & 9,635 & 1.80 & 2.02 & 1.12 & 1.57 \\
\hline 2000 & 0.755 & 9,728 & 1.82 & 2.04 & 1.12 & 1.56 \\
\hline 2001 & 0.759 & 9,804 & 1.77 & 2.00 & 1.13 & 1.58 \\
\hline 2002 & 0.767 & 9,911 & 1.78 & 2.02 & 1.14 & 1.62 \\
\hline 2003 & 0.777 & 9,939 & 1.76 & 2.03 & 1.15 & 1.64 \\
\hline 2004 & 0.791 & 10,103 & 1.78 & 2.07 & 1.17 & 1.67 \\
\hline 2005 & 0.805 & 10,093 & 1.77 & 2.10 & 1.19 & 1.68 \\
\hline 2006 & 0.818 & 10,082 & 1.77 & 2.14 & 1.21 & 1.69 \\
\hline 2007 & 0.824 & 10,037 & 1.77 & 2.14 & 1.21 & 1.70 \\
\hline 2008 & 0.820 & 9,766 & 1.78 & 2.14 & 1.20 & 1.72 \\
\hline 2009 & 0.810 & 9,617 & 1.79 & 2.12 & 1.19 & 1.78 \\
\hline 2010 & 0.802 & 9,592 & 1.79 & 2.11 & 1.18 & 1.79 \\
\hline 2011 & 0.799 & 9,467 & 1.79 & 2.10 & 1.17 & 1.78 \\
\hline 2012 & 0.801 & 9,457 & 1.75 & 2.08 & 1.19 & 1.77 \\
\hline 2013 & 0.799 & 9,450 & 1.73 & 2.06 & 1.19 & 1.76 \\
\hline 2014 & 0.810 & 9,498 & 1.74 & 2.09 & 1.21 & 1.76 \\
\hline 2015 & 0.823 & 9,646 & 1.73 & 2.10 & 1.21 & 1.78 \\
\hline & \multicolumn{6}{|c|}{ Average annual percentage change } \\
\hline 1950-2015 & $1.6 \%$ & $1.8 \%$ & $0.3 \%$ & $1.1 \%$ & $0.8 \%$ & $1.4 \%$ \\
\hline 2005-2015 & $0.2 \%$ & $-0.5 \%$ & $-0.2 \%$ & $0.0 \%$ & $0.2 \%$ & $0.6 \%$ \\
\hline
\end{tabular}

\section{Sources:}

Resident population and civilian employed persons - U.S. Department of Commerce, Bureau of the Census, Online Data Retrieval, Washington, DC, 2017. (Additional resources: www.census.gov)

Vehicles in operation - IHS Automotive. FURTHER REPRODUCTION PROHIBITED. (Additional resources: https://www.ihs.com/industry/automotive.html)

Vehicle-miles - U.S. Department of Transportation, Federal Highway Administration, Highway Statistics 2015, Table VM-1 and annual. (Additional resources: www.fhwa.dot.gov)

\footnotetext{
${ }^{\text {a }}$ Includes all vehicles (light and heavy).
} 
Household vehicle ownership shows a dramatic increase from 1960 to 1990. In 1960, nearly 79\% of households owned less than two vehicles; by 1990, it declined to 45\%. Census data prior to 1990 indicated that the majority of households owned one vehicle; in 1990 that changed to two vehicles. Since 2000, less than 10\% of households had no vehicles. The American Community Survey now collects these data on an annual basis, thus annual data are available after 2010.

Table 8.3

Household Vehicle Ownership, 1960-2015 Census (percentage)

\begin{tabular}{rrrrr}
\hline & $\begin{array}{c}\text { No } \\
\text { vehicles }\end{array}$ & $\begin{array}{c}\text { One } \\
\text { vehicle }\end{array}$ & $\begin{array}{c}\text { Two } \\
\text { vehicles }\end{array}$ & $\begin{array}{c}\text { Three or } \\
\text { more } \\
\text { vehicles }\end{array}$ \\
\hline 1960 & $21.5 \%$ & $56.9 \%$ & $19.0 \%$ & $2.5 \%$ \\
1970 & $17.5 \%$ & $47.7 \%$ & $29.3 \%$ & $5.5 \%$ \\
1980 & $12.9 \%$ & $35.5 \%$ & $34.0 \%$ & $17.5 \%$ \\
1990 & $11.5 \%$ & $33.7 \%$ & $37.4 \%$ & $17.3 \%$ \\
2000 & $9.4 \%$ & $33.8 \%$ & $38.6 \%$ & $18.3 \%$ \\
2010 & $9.1 \%$ & $33.8 \%$ & $37.6 \%$ & $19.5 \%$ \\
2011 & $9.3 \%$ & $34.1 \%$ & $37.5 \%$ & $19.1 \%$ \\
2012 & $9.2 \%$ & $34.1 \%$ & $37.3 \%$ & $19.3 \%$ \\
2013 & $9.1 \%$ & $33.9 \%$ & $37.3 \%$ & $19.7 \%$ \\
2014 & $9.1 \%$ & $33.7 \%$ & $37.3 \%$ & $19.9 \%$ \\
2015 & $8.9 \%$ & $33.5 \%$ & $37.2 \%$ & $20.3 \%$ \\
\hline
\end{tabular}

\section{Source:}

U. S. Department of Transportation, Volpe National Transportation Systems Center, Journey-to-Work Trends in the United States and its Major Metropolitan Area, 1960-1990, Cambridge, MA, 1994, p. 2-2.

2000 data - U.S. Bureau of the Census, American Fact Finder, factfinder.census.gov, Table QT-04, August 2001. (Additional resources: www.census.gov)

2010-2015 data - U.S. Bureau of the Census, American Community Survey, Table CP04, 2017. 


\section{National Household Travel Survey Daily Trip Data}

The Department of Transportation (DOT) collected data on daily trips in 1969, 1977, 1983, 1990 and 1995 via the Nationwide Personal Transportation Survey (NPTS). For 2001, the DOT combined the collection of long trip and daily trip data into one survey - the 2001 National Household Travel Survey (NHTS). The long trip data were not included in the 2009 NHTS.

The NHTS is the nation's inventory of daily travel. The survey includes demographic characteristics of households, people, vehicles, and detailed information on daily travel for all purposes by all modes. NHTS survey data are collected from a sample of U.S. households and expanded to provide national estimates of trips and miles by travel mode, trip purpose, and a host of household attributes.

The NHTS was designed to continue the NPTS series, but as with all data surveys, caution should be used when comparing statistics from one survey to another due to changes in terminology, survey procedures, and target population. The 2001 and 2009 surveys collected data on trips of children under 5 years of age, while the previous NPTS did not. Improved methodologies first used in the collection of trip information in the 1995 NPTS make it difficult to compare these data with past NPTS survey data. Thus, the 1990 NPTS trip data have been adjusted to make it comparable with the later surveys.

The next NHTS is being conducted in 2016 and data will be available in 2018.

Table 8.4

Demographic Statistics from the 1969, 1977, 1983, 1990, 1995 NPTS and 2001, 2009 NHTS

\begin{tabular}{lcccccccc}
\hline & & & & & & & & $\begin{array}{c}\text { Percent } \\
\text { change } \\
\end{array}$ \\
& 1969 & 1977 & 1983 & 1990 & 1995 & 2001 & 2009 & $1969-2009$ \\
\hline Persons per household & 3.16 & 2.83 & 2.69 & 2.56 & 2.63 & 2.58 & 2.50 & $-21 \%$ \\
Vehicles per household & 1.16 & 1.59 & 1.68 & 1.77 & 1.78 & 1.89 & 1.87 & $61 \%$ \\
Workers per household & 1.21 & 1.23 & 1.21 & 1.27 & 1.33 & 1.35 & 1.34 & $11 \%$ \\
Licensed drivers per household & 1.65 & 1.69 & 1.72 & 1.75 & 1.78 & 1.77 & 1.88 & $14 \%$ \\
Vehicles per worker & 0.96 & 1.29 & 1.39 & 1.40 & 1.34 & 1.39 & 1.40 & $46 \%$ \\
Vehicles per licensed driver & 0.70 & 0.94 & 0.98 & 1.01 & 1.00 & 1.06 & 1.00 & $42 \%$ \\
Average vehicle trip length (miles) & 8.89 & 8.34 & 7.90 & 8.98 & 9.06 & 9.87 & 9.72 & $9 \%$ \\
\hline
\end{tabular}

Note: Average vehicle trip length for 1990 and 1995 is calculated using only those records with trip mileage information present. The 1969 survey does not include pickups and other light trucks as household vehicles. Data on vehicles per household and licensed drivers per household will not match Table 8.2 because they come from a different source.

\section{Sources:}

U.S. Department of Transportation, Federal Highway Administration, 1990 Nationwide Personal Transportation Survey: Summary of Travel Trends, FHWA-PL-92-027, Washington, DC, March 1992, Table 2. Data for 1995, 2001 and 2009 were generated from the 2009 National Household Travel Survey website nhts.ornl.gov. (Additional resources: www.fhwa.dot.gov) 
Due to methodology improvements in collecting trip information, the 2001 and 1995 data should be compared only to the 1990 adjusted data. The original 1990 data are comparable to all previous surveys; however, comparisons should always be made with caution because of differing survey methodologies.

Table 8.5

\section{Average Annual Vehicle-Miles, Vehicle Trips and Trip Length per Household} 1969, 1977, 1983, 1990, 1995 NPTS and 2001, 2009 NHTS

\begin{tabular}{lcc}
\hline & Journey-to-work & All trips \\
\hline \multicolumn{3}{c}{ Average annual vehicle-miles per household } \\
\hline 1969 & 4,183 & 12,423 \\
1977 & 3,815 & 12,036 \\
1983 & 3,538 & 11,739 \\
1990 original & 4,853 & 15,100 \\
\hline 1990 adjusted & 4,853 & 18,161 \\
1995 & 6,492 & 20,895 \\
2001 & 5,724 & 21,171 \\
2009 & 5,513 & 19,850 \\
\hline \multicolumn{3}{c}{ Average annual vehicle trips per household } \\
\hline 1969 & 445 & 1,396 \\
1977 & 423 & 1,442 \\
1983 & 414 & 1,486 \\
1990 original & 448 & 1,702 \\
\hline 1990 adjusted & 448 \\
1995 & 553 & 2,077 \\
2001 & 479 & 2,321 \\
2009 & 457 & 2,171 \\
\hline \multicolumn{3}{c}{ Average vehicle trip length (miles) } \\
\hline 1969 & 9.4 \\
1977 & 9.0 & 2,068 \\
1983 & 8.5 & 8.9 \\
1990 original & 11.0 & 8.4 \\
1990 adjusted & 11.0 & 7.9 \\
1995 & 11.8 & 9.0 \\
2001 & 12.2 & 8.9 \\
2009 & 12.2 & 9.1 \\
\hline
\end{tabular}

Sources:

U.S. Department of Transportation, Federal Highway Administration, 1990 Nationwide Personal Transportation Survey: Summary of Travel Trends, FHWA-PL-92-027, Washington, DC, March 1992, Table 7. 1990 adjusted data - Oak Ridge National Laboratory, Oak Ridge, TN, August 1998. 1995 NPTS, 2001, 2009 NHTS data were generated from the 2009 National Household Travel Survey website nhts.ornl.gov. (Additional resources: www.fhwa.dot.gov, nhts.ornl.gov)

a It is believed that the methodology changes in the 1995 NPTS did not affect journey-to-work trips; therefore, no adjustment is necessary. 
In 2001 and 2009 annual vehicle-miles traveled (vmt) for a three-person household is around 28,000 miles. The number of drivers in a household makes a big difference in vmt, as does the presence of children in the household. Households with children have more than double the vmt of households without children.

Table 8.6

Average Number of Vehicles and Vehicle Travel per Household, 1990 NPTS and 2001 and 2009 NHTS

\begin{tabular}{|c|c|c|c|c|c|c|}
\hline \multirow{2}{*}{$\begin{array}{l}\text { Number of licensed } \\
\text { drivers }\end{array}$} & \multicolumn{3}{|c|}{$\begin{array}{c}\text { Average } \\
\text { number of vehicles } \\
\text { per household }\end{array}$} & \multicolumn{3}{|c|}{$\begin{array}{c}\text { Average } \\
\text { vehicle-miles traveled } \\
\text { per household }\end{array}$} \\
\hline & 1990 & 2001 & 2009 & 1990 & 2001 & 2009 \\
\hline 1 & 1.5 & 1.2 & 1.1 & 15,200 & 9,700 & 8,800 \\
\hline 2 & 2.1 & 2.2 & 2.2 & 22,900 & 25,800 & 23,500 \\
\hline 3 & 2.9 & 3.0 & 3.0 & 29,400 & 37,900 & 37,700 \\
\hline 4 or more & 3.8 & 3.8 & 3.9 & 40,500 & 47,200 & 55,200 \\
\hline \multicolumn{7}{|l|}{ Household size } \\
\hline 1 person & 1.2 & 1.0 & 1.0 & 11,400 & 7,500 & 7,100 \\
\hline 2 persons & 1.9 & 2.0 & 2.0 & 19,300 & 21,200 & 17,500 \\
\hline 3 persons & 2.2 & 2.3 & 2.3 & 23,700 & 28,400 & 27,900 \\
\hline 4 persons & 2.4 & 2.4 & 2.4 & 25,300 & 28,600 & 33,200 \\
\hline 5 persons & 2.4 & 2.4 & 2.4 & 24,900 & 33,200 & 33,700 \\
\hline 6 or more persons & 2.7 & 2.5 & 2.4 & 29,200 & 33,800 & 33,600 \\
\hline \multicolumn{7}{|c|}{ Household urban status } \\
\hline Urban & 1.9 & 1.8 & 1.7 & 19,000 & 19,300 & 17,600 \\
\hline Rural & 2.1 & 2.3 & 2.4 & 22,200 & 28,400 & 27,700 \\
\hline \multicolumn{7}{|c|}{ Household composition } \\
\hline With children & 2.2 & 2.2 & 2.2 & 24,100 & 28,300 & 30,400 \\
\hline Without children & 1.8 & 1.7 & 1.7 & 17,600 & 16,700 & 14,400 \\
\hline All households & 1.8 & 1.9 & 1.9 & 18,300 & 21,200 & 19,900 \\
\hline
\end{tabular}

Source:

Generated from the Department of Transportation, Federal Highway Administration, Nationwide Personal Transportation Survey Public Use Files, Washington, DC, 2000 and the 2009 National Household Travel Survey website nhts.ornl.gov. (Additional resources: nhts.ornl.gov) 
In 2009, 22\% of vehicle trips were traveling to and from work. Another $22 \%$ of trips were for shopping. Shopping is done close to home, as the average trip length for shopping was only 6.5 miles.

Table 8.7

Trip Statistics ${ }^{\mathrm{a}}$ by Trip Purpose, 2001 and 2009 NHTS

\begin{tabular}{|c|c|c|c|c|c|c|c|c|}
\hline \multirow[b]{2}{*}{ Trip purpose } & \multicolumn{2}{|c|}{ Share of trips } & \multicolumn{2}{|c|}{$\begin{array}{l}\text { Share of vehicle- } \\
\text { miles traveled }\end{array}$} & \multicolumn{2}{|c|}{$\begin{array}{l}\text { Trip length } \\
\text { (miles) }\end{array}$} & \multicolumn{2}{|c|}{$\begin{array}{l}\text { Trip length } \\
\text { (minutes) }\end{array}$} \\
\hline & 2001 & 2009 & 2001 & 2009 & 2001 & 2009 & 2001 & 2009 \\
\hline To/from work & $22.1 \%$ & $22.3 \%$ & $27.0 \%$ & $28.7 \%$ & 12.1 & 12.2 & 22.3 & 22.9 \\
\hline Work-related business & $4.1 \%$ & $3.9 \%$ & $8.4 \%$ & $7.2 \%$ & 20.3 & 17.2 & 30.9 & 27.5 \\
\hline Shopping & $21.1 \%$ & $22.8 \%$ & $14.5 \%$ & $15.5 \%$ & 6.7 & 6.5 & 14.4 & 14.4 \\
\hline Other family/personal business & $24.7 \%$ & $21.9 \%$ & $18.7 \%$ & $15.7 \%$ & 7.5 & 6.8 & 15.2 & 14.8 \\
\hline School/church & $4.9 \%$ & $5.0 \%$ & $3.7 \%$ & $4.6 \%$ & 7.5 & 8.8 & 15.8 & 17.5 \\
\hline Medical/dental & $2.2 \%$ & $2.6 \%$ & $2.2 \%$ & $2.6 \%$ & 9.9 & 9.9 & 20.7 & 21.2 \\
\hline Vacation & $0.4 \%$ & $0.7 \%$ & $1.8 \%$ & $2.3 \%$ & 47.4 & 31.4 & 59.6 & 41.3 \\
\hline Visit friends/relatives & $6.3 \%$ & $5.7 \%$ & $9.4 \%$ & $9.4 \%$ & 14.9 & 15.7 & 24.4 & 24.6 \\
\hline Other social/recreational & $13.7 \%$ & $14.9 \%$ & $13.2 \%$ & $13.5 \%$ & 9.6 & 8.6 & 18.2 & 17.2 \\
\hline Other & $0.5 \%$ & $0.3 \%$ & $1.0 \%$ & $0.6 \%$ & 18.1 & 19.0 & 31.4 & 29.7 \\
\hline All & $100.0 \%$ & $100.0 \%$ & $100.0 \%$ & $100.0 \%$ & 9.9 & 9.7 & 18.7 & 18.6 \\
\hline
\end{tabular}

Note: The "All" category for average trip length and duration includes records for which trip purpose was not identified.

\section{Source:}

Generated from the 2009 National Household Travel Survey website nhts.ornl.gov.

${ }^{\text {a }}$ Percentages may not sum to totals due to rounding. 
While car occupancy stayed nearly constant from 1995 to 2009, most other vehicle types showed increased occupancy. Vans and sport utility vehicles have higher vehicle occupancies than cars.

Figure 8.1. Average Vehicle Occupancy by Vehicle Type, 1995 NPTS and 2009 NHTS

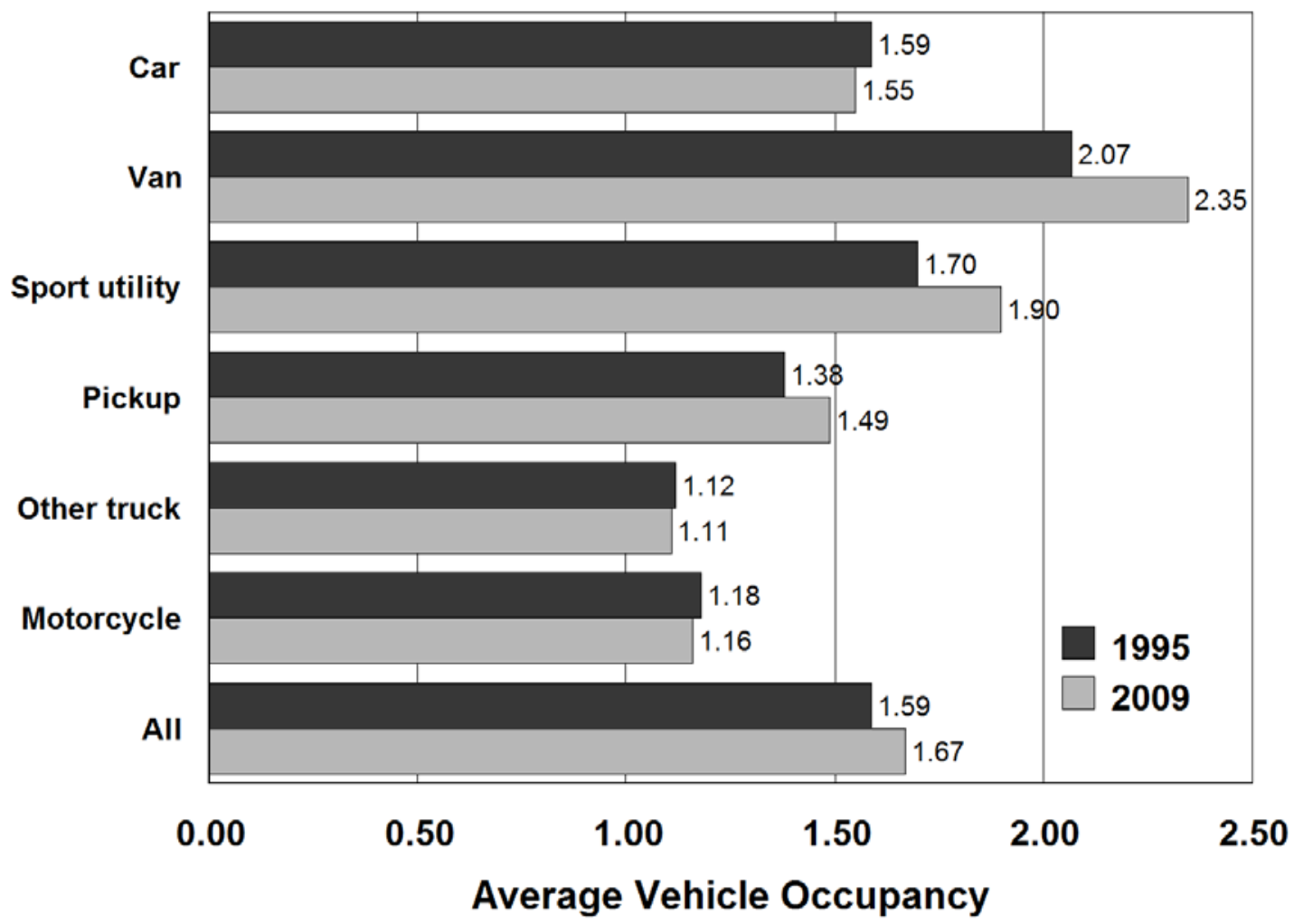

Sources:

U.S. Department of Transportation, Federal Highway Administration, 1995 Nationwide Personal Transportation Survey, Washington, DC, 1997, and 2009 National Household Travel Survey, Washington, DC. (Additional resources: www.fhwa.dot.gov, nhts.ornl.gov) 
The average vehicle occupancy, calculated as person-miles per vehicle-mile, is highest for social and recreational purposes. The highest vehicle occupancy levels for all purposes were in 1977. The increase in number of vehicles per household and the decrease in average household size could have contributed to the decline since then.

Figure 8.2. Average Vehicle Occupancy by Trip Purpose 1977 NPTS and 2009 NHTS

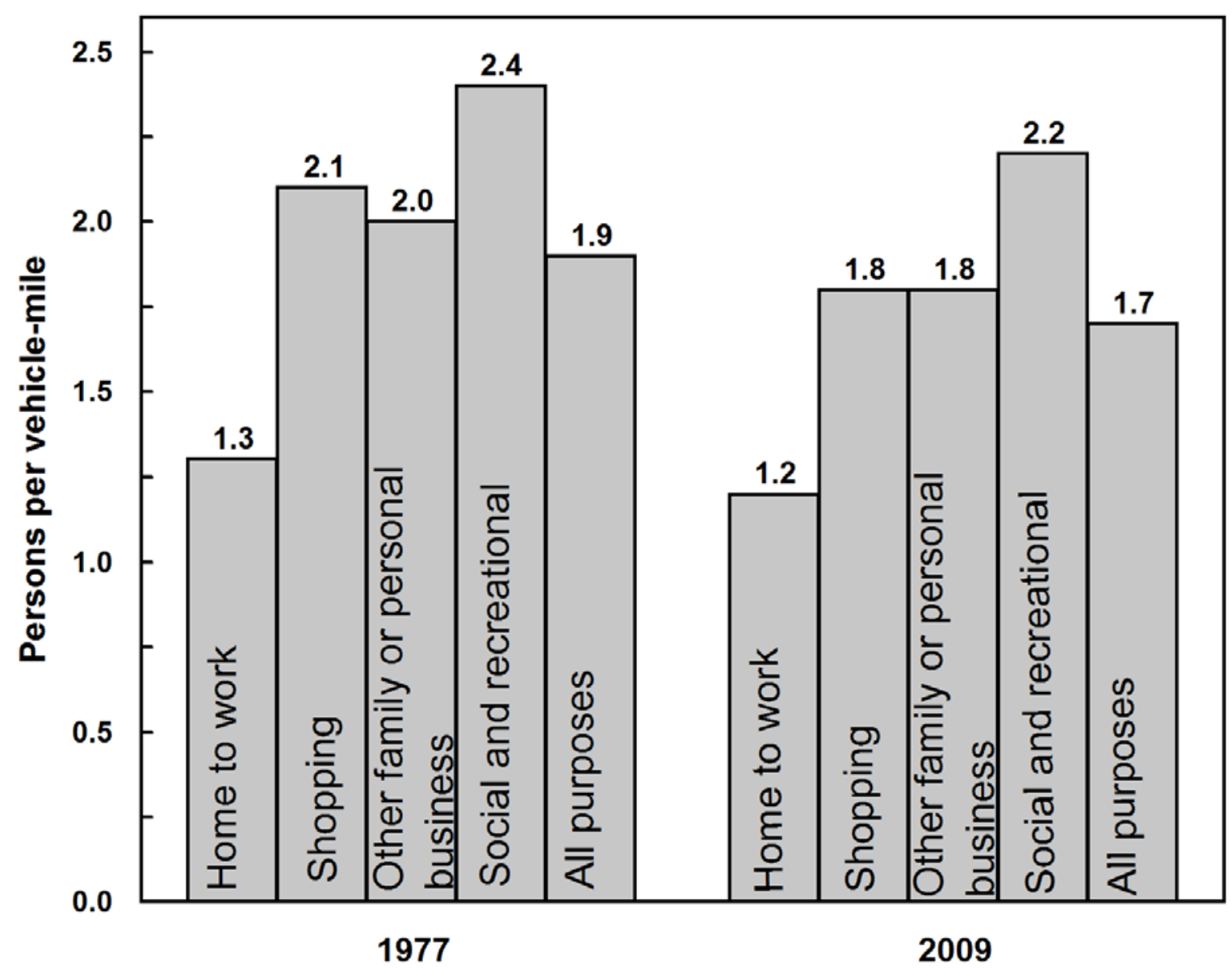

Sources:

U.S. Department of Transportation, Federal Highway Administration, 1990 Nationwide Personal Transportation Survey: Summary of Travel Trends, FHWA-PL-92027, Washington, DC, March 1992, Figure 6. Data from 2009 NHTS were generated from the 2009 National Household Travel Survey website nhts.ornl.gov, March 2011. (Additional resources: www.fhwa.dot.gov, nhts.ornl.gov) 
The 1990 household survey reports the highest average annual miles per vehicle and the 1983 survey reports the lowest. These data show that younger vehicles are typically driven more miles than older vehicles.

Table 8.8

Average Annual Miles per Household Vehicle by Vehicle Age

\begin{tabular}{cccccc}
\hline $\begin{array}{c}\text { Vehicle age } \\
\text { (years) }\end{array}$ & $\begin{array}{c}1983 \\
\text { self-reported }\end{array}$ & $\begin{array}{c}1990 \\
\text { self-reported }\end{array}$ & $\begin{array}{c}1995 \\
\text { self-reported }\end{array}$ & $\begin{array}{c}2001 \\
\text { self-reported }\end{array}$ & $\begin{array}{c}2009 \\
\text { self-reported }\end{array}$ \\
\hline Under 1 & 8,200 & 19,600 & 15,900 & 15,500 & 13,200 \\
1 & 15,200 & 16,800 & 16,800 & 14,300 & 14,600 \\
2 & 16,800 & 16,600 & 15,500 & 14,000 & 13,900 \\
3 & 14,500 & 14,700 & 14,400 & 13,100 & 12,700 \\
4 & 13,000 & 13,600 & 14,100 & 12,500 & 12,600 \\
5 & 12,100 & 12,900 & 13,500 & 12,000 & 12,800 \\
6 & 11,300 & 13,200 & 13,200 & 11,800 & 12,100 \\
7 & 10,000 & 12,400 & 12,800 & 11,600 & 11,900 \\
8 & 9,800 & 12,600 & 12,200 & 10,900 & 11,500 \\
9 & 9,000 & 11,500 & 12,200 & 10,800 & 11,300 \\
10 and older & 7,300 & 9,200 & 8,900 & 7,400 & 9,300 \\
\hline All household & \multicolumn{7}{c}{} & 11,100 & 11,300 \\
vehicles & 10,400 & 12,500 & 12,200 & & \\
\hline
\end{tabular}

Note: Data include all household vehicles, and have been rounded to the nearest hundred.

\section{Sources:}

Nationwide Personal Transportation Study-1983: D. Klinger and J. Richard Kuzmyak, COMSIS Corporation, Personal Travel in the United States, Volume 1: 1983-84 Nationwide Personal Travel Study, prepared for the U.S. Department of Transportation, Washington, DC, August 1986, Table 4-22, p. 4-21. 1990: Generated from the 1990 Nationwide Personal Transportation Study Public Use Tape, March 1992. 1995, 2001 and 2009: Generated from the 2009 NHTS datasets, version 2, February 2011. (Additional resources: nhts.ornl.gov) 
Historically, the data from the Nationwide Personal Transportation Survey (NPTS) are based on estimates reported by survey respondents. For the 1995 NPTS and the 2001 National Household Travel Survey (NHTS), odometer data were also collected. The 1995 data indicate that respondents overestimate the number of miles they drive in a year, but the 2001 data do not show that same trend.

Table 8.9

Self-Reported vs. Odometer Average Annual Miles, 1995 NPTS and 2001 NHTS

\begin{tabular}{ccccc}
\hline $\begin{array}{c}\text { Vehicle age } \\
\text { (years) }\end{array}$ & $\begin{array}{c}1995 \\
\text { self-reported }\end{array}$ & $\begin{array}{c}1995 \\
\text { odometer }\end{array}$ & $\begin{array}{c}2001 \\
\text { self-reported }\end{array}$ & $\begin{array}{c}2001 \\
\text { odometer }\end{array}$ \\
\hline Under 1 & 15,900 & 15,600 & 15,500 & 14,500 \\
1 & 16,800 & 14,500 & 14,300 & 14,200 \\
2 & 15,500 & 14,800 & 14,000 & 13,700 \\
3 & 14,400 & 13,800 & 13,100 & 14,100 \\
4 & 14,100 & 12,900 & 12,500 & 13,400 \\
5 & 13,500 & 12,700 & 12,000 & 12,900 \\
6 & 13,200 & 12,400 & 11,800 & 12,400 \\
7 & 12,800 & 11,600 & 11,600 & 12,100 \\
8 & 12,200 & 11,300 & 10,900 & 11,300 \\
9 & 12,200 & 11,200 & 10,800 & 10,500 \\
10 and older & 8,900 & 9,000 & 7,400 & 8,100 \\
\hline All household & & & & 11,800 \\
vehicles & 12,200 & 11,800 & & \\
\hline
\end{tabular}

Note: The 2009 NHTS did not collect similar data. Survey methodology on odometer reading data differs from 1995 to 2001 data.

\section{Source:}

Generated from the 2009 National Household Travel Survey website nhts.ornl.gov and 2001 NHTS public use file. 
Figure 8.3. Share of Vehicle Trips by Trip Distance, 2009 NHTS

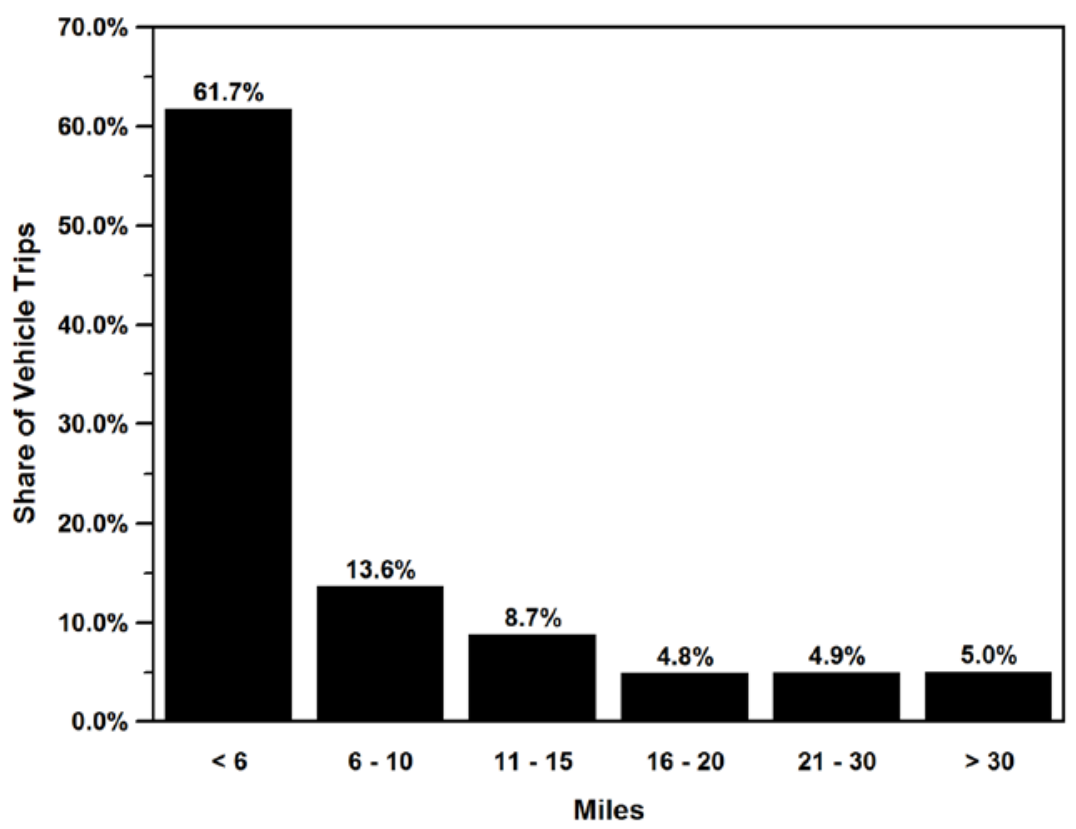

Source:

Generated from the 2009 National Household Travel Survey website nhts.ornl.gov.

Figure 8.4. Share of Vehicle Trips to Work by Trip Distance, 2009 NHTS

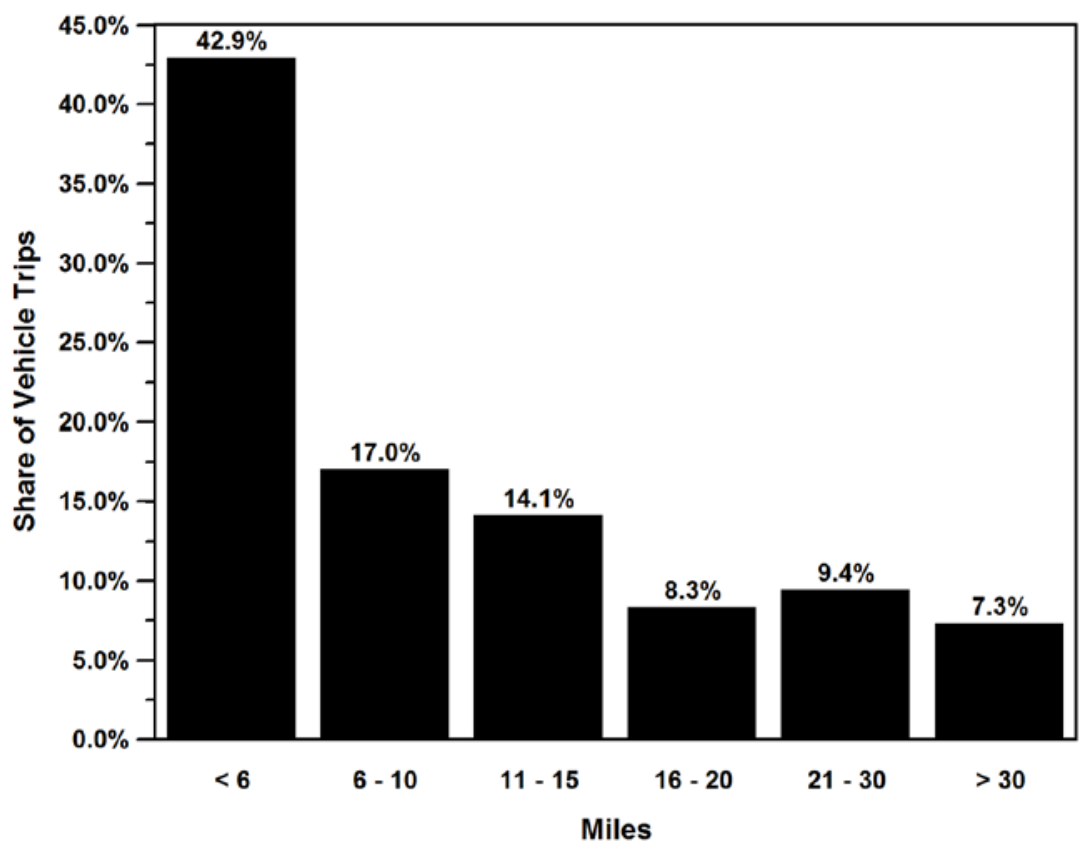

Source:

Generated from the 2009 National Household Travel Survey website nhts.ornl.gov. 
Nineteen percent of new vehicles (1-year-old and under) travel over 20,000 miles per year. Almost half of the vehicles over 20 years old travel less than 4,000 miles in a year.

Table 8.10

Share of Vehicles by Annual Miles of Travel and Vehicle Age, 2009 NHTS

\begin{tabular}{|c|c|c|c|c|c|c|c|}
\hline \multirow[b]{2}{*}{$\begin{array}{l}\text { Annual vehicle miles } \\
\text { of travel }\end{array}$} & \multicolumn{7}{|c|}{ Vehicle age (years) } \\
\hline & $\begin{array}{l}1 \text { and } \\
\text { under }\end{array}$ & 2 & 3 & 4 & 5 & 6 & 7 \\
\hline$<1,000$ miles & $2 \%$ & $3 \%$ & $3 \%$ & $3 \%$ & $3 \%$ & $4 \%$ & $3 \%$ \\
\hline 1 - 2,000 miles & $2 \%$ & $3 \%$ & $2 \%$ & $3 \%$ & $3 \%$ & $3 \%$ & $3 \%$ \\
\hline $2-4,000$ miles & $5 \%$ & $6 \%$ & $7 \%$ & $7 \%$ & $6 \%$ & $7 \%$ & $9 \%$ \\
\hline 4 - 6,000 miles & $7 \%$ & $10 \%$ & $9 \%$ & $8 \%$ & $8 \%$ & $10 \%$ & $10 \%$ \\
\hline $6-8,000$ miles & $10 \%$ & $10 \%$ & $11 \%$ & $11 \%$ & $10 \%$ & $12 \%$ & $12 \%$ \\
\hline 8 - 10,000 miles & $11 \%$ & $11 \%$ & $11 \%$ & $11 \%$ & $11 \%$ & $12 \%$ & $12 \%$ \\
\hline 10 - 12,000 miles & $9 \%$ & $11 \%$ & $11 \%$ & $11 \%$ & $12 \%$ & $11 \%$ & $11 \%$ \\
\hline 12 - 15,000 miles & $16 \%$ & $15 \%$ & $14 \%$ & $15 \%$ & $15 \%$ & $14 \%$ & $13 \%$ \\
\hline 15 - 20,000 miles & $18 \%$ & $15 \%$ & $17 \%$ & $17 \%$ & $16 \%$ & $14 \%$ & $14 \%$ \\
\hline 20 - 30,000 miles & $13 \%$ & $11 \%$ & $12 \%$ & $11 \%$ & $11 \%$ & $10 \%$ & $9 \%$ \\
\hline$>30,000$ miles & $6 \%$ & $5 \%$ & $4 \%$ & $3 \%$ & $4 \%$ & $4 \%$ & $3 \%$ \\
\hline \multirow[t]{3}{*}{ All } & $100 \%$ & $100 \%$ & $100 \%$ & $100 \%$ & $100 \%$ & $100 \%$ & $100 \%$ \\
\hline & \multicolumn{7}{|c|}{ Vehicle age (years) } \\
\hline & 8 & 9 & 10 & $11-15$ & $16-20$ & Over 20 & \\
\hline$<1,000$ miles & $4 \%$ & $4 \%$ & $4 \%$ & $6 \%$ & $9 \%$ & $19 \%$ & \\
\hline $1-2,000$ miles & $4 \%$ & $4 \%$ & $4 \%$ & $5 \%$ & $7 \%$ & $8 \%$ & \\
\hline 2 - 4,000 miles & $9 \%$ & $9 \%$ & $10 \%$ & $11 \%$ & $16 \%$ & $19 \%$ & \\
\hline 4 - 6,000 miles & $11 \%$ & $12 \%$ & $12 \%$ & $14 \%$ & $14 \%$ & $14 \%$ & \\
\hline $6-8,000$ miles & $12 \%$ & $12 \%$ & $11 \%$ & $14 \%$ & $13 \%$ & $12 \%$ & \\
\hline 8 - 10,000 miles & $13 \%$ & $11 \%$ & $12 \%$ & $12 \%$ & $10 \%$ & $7 \%$ & \\
\hline 10 - 12,000 miles & $11 \%$ & $11 \%$ & $11 \%$ & $10 \%$ & $8 \%$ & $6 \%$ & \\
\hline 12 - 15,000 miles & $13 \%$ & $13 \%$ & $12 \%$ & $10 \%$ & $8 \%$ & $5 \%$ & \\
\hline 15 - 20,000 miles & $12 \%$ & $13 \%$ & $14 \%$ & $9 \%$ & $7 \%$ & $5 \%$ & \\
\hline 20 - 30,000 miles & $9 \%$ & $8 \%$ & $7 \%$ & $7 \%$ & $4 \%$ & $3 \%$ & \\
\hline$>30,000$ miles & $3 \%$ & $3 \%$ & $3 \%$ & $3 \%$ & $2 \%$ & $2 \%$ & \\
\hline All & $100 \%$ & $100 \%$ & $100 \%$ & $100 \%$ & $100 \%$ & $100 \%$ & \\
\hline
\end{tabular}

\section{Source:}

Generated from the 2009 National Household Travel Survey website nhts.ornl.gov. (Additional resources: nhts.ornl.gov) 
The average driver makes three trips per day with an average of 9.7 miles for each trip.

Table 8.11

Household Vehicle Trips, 2009 NHTS

\begin{tabular}{cccc}
\hline & $\begin{array}{c}\text { Number of daily } \\
\text { vehicle trips } \\
\text { (per driver) }\end{array}$ & $\begin{array}{c}\text { Average } \\
\text { vehicle trip } \\
\text { length (miles) }\end{array}$ & $\begin{array}{c}\text { Daily vehicle } \\
\text { miles of travel } \\
\text { (per driver) }\end{array}$ \\
\hline 1990 & 3.3 & 8.9 & 28.5 \\
1995 & 3.6 & 9.1 & 32.1 \\
2001 & 3.4 & 9.9 & 32.7 \\
2009 & 3.0 & 9.7 & 29.0 \\
\hline
\end{tabular}

Source:

Generated from the 2009 National Household Travel Survey website nhts.ornl.gov.

Figure 8.5. Average Daily Miles Driven (per Driver), 2009 NHTS

Source:

Generated from the 2009 National Household Travel Survey website nhts.ornl.gov. 
Table 8.12

Daily Vehicle Miles of Travel (per Vehicle) by Number of Vehicles in the Household, 2009 NHTS

\begin{tabular}{ccc}
\hline & \multicolumn{2}{c}{ Daily miles per vehicle } \\
\cline { 2 - 3 } Number of household vehicles & 2001 & 2009 \\
\hline 1 & 25.6 & 29.1 \\
2 & 27.5 & 32.7 \\
3 & 24.2 & 31.3 \\
4 & 23.0 & 30.2 \\
5 & 21.1 & 27.6 \\
More than 5 & 18.4 & 27.2 \\
\hline All & 25.2 & 31.1 \\
\hline
\end{tabular}

Source:

Generated from the 2009 National Household Travel Survey website nhts.ornl.gov.

Table 8.13

Daily and Annual Vehicle Miles of Travel and Average Age for Each Vehicle in a Household, 2009 NHTS

\begin{tabular}{cccc}
\hline $\begin{array}{c}\text { Vehicle number } \\
\text { One-vehicle household }\end{array}$ & $\begin{array}{c}\text { Average } \\
\text { daily miles }\end{array}$ & $\begin{array}{c}\text { Average } \\
\text { annual miles }\end{array}$ & $\begin{array}{c}\text { Average age } \\
\text { (years) }\end{array}$ \\
$\begin{array}{c}\text { Two-vehicle household } \\
1\end{array}$ & 29.0 & 10,600 & 9.0 \\
2 & 43.6 & 15,900 & 7.6 \\
Three-vehicle household & 21.4 & 7,800 & 9.0 \\
1 & & & \\
2 & 50.7 & 18,500 & 7.9 \\
3 & 28.2 & 10,300 & 9.1 \\
Four-vehicle household & 14.0 & 5,100 & 11.8 \\
1 & & & \\
2 & 56.2 & 20,500 & 8.5 \\
3 & 33.2 & 12,100 & 8.8 \\
4 & 20.3 & 7,400 & 11.4 \\
1 & 9.9 & 3,600 & 13.2 \\
2 & & & \\
3 & 57.8 & 21,100 & 8.5 \\
4 & 34.0 & 12,400 & 9.4 \\
5 & 22.7 & 8,300 & 12.3 \\
Five-vehicle household & 14.2 & 5,200 & 12.7 \\
1 & 6.3 & 2,300 & 16.8 \\
2 & & & \\
3 & 61.4 & 22,400 & 10.2 \\
4 & 38.1 & 13,900 & 9.8 \\
5 & 26.3 & 9,600 & 12.2 \\
6 & 17.5 & 6,400 & 12.5 \\
& 10.4 & 3,800 & 14.5 \\
Six-vehicle household & 4.4 & 1,600 & 17.9 \\
\hline
\end{tabular}

Source:

Generated from the 2009 National Household Travel Survey website nhts.ornl.gov. 
Figure 8.6. Daily Vehicle Miles of Travel for Each Vehicle in a Household, 2009 NHTS

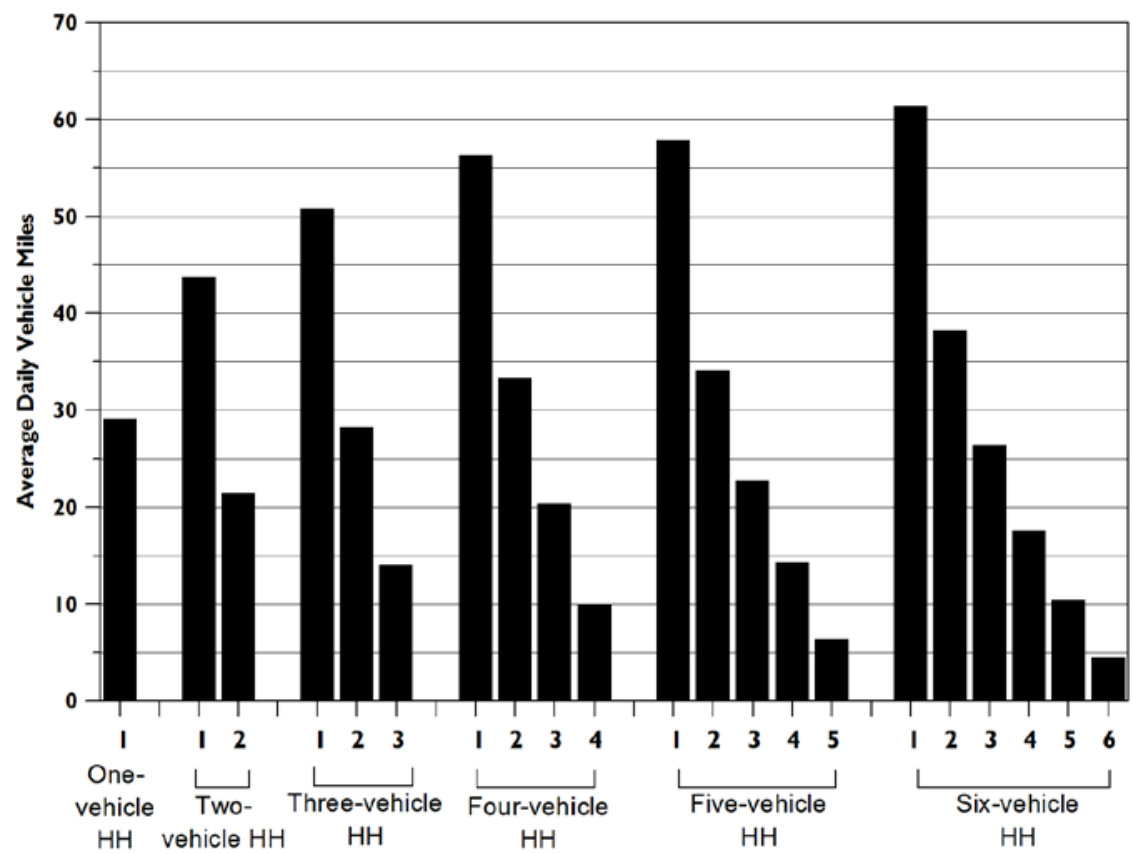

Source:

Generated from the 2009 National Household Travel Survey website nhts.ornl.gov.

Figure 8.7. Annual Vehicle Miles of Travel for Each Vehicle in a Household, 2009 NHTS

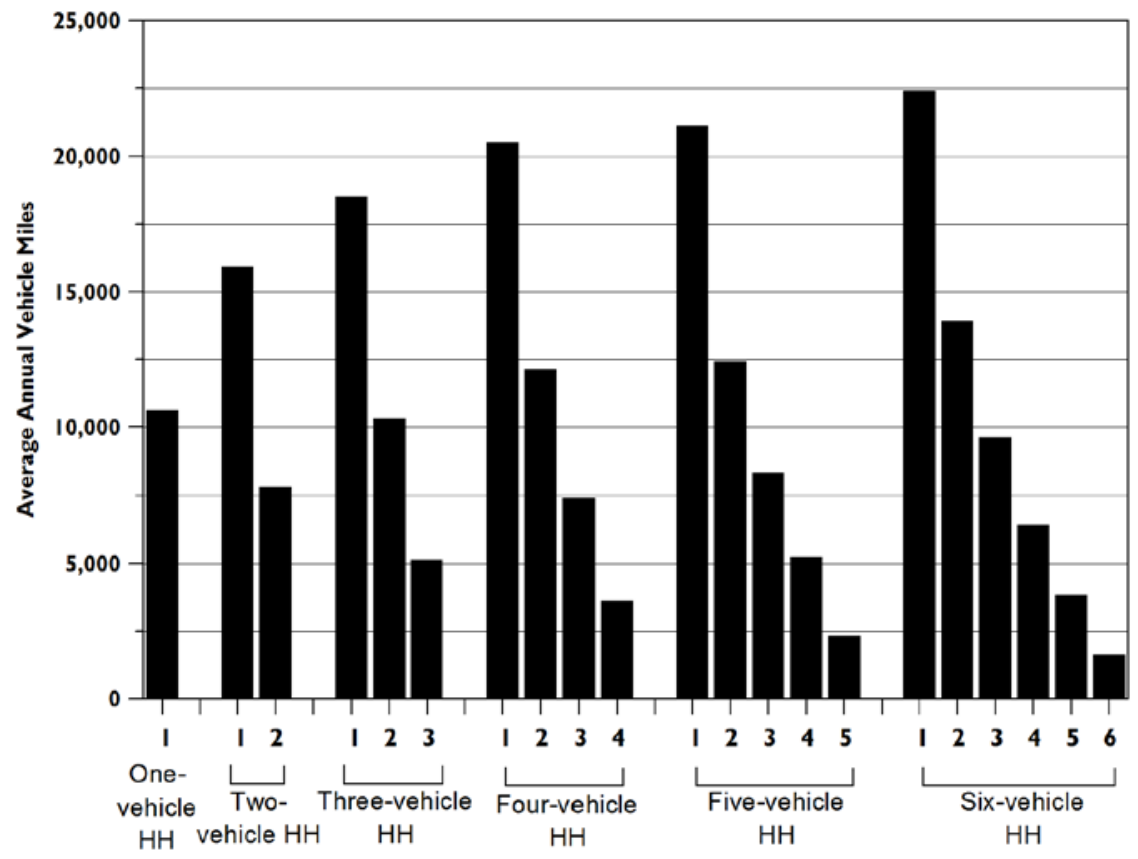

Source:

Generated from the 2009 National Household Travel Survey website nhts.ornl.gov. 
According to the U.S. Census data, the percentage of workers who car pooled has dropped from $19.7 \%$ in 1980 to $9.0 \%$ in 2015. The percent of workers using public transit declined from $6.4 \%$ to $5.3 \%$ in the ten-year period between 1980 and 1990, but stayed relatively the same from 1990 to 2014 ( 5.0\%). The average travel time increased by 4.2 minutes from 1980 to 2015. The American Community Survey (ACS) now collects journey-to-work data on an annual basis. It shows the average commute time as 25.9 minutes in 2015.

Table 8.14

Means of Transportation to Work, 1980, 1990, 2000, and 2015

\begin{tabular}{|c|c|c|c|c|c|c|c|c|}
\hline \multirow[b]{2}{*}{ Means of transportation } & \multicolumn{2}{|c|}{1980 Census } & \multicolumn{2}{|c|}{1990 Census } & \multicolumn{2}{|c|}{2000 Census } & \multicolumn{2}{|c|}{2015 ACS } \\
\hline & $\begin{array}{c}\text { Number of } \\
\text { workers } \\
\text { (thousands) }\end{array}$ & Share & $\begin{array}{c}\text { Number of } \\
\text { workers } \\
\text { (thousands) }\end{array}$ & Share & $\begin{array}{c}\text { Number of } \\
\text { workers } \\
\text { (thousands) }\end{array}$ & Share & $\begin{array}{c}\text { Number of } \\
\text { workers } \\
\text { (thousands) }\end{array}$ & Share \\
\hline Private vehicle & 81,258 & $84.1 \%$ & 99,593 & $86.5 \%$ & 112,737 & $87.9 \%$ & 126,924 & $85.6 \%$ \\
\hline Drove alone & 62,193 & $64.4 \%$ & 84,215 & $73.2 \%$ & 97,102 & $75.7 \%$ & 113,576 & $76.6 \%$ \\
\hline Car pooled & 19,065 & $19.7 \%$ & 15,378 & $13.4 \%$ & 15,635 & $12.2 \%$ & 13,348 & $9.0 \%$ \\
\hline Public transportation & 6,175 & $6.4 \%$ & 6,068 & $5.3 \%$ & 6,068 & $4.7 \%$ & 7,949 & $5.4 \%$ \\
\hline Bus or trolley bus ${ }^{a}$ & 3,925 & $4.1 \%$ & 3,445 & $3.0 \%$ & 3,207 & $2.5 \%$ & 3,882 & $2.6 \%$ \\
\hline Streetcar or trolley car ${ }^{a}$ & $b$ & $b$ & 78 & $0.1 \%$ & 73 & $0.1 \%$ & 89 & $0.1 \%$ \\
\hline Subway or elevated & 1,529 & $1.6 \%$ & 1,755 & $1.5 \%$ & 1,886 & $1.5 \%$ & 2,851 & $1.9 \%$ \\
\hline Railroad & 554 & $0.6 \%$ & 574 & $0.5 \%$ & 658 & $0.5 \%$ & 881 & $0.6 \%$ \\
\hline Ferryboat & $b$ & $b$ & 37 & $0.0 \%$ & 44 & $0.0 \%$ & 59 & $0.0 \%$ \\
\hline Taxicab & 167 & $0.2 \%$ & 179 & $0.2 \%$ & 200 & $0.2 \%$ & 188 & $0.1 \%$ \\
\hline Motorcycle & 419 & $0.4 \%$ & 237 & $0.2 \%$ & 142 & $0.1 \%$ & 266 & $0.2 \%$ \\
\hline Bicycle & 468 & $0.5 \%$ & 467 & $0.4 \%$ & 488 & $0.4 \%$ & 885 & $0.6 \%$ \\
\hline Walked only & 5,413 & $5.6 \%$ & 4,489 & $3.9 \%$ & 3,759 & $2.9 \%$ & 4,114 & $2.8 \%$ \\
\hline Other means & 703 & $0.7 \%$ & 809 & $0.7 \%$ & 901 & $0.7 \%$ & 1,343 & $0.9 \%$ \\
\hline Worked at home & 2,180 & $2.3 \%$ & 3,406 & $3.0 \%$ & 4,184 & $3.3 \%$ & 6,843 & $4.6 \%$ \\
\hline Total workers & 96,616 & $100.0 \%$ & 115,069 & $100.0 \%$ & 128,279 & $100.0 \%$ & 148,324 & $100.0 \%$ \\
\hline Average travel time (minutes) & 21.7 & & 22.4 & & 25.5 & & 25.9 & \\
\hline
\end{tabular}

\section{Sources:}

1980-1990 data - Provided by the Journey-to-Work and Migration Statistics Branch, Population Division, U.S. Bureau of the Census.

2000 data - U.S. Bureau of the Census, Journey to Work: 2000, Tables 1 and 2, 1990-2000, March 2004 (www.census.gov/population/www/socdemo/journey.html).

2015 data - U.S. Bureau of the Census, 2009-2015 American Community Survey Five-Year Estimates, Tables B08301 and GCT0801. (Additional resources: www.census.gov)

a This category was "Bus or streetcar" in 1980.

${ }^{\mathrm{b}}$ Data are not available. 
Table 8.15

Characteristics of U.S. Daily per Vehicle Driving vs. Dwelling Unit Type and Density

\begin{tabular}{lcccc}
\hline & $\begin{array}{c}\text { Share of } \\
\text { vehicles in } \\
\text { density type }\end{array}$ & $\begin{array}{c}\text { Hours per } \\
\text { vehicle } \\
\text { per day }\end{array}$ & $\begin{array}{c}\text { Average vehicle } \\
\text { speed } \\
\text { (miles/hour) }\end{array}$ & $\begin{array}{c}\text { Miles } \\
\text { per vehicle } \\
\text { per day }\end{array}$ \\
\hline All classes detached single house & $77.0 \%$ & 0.92 & 32.0 & 29.6 \\
All classes other & $23.0 \%$ & 0.99 & 27.7 & 27.4 \\
$<1,000 /$ sq. mile detached single house & $81.6 \%$ & 0.91 & 34.7 & 31.6 \\
$<1,000 /$ sq. mile all other & $18.4 \%$ & 0.91 & 32.5 & 29.5 \\
1,000-4,000/sq. mile detached single house & $75.5 \%$ & 0.94 & 27.5 & 26.0 \\
1,000-4,000/sq. mile all other & $24.5 \%$ & 1.03 & 25.1 & 25.9 \\
4,000-10,000/sq. mile detached single house & $42.5 \%$ & 0.96 & 26.1 & 25.1 \\
4,000-10,000/sq. mile all other & $57.5 \%$ & 1.15 & 21.5 & 24.6 \\
10,000-25,000/sq. mile detached single house & $17.8 \%$ & 1.02 & 18.2 & 18.5 \\
10,000-25,000/sq. mile all other & $82.2 \%$ & 1.05 & 21.3 & 22.3 \\
$>$ >25,000/sq. mile detached single house & $9.8 \%$ & 0.72 & 20.5 & 14.8 \\
$>$ 25,000/sq. mile all other & $90.2 \%$ & 1.23 & 21.9 & 26.9 \\
\hline
\end{tabular}

Source:

Generated from the 2009 National Household Travel Survey website nhts.ornl.gov.

Table 8.16

Housing Unit Characteristics, 2015

\begin{tabular}{lcc}
\hline & $\begin{array}{c}\text { Share of occupied } \\
\text { housing units }\end{array}$ & $\begin{array}{c}\text { Percent with } \\
\text { garage or carport }\end{array}$ \\
\hline Type of housing unit & & $70.8 \%$ \\
New construction (<= 4 years) & $3.2 \%$ & $33.0 \%$ \\
Manufactured/mobile homes & $5.9 \%$ & \\
\hline Geographic location (Census Region) & & $49.3 \%$ \\
$\quad$ Northeast & $18.1 \%$ & $71.9 \%$ \\
Midwest & $22.3 \%$ & $56.0 \%$ \\
South & $37.2 \%$ & $76.3 \%$ \\
West & $22.4 \%$ & $78.3 \%$ \\
\hline Tenure & & $36.8 \%$ \\
$\quad$ Owner & $62.7 \%$ & $62.9 \%$ \\
Renter & $37.3 \%$ & 118,290 units \\
\hline All occupied units & & \\
\hline
\end{tabular}

Note: The American Housing Survey is updated every two years. The 2015 data are the latest available.

\section{Source:}

U.S. Bureau of the Census, 2015 American Housing Survey, Table Creator, accessed July 28, 2017.

(Additional information: www.census.gov/programs-surveys/ahs) 
The average one-way commute time was 25.9 minutes in 2015. More than $63 \%$ of workers traveled less than 30 minutes to work in 2015. In 1990, 32.5\% of workers commuted less than 15 minutes; in 2015 that number dropped to $27.0 \%$.

Table 8.17

Workers by Commute Time, 1990, 2000 and 2015

\begin{tabular}{lcrr}
\hline \multicolumn{1}{c}{ Commute time (one-way) } & 1990 & 2000 & 2015 \\
\hline Less than 15 minutes & $32.5 \%$ & $29.4 \%$ & $27.0 \%$ \\
15-29 minutes & $37.0 \%$ & $36.1 \%$ & $36.4 \%$ \\
30-39 minutes & $15.2 \%$ & $15.8 \%$ & $16.5 \%$ \\
40-59 minutes & $9.2 \%$ & $10.7 \%$ & $11.6 \%$ \\
60 minutes or more & $6.1 \%$ & $8.0 \%$ & $8.5 \%$ \\
\hline Average travel time (minutes) & 22.4 & 25.5 & 25.9 \\
\hline
\end{tabular}

\section{Sources:}

1990-2000 - U.S. Bureau of the Census, Journey to Work: 2000, Tables 1 and 2, 1990-2000, March 2004.

2015 - U.S. Bureau of the Census, 2009-2015 American Community Survey, 5-Year Estimates, Tables S0802 and B08303. (Additional resources: www.census.gov) 
Sales of bicycles with wheel sizes of 20 inches and over have grown at an average annual rate of $1.0 \%$ from 1981 to 2015. Bicycle sales experienced a large decline in 2009, which brought total sales to 14.9 million-a new low in the 18-year series. Sales in 2015 were 17.4 million.

Table 8.18

Bicycle Sales, 1981-2015

(millions)

\begin{tabular}{|c|c|c|c|}
\hline & $\begin{array}{c}\text { Wheel sizes } \\
\text { under } 20 \\
\text { inches }\end{array}$ & $\begin{array}{c}\text { Wheel sizes of } \\
20 \text { inches and } \\
\text { over }\end{array}$ & $\begin{array}{c}\text { All } \\
\text { wheel sizes }\end{array}$ \\
\hline 1981 & $\mathrm{a}$ & 8.9 & $\mathrm{a}$ \\
\hline 1982 & a & 6.8 & $\mathrm{a}$ \\
\hline 1983 & a & 9.0 & a \\
\hline 1984 & a & 10.1 & a \\
\hline 1985 & a & 11.4 & $\mathrm{a}$ \\
\hline 1986 & a & 12.3 & $\mathrm{a}$ \\
\hline 1987 & a & 12.6 & $\mathrm{a}$ \\
\hline 1988 & $\mathrm{a}$ & 9.9 & $\mathrm{a}$ \\
\hline 1989 & a & 10.7 & a \\
\hline 1990 & a & 10.8 & a \\
\hline 1991 & a & 11.6 & $\mathrm{a}$ \\
\hline 1992 & 3.7 & 11.6 & 15.3 \\
\hline 1993 & 3.8 & 13.0 & 16.8 \\
\hline 1994 & 4.2 & 12.5 & 16.7 \\
\hline 1995 & 4.1 & 12.0 & 16.1 \\
\hline 1996 & 4.5 & 10.9 & 15.4 \\
\hline 1997 & 4.2 & 11.0 & 15.2 \\
\hline 1998 & 4.7 & 11.1 & 15.8 \\
\hline 1999 & 5.9 & 11.6 & 17.5 \\
\hline 2000 & 9.0 & 11.9 & 20.9 \\
\hline 2001 & 5.4 & 11.3 & 16.7 \\
\hline 2002 & 5.9 & 13.6 & 19.5 \\
\hline 2003 & 5.6 & 12.9 & 18.5 \\
\hline 2004 & 5.3 & 13.0 & 18.3 \\
\hline 2005 & 5.8 & 14.0 & 19.8 \\
\hline 2006 & 5.5 & 12.7 & 18.2 \\
\hline 2007 & 5.4 & 12.8 & 18.2 \\
\hline 2008 & 5.1 & 13.4 & 18.5 \\
\hline 2009 & 4.7 & 10.2 & 14.9 \\
\hline 2010 & 6.3 & 13.5 & 19.8 \\
\hline 2011 & 4.7 & 11.0 & 15.7 \\
\hline 2012 & 5.7 & 13.0 & 18.7 \\
\hline 2013 & 4.9 & 11.3 & 16.2 \\
\hline 2014 & 5.6 & 12.4 & 18.0 \\
\hline $2015^{b}$ & 4.9 & 12.5 & 17.4 \\
\hline \multicolumn{4}{|c|}{ Average annual percentage change } \\
\hline 1981-2015 & a & $1.0 \%$ & a \\
\hline $2005-2015$ & $-1.7 \%$ & $-1.1 \%$ & $-1.3 \%$ \\
\hline
\end{tabular}

\section{Source:}

1981-1996: Bicycle Manufacturers Association. 1997-on: National Bicycle Dealers Association. (Additional resources: www.nbda.com)

a Data are not available.

b Latest year available. 
In 2009, 4.5\% of walk trips and $10.9 \%$ of bike trips were to/from work. Forty-seven percent of all bike trips were for social/recreational purposes. Nearly $15 \%$ of walk trips were shopping trips.

Figure 8.8. Walk and Bike Trips by Trip Purpose, 2009 NHTS

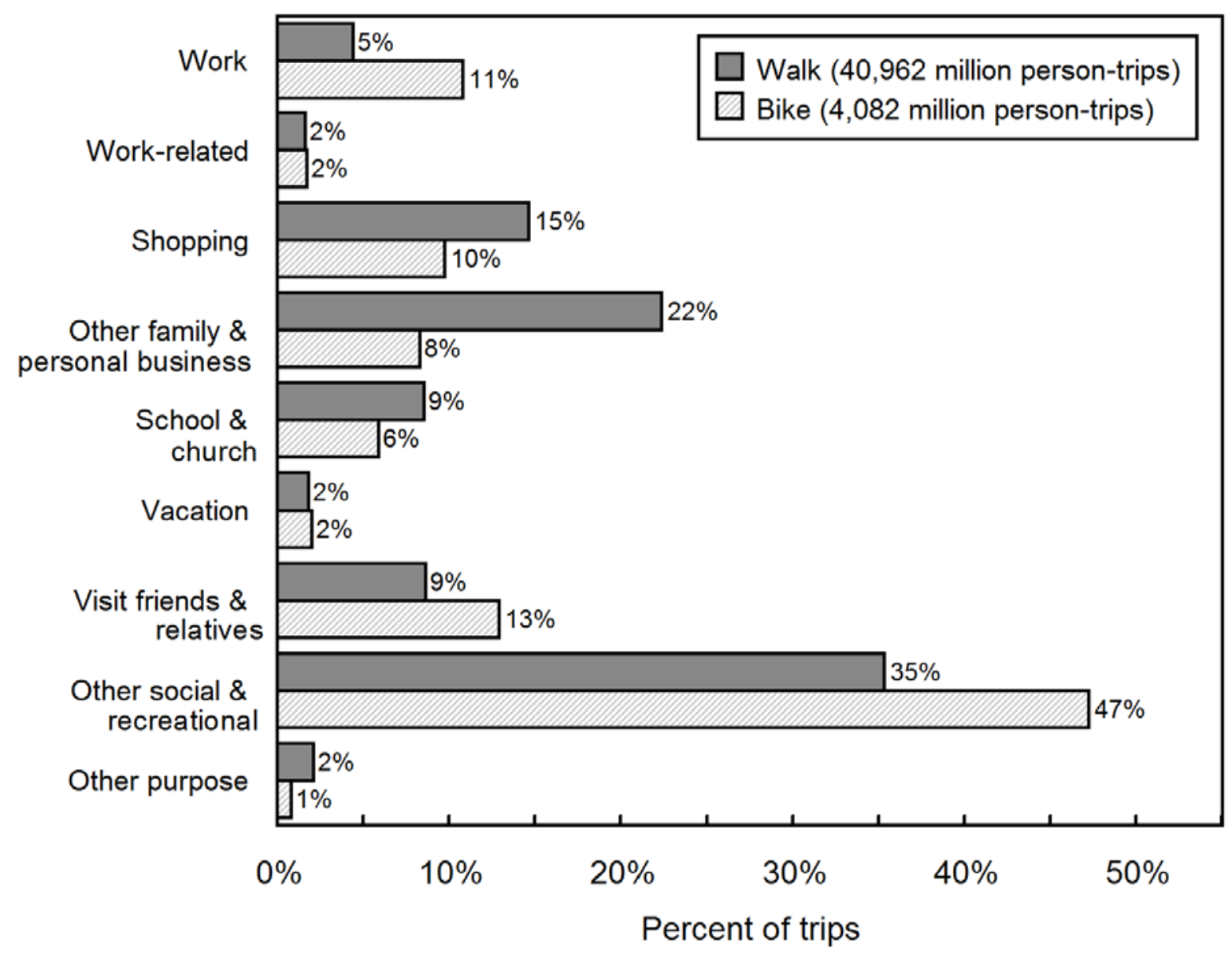

Note: Percentages may not sum to totals due to rounding.

\section{Source:}

Generated from the 2009 National Household Travel Survey website nhts.ornl.gov. 
After 2001 only data on daily trips were collected in the NHTS. The 2001 data are still the latest available on longdistance trips.

\section{Long Distance Trips - 2001 National Household Travel Survey}

The 2001 National Household Travel Survey (NHTS) collected data on long-distance trips as well as everyday travel. The everyday travel data is a continuation of the Nationwide Personal Transportation Survey (NPTS), while the long-distance travel data is a continuation of the American Travel Survey (ATS) which was collected in 1977 and 1985 . The survey collected trip-related data such as mode of transportation, duration, distance and purpose of trip. It also gathered demographic, geographic, and economic data for analysis purposes.

A long-distance trip is defined as a trip of 50 miles or more, one-way. Long-trip data from the 2001 NHTS were released in the summer of 2004. For additional information about the 2001 NHTS data, contact the Bureau of Transportation Statistics at 202-366-3282 or visit the following website: nhts.ornl.gov. 
Table 8.19

Long-Distance Trip ${ }^{a}$ Characteristics, 2001 NHTS

\begin{tabular}{|c|c|c|c|c|}
\hline \multirow[b]{2}{*}{ Trip characteristic } & \multicolumn{2}{|c|}{ Person trips } & \multicolumn{2}{|c|}{ Person miles } \\
\hline & (thousands) & (percent) & (thousands) & (percent) \\
\hline Total & $2,554,068$ & 100.0 & $1,138,322,697$ & 100.0 \\
\hline \multicolumn{5}{|l|}{ Principal means of transportation: } \\
\hline Personal use vehicles & $2,310,376$ & 90.5 & $735,882,255$ & 64.7 \\
\hline Airplane & 165,039 & 6.5 & $367,888,741$ & 32.3 \\
\hline Commercial airplane & 158,880 & 6.2 & $361,717,015$ & 31.8 \\
\hline Bus $^{\mathrm{b}}$ & 52,962 & 2.1 & $23,747,433$ & 2.1 \\
\hline Intercity bus & 3,456 & 0.1 & $1,765,696$ & 0.2 \\
\hline Charter, tour, or school bus & 45,952 & 1.8 & $21,019,942$ & 1.9 \\
\hline Train & 20,672 & 0.8 & $9,266,373$ & 0.8 \\
\hline \multicolumn{5}{|l|}{ Round trip distance: } \\
\hline 100 to 300 miles & $1,688,358$ & 66.1 & $284,586,370$ & 25.0 \\
\hline 300 to 499 miles & 373,550 & 14.6 & $143,571,597$ & 12.6 \\
\hline 500 to 999 miles & 261,802 & 10.3 & $180,669,482$ & 15.9 \\
\hline 1,000 to 1,999 miles & 125,665 & 4.9 & $178,629,838$ & 15.7 \\
\hline 2,000 miles or more & 104,694 & 4.1 & $350,865,409$ & 30.8 \\
\hline Mean (miles) & 446 & c & c & c \\
\hline Median (miles) & 206 & c & c & c \\
\hline \multicolumn{5}{|l|}{ Calendar quarter: } \\
\hline 1st quarter & 566,502 & 22.2 & $246,556,190$ & 21.7 \\
\hline 2nd quarter & 653,310 & 25.6 & $298,154,812$ & 26.2 \\
\hline 3rd quarter & 734,878 & 28.8 & $341,021,290$ & 30.0 \\
\hline 4th quarter & 599,378 & 23.5 & $252,590,405$ & 22.2 \\
\hline \multicolumn{5}{|l|}{ Main purpose of trip: } \\
\hline Commuting & 329,395 & 12.9 & 65,877,968 & 5.8 \\
\hline Other business & 405,866 & 15.9 & $242,353,212$ & 21.3 \\
\hline Personal/leisure & $1,406,411$ & 55.1 & $667,471,358$ & 58.7 \\
\hline Personal business & 322,645 & 12.6 & $130,020,982$ & 11.4 \\
\hline Other & 88,230 & 3.5 & $32,031,679$ & 2.8 \\
\hline \multicolumn{5}{|l|}{ Nights away from home: } \\
\hline None & $1,454,847$ & 57.0 & $304,469,524$ & 26.8 \\
\hline 1 to 3 nights & 808,281 & 31.7 & $414,219,147$ & 36.4 \\
\hline 4 to 7 nights & 214,464 & 8.4 & $269,265,597$ & 23.7 \\
\hline 8 or more nights & 76,475 & 3.0 & $150,368,429$ & 13.2 \\
\hline \multicolumn{5}{|l|}{ Destination: } \\
\hline Within Census division & $2,077,810$ & 81.4 & $549,651,116$ & 48.3 \\
\hline Across Census division, within Census & 196,890 & 7.7 & $134,930,113$ & 11.9 \\
\hline Across Census region & 279,367 & 10.9 & $453,741,468$ & 39.9 \\
\hline
\end{tabular}

Note: Long-distance trips were not included in the 2009 NHTS.

\section{Source:}

U.S. Bureau of Transportation Statistics and the U.S. Federal Highway Administration, 2001 National Household Transportation Survey. (Additional resources: nhts.ornl.gov)

\footnotetext{
${ }^{\text {a }}$ A long-distance trip is defined as a trip of 50 miles or more, one-way.

${ }^{\mathrm{b}}$ Includes other types of buses.

${ }^{\mathrm{c}}$ Not applicable.
} 


\section{Chapter 9 \\ Nonhighway Modes}

Summary Statistics from Tables in this Chapter

\begin{tabular}{lrr}
\hline Source & & \\
\hline & Passenger-miles & (millions) \\
Table 9.2 & Domestic and international air carrier, 2016 & 939,222 \\
Table 9.10 & Amtrak, 2015 & 6,536 \\
Table 9.11 & Commuter rail, 2015 & 11,804 \\
Table 9.12 & Transit rail, 2015 & 20,710 \\
Table 9.5 & Freight ton-miles & (millions) \\
Table 9.8 & Domestic waterborne commerce, 2015 & 491,000 \\
Table 9.2 & Class I railroad, 2015 & (trillion Btu) \\
Table 9.3 & Passenger energy use & $2,385.2$ \\
Table 9.6 & Domestic and international air carrier, 2016 \\
Table 9.10 & Recreational boats, 2015 & 208.9 \\
Table 9.11 & Amtrak, 2015 & 246.0 \\
Table 9.12 & Commuter rail, 2015 & 10.9 \\
Table 9.8 & Transit rail, 2015 & 19.4 \\
\hline & Freight energy use & 16.1 \\
& Class I railroad, 2015 & (trillion Btus) \\
& & 516.4 \\
\hline
\end{tabular}


Nonhighway transportation modes accounted for 18.0\% of total transportation energy use in 2015.

Table 9.1

Nonhighway Energy Use Shares, 1970-2015

\begin{tabular}{|c|c|c|c|c|c|c|}
\hline \multirow[b]{2}{*}{ Year } & \multicolumn{6}{|c|}{ Share of transportation energy use } \\
\hline & Air & Water & Pipeline & Rail & $\begin{array}{c}\text { Nonhighway } \\
\text { total }\end{array}$ & $\begin{array}{c}\text { Transportation } \\
\text { total (trillion Btu) }\end{array}$ \\
\hline 1970 & $8.5 \%$ & $5.5 \%$ & $5.4 \%$ & $3.5 \%$ & $22.9 \%$ & 15,192 \\
\hline 1971 & $8.2 \%$ & $4.9 \%$ & $5.4 \%$ & $3.4 \%$ & $21.8 \%$ & 15,819 \\
\hline 1972 & $7.8 \%$ & $4.7 \%$ & $5.2 \%$ & $3.3 \%$ & $21.0 \%$ & 16,845 \\
\hline 1973 & $7.7 \%$ & $5.1 \%$ & $4.7 \%$ & $3.3 \%$ & $20.8 \%$ & 17,670 \\
\hline 1974 & $7.2 \%$ & $5.2 \%$ & $4.5 \%$ & $3.5 \%$ & $20.5 \%$ & 16,968 \\
\hline 1975 & $7.2 \%$ & $5.4 \%$ & $4.0 \%$ & $3.1 \%$ & $19.7 \%$ & 17,204 \\
\hline 1976 & $7.0 \%$ & $5.9 \%$ & $3.5 \%$ & $3.1 \%$ & $19.6 \%$ & 18,266 \\
\hline 1977 & $7.1 \%$ & $6.2 \%$ & $3.3 \%$ & $3.0 \%$ & $19.7 \%$ & 18,951 \\
\hline 1978 & $7.1 \%$ & $6.9 \%$ & $3.1 \%$ & $2.9 \%$ & $20.1 \%$ & 19,922 \\
\hline 1979 & $7.6 \%$ & $5.9 \%$ & $3.6 \%$ & $3.0 \%$ & $20.2 \%$ & 19,473 \\
\hline 1980 & $7.6 \%$ & $7.4 \%$ & $3.9 \%$ & $3.0 \%$ & $22.0 \%$ & 18,760 \\
\hline 1981 & $7.8 \%$ & $6.8 \%$ & $4.0 \%$ & $2.9 \%$ & $21.6 \%$ & 18,558 \\
\hline 1982 & $8.0 \%$ & $5.9 \%$ & $3.8 \%$ & $2.5 \%$ & $20.3 \%$ & 18,055 \\
\hline 1983 & $7.9 \%$ & $5.4 \%$ & $3.2 \%$ & $2.5 \%$ & $19.0 \%$ & 18,188 \\
\hline 1984 & $8.6 \%$ & $5.1 \%$ & $3.3 \%$ & $2.7 \%$ & $19.7 \%$ & 18,773 \\
\hline 1985 & $8.8 \%$ & $4.6 \%$ & $3.1 \%$ & $2.5 \%$ & $19.0 \%$ & 19,017 \\
\hline 1986 & $9.1 \%$ & $6.6 \%$ & $2.9 \%$ & $2.3 \%$ & $20.8 \%$ & 20,086 \\
\hline 1987 & $9.2 \%$ & $6.7 \%$ & $3.0 \%$ & $2.3 \%$ & $21.2 \%$ & 20,578 \\
\hline 1988 & $9.4 \%$ & $6.7 \%$ & $3.4 \%$ & $2.3 \%$ & $21.7 \%$ & 21,131 \\
\hline 1989 & $9.2 \%$ & $7.1 \%$ & $3.4 \%$ & $2.2 \%$ & $21.9 \%$ & 21,487 \\
\hline 1990 & $9.6 \%$ & $6.7 \%$ & $3.6 \%$ & $2.2 \%$ & $22.1 \%$ & 21,383 \\
\hline 1991 & $9.1 \%$ & $7.3 \%$ & $3.3 \%$ & $2.1 \%$ & $21.8 \%$ & 20,985 \\
\hline 1992 & $9.0 \%$ & $7.4 \%$ & $3.2 \%$ & $2.1 \%$ & $21.6 \%$ & 21,646 \\
\hline 1993 & $9.0 \%$ & $6.5 \%$ & $3.3 \%$ & $2.1 \%$ & $20.9 \%$ & 22,125 \\
\hline 1994 & $9.1 \%$ & $6.1 \%$ & $3.5 \%$ & $2.2 \%$ & $20.9 \%$ & 22,729 \\
\hline 1995 & $9.2 \%$ & $6.3 \%$ & $3.5 \%$ & $2.2 \%$ & $21.2 \%$ & 23,263 \\
\hline 1996 & $9.3 \%$ & $5.9 \%$ & $3.4 \%$ & $2.3 \%$ & $20.9 \%$ & 23,773 \\
\hline 1997 & $9.5 \%$ & $5.2 \%$ & $3.5 \%$ & $2.2 \%$ & $20.5 \%$ & 24,126 \\
\hline 1998 & $9.3 \%$ & $5.0 \%$ & $3.0 \%$ & $2.2 \%$ & $19.5 \%$ & 24,461 \\
\hline 1999 & $9.6 \%$ & $5.3 \%$ & $2.9 \%$ & $2.2 \%$ & $20.0 \%$ & 25,758 \\
\hline 2000 & $9.8 \%$ & $5.6 \%$ & $2.8 \%$ & $2.1 \%$ & $20.4 \%$ & 26,069 \\
\hline 2001 & $9.3 \%$ & $4.6 \%$ & $2.8 \%$ & $2.2 \%$ & $18.9 \%$ & 25,741 \\
\hline 2002 & $8.5 \%$ & $4.7 \%$ & $2.9 \%$ & $2.1 \%$ & $18.3 \%$ & 26,331 \\
\hline 2003 & $8.5 \%$ & $4.0 \%$ & $2.6 \%$ & $2.2 \%$ & $17.3 \%$ & 26,512 \\
\hline 2004 & $9.1 \%$ & $4.8 \%$ & $2.5 \%$ & $2.3 \%$ & $18.6 \%$ & 26,970 \\
\hline 2005 & $9.2 \%$ & $5.0 \%$ & $2.5 \%$ & $2.2 \%$ & $19.0 \%$ & 27,377 \\
\hline 2006 & $9.1 \%$ & $5.3 \%$ & $2.5 \%$ & $2.3 \%$ & $19.1 \%$ & 27,554 \\
\hline 2007 & $8.6 \%$ & $5.4 \%$ & $2.5 \%$ & $2.1 \%$ & $18.6 \%$ & 29,013 \\
\hline 2008 & $8.4 \%$ & $5.1 \%$ & $2.6 \%$ & $2.1 \%$ & $18.3 \%$ & 28,381 \\
\hline 2009 & $7.9 \%$ & $5.0 \%$ & $2.9 \%$ & $1.8 \%$ & $17.6 \%$ & 26,895 \\
\hline 2010 & $8.0 \%$ & $5.5 \%$ & $2.9 \%$ & $2.0 \%$ & $18.3 \%$ & 26,974 \\
\hline 2011 & $8.2 \%$ & $5.3 \%$ & $3.0 \%$ & $2.1 \%$ & $18.6 \%$ & 26,388 \\
\hline 2012 & $8.0 \%$ & $4.5 \%$ & $3.2 \%$ & $2.1 \%$ & $17.9 \%$ & 26,001 \\
\hline 2013 & $7.9 \%$ & $4.1 \%$ & $3.6 \%$ & $2.2 \%$ & $17.7 \%$ & 25,905 \\
\hline 2014 & $7.9 \%$ & $3.5 \%$ & $3.1 \%$ & $2.2 \%$ & $16.7 \%$ & 26,109 \\
\hline 2015 & $8.2 \%$ & $4.0 \%$ & $3.0 \%$ & $2.2 \%$ & $17.4 \%$ & 25,880 \\
\hline
\end{tabular}

Source:

See Appendix A, Section 2.3. Nonhighway Energy Use.

${ }^{a}$ Only end-use energy was counted for electricity. Previous editions included primary energy use for electricity which included generation and distribution losses. 
These data include ALL international and domestic certificated route air carrier statistics; therefore, the data are different than those in Chapter 2. Revenue aircraft-miles, passenger-miles, and seat-miles began to rise in 2010. Passenger load factor was $83.0 \%$ in 2016.

Table 9.2

\section{Summary Statistics for U.S. Domestic and International Certificated Route Air Carriers (Combined Totals), 1970-2016}

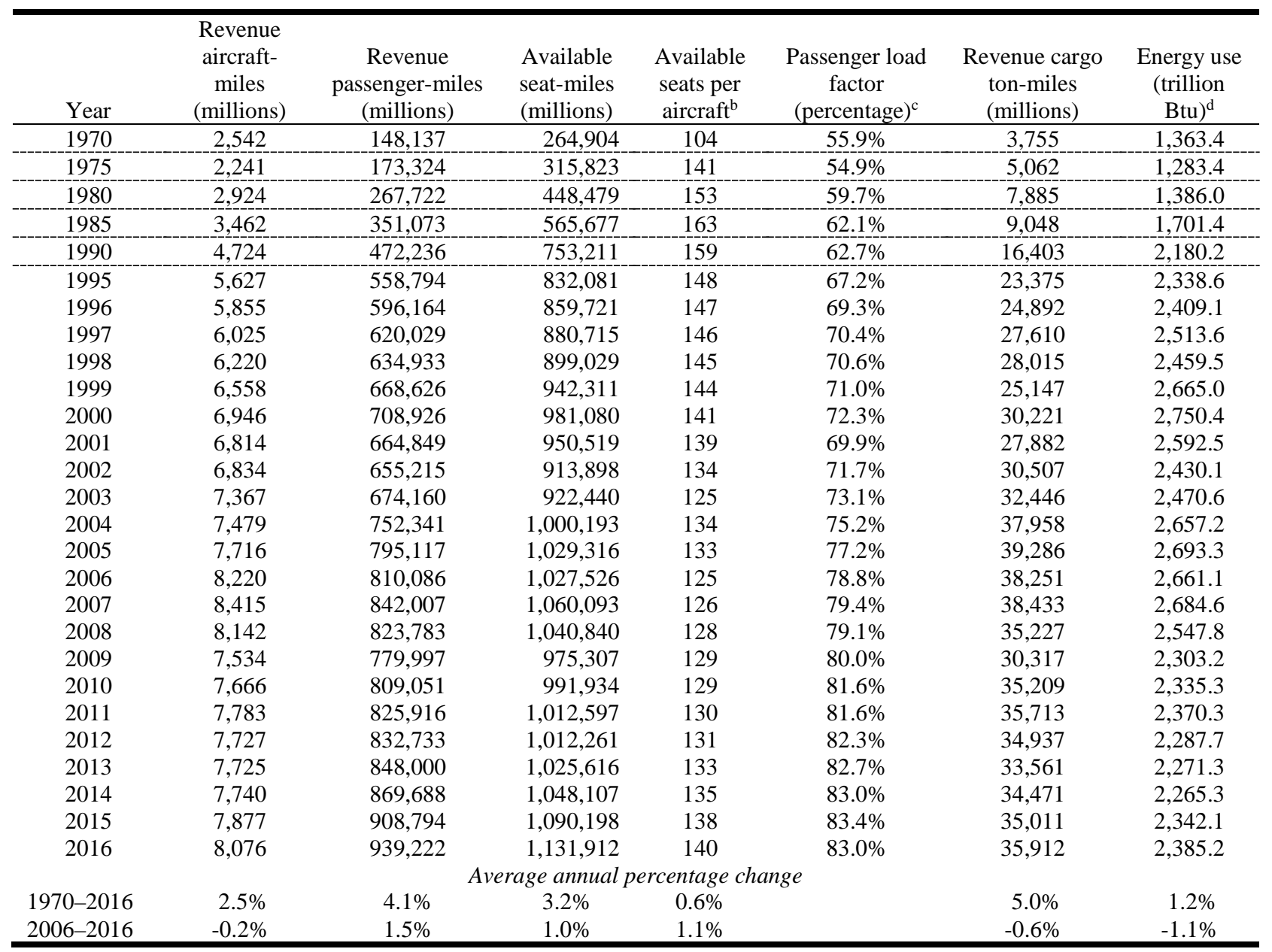

Sources:

U.S. Department of Transportation, Bureau of Transportation Statistics, www.transtats.bts.gov. (Additional resources: www.rita.dot.gov/bts)

1970-76 Energy Use - Department of Transportation, Civil Aeronautics Board, Fuel Cost and Consumption, Washington, DC, 1981, and annual.

${ }^{\text {a }}$ Data are for all U.S. air carriers reporting on Form 41.

${ }^{\mathrm{b}}$ Available seats per aircraft is calculated as the ratio of available seat-miles to revenue aircraft-miles.

c Passenger load factor is calculated as the ratio of revenue passenger-miles to available seat-miles for scheduled and nonscheduled services.

${ }^{\mathrm{d}}$ Energy use includes fuel purchased abroad for international flights. 
General aviation includes: (1) aircraft operating under general operating and flight rules; (2) not-for-hire airplanes with a seating capacity of 20 or more or a maximum payload capacity of 6,000 lbs. or more; (3) rotorcraft external load operations; (4) on-demand and commuter operations not covered under Federal Aviation Regulations

Part 121; and (5) agricultural aircraft operations.

Table 9.3

Summary Statistics for General Aviation, 1970-2015

\begin{tabular}{|c|c|c|c|}
\hline Calendar year & Total number of aircraft & $\begin{array}{c}\text { Aircraft hours flown } \\
\text { (thousands) }\end{array}$ & Energy use (trillion Btu) \\
\hline 1970 & $131,700^{\mathrm{a}}$ & $26,030^{\mathrm{b}}$ & 94.3 \\
\hline 1975 & 168,475 & 30,298 & 110.7 \\
\hline 1980 & 211,045 & 41,016 & 165.9 \\
\hline 1985 & 196,500 & 31,456 & 143.9 \\
\hline 1986 & 205,300 & 31,782 & 147.9 \\
\hline 1987 & 202,700 & 30,883 & 139.1 \\
\hline 1988 & 196,200 & 31,114 & 148.5 \\
\hline 1989 & 205,000 & 32,332 & 134.1 \\
\hline 1990 & 198,000 & 32,096 & 131.8 \\
\hline 1991 & 196,874 & 29,862 & 120.0 \\
\hline 1992 & 185,650 & 26,747 & 103.7 \\
\hline 1993 & 177,120 & 24,455 & 93.6 \\
\hline 1994 & 172,935 & 24,092 & 95.3 \\
\hline 1995 & 188,089 & 26,612 & 106.6 \\
\hline 1996 & 191,129 & 26,909 & 111.0 \\
\hline 1997 & 192,414 & 27,713 & 121.1 \\
\hline 1998 & 204,710 & 28,100 & 147.4 \\
\hline 1999 & 219,464 & 31,231 & 172.1 \\
\hline 2000 & 217,533 & 29,960 & 175.2 \\
\hline 2001 & 211,446 & 27,017 & 165.1 \\
\hline 2002 & 211,244 & 27,040 & 141.5 \\
\hline 2003 & 209,708 & 27,329 & 141.4 \\
\hline 2004 & 219,426 & 28,126 & 175.9 \\
\hline 2005 & 224,352 & 26,982 & 242.4 \\
\hline 2006 & 221,943 & 27,705 & 256.3 \\
\hline 2007 & 231,607 & 27,852 & 243.6 \\
\hline 2008 & 228,663 & 26,009 & 265.7 \\
\hline 2009 & 223,877 & 23,763 & 210.3 \\
\hline 2010 & 223,370 & 24,802 & 221.2 \\
\hline 2011 & 220,770 & 24,570 & 227.1 \\
\hline 2012 & 209,034 & 24,403 & 228.8 \\
\hline 2013 & 199,927 & 22,876 & 203.6 \\
\hline 2014 & 204,408 & 23,271 & 221.0 \\
\hline 2015 & 210,030 & 24,142 & 208.9 \\
\hline \multicolumn{4}{|c|}{ Average annual percentage change } \\
\hline 1970-2015 & $1.0 \%$ & $-0.2 \%$ & $1.8 \%$ \\
\hline 2005-2015 & $-0.7 \%$ & $-1.1 \%$ & $-1.5 \%$ \\
\hline
\end{tabular}

\section{Sources:}

U.S. Department of Transportation, Federal Aviation Administration, General Aviation and Part 135 Activity Surveys, CY 2015, Tables 1.1, 1.4, 5.1, and annual. 2011 Data: Aviation Forecasts, Tables 28 and 29, May 2013. (Additional resources: www.faa.gov/data-research/aviation_data_statistics/general_aviation)

${ }^{\text {a }}$ Active fixed-wing general aviation aircraft only.

${ }^{\mathrm{b}}$ Includes rotorcraft. 
In the early seventies, domestic waterborne commerce accounted for over $60 \%$ of total tonnage, but by 1994 foreign tonnage grew to more than half of all waterborne tonnage. Total foreign and domestic tons shipped were about 2.28 billion tons in 2015, down from a peak of 2.59 billion tons in 2006.

Table 9.4

Tonnage Statistics for Domestic and International Waterborne Commerce, 1970-2015 (million tons shipped)

\begin{tabular}{|c|c|c|c|c|}
\hline Year & $\begin{array}{c}\text { Foreign and domestic } \\
\text { total }\end{array}$ & Foreign total $^{\mathrm{a}}$ & Domestic total $^{\mathrm{b}}$ & Percent domestic of total \\
\hline 1970 & 1,532 & 581 & 951 & $62.1 \%$ \\
\hline 1975 & 1,695 & 749 & 946 & $55.8 \%$ \\
\hline 1980 & 1,999 & 921 & 1,077 & $53.9 \%$ \\
\hline 1985 & 1,788 & 774 & 1,014 & $56.7 \%$ \\
\hline 1986 & 1,874 & 837 & 1,037 & $55.3 \%$ \\
\hline 1987 & 1,967 & 891 & 1,076 & $54.7 \%$ \\
\hline 1988 & 2,088 & 976 & 1,112 & $53.3 \%$ \\
\hline 1989 & 2,140 & 1,038 & 1,103 & $51.5 \%$ \\
\hline 1990 & 2,164 & 1,042 & 1,122 & $51.8 \%$ \\
\hline 1991 & 2,092 & 1,014 & 1,079 & $51.6 \%$ \\
\hline 1992 & 2,132 & 1,037 & 1,095 & $51.4 \%$ \\
\hline 1993 & 2,128 & 1,060 & 1,068 & $50.2 \%$ \\
\hline 1994 & 2,215 & 1,116 & 1,099 & $49.6 \%$ \\
\hline 1995 & 2,240 & 1,147 & 1,093 & $48.8 \%$ \\
\hline 1996 & 2,284 & 1,183 & 1,101 & $48.2 \%$ \\
\hline 1997 & 2,333 & 1,221 & 1,113 & $47.7 \%$ \\
\hline 1998 & 2,340 & 1,245 & 1,094 & $46.8 \%$ \\
\hline 1999 & 2,323 & 1,261 & 1,062 & $45.7 \%$ \\
\hline 2000 & 2,425 & 1,355 & 1,070 & $44.1 \%$ \\
\hline 2001 & 2,393 & 1,351 & 1,042 & $43.5 \%$ \\
\hline 2002 & 2,340 & 1,319 & 1,021 & $43.6 \%$ \\
\hline 2003 & 2,394 & 1,378 & 1,016 & $42.4 \%$ \\
\hline 2004 & 2,552 & 1,505 & 1,047 & $41.0 \%$ \\
\hline 2005 & 2,527 & 1,499 & 1,029 & $40.7 \%$ \\
\hline 2006 & 2,588 & 1,565 & 1,023 & $39.5 \%$ \\
\hline 2007 & 2,564 & 1,543 & 1,022 & $39.9 \%$ \\
\hline 2008 & 2,477 & 1,521 & 956 & $38.6 \%$ \\
\hline 2009 & 2,211 & 1,354 & 857 & $38.8 \%$ \\
\hline 2010 & 2,335 & 1,441 & 894 & $38.3 \%$ \\
\hline 2011 & 2,368 & 1,480 & 888 & $37.5 \%$ \\
\hline 2012 & 2,307 & 1,422 & 885 & $38.4 \%$ \\
\hline 2013 & 2,274 & 1,383 & 891 & $39.2 \%$ \\
\hline 2014 & 2,346 & 1,409 & 937 & $39.9 \%$ \\
\hline 2015 & 2,279 & 1,374 & 905 & $39.7 \%$ \\
\hline \multicolumn{5}{|c|}{ Average annual percentage change } \\
\hline 1970-2015 & $0.9 \%$ & $1.9 \%$ & $-0.1 \%$ & \\
\hline 2005-2015 & $-1.0 \%$ & $-0.9 \%$ & $-1.3 \%$ & \\
\hline
\end{tabular}

\section{Source:}

U.S. Department of the Army, Corps of Engineers, The U.S. Waterway System, Transportation Facts and Information Fact Card, December 2016. (Additional resources: www.navigationdatacenter.us/index.htm)

a All movements between the United States and foreign countries and between Puerto Rico and the Virgin Islands and foreign countries are classified as foreign trade.

${ }^{\mathrm{b}}$ All movements between U.S. ports, continental and noncontiguous, and on the inland rivers, canals, and connecting channels of the United States, Puerto Rico, and the Virgin Islands, excluding the Panama Canal. Beginning in 1996, fish was excluded for internal and intra-port domestic traffic. 
The U.S. Army Corps of Engineers Navigation Data Center collects a wealth of waterborne commerce data. Energy use data, however, have never been collected as part of this effort. The energy use data collected by the Energy Information Administration (EIA) on vessel bunkering was formerly displayed on this table. The EIA data include different uses of fuel, not just fuel for domestic waterborne commerce; therefore it was misleading to display those data together.

Table 9.5

Summary Statistics for Domestic Waterborne Commerce, 1970-2015

\begin{tabular}{|c|c|c|c|c|}
\hline Year & Number of vessels ${ }^{\mathrm{a}}$ & $\begin{array}{c}\text { Ton-miles } \\
\text { (billions) }\end{array}$ & Tons shipped ${ }^{\mathrm{b}}$ (millions) & $\begin{array}{c}\text { Average length of haul } \\
\text { (miles) }\end{array}$ \\
\hline 1970 & 25,832 & 596 & 949 & 628.2 \\
\hline 1975 & 31,666 & 566 & 944 & 599.9 \\
\hline 1980 & 38,792 & 922 & 1,074 & 856.4 \\
\hline 1985 & 41,672 & 893 & 1,011 & 883.5 \\
\hline 1990 & 41,119 & 834 & 1,118 & 745.7 \\
\hline 1995 & 39,445 & 808 & 1,086 & 743.6 \\
\hline 1996 & 41,104 & 765 & 1,093 & 699.4 \\
\hline 1997 & 41,419 & 707 & 1,106 & 639.5 \\
\hline 1998 & 42,032 & 673 & 1,087 & 619.0 \\
\hline 1999 & 41,766 & 656 & 1,056 & 621.1 \\
\hline 2000 & 39,641 & 646 & 1,064 & 606.8 \\
\hline 2001 & 41,588 & 622 & 1,037 & 599.7 \\
\hline 2002 & 41,002 & 612 & 1,016 & 602.5 \\
\hline 2003 & 39,983 & 606 & 1,010 & 600.3 \\
\hline 2004 & 40,290 & 621 & 1,042 & 596.4 \\
\hline 2005 & 41,354 & 591 & 1,024 & 577.4 \\
\hline 2006 & 41,109 & 562 & 1,018 & 548.7 \\
\hline 2007 & 40,695 & 553 & 1,016 & 544.2 \\
\hline 2008 & 40,301 & 521 & 952 & 546.7 \\
\hline 2009 & 40,109 & 477 & 852 & 559.7 \\
\hline 2010 & 40,512 & 503 & 894 & 562.8 \\
\hline 2011 & 40,521 & 500 & 888 & 563.5 \\
\hline 2012 & 40,530 & 475 & 885 & 536.5 \\
\hline 2013 & 39,999 & 465 & 891 & 521.8 \\
\hline 2014 & 40,082 & 505 & 937 & 538.4 \\
\hline \multirow[t]{2}{*}{2015} & 40,555 & 491 & 905 & 542.2 \\
\hline & \multicolumn{4}{|c|}{ Average annual percentage change } \\
\hline 1970-2015 & $1.0 \%$ & $-0.4 \%$ & $-0.1 \%$ & $-0.3 \%$ \\
\hline 2005-2015 & $-0.2 \%$ & $-1.8 \%$ & $-1.2 \%$ & $-0.6 \%$ \\
\hline
\end{tabular}

\section{Sources:}

Number of vessels 1970-92, 1995-2010 - U.S. Department of the Army, Corps of Engineers, Waterborne Transportation Lines of the United States, 2011, New Orleans, LA, 2012, Table 2, p. 6, and annual. 1993-94 - U.S. Department of the Army, Corps of Engineers, The U.S. Waterway System-Facts, Navigation Data Center, New Orleans, Louisiana, January 1996.

Ton-miles, tons shipped, average length of haul - U.S. Department of the Army, Corps of Engineers, Waterborne Commerce of the United States, Calendar Year 2011, Part 5: National Summaries, New Orleans, LA, 2012, Table 1-4, pp. 1-6, 1-7, and annual. (Additional resources: www.navigationdatacenter.us/index.htm)

Number of vessels, ton-miles, tons shipped and average length of haul, 2011-2015 - U.S. Department of the Army, Corps of Engineers, The U.S. Waterway System, Transportation Facts and Information Fact Card, December 2016.

${ }^{\text {a }}$ Grand total for self-propelled and non-self-propelled.

${ }^{\mathrm{b}}$ These figures are not consistent with the figures on Table 9.4 because intra-territory tons are not included in this table. Intra-territory traffic is traffic between ports in Puerto Rico and the Virgin Islands. 
The data displayed in this table come from the Environmental Protection Agency's MOVES2014a model.

Table 9.6

Recreational Boat Energy Use, 1970-2015

\begin{tabular}{|c|c|c|c|c|}
\hline & Number of boats & Diesel fuel & Gasoline & Total energy use \\
\hline Year & (thousands) & & (trillion Btu) & \\
\hline 1970 & 10,087 & 5.5 & 151.7 & 157.2 \\
\hline 1975 & 10,337 & 10.7 & 156.4 & 167.1 \\
\hline 1976 & 10,387 & 11.8 & 157.4 & 169.1 \\
\hline 1977 & 10,437 & 12.8 & 158.3 & 171.1 \\
\hline 1978 & 10,487 & 13.9 & 159.3 & 173.1 \\
\hline 1979 & 10,537 & 14.9 & 160.2 & 175.1 \\
\hline 1980 & 10,587 & 16.0 & 161.2 & 177.1 \\
\hline 1981 & 10,637 & 17.0 & 162.1 & 179.1 \\
\hline 1982 & 10,687 & 18.0 & 163.1 & 181.1 \\
\hline 1983 & 10,737 & 19.1 & 164.0 & 183.1 \\
\hline 1984 & 10,787 & 20.1 & 165.0 & 185.1 \\
\hline 1985 & 10,837 & 21.2 & 165.9 & 187.1 \\
\hline 1986 & 10,887 & 22.2 & 166.9 & 189.1 \\
\hline 1987 & 10,937 & 23.3 & 167.8 & 191.1 \\
\hline 1988 & 11,030 & 24.3 & 170.4 & 194.7 \\
\hline 1989 & 11,122 & 25.4 & 172.9 & 198.3 \\
\hline 1990 & 11,215 & 26.4 & 175.4 & 201.8 \\
\hline 1991 & 11,327 & 27.5 & 178.7 & 206.2 \\
\hline 1992 & 11,440 & 28.5 & 182.0 & 210.5 \\
\hline 1993 & 11,553 & 29.5 & 185.3 & 214.8 \\
\hline 1994 & 11,770 & 30.6 & 192.5 & 223.1 \\
\hline 1995 & 11,988 & 31.6 & 199.7 & 231.3 \\
\hline 1996 & 12,206 & 32.7 & 206.8 & 239.5 \\
\hline 1997 & 12,244 & 33.7 & 207.2 & 240.9 \\
\hline 1998 & 12,283 & 34.8 & 207.4 & 242.2 \\
\hline 1999 & 12,321 & 35.8 & 207.1 & 243.0 \\
\hline 2000 & 12,359 & 36.8 & 206.6 & 243.4 \\
\hline 2001 & 12,464 & 37.9 & 206.9 & 244.9 \\
\hline 2002 & 12,568 & 39.0 & 206.7 & 245.7 \\
\hline 2003 & 12,673 & 40.2 & 206.0 & 246.2 \\
\hline 2004 & 12,777 & 41.3 & 205.0 & 246.2 \\
\hline 2005 & 12,882 & 42.4 & 203.7 & 246.1 \\
\hline 2006 & 12,984 & 43.5 & 202.5 & 245.9 \\
\hline 2007 & 13,086 & 44.6 & 201.2 & 245.8 \\
\hline 2008 & 13,189 & 45.7 & 200.0 & 245.7 \\
\hline 2009 & 13,291 & 46.8 & 198.8 & 245.6 \\
\hline 2010 & 13,393 & 47.9 & 197.3 & 245.2 \\
\hline 2011 & 13,497 & 49.0 & 195.9 & 244.9 \\
\hline 2012 & 13,602 & 50.1 & 194.7 & 244.8 \\
\hline 2013 & 13,707 & 51.2 & 193.8 & 245.0 \\
\hline 2014 & 13,811 & 52.3 & 193.1 & 245.4 \\
\hline 2015 & 13,916 & 53.4 & 192.6 & 246.0 \\
\hline \multicolumn{5}{|c|}{ Average annual percentage change } \\
\hline 1970-2015 & $0.7 \%$ & $5.2 \%$ & $0.5 \%$ & $1.0 \%$ \\
\hline $2005-2015$ & $0.8 \%$ & $2.3 \%$ & $-0.6 \%$ & $0.0 \%$ \\
\hline
\end{tabular}

Source:

U.S. Environmental Protection Agency, MOVES2014a model, www3.epa.gov/otaq/models/moves. 
The Interstate Commerce Commission designates Class I railroads on the basis of annual gross revenues. In 2015, seven railroads were given this designation. The number of railroads designated as Class I has changed considerably in the last 30 years; in 1976 there were 52 railroads given Class I designation.

Table 9.7

Class I Railroad Freight Systems in the United States Ranked by Revenue Ton-Miles, 2015

\begin{tabular}{lcr}
\hline \multicolumn{1}{c}{ Railroad } & $\begin{array}{c}\text { Revenue ton-miles } \\
\text { (billions) }\end{array}$ & Percent \\
\hline Burlington Northern and Santa Fe Railway Company & 702 & $40.2 \%$ \\
Union Pacific Railroad Company & 485 & $27.8 \%$ \\
CSX Transportation & 230 & $13.2 \%$ \\
Norfolk Southern Railway & 200 & $11.4 \%$ \\
Canadian National, Grand Trunk Corporation & 61 & $3.5 \%$ \\
Canadian Pacific Soo Railway & 36 & $2.1 \%$ \\
Kansas City Southern Railway Company & 31 & $1.8 \%$ \\
\hline Total & $\mathbf{1 , 7 4 5}$ & $\mathbf{1 0 0 . 0 \%}$ \\
\hline
\end{tabular}

\section{Source:}

Association of American Railroads, Railroad Facts, 2016 Edition, Washington, DC, September 2016, p. 68. (Additional resources: www.aar.org) 
Revenue ton-miles for Class I freight railroads was over 1.7 trillion in 2015. Though there are many regional and local freight railroads, the Class I freight railroads accounted for $94 \%$ of the railroad industry's freight revenue in 2015 and 69\% of the industry's mileage operated. The energy intensity of Class I railroads hit an all-time low of 289 Btu/ton-mile in 2010 and continued to be below 300 Btu/ton-mile in 2015.

Table 9.8

Summary Statistics for Class I Freight Railroads, 1970-2015

\begin{tabular}{|c|c|c|c|c|c|c|c|c|c|}
\hline Year & $\begin{array}{c}\text { Number of } \\
\text { locomotives } \\
\text { in service }^{\mathrm{a}}\end{array}$ & $\begin{array}{l}\text { Number of } \\
\text { freight cars } \\
\text { (thousands) }^{\mathrm{b}}\end{array}$ & $\begin{array}{c}\text { Train- } \\
\text { miles } \\
\text { (millions) }\end{array}$ & $\begin{array}{l}\text { Car-miles } \\
\text { (millions) }\end{array}$ & $\begin{array}{c}\text { Tons } \\
\text { originated }^{\mathrm{c}} \\
\text { (millions) }\end{array}$ & $\begin{array}{l}\text { Average } \\
\text { length of } \\
\text { haul } \\
\text { (miles) }\end{array}$ & $\begin{array}{l}\text { Revenue } \\
\text { ton-miles } \\
\text { (millions) }\end{array}$ & $\begin{array}{c}\text { Energy } \\
\text { intensity } \\
\text { (Btu/ton- } \\
\text { mile) }\end{array}$ & $\begin{array}{c}\text { Energy } \\
\text { use } \\
\text { (trillion } \\
\text { Btu) }\end{array}$ \\
\hline 1970 & $27,077^{d}$ & 1,424 & 427 & 29,890 & 1,485 & 515 & 764,809 & 691 & 528.1 \\
\hline 1975 & 27,846 & 1,359 & 403 & 27,656 & 1,395 & 541 & 754,252 & 687 & 518.3 \\
\hline 1980 & 28,094 & 1,168 & 428 & 29,277 & 1,492 & 616 & 918,958 & 597 & 548.7 \\
\hline 1985 & 22,548 & 867 & 347 & 24,920 & 1,320 & 665 & 876,984 & 497 & 436.1 \\
\hline 1987 & 19,647 & 749 & 361 & 25,627 & 1,372 & 688 & 943,747 & 456 & 430.3 \\
\hline 1988 & 19,364 & 725 & 379 & 26,339 & 1,430 & 697 & 996,182 & 443 & 441.4 \\
\hline 1989 & 19,015 & 682 & 383 & 26,196 & 1,403 & 723 & $1,013,841$ & 437 & 442.6 \\
\hline 1990 & 18,835 & 659 & 380 & 26,159 & 1,425 & 726 & $1,033,969$ & 420 & 434.7 \\
\hline 1991 & 18,344 & 633 & 375 & 25,628 & 1,383 & 751 & $1,038,875$ & 391 & 405.8 \\
\hline 1992 & 18,004 & 605 & 390 & 26,128 & 1,399 & 763 & $1,066,781$ & 393 & 419.2 \\
\hline 1993 & 18,161 & 587 & 405 & 26,883 & 1,397 & 794 & $1,109,309$ & 389 & 431.6 \\
\hline 1994 & 18,505 & 591 & 441 & 28,485 & 1,470 & 817 & $1,200,701$ & 388 & 465.4 \\
\hline 1996 & 19,269 & 571 & 469 & 31,715 & 1,611 & 842 & $1,355,975$ & 368 & 499.4 \\
\hline 1997 & 19,684 & 568 & 475 & 31,660 & 1,585 & 851 & $1,348,926$ & 370 & 499.7 \\
\hline 1998 & 20,261 & 576 & 475 & 32,657 & 1,649 & 835 & $1,376,802$ & 365 & 502.0 \\
\hline 1999 & 20,256 & 579 & 490 & 33,851 & 1,717 & 835 & $1,433,461$ & 363 & 520.0 \\
\hline 2000 & 20,028 & 560 & 504 & 34,590 & 1,738 & 843 & $1,465,960$ & 352 & 516.0 \\
\hline 2001 & 19,745 & 500 & 500 & 34,243 & 1,742 & 859 & $1,495,472$ & 346 & 517.3 \\
\hline 2002 & 20,506 & 478 & 500 & 34,680 & 1,767 & 853 & $1,507,011$ & 345 & 520.3 \\
\hline 2003 & 20,774 & 467 & 516 & 35,555 & 1,799 & 862 & $1,551,438$ & 344 & 533.9 \\
\hline 2004 & 22,015 & 474 & 535 & 37,071 & 1,844 & 902 & $1,662,598$ & 341 & 566.2 \\
\hline 2005 & 22,779 & 475 & 548 & 37,712 & 1,899 & 894 & $1,696,425$ & 337 & 571.4 \\
\hline 2006 & 23,732 & 475 & 563 & 38,995 & 1,957 & 906 & $1,771,897$ & 330 & 584.5 \\
\hline 2007 & 24,143 & 460 & 543 & 38,186 & 1,940 & 913 & $1,770,545$ & 320 & 566.9 \\
\hline 2008 & 24,003 & 450 & 524 & 37,226 & 1,934 & 919 & $1,777,236$ & 305 & 542.5 \\
\hline 2009 & 24,045 & 416 & 436 & 32,115 & 1,668 & 919 & $1,532,214$ & 291 & 446.6 \\
\hline 2010 & 23,893 & 398 & 476 & 35,541 & 1,851 & 914 & $1,691,004$ & 289 & 488.1 \\
\hline 2011 & 24,250 & 381 & 493 & 36,649 & 1,885 & 917 & $1,729,256$ & 298 & 514.6 \\
\hline 2014 & 25,916 & 372 & 518 & 37,193 & 1,840 & 1,006 & $1,851,229$ & 292 & 540.5 \\
\hline 2015 & 26,574 & 331 & 495 & 35,861 & 1,731 & 1,008 & $1,744,731$ & 296 & 516.4 \\
\hline \multicolumn{10}{|c|}{ Average annual percentage change } \\
\hline 1970-2015 & $0.0 \%$ & $-3.2 \%$ & $0.3 \%$ & $0.4 \%$ & $0.3 \%$ & $1.5 \%$ & $1.8 \%$ & $-1.9 \%$ & $0.0 \%$ \\
\hline 2005-2015 & $1.6 \%$ & $-3.5 \%$ & $-1.0 \%$ & $-0.5 \%$ & $-0.9 \%$ & $1.2 \%$ & $0.3 \%$ & $-1.3 \%$ & $-1.0 \%$ \\
\hline
\end{tabular}

Source:

Association of American Railroads, Railroad Facts, 2016 Edition, Washington, DC, September 2016, pp. 30, 31, 36, 37, 39, 51, and 69. (Additional resources: www.aar.org)

${ }^{\text {a }}$ Does not include self-powered units. excluded.

${ }^{\mathrm{b}}$ Does not include private or shipper-owned cars. Beginning in 2001, Canadian-owned U.S. railroads are

${ }^{\mathrm{c}}$ Tons originated is a more accurate representation of total tonnage than revenue tons. Revenue tons often produces double-counting of loads switched between rail companies.

${ }^{\mathrm{d}}$ Data represent total locomotives used in freight and passenger service. Separate estimates are not available. 
According to the 2012 Commodity Flow Survey, 7\% of all freight ton-miles are rail intermodal shipments (truck/rail or rail/water). See Table 5.16 for details. The number of trailers and containers moved by railroads has increased more than eight-fold from 1965 to 2015. Containerization has increased in the last two decades, evidenced by the 432\% increase in the number of containers from 1988 to 2015. The number of trailers moved by rail fell to an alltime low in 2013 but has increased for the last two years.

\section{Table 9.9}

Intermodal Rail Traffic, 1965-2015

\begin{tabular}{|c|c|c|c|}
\hline Year & Trailers \& containers & Trailers & Containers \\
\hline 1965 & $1,664,929$ & $\mathrm{~b}$ & $\mathrm{~b}$ \\
\hline 1970 & $2,363,200$ & $\mathrm{~b}$ & b \\
\hline 1975 & $2,238,117$ & $\mathrm{~b}$ & $\mathrm{~b}$ \\
\hline 1980 & $3,059,402$ & b & $\mathrm{b}$ \\
\hline 1985 & $4,590,952$ & b & $\mathrm{b}$ \\
\hline 1986 & $4,997,229$ & $\mathrm{~b}$ & $\mathrm{~b}$ \\
\hline 1987 & $5,503,819$ & $\mathrm{~b}$ & $\mathrm{~b}$ \\
\hline 1988 & $5,779,547$ & $3,481,020$ & $2,298,527$ \\
\hline 1989 & $5,987,355$ & $3,496,262$ & $2,491,093$ \\
\hline 1990 & $6,206,782$ & $3,451,953$ & $2,754,829$ \\
\hline 1991 & $6,246,134$ & $3,201,560$ & $3,044,574$ \\
\hline 1992 & $6,627,841$ & $3,264,597$ & $3,363,244$ \\
\hline 1993 & $7,156,628$ & $3,464,126$ & $3,692,502$ \\
\hline 1994 & $8,128,228$ & $3,752,502$ & $4,375,726$ \\
\hline 1995 & $7,936,172$ & $3,492,463$ & $4,443,709$ \\
\hline 1996 & $8,143,258$ & $3,302,128$ & $4,841,130$ \\
\hline 1997 & $8,698,308$ & $3,453,907$ & $5,244,401$ \\
\hline 1998 & $8,772,663$ & $3,353,032$ & $5,419,631$ \\
\hline 1999 & $8,907,626$ & $3,207,407$ & $5,700,219$ \\
\hline 2000 & $9,176,890$ & $2,888,630$ & $6,288,260$ \\
\hline 2001 & $8,935,444$ & $2,603,423$ & $6,332,021$ \\
\hline 2002 & $9,312,360$ & $2,531,338$ & $6,781,022$ \\
\hline 2003 & $9,955,605$ & $2,625,837$ & $7,329,768$ \\
\hline 2004 & $10,993,662$ & $2,928,123$ & $8,065,539$ \\
\hline 2005 & $11,693,512$ & $2,979,906$ & $8,713,606$ \\
\hline 2006 & $12,282,221$ & 2,882,699 & $9,399,522$ \\
\hline 2007 & $12,026,631$ & $2,600,635$ & $9,425,996$ \\
\hline 2008 & $11,499,978$ & $2,478,890$ & $9,021,088$ \\
\hline 2009 & $9,875,967$ & $1,639,603$ & $8,236,364$ \\
\hline 2010 & $11,283,151$ & $1,684,684$ & $9,598,467$ \\
\hline 2011 & $11,892,418$ & 1,698,615 & $10,193,803$ \\
\hline 2012 & $12,267,416$ & $1,518,323$ & $10,749,093$ \\
\hline 2013 & $12,831,311$ & $1,483,938$ & $11,347,373$ \\
\hline 2014 & $13,496,822$ & $1,550,124$ & $11,946,698$ \\
\hline 2015 & $13,710,646$ & $1,475,754$ & $12,234,892$ \\
\hline \multicolumn{4}{|c|}{ Average annual percentage change } \\
\hline 1965-2015 & $4.3 \%$ & $\mathrm{~b}$ & $\mathrm{~b}$ \\
\hline $2005-2015$ & $1.6 \%$ & $-6.8 \%$ & $3.5 \%$ \\
\hline
\end{tabular}

\section{Source:}

Association of American Railroads, Railroad Facts, 2016 Edition, Washington, DC, September 2016, p. 29. (Additional resources: www.aar.org)

a Beginning in 1995, the Grand Trunk Western Railroad and the Soo Line Railroad Company are excluded. Beginning in 1999, the Illinois Central data are excluded. Beginning in 2002, the Wisconsin Central data are excluded.

${ }^{\mathrm{b}}$ Data are not available. 
The National Railroad Passenger Corporation, known as Amtrak, began operation in 1971. Amtrak revenue passenger-miles have grown at an average annual rate of 2.7\% from 1971 to 2015.

Table 9.10

Summary Statistics for the National Railroad Passenger Corporation (Amtrak), 1971-2015

\begin{tabular}{|c|c|c|c|c|c|c|c|c|}
\hline Year & $\begin{array}{l}\text { Number of } \\
\text { locomotives } \\
\text { in service } \\
\end{array}$ & $\begin{array}{c}\text { Number of } \\
\text { passenger } \\
\text { cars } \\
\end{array}$ & $\begin{array}{l}\text { Train-miles } \\
\text { (thousands) }\end{array}$ & $\begin{array}{c}\text { Car-miles } \\
\text { (thousands) }\end{array}$ & $\begin{array}{c}\text { Revenue } \\
\text { passenger- } \\
\text { miles } \\
\text { (millions) } \\
\end{array}$ & $\begin{array}{l}\text { Average } \\
\text { trip length } \\
\text { (miles) }\end{array}$ & $\begin{array}{l}\text { Energy intensity } \\
\text { (Btu per revenue } \\
\text { passenger-mile) }\end{array}$ & $\begin{array}{l}\text { Energy } \\
\text { use } \\
\text { (trillion } \\
\text { Btu) } \\
\end{array}$ \\
\hline 1971 & a & 1,165 & 16,537 & 140,147 & 1,993 & 188 & $\mathrm{~b}$ & $\mathrm{~b}$ \\
\hline 1975 & 355 & 1,913 & 30,166 & 253,898 & 3,753 & 224 & 3,311 & 12.4 \\
\hline 1980 & 448 & 2,128 & 29,487 & 235,235 & 4,503 & 217 & 2,859 & 12.9 \\
\hline 1985 & 382 & 1,818 & 30,038 & 250,642 & 4,785 & 238 & 2,237 & 10.7 \\
\hline 1991 & 316 & 1,786 & 34,000 & 312,484 & 6,273 & 285 & 2,011 & 12.6 \\
\hline 1992 & 336 & 1,796 & 34,000 & 307,282 & 6,091 & 286 & 2,117 & 12.9 \\
\hline 1993 & 360 & 1,853 & 34,936 & 302,739 & 6,199 & 280 & 2,142 & 13.3 \\
\hline 1994 & 411 & 1,874 & 34,940 & 305,600 & 5,869 & 276 & 1,917 & 11.3 \\
\hline 1995 & 422 & 1,907 & 31,579 & 282,579 & 5,401 & 266 & 2,071 & 11.2 \\
\hline 1996 & 348 & 1,501 & 30,542 & 277,750 & 5,066 & 257 & 2,194 & 11.1 \\
\hline 1997 & 292 & 1,572 & 32,000 & 287,760 & 5,166 & 255 & 2,289 & 11.8 \\
\hline 1998 & 362 & 1,347 & 32,926 & 315,823 & 5,325 & 251 & 2,246 & 12.0 \\
\hline 2000 & 385 & 1,891 & 35,404 & 371,215 & 5,574 & 243 & 2,651 & 14.8 \\
\hline 2001 & 401 & 2,084 & 36,512 & 377,705 & 5,571 & 238 & 2,690 & 15.0 \\
\hline 2002 & 372 & 2,896 & 37,624 & 378,542 & 5,314 & 228 & 2,537 & 13.5 \\
\hline 2003 & 442 & 1,623 & 37,459 & 331,864 & 5,680 & 231 & 2,145 & 12.2 \\
\hline 2004 & 276 & 1,211 & 37,159 & 308,437 & 5,511 & 219 & 2,068 & 11.4 \\
\hline 2005 & 258 & 1,186 & 36,199 & 264,796 & 5,381 & 215 & 2,025 & 10.9 \\
\hline 2006 & 319 & 1,191 & 36,083 & 263,908 & 5,410 & 220 & 1,948 & 10.5 \\
\hline 2007 & 270 & 1,164 & 37,484 & 266,545 & 5,784 & 218 & 1,824 & 10.5 \\
\hline 2008 & 278 & 1,177 & 37,736 & 271,762 & 6,179 & 215 & 1,745 & 10.8 \\
\hline 2009 & 274 & 1,214 & 38,300 & 282,764 & 5,914 & 217 & 1,773 & 10.5 \\
\hline 2010 & 282 & 1,274 & 37,453 & 294,820 & 6,420 & 220 & 1,668 & 10.7 \\
\hline 2011 & 287 & 1,301 & 37,090 & 296,315 & 6,568 & 213 & 1,628 & 10.7 \\
\hline 2012 & 485 & 2,090 & 37,640 & 319,088 & 6,804 & 218 & 1,561 & 10.6 \\
\hline 2013 & 418 & 1,447 & 38,410 & 324,949 & 6,810 & 218 & 1,559 & 10.6 \\
\hline 2014 & 428 & 1,419 & 38,013 & 324,683 & 6,675 & 218 & 1,641 & 11.0 \\
\hline 2015 & 423 & 1,428 & 37,798 & 319,464 & 6,536 & 218 & 1,663 & 10.9 \\
\hline \multicolumn{9}{|c|}{ Average annual percentage change } \\
\hline 2005-2015 & $5.1 \%$ & $1.9 \%$ & $0.4 \%$ & $1.9 \%$ & $2.0 \%$ & $0.1 \%$ & $-2.0 \%$ & $0.0 \%$ \\
\hline
\end{tabular}

\section{Sources:}

1971-83 - Association of American Railroads, Economics and Finance Department, Statistics of Class I Railroads, Washington, DC, and annual.

1984-88 - Association of American Railroads, Railroad Facts, 1988 Edition, Washington, DC, December 1989, p. 61 , and annual.

1989-93 - Personal communication with the Corporate Accounting Office of Amtrak, Washington, DC.

1994-2015 - Number of locomotives in service, number of passenger cars, train-miles, car-miles, revenue passengermiles, and average trip length - Association of American Railroads, Railroad Facts, 2016 Edition, Washington, DC, 2016, p. 77.

Energy use - Personal communication with the Amtrak, Washington, DC. (Additional resources: www.amtrak.com, www.aar.org)

${ }^{a}$ Only end-use energy was counted for electricity. Previous editions included primary energy use for electricity which included generation and distribution losses.

${ }^{\mathrm{b}}$ Data are not available.

c Energy use for 1994 on is not directly comparable to earlier years. Some commuter rail energy use may have been inadvertently included in earlier years. 
Commuter rail, which is also known as regional rail or suburban rail, is long-haul rail passenger service operating between metropolitan and suburban areas, whether within or across state lines. Commuter rail lines usually have reduced fares for multiple rides and commutation tickets for regular, recurring riders.

Table 9.11

Summary Statistics for Commuter Rail Operations, 1984-2015

\begin{tabular}{|c|c|c|c|c|c|c|c|}
\hline Year & $\begin{array}{l}\text { Number of } \\
\text { passenger } \\
\text { vehicles }\end{array}$ & $\begin{array}{l}\text { Vehicle- } \\
\text { miles } \\
\text { (millions) }\end{array}$ & $\begin{array}{l}\text { Passenger } \\
\text { trips } \\
\text { (millions) }\end{array}$ & $\begin{array}{l}\text { Passenger- } \\
\text { miles } \\
\text { (millions) }\end{array}$ & $\begin{array}{l}\text { Average } \\
\text { trip length } \\
\text { (miles) }\end{array}$ & $\begin{array}{c}\text { Energy intensity } \\
\text { (Btu/passenger- } \\
\text { mile) }{ }^{\mathrm{a}}\end{array}$ & $\begin{array}{l}\text { Energy } \\
\text { use } \\
\text { (trillion } \\
\text { Btu) }\end{array}$ \\
\hline 1984 & 4,075 & 167.9 & 267 & 6,207 & 23.2 & 1,798 & 11.2 \\
\hline 1985 & 4,035 & 182.7 & 275 & 6,534 & 23.8 & 1,720 & 11.2 \\
\hline 1986 & 4,440 & 188.6 & 306 & 6,723 & 22.0 & 1,720 & 11.6 \\
\hline 1987 & 4,686 & 188.9 & 311 & 6,818 & 21.9 & 1,628 & 11.1 \\
\hline 1988 & 4,649 & 202.2 & 325 & 6,964 & 21.4 & 1,666 & 11.4 \\
\hline 1989 & 4,472 & 209.6 & 330 & 7,211 & 21.9 & 1,622 & 11.7 \\
\hline 1990 & 4,982 & 212.7 & 328 & 7,082 & 21.6 & 1,622 & 11.5 \\
\hline 1991 & 5,126 & 214.9 & 318 & 7,344 & 23.1 & 1,601 & 11.8 \\
\hline 1992 & 5,164 & 218.8 & 314 & 7,320 & 23.3 & 1,565 & 11.5 \\
\hline 1993 & 4,982 & 223.9 & 322 & 6,940 & 21.6 & 1,782 & 12.4 \\
\hline 1994 & 5,126 & 230.8 & 339 & 7,996 & 23.6 & 1,605 & 12.8 \\
\hline 1995 & 5,164 & 237.7 & 344 & 8,244 & 24.0 & 1,580 & 13.0 \\
\hline 1996 & 5,240 & 241.9 & 352 & 8,351 & 23.7 & 1,541 & 12.9 \\
\hline 1997 & 5,426 & 250.7 & 357 & 8,038 & 22.5 & 1,630 & 13.1 \\
\hline 1998 & 5,536 & 259.5 & 381 & 8,704 & 22.8 & 1,612 & 14.0 \\
\hline 1999 & 5,550 & 265.9 & 396 & 8,766 & 22.1 & 1,670 & 14.6 \\
\hline 2000 & 5,498 & 270.9 & 413 & 9,402 & 22.8 & 1,542 & 14.5 \\
\hline 2001 & 5,572 & 277.3 & 419 & 9,548 & 22.8 & 1,533 & 14.6 \\
\hline 2002 & 5,724 & 283.7 & 414 & 9,504 & 22.9 & 1,542 & 14.7 \\
\hline 2003 & 5,959 & 286.0 & 410 & 9,559 & 23.3 & 1,542 & 14.7 \\
\hline 2004 & 6,228 & 294.7 & 414 & 9,719 & 23.5 & 1,536 & 14.9 \\
\hline 2005 & 6,392 & 303.4 & 423 & 9,473 & 22.4 & 1,658 & 15.7 \\
\hline 2006 & 6,403 & 314.7 & 441 & 10,361 & 23.5 & 1,539 & 15.9 \\
\hline 2007 & 6,391 & 325.7 & 459 & 11,153 & 24.3 & 1,543 & 17.2 \\
\hline 2008 & 6,617 & 310.2 & 472 & 11,049 & 23.4 & 1,579 & 17.4 \\
\hline 2009 & 6,941 & 343.5 & 468 & 11,232 & 24.0 & 1,714 & 19.2 \\
\hline 2010 & 6,927 & 345.3 & 464 & 10,874 & 23.4 & 1,753 & 19.1 \\
\hline 2011 & 7,193 & 345.2 & 466 & 11,427 & 24.5 & 1,681 & 19.2 \\
\hline 2012 & 7,059 & 346.4 & 471 & 11,181 & 23.7 & 1,703 & 19.0 \\
\hline 2013 & 7,310 & 359.1 & 480 & 11,862 & 24.7 & 1,676 & 19.9 \\
\hline 2014 & 7,337 & 370.8 & 490 & 11,718 & 23.9 & 1,638 & 19.2 \\
\hline 2015 & 7,301 & 373.7 & 498 & 11,804 & 23.7 & 1,643 & 19.4 \\
\hline \multicolumn{8}{|c|}{ Average annual percentage change } \\
\hline 1984-2015 & $1.9 \%$ & $2.6 \%$ & $2.0 \%$ & $2.1 \%$ & $0.1 \%$ & $-0.3 \%$ & $1.8 \%$ \\
\hline 2005-2015 & $1.3 \%$ & $2.1 \%$ & $1.6 \%$ & $2.2 \%$ & $0.6 \%$ & $-0.1 \%$ & $2.1 \%$ \\
\hline
\end{tabular}

\section{Sources:}

1984-2014: American Public Transportation Association, 2016 Public Transportation Fact Book, Washington, DC, April 2016, Appendix A. (Additional resources: www.apta.com)

2015: U.S. Department of Transportation, Federal Transit Administration, 2015 National Transit Database, accessed September 2017. (Additional resources: www.transit.gov/ntd)

a Only end-use energy was counted for electricity. Previous editions included primary energy use for electricity which included generation and distribution losses. 
This table on transit rail operations includes data on light rail and heavy rail systems. Light rail vehicles are usually single vehicles driven electrically with power drawn from overhead wires. Heavy rail is characterized by high speed and rapid acceleration of rail cars operating on a separate right-of-way.

Table 9.12

Summary Statistics for Rail Transit Operations, 1970-2015

\begin{tabular}{|c|c|c|c|c|c|c|c|}
\hline Year & $\begin{array}{c}\text { Number of } \\
\text { passenger } \\
\text { vehicles }\end{array}$ & $\begin{array}{c}\text { Vehicle- } \\
\text { miles } \\
\text { (millions) } \\
\end{array}$ & $\begin{array}{l}\text { Passenger } \\
\text { trips } \\
\text { (millions) }^{\mathrm{b}}\end{array}$ & $\begin{array}{l}\text { Passenger- } \\
\text { miles } \\
\text { (millions) }^{\mathrm{C}}\end{array}$ & $\begin{array}{c}\text { Average trip } \\
\text { length } \\
\text { (miles) }^{\mathrm{d}}\end{array}$ & $\begin{array}{c}\text { Energy intensity } \\
\text { (Btu/passenger- } \\
\text { mile) }{ }^{\mathrm{e}}\end{array}$ & $\begin{array}{c}\text { Energy use } \\
\text { (trillion Btu) }^{\mathrm{e}}\end{array}$ \\
\hline 1970 & 10,548 & 440.8 & 2,116 & 12,273 & $f$ & 712 & 8.7 \\
\hline 1975 & 10,617 & 446.9 & 1,797 & 10,423 & $\bar{f}$ & 866 & 9.0 \\
\hline 1980 & 10,654 & 402.2 & 2,241 & 10,939 & 4.9 & 763 & 8.3 \\
\hline 1985 & 11,109 & 467.8 & 2,422 & 10,777 & 4.4 & 927 & 10.0 \\
\hline 1990 & 11,332 & 560.9 & 2,521 & 12,046 & 4.8 & 998 & 12.0 \\
\hline 1995 & 11,156 & 571.8 & 2,284 & 11,419 & 5.0 & 1,102 & 12.6 \\
\hline 1996 & 11,341 & 580.7 & 2,418 & 12,487 & 5.2 & 996 & 12.4 \\
\hline 1997 & 11,471 & 598.9 & 2,692 & 13,091 & 4.9 & 943 & 12.3 \\
\hline 1998 & 11,521 & 609.5 & 2,669 & 13,412 & 5.0 & 931 & 12.5 \\
\hline 1999 & 11,603 & 626.4 & 2,813 & 14,108 & 5.0 & 919 & 13.0 \\
\hline 2000 & 12,168 & 648.0 & 2,952 & 15,200 & 5.1 & 923 & 14.0 \\
\hline 2001 & 12,084 & 662.4 & 3,064 & 15,615 & 5.1 & 925 & 14.4 \\
\hline 2002 & 12,479 & 681.9 & 3,025 & 15,095 & 5.0 & 948 & 14.3 \\
\hline 2003 & 12,236 & 694.2 & 3,005 & 15,082 & 5.0 & 936 & 14.1 \\
\hline 2004 & 12,480 & 709.7 & 3,098 & 15,930 & 5.1 & 907 & 14.5 \\
\hline 2005 & 12,755 & 715.4 & 3,189 & 16,118 & 5.1 & 919 & 14.8 \\
\hline 2006 & 12,853 & 726.4 & 3,334 & 16,587 & 5.0 & 893 & 14.8 \\
\hline 2007 & 13,032 & 741.2 & 3,879 & 18,070 & 4.7 & 851 & 15.4 \\
\hline 2008 & 13,346 & 762.8 & 4,001 & 18,941 & 4.7 & 832 & 15.8 \\
\hline 2009 & 13,529 & 775.3 & 3,955 & 19,004 & 4.8 & 830 & 15.8 \\
\hline 2010 & 13,614 & 759.6 & 4,007 & 18,580 & 4.6 & 832 & 15.5 \\
\hline 2011 & 13,328 & 744.1 & 4,083 & 19,520 & 4.8 & 812 & 15.8 \\
\hline 2012 & 12,455 & 749.5 & 4,192 & 19,835 & 4.7 & 791 & 15.7 \\
\hline 2013 & 12,434 & 774.3 & 4,275 & 20,381 & 4.8 & 793 & 16.2 \\
\hline 2014 & 12,608 & 780.9 & 4,411 & 20,829 & 4.7 & 786 & 16.4 \\
\hline 2015 & 12,820 & 803.2 & 4,339 & 20,710 & 4.8 & 776 & 16.1 \\
\hline \multicolumn{8}{|c|}{ Average annual percentage change } \\
\hline 1970-2015 & $0.4 \%$ & $1.3 \%$ & $1.6 \%$ & $1.2 \%$ & $-0.1 \% \mathrm{~g}$ & $0.2 \%$ & $1.4 \%$ \\
\hline 2005-2015 & $0.1 \%$ & $1.2 \%$ & $3.1 \%$ & $2.5 \%$ & $-0.6 \%$ & $-1.7 \%$ & $0.8 \%$ \\
\hline
\end{tabular}

\section{Sources:}

1970-2014: American Public Transportation Association, 2016 Public Transportation Fact Book, Washington, DC, April 2016, Appendix A. (Additional resources: www.apta.com)

2015: U.S. Department of Transportation, Federal Transit Administration, 2015 National Transit Database, accessed September 2017. (Additional resources: www.transit.gov/ntd)

Energy use - See Appendix A for Rail Transit Energy Use.

${ }^{a}$ Heavy rail and light rail. Series not continuous between 1983 and 1984 because of a change in data source by the American Public Transit Association (APTA). Beginning in 1984, data provided by APTA are taken from mandatory reports filed with the Urban Mass Transit Administration (UMTA). Data for prior years were provided on a voluntary basis by APTA members and expanded statistically.

b 1970-79 data represents total passenger rides; after 1979, data represents unlinked passenger trips.

c Estimated for years 1970-76 based on an average trip length of 5.8 miles.

${ }^{\mathrm{d}}$ Calculated as the ratio of passenger-miles to passenger trips.

e Only end-use energy was counted for electricity. Previous editions included primary energy use for electricity which included generation and distribution losses. Large system-to-system variations exist for energy intensities.

${ }^{\mathrm{f}}$ Data are not available.

g Average annual percentage change is calculated for years 1980-2015. 
9-14 


\section{Chapter 10 Transportation and the Economy}

Summary Statistics from Tables/Figures in this Chapter

\begin{tabular}{|c|c|c|}
\hline Source & & \\
\hline Table 10.1 & Average household transportation expenditures, 2015 & $17.0 \%$ \\
\hline \multirow[t]{7}{*}{ Figure 10.2} & Share of gasoline cost attributed to taxes, 2016 & \\
\hline & Canada & $38 \%$ \\
\hline & France & $66 \%$ \\
\hline & Germany & $66 \%$ \\
\hline & Japan & $54 \%$ \\
\hline & United Kingdom & $70 \%$ \\
\hline & United States & $21 \%$ \\
\hline \multirow[t]{3}{*}{ Table 10.13} & Average price of a new car, 2016 (current dollars) & 25,774 \\
\hline & Domestic & 24,414 \\
\hline & Import & 29,913 \\
\hline \multirow[t]{3}{*}{ Table 10.14} & Car operating costs, 2016 & \\
\hline & Variable costs (constant 2016 dollars per 10,000 miles) & 1,473 \\
\hline & Fixed costs (constant 2016 dollars per 10,000 miles) & 6,072 \\
\hline \multirow[t]{3}{*}{ Table 10.18} & Transportation sector share of total employment & \\
\hline & 2000 & $8.3 \%$ \\
\hline & 2016 & $7.5 \%$ \\
\hline
\end{tabular}




\section{Adjusting Dollar Amounts for Inflation}

A dollar spent in 1970 does not have the purchasing power of a dollar spent in 2016 due to the inflation of prices for all goods and services. Thus, prices in a historical series must be adjusted in order to provide proper comparison. The term "current dollars" is used in this report for dollar amounts that were current as of the year listed - this can also be referred to as "nominal dollars." The term "constant 2016 dollars" is used in this report for dollar amounts that have been adjusted to a constant purchasing power (2016, in this example) and thus the data are comparable historically - this can also be referred to as "real dollars."

Appendix B, Table B.17 contains the Consumer Price Inflation Index and Table B.18 contains the Gross National Product Implicit Price Deflator for years 1970 to 2016. Tables in the report with constant dollars have a footnote indicating which of these inflation adjustment indices were used. 
The Transportation Services Index (TSI) was created by the U.S. Department of Transportation Bureau of Transportation Statistics (BTS). It is an index that measures the movement of freight and passengers.

The Freight TSI consists of:

- for-hire trucking (parcel services are not included);

- freight railroad services (including rail-based intermodal shipments such as containers on flat cars);

- inland waterway traffic;

- pipeline movements (including principally petroleum and petroleum products and natural gas); and

- air freight.

The index does not include international or coastal steamship movements, private trucking, courier services, or the United States Postal Services.

The Passenger TSI consists of:

- local mass transit;

- intercity passenger rail; and

- passenger air transportation.

The index does not include intercity bus, sightseeing services, taxi service, private car usage, or bicycling and other nonmotorized means of transportation.

Figure 10.1. Transportation Services Index, January 1990-January 2017

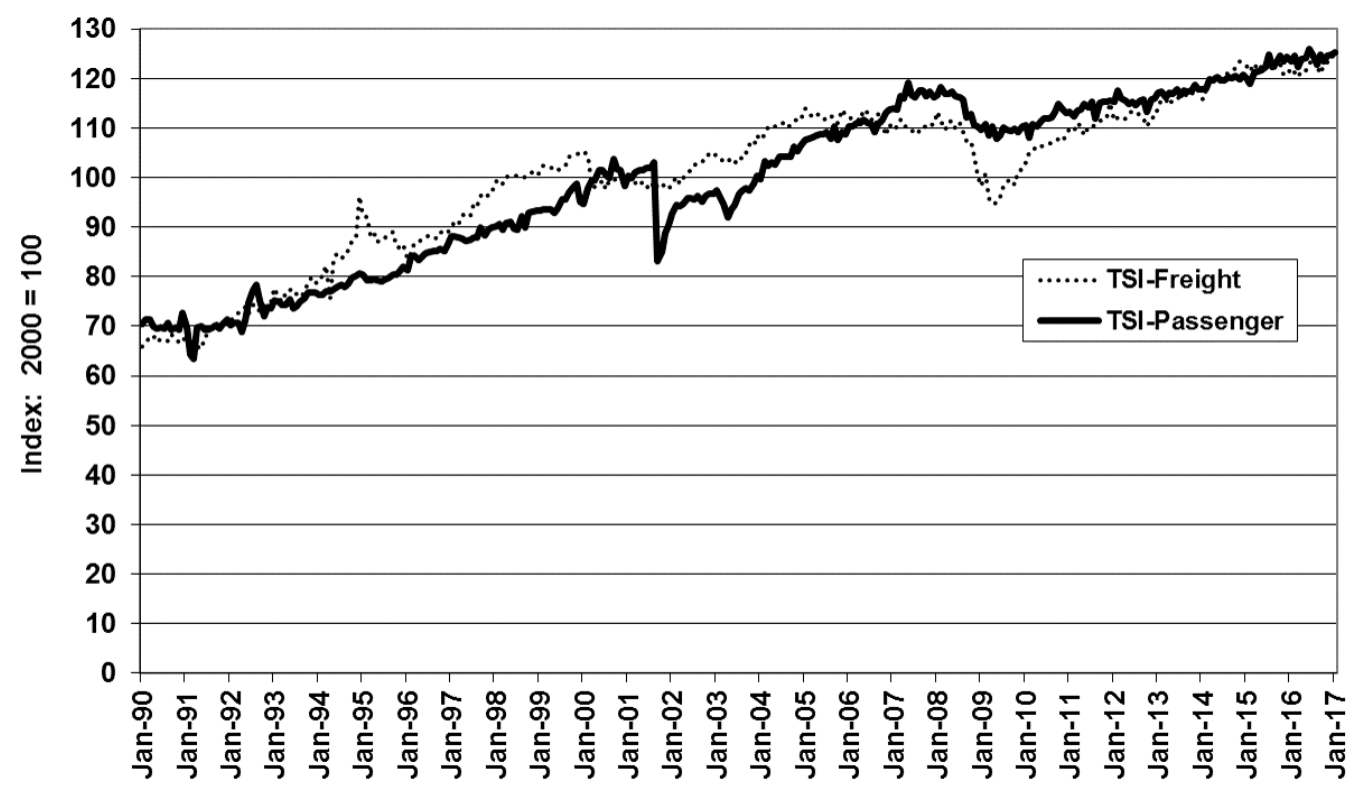

\section{Source:}

U.S. Department of Transportation, Bureau of Transportation Statistics, Transportation Services Index website, www.transtats.bts.gov/OSEA/TSI/. (Additional resources: www.bts.gov) 
Table 10.1

Average Annual Expenditures of Households by Income, 2015a

\begin{tabular}{|c|c|c|c|c|c|}
\hline & \multirow[b]{2}{*}{$\begin{array}{c}\text { All } \\
\text { households }\end{array}$} & \multicolumn{4}{|c|}{ Income before taxes } \\
\hline & & $\begin{array}{r}\text { Less than } \\
\$ 15,000 \\
\end{array}$ & $\begin{array}{r}\$ 15,000- \\
\$ 29,999 \\
\end{array}$ & $\begin{array}{r}\$ 30,000- \\
\$ 39,999 \\
\end{array}$ & $\begin{array}{r}\$ 40,000- \\
\$ 49,999 \\
\end{array}$ \\
\hline \multirow[t]{2}{*}{ Total expenditures } & $\$ 55,978$ & $\$ 23,479$ & $\$ 30,974$ & $\$ 38,634$ & $\$ 42,529$ \\
\hline & \multicolumn{5}{|c|}{ Percentage of total expenditures ${ }^{\mathrm{b}}$} \\
\hline Food $^{\mathrm{c}}$ & $12.5 \%$ & $16.0 \%$ & $14.2 \%$ & $14.4 \%$ & $12.3 \%$ \\
\hline Housing & $32.9 \%$ & $40.5 \%$ & $38.2 \%$ & $35.5 \%$ & $35.5 \%$ \\
\hline Apparel and services & $3.3 \%$ & $3.1 \%$ & $3.2 \%$ & $3.3 \%$ & $2.7 \%$ \\
\hline Transportation & $17.0 \%$ & $14.5 \%$ & $16.4 \%$ & $16.8 \%$ & $19.3 \%$ \\
\hline Vehicle purchases (net outlay) & $7.1 \%$ & $4.5 \%$ & $6.0 \%$ & $6.3 \%$ & $8.6 \%$ \\
\hline Gasoline and motor oil & $3.7 \%$ & $3.8 \%$ & $4.2 \%$ & $4.5 \%$ & $4.7 \%$ \\
\hline Other vehicle expenditures & $4.9 \%$ & $5.4 \%$ & $5.3 \%$ & $5.3 \%$ & $5.2 \%$ \\
\hline Public transportation & $1.2 \%$ & $0.8 \%$ & $0.9 \%$ & $0.7 \%$ & $0.9 \%$ \\
\hline Health care & $7.8 \%$ & $7.3 \%$ & $9.7 \%$ & $9.3 \%$ & $8.8 \%$ \\
\hline Entertainment & $5.1 \%$ & $4.9 \%$ & $5.1 \%$ & $5.0 \%$ & $4.9 \%$ \\
\hline Personal Insurance \& pensions & $11.3 \%$ & $2.0 \%$ & $3.8 \%$ & $6.6 \%$ & $7.6 \%$ \\
\hline Others $^{\mathrm{d}}$ & $9.1 \%$ & $10.8 \%$ & $8.8 \%$ & $8.5 \%$ & $7.9 \%$ \\
\hline Households $^{\mathrm{e}}$ (thousands) & 128,437 & 17,946 & 23,162 & 12,536 & 10,914 \\
\hline Percentage of households & $100.0 \%$ & $14.0 \%$ & $18.0 \%$ & $9.8 \%$ & $8.5 \%$ \\
\hline \multirow[t]{3}{*}{ Average number of vehicles in $\mathrm{HH}$} & 1.9 & 0.8 & 1.3 & 1.7 & 1.9 \\
\hline & \multicolumn{5}{|c|}{ Income before taxes } \\
\hline & $\begin{array}{r}\$ 50,000- \\
\$ 69,999 \\
\end{array}$ & $\begin{array}{r}\$ 70,000- \\
\$ 99,999 \\
\end{array}$ & $\begin{array}{r}\$ 100,000- \\
\$ 149,999 \\
\end{array}$ & $\begin{array}{r}\$ 150,000- \\
\$ 199,999 \\
\end{array}$ & $\begin{array}{r}\$ 200,000 \\
\text { and over }\end{array}$ \\
\hline \multirow[t]{2}{*}{ Total expenditures } & $\$ 51,394$ & $\$ 66,008$ & $\$ 86,140$ & $\$ 113,272$ & $\$ 158,731$ \\
\hline & \multicolumn{5}{|c|}{ Percentage of total expenditures ${ }^{\mathrm{b}}$} \\
\hline Food $^{\mathrm{C}}$ & $12.8 \%$ & $12.9 \%$ & $12.0 \%$ & $11.9 \%$ & $9.6 \%$ \\
\hline Housing & $33.5 \%$ & $31.6 \%$ & $30.2 \%$ & $29.3 \%$ & $30.2 \%$ \\
\hline Apparel and services & $2.8 \%$ & $3.2 \%$ & $3.0 \%$ & $3.7 \%$ & $4.4 \%$ \\
\hline Transportation & $19.1 \%$ & $18.0 \%$ & $18.3 \%$ & $14.9 \%$ & $13.9 \%$ \\
\hline Vehicle purchases (net outlay) & $8.1 \%$ & $8.0 \%$ & $8.4 \%$ & $5.9 \%$ & $6.0 \%$ \\
\hline Gasoline and motor oil & $4.5 \%$ & $4.0 \%$ & $3.6 \%$ & $2.8 \%$ & $2.1 \%$ \\
\hline Other vehicle expenditures & $5.5 \%$ & $5.0 \%$ & $5.1 \%$ & $4.7 \%$ & $3.6 \%$ \\
\hline Public transportation & $1.0 \%$ & $1.0 \%$ & $1.2 \%$ & $1.5 \%$ & $2.2 \%$ \\
\hline Health care & $8.6 \%$ & $8.2 \%$ & $7.3 \%$ & $6.5 \%$ & $5.2 \%$ \\
\hline Entertainment & $4.7 \%$ & $4.9 \%$ & $5.2 \%$ & $5.8 \%$ & $5.2 \%$ \\
\hline Personal Insurance \& pensions & $9.9 \%$ & $12.1 \%$ & $14.0 \%$ & $16.4 \%$ & $18.5 \%$ \\
\hline Others $^{\mathrm{d}}$ & $7.7 \%$ & $8.1 \%$ & $9.2 \%$ & $10.5 \%$ & $12.0 \%$ \\
\hline Households $^{\mathrm{e}}$ (thousands) & 18,112 & 18,168 & 15,616 & 6,020 & 5,964 \\
\hline Percentage of households & $14.1 \%$ & $14.1 \%$ & $12.2 \%$ & $4.7 \%$ & $4.6 \%$ \\
\hline Average number of vehicles in $\mathrm{HH}$ & 2.1 & 2.4 & 2.7 & 2.8 & 2.8 \\
\hline
\end{tabular}

\section{Source:}

U.S. Department of Labor, Bureau of Labor Statistics, website: www.bls.gov/cex, July 2017. (Additional resources: www.bls.gov)

${ }^{a}$ Public assistance monies are included in reported income. Data for those reporting incomes.

${ }^{\mathrm{b}}$ Percentages may not sum to totals due to rounding.

${ }^{\mathrm{c}}$ Includes alcoholic beverages.

${ }^{\mathrm{d}}$ Includes personal care, reading, education, tobacco and smoking supplies, cash contributions, and miscellaneous items.

e The term household refers to a "consumer unit," which is defined differently than households on Error! Reference source not found.. 
The average amount of money that a household spends in a year has changed very little between 1985 and 2015 in constant dollar terms. Expenditures on transportation were 19.4\% of the total in 1985, but were 17.0\% in 2015. Vehicle purchases made up more than one-third of transportation expenditures in 2015, while gas and oil were $22 \%$.

Table 10.2

Annual Household Expenditures for Transportation, 1985-2015 (constant 2015 dollars)

\begin{tabular}{|c|c|c|c|c|c|c|c|}
\hline \multirow[b]{2}{*}{ Year } & \multicolumn{5}{|c|}{ Transportation expenditures } & \multirow{2}{*}{$\begin{array}{c}\text { Average } \\
\text { annual } \\
\text { household } \\
\text { expenditures }\end{array}$} & \multirow{2}{*}{$\begin{array}{c}\text { Transportation } \\
\text { share of } \\
\text { annual } \\
\text { expenditures }\end{array}$} \\
\hline & $\begin{array}{c}\text { Vehicle } \\
\text { purchases }\end{array}$ & $\begin{array}{c}\text { Gas \& } \\
\text { Oil }\end{array}$ & $\begin{array}{c}\text { Other } \\
\text { vehicle } \\
\text { expenses }^{\mathrm{a}}\end{array}$ & $\begin{array}{c}\text { Public } \\
\text { transportation }\end{array}$ & $\begin{array}{c}\text { Total } \\
\text { transportation }\end{array}$ & & \\
\hline 1985 & 4,544 & 2,304 & 2,811 & 584 & 10,241 & 52,813 & $19.4 \%$ \\
\hline 1986 & 5,056 & 1,996 & 2,971 & 541 & 10,564 & 52,851 & $20.0 \%$ \\
\hline 1987 & 4,206 & 1,838 & 2,986 & 540 & 9,572 & 51,693 & $18.5 \%$ \\
\hline 1988 & 4,784 & 1,871 & 3,111 & 533 & 10,298 & 52,871 & $19.5 \%$ \\
\hline 1989 & 4,488 & 1,887 & 3,181 & 531 & 10,087 & 54,137 & $18.6 \%$ \\
\hline 1990 & 3,937 & 1,911 & 3,025 & 549 & 9,423 & 52,702 & $17.9 \%$ \\
\hline 1991 & 3,748 & 1,737 & 3,091 & 536 & 9,110 & 53,054 & $17.2 \%$ \\
\hline 1992 & 3,661 & 1,644 & 3,051 & 485 & 8,839 & 51,571 & $17.1 \%$ \\
\hline 1993 & 3,796 & 1,603 & 3,097 & 522 & 9,015 & 51,563 & $17.5 \%$ \\
\hline 1994 & 4,323 & 1,583 & 3,181 & 629 & 9,717 & 52,361 & $18.6 \%$ \\
\hline 1995 & 4,162 & 1,577 & 3,208 & 571 & 9,520 & 52,251 & $18.2 \%$ \\
\hline 1996 & 4,414 & 1,671 & 3,243 & 647 & 9,973 & 53,765 & $18.5 \%$ \\
\hline 1997 & 4,218 & 1,639 & 3,414 & 576 & 9,848 & 53,378 & $18.5 \%$ \\
\hline 1998 & 4,425 & 1,498 & 3,323 & 621 & 9,866 & 54,179 & $18.2 \%$ \\
\hline 1999 & 4,847 & 1,524 & 3,322 & 580 & 10,275 & 55,688 & $18.5 \%$ \\
\hline 2000 & 4,771 & 1,811 & 3,228 & 607 & 10,417 & 55,384 & $18.8 \%$ \\
\hline 2001 & 5,056 & 1,726 & 3,275 & 542 & 10,598 & 55,400 & $19.1 \%$ \\
\hline 2002 & 4,977 & 1,650 & 3,360 & 532 & 10,519 & 56,069 & $18.8 \%$ \\
\hline 2003 & 4,986 & 1,743 & 3,112 & 515 & 10,358 & 55,058 & $18.8 \%$ \\
\hline 2004 & 4,262 & 2,005 & 2,967 & 553 & 9,788 & 54,449 & $18.0 \%$ \\
\hline 2005 & 4,301 & 2,443 & 2,839 & 544 & 10,126 & 56,322 & $18.0 \%$ \\
\hline 2006 & 4,022 & 2,618 & 2,769 & 594 & 10,003 & 56,901 & $17.6 \%$ \\
\hline 2007 & 3,708 & 2,725 & 2,963 & 615 & 10,011 & 56,742 & $17.6 \%$ \\
\hline 2008 & 3,033 & 2,989 & 2,885 & 565 & 9,472 & 55,578 & $17.0 \%$ \\
\hline 2009 & 2,935 & 2,194 & 2,802 & 529 & 8,460 & 54,208 & $15.6 \%$ \\
\hline 2010 & 2,813 & 2,317 & 2,678 & 536 & 8,345 & 52,292 & $16.0 \%$ \\
\hline 2011 & 2,812 & 2,798 & 2,586 & 544 & 8,738 & 52,374 & $16.7 \%$ \\
\hline 2012 & 3,314 & 2,845 & 2,571 & 560 & 9,289 & 53,105 & $17.5 \%$ \\
\hline 2013 & 3,328 & 2,657 & 2,629 & 546 & 9,161 & 51,991 & $17.6 \%$ \\
\hline 2014 & 3,305 & 2,471 & 2,726 & 582 & 9,084 & 53,558 & $17.0 \%$ \\
\hline 2015 & 3,997 & 2,090 & 2,756 & 661 & 9,503 & 55,978 & $17.0 \%$ \\
\hline
\end{tabular}

Source:

U.S. Department of Labor, Bureau of Labor Statistics, Consumer Expenditure Survey, www.bls.gov/cex, July 2017. (Additional resources: www.bls.gov)

a Other vehicle expenses include vehicle finance charges, maintenance and repairs, insurance, licenses, and other vehicle charges. 
The United States prices are the lowest of these listed countries. Those in France, the United Kingdom, and Germany paid, on average, over \$5 per gallon in 2016. Data for China and India have been discontinued by the International Energy Agency.

Table 10.3

Gasoline Prices $^{\text {a }}$ for Selected Countries, 1990-2016

\begin{tabular}{|c|c|c|c|c|c|c|c|}
\hline & \multicolumn{6}{|c|}{ Current dollars per gallon } & $\begin{array}{c}\text { Average annual } \\
\text { percentage change }\end{array}$ \\
\hline & 1990 & 1995 & 2000 & 2005 & 2010 & 2016 & 1990-2016 \\
\hline China & $\mathrm{b}$ & 1.03 & $\mathrm{~b}$ & 1.70 & 3.71 & $\mathrm{~b}$ & $\mathrm{~b}$ \\
\hline Japan & 3.16 & 4.43 & 3.65 & 4.28 & 5.73 & 4.53 & $1.5 \%$ \\
\hline India & b & b & $\mathrm{b}$ & 3.71 & 4.29 & $\mathrm{~b}$ & $\mathrm{~b}$ \\
\hline Korea & $\mathrm{b}$ & $\mathrm{b}$ & b & 5.28 & 5.60 & 4.80 & $\mathrm{~b}$ \\
\hline France $^{c}$ & 3.63 & 4.26 & 3.80 & 5.46 & 6.74 & 5.47 & $1.7 \%$ \\
\hline United Kingdom ${ }^{c}$ & 2.82 & 3.21 & 4.58 & 5.97 & 6.83 & 5.56 & $2.8 \%$ \\
\hline Germany $^{c}$ & 2.65 & 3.96 & 3.45 & 5.75 & 7.11 & 5.47 & $2.9 \%$ \\
\hline Canada & 1.87 & 1.53 & 1.86 & 2.89 & 3.79 & 2.94 & $1.8 \%$ \\
\hline \multirow[t]{3}{*}{ United States ${ }^{\mathrm{d}}$} & 1.16 & 1.15 & 1.51 & 2.27 & 2.78 & 2.15 & $2.5 \%$ \\
\hline & \multicolumn{6}{|c|}{ Constant 2016 dollars ${ }^{\mathrm{e}}$ per gallon } & $\begin{array}{c}\text { Average annual } \\
\text { percentage change }\end{array}$ \\
\hline & 1990 & 1995 & 2000 & 2005 & 2010 & 2016 & 1990-2016 \\
\hline China & $\mathrm{b}$ & 1.03 & $\mathrm{~b}$ & 2.09 & 4.08 & $\mathrm{~b}$ & $\mathrm{~b}$ \\
\hline Japan & 5.80 & 6.98 & 5.09 & 5.26 & 6.31 & 4.53 & $-1.0 \%$ \\
\hline India & $\mathrm{b}$ & b & $\mathrm{b}$ & 4.56 & 4.73 & $\mathrm{~b}$ & $\mathrm{~b}$ \\
\hline Korea & $\mathrm{b}$ & $\mathrm{b}$ & $\mathrm{b}$ & 6.49 & 6.16 & 4.80 & b \\
\hline France $^{c}$ & 6.67 & 6.71 & 5.30 & 6.71 & 7.42 & 5.47 & $-0.8 \%$ \\
\hline United Kingdomc ${ }^{c}$ & 5.18 & 5.06 & 6.38 & 7.33 & 7.52 & 5.56 & $0.3 \%$ \\
\hline Germany $^{\mathrm{c}}$ & 4.87 & 6.24 & 4.81 & 7.07 & 7.82 & 5.47 & $0.5 \%$ \\
\hline Canada & 3.43 & 2.41 & 2.59 & 3.55 & 4.19 & 2.94 & $-0.6 \%$ \\
\hline United States ${ }^{\mathrm{d}}$ & 2.13 & 1.81 & 2.10 & 2.79 & 3.06 & 2.15 & $0.0 \%$ \\
\hline
\end{tabular}

Note: Comparisons between prices and price trends in different countries require care. They are of limited validity because of fluctuations in exchange rates; differences in product quality, marketing practices, and market structures; and the extent to which the standard categories of sales are representative of total national sales for a given period.

\section{Source:}

International Energy Agency, Energy Prices and Taxes, First Quarter, 2017, Paris, France, 2017. (Additional resources: www.iea.org)

${ }^{a}$ Prices represent the retail prices (including taxes) for regular unleaded gasoline, except for France, Germany and the United Kingdom which are premium unleaded gasoline.

${ }^{\mathrm{b}}$ Data are not available.

c Premium gasoline.

d These estimates are international comparisons only and do not necessarily correspond to gasoline price estimates in other sections of the book.

e Adjusted by the U.S. Consumer Price Inflation Index. 
Of these selected countries, the United Kingdom had the highest diesel fuel price average in 2016, while the United States had the lowest. All of the countries listed except the United States had diesel prices over \$3 per gallon in 2016.

Table 10.4

Diesel Fuel Prices ${ }^{\text {a }}$ for Selected Countries, 1990-2016

\begin{tabular}{|c|c|c|c|c|c|c|c|}
\hline & \multicolumn{6}{|c|}{ Current dollars per gallon } & $\begin{array}{l}\text { Average annual } \\
\text { percentage } \\
\text { change }\end{array}$ \\
\hline & 1990 & 2000 & 2005 & 2010 & 2015 & 2016 & 1990-2016 \\
\hline China & $\mathrm{b}$ & $\mathrm{b}$ & 1.69 & 3.65 & $\mathrm{~b}$ & $\mathrm{~b}$ & $\mathrm{~b}$ \\
\hline Japan & 1.75 & 2.85 & 3.44 & 4.86 & 3.66 & 3.55 & $2.8 \%$ \\
\hline Korea & $\mathrm{b}$ & 2.05 & 3.98 & 4.92 & 4.35 & 3.86 & $\mathrm{~b}$ \\
\hline France & 1.78 & 2.95 & 4.81 & 5.74 & 4.83 & 4.63 & $3.7 \%$ \\
\hline United Kingdom & 2.04 & 4.66 & 6.25 & 6.97 & 6.65 & 5.63 & $4.0 \%$ \\
\hline Germany & 2.72 & 2.79 & 5.01 & 6.15 & 4.99 & 4.61 & $2.0 \%$ \\
\hline \multirow[t]{3}{*}{ United States $^{\mathrm{c}}$} & 0.99 & 1.50 & 2.40 & 2.99 & 2.71 & 2.31 & $3.3 \%$ \\
\hline & \multicolumn{6}{|c|}{ Constant 2016 dollars ${ }^{\mathrm{d}}$ per gallon } & $\begin{array}{c}\text { Average annual } \\
\text { percentage } \\
\text { change }\end{array}$ \\
\hline & 1990 & 2000 & 2005 & 2010 & 2015 & 2016 & 1990-2016 \\
\hline China & $\mathrm{b}$ & $\mathrm{b}$ & 2.08 & 4.02 & $\mathrm{~b}$ & $\mathrm{~b}$ & $\mathrm{~b}$ \\
\hline Japan & 3.21 & 3.98 & 4.23 & 5.36 & 3.71 & 3.55 & $0.4 \%$ \\
\hline Korea & $\mathrm{b}$ & 2.86 & 4.89 & 5.42 & 4.41 & 3.86 & $\mathrm{~b}$ \\
\hline France & 3.27 & 4.11 & 5.91 & 6.32 & 4.89 & 4.63 & $1.3 \%$ \\
\hline United Kingdom & 3.75 & 6.49 & 7.69 & 7.68 & 6.73 & 5.63 & $1.6 \%$ \\
\hline Germany & 4.99 & 3.89 & 6.15 & 6.77 & 5.06 & 4.61 & $-0.3 \%$ \\
\hline United States ${ }^{c}$ & 1.82 & 2.08 & 2.94 & 3.30 & 2.75 & 2.31 & $0.9 \%$ \\
\hline
\end{tabular}

Note: Comparisons between prices and price trends in different countries require care. They are of limited validity because of fluctuations in exchange rates; differences in product quality, marketing practices, and market structures; and the extent to which the standard categories of sales are representative of total national sales for a given period.

\section{Source:}

International Energy Agency, Energy Prices and Taxes, First Quarter, 2017, Paris, France, 2017. (Additional resources: www.iea.org)

${ }^{a}$ Prices represent the retail prices (including taxes) for car diesel fuel for non-commercial (household) use.

${ }^{\mathrm{b}}$ Data are not available.

c These estimates are for international comparisons only and do not necessarily correspond to gasoline price estimates in other sections of the book.

d Adjusted by the U.S. Consumer Price Inflation Index. 
In 2016 over sixty percent of the cost of gasoline in France, Germany, and the United Kingdom went for taxes. Of the listed countries, the United States has the lowest percentage of taxes.

Figure 10.2. Gasoline Prices for Selected Countries, 1990 and 2016

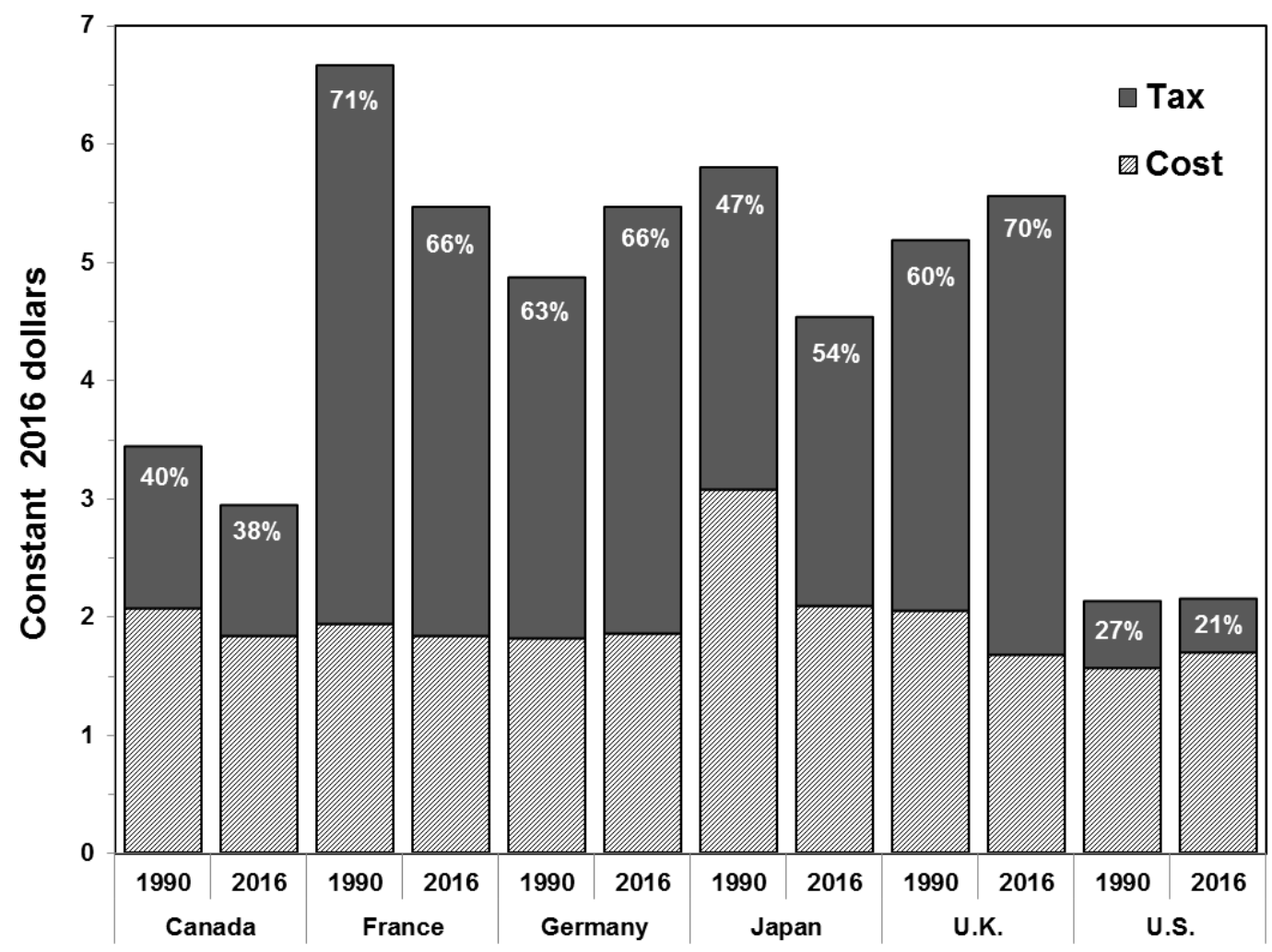

\section{Source:}

Table 10.3 and International Energy Agency, Energy Prices \& Taxes, First Quarter, 2017, Paris, France, 2017. (Additional resources: www.iea.org) 
Diesel fuel is taxed heavily in the European countries shown here. The U.S. diesel fuel tax share is the lowest of the listed countries.

Figure 10.3. Diesel Prices for Selected Countries, 1990 and 2016

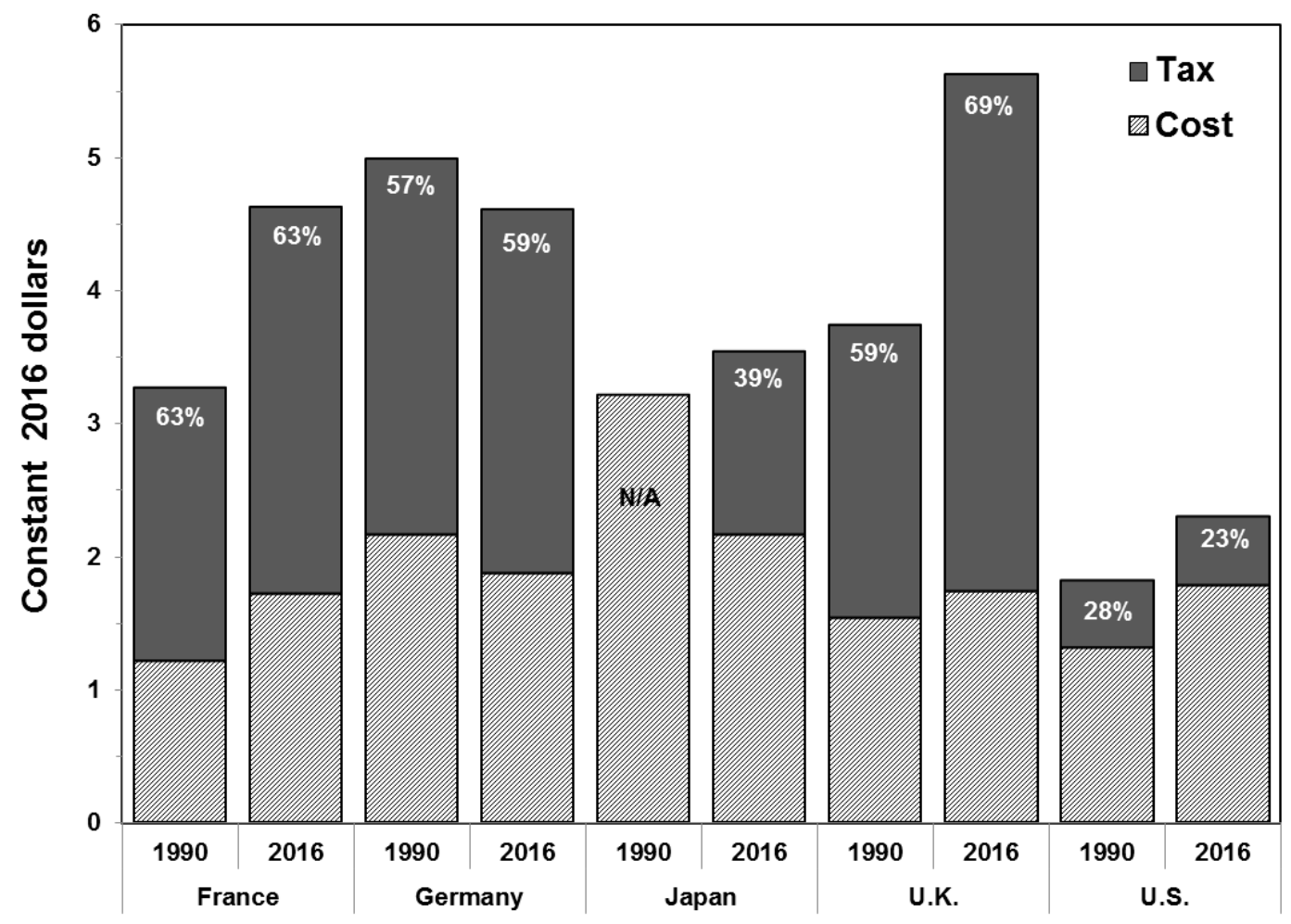

Note: Data for Canada are not available.

\section{Source:}

Table 10.4 and International Energy Agency, Energy Prices \& Taxes, First Quarter, 2017, Paris, France, 2017. (Additional resources: www.iea.org) 
Though the cost of crude oil certainly influences the price of gasoline, it is not the only factor which determines the price at the pump. Processing cost, transportation cost, and taxes also play a major part of the cost of a gallon of gasoline. The average price of a barrel of crude oil (in constant 2016 dollars) increased by 3\% from 2000 to 2016, while the average price of a gallon of gasoline increased $1 \%$ in this same time period.

Table 10.5

Prices for a Barrel of Crude Oil and a Gallon of Gasoline, 1978-2016

\begin{tabular}{|c|c|c|c|c|c|}
\hline \multirow[b]{2}{*}{ Year } & \multicolumn{2}{|c|}{$\begin{array}{c}\text { Crude oil }{ }^{\mathrm{a}} \\
\text { (dollars per barrel) }^{\text {(d) }}\end{array}$} & \multicolumn{2}{|c|}{$\begin{array}{c}\text { Gasoline }^{\mathrm{b}} \\
\text { (dollars per gallon) }\end{array}$} & \multirow{2}{*}{$\begin{array}{l}\text { Ratio of } \\
\text { gasoline price to } \\
\text { crude oil price }\end{array}$} \\
\hline & Current & Constant $2016^{\mathrm{c}}$ & Current & Constant $2016^{\mathrm{c}}$ & \\
\hline 1978 & 12.5 & 45.9 & 0.65 & 2.4 & 2.2 \\
\hline 1980 & 28.1 & 81.8 & 1.22 & 3.6 & 1.8 \\
\hline 1985 & 26.8 & 59.7 & 1.20 & 2.7 & 1.9 \\
\hline 1990 & 22.2 & 40.8 & 1.22 & 2.2 & 2.3 \\
\hline 1991 & 19.1 & 33.6 & 1.20 & 2.1 & 2.6 \\
\hline 1992 & 18.4 & 31.5 & 1.19 & 2.0 & 2.7 \\
\hline 1993 & 16.4 & 27.3 & 1.17 & 1.9 & 3.0 \\
\hline 1994 & 15.6 & 25.2 & 1.17 & 1.9 & 3.2 \\
\hline 1995 & 17.2 & 27.1 & 1.21 & 1.9 & 2.9 \\
\hline 1996 & 20.7 & 31.7 & 1.29 & 2.0 & 2.6 \\
\hline 1997 & 19.0 & 28.5 & 1.29 & 1.9 & 2.8 \\
\hline 1998 & 12.5 & 18.4 & 1.12 & 1.6 & 3.7 \\
\hline 1999 & 17.5 & 25.2 & 1.22 & 1.8 & 2.9 \\
\hline 2000 & 28.3 & 39.4 & 1.56 & 2.2 & 2.3 \\
\hline 2001 & 23.0 & 31.1 & 1.53 & 2.1 & 2.8 \\
\hline 2002 & 24.1 & 32.1 & 1.44 & 1.9 & 2.5 \\
\hline 2003 & 28.5 & 37.2 & 1.64 & 2.1 & 2.4 \\
\hline 2004 & 37.0 & 47.0 & 1.92 & 2.4 & 2.2 \\
\hline 2005 & 50.2 & 61.7 & 2.34 & 2.9 & 2.0 \\
\hline 2006 & 60.2 & 71.7 & 2.64 & 3.1 & 1.8 \\
\hline 2007 & 67.9 & 78.7 & 2.85 & 3.3 & 1.8 \\
\hline 2008 & 94.7 & 105.6 & 3.32 & 3.7 & 1.5 \\
\hline 2009 & 59.3 & 66.3 & 2.40 & 2.7 & 1.7 \\
\hline 2010 & 76.7 & 84.4 & 2.84 & 3.1 & 1.6 \\
\hline 2011 & 101.9 & 108.7 & 3.58 & 3.8 & 1.5 \\
\hline 2012 & 100.9 & 105.5 & 3.70 & 3.9 & 1.5 \\
\hline 2013 & 100.5 & 103.5 & 3.58 & 3.7 & 1.5 \\
\hline 2014 & 92.0 & 93.3 & 3.43 & 3.5 & 1.6 \\
\hline 2015 & 48.4 & 49.0 & 2.51 & 2.5 & 2.2 \\
\hline 2016 & 40.7 & 40.7 & 2.20 & 2.2 & 2.3 \\
\hline \multicolumn{6}{|c|}{ Average annual percentage change } \\
\hline 1978-2016 & $3.2 \%$ & $-0.3 \%$ & $3.3 \%$ & $-0.2 \%$ & \\
\hline $2006-2016$ & $-3.8 \%$ & $-5.5 \%$ & $-1.8 \%$ & $-3.4 \%$ & \\
\hline
\end{tabular}

\section{Sources:}

Crude oil - U.S. Department of Energy, Energy Information Administration, Monthly Energy Review, July 2017, Washington, DC, Table 9.1.

Gasoline - U.S. Department of Energy, Energy Information Administration, Monthly Energy Review, July 2017, Washington, DC, Table 9.4. (Additional resources: www.eia.doe.gov)

${ }^{a}$ Refiner acquisition cost of composite (domestic and imported) crude oil.

${ }^{\mathrm{b}}$ Average for all types. These prices were collected from a sample of service stations in 85 urban areas selected to represent all urban consumers. Urban consumers make up about $80 \%$ of the total U.S. population.

${ }^{c}$ Adjusted by the Consumer Price Inflation Index. 
The price of diesel fuel was lower than gasoline in constant dollars prior to 2005 but since that time the price of diesel fuel has increased to become higher than gasoline. Prices for both fuels declined substantially since 2015.

Table 10.6

Retail Prices for Motor Fuel, 1978-2016 (cents per gallon, including tax)

\begin{tabular}{|c|c|c|c|c|}
\hline \multirow[b]{2}{*}{ Year } & \multicolumn{2}{|c|}{ Diesel fuel $^{\mathrm{a}}$} & \multicolumn{2}{|c|}{ Average for all gasoline types } \\
\hline & Current & $\begin{array}{c}\text { Constant } \\
2016^{c} \\
\end{array}$ & Current & $\begin{array}{c}\text { Constant } \\
2016^{c}\end{array}$ \\
\hline 1978 & d & $\mathrm{d}$ & 65 & 240 \\
\hline 1980 & 101 & 294 & 122 & 356 \\
\hline 1985 & 122 & 272 & 120 & 267 \\
\hline 1986 & 94 & 206 & 93 & 204 \\
\hline 1987 & 96 & 203 & 96 & 202 \\
\hline 1988 & 95 & 193 & 96 & 195 \\
\hline 1989 & 102 & 197 & 106 & 205 \\
\hline 1990 & 107 & 196 & 122 & 223 \\
\hline 1991 & 91 & 160 & 120 & 211 \\
\hline 1992 & 106 & 181 & 119 & 204 \\
\hline 1993 & 98 & 163 & 117 & 195 \\
\hline 1994 & 111 & 180 & 117 & 190 \\
\hline 1995 & 111 & 175 & 121 & 190 \\
\hline 1996 & 124 & 189 & 129 & 197 \\
\hline 1997 & 120 & 179 & 129 & 193 \\
\hline 1998 & 104 & 154 & 112 & 164 \\
\hline 1999 & 112 & 162 & 122 & 176 \\
\hline 2000 & 149 & 208 & 156 & 218 \\
\hline 2001 & 140 & 190 & 153 & 207 \\
\hline 2002 & 132 & 176 & 144 & 192 \\
\hline 2003 & 151 & 197 & 164 & 214 \\
\hline 2004 & 181 & 230 & 192 & 244 \\
\hline 2005 & 240 & 295 & 234 & 287 \\
\hline 2006 & 271 & 322 & 264 & 314 \\
\hline 2007 & 289 & 334 & 285 & 330 \\
\hline 2008 & 380 & 424 & 332 & 370 \\
\hline 2009 & 247 & 276 & 240 & 269 \\
\hline 2010 & 299 & 330 & 284 & 313 \\
\hline 2011 & 384 & 410 & 358 & 382 \\
\hline 2012 & 397 & 415 & 370 & 386 \\
\hline 2013 & 392 & 404 & 358 & 369 \\
\hline 2014 & 383 & 388 & 343 & 347 \\
\hline 2015 & 271 & 274 & 251 & 254 \\
\hline 2016 & 230 & 230 & 220 & 220 \\
\hline \multicolumn{5}{|c|}{ Average annual percentage change } \\
\hline 1978-2016 & $2.3 \%{ }^{\mathrm{e}}$ & $-0.7 \%^{\mathrm{c}}$ & $3.3 \%$ & $-0.2 \%$ \\
\hline 2006-2016 & $-1.6 \%$ & $-3.3 \%$ & $-1.8 \%$ & $-3.5 \%$ \\
\hline
\end{tabular}

\section{Sources:}

Gasoline - U.S. Department of Energy, Energy Information Administration, Monthly Energy Review, July 2017, Washington, DC, Table 9.4.

Diesel - U.S. Department of Energy, Energy Information Administration, International Energy Annual 2004, Washington, DC, June 2004, Table 7.2. 2005-2016 data from EIA website. (Additional resources: www.eia.doe.gov)

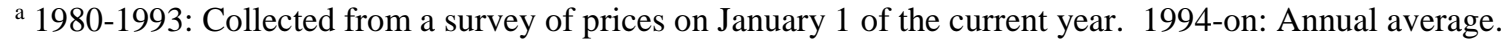

b These prices were collected from a sample of service stations in 85 urban areas selected to represent all urban consumers. Urban consumers make up about 80 percent of the total U.S. population.

${ }^{c}$ Adjusted by the Consumer Price Inflation Index.

${ }^{\mathrm{d}}$ Data are not available.

e Average annual percentage change is from the earliest year possible to 2016. 
Major oil price shocks have disrupted world energy markets five times in the past 30 years (1973-74, 1979-80, 1990-91, 1999-2000, 2008). Most of the oil price shocks were followed by an economic recession in the United States.

Figure 10.4. Oil Price and Economic Growth, 1970-2016

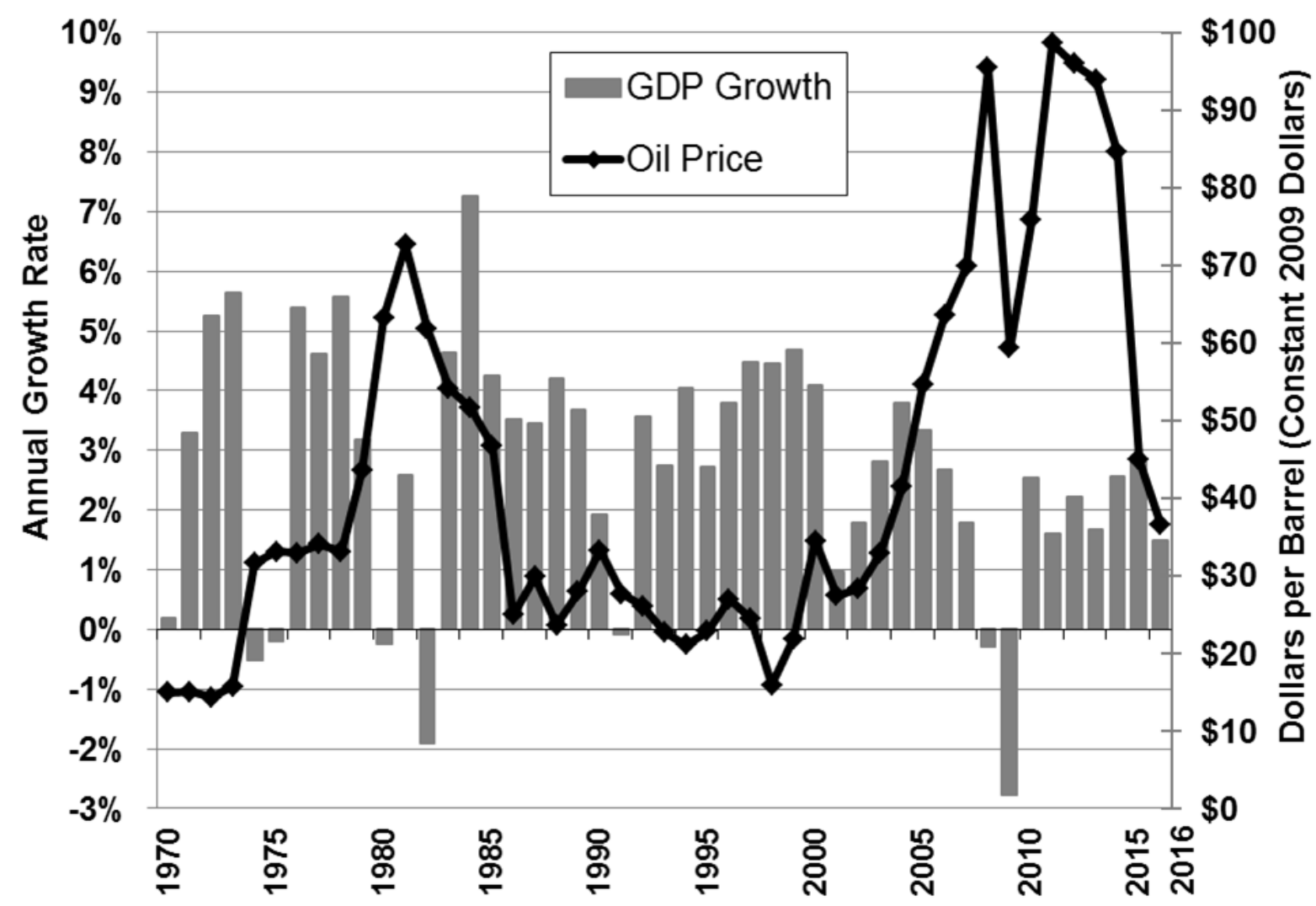

Source:

Greene, D.L. and N. I. Tishchishyna, Costs of Oil Dependence: A 2000 Update, Oak Ridge National Laboratory, ORNL/TM-2000/152, Oak Ridge, TN, 2000, and data updates, 2017. (Additional resources: cta.ornl.gov/cta/publications.shtml) 
The United States has long recognized the problem of oil dependence and the economic problems that arise from it. Greene, Lee and Hopson define oil dependence as a combination of four factors: (1) a noncompetitive world oil market strongly influenced by the Organization of the Petroleum Exporting Countries (OPEC) cartel, (2) high levels of U.S. imports, (3) the importance of oil to the U.S. economy, and (4) the lack of economical and readily available substitutes for oil. The most recent study shows that the U.S. economy suffered the greatest losses in 2008 when wealth transfer and gross domestic product (GDP) losses (combined) amounted to nearly half a trillion dollars. However, when comparing oil dependence to the size of the economy, the year 1980 is the highest. Low oil prices in 2009-2010 and 2013-2014 caused total dependence cost to drop; in 2015, the total cost was about $\$ 104$ billion.

Figure 10.5. Costs of Oil Dependence to the U.S. Economy, 1970-2015

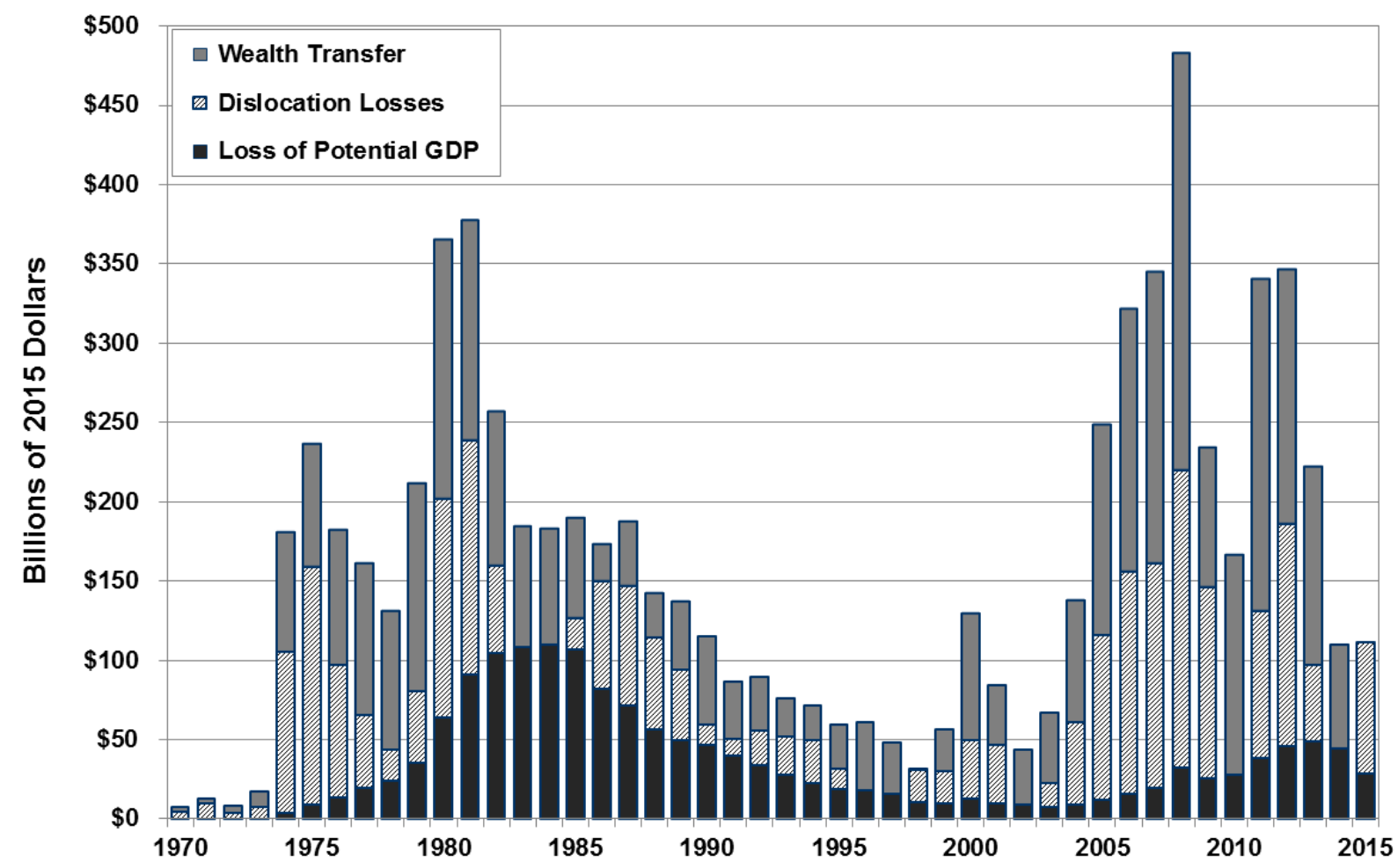

Notes:

Wealth Transfer is the product of total U.S. oil imports and the difference between the actual market price of oil (influenced by market power) and what the price would have been in a competitive market.

Dislocation Losses are temporary reductions in GDP as a result of oil price shocks.

Loss of Potential Gross Domestic Product (GDP) results because a basic resource used by the economy to produce output has become more expensive. As a consequence, with the same endowment of labor, capital, and other resources, our economy cannot produce quite as much as it could have at a lower oil price.

\section{Source:}

Greene, David L., Roderick Lee, and Janet L. Hopson, “OPEC and the Costs to the U.S. Economy of Oil Dependence: 1970-2010,” Oak Ridge National Laboratory Memorandum, 2011, and updates from the ORNL Transportation Energy Evolution Modeling Team. 
The fuel prices shown here are refiner sales prices of transportation fuels to end users, excluding tax. Sales to end users are those made directly to the ultimate consumer, including bulk consumers. Bulk sales to utility, industrial, and commercial accounts previously included in the wholesale category are now counted as sales to end users.

Both propane and diesel prices fell drastically in 2015 and diesel continued to fall in 2016.

Table 10.7

Refiner Sales Prices for Propane and No. 2 Diesel, 1978-2016 (cents per gallon, excluding tax)

\begin{tabular}{|c|c|c|c|c|}
\hline \multirow[b]{3}{*}{ Year } & \multicolumn{2}{|c|}{ Propane $^{\mathrm{a}}$} & \multicolumn{2}{|c|}{ No 2. diesel fuel } \\
\hline & & Constant & & Constant \\
\hline & Current & $2016^{\mathrm{b}}$ & Current & $2016^{\mathrm{b}}$ \\
\hline 1978 & 33.5 & 123.3 & 37.7 & 138.8 \\
\hline 1980 & 48.2 & 140.4 & 81.8 & 238.3 \\
\hline 1985 & 71.7 & 159.9 & 78.9 & 176.0 \\
\hline 1986 & 74.5 & 163.1 & 47.8 & 104.7 \\
\hline 1987 & 70.1 & 148.1 & 55.1 & 116.4 \\
\hline 1988 & 71.4 & 144.9 & 50.0 & 101.4 \\
\hline 1989 & 61.5 & 119.0 & 58.5 & 113.2 \\
\hline 1990 & 74.5 & 136.8 & 72.5 & 133.1 \\
\hline 1991 & 73.0 & 128.6 & 64.8 & 114.2 \\
\hline 1992 & 64.3 & 110.0 & 61.9 & 105.9 \\
\hline 1993 & 67.3 & 111.8 & 60.2 & 100.0 \\
\hline 1994 & 53.0 & 85.8 & 55.4 & 89.7 \\
\hline 1995 & 49.2 & 77.5 & 56.0 & 88.2 \\
\hline 1996 & 60.5 & 92.5 & 68.1 & 104.2 \\
\hline 1997 & 55.2 & 82.5 & 64.2 & 96.0 \\
\hline 1998 & 40.5 & 59.6 & 49.4 & 72.7 \\
\hline 1999 & 45.8 & 66.0 & 58.4 & 84.1 \\
\hline 2000 & 60.3 & 84.0 & 93.5 & 130.3 \\
\hline 2001 & 50.6 & 68.6 & 84.2 & 114.1 \\
\hline 2002 & 41.9 & 55.9 & 76.2 & 101.7 \\
\hline 2003 & 57.7 & 75.3 & 94.4 & 123.1 \\
\hline 2004 & 83.9 & 106.6 & 124.3 & 157.9 \\
\hline 2005 & 108.9 & 133.8 & 178.6 & 219.5 \\
\hline 2006 & 135.8 & 161.7 & 209.6 & 249.5 \\
\hline 2007 & 148.9 & 172.4 & 226.7 & 262.4 \\
\hline 2008 & 189.2 & 210.9 & 315.0 & 351.1 \\
\hline 2009 & 122.0 & 136.5 & 183.4 & 205.2 \\
\hline 2010 & 148.1 & 163.0 & 213.4 & 234.9 \\
\hline 2011 & 170.9 & 182.3 & 311.7 & 332.6 \\
\hline 2012 & 113.9 & 119.1 & 320.2 & 334.7 \\
\hline 2013 & 102.8 & 105.9 & 312.2 & 321.6 \\
\hline 2014 & 109.7 & 111.2 & 292.3 & 296.3 \\
\hline 2015 & 48.1 & 48.7 & 181.9 & 184.2 \\
\hline 2016 & 49.8 & 49.8 & 151.1 & 151.1 \\
\hline \multicolumn{5}{|c|}{ Average annual percentage change } \\
\hline 1978-2016 & $1.0 \%$ & $-2.4 \%$ & $3.7 \%$ & $0.2 \%$ \\
\hline 2006-2016 & $-9.5 \%$ & $-11.1 \%$ & $-3.2 \%$ & $-4.3 \%$ \\
\hline
\end{tabular}

Source:

U.S. Department of Energy, Energy Information Administration, Monthly Energy Review, Washington, DC, July 2017, Table 9.7. (Additional resources: www.eia.doe.gov)

${ }^{\mathrm{a}}$ Consumer grade.

${ }^{\mathrm{b}}$ Adjusted by the Consumer Price Inflation Index. 
Prices of finished aviation gasoline (current dollars) dropped in 2009 but then began to climb. In 2012 both finished aviation gasoline and kerosene-type jet fuel reached their all-time high.

Table 10.8

Refiner Sales Prices for Aviation Gasoline and Jet Fuel, 1978-2016 (cents per gallon, excluding tax)

\begin{tabular}{|c|c|c|c|c|}
\hline \multirow[b]{2}{*}{ Year } & \multicolumn{2}{|c|}{ Finished aviation gasoline } & \multicolumn{2}{|c|}{ Kerosene-type jet fuel } \\
\hline & Current & Constant 2016 & Current & Constant 2016 \\
\hline 1978 & 51.6 & 189.9 & 38.7 & 142.5 \\
\hline 1980 & 108.4 & 315.8 & 86.8 & 252.8 \\
\hline 1985 & 120.1 & 267.9 & 79.6 & 177.6 \\
\hline 1986 & 101.1 & 221.4 & 52.9 & 115.9 \\
\hline 1987 & 90.7 & 191.6 & 54.3 & 114.7 \\
\hline 1988 & 89.1 & 180.8 & 51.3 & 104.1 \\
\hline 1989 & 99.5 & 192.6 & 59.2 & 114.6 \\
\hline 1990 & 112.0 & 205.6 & 76.6 & 140.6 \\
\hline 1991 & 104.7 & 184.5 & 65.2 & 114.9 \\
\hline 1992 & 102.7 & 175.7 & 61.0 & 104.4 \\
\hline 1993 & 99.0 & 164.4 & 58.0 & 96.3 \\
\hline 1994 & 95.7 & 154.9 & 53.4 & 86.5 \\
\hline 1995 & 100.5 & 158.3 & 54.0 & 85.1 \\
\hline 1996 & 111.6 & 170.7 & 65.1 & 99.6 \\
\hline 1997 & 112.8 & 168.6 & 61.3 & 91.6 \\
\hline 1998 & 95.7 & 140.9 & 45.2 & 66.5 \\
\hline 1999 & 105.9 & 152.6 & 54.3 & 78.2 \\
\hline 2000 & 130.6 & 182.1 & 89.9 & 125.3 \\
\hline 2001 & 132.3 & 179.3 & 77.5 & 105.0 \\
\hline 2002 & 128.8 & 171.8 & 72.1 & 96.2 \\
\hline 2003 & 149.3 & 194.7 & 87.2 & 113.7 \\
\hline 2004 & 181.9 & 231.2 & 120.7 & 153.4 \\
\hline 2005 & 223.1 & 274.2 & 173.5 & 213.2 \\
\hline 2006 & 268.2 & 319.4 & 199.8 & 238.0 \\
\hline 2007 & 284.9 & 329.9 & 216.5 & 250.7 \\
\hline 2008 & 327.3 & 364.9 & 305.2 & 340.3 \\
\hline 2009 & 244.2 & 273.3 & 170.4 & 190.7 \\
\hline 2010 & 302.8 & 333.4 & 220.1 & 242.3 \\
\hline 2011 & 380.3 & 405.8 & 305.4 & 325.9 \\
\hline 2012 & 397.1 & 415.0 & 310.4 & 324.4 \\
\hline 2013 & 393.2 & 405.0 & 297.9 & 306.8 \\
\hline 2014 & 398.6 & 404.2 & 277.2 & 281.1 \\
\hline 2015 & b & b & 162.9 & 165.0 \\
\hline 2016 & b & b & 131.9 & 131.9 \\
\hline \multicolumn{5}{|c|}{ Average annual percentage change } \\
\hline 1978-2016 & $5.8 \%{ }^{\mathrm{c}}$ & $2.1 \%^{c}$ & $3.3 \%$ & $-0.2 \%$ \\
\hline 2006-2016 & $5.1 \%^{\mathrm{c}}$ & $3.0 \%{ }^{c}$ & $-4.1 \%$ & $-5.7 \%$ \\
\hline
\end{tabular}

\section{Source:}

U.S. Department of Energy, Energy Information Administration, Petroleum Data Analysis Tools, Refiner Petroleum Product Prices by Sales Type, August 2017, Washington, DC. (Additional resources: www.eia.doe.gov)

a Adjusted by the Consumer Price Inflation Index.

b EIA withheld value to avoid disclosure of individual company data.

c Data through 2014. 
The federal government taxes highway motor fuel and uses the money to pay for roadway upkeep and improvement, as well as other related expenditures. Compressed natural gas (CNG) and liquefied petroleum gas (LPG) have the lowest taxes, while diesel fuel and liquefied natural gas (LNG) have the highest.

Table 10.9

Federal Excise Taxes on Motor Fuels, 2015

\begin{tabular}{lcc}
\hline Fuel & Cents per gallon & Effective Date \\
\hline Gasoline & 18.4 & October 1, 1997 \\
Diesel and kerosene & 24.4 & October 1, 1997 \\
Gasohol $^{\mathrm{a}}$ & 18.4 & January 1, 2005 \\
CNG & $18.3^{\mathrm{c}}$ & October 1, 2006 \\
LNG & 24.3 & October 1, 2006 \\
LPG & 18.3 & October 1, 2006 \\
Other alternative fuels & bctober 1, 1997 \\
\hline
\end{tabular}

\section{Source:}

U.S. Department of Transportation, Federal Highway Administration, Highway Statistics 2015, Washington, DC, 2016, Table FE-21B. (Additional resources: www.fhwa.dot.gov)

${ }^{a}$ All gasohol blends are taxed at the same rate.

b Includes benzol, benzene, naphtha, and other liquids used as a motor fuel.

c Compressed natural gas is 18.3 cents per energy equivalent of a gallon of gasoline. 
Federal, state, and local jurisdictions have laws and incentives for alternative fuels production use.

Table 10.10

Federal, State, and Local Alternative Fuel Incentives, 2017 (number of incentives)

\begin{tabular}{|c|c|c|c|c|c|c|c|c|}
\hline $\begin{array}{l}\text { State (including } \\
\text { jurisdictions in the } \\
\text { State) }\end{array}$ & Biodiesel & Ethanol & $\begin{array}{c}\text { Natural } \\
\text { Gas }\end{array}$ & $\begin{array}{l}\text { Liquefied } \\
\text { petroleum } \\
\text { gas (LPG) }\end{array}$ & $\begin{array}{c}\text { Electric } \\
\text { vehicles } \\
\text { (EVs) }\end{array}$ & $\begin{array}{c}\text { Neighborhood } \\
\text { electric } \\
\text { vehicles } \\
\text { (NEVs) } \\
\end{array}$ & $\begin{array}{l}\text { Hydrogen } \\
\text { fuel cells }\end{array}$ & $\begin{array}{l}\text { Aftermarket } \\
\text { conversions }\end{array}$ \\
\hline Federal & 30 & 26 & 28 & 27 & 26 & 3 & 23 & 6 \\
\hline Alabama & 2 & 2 & 4 & 4 & 2 & 0 & 1 & 2 \\
\hline Alaska & 1 & 2 & 1 & 1 & 1 & 1 & 1 & 1 \\
\hline Arizona & 4 & 4 & 14 & 14 & 15 & 1 & 12 & 0 \\
\hline Arkansas & 4 & 3 & 6 & 5 & 2 & 0 & 2 & 2 \\
\hline California & 18 & 16 & 29 & 19 & 56 & 3 & 29 & 6 \\
\hline Colorado & 10 & 9 & 18 & 13 & 17 & 1 & 8 & 2 \\
\hline Connecticut & 3 & 4 & 4 & 2 & 15 & 0 & 6 & 3 \\
\hline Delaware & 2 & 2 & 4 & 5 & 4 & 1 & 1 & 1 \\
\hline Dist. of Columbia & 2 & 3 & 4 & 4 & 5 & 0 & 4 & 1 \\
\hline Florida & 7 & 6 & 5 & 5 & 11 & 1 & 2 & 1 \\
\hline Georgia & 4 & 4 & 5 & 4 & 10 & 0 & 4 & 1 \\
\hline Hawaii & 8 & 8 & 4 & 4 & 10 & 2 & 7 & 0 \\
\hline Idaho & 3 & 1 & 2 & 2 & 5 & 2 & 1 & 0 \\
\hline Illinois & 13 & 11 & 8 & 8 & 18 & 1 & 6 & 4 \\
\hline Indiana & 12 & 11 & 13 & 9 & 7 & 1 & 5 & 3 \\
\hline Iowa & 8 & 10 & 5 & 4 & 5 & 1 & 2 & 1 \\
\hline Kansas & 7 & 12 & 5 & 3 & 0 & 1 & 0 & 3 \\
\hline Kentucky & 6 & 6 & 5 & 4 & 2 & 1 & 2 & 1 \\
\hline Louisiana & 5 & 4 & 8 & 6 & 2 & 1 & 0 & 2 \\
\hline Maine & 5 & 4 & 3 & 3 & 4 & 3 & 2 & 0 \\
\hline Maryland & 3 & 3 & 3 & 3 & 15 & 2 & 2 & 2 \\
\hline Massachusetts & 5 & 4 & 4 & 3 & 14 & 1 & 5 & 1 \\
\hline Michigan & 2 & 1 & 6 & 5 & 8 & 0 & 5 & 0 \\
\hline Minnesota & 8 & 13 & 5 & 2 & 11 & 4 & 2 & 0 \\
\hline Mississippi & 2 & 2 & 6 & 5 & 1 & 0 & 1 & 2 \\
\hline Missouri & 8 & 7 & 9 & 9 & 6 & 1 & 6 & 0 \\
\hline Montana & 6 & 5 & 3 & 3 & 1 & 2 & 1 & 1 \\
\hline Nebraska & 1 & 3 & 8 & 5 & 3 & 1 & 3 & 2 \\
\hline Nevada & 4 & 2 & 7 & 7 & 8 & 1 & 6 & 0 \\
\hline New Hampshire & 4 & 1 & 3 & 3 & 2 & 2 & 1 & 2 \\
\hline New Jersey & 4 & 3 & 4 & 4 & 9 & 1 & 1 & 1 \\
\hline New Mexico & 8 & 4 & 4 & 4 & 4 & 1 & 5 & 0 \\
\hline New York & 3 & 3 & 7 & 2 & 12 & 1 & 4 & 2 \\
\hline North Carolina & 10 & 9 & 7 & 6 & 12 & 0 & 5 & 1 \\
\hline North Dakota & 11 & 9 & 3 & 2 & 1 & 1 & 2 & 0 \\
\hline Ohio & 6 & 6 & 10 & 8 & 8 & 0 & 4 & 2 \\
\hline Oklahoma & 9 & 11 & 16 & 9 & 8 & 1 & 8 & 7 \\
\hline Oregon & 10 & 10 & 10 & 9 & 14 & 1 & 6 & 3 \\
\hline Pennsylvania & 3 & 3 & 1 & 2 & 5 & 0 & 2 & 1 \\
\hline Rhode Island & 5 & 4 & 5 & 4 & 12 & 2 & 5 & 2 \\
\hline South Carolina & 9 & 7 & 5 & 6 & 5 & 2 & 7 & 3 \\
\hline South Dakota & 5 & 7 & 2 & 2 & 0 & 0 & 0 & 0 \\
\hline Tennessee & 6 & 6 & 7 & 3 & 2 & 1 & 1 & 0 \\
\hline Texas & 6 & 6 & 15 & 9 & 11 & 1 & 7 & 4 \\
\hline Utah & 2 & 1 & 15 & 8 & 12 & 1 & 7 & 3 \\
\hline Vermont & 3 & 3 & 4 & 3 & 6 & 2 & 4 & 1 \\
\hline Virginia & 17 & 12 & 16 & 10 & 14 & 1 & 11 & 3 \\
\hline Washington & 12 & 9 & 9 & 6 & 20 & 1 & 4 & 2 \\
\hline West Virginia & 4 & 4 & 8 & 7 & 5 & 1 & 5 & 2 \\
\hline Wisconsin & 11 & 8 & 7 & 8 & 7 & 1 & 6 & 0 \\
\hline Wyoming & 4 & 5 & 8 & 5 & 5 & 0 & 1 & 0 \\
\hline Totals & 345 & 319 & 392 & 308 & 458 & 57 & 245 & 87 \\
\hline
\end{tabular}

Source:

U.S. Department of Energy, Energy Efficiency and Renewable Energy, Alternative Fuels Data Center. Data downloaded August 2017. (Additional resources: www.eere.energy.gov/afdc/laws/matrix/tech) 
Table 10.11

Federal, State, and Local Advanced Technology Incentives, 2017 (number of incentives)

\begin{tabular}{|c|c|c|c|c|}
\hline $\begin{array}{l}\text { State (including jurisdictions in } \\
\text { the State) }\end{array}$ & $\begin{array}{l}\text { Hybrid electric vehicles (HEV) or } \\
\text { plug-in hybrid vehicles (PHEVs) }\end{array}$ & $\begin{array}{c}\text { Fuel economy or } \\
\text { efficiency }\end{array}$ & Idle reduction & Other $^{\mathrm{a}}$ \\
\hline Federal & 38 & 13 & 7 & 6 \\
\hline Alabama & 3 & 1 & 4 & 0 \\
\hline Alaska & 1 & 1 & 1 & 0 \\
\hline Arizona & 16 & 0 & 2 & 1 \\
\hline Arkansas & 2 & 0 & 1 & 1 \\
\hline California & 71 & 7 & 5 & 12 \\
\hline Colorado & 22 & 2 & 3 & 3 \\
\hline Connecticut & 15 & 1 & 2 & 2 \\
\hline Delaware & 5 & 2 & 2 & 2 \\
\hline Dist. of Columbia & 5 & 3 & 1 & 0 \\
\hline Florida & 12 & 1 & 1 & 0 \\
\hline Georgia & 11 & 0 & 2 & 1 \\
\hline Hawaii & 13 & 1 & 1 & 1 \\
\hline Idaho & 8 & 1 & 0 & 0 \\
\hline Illinois & 15 & 3 & 5 & 0 \\
\hline Indiana & 9 & 3 & 4 & 2 \\
\hline Iowa & 5 & 0 & 1 & 0 \\
\hline Kansas & 0 & 0 & 1 & 0 \\
\hline Kentucky & 1 & 0 & 0 & 1 \\
\hline Louisiana & 3 & 1 & 0 & 0 \\
\hline Maine & 5 & 2 & 3 & 1 \\
\hline Maryland & 11 & 1 & 3 & 2 \\
\hline Massachusetts & 12 & 0 & 3 & 1 \\
\hline Michigan & 7 & 0 & 0 & 0 \\
\hline Minnesota & 11 & 1 & 3 & 1 \\
\hline Mississippi & 2 & 1 & 1 & 0 \\
\hline Missouri & 6 & 0 & 1 & 0 \\
\hline Montana & 1 & 1 & 0 & 0 \\
\hline Nebraska & 2 & 0 & 1 & 0 \\
\hline Nevada & 11 & 0 & 1 & 0 \\
\hline New Hampshire & 1 & 2 & 5 & 3 \\
\hline New Jersey & 9 & 4 & 1 & 4 \\
\hline New Mexico & 5 & 1 & 1 & 0 \\
\hline New York & 13 & 3 & 3 & 4 \\
\hline North Carolina & 12 & 1 & 3 & 0 \\
\hline North Dakota & 0 & 0 & 0 & 1 \\
\hline Ohio & 5 & 0 & 2 & 0 \\
\hline Oklahoma & 9 & 0 & 1 & 3 \\
\hline Oregon & 15 & 1 & 3 & 2 \\
\hline Pennsylvania & 4 & 1 & 3 & 1 \\
\hline Rhode Island & 12 & 3 & 5 & 6 \\
\hline South Carolina & 10 & 1 & 3 & 1 \\
\hline South Dakota & 0 & 0 & 0 & 1 \\
\hline Tennessee & 3 & 1 & 1 & 0 \\
\hline Texas & 11 & 1 & 3 & 1 \\
\hline Utah & 11 & 3 & 3 & 5 \\
\hline Vermont & 7 & 2 & 4 & 2 \\
\hline Virginia & 16 & 2 & 2 & 3 \\
\hline Washington & 18 & 2 & 2 & 3 \\
\hline West Virginia & 4 & 0 & 2 & 1 \\
\hline Wisconsin & 9 & 0 & 1 & 0 \\
\hline Wyoming & 4 & 0 & 1 & 0 \\
\hline Totals & 501 & 74 & 108 & 78 \\
\hline
\end{tabular}

Source:

U.S. Department of Energy, Energy Efficiency and Renewable Energy, Alternative Fuels Data Center. Data downloaded August 2017. (Additional resources: www.eere.energy.gov/afdc/laws/matrix/tech)

a Includes Clean Fuel Initiatives and Pollution Prevention. 
The average price of a new car in 2016 (\$25,774) was close to the average price in $1916(\$ 23,798)$ when adjusted for inflation. Average new car prices were at their lowest in 1940 (\$13,311). Since 1914 the highest average price was in the year $1998(\$ 32,102)$.

Table 10.12

Average Price of a New Car, 1913-2016

\begin{tabular}{ll|lc|cc|cc}
\hline Year & $\begin{array}{c}2016 \\
\text { Constant } \\
\text { dollars }\end{array}$ & Year & $\begin{array}{c}2016 \\
\text { Constant } \\
\text { dollars }\end{array}$ & Year & $\begin{array}{c}2016 \\
\text { Constant } \\
\text { dollars }\end{array}$ & $\begin{array}{c}\text { Year } \\
\text { Constant } \\
\text { dollars }\end{array}$ \\
\hline 1913 & $\$ 34,689$ & 1939 & $\$ 14,319$ & 1965 & $\$ 21,048$ & 1991 & $\$ 27,270$ \\
1914 & $\$ 35,899$ & 1940 & $\$ 13,311$ & 1966 & $\$ 21,032$ & 1992 & $\$ 28,459$ \\
1915 & $\$ 29,848$ & 1941 & $\$ 13,483$ & 1967 & $\$ 23,110$ & 1993 & $\$ 28,022$ \\
1916 & $\$ 23,798$ & 1942 & $\$ 13,656$ & 1968 & $\$ 21,776$ & 1994 & $\$ 28,994$ \\
1917 & $\$ 21,983$ & 1943 & $\$ 13,829$ & 1969 & $\$ 23,262$ & 1995 & $\$ 28,283$ \\
1918 & $\$ 20,168$ & 1944 & $\$ 14,001$ & 1970 & $\$ 21,910$ & 1996 & $\$ 28,723$ \\
1919 & $\$ 19,966$ & 1945 & $\$ 14,174$ & 1971 & $\$ 22,175$ & 1997 & $\$ 31,442$ \\
1920 & $\$ 19,764$ & 1946 & $\$ 14,346$ & 1972 & $\$ 22,272$ & 1998 & $\$ 32,102$ \\
1921 & $\$ 20,974$ & 1947 & $\$ 14,519$ & 1973 & $\$ 21,903$ & 1999 & $\$ 31,297$ \\
1922 & $\$ 22,185$ & 1948 & $\$ 15,206$ & 1974 & $\$ 21,615$ & 2000 & $\$ 29,860$ \\
1923 & $\$ 20,168$ & 1949 & $\$ 17,719$ & 1975 & $\$ 22,082$ & 2001 & $\$ 28,879$ \\
1924 & $\$ 18,151$ & 1950 & $\$ 18,159$ & 1976 & $\$ 22,853$ & 2002 & $\$ 28,291$ \\
1925 & $\$ 17,949$ & 1951 & $\$ 18,468$ & 1977 & $\$ 23,026$ & 2003 & $\$ 27,417$ \\
1926 & $\$ 17,748$ & 1952 & $\$ 20,005$ & 1978 & $\$ 23,482$ & 2004 & $\$ 27,277$ \\
1927 & $\$ 17,546$ & 1953 & $\$ 20,030$ & 1979 & $\$ 22,635$ & 2005 & $\$ 27,594$ \\
1928 & $\$ 17,344$ & 1954 & $\$ 19,666$ & 1980 & $\$ 22,061$ & 2006 & $\$ 27,485$ \\
1929 & $\$ 17,143$ & 1955 & $\$ 19,559$ & 1981 & $\$ 23,525$ & 2007 & $\$ 26,986$ \\
1930 & $\$ 16,941$ & 1956 & $\$ 20,123$ & 1982 & $\$ 24,598$ & 2008 & $\$ 25,421$ \\
1931 & $\$ 18,958$ & 1957 & $\$ 22,354$ & 1983 & $\$ 25,557$ & 2009 & $\$ 24,894$ \\
1932 & $\$ 20,974$ & 1958 & $\$ 23,648$ & 1984 & $\$ 26,276$ & 2010 & $\$ 26,485$ \\
1933 & $\$ 19,764$ & 1959 & $\$ 23,698$ & 1985 & $\$ 26,405$ & 2011 & $\$ 26,156$ \\
1934 & $\$ 18,554$ & 1960 & $\$ 22,804$ & 1986 & $\$ 27,706$ & 2012 & $\$ 25,653$ \\
1935 & $\$ 16,538$ & 1961 & $\$ 21,714$ & 1987 & $\$ 28,281$ & 2013 & $\$ 25,194$ \\
1936 & $\$ 14,521$ & 1962 & $\$ 21,586$ & 1988 & $\$ 28,265$ & 2014 & $\$ 24,703$ \\
1937 & $\$ 14,924$ & 1963 & $\$ 21,348$ & 1989 & $\$ 27,816$ & 2015 & $\$ 24,662$ \\
1938 & $\$ 15,327$ & 1964 & $\$ 21,454$ & 1990 & $\$ 27,622$ & 2016 & $\$ 25,774$ \\
\hline
\end{tabular}

Note: These data are based on an average car and do not include prices for pickups, vans, or sport utility vehicles. Estimations were used for years 1941-1946.

\section{Sources:}

Compiled by Jacob Ward, Vehicle Technologies Program, U.S. Department of Energy, from the following sources. Raff, D.M.G. \& Trajtenberg, M. (1995), "Quality-Adjusted Prices for the American Automobile Industry: 1906-1940," National Bureau of Economic Research, Inc.; Gordon, R.J. (1990), The Measurement of Durable Goods Prices, National Bureau of Economic Research, Inc.; and U.S. Department of Commerce, Bureau of Economic Analysis (2017), National Income and Product Accounts. 
In current dollars, import cars, on average, were less expensive than domestic cars until 1982. Since then, import prices have more than tripled, while domestic prices have more than doubled (current dollars).

Table 10.13

Average Price of a New Car (Domestic and Import), 1970-2016

\begin{tabular}{|c|c|c|c|c|c|c|}
\hline \multirow[b]{2}{*}{ Year } & \multicolumn{2}{|c|}{ Domestic $^{\mathrm{a}}$} & \multicolumn{2}{|c|}{ Import } & \multicolumn{2}{|c|}{ Total } \\
\hline & $\begin{array}{l}\text { Current } \\
\text { dollars }\end{array}$ & $\begin{array}{c}\text { Constant } 2016 \\
\text { dollars }^{\mathrm{b}}\end{array}$ & $\begin{array}{l}\text { Current } \\
\text { dollars }\end{array}$ & $\begin{array}{c}\text { Constant } 2016 \\
\text { dollars }^{\mathrm{b}}\end{array}$ & $\begin{array}{l}\text { Current } \\
\text { dollars }\end{array}$ & $\begin{array}{c}\text { Constant } 2016 \\
\text { dollars }^{\mathrm{b}}\end{array}$ \\
\hline 1970 & 3,708 & 22,938 & 2,648 & 16,381 & 3,542 & 21,911 \\
\hline 1975 & 5,084 & 22,680 & 4,384 & 19,557 & 4,950 & 22,082 \\
\hline 1980 & 7,609 & 22,165 & 7,482 & 21,795 & 7,574 & 22,063 \\
\hline 1985 & 11,589 & 25,855 & 12,853 & 28,675 & 11,838 & 26,411 \\
\hline 1986 & 12,319 & 26,979 & 13,670 & 29,937 & 12,652 & 27,708 \\
\hline 1987 & 12,922 & 27,304 & 14,470 & 30,575 & 13,386 & 28,285 \\
\hline 1988 & 13,418 & 27,225 & 15,221 & 30,883 & 13,932 & 28,268 \\
\hline 1989 & 13,936 & 26,980 & 15,510 & 30,027 & 14,371 & 27,822 \\
\hline 1990 & 14,489 & 26,602 & 16,640 & 30,551 & 15,042 & 27,617 \\
\hline 1991 & 15,192 & 26,768 & 16,327 & 28,768 & 15,475 & 27,267 \\
\hline 1992 & 15,644 & 26,767 & 18,593 & 31,813 & 16,636 & 28,464 \\
\hline 1993 & 15,976 & 26,536 & 20,261 & 33,654 & 16,871 & 28,023 \\
\hline 1994 & 16,930 & 27,410 & 21,989 & 35,600 & 17,903 & 28,985 \\
\hline 1995 & 16,864 & 26,561 & 23,202 & 36,543 & 17,959 & 28,285 \\
\hline 1996 & 17,468 & 26,726 & 26,205 & 40,094 & 18,777 & 28,729 \\
\hline 1997 & 17,532 & 26,210 & 27,718 & 41,438 & 21,026 & 31,434 \\
\hline 1998 & 18,501 & 27,233 & 28,695 & 42,239 & 21,802 & 32,093 \\
\hline 1999 & 19,006 & 27,388 & 27,472 & 39,587 & 21,725 & 31,306 \\
\hline 2000 & 19,561 & 27,268 & 26,008 & 36,255 & 21,424 & 29,865 \\
\hline 2001 & 20,004 & 27,105 & 25,809 & 34,971 & 21,310 & 28,875 \\
\hline 2002 & 20,431 & 27,255 & 25,612 & 34,166 & 21,206 & 28,289 \\
\hline 2003 & 19,961 & 26,029 & 26,136 & 34,081 & 21,019 & 27,409 \\
\hline 2004 & 20,509 & 26,067 & 25,942 & 32,972 & 21,469 & 27,287 \\
\hline 2005 & 21,565 & 26,503 & 26,622 & 32,718 & 22,454 & 27,596 \\
\hline 2006 & 22,139 & 26,368 & 27,061 & 32,230 & 23,087 & 27,497 \\
\hline 2007 & 22,264 & 25,782 & 27,463 & 31,802 & 23,313 & 26,996 \\
\hline 2008 & 22,192 & 24,744 & 25,902 & 28,881 & 22,804 & 25,426 \\
\hline 2009 & 22,080 & 24,708 & 25,223 & 28,225 & 22,252 & 24,900 \\
\hline 2010 & 23,768 & 26,169 & 27,232 & 29,982 & 24,063 & 26,493 \\
\hline 2011 & 24,167 & 25,786 & 28,284 & 30,179 & 24,514 & 26,156 \\
\hline 2012 & 24,131 & 25,217 & 28,973 & 30,277 & 24,540 & 25,644 \\
\hline 2013 & 23,921 & 24,639 & 29,769 & 30,662 & 24,454 & 25,188 \\
\hline 2014 & 23,787 & 24,120 & 29,935 & 30,354 & 24,366 & 24,707 \\
\hline 2015 & 23,981 & 24,293 & 29,284 & 29,665 & 24,355 & 24,672 \\
\hline 2016 & 24,414 & 24,414 & 29,913 & 29,913 & 25,774 & 25,774 \\
\hline \multicolumn{7}{|c|}{ Average annual percentage change } \\
\hline 1970-2016 & $4.2 \%$ & $0.1 \%$ & $5.4 \%$ & $1.3 \%$ & $4.4 \%$ & $0.4 \%$ \\
\hline 2006-2016 & $1.0 \%$ & $-0.8 \%$ & $1.0 \%$ & $-0.7 \%$ & $1.1 \%$ & $-0.6 \%$ \\
\hline
\end{tabular}

Note: These data are based on an average car and do not include prices for pickups, vans, or sport utility vehicles.

Source:

U.S. Department of Commerce, Bureau of Economic Analysis, Average Transaction Price per New Car, Washington, DC, 2017. (Additional resources: www.bea.gov)

${ }^{a}$ Includes all vehicles produced in the United States regardless of manufacturer.

${ }^{\mathrm{b}}$ Adjusted by the Consumer Price Inflation Index. 
The total cost of operating a car is the sum of the fixed cost (depreciation, insurance, finance charge, and license fee) and the variable cost (gas and oil, tires, and maintenance), which is related to the amount of travel. The gas and oil share of total cost was 11.2\% in 2016 which is down from 18.4\% in 2012.

Table 10.14

Car Operating Cost per Mile, 1985-2016

\begin{tabular}{|c|c|c|c|c|c|}
\hline \multirow[b]{2}{*}{ Model year } & \multicolumn{3}{|c|}{ Constant 2016 dollars per 10,000 miles $^{a}$} & \multirow{2}{*}{$\begin{array}{l}\text { Total cost per } \\
\text { mile }^{\mathrm{b}} \text { (constant } \\
2016 \text { cents }^{\mathrm{a}} \text { ) }\end{array}$} & \multirow{2}{*}{$\begin{array}{l}\text { Percentage gas } \\
\text { and oil of total } \\
\text { cost }\end{array}$} \\
\hline & Variable cost & Fixed cost & Total cost & & \\
\hline 1985 & 1,655 & 4,597 & 6,252 & 62.52 & $19.9 \%$ \\
\hline 1986 & 1,428 & 5,052 & 6,480 & 64.80 & $15.1 \%$ \\
\hline 1987 & 1,416 & 4,918 & 6,334 & 63.34 & $14.7 \%$ \\
\hline 1988 & 1,603 & 6,147 & 7,750 & 77.50 & $13.6 \%$ \\
\hline 1989 & 1,548 & 5,652 & 7,200 & 72.00 & $14.2 \%$ \\
\hline 1990 & 1,543 & 5,979 & 7,522 & 75.22 & $13.2 \%$ \\
\hline 1991 & 1,709 & 6,284 & 7,993 & 79.93 & $14.6 \%$ \\
\hline 1992 & 1,540 & 6,473 & 8,013 & 80.13 & $12.6 \%$ \\
\hline 1993 & 1,528 & 6,182 & 7,710 & 77.10 & $12.7 \%$ \\
\hline 1994 & 1,474 & 6,212 & 7,686 & 76.86 & $11.8 \%$ \\
\hline 1995 & 1,512 & 6,307 & 7,819 & 78.19 & $11.7 \%$ \\
\hline 1996 & 1,468 & 6,414 & 7,882 & 78.82 & $10.9 \%$ \\
\hline 1997 & 1,615 & 6,502 & 8,117 & 81.17 & $12.2 \%$ \\
\hline 1998 & 1,576 & 6,667 & 8,243 & 82.43 & $11.1 \%$ \\
\hline 1999 & 1,527 & 6,713 & 8,240 & 82.40 & $9.8 \%$ \\
\hline 2000 & 1,700 & 6,584 & 8,285 & 82.85 & $11.6 \%$ \\
\hline 2001 & 1,843 & 6,262 & 8,105 & 81.05 & $13.2 \%$ \\
\hline 2002 & 1,574 & 6,502 & 8,077 & 80.77 & $9.7 \%$ \\
\hline 2003 & 1,709 & 6,371 & 8,079 & 80.79 & $11.6 \%$ \\
\hline 2004 & 1,601 & 7,157 & 8,758 & 87.58 & $9.4 \%$ \\
\hline 2005 & 1,733 & 6,651 & 8,384 & 83.84 & $12.0 \%$ \\
\hline 2006 & 1,798 & 5,579 & 7,376 & 73.76 & $15.3 \%$ \\
\hline 2007 & 1,678 & 5,516 & 7,194 & 71.94 & $14.3 \%$ \\
\hline 2008 & 1,891 & 6,018 & 7,909 & 79.09 & $16.4 \%$ \\
\hline 2009 & 1,725 & 6,182 & 7,907 & 79.07 & $14.3 \%$ \\
\hline 2010 & 1,841 & 6,295 & 8,136 & 81.36 & $15.4 \%$ \\
\hline 2011 & 1,893 & 6,249 & 8,142 & 81.42 & $16.2 \%$ \\
\hline 2012 & 2,053 & 6,007 & 8,060 & 80.60 & $18.4 \%$ \\
\hline 2013 & 2,104 & 5,967 & 8,071 & 80.71 & $18.4 \%$ \\
\hline 2014 & 1,929 & 5,855 & 7,784 & 77.84 & $16.9 \%$ \\
\hline 2015 & 1,752 & 5,925 & 7,677 & 76.77 & $14.80 \%$ \\
\hline 2016 & 1,473 & 6,072 & 7,545 & 75.45 & $11.20 \%$ \\
\hline \multicolumn{6}{|c|}{ Average annual percentage change } \\
\hline 1985-2016 & $-0.4 \%$ & $0.9 \%$ & $0.6 \%$ & $0.6 \%$ & \\
\hline 2006-2016 & $-2.0 \%$ & $0.9 \%$ & $0.2 \%$ & $0.2 \%$ & \\
\hline
\end{tabular}

\section{Source:}

Ward's Communications, Motor Vehicle Facts and Figures 2016, Southfield, Michigan, 2016, p. 55, and annual. Original data from AAA “Your Driving Costs.” (Additional resources: newsroom.aaa.com)

${ }^{\text {a }}$ Adjusted by the Consumer Price Inflation Index.

${ }^{\mathrm{b}}$ Based on 10,000 miles per year. 
While the previous table shows costs per mile, this table presents costs per year for fixed costs associated with car operation. For 2016 model year cars, the fixed cost is over \$17 per day.

Table 10.15

Fixed Car Operating Costs per Year, 1975-2016 (constant 2016 dollars) ${ }^{\mathrm{a}}$

\begin{tabular}{|c|c|c|c|c|c|c|}
\hline Model year & Insurance $^{\mathrm{b}}$ & $\begin{array}{l}\text { License, } \\
\text { registration } \\
\& \text { taxes }\end{array}$ & Depreciation & $\begin{array}{c}\text { Finance } \\
\text { charge }\end{array}$ & Total & $\begin{array}{l}\text { Average } \\
\text { fixed cost } \\
\text { per day }\end{array}$ \\
\hline 1975 & 1,709 & 134 & 3,448 & c & 5,291 & 14.50 \\
\hline 1980 & 1,455 & 239 & 3,023 & $\bar{c}$ & 5,922 & 16.22 \\
\hline 1981 & 1,367 & 232 & 3,398 & c & 6,271 & 17.19 \\
\hline 1982 & 1,125 & 134 & 3,373 & c & 5,964 & 16.34 \\
\hline 1983 & 1,138 & 234 & 3,128 & c & 5,769 & 15.81 \\
\hline 1984 & 1,175 & 245 & 2,788 & c & 5,419 & 14.85 \\
\hline 1985 & 1,043 & 245 & 2,815 & 1,191 & 5,289 & 14.50 \\
\hline 1986 & 1,118 & 285 & 2,891 & 1,395 & 5,685 & 15.57 \\
\hline 1987 & 1,137 & 270 & 3,156 & 1,111 & 5,668 & 15.53 \\
\hline 1988 & 1,170 & 282 & 3,619 & 1,146 & 6,210 & 17.02 \\
\hline 1989 & 1,258 & 279 & 3,906 & 1,138 & 6,571 & 18.00 \\
\hline 1990 & 1,247 & 303 & 4,328 & 1,249 & 7,116 & 19.50 \\
\hline 1991 & 1,256 & 296 & 4,412 & 469 & 6,425 & 17.60 \\
\hline 1992 & 1,353 & 298 & 4,648 & 1,362 & 7,654 & 20.97 \\
\hline 1993 & 1,242 & 296 & 4,700 & 1,113 & 7,345 & 20.13 \\
\hline 1994 & 1,250 & 314 & 4,761 & 1,049 & 7,370 & 20.19 \\
\hline 1995 & 1,239 & 320 & 4,840 & 1,080 & 7,473 & 20.47 \\
\hline 1996 & 1,299 & 329 & 4,849 & 1,098 & 7,569 & 20.74 \\
\hline 1997 & 1,271 & 323 & 4,893 & 1,148 & 7,631 & 20.91 \\
\hline 1998 & 1,328 & 333 & 4,953 & 1,197 & 7,808 & 21.39 \\
\hline 1999 & 1,403 & 326 & 4,950 & 1,193 & 7,866 & 21.55 \\
\hline 2000 & 1,360 & 311 & 4,867 & 1,183 & 7,713 & 21.13 \\
\hline 2001 & 1,349 & 282 & 4,808 & 1,174 & 7,607 & 20.84 \\
\hline 2002 & 1,356 & 268 & 4,964 & 1,105 & 7,690 & 21.07 \\
\hline 2003 & 1,443 & 267 & 4,876 & 970 & 7,551 & 20.69 \\
\hline 2004 & 2,037 & 527 & 4,805 & 941 & 8,311 & 22.77 \\
\hline 2005 & 1,583 & 478 & 4,767 & 908 & 7,736 & 21.20 \\
\hline 2006 & 1,102 & 637 & 4,038 & 852 & 6,630 & 18.17 \\
\hline 2007 & 1,140 & 623 & 3,926 & 848 & 6,538 & 17.91 \\
\hline 2008 & 1,051 & 618 & 3,702 & 845 & 6,216 & 17.03 \\
\hline 2009 & 1,092 & 634 & 3,872 & 871 & 6,470 & 17.72 \\
\hline 2010 & 1,135 & 644 & 3,912 & 887 & 6,578 & 18.02 \\
\hline 2011 & 1,033 & 635 & 3,978 & 878 & 6,524 & 17.87 \\
\hline 2012 & 1,046 & 638 & 3,705 & 884 & 6,273 & 17.19 \\
\hline 2013 & 1,060 & 629 & 3,679 & 874 & 6,242 & 17.10 \\
\hline 2014 & 1,037 & 650 & 3,558 & 859 & 6,104 & 16.73 \\
\hline 2015 & 1,129 & 673 & 3,700 & 677 & 6,180 & 16.93 \\
\hline 2016 & 1,222 & 687 & 3,759 & 683 & 6,351 & 17.40 \\
\hline \multicolumn{7}{|c|}{ Average annual percentage change } \\
\hline 1975-2016 & $-0.8 \%$ & $4.1 \%$ & $0.2 \%$ & c & $0.4 \%$ & $0.4 \%$ \\
\hline 2006-2016 & $-1.0 \%$ & $0.8 \%$ & $-0.7 \%$ & $-2.2 \%$ & $-0.4 \%$ & $-0.4 \%$ \\
\hline
\end{tabular}

Source:

Ward's Communications, Motor Vehicle Facts and Figures 2016, Southfield, Michigan, 2016, p. 55 and annual. Original data from AAA "Your Driving Costs.” (Additional resources: newsroom.aaa.com)

${ }^{a}$ Adjusted by the Consumer Price Inflation Index.

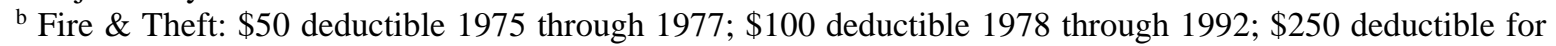
1993 - 2003; \$100 deductible 2004-2015. Collision: \$100 deductible through 1979; \$250 deductible 1980-1992; $\$ 500$ deductible for 1993 - on. Property Damage \& Liability: coverage $=\$ 100,000 / \$ 300,000$.

${ }^{\mathrm{c}}$ Data are not available. 
Table 10.16

Personal Consumption Expenditures, 1970-2016

(billion dollars)

\begin{tabular}{|c|c|c|c|c|c|}
\hline \multirow[b]{2}{*}{ Year } & \multicolumn{2}{|c|}{ Personal consumption expenditures } & \multicolumn{2}{|c|}{$\begin{array}{c}\text { Transportation personal } \\
\text { consumption expenditures }\end{array}$} & \multirow[b]{2}{*}{$\begin{array}{l}\text { Transportation PCE } \\
\text { as a percent of PCE }\end{array}$} \\
\hline & Current & $\begin{array}{c}\text { Constant } \\
2016^{\mathrm{a}}\end{array}$ & Current & $\begin{array}{c}\text { Constant } \\
2016^{\mathrm{a}}\end{array}$ & \\
\hline 1970 & 647.7 & $3,175.7$ & 80.8 & 396.2 & $12.5 \%$ \\
\hline 1980 & $1,754.6$ & $4,416.3$ & 241.7 & 608.4 & $13.8 \%$ \\
\hline 1990 & $3,825.6$ & $6,392.6$ & 455.7 & 761.5 & $11.9 \%$ \\
\hline 2000 & $6,792.4$ & $9,251.2$ & 811.2 & $1,104.9$ & $11.9 \%$ \\
\hline 2005 & $8,794.1$ & $10,667.2$ & 983.2 & $1,192.6$ & $11.2 \%$ \\
\hline 2010 & $10,202.2$ & $11,222.4$ & 968.3 & $1,065.1$ & $9.5 \%$ \\
\hline 2011 & $10,689.3$ & $11,523.1$ & $1,081.2$ & $1,165.5$ & $10.1 \%$ \\
\hline 2012 & $11,050.6$ & $11,702.6$ & $1,132.2$ & $1,199.0$ & $10.2 \%$ \\
\hline 2013 & $11,361.2$ & $11,838.4$ & $1,162.8$ & $1,211.6$ & $10.2 \%$ \\
\hline 2014 & $11,863.7$ & $12,148.4$ & 1,197.2 & $1,225.9$ & $10.1 \%$ \\
\hline 2015 & $12,332.3$ & $12,492.6$ & $1,151.5$ & $1,166.5$ & $9.3 \%$ \\
\hline 2016 & $12,820.7$ & $12,820.7$ & $1,147.0$ & $1,147.0$ & $8.9 \%$ \\
\hline
\end{tabular}

Note: Transportation PCE includes the following categories: transportation, motor vehicles and parts, and gasoline and oil.

\section{Source:}

U.S. Department of Commerce, Bureau of Economic Analysis, National Income and Product Accounts, Table 2.3.5, www.bea.gov

Table 10.17

Consumer Price Indices, 1970-2016

$(1970=1.000)$

\begin{tabular}{|c|c|c|c|c|c|}
\hline Year & $\begin{array}{c}\text { Consumer price } \\
\text { index }\end{array}$ & $\begin{array}{c}\text { Transportation } \\
\text { consumer price index }\end{array}$ & $\begin{array}{c}\text { New car } \\
\text { consumer price } \\
\text { index } \\
\end{array}$ & $\begin{array}{c}\text { Used car } \\
\text { consumer price } \\
\text { index } \\
\end{array}$ & $\begin{array}{c}\text { Gross national product } \\
\text { index }\end{array}$ \\
\hline 1970 & 1.000 & 1.000 & 1.000 & 1.000 & 1.000 \\
\hline 1980 & 2.124 & 2.216 & 1.667 & 1.997 & 2.676 \\
\hline 1990 & 3.369 & 3.213 & 2.286 & 3.769 & 5.557 \\
\hline 2000 & 4.438 & 4.088 & 2.689 & 4.994 & 9.537 \\
\hline 2005 & 5.034 & 4.637 & 2.597 & 4.468 & 12.184 \\
\hline 2010 & 5.620 & 5.157 & 2.599 & 4.587 & 14.017 \\
\hline 2011 & 5.797 & 5.663 & 2.672 & 4.776 & 14.566 \\
\hline 2012 & 5.917 & 5.796 & 2.716 & 4.818 & 15.146 \\
\hline 2013 & 6.004 & 5.798 & 2.745 & 4.804 & 15.648 \\
\hline 2014 & 6.101 & 5.758 & 2.755 & 4.779 & 16.319 \\
\hline 2015 & 6.109 & 5.308 & 2.771 & 4.715 & 16.932 \\
\hline 2016 & 6.186 & 5.197 & 2.775 & 4.599 & 17.390 \\
\hline
\end{tabular}

\section{Sources:}

Bureau of Labor Statistics, Consumer Price Index Table 1A for 2016, and annual.

(Additional resources: www.bls.gov)

GNP - U.S. Department of Commerce, Bureau of Economic Analysis, National Income and Product Accounts, Table 1.7.5. (Additional resources: www.bea.gov)

a Adjusted by the GNP price deflator.

b Transportation Consumer Price Index includes new and used cars, gasoline, car insurance rates, intracity mass transit, intracity bus fare, and airline fares. 
The data below were summarized from the Bureau of Labor Statistics (BLS) Current Employment Statistics Survey data using the North American Industry Classification System (NAICS). Transportation-related employment was 7.5\% of total employment in 2016.

Table 10.18

Transportation-Related Employment, 2000 and 2016 (thousands)

\begin{tabular}{lrrr}
\hline & & & \\
& 2000 & \multicolumn{1}{c}{$\begin{array}{c}\text { Percent } \\
\text { change }\end{array}$} \\
\hline Truck transportation (includes drivers) & $1,405.8$ & $1,453.6$ & $3.4 \%$ \\
Transit and ground transportation & 372.1 & 478.4 & $28.6 \%$ \\
Air transportation & 614.4 & 475.9 & $-22.5 \%$ \\
Rail transportation & 231.7 & 214.7 & $-7.3 \%$ \\
Water transportation & 56.0 & 65.5 & $17.0 \%$ \\
Pipeline transportation & 46.0 & 49.5 & $7.6 \%$ \\
Motor vehicle and parts - retail & $1,846.9$ & $1,981.8$ & $7.3 \%$ \\
Motor vehicles and parts - wholesale & 355.7 & 331.9 & $-6.7 \%$ \\
Gasoline stations - retail & 935.7 & 925.7 & $-1.1 \%$ \\
Automotive repair and maintenance & 888.1 & 913.3 & $2.8 \%$ \\
Automotive equipment rental and leasing & 208.3 & 206.4 & $-0.9 \%$ \\
Manufacturing & $2,143.9$ & $1,679.8$ & $-21.6 \%$ \\
$\quad$ Cars and light trucks & 237.4 & 180.9 & $-23.8 \%$ \\
$\quad$ Heavy-duty trucks & 54.0 & 27.9 & $-48.3 \%$ \\
$\quad$ Motor vehicle bodies and trailers & 182.7 & 151.2 & $-17.2 \%$ \\
$\quad$ Motor vehicle parts & 839.5 & 580.0 & $-30.9 \%$ \\
$\quad$ Aerospace products and parts & 516.7 & 489.0 & $-5.4 \%$ \\
$\quad$ Railroad rolling stock \& other transportation equipment & 72.7 & 60.6 & $-16.6 \%$ \\
$\quad$ Ship \& boat building & 154.1 & 135.2 & $-12.3 \%$ \\
Tires & 86.8 & 55.0 & $-36.6 \%$ \\
Oil and gas pipeline construction & 72.2 & 124.7 & $72.7 \%$ \\
Highway street and bridge construction & 340.1 & 321.5 & $-5.5 \%$ \\
Scenic \& sightseeing & 27.5 & 34.6 & $25.8 \%$ \\
Support activities for transportation & 537.4 & 660.1 & $22.8 \%$ \\
Couriers and messengers & 605.0 & 641.9 & $6.1 \%$ \\
Travel arrangement and reservation services & 298.6 & 216.3 & $-27.6 \%$ \\
\hline Total transportation-related employment & $\mathbf{1 0 , 9 8 5 . 4}$ & $\mathbf{1 0 , 7 7 5 . 6}$ & $\mathbf{- 1 . 9 \%}$ \\
\hline Total nonfarm employment & $132,019.0$ & $144,306.0$ & $9.3 \%$ \\
Transportation-related to total employment & $8.3 \%$ & $7.5 \%$ & \\
\hline & & & \\
& & & \\
& & &
\end{tabular}

Source:

Bureau of Labor Statistics website query system: www.bls.gov/data/. (Additional resources: www.bls.gov)

${ }^{a}$ Not seasonally adjusted. 
The total number of employees involved in the manufacture of motor vehicles decreased by over $74 \%$ from 1990 to 2016 and by almost 89\% for those involved in the manufacture of motor vehicle parts. Beginning in 2008, the share of production workers fell below 80\% for manufacturers of both vehicles and parts and remained below $80 \%$ for motor vehicle parts.

Table 10.19 U.S. Employment for Motor Vehicles and Motor Vehicle Parts Manufacturing, 1990-2016 ${ }^{\mathrm{a}}$

\begin{tabular}{|c|c|c|c|}
\hline Year & $\begin{array}{l}\text { All employees } \\
\text { (thousands) }\end{array}$ & $\begin{array}{c}\text { Production workers } \\
\text { (thousands) }\end{array}$ & $\begin{array}{c}\text { Share of production workers } \\
\text { to total employees }\end{array}$ \\
\hline \multicolumn{4}{|c|}{ Motor vehicles } \\
\hline 1990 & 271.4 & 243.4 & 89.7\% \\
\hline 1995 & 294.7 & 273.7 & $92.9 \%$ \\
\hline 1996 & 285.3 & 271.2 & $95.1 \%$ \\
\hline 1997 & 286.8 & 273.6 & $95.4 \%$ \\
\hline 1998 & 283.6 & 254.8 & $89.8 \%$ \\
\hline 1999 & 291.3 & 254.3 & $87.3 \%$ \\
\hline 2000 & 291.4 & 251.0 & $86.1 \%$ \\
\hline 2001 & 278.7 & 236.4 & $84.8 \%$ \\
\hline 2002 & 265.4 & 220.8 & $83.2 \%$ \\
\hline 2003 & 264.6 & 217.1 & $82.0 \%$ \\
\hline 2004 & 255.9 & 208.0 & $81.3 \%$ \\
\hline 2005 & 247.6 & 198.6 & $80.2 \%$ \\
\hline 2006 & 236.5 & 191.8 & $81.1 \%$ \\
\hline 2007 & 220.0 & 177.3 & $80.6 \%$ \\
\hline 2008 & 191.6 & 151.1 & $78.9 \%$ \\
\hline 2009 & 146.4 & 114.2 & $78.0 \%$ \\
\hline 2010 & 152.6 & 120.7 & $79.1 \%$ \\
\hline 2011 & 157.9 & 124.7 & $79.0 \%$ \\
\hline 2012 & 167.6 & 134.7 & $80.4 \%$ \\
\hline 2013 & 181.5 & 150.1 & $82.7 \%$ \\
\hline 2014 & 194.0 & 160.8 & $82.9 \%$ \\
\hline 2015 & 200.8 & 161.6 & $80.5 \%$ \\
\hline 2016 & 208.9 & 166.5 & $79.7 \%$ \\
\hline \multicolumn{4}{|c|}{ Motor vehicle parts } \\
\hline 1990 & 653.0 & 527.4 & 80.8\% \\
\hline 1995 & 786.9 & 647.7 & $82.3 \%$ \\
\hline 1996 & 799.9 & 657.4 & $82.2 \%$ \\
\hline 1997 & 808.9 & 662.4 & $81.9 \%$ \\
\hline 1998 & 818.2 & 660.3 & $80.7 \%$ \\
\hline 1999 & 837.1 & 674.2 & $80.5 \%$ \\
\hline 2000 & 839.5 & 676.7 & $80.6 \%$ \\
\hline 2001 & 774.7 & 624.9 & $80.7 \%$ \\
\hline 2002 & 733.6 & 590.9 & $80.5 \%$ \\
\hline 2003 & 707.8 & 567.6 & $80.2 \%$ \\
\hline 2004 & 692.1 & 561.6 & $81.1 \%$ \\
\hline 2005 & 678.1 & 553.9 & $81.7 \%$ \\
\hline 2006 & 654.7 & 533.7 & $81.5 \%$ \\
\hline 2007 & 607.9 & 488.9 & $80.4 \%$ \\
\hline 2008 & 543.7 & 430.6 & $79.2 \%$ \\
\hline 2009 & 413.7 & 317.8 & $76.8 \%$ \\
\hline 2010 & 418.9 & 323.3 & $77.2 \%$ \\
\hline 2011 & 445.5 & 345.0 & $77.4 \%$ \\
\hline 2012 & 482.8 & 365.3 & $75.7 \%$ \\
\hline 2013 & 508.7 & 385.2 & $75.7 \%$ \\
\hline 2014 & 537.0 & 415.9 & $77.4 \%$ \\
\hline 2015 & 564.9 & 436.7 & $77.3 \%$ \\
\hline 2016 & 580.0 & 447.7 & $77.2 \%$ \\
\hline
\end{tabular}

Source:

Tabulated from the U.S. Department of Labor, Bureau of Labor Statistics, www.bls.gov, August 2017.

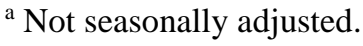


$10-26$ 


\section{Chapter 11 \\ Greenhouse Gas Emissions}

Summary Statistics from Tables/Figures in this Chapter

\begin{tabular}{|c|c|c|c|}
\hline Source & & & \\
\hline \multirow[t]{8}{*}{ Table 11.1} & Carbon dioxide emissions (million metric tons) & 1990 & 2016 \\
\hline & United States & 4,989 & 5,436 \\
\hline & OECD Europe & 4,149 & 4,100 \\
\hline & China & 2,293 & 9,285 \\
\hline & Russia & 2,393 & 1,734 \\
\hline & Japan & 1,054 & 1,194 \\
\hline & Non-OECD Europe and Eurasia & 4,246 & 2,804 \\
\hline & India & 573 & 1,982 \\
\hline \multirow[t]{4}{*}{ Table 11.5} & \multicolumn{3}{|c|}{$\begin{array}{l}\text { Transportation share of U.S. carbon dioxide emissions from fossil fuel } \\
\text { consumption }\end{array}$} \\
\hline & 1990 & & $31.8 \%$ \\
\hline & 2005 & & $33.2 \%$ \\
\hline & 2015 & & $34.7 \%$ \\
\hline Table 11.7 & \multicolumn{2}{|c|}{$\begin{array}{l}\text { Motor gasoline share of transportation carbon dioxide } \\
\text { emissions, } 2015\end{array}$} & $61.5 \%$ \\
\hline \multirow[t]{3}{*}{ Table 11.11} & \multicolumn{3}{|c|}{ Average annual carbon footprint, 2016 (metric tons of $\mathrm{CO}_{2}$ ) } \\
\hline & New Cars & & 5.9 \\
\hline & New Light trucks & & 7.9 \\
\hline
\end{tabular}


The U.S. accounted for 23\% of the World's carbon dioxide emissions in 1990, 21\% in 2005, and only 16\% in 2016. About $42 \%$ of the U.S. carbon emissions are from oil use.

Table 11.1

World Carbon Dioxide Emissions, 1990, 2005, and 2016

\begin{tabular}{|c|c|c|c|c|c|c|}
\hline \multirow[b]{2}{*}{ Country/Region } & \multicolumn{2}{|c|}{1990} & \multicolumn{2}{|c|}{2005} & \multicolumn{2}{|c|}{2016} \\
\hline & $\begin{array}{c}\text { Million } \\
\text { metric } \\
\text { tons }\end{array}$ & $\begin{array}{l}\text { Percent of } \\
\text { emissions } \\
\text { from oil use }\end{array}$ & $\begin{array}{c}\text { Million } \\
\text { metric } \\
\text { tons }\end{array}$ & $\begin{array}{l}\text { Percent of } \\
\text { emissions } \\
\text { from oil use }\end{array}$ & $\begin{array}{c}\text { Million } \\
\text { metric } \\
\text { tons }\end{array}$ & $\begin{array}{l}\text { Percent of } \\
\text { emissions } \\
\text { from oil use } \\
\end{array}$ \\
\hline \multicolumn{7}{|l|}{ OECD $^{\mathrm{a}}$ Americas } \\
\hline United States & 4,989 & $44 \%$ & 5,985 & $44 \%$ & 5,436 & $42 \%$ \\
\hline Canada & 471 & $48 \%$ & 620 & $49 \%$ & 558 & $52 \%$ \\
\hline Mexico/Chile & 302 & $77 \%$ & 461 & $66 \%$ & 495 & $59 \%$ \\
\hline Total & 5,762 & $46 \%$ & 7,066 & $46 \%$ & 6,490 & $44 \%$ \\
\hline OECD ${ }^{\mathrm{a}}$ Europe & 4,149 & $45 \%$ & 4,488 & $49 \%$ & 4,100 & $45 \%$ \\
\hline \multicolumn{7}{|l|}{ OECD $^{\text {a }}$ Asia } \\
\hline Japan & 1,054 & $65 \%$ & 1,241 & $52 \%$ & 1,194 & $41 \%$ \\
\hline Australia/New Zealand & 298 & $38 \%$ & 438 & $55 \%$ & 439 & $39 \%$ \\
\hline Other & 243 & $59 \%$ & 494 & $30 \%$ & 717 & $37 \%$ \\
\hline Total & 1,595 & $59 \%$ & 2,173 & $47 \%$ & 2,350 & $40 \%$ \\
\hline \multicolumn{7}{|l|}{ Non-OECD Europe \& } \\
\hline \multicolumn{7}{|l|}{ Eurasia } \\
\hline Russia & 2,393 & $33 \%$ & 1,548 & $25 \%$ & 1,734 & $25 \%$ \\
\hline Other & 1,853 & $32 \%$ & 1,120 & $26 \%$ & 1,070 & $30 \%$ \\
\hline Total & 4,246 & $32 \%$ & 2,668 & $25 \%$ & 2,804 & $27 \%$ \\
\hline \multicolumn{7}{|l|}{ Non-OECD Asia } \\
\hline China & 2,293 & $15 \%$ & 5,490 & $16 \%$ & 9,285 & $16 \%$ \\
\hline India & 573 & $28 \%$ & 1,182 & $27 \%$ & 1,982 & $27 \%$ \\
\hline Other & 811 & $57 \%$ & 1,665 & $53 \%$ & 2,199 & $51 \%$ \\
\hline Total & 3,677 & $26 \%$ & 8,337 & $25 \%$ & 13,465 & $23 \%$ \\
\hline \multicolumn{7}{|l|}{ Other Non-OECD } \\
\hline Middle East & 704 & $70 \%$ & 1,333 & $59 \%$ & 2,156 & $56 \%$ \\
\hline Africa & 659 & $46 \%$ & 978 & $43 \%$ & 1,309 & $43 \%$ \\
\hline Central \& South America & 695 & $76 \%$ & 1,011 & $72 \%$ & 1,290 & $72 \%$ \\
\hline Total & 2,058 & $64 \%$ & 3,322 & $58 \%$ & 4,755 & $57 \%$ \\
\hline Total World & 21,487 & $42 \%$ & 28,054 & $40 \%$ & 33,964 & $36 \%$ \\
\hline
\end{tabular}

Source:

U.S. Department of Energy, Energy Information Administration, International Energy Statistics Databases, and International Energy Outlook, Washington, DC, May 2016. (Additional resources: www.eia.doe.gov) countries.

a OECD is the Organization for Economic Cooperation and Development. See Glossary for included

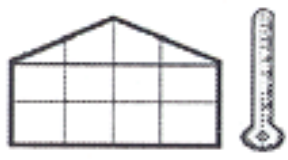


Since 1990, China shows the greatest increase of carbon dioxide $\left(\mathrm{CO}_{2}\right)$ emissions. The Americas have about the same $\mathrm{CO}_{2}$ emissions in 2014 as in 1990. Europe and Eurasia have fewer $\mathrm{CO}_{2}$ emissions in 2016 than 1990.

Figure 11.1. World Carbon Dioxide Emissions, 1990-2016

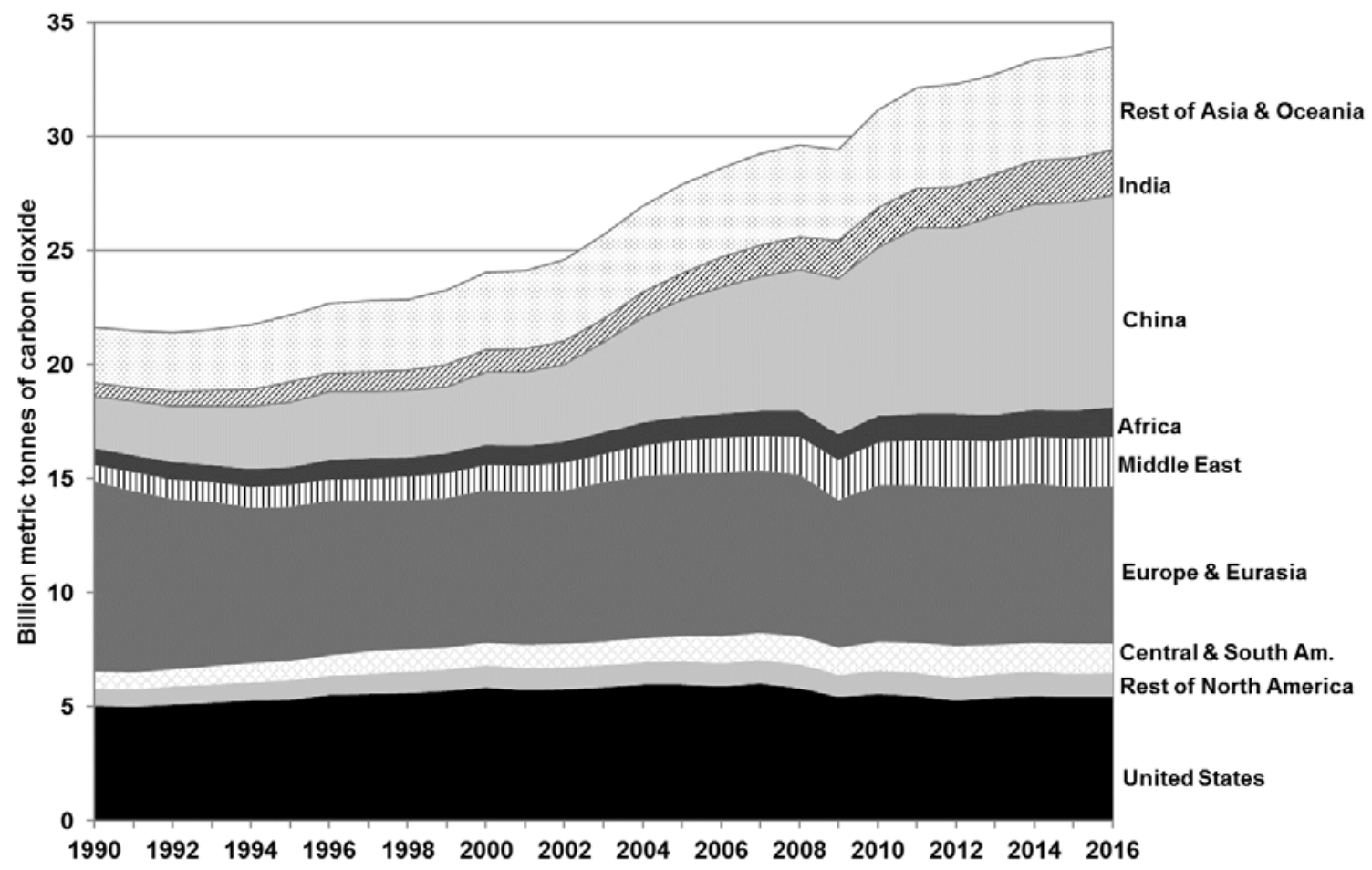

\section{Source:}

1990-2012: U.S. Department of Energy, Energy Information Administration, International Energy Statistics, Total Carbon Dioxide Emissions from the Consumption of Energy, www.eia.doe.gov/cfapps/ipdbproject/IEDIndex3.cfm, September 2016.

2013-2016: U.S. Department of Energy, Energy Information Administration, International Energy Outlook 2016, www.eia.gov/forecasts/aeo/index.cfm, accessed August 2017. (Additional resources: www.eia.doe.gov) 
Global Warming Potentials (GWP) were developed to allow comparison of the ability of each greenhouse gas to trap heat in the atmosphere relative to carbon dioxide. Extensive research has been performed and it has been discovered that the effects of various gases on global warming are too complex to be precisely summarized by a single number. Further understanding of the subject also causes frequent changes to estimates. Despite that, the scientific community has developed approximations, the latest of which are shown below. Most analysts use the 100-year time horizon.

\section{Table 11.2}

Numerical Estimates of Global Warming Potentials Compared with Carbon Dioxide (kilogram of gas per kilogram of carbon dioxide)

\begin{tabular}{|c|c|c|c|}
\hline \multirow[b]{2}{*}{ Gas } & \multirow{2}{*}{$\begin{array}{l}\text { Lifetime } \\
\text { (years) }\end{array}$} & \multicolumn{2}{|c|}{$\begin{array}{c}\text { Global warming potential } \\
\text { direct effect for time horizons of }\end{array}$} \\
\hline & & 20 years & 100 years \\
\hline Carbon Dioxide $\left(\mathrm{CO}_{2}\right)$ & $5-200^{\mathrm{a}}$ & 1 & 1 \\
\hline Methane $\left(\mathrm{CH}_{4}\right)^{\mathrm{b}}$ & 12.4 & 86 & 34 \\
\hline Tetrafluoroethane (HFC-134a) & 13.4 & 3,790 & 1,550 \\
\hline Trichlorofluoromethane (CFC-11) & 45 & 7,020 & 5,350 \\
\hline Nitrous Oxide $\left(\mathrm{N}_{2} \mathrm{O}\right)$ & 121 & 268 & 298 \\
\hline Perfluoromethane $\left(\mathrm{CF}_{4}\right)$ & 50,000 & 4,950 & 7,350 \\
\hline
\end{tabular}

Note: Includes climate-carbon feedbacks.

Source:

Myhre, G., D. Shindell, F.-M. Breon, W. Collins, J. Fuglestvedt, J. Huang, D. Koch, J.-F. Lamarque, D. Lee, B. Mendoza, T. Nakajima, A. Robock, G. Stephens, T. Takemura and H. Zhang, 2013: Anthropogenic and Natural Radiative Forcing. In: Climate Change 2013: The Physical Science Basis. Contribution of Working Group 1 to the Fifth Assessment Report of the Intergovernmental Panel on Climate Change [Stocker, R.F., D. Qin, G.-K. Plattner, M. Tignor, S.K. Allen, J. Boschung, A. Nauels, Y. Zia, V. Bex and P.M. Midgley (eds)]. Cambridge University Press, Cambridge, United Kingdom and New York, NY, USA.

a No single lifetime can be defined for carbon dioxide due to different rates of uptake by different removal processes.

b These values do not include carbon dioxide from methane oxidation. Perturbation lifetime is used in the calculation of metrics. 
Carbon dioxide emissions in 2015 were 5\% higher than in 1990, but down from the highest annual emissions of this data series in 2007. Carbon dioxide accounts for the majority (82\%) of greenhouse gases.

Table 11.3

U.S. Emissions of Greenhouse Gases, Based on Global Warming Potential, 1990-2015 (million metric tons of carbon dioxide equivalent ${ }^{\mathrm{a}}$ )

\begin{tabular}{cccccc}
\hline Year & $\begin{array}{c}\text { Carbon } \\
\text { dioxide }\end{array}$ & Methane & $\begin{array}{c}\text { Nitrous } \\
\text { oxide }\end{array}$ & $\begin{array}{c}\text { High } \\
\text { GWP gases }\end{array}$ & Total \\
\hline 1990 & $5,089.8$ & 780.7 & 359.4 & 99.6 & $6,329.5$ \\
1991 & $5,034.7$ & 785.1 & 358.6 & 90.6 & $6,269.0$ \\
1992 & $5,141.3$ & 784.2 & 361.7 & 95.2 & $6,382.4$ \\
1993 & $5,253.8$ & 769.0 & 373.9 & 94.9 & $6,491.6$ \\
1994 & $5,344.6$ & 772.8 & 364.9 & 97.9 & $6,580.2$ \\
1995 & $5,410.0$ & 765.1 & 377.0 & 116.6 & $6,668.7$ \\
1996 & $5,596.2$ & 756.4 & 383.3 & 127.0 & $6,862.9$ \\
1997 & $5,671.6$ & 740.3 & 374.1 & 133.8 & $6,919.8$ \\
1998 & $5,711.7$ & 725.6 & 388.7 & 149.2 & $6,975.2$ \\
1999 & $5,784.4$ & 713.8 & 362.8 & 145.6 & $7,006.6$ \\
2000 & $5,955.8$ & 704.0 & 361.8 & 146.3 & $7,167.9$ \\
2001 & $5,849.8$ & 695.6 & 363.7 & 132.8 & $7,041.9$ \\
2002 & $5,892.4$ & 684.1 & 362.0 & 140.6 & $7,079.1$ \\
2003 & $5,932.9$ & 684.3 & 365.8 & 130.4 & $7,113.4$ \\
2004 & $6,046.0$ & 675.8 & 385.8 & 137.4 & $7,245.0$ \\
2005 & $6,074.0$ & 680.9 & 361.5 & 138.8 & $7,255.2$ \\
2006 & $5,992.8$ & 682.1 & 371.2 & 141.7 & $7,187.8$ \\
2007 & $6,078.7$ & 685.9 & 378.7 & 153.5 & $7,296.8$ \\
2008 & $5,883.5$ & 695.0 & 361.4 & 155.6 & $7,095.5$ \\
2009 & $5,448.6$ & 690.4 & 362.1 & 151.8 & $6,652.9$ \\
2010 & $5,653.4$ & 692.0 & 370.3 & 163.0 & $6,878.7$ \\
2011 & $5,523.6$ & 672.1 & 363.9 & 170.9 & $6,730.5$ \\
2012 & $5,313.7$ & 666.0 & 340.5 & 169.3 & $6,489.5$ \\
2013 & $5,466.1$ & 658.8 & 335.5 & 171.7 & $6,632.1$ \\
2014 & $5,519.1$ & 659.1 & 335.4 & 179.5 & $6,693.1$ \\
2015 & $5,364.9$ & 655.7 & 334.6 & 184.6 & $6,539.8$ \\
\hline
\end{tabular}

Note: This greenhouse gas emissions inventory includes fossil fuel combustion, use of fluorinated gases and other transportation categories.

Source:

U.S. Environmental Protection Agency, Inventory of U. S. Greenhouse Gas Emissions and Sinks: 1990-2015, April 13, 2017, EPA 430-P-17-001. (Additional resources: www.epa.gov/ghgemissions/inventory-us-greenhouse-gasemissions-and-sinks-1990-2015)

${ }^{a}$ Carbon dioxide equivalents are computed by multiplying the weight of the gas being measured by its estimated Global Warming Potential (See Table 11.2).

${ }^{\mathrm{b}}$ GWP = Global warming potential. Includes HFC-hydrofluorocarbons; PFC-perfluorocarbons; and $\mathrm{SF}_{6}$-sulfur hexaflouride. 
The transportation sector accounts for 32.6\% of carbon dioxide emissions. The industrial sector is the only sector that accounts for more greenhouse gas emissions than the transportation sector.

Table 11.4

Total U.S. Greenhouse Gas Emissions by End-Use Sector, 2015 (million metric tons of carbon dioxide equivalent ${ }^{\mathrm{a}}$ )

\begin{tabular}{lrrrrr}
\hline & & & & \multicolumn{2}{c}{ Total } \\
& $\begin{array}{c}\text { Carbon } \\
\text { dioxide }\end{array}$ & Methane & $\begin{array}{c}\text { Nitrous } \\
\text { oxide }\end{array}$ & $\begin{array}{c}\text { Hydroflurocarbons, } \\
\text { perflurocarbons, } \\
\text { sulfur hexafluoride }\end{array}$ & $\begin{array}{c}\text { greenhouse } \\
\text { emissions }\end{array}$ \\
\hline Residential & $1,009.7$ & 4.1 & 7.9 & 49.9 & $1,071.6$ \\
Commercial & 915.0 & 133.9 & 14.1 & 51.6 & $1,114.6$ \\
Agricultural & 97.5 & 244.5 & 269.9 & 0.1 & 612.0 \\
Industrial & $1,592.6$ & 271.6 & 29.1 & 37.9 & $1,931.2$ \\
Transportation & $1,750.1$ & 1.6 & 13.6 & 45.1 & $1,810.4$ \\
Transportation share of total & $32.6 \%$ & $0.2 \%$ & $4.1 \%$ & $24.4 \%$ & $27.7 \%$ \\
\hline Total greenhouse gas emissions & $5,364.9$ & 655.7 & 334.6 & 184.6 & $6,539.8$ \\
\hline
\end{tabular}

Note: Totals may not sum due to rounding.

\section{Source:}

U.S. Environmental Protection Agency, Inventory of U. S. Greenhouse Gas Emissions and Sinks: 1990-2015, April 13, 2017, EPA 430-P-17-001. (Additional resources: www.epa.gov/ghgemissions/inventory-us-greenhouse-gasemissions-and-sinks-1990-2015)

${ }^{\text {a }}$ Carbon dioxide equivalents are computed by multiplying the weight of the gas being measured by its estimated Global Warming Potential (See Table 11.2). 
Gases which contain carbon can be measured in terms of the full molecular weight of the gas or just in terms of their carbon content. This table presents carbon dioxide gas. The ratio of the weight of carbon to carbon dioxide is 0.2727 . The transportation sector accounts for approximately one-third of carbon emissions.

Table 11.5

\section{U.S. Carbon Emissions from Fossil Fuel Consumption by End-Use Sector, 1990-2015 (million metric tons of carbon dioxide)}

\begin{tabular}{|c|c|c|c|c|c|c|}
\hline & \multicolumn{4}{|c|}{ End use sector } & \multirow{2}{*}{$\begin{array}{l}\text { Transportation } \\
\text { percentage }\end{array}$} & \multirow{2}{*}{$\begin{array}{l}\mathrm{CO}_{2} \text { from } \\
\text { all sectors }\end{array}$} \\
\hline & Residential & Commercial & Industrial & Transportation & & \\
\hline 1990 & 931.4 & 755.4 & 1529.2 & 1496.8 & $31.8 \%$ & $4,712.8$ \\
\hline 1991 & 949.1 & 762.1 & 1497.0 & 1450.6 & $31.1 \%$ & $4,658.8$ \\
\hline 1992 & 945.2 & 758.0 & 1557.0 & 1499.8 & $31.5 \%$ & $4,760.0$ \\
\hline 1993 & 998.0 & 782.3 & 1565.1 & 1535.4 & $31.5 \%$ & $4,880.8$ \\
\hline 1994 & 989.0 & 794.8 & 1588.4 & 1580.1 & $31.9 \%$ & $4,952.3$ \\
\hline 1995 & 994.7 & 812.4 & 1586.7 & 1612.9 & $32.2 \%$ & $5,006.7$ \\
\hline 1996 & 1055.4 & 843.2 & 1644.2 & 1657.4 & $31.9 \%$ & $5,200.2$ \\
\hline 1997 & 1045.2 & 883.0 & 1662.3 & 1673.2 & $31.8 \%$ & $5,263.7$ \\
\hline 1998 & 1049.7 & 901.2 & 1639.6 & 1709.8 & $32.3 \%$ & $5,300.3$ \\
\hline 1999 & 1070.5 & 912.8 & 1619.2 & 1764.3 & $32.9 \%$ & $5,366.8$ \\
\hline 2000 & 1133.2 & 972.2 & 1643.8 & 1808.9 & $32.5 \%$ & $5,558.1$ \\
\hline 2001 & 1124.9 & 980.7 & 1578.8 & 1793.1 & $32.7 \%$ & $5,477.5$ \\
\hline 2002 & 1151.7 & 978.8 & 1553.5 & 1834.1 & $33.2 \%$ & $5,518.1$ \\
\hline 2003 & 1181.7 & 989.4 & 1572.5 & 1826.5 & $32.8 \%$ & $5,570.1$ \\
\hline 2004 & 1179.6 & 1007.7 & 1598.1 & 1871.7 & $33.1 \%$ & $5,657.1$ \\
\hline 2005 & 1214.1 & 1026.8 & 1564.6 & 1891.8 & $33.2 \%$ & $5,697.3$ \\
\hline 2006 & 1151.8 & 1007.2 & 1564.2 & 1887.2 & $33.6 \%$ & $5,610.4$ \\
\hline 2007 & 1204.5 & 1047.3 & 1563.1 & 1891.1 & $33.1 \%$ & $5,706.0$ \\
\hline 2008 & 1190.4 & 1039.3 & 1499.8 & 1796.5 & $32.5 \%$ & $5,526.0$ \\
\hline 2009 & 1122.7 & 976.5 & 1329.5 & 1721.4 & $33.4 \%$ & $5,150.1$ \\
\hline 2010 & 1174.6 & 993.0 & 1416.5 & 1732.7 & $32.6 \%$ & $5,316.8$ \\
\hline 2011 & 1116.2 & 958.4 & 1399.6 & 1711.9 & $33.0 \%$ & $5,186.1$ \\
\hline 2012 & 1007.8 & 897.0 & 1375.7 & 1700.6 & $34.1 \%$ & $4,981.1$ \\
\hline 2013 & 1064.6 & 925.5 & 1407.0 & 1717.0 & $33.6 \%$ & $5,114.1$ \\
\hline 2014 & 1080.1 & 934.7 & 1399.3 & 1746.9 & $33.8 \%$ & $5,161.0$ \\
\hline 2015 & 1003.9 & 909.4 & 1355.0 & 1740.1 & $34.7 \%$ & $5,008.4$ \\
\hline \multicolumn{7}{|c|}{ Average annual percentage change } \\
\hline 1990-2015 & $0.3 \%$ & $0.7 \%$ & $-0.5 \%$ & $0.6 \%$ & & $0.2 \%$ \\
\hline 2005-2015 & $-1.9 \%$ & $-1.2 \%$ & $-1.4 \%$ & $-0.8 \%$ & & $-1.3 \%$ \\
\hline
\end{tabular}

Note: The $\mathrm{CO}_{2}$ from all sectors does not match Table 11.3 since it is only from fossil fuel consumption and does not include the use of fluorinated gases and other transportation categories.

\section{Source:}

U.S. Environmental Protection Agency, Inventory of U. S. Greenhouse Gas Emissions and Sinks: 1990-2015, April 13, 2017, EPA 430-P-17-001. (Additional resources: www.epa.gov/ghgemissions/inventory-us-greenhouse-gasemissions-and-sinks-1990-2015)

${ }^{a}$ Includes energy from petroleum, coal, and natural gas. Electric utility emissions are distributed across consumption sectors. 
This report has typically displayed carbon and carbon dioxide data from the Environmental Protection Agency (EPA). However, the Energy Information Administration's (EIA's) Monthly Energy Review also includes carbon dioxide emission data. The differences in the two-data series have been about 3-4\%, but as high as 6.8\% in 1991 . Reasons for the differences include the treatment of international bunker fuel, nonfuel use of fossil fuels, and the agencies' use of different fuel consumption control totals.

Table 11.6

Transportation Sector Carbon Dioxide Emissions from

Energy Consumption, 1973-2016 (million metric tons of carbon dioxide)

\begin{tabular}{|c|c|c|c|}
\hline Year & $\begin{array}{c}\text { Energy Information } \\
\text { Administration's Monthly } \\
\text { Energy Review }\end{array}$ & $\begin{array}{c}\text { Environmental Protection } \\
\text { Agency’s Greenhouse Gas } \\
\text { Inventory Report }\end{array}$ & Percentage difference \\
\hline 1973 & $1,315.2$ & a & $\mathrm{a}$ \\
\hline 1975 & $1,291.6$ & a & a \\
\hline 1980 & $1,400.2$ & a & a \\
\hline 1985 & $1,421.2$ & a & a \\
\hline 1990 & $1,587.7$ & $1,508.7$ & $5.0 \%$ \\
\hline 1991 & $1,567.9$ & $1,461.2$ & $6.8 \%$ \\
\hline 1992 & $1,591.6$ & $1,510.6$ & $5.1 \%$ \\
\hline 1993 & $1,607.2$ & $1,546.5$ & $3.8 \%$ \\
\hline 1994 & $1,647.4$ & $1,591.7$ & $3.4 \%$ \\
\hline 1995 & $1,681.3$ & $1,624.3$ & $3.4 \%$ \\
\hline 1996 & $1,725.2$ & $1,668.4$ & $3.3 \%$ \\
\hline 1997 & $1,744.2$ & $1,684.8$ & $3.4 \%$ \\
\hline 1998 & $1,782.0$ & $1,722.0$ & $3.4 \%$ \\
\hline 1999 & $1,828.0$ & $1,776.5$ & $2.8 \%$ \\
\hline 2000 & $1,872.5$ & $1,821.0$ & $2.8 \%$ \\
\hline 2001 & $1,852.0$ & $1,804.2$ & $2.6 \%$ \\
\hline 2002 & $1,892.5$ & $1,845.1$ & $2.5 \%$ \\
\hline 2003 & $1,892.1$ & $1,836.7$ & $2.9 \%$ \\
\hline 2004 & $1,958.6$ & $1,882.0$ & $3.9 \%$ \\
\hline 2005 & $1,985.6$ & $1,902.0$ & $4.2 \%$ \\
\hline 2006 & $2,013.7$ & 1,897.1 & $5.8 \%$ \\
\hline 2007 & $2,021.0$ & $1,901.5$ & $5.9 \%$ \\
\hline 2008 & $1,897.9$ & $1,806.0$ & $4.8 \%$ \\
\hline 2009 & $1,831.6$ & $1,730.0$ & $5.5 \%$ \\
\hline 2010 & $1,849.0$ & $1,742.3$ & $5.8 \%$ \\
\hline 2011 & $1,817.5$ & $1,720.9$ & $5.3 \%$ \\
\hline 2012 & $1,780.2$ & $1,708.9$ & $4.0 \%$ \\
\hline 2013 & $1,807.0$ & $1,725.8$ & $4.5 \%$ \\
\hline 2014 & $1,825.1$ & $1,756.1$ & $3.8 \%$ \\
\hline 2015 & $1,882.8$ & a & $5.3 \%$ \\
\hline 2016 & $1,882.8$ & a & $\mathrm{a}$ \\
\hline
\end{tabular}

\section{Sources:}

U.S. Department of Energy, Energy Information Administration, Monthly Energy Review, July 2017, Washington, DC, Table 12.5.

U.S. Environmental Protection Agency, Inventory of U. S. Greenhouse Gas Emissions and Sinks: 1990-2015, April 13, 2017, EPA 430-P-17-001. (Additional resources: www.epa.gov/ghgemissions/inventory-usgreenhouse-gas-emissions-and-sinks-1990-2015)

${ }^{\text {a }}$ Data are not available. 
Most U.S. transportation sector carbon dioxide emissions come from petroleum fuels. Motor gasoline has been responsible for $60 \%-67 \%$ of U.S. carbon dioxide emissions over the last twenty-five years.

Table 11.7

\section{U.S. Carbon Emissions from Fossil Fuel Combustion in the Transportation End-Use Sector, 1990-2015 (million metric tons of carbon dioxide equivalent)}

\begin{tabular}{|c|c|c|c|c|c|c|c|c|c|}
\hline Year & $\begin{array}{c}\text { Motor } \\
\text { gasoline }\end{array}$ & $\mathrm{LPG}^{\mathrm{a}}$ & Jet fuel & $\begin{array}{l}\text { Distillate } \\
\text { fuel }\end{array}$ & $\begin{array}{l}\text { Residual } \\
\text { fuel }\end{array}$ & $\begin{array}{l}\text { Aviation } \\
\text { gas }\end{array}$ & $\begin{array}{c}\text { Natural } \\
\text { gas }\end{array}$ & Electricity ${ }^{\mathrm{b}}$ & Total \\
\hline 1990 & 983.5 & 1.4 & 184.2 & 262.9 & 22.6 & 3.1 & 36.0 & 3.0 & $1,496.7$ \\
\hline 1991 & 969.7 & 1.3 & 168.8 & 255.2 & 16.9 & 2.9 & 32.9 & 3.0 & $1,450.6$ \\
\hline 1992 & 992.8 & 1.2 & 166.6 & 271.2 & 30.0 & 2.8 & 32.2 & 3.0 & $1,499.7$ \\
\hline 1993 & $1,010.9$ & 1.2 & 168.2 & 287.6 & 27.6 & 2.7 & 34.2 & 3.0 & $1,535.4$ \\
\hline 1994 & $1,022.9$ & 2.1 & 175.5 & 309.4 & 26.9 & 2.6 & 37.6 & 3.1 & $1,580.1$ \\
\hline 1995 & $1,042.4$ & 1.1 & 172.2 & 323.8 & 29.1 & 2.7 & 38.4 & 3.1 & $1,612.9$ \\
\hline 1996 & 1,063.6 & 1.0 & 184.5 & 339.9 & 23.6 & 2.6 & 39.1 & 3.1 & $1,657.4$ \\
\hline 1997 & $1,075.6$ & 0.9 & 184.5 & 354.8 & 10.3 & 2.7 & 41.4 & 3.1 & 1,673.2 \\
\hline 1998 & $1,107.5$ & 1.1 & 188.4 & 366.0 & 5.9 & 2.5 & 35.3 & 3.2 & $1,710.0$ \\
\hline 1999 & $1,128.0$ & 0.9 & 192.4 & 387.9 & 13.2 & 2.7 & 35.8 & 3.2 & $1,764.3$ \\
\hline 2000 & $1,136.2$ & 0.7 & 194.9 & 402.1 & 33.3 & 2.5 & 35.6 & 3.4 & $1,808.8$ \\
\hline 2001 & $1,149.4$ & 0.8 & 189.6 & 400.3 & 12.0 & 2.4 & 34.9 & 3.6 & 1,793.0 \\
\hline 2002 & $1,174.6$ & 0.9 & 185.3 & 413.4 & 17.1 & 2.3 & 37.0 & 3.5 & 1,834.1 \\
\hline 2003 & $1,177.2$ & 1.1 & 179.3 & 421.9 & 7.4 & 2.1 & 33.2 & 4.3 & $1,826.5$ \\
\hline 2004 & $1,194.1$ & 1.2 & 186.6 & 437.1 & 14.0 & 2.2 & 31.9 & 4.5 & $1,871.7$ \\
\hline 2005 & $1,183.7$ & 1.7 & 189.4 & 457.5 & 19.3 & 2.4 & 33.1 & 4.7 & $1,891.7$ \\
\hline 2006 & $1,171.8$ & 1.7 & 182.3 & 468.5 & 23.0 & 2.3 & 33.1 & 4.5 & $1,887.2$ \\
\hline 2007 & $1,166.0$ & 1.4 & 179.5 & 472.9 & 29.0 & 2.2 & 35.2 & 5.1 & 1,891.3 \\
\hline 2008 & $1,109.2$ & 2.5 & 173.0 & 448.1 & 20.4 & 2.0 & 36.7 & 4.7 & 1,796.6 \\
\hline 2009 & $1,101.5$ & 1.7 & 154.1 & 405.9 & 13.9 & 1.8 & 37.9 & 4.5 & 1,721.3 \\
\hline 2010 & $1,092.5$ & 1.8 & 151.5 & 422.0 & 20.4 & 1.9 & 38.1 & 4.5 & $1,732.7$ \\
\hline 2011 & $1,068.8$ & 2.1 & 146.6 & 430.0 & 19.4 & 1.9 & 38.9 & 4.3 & $1,712.0$ \\
\hline 2012 & $1,064.7$ & 2.3 & 143.4 & 427.5 & 15.8 & 1.7 & 41.3 & 3.9 & $1,700.6$ \\
\hline 2013 & $1,065.6$ & 2.7 & 147.1 & 433.9 & 15.1 & 1.5 & 47.0 & 4.0 & $1,716.9$ \\
\hline 2014 & $1,096.1$ & 2.9 & 148.6 & 447.7 & 5.8 & 1.5 & 40.3 & 4.1 & $1,747.0$ \\
\hline 2015 & $1,070.5$ & 3.0 & 157.7 & 460.7 & 4.2 & 1.5 & 38.8 & 3.7 & $1,740.1$ \\
\hline & \multicolumn{9}{|c|}{ Average annual percentage change } \\
\hline $1990-2015$ & $0.3 \%$ & $3.1 \%$ & $-0.6 \%$ & $2.3 \%$ & $-6.5 \%$ & $-2.9 \%$ & $0.3 \%$ & $0.8 \%$ & $0.6 \%$ \\
\hline $2005-2015$ & $-1.0 \%$ & $5.8 \%$ & $-1.8 \%$ & $0.1 \%$ & $-14.2 \%$ & $-4.6 \%$ & $1.6 \%$ & $-2.4 \%$ & $-0.8 \%$ \\
\hline
\end{tabular}

Note: Emissions from U.S. Territories are not included. Emissions from International Bunker Fuels are not included.

\section{Source:}

U.S. Environmental Protection Agency, Inventory of U. S. Greenhouse Gas Emissions and Sinks: 1990-2015, April 13, 2017, EPA 430-P-17-001. (Additional resources: www.epa.gov/ghgemissions/inventory-us-greenhouse-gasemissions-and-sinks-1990-2015)

${ }^{a}$ Liquefied petroleum gas.

${ }^{\mathrm{b}}$ Share of total electric utility carbon dioxide emissions weighted by sales to the transportation sector. 
Highway vehicles are responsible for the majority of greenhouse gas emissions in the transportation sector.

Table 11.8

Transportation Carbon Dioxide Emissions by Mode, 1990-2015 (Million metric tons of carbon dioxide equivalent)

\begin{tabular}{|c|c|c|c|c|c|c|c|c|}
\hline Year & $\begin{array}{c}\text { Passenger } \\
\text { Vehicles }\end{array}$ & $\begin{array}{l}\text { Heavy } \\
\text { Trucks }\end{array}$ & $\begin{array}{c}\text { Highway } \\
\text { Total }\end{array}$ & Water & Air & Rail & Pipeline & Total \\
\hline 1990 & 951.8 & 238.7 & $1,190.5$ & 44.3 & 187.4 & 38.5 & 36.0 & $1,496.7$ \\
\hline 1991 & 936.9 & 233.3 & $1,170.2$ & 39.5 & 171.7 & 36.4 & 32.9 & $1,450.7$ \\
\hline 1992 & 968.5 & 243.8 & $1,212.3$ & 48.5 & 169.4 & 37.4 & 32.2 & $1,499.8$ \\
\hline 1993 & 988.1 & 256.9 & $1,245.0$ & 47.1 & 170.9 & 38.3 & 34.2 & $1,535.5$ \\
\hline 1994 & $1,001.6$ & 273.9 & $1,275.5$ & 47.7 & 178.1 & 41.2 & 37.6 & $1,580.1$ \\
\hline 1995 & $1,015.1$ & 284.2 & 1,299.3 & 57.7 & 174.9 & 42.7 & 38.4 & $1,613.0$ \\
\hline 1996 & $1,037.7$ & 296.5 & 1,334.2 & 53.7 & 187.1 & 43.4 & 39.1 & $1,657.5$ \\
\hline 1997 & $1,051.4$ & 310.5 & 1,361.9 & 39.4 & 187.2 & 43.5 & 41.4 & $1,673.4$ \\
\hline 1998 & $1,082.5$ & 323.9 & $1,406.4$ & 33.4 & 190.9 & 43.9 & 35.2 & $1,709.8$ \\
\hline 1999 & $1,116.1$ & 342.6 & $1,458.7$ & 29.3 & 195.1 & 45.4 & 35.7 & $1,764.2$ \\
\hline 2000 & $1,113.4$ & 356.6 & $1,470.0$ & 60.3 & 197.4 & 45.5 & 35.4 & 1,808.6 \\
\hline 2001 & $1,124.3$ & 354.6 & $1,478.9$ & 41.8 & 192.0 & 45.8 & 34.6 & 1,793.1 \\
\hline 2002 & $1,150.1$ & 367.7 & $1,517.8$ & 46.5 & 187.6 & 45.4 & 36.6 & $1,833.9$ \\
\hline 2003 & $1,163.8$ & 365.0 & $1,528.8$ & 36.5 & 181.4 & 47.1 & 32.7 & $1,826.5$ \\
\hline 2004 & $1,182.2$ & 380.3 & $1,562.5$ & 39.3 & 188.8 & 49.6 & 31.3 & $1,871.5$ \\
\hline 2005 & $1,165.0$ & 408.0 & $1,573.0$ & 44.4 & 191.8 & 50.2 & 32.4 & 1,891.8 \\
\hline 2006 & 1,153.6 & 417.0 & $1,570.6$ & 47.4 & 184.6 & 52.2 & 32.4 & $1,887.2$ \\
\hline 2007 & 1,123.7 & 446.0 & $1,569.7$ & 53.9 & 181.7 & 51.3 & 34.4 & $1,891.0$ \\
\hline 2008 & $1,065.3$ & 428.0 & $1,493.3$ & 44.7 & 175.1 & 47.6 & 35.9 & $1,796.6$ \\
\hline 2009 & $1,061.3$ & 388.5 & $1,449.8$ & 38.0 & 155.9 & 40.4 & 37.1 & $1,721.2$ \\
\hline 2010 & $1,053.4$ & 401.4 & $1,454.8$ & 44.0 & 153.4 & 43.1 & 37.3 & $1,732.6$ \\
\hline 2011 & $1,034.3$ & 400.8 & $1,435.1$ & 45.5 & 148.5 & 44.7 & 38.1 & $1,711.9$ \\
\hline 2012 & $1,030.1$ & 402.2 & $1,432.3$ & 39.3 & 145.1 & 43.4 & 40.5 & $1,700.6$ \\
\hline 2013 & $1,030.3$ & 409.1 & $1,439.4$ & 38.6 & 148.6 & 44.1 & 46.2 & $1,716.9$ \\
\hline 2014 & $1,071.9$ & 422.8 & $1,494.7$ & 16.9 & 150.1 & 45.7 & 39.4 & $1,746.8$ \\
\hline 2015 & $1,037.4$ & 429.7 & $1,467.1$ & 32.3 & 159.2 & 43.6 & 38.0 & $1,740.2$ \\
\hline \multicolumn{9}{|c|}{ Average annual percentage change } \\
\hline 1990-2015 & $0.3 \%$ & $2.4 \%$ & $0.8 \%$ & $-1.3 \%$ & $-0.7 \%$ & $0.5 \%$ & $0.2 \%$ & $0.6 \%$ \\
\hline 2005-2015 & $-1.2 \%$ & $0.5 \%$ & $-0.7 \%$ & $-3.1 \%$ & $-1.8 \%$ & $-1.4 \%$ & $1.6 \%$ & $-0.8 \%$ \\
\hline
\end{tabular}

Note: Emissions from U.S. Territories are not included. Emissions from International Bunker Fuels are not included. Passenger vehicles include cars, light trucks and motorcycles. Heavy trucks include medium and heavy trucks and buses.

Source:

U.S. Environmental Protection Agency, Inventory of U. S. Greenhouse Gas Emissions and Sinks: 1990-2015, April 13, 2017, EPA 430-P-17-001. (Additional resources: www.epa.gov/ghgemissions/inventory-us-greenhouse-gasemissions-and-sinks-1990-2015) 


\section{The Greenhouse Gases, Regulated Emissions, and Energy Use in Transportation (GREET) Model}

\section{greet.es.anl.gov}

Sponsored by the U.S. Department of Energy's Office of Energy Efficiency and Renewable Energy (EERE), Argonne has developed a full life-cycle model called GREET ${ }^{\circledR}$ (Greenhouse gases, Regulated Emissions, and Energy use in Transportation). It allows researchers and analysts to evaluate energy and environmental impacts of various vehicle and fuel combinations on a life-cycle basis. The first version of GREET was released in 1996. Since then, Argonne has continued to update and expand the model. The most recent GREET versions are GREET 12015 version for fuel-cycle analysis and GREET 22015 version for vehicle-cycle analysis.

Figure 11.2. GREET Model

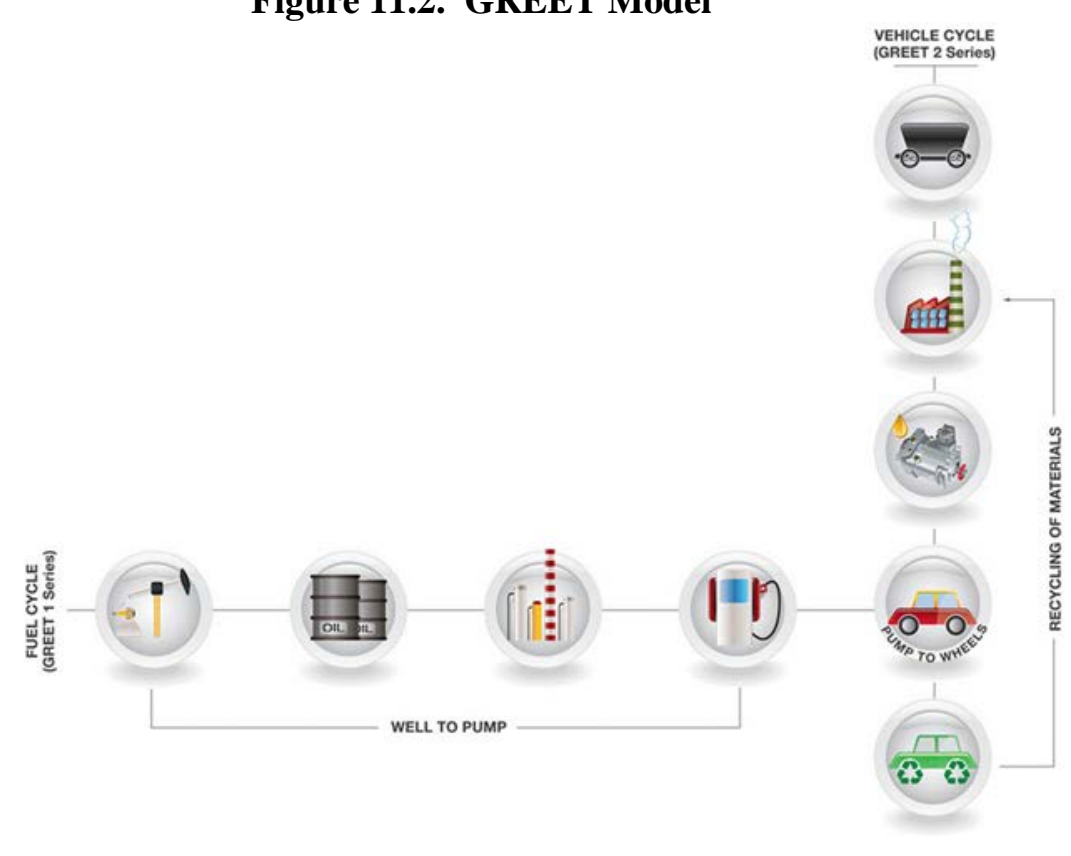

For a given vehicle and fuel system, GREET separately calculates the following:

- Consumption of total energy (energy in non-renewable and renewable sources), fossil fuels (petroleum, natural gas, and coal together), petroleum, coal and natural gas.

- Emissions of $\mathrm{CO}_{2}$-equivalent greenhouse gases - primarily carbon dioxide $\left(\mathrm{CO}_{2}\right)$, methane $\left(\mathrm{CH}_{4}\right)$, and nitrous oxide $\left(\mathrm{N}_{2} \mathrm{O}\right)$.

- Emissions of six criteria pollutants: volatile organic compounds (VOCs), carbon monoxide (CO), nitrogen oxide (NOx), particulate matter with size smaller than 10 micron $\left(\mathrm{PM}_{10}\right)$, particulate matter with size smaller than 2.5 micron ( $\mathrm{PM}_{2.5}$ ), and sulfur oxides (SOx). 
GREET includes more than 100 fuel production pathways and more than 80 vehicle/fuel systems. These vehicle/fuel systems cover current and advanced vehicle technologies such as conventional sparkignition engine vehicles, compression-ignition engine vehicles, hybrid electric vehicles, plug-in hybrid electric vehicles, battery-powered electric vehicles and fuel-cell electric vehicles. GREET also evaluates transportation modes other than light-duty vehicles, such as heavy-duty vehicles, aviation, rail and marine.

\section{Figure 11.3. GREET Model Feedstocks and Fuels}
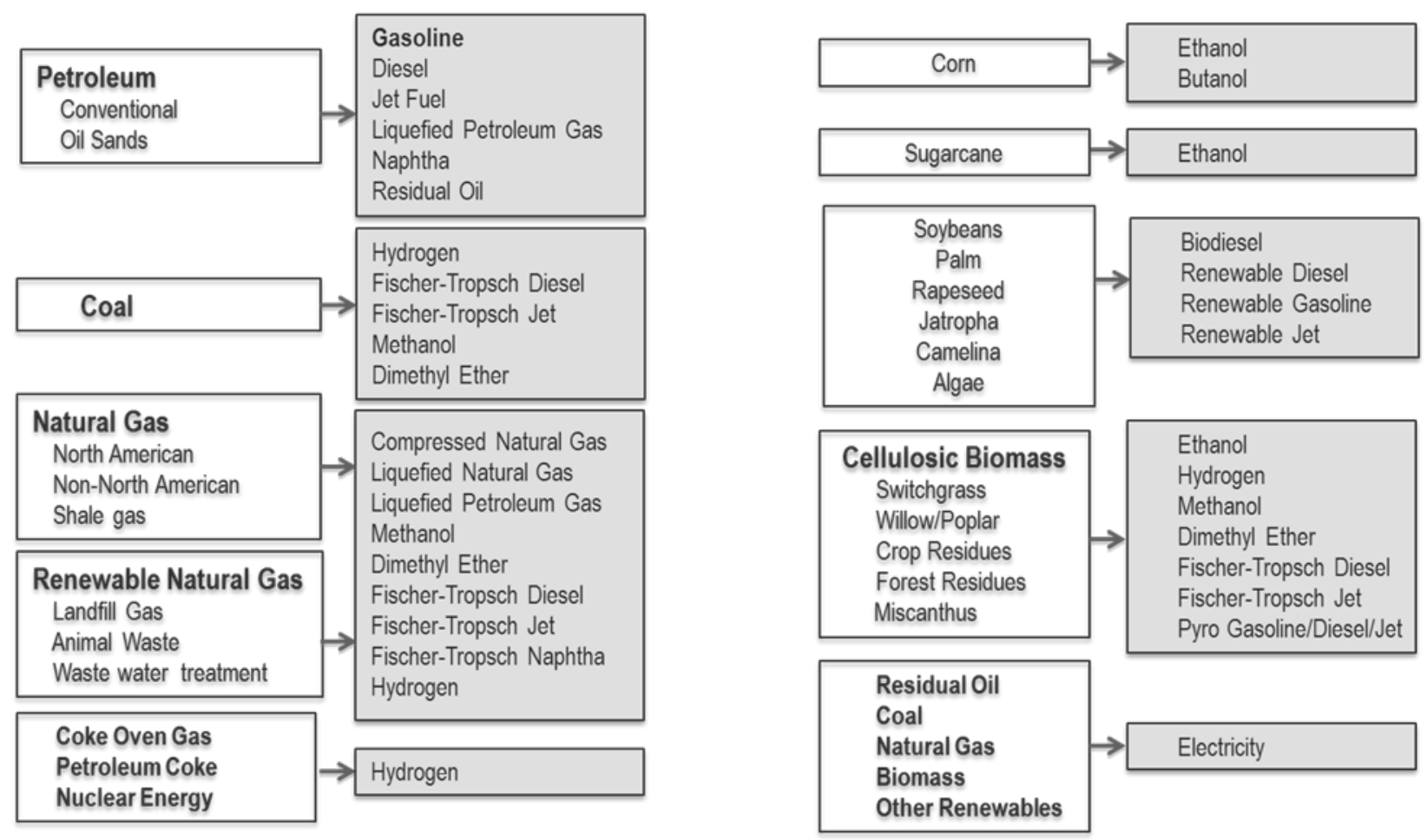

To address technology improvements over time, GREET 2015 simulates current and future vehicle/fuel systems up to year 2040.

For additional information about the GREET model and associated documentation, please visit the GREET website www.greet.es.anl.gov, or contact greet@anl.gov. 
Results from the GREET 2015 model on emissions of carbon dioxide per mile are shown below for various fuels and vehicle technologies. A full description of the model is on the preceding pages.

Figure 11.4. Well-to-Wheel Emissions for Various Fuels and Vehicle Technologies

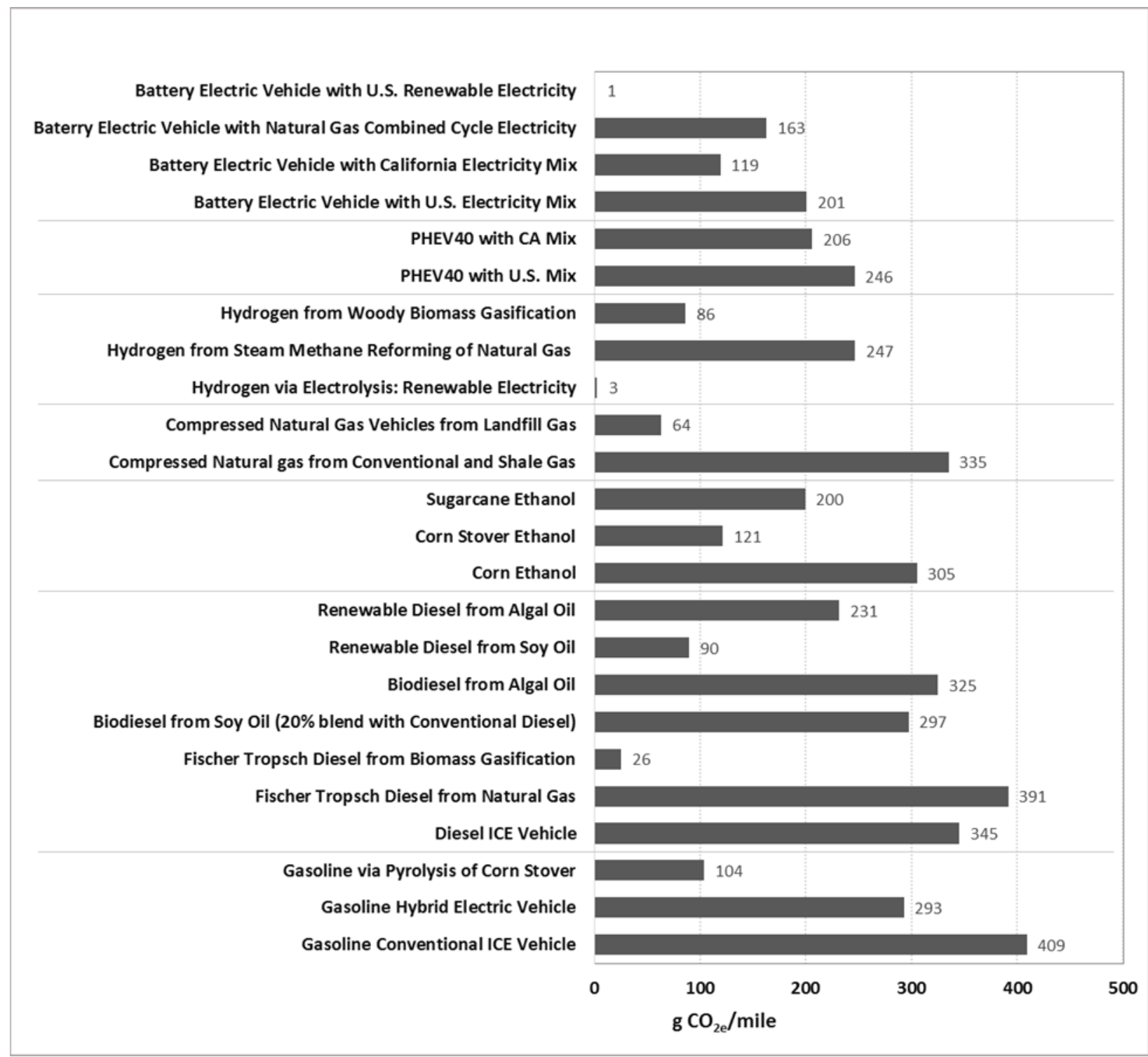

Note: PHEV40 = Plug-in hybrid electric vehicle with 40-mile electric range.

Source: Argonne National Laboratory, GREET 12015 Model. 


\section{Carbon Footprint}

The carbon footprint measures a vehicle's impact on climate change in tons of carbon dioxide $\left(\mathrm{CO}_{2}\right)$ emitted annually. The following three tables show the carbon footprint for various vehicle classes. The sales-weighted average fuel economy rating for each vehicle class, based on $45 \%$ highway and 55\% city driving, is used to determine the average annual carbon footprint for vehicles in the class. An estimate of 15,000 annual miles is used for each vehicle class and for each year in the series.

CarbonFootprint $=\left(\mathrm{CO}_{2} \times L \mathrm{HV} \times \frac{\text { AnnualMiles }}{\text { CombinedMPG }}\right)+\left(\mathrm{CH}_{4}+\mathrm{N}_{2} \mathrm{O}\right) \times$ AnnualMiles

where:

$\mathrm{CO}_{2}=$ (Tailpipe $\mathrm{CO}_{2}+$ Upstream Greenhouse Gases) in grams per million Btu

LHV = Lower (or net) Heating Value in million Btu per gallon

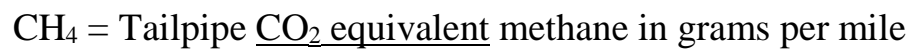

$\mathrm{N}_{2} \mathrm{O}=$ Tailpipe $\underline{\mathrm{CO}}_{2}$ equivalent nitrous oxide in grams per mile

Note: The Environmental Protection Agency publishes tailpipe emissions in terms of grams of $\mathrm{CO}_{2}$ per mile in the Light-Duty Automotive Technology, Carbon Dioxide Emissions, and Fuel Economy Trends: 1975 through 2016, www.epa.gov/fueleconomy/trends-report. 
The production-weighted average annual carbon footprint for cars and car SUVs declined by an average of 1.9\% annually between 1975 and 2016.

Table 11.9

Production-Weighted Annual Carbon Footprint of New Domestic and Import Cars

Model Years 1975-2016

(metric tons of $\mathrm{CO}_{2}$ )

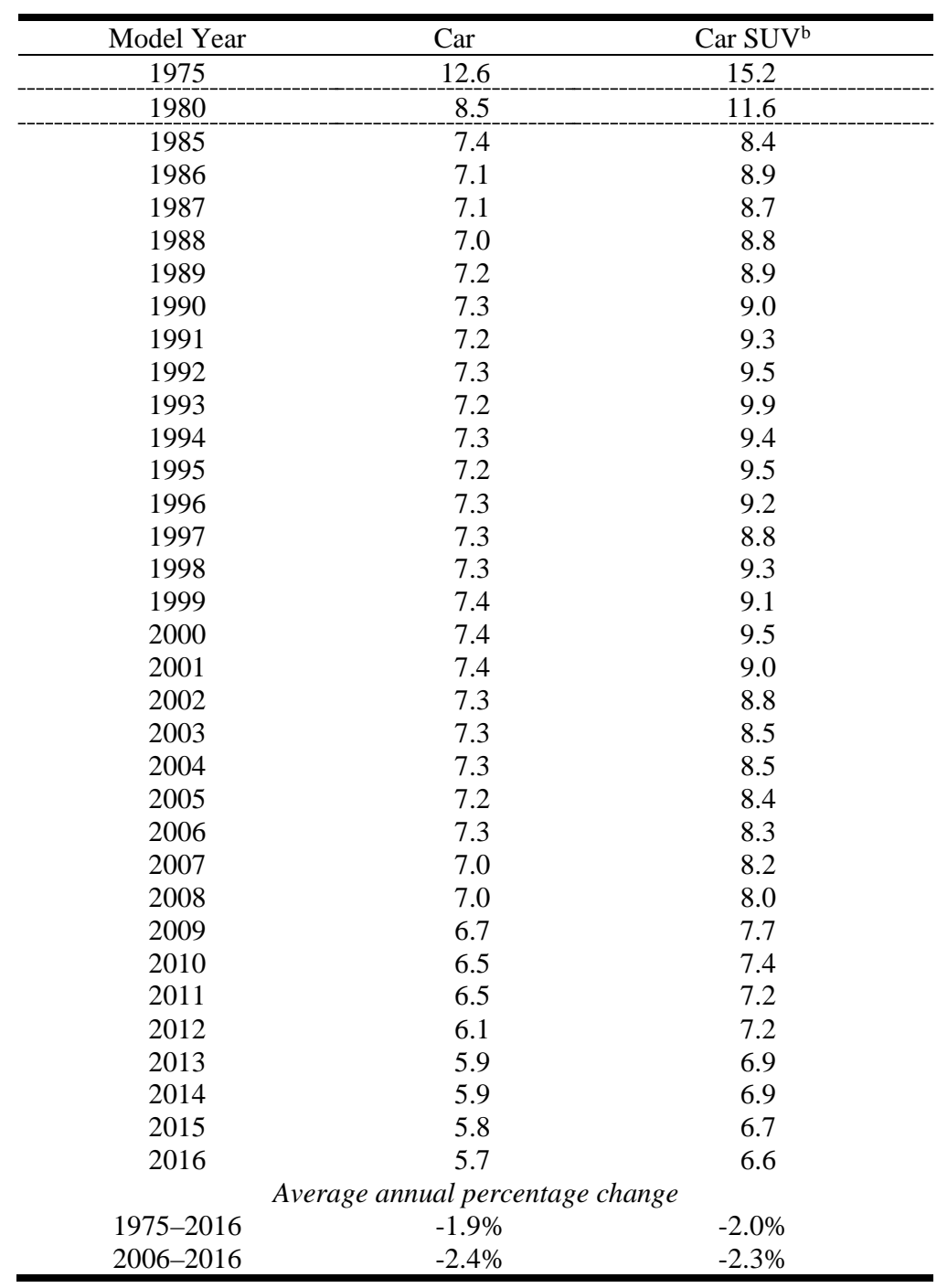

\section{Source:}

Calculated using fuel economy from the U.S. Environmental Protection Agency, Light-Duty Automotive Technology, Carbon Dioxide Emissions, and Fuel Economy Trends: 1975 Through 2016, December 2017. See page 11-14 for details. (Additional resources: www.epa.gov/otaq/fetrends.htm)

a Annual carbon footprint is based on 15,000 miles of annual driving. Includes tailpipe plus upstream emissions.

${ }^{\mathrm{b}}$ Car SUV category is defined in Table 4.9.

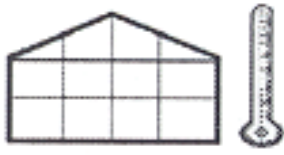


The production-weighted average annual footprint of pickups, vans, and truck SUVs decreased from 1975 to 2016.

Truck SUVs and vans experienced the greatest decline from 2005 to 2016.

Table 11.10

Production-Weighted Annual Carbon Footprint of New Domestic and Import Trucks Model Years 1975-2016

(metric tons of $\mathrm{CO}_{2}$ )

\begin{tabular}{|c|c|c|c|}
\hline Model Year & Pickup & Van & Truck SUV \\
\hline 1975 & 14.2 & 15.2 & 15.3 \\
\hline 1980 & 10.2 & 12.0 & 12.8 \\
\hline 1985 & 9.3 & 10.2 & 10.2 \\
\hline 1990 & 9.7 & 9.5 & 10.3 \\
\hline 1991 & 9.3 & 9.4 & 10.1 \\
\hline 1992 & 9.7 & 9.4 & 10.4 \\
\hline 1993 & 9.6 & 9.3 & 10.4 \\
\hline 1994 & 9.7 & 9.5 & 10.6 \\
\hline 1995 & 10.0 & 9.4 & 10.6 \\
\hline 1996 & 9.9 & 9.2 & 10.4 \\
\hline 1997 & 10.0 & 9.3 & 10.5 \\
\hline 1998 & 10.0 & 9.1 & 10.5 \\
\hline 1999 & 10.4 & 9.3 & 10.5 \\
\hline 2000 & 10.2 & 9.1 & 10.6 \\
\hline 2001 & 10.6 & 9.4 & 10.3 \\
\hline 2002 & 10.7 & 9.1 & 10.4 \\
\hline 2003 & 10.5 & 8.9 & 10.3 \\
\hline 2004 & 10.7 & 8.8 & 10.3 \\
\hline 2005 & 10.7 & 8.8 & 10.1 \\
\hline 2006 & 10.5 & 8.7 & 9.9 \\
\hline 2007 & 10.5 & 8.7 & 9.6 \\
\hline 2008 & 10.3 & 8.5 & 9.3 \\
\hline 2009 & 10.0 & 8.4 & 8.8 \\
\hline 2010 & 10.0 & 8.4 & 8.6 \\
\hline 2011 & 9.8 & 8.1 & 8.5 \\
\hline 2012 & 9.9 & 7.9 & 8.5 \\
\hline 2013 & 9.7 & 8.0 & 8.1 \\
\hline 2014 & 9.4 & 7.9 & 7.8 \\
\hline 2015 & 9.0 & 7.8 & 7.7 \\
\hline 2016 & 8.9 & 7.7 & 7.5 \\
\hline \multicolumn{4}{|c|}{ Average annual percentage change } \\
\hline 1975-2016 & $-1.1 \%$ & $-1.6 \%$ & $-1.7 \%$ \\
\hline 2006-2016 & $-1.6 \%$ & $-1.2 \%$ & $-2.7 \%$ \\
\hline
\end{tabular}

Note: Includes light trucks of 8,500 lbs. or less.

\section{Source:}

Calculated using fuel economy from the U.S. Environmental Protection Agency, Light-Duty Automotive Technology, Carbon Dioxide Emissions, and Fuel Economy Trends: 1975 Through 2016, December 2017. See page 11-14 for details. (Additional resources: www.epa.gov/otaq/fetrends.htm)

${ }^{\text {a }}$ Annual carbon footprint is based on 15,000 miles of annual driving. Includes tailpipe plus upstream emissions.

b Truck SUV category includes all SUV not in the Car SUV category. Car SUV category is defined in Table 4.9. 
Between 1975 and 2016, the production-weighted average annual carbon footprint for new light vehicles dropped dramatically. Total new cars experienced a decrease of 53.4\% while the carbon footprint for light trucks decreased by $45.4 \%$.

Table 11.11

Average Annual Carbon Footprint of New Vehicles by Vehicle Classification, Model Years 1975 and 2016 (metric tons of $\mathrm{CO}_{2}$ )

\begin{tabular}{|c|c|c|c|c|c|}
\hline \multirow[b]{2}{*}{ Vehicle class } & \multicolumn{2}{|c|}{ Production share } & \multicolumn{2}{|c|}{ Carbon footprint } & \multirow[b]{2}{*}{$\begin{array}{r}\text { Percent change } \\
1975 \text { - } 2016\end{array}$} \\
\hline & $\begin{array}{l}\text { Model year } \\
1975\end{array}$ & $\begin{array}{l}\text { Model year } \\
2016\end{array}$ & $\begin{array}{c}\text { Model year } \\
1975\end{array}$ & $\begin{array}{l}\text { Model year } \\
2016\end{array}$ & \\
\hline \multicolumn{6}{|c|}{ Cars } \\
\hline Car & $80.6 \%$ & $51.4 \%$ & 12.6 & 5.7 & $-54.6 \%$ \\
\hline Car SUV & $0.1 \%$ & $10.7 \%$ & 15.2 & 6.6 & $-56.3 \%$ \\
\hline Total cars & $80.7 \%$ & $62.1 \%$ & 12.6 & 5.9 & $-53.4 \%$ \\
\hline \multicolumn{6}{|c|}{ Light trucks } \\
\hline Van & $4.5 \%$ & $3.7 \%$ & 15.2 & 7.7 & $-49.1 \%$ \\
\hline Truck SUV ${ }^{b}$ & $1.7 \%$ & $23.4 \%$ & 15.3 & 7.5 & $-51.1 \%$ \\
\hline Pickup & $13.1 \%$ & $10.8 \%$ & 14.2 & 8.9 & $-37.1 \%$ \\
\hline Total light trucks & $19.3 \%$ & $37.9 \%$ & 14.5 & 7.9 & $-45.4 \%$ \\
\hline
\end{tabular}

\section{Source:}

Calculated using fuel economy from the U.S. Environmental Protection Agency, Light-Duty Automotive Technology, Carbon Dioxide Emissions, and Fuel Economy Trends: 1975 Through 2016, December 2017. See page 11-14 for details. (Additional resources: www.epa.gov/otaq/fetrends.htm) emissions.

${ }^{a}$ Annual carbon footprint is based on 15,000 miles of annual driving. Includes tailpipe and upstream

${ }^{\mathrm{b}}$ Car SUV category is defined in Table 4.9. Truck SUV category includes all SUVs not in the Car SUV category. 
The amount of carbon dioxide released into the atmosphere by a vehicle is primarily determined by the carbon content of the fuel. However, there is a small portion of the fuel that is not oxidized into carbon dioxide when the fuel is burned. The Environmental Protection Agency (EPA) has published information on carbon dioxide emissions from gasoline and diesel which takes the oxidation factor into account and is based on the carbon content used in EPA's fuel economy analyses. The other fuels listed come from the Energy Information Administration.

Table 11.12

Direct Carbon Dioxide Emissions from a Gallon of Fuel ${ }^{\mathrm{a}}$

\begin{tabular}{lccc}
\hline & $\begin{array}{c}\text { Grams } \\
\text { per gallon }\end{array}$ & $\begin{array}{c}\text { Kilograms } \\
\text { per gallon }\end{array}$ & $\begin{array}{c}\text { Pounds } \\
\text { per gallon }\end{array}$ \\
\hline Gasoline & 8,887 & 8.9 & 19.6 \\
Diesel & 10,180 & 10.2 & 22.4 \\
E85 & 1,340 & 1.3 & 3.0 \\
B20 & 8,120 & 8.1 & 17.9 \\
LPG & 5,805 & 5.8 & 12.8 \\
Propane & 5,740 & 5.8 & 12.7 \\
Aviation gasoline & 8,320 & 8.3 & 18.3 \\
Jet fuel & 9,751 & 9.6 & 21.5 \\
Kerosene & 9,751 & 9.8 & 21.5 \\
Residual fuel & 11,791 & 11.8 & 26.0 \\
\hline
\end{tabular}

\section{Sources:}

Gasoline and Diesel: U.S. Environmental Protection Agency, "Greenhouse Gas Emissions from a Typical Passenger Vehicle,” May 2014. (Additional resources: www.epa.gov/otaq)

All others: Energy Information Administration, Voluntary Reporting of Greenhouse Gases Program, Fuel and Energy Source Codes and Emission Coefficients.

a Direct emissions are from the "tank-to-wheels" process. No upstream emissions are included. 
The average carbon content of 11 different transportation fuels comes from the GREET Model. Residual oil (used in ships) has the highest carbon content of those listed. Ethanol has the lowest carbon content per gallon.

Table 11.13

Carbon Content of Transportation Fuels

\begin{tabular}{lcccc}
\hline Fuel Type & $\begin{array}{c}\text { Density } \\
\text { (grams/gallon) }\end{array}$ & $\begin{array}{c}\text { Carbon ratio } \\
\text { (grams of carbon } \\
\text { per grams of fuel) }\end{array}$ & $\begin{array}{c}\text { Carbon content } \\
\text { (grams/gallon) }\end{array}$ & $\begin{array}{c}\text { Carbon content }^{\mathrm{a}} \\
\text { (grams per Btu) }^{\text {(grams }}\end{array}$ \\
\hline Gasoline blendstock & 2,819 & 0.863 & 2,433 & 0.0196 \\
Ethanol & 2,988 & 0.522 & 1,560 & 0.0185 \\
Gasoline (E10) & 2,836 & 0.828 & 2,347 & 0.0195 \\
U.S. conventional diesel & 3,167 & 0.865 & 2,739 & 0.0199 \\
Low-sulfur diesel & 3,206 & 0.871 & 2,792 & 0.0202 \\
Conventional jet fuel & 3,036 & 0.862 & 2,617 & 0.0197 \\
Ultra low-sulfur jet fuel & 2,998 & 0.860 & 2,578 & 0.0196 \\
Residual oil & 3,752 & 0.868 & 3,257 & 0.0217 \\
Liquefied petroleum gas (LPG) & 1,923 & 0.820 & 1,577 & 0.0173 \\
Methyl ester (biodiesel, BD) & 3,361 & 0.776 & 2,608 & 0.0204 \\
\hline
\end{tabular}

Source:

Argonne National Laboratory, GREET 12015 Model.

${ }^{a}$ Based on higher heating values. 
$11-20$ 


\section{Chapter 12 \\ Criteria Air Pollutants}

Summary Statistics from Tables in this Chapter

\begin{tabular}{ccc}
\hline Source & & \\
\hline Table 12.1 & Transportation's share of U.S. emissions, 2016 & $52.3 \%$ \\
& $\mathrm{CO}$ & $57.4 \%$ \\
$\mathrm{NO}$ & & $21.1 \%$ \\
$\mathrm{VOC}$ & $4.6 \%$ \\
$\mathrm{PM}-2.5$ & $1.8 \%$ \\
& $P M-10$ & $1.8 \%$ \\
\hline
\end{tabular}


Transportation accounts for the majority of carbon monoxide and nitrogen oxide emissions. Highway vehicles are responsible for the largest share of transportation emission.

Table 12.1

Total National Emissions of the Criteria Air Pollutants by Sector, 2016 (millions of short tons/percentage)

\begin{tabular}{lrrrrrr}
\hline Sector & CO & NOx & \multicolumn{1}{c}{ VOC } & PM-10 & PM-2.5 & \multicolumn{1}{c}{ SO $_{2}$} \\
\hline Highway vehicles & $\mathbf{1 7 . 8 7}$ & $\mathbf{3 . 6 1}$ & $\mathbf{1 . 7 7}$ & $\mathbf{0 . 2 9}$ & $\mathbf{0 . 1 3}$ & $\mathbf{0 . 0 2}$ \\
& $30.3 \%$ & $34.4 \%$ & $11.1 \%$ & $1.2 \%$ & $2.2 \%$ & $0.7 \%$ \\
Other off-highway & $\mathbf{1 2 . 9 9}$ & $\mathbf{2 . 4 2}$ & $\mathbf{1 . 5 8}$ & $\mathbf{0 . 1 6}$ & $\mathbf{0 . 1 5}$ & $\mathbf{0 . 0 3}$ \\
& $22.0 \%$ & $23.0 \%$ & $9.9 \%$ & $0.7 \%$ & $2.5 \%$ & $1.1 \%$ \\
Transportation total & $\mathbf{3 0 . 8 6}$ & $\mathbf{6 . 0 2}$ & 3.34 & $\mathbf{0 . 4 5}$ & $\mathbf{0 . 2 8}$ & $\mathbf{0 . 0 5}$ \\
& $52.3 \%$ & $57.4 \%$ & $21.1 \%$ & $1.8 \%$ & $4.6 \%$ & $1.8 \%$ \\
Stationary source fuel combustion & $\mathbf{4 . 1 3}$ & $\mathbf{2 . 7 8}$ & $\mathbf{0 . 5 3}$ & $\mathbf{0 . 9 2}$ & $\mathbf{0 . 7 8}$ & $\mathbf{1 . 9 7}$ \\
& $7.0 \%$ & $26.5 \%$ & $3.4 \%$ & $3.8 \%$ & $12.7 \%$ & $72.8 \%$ \\
Industrial processes & $\mathbf{2 . 2 2}$ & $\mathbf{1 . 2 9}$ & 7.30 & $\mathbf{0 . 9 6}$ & $\mathbf{0 . 4 2}$ & $\mathbf{0 . 5 0}$ \\
& $3.8 \%$ & $12.3 \%$ & $46.0 \%$ & $3.9 \%$ & $6.8 \%$ & $18.5 \%$ \\
Waste disposal and recycling total & $\mathbf{2 . 1 6}$ & $\mathbf{0 . 1 1}$ & $\mathbf{0 . 2 0}$ & $\mathbf{0 . 3 1}$ & $\mathbf{0 . 2 5}$ & $\mathbf{0 . 0 4}$ \\
& $3.7 \%$ & $1.1 \%$ & $1.2 \%$ & $1.2 \%$ & $4.1 \%$ & $1.4 \%$ \\
Miscellaneous & $\mathbf{1 9 . 6 2}$ & $\mathbf{0 . 2 9}$ & $\mathbf{4 . 4 9}$ & $\mathbf{2 1 . 8 0}$ & $\mathbf{4 . 4 3}$ & $\mathbf{0 . 1 5}$ \\
Total of all sources & $33.3 \%$ & $2.8 \%$ & $28.3 \%$ & $89.2 \%$ & $71.8 \%$ & $5.5 \%$ \\
& $\mathbf{5 8 . 9 8}$ & $\mathbf{1 0 . 4 9}$ & $\mathbf{1 5 . 8 6}$ & $\mathbf{2 4 . 4 4}$ & $\mathbf{6 . 1 6}$ & $\mathbf{2 . 7 1}$ \\
& $100.0 \%$ & $100.0 \%$ & $100.0 \%$ & $100.0 \%$ & $100.0 \%$ & $100.0 \%$ \\
\hline
\end{tabular}

Note: $\mathrm{CO}=$ Carbon monoxide. $\mathrm{NO}_{\mathrm{x}}=$ Nitrogen oxides. $\mathrm{VOC}=$ Volatile organic compounds. $\mathrm{PM}-10=$ Particulate matter less than 10 microns. PM-2.5 = Particulate matter less than 2.5 microns. $\mathrm{SO}_{2}=$ Sulfur dioxide.

\section{Source:}

U. S. Environmental Protection Agency, National Emission Inventory Air Pollutant Emission Trends website www.epa.gov/air-emissions-inventories/air-pollutant-emissions-trends-data. (Additional resources: www.epa.gov/air-emissions-inventories/national-emissions-inventory) 
The transportation sector accounted for almost 53\% of the nation's carbon monoxide (CO) emissions in 2016. Highway vehicles are by far the source of the greatest amount of $\mathrm{CO}$. For details on the highway emissions of CO, see Table 12.3.

Table 12.2

Total National Emissions of Carbon Monoxide, 1970-2016 (million short tons)

\begin{tabular}{lrrrrrrr}
\hline & & & & & & & $\begin{array}{c}\text { Percent } \\
\text { of total, }\end{array}$ \\
Source category & 1970 & \multicolumn{1}{c}{1980} & \multicolumn{1}{c}{1990} & 2000 & 2010 & 2016 & \multicolumn{1}{c}{2016} \\
\hline Highway vehicles & 163.23 & 143.83 & 110.26 & 68.06 & 28.24 & 17.87 & $30.3 \%$ \\
$\quad$ Other off-highway & 11.37 & 16.69 & 21.45 & 24.18 & 15.35 & 12.99 & $22.0 \%$ \\
Transportation total & 174.60 & 160.52 & 131.71 & 92.24 & 43.59 & 30.86 & $52.3 \%$ \\
Stationary fuel combustion total & 4.63 & 7.30 & 5.51 & 4.78 & 4.52 & 4.13 & $7.0 \%$ \\
Industrial processes total & 9.84 & 6.95 & 4.77 & 2.63 & 1.90 & 2.22 & $3.8 \%$ \\
Waste disposal and recycling total & 7.06 & 2.30 & 1.08 & 1.85 & 1.20 & 2.16 & $3.7 \%$ \\
Miscellaneous total & 7.91 & 8.34 & 11.12 & 12.96 & 22.56 & 19.62 & $33.3 \%$ \\
\hline Total of all sources & $\mathbf{2 0 4 . 0 4}$ & $\mathbf{1 8 5 . 4 1}$ & $\mathbf{1 5 4 . 1 9}$ & $\mathbf{1 1 4 . 4 6}$ & $\mathbf{7 3 . 7 7}$ & $\mathbf{5 8 . 9 8}$ & $\mathbf{1 0 0 . 0 \%}$ \\
\hline
\end{tabular}

Source:

U. S. Environmental Protection Agency, National Emission Inventory Air Pollutant Emission Trends website www.epa.gov/air-emissions-inventories/air-pollutant-emissions-trends-data. (Additional resources: www.epa.gov/air-emissions-inventories/national-emissions-inventory)

a The sums of subcategories may not equal total due to rounding. 
Though gasoline-powered light vehicles continue to be responsible for the majority of carbon monoxide emissions from highway vehicles, the total pollution from light vehicles in 2005 is less than a fifth of what it was in 1970. This is despite the fact that there were many more light vehicles on the road in 2005. Between 2005 and 2011 the Environmental Protection Agency updated their source from the MOBILE 6.2 emissions model to the MOVES emission model. MOVES results typically show higher emissions, especially for heavy trucks. The 2014 data are the latest available.

Table 12.3

Emissions of Carbon Monoxide from Highway Vehicles, 1970-2014 (million short tons)

\begin{tabular}{|c|c|c|c|c|c|c|c|c|}
\hline Source category & 1970 & 1980 & 1990 & 2000 & 2005 & $2011^{b}$ & $2014^{\mathrm{b}}$ & $\begin{array}{c}\text { Percent of } \\
\text { total, } \\
2014 \\
\end{array}$ \\
\hline \multicolumn{9}{|c|}{ Gasoline powered } \\
\hline $\begin{array}{l}\text { Light vehicles \& } \\
\text { motorcycles }\end{array}$ & 119.14 & 98.21 & 67.24 & 36.40 & 24.19 & c & c & c \\
\hline Light trucks ${ }^{\mathrm{d}}$ & 22.27 & 28.83 & 32.23 & 27.04 & 21.19 & c & c & c \\
\hline Subtotal light vehicles & 141.41 & 127.04 & 99.47 & 63.44 & 45.38 & 25.34 & 20.03 & $91.7 \%$ \\
\hline Heavy vehicles & 21.27 & 15.35 & 8.92 & 3.42 & 1.97 & 0.86 & 0.90 & $4.1 \%$ \\
\hline Subtotal gasoline vehicles & 162.68 & 142.39 & 108.39 & 66.86 & 47.35 & 26.20 & 20.93 & $95.8 \%$ \\
\hline \multicolumn{9}{|c|}{ Diesel powered } \\
\hline Light vehicles & 0.01 & 0.03 & 0.04 & 0.01 & 0.01 & c & c & c \\
\hline Light trucks ${ }^{\mathrm{d}}$ & 0.06 & 0.05 & 0.03 & 0.01 & 0.01 & c & c & c \\
\hline Subtotal light vehicles & 0.07 & 0.08 & 0.07 & 0.02 & 0.02 & 0.38 & 0.24 & $1.1 \%$ \\
\hline Heavy vehicles & 0.49 & 1.36 & 1.81 & 1.19 & 0.85 & 0.77 & 0.67 & $3.1 \%$ \\
\hline Subtotal diesel vehicles & 0.56 & 1.43 & 1.87 & 1.20 & 0.87 & 1.15 & 0.91 & $4.2 \%$ \\
\hline \multicolumn{9}{|c|}{ Total } \\
\hline Highway vehicle total & 163.23 & 143.83 & 110.26 & 68.06 & 48.22 & 27.36 & 21.84 & $100.0 \%$ \\
\hline Percent diesel & $0.3 \%$ & $1.0 \%$ & $1.7 \%$ & $1.8 \%$ & $1.8 \%$ & $4.2 \%$ & $4.2 \%$ & \\
\hline
\end{tabular}

\section{Source:}

U. S. Environmental Protection Agency, National Emission Inventory Air Pollutant Emission Trends website www.epa.gov/air-emissions-inventories/national-emissions-inventory-nei. www.epa.gov/air-emissions-inventories/national-emissions-inventory)

(Additional resources:

${ }^{\text {a }}$ The sums of subcategories may not equal total due to rounding.

${ }^{\mathrm{b}}$ These data are not directly comparable to the older data due to the change in source from the MOBILE emissions model to the MOVES emissions model.

${ }^{\mathrm{c}}$ Data are not available.

${ }^{\mathrm{d}}$ Less than 8,500 pounds. 
The transportation sector accounted for almost $60 \%$ of the nation's nitrogen oxide (NOx) emissions in 2016, with the majority coming from highway vehicles. For details on the highway emissions of NOx, see Table 12.5.

Table 12.4

Total National Emissions of Nitrogen Oxides, 1970-2016

(million short tons)

\begin{tabular}{lrrrrrrr}
\hline & & & & & & & $\begin{array}{c}\text { Percent } \\
\text { of total, }\end{array}$ \\
Source category & 1970 & 1980 & 1990 & 2000 & 2010 & 2016 & 2016 \\
\hline Highway vehicles & 12.62 & 11.49 & 9.59 & 8.39 & 5.70 & 3.61 & $34.4 \%$ \\
$\quad$ Other off-highway & 2.65 & 3.35 & 3.78 & 4.17 & 3.32 & 2.42 & $23.0 \%$ \\
Transportation total & 15.27 & 14.84 & 13.37 & 12.56 & 9.02 & 6.02 & $57.4 \%$ \\
Stationary fuel combustion total & 10.06 & 11.32 & 10.89 & 8.82 & 4.33 & 2.78 & $26.5 \%$ \\
Industrial processes total & 0.78 & 0.56 & 0.80 & 0.81 & 1.12 & 1.29 & $12.3 \%$ \\
Waste disposal and recycling total & 0.44 & 0.11 & 0.09 & 0.13 & 0.09 & 0.11 & $1.1 \%$ \\
Miscellaneous total & 0.33 & 0.25 & 0.37 & 0.28 & 0.30 & 0.29 & $2.8 \%$ \\
\hline Total of all sources & $\mathbf{2 6 . 8 8}$ & $\mathbf{2 7 . 0 8}$ & $\mathbf{2 5 . 5 2}$ & $\mathbf{2 2 . 6 0}$ & $\mathbf{1 4 . 8 6}$ & $\mathbf{1 0 . 4 9}$ & $\mathbf{1 0 0 . 0 \%}$ \\
\hline
\end{tabular}

Source:

U. S. Environmental Protection Agency, National Emission Inventory Air Pollutant Emission Trends website www.epa.gov/air-emissions-inventories/air-pollutant-emissions-trends-data. (Additional resources: www.epa.gov/air-emissions-inventories/national-emissions-inventory)

${ }^{a}$ The sums of subcategories may not equal total due to rounding. 
Diesel-powered vehicles were responsible for nearly one-half (49\%) of highway vehicle nitrogen oxide emissions in 2014, while light gasoline vehicles were responsible for the rest. Between 2005 and 2011 the Environmental Protection Agency updated their source from the MOBILE 6.2 emissions model to the MOVES emission model. MOVES results typically show higher emissions, especially for heavy trucks. The 2014 data are the latest available.

Table 12.5

Emissions of Nitrogen Oxides from Highway Vehicles, 1970-2014 (million short tons)

\begin{tabular}{|c|c|c|c|c|c|c|c|c|}
\hline Source category & 1970 & 1980 & 1990 & 2000 & 2005 & $2011^{\mathrm{b}}$ & $2014^{\mathrm{b}}$ & $\begin{array}{c}\text { Percent of } \\
\text { total, } \\
2014 \\
\end{array}$ \\
\hline \multicolumn{9}{|c|}{ Gasoline powered } \\
\hline $\begin{array}{l}\text { Light vehicles \& } \\
\text { motorcycles }\end{array}$ & 8.54 & 6.63 & 4.26 & 2.31 & 1.63 & c & c & c \\
\hline Light trucks ${ }^{\mathrm{d}}$ & 1.54 & 1.58 & 1.50 & 1.44 & 1.56 & c & c & c \\
\hline Subtotal light vehicles & 10.08 & 8.21 & 5.76 & 3.75 & 3.19 & 3.09 & 2.29 & $49.1 \%$ \\
\hline Heavy vehicles & 0.72 & 0.62 & 0.57 & 0.45 & 0.38 & 0.09 & 0.09 & $1.9 \%$ \\
\hline Subtotal gasoline vehicles & 10.81 & 8.83 & 6.33 & 4.20 & 3.57 & 3.18 & 2.38 & $51.0 \%$ \\
\hline \multicolumn{9}{|c|}{ Diesel powered } \\
\hline Light vehicles & 0.00 & 0.03 & 0.04 & 0.01 & 0.00 & c & c & c \\
\hline Light trucks ${ }^{\mathrm{d}}$ & 0.07 & 0.05 & 0.02 & 0.01 & 0.01 & c & c & c \\
\hline Subtotal light vehicles & 0.07 & 0.08 & 0.06 & 0.02 & 0.01 & 0.13 & 0.11 & $2.4 \%$ \\
\hline Heavy vehicles & 1.76 & 2.59 & 3.19 & 4.18 & 2.81 & 2.56 & 2.17 & $46.6 \%$ \\
\hline Subtotal diesel vehicles & 1.83 & 2.66 & 3.26 & 4.19 & 2.82 & 2.69 & 2.28 & $49.0 \%$ \\
\hline \multicolumn{9}{|c|}{ Total } \\
\hline Highway vehicle total & 12.64 & 11.49 & 9.59 & 8.39 & 6.39 & 5.87 & 4.67 & $100.0 \%$ \\
\hline Percent diesel & $14.5 \%$ & $23.1 \%$ & $34.0 \%$ & $49.9 \%$ & $44.1 \%$ & $45.8 \%$ & $49.0 \%$ & \\
\hline
\end{tabular}

Source:

U. S. Environmental Protection Agency, National Emission Inventory Air Pollutant Emission Trends website www.epa.gov/air-emissions-inventories/national-emissions-inventory-nei. (Additional resources: www.epa.gov/air-emissions-inventories/national-emissions-inventory)

${ }^{\mathrm{a}}$ The sums of subcategories may not equal total due to rounding.

${ }^{\mathrm{b}}$ These data are not directly comparable to the older data due to the change in source from the MOBILE emissions model to the MOVES emissions model.

${ }^{\mathrm{c}}$ Data are not available.

${ }^{\mathrm{d}}$ Less than 8,500 pounds. 
The transportation sector accounted for about $21 \%$ of the nation's volatile organic compound (VOC) emissions in 2016, with the majority coming from highway vehicles. For details on the highway emissions of VOC, see Table 12.7.

Table 12.6

Total National Emissions of Volatile Organic Compounds, 1970-2016 (million short tons)

\begin{tabular}{lrrrrrrr}
\hline & & & & & & & $\begin{array}{c}\text { Percent } \\
\text { of total, }\end{array}$ \\
Source category & 1970 & 1980 & 1990 & 2000 & 2010 & 2016 & \multicolumn{1}{c}{2016} \\
\hline Highway vehicles & 16.91 & 13.87 & 9.39 & 5.33 & 2.77 & 1.77 & $11.1 \%$ \\
$\quad$ Off-highway & 1.62 & 2.19 & 2.66 & 2.64 & 2.30 & 1.58 & $9.9 \%$ \\
Transportation total & 18.53 & 16.06 & 12.05 & 7.97 & 5.07 & 3.34 & $21.1 \%$ \\
Stationary fuel combustion total & 0.72 & 1.05 & 1.01 & 1.18 & 0.61 & 0.53 & $3.4 \%$ \\
Industrial processes total & 12.33 & 12.10 & 9.01 & 7.21 & 6.96 & 7.30 & $46.0 \%$ \\
Waste disposal and recycling total & 1.98 & 0.76 & 0.99 & 0.42 & 0.15 & 0.20 & $1.2 \%$ \\
Miscellaneous total & 1.10 & 1.13 & 1.06 & 0.73 & 5.06 & 4.49 & $28.3 \%$ \\
\hline Total of all sources & $\mathbf{3 4 . 6 6}$ & $\mathbf{3 1 . 1 0}$ & $\mathbf{2 4 . 1 2}$ & $\mathbf{1 7 . 5 1}$ & $\mathbf{1 7 . 8 5}$ & $\mathbf{1 5 . 8 6}$ & $\mathbf{1 0 0 . 0 \%}$ \\
\hline
\end{tabular}

Source:

U. S. Environmental Protection Agency, National Emission Inventory Air Pollutant Emission Trends website www.epa.gov/air-emissions-inventories/air-pollutant-emissions-trends-data. (Additional resources: www.epa.gov/air-emissions-inventories/national-emissions-inventory)

a The sum of subcategories may not equal total due to rounding. The EPA's definition of volatile organic compounds excludes methane, ethane, and certain other nonphotochemically reactive organic compounds. 
Gasoline-powered vehicles are responsible for over $90 \%$ of highway vehicle emissions of volatile organic compounds. VOC emissions from highway vehicles in 2014 were less than one-quarter of the 1990 level. Between 2005 and 2011 the Environmental Protection Agency updated their source from the MOBILE 6.2 emissions model to the MOVES emission model. MOVES results typically show higher emissions, especially for heavy trucks. The 2014 data are the latest available.

Table 12.7

Emissions of Volatile Organic Compounds from Highway Vehicles, 1970-2014 ${ }^{\text {a }}$ (thousand short tons)

\begin{tabular}{|c|c|c|c|c|c|c|c|c|}
\hline Source category & 1970 & 1980 & 1990 & 2000 & 2005 & $2011^{\mathrm{b}}$ & $2014^{\mathrm{b}}$ & $\begin{array}{c}\text { Percent } \\
\text { of total, } \\
2014 \\
\end{array}$ \\
\hline \multicolumn{9}{|c|}{ Gasoline powered } \\
\hline Light vehicles \& motorcycles & 11,996 & 9,304 & 5,690 & 2,903 & 2,111 & c & c & c \\
\hline Light trucks ${ }^{\mathrm{d}}$ & 2,776 & 2,864 & 2,617 & 1,929 & 1,629 & c & c & c \\
\hline Subtotal light vehicles & 14,772 & 12,168 & 8,307 & 4,832 & 3,740 & 2,345 & 1,811 & $88.2 \%$ \\
\hline Heavy vehicles & 1,679 & 1,198 & 633 & 256 & 171 & 40 & 41 & $2.0 \%$ \\
\hline Subtotal gasoline vehicles & 16,451 & 13,366 & 8,940 & 5,088 & 3,911 & 2,386 & 1,853 & $90.3 \%$ \\
\hline \multicolumn{9}{|c|}{ Diesel powered } \\
\hline Light vehicles & 8 & 16 & 18 & 3 & 2 & c & c & c \\
\hline Light trucks ${ }^{\mathrm{d}}$ & 41 & 28 & 15 & 4 & 6 & c & c & c \\
\hline Subtotal light vehicles & 49 & 44 & 33 & 7 & 8 & 43 & 26 & $1.3 \%$ \\
\hline Heavy vehicles & 411 & 459 & 415 & 230 & 159 & 213 & 174 & $8.4 \%$ \\
\hline Subtotal diesel vehicles & 460 & 503 & 448 & 238 & 167 & 256 & 200 & $9.7 \%$ \\
\hline \multicolumn{9}{|c|}{ Total } \\
\hline Highway vehicle total & 16,911 & 13,869 & 9,388 & 5,326 & 4,078 & 2,642 & 2,053 & $100.0 \%$ \\
\hline Percent diesel & $2.7 \%$ & $3.6 \%$ & $4.8 \%$ & $4.5 \%$ & $4.1 \%$ & $9.7 \%$ & $9.7 \%$ & \\
\hline
\end{tabular}

\section{Source:}

U. S. Environmental Protection Agency, National Emission Inventory Air Pollutant Emission Trends website www.epa.gov/air-emissions-inventories/national-emissions-inventory-nei. (Additional resources: www.epa.gov/air-emissions-inventories/national-emissions-inventory)

${ }^{a}$ The sums of subcategories may not equal total due to rounding.

b These data are not directly comparable to the older data due to the change in source from the MOBILE emissions model to the MOVES emissions model.

${ }^{\mathrm{c}}$ Data are not available.

${ }^{\mathrm{d}}$ Less than 8,500 pounds. 
The transportation sector accounted for less than 2\% of the nation's particulate matter (PM-10) emissions in 2016.

For details on the highway emissions of PM-10, see Table 12.9.

Table 12.8

Total National Emissions of Particulate Matter (PM-10), 1970-2016 ${ }^{\mathrm{a}}$ (million short tons)

\begin{tabular}{|c|c|c|c|c|c|c|c|}
\hline Source category & 1970 & 1980 & 1990 & 2000 & 2010 & 2016 & $\begin{array}{l}\text { Percent of } \\
\text { total, } 2016 \\
\end{array}$ \\
\hline Highway vehicles & 0.48 & 0.43 & 0.39 & 0.23 & 0.28 & 0.29 & $1.2 \%$ \\
\hline Off-highway & 0.16 & 0.26 & 0.33 & 0.32 & 0.23 & 0.16 & $0.7 \%$ \\
\hline Transportation total & 0.64 & 0.69 & 0.72 & 0.55 & 0.51 & 0.45 & $1.8 \%$ \\
\hline Stationary fuel combustion total & 2.87 & 2.45 & 1.20 & 1.47 & 0.98 & 0.92 & $3.8 \%$ \\
\hline Industrial processes total & 7.67 & 2.75 & 1.04 & 0.71 & 1.05 & 0.96 & $3.9 \%$ \\
\hline Waste disposal and recycling total & 1.00 & 0.27 & 0.27 & 0.36 & 0.21 & 0.31 & $1.2 \%$ \\
\hline Miscellaneous total & 0.84 & 0.85 & 24.54 & 20.65 & 18.08 & 21.80 & $89.2 \%$ \\
\hline Total of all sources & 13.02 & 7.01 & 27.77 & 23.74 & 20.83 & 24.44 & $100.0 \%$ \\
\hline
\end{tabular}

Note: Because PM-10 is fine particle matter less than 10 microns, it also includes PM-2.5. Specific data for PM-2.5 are shown on Tables 12.10 and 12.11 .

\section{Source:}

U. S. Environmental Protection Agency, National Emission Inventory Air Pollutant Emission Trends website www.epa.gov/air-emissions-inventories/air-pollutant-emissions-trends-data. (Additional resources: www.epa.gov/air-emissions-inventories/national-emissions-inventory)

\footnotetext{
${ }^{\text {a }}$ Fine particle matter less than 10 microns. The sums of subcategories may not equal total due to rounding.
} 
In 2014, diesel-powered vehicles were responsible for 45\% of highway vehicle emissions of particulate matter (PM10); in 1990 diesels were responsible for 73.4\%. Between 2005 and 2011 the Environmental Protection Agency updated their source from the MOBILE 6.2 emissions model to the MOVES emission model. MOVES results typically show higher emissions, especially for heavy trucks. The 2014 data are the latest available.

Table 12.9

Emissions of Particulate Matter (PM-10) from Highway Vehicles, 1970-2014 ${ }^{\text {a }}$ (thousand short tons)

\begin{tabular}{|c|c|c|c|c|c|c|c|c|}
\hline Source category & 1970 & 1980 & 1990 & 2000 & 2005 & $2011^{b}$ & $2014^{\mathrm{b}}$ & $\begin{array}{l}\text { Percent of } \\
\text { total, } 2014 \\
\end{array}$ \\
\hline \multicolumn{9}{|c|}{ Gasoline powered } \\
\hline $\begin{array}{l}\text { Light vehicles \& } \\
\text { motorcycles }\end{array}$ & 249 & 141 & 56 & 51 & 46 & c & c & c \\
\hline Light trucks ${ }^{\mathrm{d}}$ & 74 & 49 & 31 & 31 & 35 & c & c & c \\
\hline Subtotal light vehicles & 323 & 190 & 87 & 82 & 81 & 199 & 166 & $53.5 \%$ \\
\hline Heavy vehicles & 44 & 30 & 17 & 10 & 8 & 3 & 5 & $1.5 \%$ \\
\hline Subtotal gasoline vehicles & 367 & 220 & 104 & 92 & 89 & 203 & 171 & $55.0 \%$ \\
\hline \multicolumn{9}{|c|}{ Diesel powered } \\
\hline Light vehicles & 2 & 9 & 11 & 1 & 1 & c & c & c \\
\hline Light trucks ${ }^{\mathrm{d}}$ & 19 & 12 & 5 & 1 & 1 & c & c & c \\
\hline Subtotal light vehicles & 21 & 21 & 16 & 2 & 2 & 10 & 7 & $2.2 \%$ \\
\hline Heavy vehicles & 92 & 191 & 268 & 135 & 92 & 159 & 133 & $42.8 \%$ \\
\hline Subtotal diesel vehicles & 113 & 212 & 284 & 137 & 94 & 168 & 140 & $45.0 \%$ \\
\hline \multicolumn{9}{|c|}{ Total } \\
\hline Highway vehicle total & 480 & 432 & 387 & 230 & 183 & 371 & 311 & $100.0 \%$ \\
\hline Percent diesel & $23.5 \%$ & $49.1 \%$ & $73.4 \%$ & $59.6 \%$ & $51.4 \%$ & $45.3 \%$ & $45.0 \%$ & \\
\hline
\end{tabular}

Note: Because PM-10 is fine particle matter less than 10 microns, it also includes PM-2.5. Specific data for PM-2.5 are shown on Tables 12.10 and 12.11.

\section{Source:}

U. S. Environmental Protection Agency, National Emission Inventory Air Pollutant Emission Trends website www.epa.gov/air-emissions-inventories/national-emissions-inventory-nei. (Additional resources: www.epa.gov/air-emissions-inventories/national-emissions-inventory)

${ }^{a}$ The sums of subcategories may not equal total due to rounding.

${ }^{\mathrm{b}}$ These data are not directly comparable to the older data due to the change in source from the MOBILE emissions model to the MOVES emissions model.

${ }^{\mathrm{c}}$ Data are not available.

${ }^{\mathrm{d}}$ Less than 8,500 pounds. 
The transportation sector accounted for almost 5\% of the nation's particulate matter (PM-2.5) emissions in 2016.

For details on the highway emissions of PM-2.5, see Table 12.11.

Table 12.10

Total National Emissions of Particulate Matter (PM-2.5), 1990-2016 ${ }^{\mathrm{a}}$ (million short tons)

\begin{tabular}{lccccccc}
\hline & & & & & & \multicolumn{3}{c}{$\begin{array}{c}\text { Percent } \\
\text { of total, }\end{array}$} \\
Source category & 1990 & 1995 & 2000 & 2005 & 2010 & 2016 & \multicolumn{2}{c}{2016} \\
\hline Highway vehicles & 0.32 & 0.25 & 0.17 & 0.31 & 0.20 & 0.13 & $2.2 \%$ \\
$\quad$ Off-highway & 0.30 & 0.31 & 0.30 & 0.29 & 0.21 & 0.15 & $2.5 \%$ \\
Transportation total & 0.62 & 0.56 & 0.47 & 0.60 & 0.41 & 0.28 & $4.6 \%$ \\
Stationary fuel combustion total & 0.91 & 0.90 & 1.29 & 1.13 & 0.84 & 0.78 & $12.7 \%$ \\
Industrial processes total & 0.56 & 0.50 & 0.50 & 0.53 & 0.42 & 0.42 & $6.8 \%$ \\
Waste disposal and recycling total & 0.23 & 0.25 & 0.33 & 0.27 & 0.18 & 0.25 & $4.1 \%$ \\
Miscellaneous total & 5.23 & 4.73 & 4.69 & 3.07 & 4.15 & 4.43 & $71.8 \%$ \\
\hline Total of all sources & $\mathbf{7 . 5 5}$ & $\mathbf{6 . 9 4}$ & $\mathbf{7 . 2 8}$ & $\mathbf{5 . 6 0}$ & $\mathbf{6 . 0 0}$ & $\mathbf{6 . 1 6}$ & $\mathbf{1 0 0 . 0 \%}$ \\
\hline
\end{tabular}

\section{Source:}

U. S. Environmental Protection Agency, National Emission Inventory Air Pollutant Emission Trends website www.epa.gov/air-emissions-inventories/air-pollutant-emissions-trends-data. (Additional resources: www.epa.gov/air-emissions-inventories/national-emissions-inventory)

${ }^{a}$ The sums of subcategories may not equal total due to rounding. 
Diesel vehicles are responsible for the majority of highway vehicle PM-2.5 emissions. Nearly two-thirds of the highway vehicles' PM-2.5 emissions are from heavy diesel trucks. Between 2005 and 2011 the Environmental Protection Agency updated their source from the MOBILE 6.2 emissions model to the MOVES emission model. MOVES results typically show higher emissions, especially for heavy trucks. The 2014 data are the latest available.

Table 12.11

Emissions of Particulate Matter (PM-2.5) from Highway Vehicles, 1990-2014 (thousand short tons)

\begin{tabular}{|c|c|c|c|c|c|c|c|}
\hline Source category & 1990 & 1995 & 2000 & 2005 & $2011^{\mathrm{b}}$ & $2014^{\mathrm{b}}$ & $\begin{array}{c}\text { Percent } \\
\text { of total, } \\
2014\end{array}$ \\
\hline & \multicolumn{7}{|c|}{ Gasoline powered } \\
\hline Light vehicles \& motorcycles & 35 & 30 & 27 & 23 & c & c & c \\
\hline Light trucks ${ }^{\mathrm{d}}$ & 21 & 20 & 18 & 18 & c & c & c \\
\hline Subtotal light vehicles & 56 & 50 & 45 & 41 & 68 & 59 & $37.2 \%$ \\
\hline Heavy vehicles & 11 & 9 & 7 & 6 & 2 & 2 & $1.1 \%$ \\
\hline \multirow{2}{*}{ Subtotal gasoline vehicles } & 67 & 59 & 52 & 47 & 70 & 61 & $38.3 \%$ \\
\hline & \multicolumn{7}{|c|}{ Diesel powered } \\
\hline Light vehicles & 9 & 4 & 1 & 1 & c & c & c \\
\hline Light trucks ${ }^{\mathrm{d}}$ & 4 & 2 & 1 & 1 & c & c & c \\
\hline Subtotal light vehicles & 13 & 6 & 2 & 2 & 6 & 4 & $2.8 \%$ \\
\hline Heavy vehicles & 243 & 179 & 119 & 79 & 120 & 93 & $58.9 \%$ \\
\hline \multirow[t]{2}{*}{ Subtotal diesel vehicles } & 256 & 185 & 121 & 81 & 126 & 97 & $61.7 \%$ \\
\hline & \multicolumn{7}{|c|}{ Total } \\
\hline Highway vehicle total & 323 & 244 & 173 & 128 & 196 & 159 & $100.0 \%$ \\
\hline Percent diesel & $79.3 \%$ & $75.8 \%$ & $69.9 \%$ & $63.3 \%$ & $64.6 \%$ & $61.7 \%$ & \\
\hline
\end{tabular}

\section{Source:}

U. S. Environmental Protection Agency, National Emission Inventory Air Pollutant Emission Trends website www.epa.gov/air-emissions-inventories/national-emissions-inventory-nei. (Additional resources: www.epa.gov/air-emissions-inventories/national-emissions-inventory)

a The sums of subcategories may not equal total due to rounding.

${ }^{\mathrm{b}}$ These data are not directly comparable to the older data due to the change in source from the MOBILE emissions model to the MOVES emissions model. The 2011 data include condensable plus filterable PM-2.5.

${ }^{\mathrm{c}}$ Data are not available.

${ }^{\mathrm{d}}$ Less than 8,500 pounds. 
The transportation sector accounted for only $2 \%$ of the nation's sulfur dioxide $\left(\mathrm{SO}_{2}\right)$ emissions in 2016, with offhighway vehicles responsible for most of the emissions. Stationary fuel combustion (e.g., factories) was responsible for nearly $73 \%$ of all $\mathrm{SO}_{2}$ emissions in 2016.

Table 12.12

Total National Emissions of Sulfur Dioxide, 1970-2016

(million short tons)

\begin{tabular}{lrrrrrrrr}
\hline & & & & & & & \multicolumn{2}{c}{$\begin{array}{c}\text { Percent } \\
\text { of total, }\end{array}$} \\
Source category & \multicolumn{1}{c}{ 1970 } & \multicolumn{1}{c}{ 1980 } & \multicolumn{1}{c}{1990} & 2000 & 2010 & 2016 & \multicolumn{2}{c}{2016} \\
\hline Highway vehicles & 0.27 & 0.39 & 0.50 & 0.26 & 0.04 & 0.02 & $0.7 \%$ \\
$\quad$ Other off-highway & 0.28 & 0.32 & 0.37 & 0.44 & 0.12 & 0.03 & $1.1 \%$ \\
Transportation total & 0.55 & 0.72 & 0.87 & 0.70 & 0.16 & 0.05 & $1.8 \%$ \\
Stationary fuel combustion total & 23.46 & 21.39 & 20.21 & 14.16 & 6.75 & 1.97 & $72.8 \%$ \\
Industrial processes total & 7.10 & 3.81 & 1.90 & 1.42 & 0.68 & 0.50 & $18.5 \%$ \\
Waste disposal and recycling total & 0.01 & 0.03 & 0.04 & 0.03 & 0.02 & 0.04 & $1.4 \%$ \\
Miscellaneous total & 0.11 & 0.01 & 0.01 & 0.07 & 0.16 & 0.15 & $5.5 \%$ \\
\hline Total of all sources & $\mathbf{3 1 . 2 2}$ & $\mathbf{2 5 . 9 3}$ & $\mathbf{2 3 . 0 8}$ & $\mathbf{1 6 . 3 5}$ & $\mathbf{7 . 7 3}$ & $\mathbf{2 . 7 1}$ & $\mathbf{1 0 0 . 0 \%}$ \\
\hline
\end{tabular}

Source:

U. S. Environmental Protection Agency, National Emission Inventory Air Pollutant Emission Trends website www.epa.gov/air-emissions-inventories/air-pollutant-emissions-trends-data.

(Additional resources: www.epa.gov/air-emissions-inventories/national-emissions-inventory)

${ }^{a}$ The sums of subcategories may not equal total due to rounding. 


\section{EMISSION STANDARDS}

The U.S. Environmental Protection Agency (EPA) regulates emissions from mobile sources including vehicles, engines, and motorized equipment that produce exhaust and evaporative emissions. Mobile sources contribute to four main air pollutants: carbon monoxide, hydrocarbons, nitrogen oxides, and particulate matter. The EPA not only sets standards for the vehicles, engines, and equipment, but also the fuels that they use. Tables 12.13 through Table 12.30 contain summaries of the current standards.

\begin{tabular}{|llll|}
\hline \multicolumn{3}{|c|}{ Acronyms Used on Tables 12.13 through } & Table 12.30 \\
ABT & Averaging, banking, and credit trading program & LLDT & Light light-duty truck \\
ATV & All-terrain vehicle & LPG & Liquefied petroleum gas \\
bhp & Brake horsepower-hour & LVW & Loaded vehicle weight \\
CFR & Code of Federal Regulations & MDPV & Medium-duty passenger vehicle \\
CI & Compression-ignition & & (8,500-10,000 lbs. GVWR) \\
CO & Carbon Monoxide & MDV & Medium-duty vehicle \\
DE & Diesel engine & MY & Model year \\
EPA & Environmental Protection Agency & NMHC & Non-methane hydrocarbon \\
FEL & Family emission limit & NMOG & Non-methane organic gases \\
FTP & Federal test procedure & NR & Nonroad \\
g & Gram & NRLM & Nonroad, locomotive and marine \\
g/kN & Grams per kilonewton & NOx & Nitrogen oxides \\
g/kW-hr & Grams per kilowatt-hour & NTE & Not-to-exceed \\
g/mi & Grams per mile & OEM & Original equipment manufacturer \\
GPA & Geographic Phase-in Area & PM & Particulate matter \\
GVW & Gross vehicle weight & ppm & Parts per million \\
HC & Hydrocarbons & PWC & Personal watercraft \\
HCHO & Formaldehyde & rO & Rated output \\
HLDT & Heavy light-duty truck & rPR & Rated pressure ratio \\
Hp-hr & Horsepower-hour & SI & Spark-ignition \\
ICAO & International Civil Aviation Organization & SULEV & Super-ultra-low-emission vehicle \\
kN & Kilonewton & THC & Total hydrocarbons \\
kW & Kilowatt & THCE & Total hydrocarbon equivalent \\
kW-hr & Kilowatt-hour & ULEV & Ultra-low-emission vehicle \\
LDT & Light-duty truck & ULSD & Ultra-low sulfur diesel \\
LDV & Light-duty vehicle & ZEV & Zero-emission vehicle \\
LEV & Low-emission vehicle & & \\
\hline & & & \\
\hline
\end{tabular}


The Environmental Protection Agency issued final Tier 3 emission standards in 2014. The combined emissions of non-methane organic gases (NMOG) and nitrogen oxides (NOx) that new gasoline engines are allowed to produce from model years 2017 to 2025 are regulated in these new standards. These standards apply to a corporate average, meaning that some vehicles produced in those model years will emit more than the standard, while others will emit less, so long as the average for each Original Equipment Manufacturer (OEM) product offerings meets the standard.

Table 12.13

Tier 3 Non-Methane Organic Gases and Nitrogen Oxide Standards (milligrams per mile)

\begin{tabular}{ccccc}
\hline Light-duty & $\begin{array}{c}\text { LDT2, 3, 4, and } \\
\text { vehicles and } \\
\text { medium-duty } \\
\text { Model Year }\end{array}$ & passenger vehicles & Class 2b trucks & Class 3 trucks \\
\hline 2016 & $\mathrm{a}$ & 101 & $333^{\mathrm{b}}$ & $548^{\mathrm{b}}$ \\
2017 & 86 & 92 & $310^{\mathrm{b}}$ & $508^{\mathrm{b}}$ \\
2018 & 79 & 83 & 278 & 451 \\
2019 & 72 & 74 & 253 & 400 \\
2020 & 65 & 65 & 228 & 349 \\
2021 & 58 & 56 & 203 & 298 \\
2022 & 51 & 47 & 178 & 247 \\
2023 & 44 & 38 & 178 & 247 \\
2024 & 37 & 30 & 178 & 247 \\
2025 and later & 30 & & 178 & 247 \\
\hline
\end{tabular}

Notes: Standards are for the Federal Test Procedure. Different standards apply for the Supplemental Federal Test Procedure. For vehicles over 6,000 lbs. gross vehicle weight rating (GVWR), the standards apply beginning in MY 2018.

LDT1 = Light trucks less than 6,000 lbs. GVWR and less than 3,750 lbs. loaded vehicle weight (LVW).

LDT2, 3, 4 = Light trucks less than 8,500 lbs. GVWR and more than 3,750 lbs. LVW.

Class $2 \mathrm{~b}$ trucks $=$ trucks 8,501-10,000 lbs. GVWR.

Class 3 trucks $=$ trucks 10,001-14,000 lbs. GVWR.

\section{Source:}

Federal Register Vol. 79, No. 81, Monday, April 28, 2014.

\footnotetext{
a Not applicable.

${ }^{\mathrm{b}}$ Voluntary standard.
} 
Table 12.14

Tier 3 Particulate Matter Emission Standards for Light Gasoline Vehicles, MY 2017 and Beyond (milligrams per mile)

\begin{tabular}{cccc}
\hline Model Year & $\begin{array}{c}\text { Certification standard } \\
\text { (milligrams per mile) }\end{array}$ & $\begin{array}{c}\text { In-use standard } \\
\text { (milligrams per mile) }\end{array}$ & $\begin{array}{c}\text { Phase-in (percent of } \\
\text { U.S. sales) }\end{array}$ \\
\hline 2017 & 3 & 6 & $20^{\mathrm{a}}$ \\
2018 & 3 & 6 & 20 \\
2019 & 3 & 6 & 40 \\
2020 & 3 & 6 & 70 \\
2021 & 3 & 6 & 100 \\
2022 -on & 3 & 3 & 100 \\
\hline
\end{tabular}

Note: Standards are for the Federal Test Procedure. The standards apply to all light-duty vehicles, light-duty trucks, and medium-duty passenger vehicles. For vehicles over 6,000 lbs. gross vehicle weight rating, the standards apply beginning in MY 2018.

Source:

Federal Register Vol. 79, No. 81, Monday, April 28, 2014.

Table 12.15

Tier 3 Evaporative Emission Standards (grams per test)

\begin{tabular}{lc}
\hline \multicolumn{1}{c}{ Vehicle class } & $\begin{array}{c}\text { Highest hot soak + diurnal level } \\
\text { (over both 2-day and 3-day diurnal tests) }\end{array}$ \\
\hline Light-duty vehicles and LDT1 & 0.3 \\
LDT2 & 0.4 \\
LDT3, LT4, and medium-duty passenger vehicles & 0.5 \\
Heavy-duty gasoline vehicles & 0.6 \\
\hline
\end{tabular}

Note: LDT1 = Light trucks less than 6,000 lbs. gross vehicle weight rating (GVWR) and less than 3,750 lbs. loaded vehicle weight (LVW).

LDT2 $=$ Light trucks less than 6,000 lbs. GVWR and less than 3,750 lbs. LVW.

LDT3, 4 = Light trucks less than 8,500 lbs. GVWR and more than 3,750 lbs. LVW.

Heavy-duty gasoline vehicles $=$ trucks over 10,000 lbs. GVWR.

\section{Source:}

Federal Register Vol. 79, No. 81, Monday, April 28, 2014.

${ }^{a}$ Manufacturers comply with 20\% of their light-duty truck fleet under 6,000 lbs. gross vehicle weight, alternatively with $10 \%$ of their total light-duty vehicle, light-duty trucks and medium-duty passenger vehicle fleet. 
Table 12.16

Light-Duty Vehicle, Light-Duty Truck, and Medium-Duty Passenger Vehicle - Tier 2 Exhaust Emission Standards

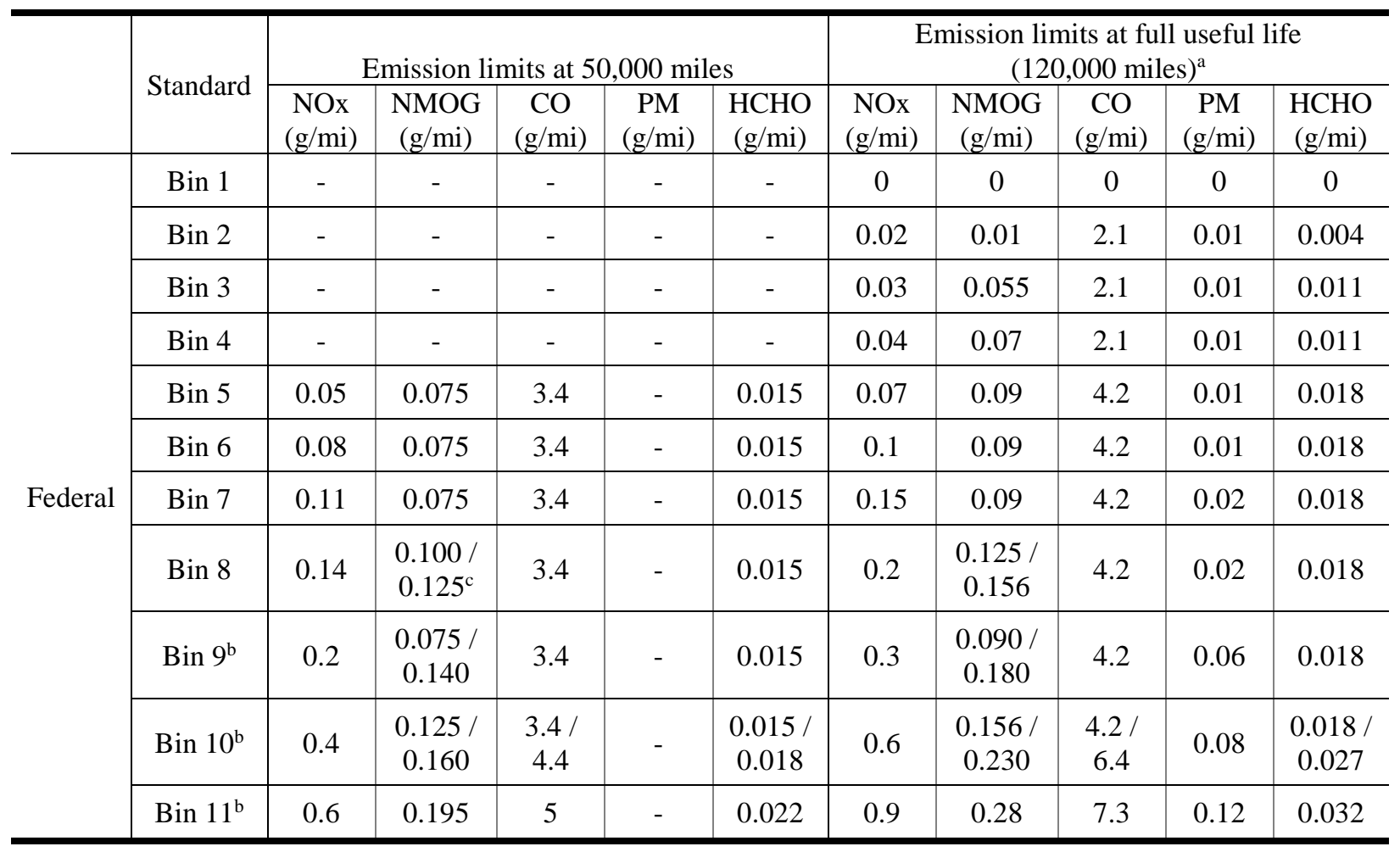

Note: Tests Covered: Federal Test Procedure (FTP), cold carbon monoxide, highway, and idle. Definitions of acronyms are on page 12-14.

\section{Source:}

40 CR 86 Subpart S. (Additional resources: www.epa.gov/emission-standards-reference-guide/light-duty-vehiclesand-trucks-emission-standards)

${ }^{a}$ In lieu of intermediate useful life standards (50,000 miles) or to gain additional nitrogen oxides credit, manufacturers may optionally certify to the Tier 2 exhaust emission standards with a useful life of 150,000 miles.

${ }^{\mathrm{b}}$ Bins 9-11 expired in 2006 for light-duty vehicles and light light-duty trucks and 2008 for heavy light-duty trucks and medium-duty passenger vehicles. number).

c Pollutants with two numbers have a separate certification standard (1st number) and in-use standard (2nd 
Table 12.17

Light-Duty Vehicle, Light-Duty Truck, and Medium-Duty Passenger Vehicle - Tier 2 Evaporative Emission Standards

\begin{tabular}{lllccc}
\hline \multirow{6}{*}{ Federal } & Model & $\begin{array}{c}\text { 3 Day diurnal } \\
\text { + hot soak } \\
\text { (g/test) }\end{array}$ & $\begin{array}{c}\text { Supplemental } \\
\text { 2 day diurnal } \\
\text { + hot soak } \\
\text { (g/test) }\end{array}$ & $\begin{array}{c}\text { Running } \\
\text { loss } \\
(\mathrm{g} / \mathrm{mi})\end{array}$ \\
\hline & Vehicle type & year & 0.95 & 1.20 & 0.05 \\
& LDV/LLDTs $^{\mathrm{a}}$ & 2004 & 1.20 & 1.50 & 0.05 \\
& HLDTs $^{\mathrm{b}}$ & 2004 & 1.40 & 1.75 & 0.05 \\
& MDPVs $^{\mathrm{a}, \mathrm{b}}$ & 2004 & 0.50 & 0.65 & 0.05 \\
& LDV $^{\mathrm{a}}$ & 2009 & 0.65 & 0.85 & 0.05 \\
& LLDT $^{\mathrm{a}}$ & 2009 & 0.90 & 1.15 & 0.05 \\
& HLDT $^{\mathrm{b}}$ & 2010 & 1.00 & 1.25 & 0.05 \\
\hline
\end{tabular}

Note: Multi-fuel vehicle phase-in applies. Definitions of acronyms are on page 12-14.

Source:

40 CR 86 Subpart S. (Additional resources: www.epa.gov/emission-standards-reference-guide/light-duty-vehiclesand-trucks-emission-standards)

${ }^{a}$ For liquefied petroleum gas-fueled light-duty vehicles (LDV), light-duty trucks (LDT), and medium-duty passenger vehicles (MDPV): 0.15 grams hydrocarbon per gallon (0.04 grams per liter) of fuel dispensed.

${ }^{\mathrm{b}}$ Refueling standards for heavy light-duty trucks (HLDT) are subject to phase-in requirements. MDPVs must also comply with the phase-in requirement and must be grouped with HLDTs to determine phase-in compliance. 
Table 12.18

\section{Heavy-Duty Highway Compression-Ignition Engines and Urban Buses - Exhaust} Emission Standards

\begin{tabular}{|c|c|c|c|c|c|c|c|c|c|c|}
\hline & Year & $\begin{array}{c}\mathrm{HC} \\
(\mathrm{g} / \mathrm{bhp}- \\
\mathrm{hr})\end{array}$ & $\begin{array}{c}\text { NMHC } \\
\text { (g/bhp- } \\
\text { hr) }\end{array}$ & $\begin{array}{c}\text { NMHC + NOx } \\
(\mathrm{g} / \mathrm{bhp}- \\
\mathrm{hr})\end{array}$ & $\begin{array}{c}\text { NOx } \\
\text { (g/bhp- } \\
\text { hr) }\end{array}$ & $\begin{array}{c}\text { PM } \\
\text { (g/bhp- } \\
\text { hr) }\end{array}$ & $\begin{array}{c}\mathrm{CO} \\
\text { (g/bhp- } \\
\mathrm{hr})\end{array}$ & $\begin{array}{l}\text { Idle CO } \\
\text { (percent } \\
\text { Exhaust } \\
\text { gas flow) } \\
\end{array}$ & $\begin{array}{c}\text { Smoke }^{\mathrm{a}} \\
\text { (percentage) }\end{array}$ & $\begin{array}{c}\text { Useful life } \\
\text { (hours/years/miles) }\end{array}$ \\
\hline \multirow{9}{*}{ Federal $^{\mathrm{b}}$} & $1974-78$ & - & - & 16 & - & - & 40 & - & $20 / 15 / 50$ & - \\
\hline & $1979-84$ & 1.5 & - & 10 & - & - & 25 & - & $20 / 15 / 50$ & - \\
\hline & $1985-87$ & 1.3 & - & - & 10.7 & - & 15.5 & - & $20 / 15 / 50$ & $\begin{array}{l}\text { LHDDE: - / } 8 \text { / 110,000 } \\
\text { MHDDE: - / } 8 \text { / 185,000 } \\
\text { HHDDE: - / } 8 \text { / 290,000 }\end{array}$ \\
\hline & $1988-89$ & $1.3^{d}$ & - & - & 10.7 & 0.6 & 15.5 & $0.5^{c}$ & $20 / 15 / 50$ & \multirow{5}{*}{$\begin{array}{c}\text { 1990-97 and 1998+ for } \\
\text { HC, CO, and PM: } \\
\text { LHDDE: - / } 8 \text { / 110,000 } \\
\text { MHDDE: - / } 8 \text { / 185,000 } \\
\text { HHDDE: - / } 8 \text { / 290,000 } \\
\text { 1994+ urban buses for PM } \\
\text { only: } \\
\text { LHDDE: - / } 10 \text { / 110,000 } \\
\text { 1998+ for NOx: } \\
\text { LHDDE: - / } 10 \text { / 110,000 } \\
\text { MHDDE: - / } 10 \text { / 185,000 } \\
\text { HHDDE: - / } 10 \text { / 290,000 }\end{array}$} \\
\hline & 1990 & $1.3^{\mathrm{d}}$ & - & - & 6.0 & 0.6 & 15.5 & $0.5^{c}$ & $20 / 15 / 50$ & \\
\hline & 1991-93 & 1.3 & - & - & $5.0[\mathrm{ABT}]$ & $\begin{array}{c}0.25[\mathrm{ABT}] \\
0.10^{\mathrm{e}}\end{array}$ & 15.5 & $0.5^{c}$ & $20 / 15 / 50$ & \\
\hline & $1994-97$ & 1.3 & - & - & $5.0[\mathrm{ABT}]$ & $\begin{array}{c}0.1[\mathrm{ABT}] \\
0.07^{\mathrm{f}}, 0.05^{\mathrm{g}}\end{array}$ & 15.5 & $0.5^{c}$ & $20 / 15 / 50$ & \\
\hline & $1998-2003$ & 1.3 & - & - & $4.0[\mathrm{ABT}]$ & $\begin{array}{c}0.1[\mathrm{ABT}] \\
0.05^{\mathrm{g}}\end{array}$ & 15.5 & $0.5^{c}$ & $20 / 15 / 50$ & \\
\hline & $\begin{array}{l}2004-2006^{\mathrm{h}} \\
2007++^{\mathrm{h}, \mathrm{k}, \mathrm{l}, \mathrm{m}, \mathrm{n}}\end{array}$ & - & $0.14^{\circ}$ & $\begin{array}{c}2.4 \text { (or } 2.5 \\
\text { with a limit of } \\
0.5 \text { on } \\
\mathrm{NMHC}^{\circ} \\
\left.\text { [ABT }^{\mathrm{i}, j}\right] \\
\\
2.4 \text { (or } 2.5 \\
\text { with a limit of } \\
0.5 \text { on NMHC) } \\
\text { [ABT] }\end{array}$ & $0.2^{\circ}$ & $\begin{array}{c}0.1 \\
0.05^{\mathrm{g}}\end{array}$ & 15.5 & 0.5 & $20 / 15 / 50$ & $\begin{array}{c}\text { For all pollutants: } \\
\text { LHDDE: - / 10 / 110,000 } \\
\text { MHDDE: - / } 10 \text { / 185,000 } \\
\text { HHDDE: 22,000 / } 10 \text { / } \\
\text { 435,000 }\end{array}$ \\
\hline
\end{tabular}

Note: The test procedures are the EPA Transient Test Procedure and the EPA Smoke Test Procedure. Definitions of acronyms are on page 12-14.

\section{Sources:}

40 CFR 86.099-11 Emission standards for 1999 and later model year diesel heavy-duty engines and vehicles.

40 CFR 86.004-11 Emission standards for 2004 and later model year diesel heavy-duty engines and vehicles.

40 CFR 86.007-11 Emission standards and supplemental requirements for 2007 and later model year diesel heavyduty engines and vehicles. (Additional resources: www.epa.gov/emission-standards-reference-guide/lightduty-vehicles-and-trucks-emission-standards)

${ }^{a}$ Percentages apply to smoke opacity at acceleration/lug/peak modes.

b Standards for 1990 apply only to diesel-fueled heavy-duty engines (HDE). Standards for 1991+ apply to both diesel- and methanol-fueled HDEs. Standards that apply to urban buses specifically are footnoted.

${ }^{\mathrm{c}}$ This standard applies to the following fueled engines for the following model years: methanol - 1990+, natural gas and liquefied petroleum gas (LPG) - 1994+.

${ }^{d}$ For petroleum-fueled engines, the standard is for hydrocarbons (HC). For methanol-fueled engines, the standard is for total hydrocarbon equivalent (THCE).

e Certification standard for urban buses for 1993.

${ }^{\mathrm{f}}$ Certification standard for urban buses from 1994-95.

${ }^{g}$ Certification standard for urban buses from 1996 and later. The in-use standard is 0.07 .

${ }^{\mathrm{h}}$ Load Response Test certification data submittal requirements take effect for heavy-duty diesel engines beginning in model year 2004. The following requirements take effect with the 2007 model year: steady-state test requirement and Not-to-Exceed (NTE) test procedures for testing of in-use engines. On-board diagnostic requirements applicable to heavy-duty diesel vehicles and engines up to 14,000 pounds gross vehicle weight rating (GVWR) phase in from the 2005 through 2007 model years. 


\section{Table 12.18 (continued) \\ Heavy-Duty Highway Compression-Ignition Engines and Urban Buses - Exhaust Emission Standards}

i The modified averaging, banking, and trading program for 1998 and later model year engines applies only to diesel cycle engines. Credits generated under the modified program may be used only in 2004 and later model years.

${ }^{\mathrm{j}}$ For heavy-duty diesel engines, there are three options to the measurement procedures currently in place for alternative fueled engines: (1) use a THC measurement in place of an non-methane hydrocarbon (NMHC) measurement; (2) use a measurement procedure specified by the manufacturer with prior approval of the Administrator; or (3) subtract two percent from the measured THC value to obtain an NMHC value. The methodology must be specified at time of certification and will remain the same for the engine family throughout the engines' useful life. For natural gas vehicles, EPA allows the option of measuring NMHC through direct quantification of individual species by gas chromatography.

k Starting in 2006, refiners must begin producing highway diesel fuel that meets a maximum sulfur standard of 15 parts per million (ppm).

${ }^{1}$ Subject to a Supplemental Emission Test (1.0 x Federal Test Procedure [FTP] standard (or Family Emission Limit [FEL]) for nitrogen oxides [NOx], NMHC, and particulate matter [PM]) and a NTE test (1.5 x FTP standard [or FEL] for NOx, NMHC, and PM).

m EPA adopted the lab-testing and field-testing specifications in 40 CFR Part 1065 for heavy-duty highway engines, including both diesel and Otto-cycle engines. These procedures replace those previously published in 40 Code of Federal Regulations (CFR) Part 86, Subpart N. Any new testing for 2010 and later model years must be done using the 40 CFR Part 1065 procedures.

${ }^{n}$ Two-phase in-use NTE testing program for heavy-duty diesel vehicles. The program begins with the 2007 model year for gaseous pollutants and 2008 for PM. The requirements apply to diesel engines certified for use in heavy-duty vehicles (including buses) with GVWRs greater than 8,500 pounds. However, the requirements do not apply to any heavy-duty diesel vehicle that was certified using a chassis dynamometer, including medium-duty passenger vehicles with GVWRs of between 8,500 and 10,000 pounds.

${ }^{\circ}$ NOx and NMHC standards will be phased in together between 2007 and 2010. The phase-in will be on a percent-of-sales basis: 50 percent from 2007 to 2009 and 100 percent in 2010.

p Note that for an individual engine, if the useful life hours interval is reached before the engine reaches 10 years or 100,000 miles, the useful life shall become 10 years or 100,000 miles, whichever occurs first, as required under Clean Air Act section 202(d). 
Table 12.19

Heavy-Duty Highway Spark-Ignition Engines - Exhaust Emission Standards

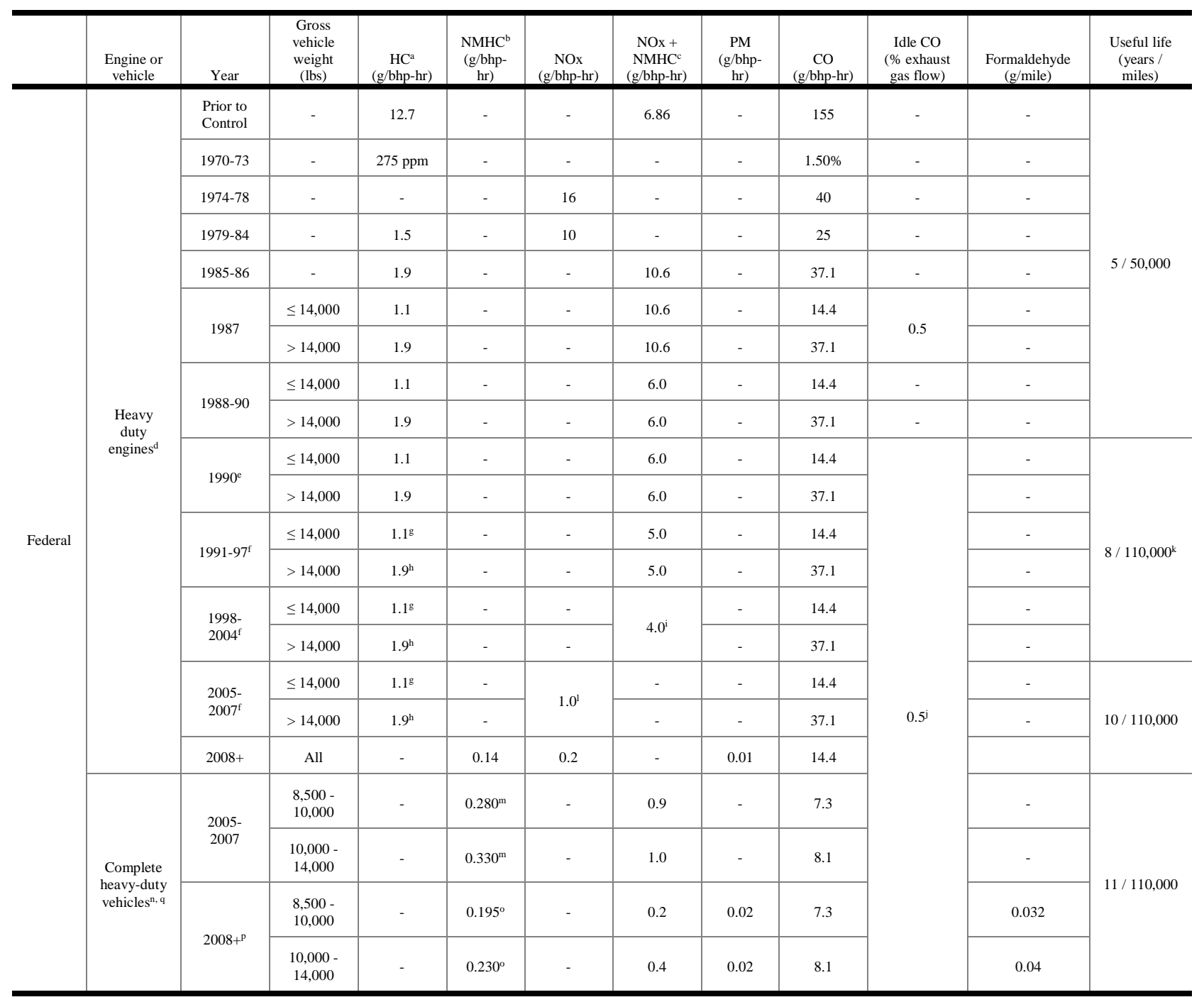

Note: Definitions of acronyms are on page 12-14.

\section{Sources:}

40 CFR 86.1816-05, 86.1816-08 Emission standards for complete heavy-duty vehicles

40 CFR 86.1806-01, 86.1806-04, 86.1806-05 Onboard diagnostics requirements

40 CFR 86.1817-05, 86.1817-08 Complete heavy-duty vehicle averaging, banking, and trading program

40 CFR 86.091-10 Heavy-duty engine averaging, banking, and trading program for 1991 and later - Not available in the e-CFR

40 CFR Part 86 Subpart B Vehicle test procedures (Additional resources: www.epa.gov/emission-standardsreference-guide/light-duty-vehicles-and-trucks-emission-standards)

${ }^{\text {a }}$ For methanol-fueled engines, the standard is for total hydrocarbon equivalent (THCE).

${ }^{\mathrm{b}}$ For methanol and alcohol fueled vehicles the standard is for non-methane hydrocarbon equivalent (NMHCE).

${ }^{\mathrm{c}}$ For methanol fueled engines the standard is for nitrogen oxides (NOx) plus NMHCE.

${ }^{\mathrm{d}}$ Standards for heavy-duty engines are expressed in grams per brake horsepower-hour (g/bhp-hr). Starting with the 1998 model year, crankcase emissions are not allowed.

e Standards for 1990 apply to gasoline and methanol-fueled engines.

${ }^{\mathrm{f}}$ Standards for 1991 and later apply to gasoline and methanol engines and are optional for natural gas and Liquefied Petroleum Gas-fueled engines through the 1996 model year.

${ }^{\mathrm{g}}$ For natural gas fueled engines the standard is $0.9 \mathrm{~g} / \mathrm{bhp}-\mathrm{hr}$ non-methane hydrocarbon (NMHC). 


\section{Table 12.19 (continued) Heavy-Duty Highway Spark-Ignition Engines - Exhaust Emission Standards}

${ }^{\mathrm{h}}$ For natural gas fueled engines the standard is $1.7 \mathrm{~g} / \mathrm{bhp}-\mathrm{hr}$ NMHC.

${ }^{\mathrm{i}}$ The NOx standard is 5.0 for all natural gas-fueled engines.

$\mathrm{j}$ This standard applies to the following engines utilizing aftertreatment technology (except for methanol) for the following model years: gasoline/1990+; natural gas and LPG/1991+; methanol/1990+. Starting in 2005, engines certified to on-board diagnostics requirements are not required to meet the idle carbon monoxide (CO) standard.

${ }^{\mathrm{k}}$ Useful life is expressed in years or miles, whichever comes first. Useful life for the 1998 and later NOx standard and for all 2004 standards is 10 years or 110,000 miles, whichever comes first.

${ }^{1}$ Manufacturers can choose this standard or one of the following options: (1) a standard of $1.5 \mathrm{~g} / \mathrm{bhp}-\mathrm{hr}$ NMHC+NOX that applies to the 2004 through 2007 model years, with complete heavy-duty vehicle standards taking effect in 2005; or (2) a standard of $1.5 \mathrm{~g} / \mathrm{bhp}-\mathrm{hr}$ NMHC + NOX that would apply to the 2003 through 2007 heavyduty engines and optionally to 2003 through 2006 complete heavy-duty vehicles.

$\mathrm{m}$ Standard is expressed as non-methane organic gas, but compliance can optionally be shown using measurement of NMHC or total hydrocarbon (THC).

${ }^{\mathrm{n}}$ Complete heavy-duty vehicles have the primary load-carrying container or device attached. Incomplete heavyduty vehicles are certified to heavy-duty engine standards. Standards for complete heavy-duty vehicles are expressed in grams per mile (g/mi). Starting in 2005 (or 2003 or 2004 depending on the selected phase in option; see footnote l), complete heavy-duty vehicles under 14,000 lbs gross vehicle weight are tested on chassis-based rather than enginebased procedures and must meet these complete heavy-duty vehicle standards.

${ }^{\circ}$ Although expressed as NMHC, compliance can optionally be shown using measurement of NMOG or THC.

${ }^{p}$ At least 50 percent of a manufacturer's sales must meet these standards in 2008, with 100 percent required in 2009.

${ }^{\mathrm{q}}$ Gross vehicle weight ranges are more accurately specified as follows: 8,500 $\leq \mathrm{GVW} \leq 10,000$ and $10,000<$ GVW $<14,000$. 
Table 12.20

\section{Heavy-Duty Highway Compression-Ignition and Spark-Ignition Engines - Evaporative Emission Standards}

\begin{tabular}{|c|c|c|c|c|c|c|c|c|c|}
\hline & $\begin{array}{c}\text { Engine } \\
\text { type }\end{array}$ & Year & $\begin{array}{c}\text { Gross vehicle } \\
\text { weight (lbs) }\end{array}$ & $\begin{array}{l}\text { Conventional } \\
\text { diurnal + hot } \\
\text { soak (g/test) }\end{array}$ & $\begin{array}{c}\text { Three-diurnal } \\
\text { test sequence } \\
(\mathrm{g} / \text { test })^{\mathrm{b}}\end{array}$ & $\begin{array}{l}\text { Supplemental } \\
\text { two-diurnal } \\
\text { test sequence } \\
\text { (g/test) }^{\mathrm{c}} \\
\end{array}$ & $\begin{array}{c}\text { Running } \\
\text { loss } \\
(\mathrm{g} / \mathrm{mi})^{\mathrm{c}}\end{array}$ & $\begin{array}{l}\text { Spitback } \\
\text { (g/test) }^{c}\end{array}$ & Useful life $^{\mathrm{d}}$ \\
\hline \multirow{10}{*}{ Federal } & \multirow{6}{*}{ SI } & \multirow{2}{*}{ 1991-95 } & $\leq 14,000$ & 3.0 & - & - & - & - & \multirow{2}{*}{$8 / 110,000$} \\
\hline & & & $>14,000^{\mathrm{e}}$ & 4.0 & - & - & - & - & \\
\hline & & \multirow{2}{*}{$\begin{array}{c}\text { 1996-2007 } \\
\text { (Enhanced) }^{\mathrm{f}}\end{array}$} & $\leq 14,000$ & - & 3.0 & 3.5 & \multirow{4}{*}{0.05} & 1.0 & \multirow{2}{*}{$10 / 120,000$} \\
\hline & & & $>14,000^{\mathrm{e}}$ & - & 4.0 & 4.5 & & - & \\
\hline & & \multirow{2}{*}{$\begin{array}{c}2008+ \\
\text { (Enhanced) }\end{array}$} & $8500-14,000$ & - & 1.4 & 1.75 & & 1.0 & \multirow{2}{*}{$11 / 110,000$} \\
\hline & & & $>14,000^{\mathrm{e}}$ & - & 1.9 & 2.3 & & - & \\
\hline & \multirow{4}{*}{ CI } & \multirow{2}{*}{ 1996-97 } & $\leq 14,000$ & - & 3.0 & - & - & - & \multirow{4}{*}{$\begin{array}{l}\text { MHDDE: } 8 \text { / 185,000 } \\
\text { HHDDE: } 8 \text { / 290,000 } \\
\text { MHDDE: } 8 \text { / 185,000 } \\
\text { HHDDE: } 8 \text { / 290,000 }\end{array}$} \\
\hline & & & $>14,000^{\mathrm{e}}$ & - & 4.0 & - & - & - & \\
\hline & & \multirow{2}{*}{$\begin{array}{c}\text { 1998+ } \\
\text { (Enhanced) }^{\mathrm{g}}\end{array}$} & $\leq 14,000$ & - & 3.0 & 3.5 & \multirow{2}{*}{0.05} & 1.0 & \\
\hline & & & $>14,000^{\mathrm{e}}$ & - & 4.0 & 4.5 & & - & \\
\hline
\end{tabular}

Note: Definitions of acronyms are on page 12-14.

\section{Sources:}

40 CFR 86.099-11 Emission standards for 1999 and later model year diesel heavy-duty engines and vehicles.

40 CFR 86.004-11 Emission standards for 2004 and later model year diesel heavy-duty engines and vehicles.

CFR 86.007-11 Emission standards and supplemental requirements for 2007 and later model year diesel heavy-duty engines and vehicles. (Additional resources: www.epa.gov/emission-standards-reference-guide/light-dutyvehicles-and-trucks-emission-standards)

a Applies to gasoline and methanol engines. Standard is hydrocarbon (HC) for gasoline engines, total hydrocarbon equivalent (THCE) for methanol engines.

${ }^{\mathrm{b}}$ For spark-ignition (SI) engines, standard applies to gasoline, methanol, natural gas, and liquefied petroleum gas engines. For compression-ignition (CI) engines, standard applies to methanol, natural gas, and liquefied petroleum gas engines. Standard is THCE for methanol engines, HC for others.

${ }^{c}$ For SI engines, standard applies to gasoline and methanol engines. For CI engines, standard applies to methanol engines. Standard is THCE for methanol engines, HC for others.

${ }^{\mathrm{d}}$ Useful life is expressed in years or miles, whichever comes first.

e Vehicles over 26,000 pounds gross vehicle weight may demonstrate compliance with an engineering design evaluation in lieu of testing.

${ }^{\mathrm{f}}$ A new enhanced evaporative test procedure applies, which is considerably more stringent than the previous test procedure despite the fact that the standard values do not change from prior years. Gasoline and methanol engines are phased in at the following rates of a manufacturer's sales for the specified model year: 1996: 20 percent; 1997: 40 percent; 1998: 90 percent; 1999: 100 percent.

${ }^{g}$ A new enhanced evaporative test procedure applies, which is considerably more stringent than the previous test procedure despite the fact that the standard values do not change from prior years. Methanol-fueled vehicles are phased in at a rate of 90 percent of a manufacturer's production in 1998 and 100 percent in 1999. 
The LEV III exhaust standards apply to new cars, light trucks, and medium vehicles, including fuel-flexible, bi-fuel, and dual-fuel vehicles from model year 2015-on.

Table 12.21

California New Car, Light Truck and Medium Truck Emission Certification Standards, Model Year 2015-On

\begin{tabular}{|c|c|c|c|c|c|}
\hline Vehicle type & $\begin{array}{l}\text { Vehicle } \\
\text { emission } \\
\text { category }\end{array}$ & $\begin{array}{l}\text { Non-methane } \\
\text { organic gases } \\
\text { + nitrogen } \\
\text { oxides }(\mathrm{g} / \mathrm{mi})\end{array}$ & $\begin{array}{l}\text { Carbon } \\
\text { monoxide } \\
(\mathrm{g} / \mathrm{mi})\end{array}$ & $\begin{array}{l}\text { Formaldehyde } \\
(\mathrm{mg} / \mathrm{mi})\end{array}$ & $\begin{array}{l}\text { Particulates } \\
(\mathrm{g} / \mathrm{mi})\end{array}$ \\
\hline \multirow{3}{*}{$\begin{array}{l}\text { All passenger cars; } \\
\text { LDTs } 8,500 \text { lbs. GVW or } \\
\text { less }\end{array}$} & LEV160 & 0.16 & 4.2 & 4 & 0.01 \\
\hline & ULEV125 & 0.125 & 2.1 & 4 & 0.01 \\
\hline & ULEV70 & 0.07 & 1.7 & 4 & 0.01 \\
\hline \multirow[t]{2}{*}{ All MDPVs } & ULEV50 & 0.05 & 1.7 & 4 & 0.01 \\
\hline & SULEV30 & 0.03 & 1.0 & 4 & 0.01 \\
\hline $\begin{array}{l}\text { Vehicles in this category } \\
\text { are tested at their loaded } \\
\text { vehicle weight }\end{array}$ & SULEV20 & 0.02 & 1.0 & 4 & 0.01 \\
\hline \multirow{2}{*}{$\begin{array}{l}\text { MDVs } \\
8,501-10,000 \text { lbs. GVW }\end{array}$} & LEV395 & 0.395 & 6.4 & 6 & 0.12 \\
\hline & ULEV340 & 0.34 & 6.4 & 6 & 0.06 \\
\hline \multirow{4}{*}{$\begin{array}{l}\text { Vehicles in this category } \\
\text { are tested at their adjusted } \\
\text { loaded vehicle weight }\end{array}$} & ULEV250 & 0.25 & 6.4 & 6 & 0.06 \\
\hline & ULEV200 & 0.2 & 4.2 & 6 & 0.06 \\
\hline & SULEV170 & 0.17 & 4.2 & 6 & 0.06 \\
\hline & SULEV150 & 0.15 & 3.2 & 6 & 0.06 \\
\hline \multirow{2}{*}{$\begin{array}{l}\text { MDVs } \\
10,000-14,000 \text { lbs. GVW }\end{array}$} & LEV630 & 0.63 & 7.3 & 6 & 0.12 \\
\hline & ULEV570 & 0.57 & 7.3 & 6 & 0.06 \\
\hline \multirow{4}{*}{$\begin{array}{l}\text { Vehicles in this category } \\
\text { are tested at their adjusted } \\
\text { loaded vehicle weight }\end{array}$} & ULEV400 & 0.4 & 7.3 & 6 & 0.06 \\
\hline & ULEV270 & 0.27 & 4.2 & 6 & 0.06 \\
\hline & SULEV230 & 0.23 & 4.2 & 6 & 0.06 \\
\hline & SULEV200 & 0.2 & 3.7 & 6 & 0.06 \\
\hline
\end{tabular}

Note: Definitions of acronyms are on page 12-14.

\section{Source:}

California LEV III Regulations with amendments effective January 1, 2016, www.arb.ca.gov/msprog/levprog/cleandoc/cleancomplete\%201ev-ghg\%20regs\%201-16.pdf. (Additional resources: www.arb.ca.gov) 
Table 12.22

Aircraft - Exhaust Emission Standards

\begin{tabular}{|c|c|c|c|c|c|c|c|}
\hline & Year & $\begin{array}{c}\text { Pressure } \\
\text { ratio (PR) }\end{array}$ & Applicability $^{\mathrm{a}}$ & $\mathrm{HC}(\mathrm{g} / \mathrm{kN})$ & NOx & $\begin{array}{c}\mathrm{CO} \\
(\mathrm{g} / \mathrm{kN})\end{array}$ & Smoke \\
\hline \multirow{16}{*}{ Federal $^{\mathrm{b}}$} & 1974+ & - & T8 & - & - & - & 30 \\
\hline & $1976+$ & - & TF with $\mathrm{rO}^{\mathrm{c}} \geq 129 \mathrm{kN}$ & - & - & - & $83.6(\mathrm{rO})^{-0.274}$ \\
\hline & $1978+$ & - & $\mathrm{T}^{\mathrm{d}}$ & - & - & - & 25 \\
\hline & $1983+$ & - & TF with $\mathrm{rO}<26.7 \mathrm{kN}$ & - & - & - & $\begin{array}{c}\text { 83.6(rO) })^{-0.274} \mathrm{NTE} \\
\text { max of } \mathrm{SN}=50\end{array}$ \\
\hline & \multirow{4}{*}{$1984+$} & - & $\begin{array}{c}\text { T3, T8, TF with } \mathrm{rO} \geq 26.7 \\
\mathrm{kN}\end{array}$ & 19.6 & - & - & $\begin{array}{c}\text { 83.6(rO) })^{-0.274} \mathrm{NTE} \\
\text { max of } \mathrm{SN}=50\end{array}$ \\
\hline & & - & TSS & $140(.92)^{\mathrm{rPR}}$ & - & - & $\begin{array}{c}83.6(\mathrm{rO})^{-0.274} \mathrm{NTE} \\
\text { max of } \mathrm{SN}=50\end{array}$ \\
\hline & & - & TSS with $\mathrm{rO} \geq 26.7 \mathrm{kN}$ & $140(.92)^{\mathrm{rPR}}$ & - & - & $\begin{array}{c}\text { 83.6(rO) })^{-0.274} \mathrm{NTE} \\
\text { max of } \mathrm{SN}=50\end{array}$ \\
\hline & & - & $\mathrm{TP}$ with $\mathrm{rO} \geq 1,000 \mathrm{~kW}$ & - & - & - & $187(\mathrm{rO})^{-0.168}$ \\
\hline & \multirow[b]{2}{*}{$1997+$} & - & $\begin{array}{c}\text { T3, T8, TF with } \mathrm{rO}>26.7 \\
\text { kN }\end{array}$ & 19.6 & $40+2(\mathrm{rPR})$ & 118 & $\begin{array}{c}\text { 83.6(rO) })^{-0.274} \mathrm{NTE} \\
\text { max of } \mathrm{SN}=50\end{array}$ \\
\hline & & - & $\begin{array}{c}\text { T3, T8, TF newly } \\
\text { certified with rO > } 26.7 \\
\text { kN }\end{array}$ & 19.6 & $32+1.6(\mathrm{rPR})$ & 118 & $\begin{array}{c}\text { 83.6(rO) })^{-0.274} \mathrm{NTE} \\
\text { max of } \mathrm{SN}=50\end{array}$ \\
\hline & $2000+$ & - & $\begin{array}{c}\text { T3, T8, TF newly } \\
\text { manufactured with rO > } \\
26.7 \mathrm{kN}\end{array}$ & 19.6 & $32+1.6(\mathrm{rPR})$ & 118 & $\begin{array}{c}\text { 83.6(rO) })^{-0.274} \mathrm{NTE} \\
\text { max of } \mathrm{SN}=50\end{array}$ \\
\hline & \multirow{5}{*}{$2005+$} & \multirow[b]{2}{*}{$\mathrm{PR} \leq 30$} & $\begin{array}{c}\text { T3, T8, TF newly } \\
\text { certified with } \mathrm{rO}>89 \mathrm{kN}\end{array}$ & - & 19+1.6(rPR) & - & - \\
\hline & & & $\begin{array}{c}\text { T3, T8, TF newly } \\
\text { certified with } 26.7 \mathrm{kN}< \\
\text { rO } \leq 89 \mathrm{kN}\end{array}$ & - & $\begin{array}{l}37.572+1.6(\mathrm{rPR})- \\
0.2087(\mathrm{rO})\end{array}$ & - & - \\
\hline & & \multirow{2}{*}{$\begin{array}{c}30<\mathrm{PR}< \\
\quad 62.5\end{array}$} & $\begin{array}{c}\text { T3, T8, TF newly } \\
\text { certified with rO>89 kN }\end{array}$ & - & $7+2.0(\mathrm{rPR})$ & - & - \\
\hline & & & $\begin{array}{c}\text { T3, T8, TF newly } \\
\text { certified with } 26.7 \mathrm{kN}<\mathrm{r} 0 \\
\leq 89 \mathrm{kN}\end{array}$ & - & $\begin{array}{c}\text { 42.71+1.4286(rPR)- } \\
0.4013(\mathrm{rO})+0.00642(\mathrm{rP} \\
\mathrm{R})(\mathrm{rO})\end{array}$ & - & - \\
\hline & & $\mathrm{PR} \leq 62.5$ & T3, T8, TF & - & $32+1.6(\mathrm{rPR})$ & - & - \\
\hline
\end{tabular}

Note: The test procedures are the International Civil Aviation Organization (ICAO) Smoke Emission Test Procedure and the ICAO Gaseous Emissions Test Procedure. There is no useful life or warranty period for purposes of compliance with aircraft emissions standards. Definitions of acronyms are on page 12-14.

\section{Source:}

40 CFR Part 87, Aircraft emission standards, test procedures, certification requirements (Additional resources: www.epa.gov/emission-standards-reference-guide/nonroad-engines-and-vehicles-emission-standards)

a T8=all aircraft gas turbine engines of the JT8D model family

$\mathrm{TF}=$ all turbofan and turbojet aircraft engines except engines of Class T3, T8, and TSS

T3=all aircraft gas turbine engines of the JT3D model family

TSS=all aircraft gas turbine engines for aircraft operations at supersonic flight speeds

$\mathrm{TP}=$ all aircraft turboprop engines

${ }^{\mathrm{b}}$ Federal standards apply to planes operating in the United States, regardless of where they were manufactured.

${ }^{\mathrm{c}}$ Rated output (rO) is the maximum power/thrust available for takeoff.

$\mathrm{d}$ T3 engines are no longer manufactured but are in the existing fleet. 
These standards apply to construction and agricultural equipment, such as excavators, paving equipment, tractors, combines, bulldozers, and skidders.

Table 12.23

Nonroad Compression-Ignition Engines - Exhaust Emission Standards

\begin{tabular}{|c|c|c|c|c|c|c|c|c|c|c|}
\hline & $\begin{array}{c}\text { Rated } \\
\text { power } \\
(\mathrm{kW})\end{array}$ & Tier & $\begin{array}{l}\text { Model } \\
\text { year }\end{array}$ & $\begin{array}{c}\text { NMHC } \\
(\mathrm{g} / \mathrm{kW} \\
-\mathrm{hr})\end{array}$ & $\begin{array}{c}\text { NMHC } \\
+ \text { NOx } \\
\text { (g/kW } \\
\text {-hr) }\end{array}$ & $\begin{array}{c}\text { NOx } \\
(\mathrm{g} / \mathrm{kW} \\
-\mathrm{hr})\end{array}$ & $\begin{array}{c}\text { PM } \\
\text { (g/kW } \\
\text {-hr) }\end{array}$ & $\begin{array}{c}\mathrm{CO} \\
\text { (g/kW } \\
\text {-hr) }\end{array}$ & $\begin{array}{c}\text { Smoke }^{\mathrm{a}} \\
\text { percentage }\end{array}$ & $\begin{array}{c}\begin{array}{c}\text { Useful life } \\
\text { (hours/years) }^{\mathrm{b}}\end{array}\end{array}$ \\
\hline \multirow{45}{*}{ Federal } & \multirow{3}{*}{$\mathrm{kW}<8$} & 1 & $2000-2004$ & -- & 10.5 & -- & 1.0 & 8.0 & \multirow{45}{*}{$20 / 15 / 50$} & \multirow{3}{*}{$3,000 / 5$} \\
\hline & & 2 & $2005-2007$ & -- & 7.5 & -- & 0.80 & 8.0 & & \\
\hline & & 4 & $2008+$ & -- & 7.5 & -- & $0.40^{c}$ & 8.0 & & \\
\hline & \multirow{3}{*}{$8 \leq \mathrm{kW}<19$} & 1 & 2000-2004 & -- & 9.5 & -- & 0.80 & 6.6 & & \multirow{3}{*}{$3,000 / 5$} \\
\hline & & 2 & $2005-2007$ & -- & 7.5 & -- & 0.80 & 6.6 & & \\
\hline & & 4 & $2008+$ & -- & 7.5 & -- & 0.40 & 6.6 & & \\
\hline & \multirow{4}{*}{$19 \leq \mathrm{kW}<37$} & 1 & $1999-2003$ & -- & 9.5 & -- & 0.80 & 5.5 & & \multirow{4}{*}{$5,000 / 7^{\mathrm{d}}$} \\
\hline & & 2 & 2004-2007 & -- & 7.5 & -- & 0.60 & 5.5 & & \\
\hline & & \multirow{2}{*}{4} & 2008-2012 & -- & 7.5 & -- & 0.30 & 5.5 & & \\
\hline & & & $2013+$ & -- & 4.7 & -- & 0.03 & 5.5 & & \\
\hline & \multirow{6}{*}{$37 \leq \mathrm{kW}<56$} & 1 & $1998-2003$ & -- & -- & 9.2 & -- & -- & & \multirow{35}{*}{$8,000 / 10$} \\
\hline & & 2 & $2004-2007$ & -- & 7.5 & -- & 0.40 & 5.0 & & \\
\hline & & $3^{e}$ & $2008-2011$ & -- & 4.7 & -- & 0.40 & 5.0 & & \\
\hline & & $4(\text { Option } 1)^{\mathrm{f}}$ & $2008-2012$ & -- & 4.7 & -- & 0.30 & 5.0 & & \\
\hline & & $4(\text { Option } 2)^{\mathrm{f}}$ & 2012 & -- & 4.7 & -- & 0.03 & 5.0 & & \\
\hline & & 4 & $2013+$ & -- & 4.7 & -- & 0.03 & 5.0 & & \\
\hline & \multirow{5}{*}{$56 \leq \mathrm{kW}<75$} & 1 & 1998-2003 & -- & -- & 9.2 & -- & -- & & \\
\hline & & 2 & 2004-2007 & -- & 7.5 & -- & 0.40 & 5.0 & & \\
\hline & & 3 & $2008-2011$ & -- & 4.7 & -- & 0.40 & 5.0 & & \\
\hline & & \multirow{2}{*}{4} & 2012-2103 & -- & 4.7 & -- & 0.02 & 5.0 & & \\
\hline & & & $2014+^{\mathrm{h}}$ & 0.19 & -- & 0.4 & 0.02 & 5.0 & & \\
\hline & \multirow{5}{*}{$75 \leq \mathrm{kW}<130$} & 1 & $1997-2002$ & -- & -- & 9.2 & -- & -- & & \\
\hline & & 2 & 2003-2006 & -- & 6.6 & -- & 0.3 & 5.0 & & \\
\hline & & 3 & 2007-2011 & -- & 4.0 & -- & 0.3 & 5.0 & & \\
\hline & & \multirow{2}{*}{4} & $2012-2013^{g}$ & -- & 4.0 & -- & 0.02 & 5.0 & & \\
\hline & & & $2014+$ & 0.19 & -- & 0.4 & 0.02 & 5.0 & & \\
\hline & \multirow{5}{*}{$\begin{array}{c}130 \leq \mathrm{kW}< \\
225\end{array}$} & 1 & 1996-2002 & $1.3^{\mathrm{i}}$ & -- & 9.2 & 0.54 & 11.4 & & \\
\hline & & 2 & 2003-2005 & -- & 6.6 & -- & 0.20 & 3.5 & & \\
\hline & & 3 & 2006-2010 & -- & 4.0 & -- & 0.20 & 3.5 & & \\
\hline & & \multirow{2}{*}{4} & $2011-2013^{g}$ & -- & 4.0 & -- & 0.02 & 3.5 & & \\
\hline & & & $2014+^{\mathrm{h}}$ & 0.19 & -- & 0.4 & 0.02 & 3.5 & & \\
\hline & \multirow{5}{*}{$\begin{array}{c}225 \leq \mathrm{kW}< \\
450\end{array}$} & 1 & $1996-2000$ & $1.3^{\mathrm{i}}$ & -- & 9.2 & 0.54 & 11.4 & & \\
\hline & & 2 & 2001-2005 & -- & 6.4 & -- & 0.20 & 3.5 & & \\
\hline & & 3 & 2006-2010 & -- & 4.0 & -- & 0.20 & 3.5 & & \\
\hline & & \multirow{2}{*}{4} & $2011-2013^{g}$ & -- & 4.0 & -- & 0.02 & 3.5 & & \\
\hline & & & $2014+^{\mathrm{h}}$ & 0.19 & -- & 0.4 & 0.02 & 3.5 & & \\
\hline & \multirow{5}{*}{$\begin{array}{c}450 \leq \mathrm{kW}< \\
560\end{array}$} & 1 & 1996-2001 & $1.3^{\mathrm{i}}$ & -- & 9.2 & 0.54 & 11.4 & & \\
\hline & & 2 & $2002-2005$ & -- & 6.4 & -- & 0.20 & 3.5 & & \\
\hline & & 3 & $2006-2010$ & -- & 4.0 & -- & 0.20 & 3.5 & & \\
\hline & & \multirow{2}{*}{4} & $2011-2013^{g}$ & -- & 4.0 & -- & 0.02 & 3.5 & & \\
\hline & & & $2014+^{\mathrm{h}}$ & 0.19 & -- & 0.4 & 0.02 & 3.5 & & \\
\hline & & 1 & $2000-2005$ & $1.3^{\mathrm{i}}$ & -- & 9.2 & 0.54 & 11.4 & & \\
\hline & $560 \leq \mathrm{kW}<$ & 2 & 2006-2010 & -- & 6.4 & -- & 0.20 & 3.5 & & \\
\hline & 900 & 4 & $2011-2014$ & 0.4 & -- & 3.5 & 0.10 & 3.5 & & \\
\hline & & 4 & $2015^{+^{\mathrm{h}}}$ & 0.19 & -- & $3.5^{j}$ & $0.04^{\mathrm{k}}$ & 3.5 & & \\
\hline
\end{tabular}


Table 12.23 (continued)

Nonroad Compression-Ignition Engines - Exhaust Emission Standards

\begin{tabular}{|c|c|c|c|c|c|c|c|c|c|c|}
\hline & $\begin{array}{c}\text { Rated } \\
\text { power } \\
(\mathrm{kW})\end{array}$ & Tier & $\begin{array}{c}\text { Model } \\
\text { year }\end{array}$ & $\begin{array}{c}\text { NMHC } \\
(\mathrm{g} / \mathrm{kW} \\
-\mathrm{hr})\end{array}$ & $\begin{array}{c}\text { NMHC } \\
+ \text { NOx } \\
(\mathrm{g} / \mathrm{kW} \\
-\mathrm{hr})\end{array}$ & $\begin{array}{c}\text { NOx } \\
(\mathrm{g} / \mathrm{kW} \\
-\mathrm{hr})\end{array}$ & $\begin{array}{c}\text { PM } \\
\text { (g/kW } \\
\text {-hr) }\end{array}$ & $\begin{array}{c}\mathrm{CO} \\
\text { (g/kW } \\
-\mathrm{hr})\end{array}$ & $\begin{array}{c}\text { Smoke }^{\mathrm{a}} \\
\text { percentage }\end{array}$ & $\begin{array}{c}\text { Useful life } \\
\text { (hours/years) }^{\mathrm{b}}\end{array}$ \\
\hline \multirow{4}{*}{ Federal } & \multirow{4}{*}{$\mathrm{kW}>900$} & 1 & $2000-2005$ & $1.3^{\mathrm{i}}$ & -- & 9.2 & 0.54 & 11.4 & \multirow{4}{*}{$20 / 15 / 50$} & \multirow{4}{*}{$8,000 / 10$} \\
\hline & & 2 & $2006-2010$ & -- & 6.4 & -- & 0.20 & 3.5 & & \\
\hline & & \multirow{2}{*}{4} & 2011-2014 & 0.4 & -- & $3.5^{\mathrm{j}}$ & 0.10 & 3.5 & & \\
\hline & & & $2015^{+^{\mathrm{h}}}$ & 0.19 & -- & $3.5^{\mathrm{j}}$ & $0.04^{\mathrm{k}}$ & 3.5 & & \\
\hline
\end{tabular}

Note: Definitions of acronyms are on page 12-14.

\section{Sources:}

40 CFR 98.112 = Exhaust emission standards

40 CFR 1039.101 = Exhaust emission standards for after 2014 model year

40 CFR 1039.102 = Exhaust emission standards for model year 2014 and earlier

40 CFR 1039 Subpart $\mathrm{F}=$ Exhaust emissions transient and steady state test procedures

40 CFR 86 Subpart I = Smoke emission test procedures

40 CFR 1065 = Test equipment and emissions measurement procedures (Additional resources:

www.epa.gov/emission-standards-reference-guide/nonroad-engines-and-vehicles-emission-standards)

a Smoke emissions may not exceed 20 percent during the acceleration mode, 15 percent during the lugging mode, and 50 percent during the peaks in either mode. Smoke emission standards do not apply to single-cylinder engines, constant-speed engines, or engines certified to a PM emission standard of 0.07 grams per kilowatt-hour (g/kW-hr) or lower. Smoke emissions are measured using procedures in 40 CFR Part 86 Subpart I.

${ }^{\mathrm{b}}$ Useful life and warranty period are expressed hours and years, whichever comes first.

${ }^{c}$ Hand-startable air-cooled direct injection engines may optionally meet a PM standard of $0.60 \mathrm{~g} / \mathrm{kW}$ hr. These engines may optionally meet Tier 2 standards through the 2009 model years. In 2010 these engines are required to meet a PM standard of $0.60 \mathrm{~g} / \mathrm{kW}$-hr.

${ }^{d}$ Useful life for constant speed engines with rated speed 3,000 revolutions per minute (rpm) or higher is 5 years or 3,000 hours, whichever comes first.

e These Tier 3 standards apply only to manufacturers selecting Tier 4 Option 2. Manufacturers selecting Tier 4 Option 1 will be meeting those standards in lieu of Tier 3 standards.

${ }_{\mathrm{f}}^{\mathrm{f}}$ A manufacturer may certify all their engines to either Option 1 or Option 2 sets of standards starting in the indicated model year. Manufacturers selecting Option 2 must meet Tier 3 standards in the 2008-2011 model years.

${ }^{\mathrm{g}}$ These standards are phase-out standards. Not more than 50 percent of a manufacturer's engine production is allowed to meet these standards in each model year of the phase out period. Engines not meeting these standards must meet the final Tier 4 standards.

${ }^{\mathrm{h}}$ These standards are phased in during the indicated years. At least 50 percent of a manufacturer's engine production must meet these standards during each year of the phase in. Engines not meeting these standards must meet the applicable phase-out standards.

${ }^{\mathrm{i}}$ For Tier 1 engines the standard is for total hydrocarbons.

${ }^{\mathrm{j}}$ The NOx standard for generator sets is $0.67 \mathrm{~g} / \mathrm{kW}-\mathrm{hr}$.

${ }^{\mathrm{k}}$ The PM standard for generator sets is $0.03 \mathrm{~g} / \mathrm{kW}-\mathrm{hr}$. 
These standards apply to gasoline and propane industrial equipment such as forklifts, generators, airport service equipment, compressors and ice-grooming machines.

Table 12.24

Nonroad Large Spark-Ignition Engines - Exhaust and Evaporative Emission Standards

\begin{tabular}{|c|c|c|c|c|c|c|c|c|c|}
\hline & \multirow[b]{2}{*}{ Tier } & \multirow[b]{2}{*}{ Year } & \multicolumn{2}{|c|}{$\begin{array}{c}\text { General duty-cycle } \\
\text { standards }\end{array}$} & \multicolumn{2}{|c|}{$\begin{array}{c}\text { Alternative standards for } \\
\text { severe-duty engines }\end{array}$} & \multicolumn{2}{|c|}{ Field testing standards } & \multirow[b]{2}{*}{$\begin{array}{c}\text { Useful life } \\
\text { (years/hours) }\end{array}$} \\
\hline & & & $\begin{array}{l}\mathrm{HC}+\mathrm{NOx}^{\mathrm{a}} \\
(\mathrm{g} / \mathrm{kW}-\mathrm{hr}) \\
\end{array}$ & $\begin{array}{c}\mathrm{CO} \\
(\mathrm{g} / \mathrm{kW}-\mathrm{hr}) \\
\end{array}$ & $\begin{array}{l}\mathrm{HC}+\mathrm{NOx}^{\mathrm{a}} \\
\text { (g/kW-hr) }\end{array}$ & $\begin{array}{c}\mathrm{CO} \\
\text { (g/kW-hr) } \\
\end{array}$ & $\begin{array}{l}\mathrm{HC}+\mathrm{NOx}^{\mathrm{a}} \\
(\mathrm{g} / \mathrm{kW}-\mathrm{hr}) \\
\end{array}$ & $\begin{array}{c}\mathrm{CO} \\
(\mathrm{g} / \mathrm{kW}-\mathrm{hr}) \\
\end{array}$ & \\
\hline \multirow{6}{*}{$\underset{\mathrm{b}}{\text { Federal }}$} & $1^{\mathrm{c}}$ & $\begin{array}{l}2004- \\
2006\end{array}$ & $4.0^{\mathrm{d}}$ & 50.0 & $4.0^{\mathrm{d}}$ & 130.0 & - & - & $7 / 5,000^{e}$ \\
\hline & \multirow{5}{*}{$2^{f}$} & \multirow{5}{*}{$2007+$} & $2.7^{\mathrm{f}}$ & $4.4^{\mathrm{f}}$ & 2.7 & 130.0 & $3.8^{f}$ & $6.5^{\mathrm{f}}$ & $7 / 5,000^{\mathrm{e}}$ \\
\hline & & & \multicolumn{7}{|c|}{ Evaporative emission standards (for engines fueled by a volatile liquid fuel) } \\
\hline & & & $\begin{array}{c}\text { Fuel line } \\
\text { permeation }\end{array}$ & \multicolumn{5}{|c|}{$\begin{array}{l}\text { Nonmetallic fuel lines must meet the permeation specifications of SAE } \\
\text { J2260 (November 1996) }\end{array}$} & \multirow{3}{*}{$5 /-$} \\
\hline & & & $\begin{array}{c}\text { Diurnal } \\
\text { emissions }\end{array}$ & \multicolumn{5}{|c|}{$\begin{array}{l}\text { Evaporative HC emissions may not exceed } 0.2 \text { grams per gallon of fuel } \\
\text { tank capacity }\end{array}$} & \\
\hline & & & $\begin{array}{l}\text { Running } \\
\text { loss }\end{array}$ & \multicolumn{5}{|c|}{$\begin{array}{l}\text { Liquid fuel in the fuel tank may not reach boiling during continuous } \\
\text { engine operation in the final installation at an ambient temperature of } \\
\qquad 30^{\circ} \mathrm{C}\end{array}$} & \\
\hline
\end{tabular}

\section{Sources:}

40 CFR 1048.101 = Exhaust emission standards

40 CFR 1048.105 = Evaporative emission standards

40 CFR 1048.110 = Engine diagnostic requirements (Additional resources: www.epa.gov/emission-standardsreference-guide/nonroad-engines-and-vehicles-emission-standards)

${ }^{a}$ The numerical emission standards for hydrocarbons (HC) must be met based on the following types of hydrocarbon emissions for engines powered by the following fuels: (1) non-methane hydrocarbons (NMHC) for natural gas; (2) total hydrocarbon equivalent (THCE) for alcohol; and (3) total hydrocarbons (THC) for other fuels.

${ }^{\mathrm{b}}$ Voluntary Blue Sky standards for large spark-ignition (SI) engines are available. Engines with displacement at or below 1,000 cubic centimeters (cc) and maximum power at or below 30 kilowatts (kW) may be certified under the program for small SI engines.

${ }^{\mathrm{c}}$ Emission standards are based on testing over a steady-state duty-cycle. (g/kW-hr).

${ }^{\mathrm{d}}$ The Tier 1 HC plus nitrogen oxides (NOx) emission standard for in-use testing is 5.4 grams per kW-hour

e Useful life is expressed in years and hours, whichever comes first. These are the minimum useful life requirements. For severe-duty engines, the minimum useful life is seven years or 1,500 hours of operation, whichever comes first. A longer useful life in hours is required if: (a) the engine is designed to operate longer than the minimum useful life based on the recommended rebuild interval; or (b) the basic mechanical warranty is longer than the minimum useful life.

${ }^{\mathrm{f}}$ Optional engine certification is allowed according to the following formula: $(\mathrm{HC}+\mathrm{NOx}) \times \mathrm{CO}^{0.784} \leq 8.57$. The $\mathrm{HC}+\mathrm{NOx}$ and carbon monoxide (CO) emission levels selected to satisfy this formula, rounded to the nearest $0.1 \mathrm{~g} / \mathrm{kW}$ $\mathrm{hr}$, become the emission standards that apply for those engines. One may not select an HC+NOx emission standard higher than $2.7 \mathrm{~g} / \mathrm{kW}$-hr or a CO emission standard higher than $20.6 \mathrm{~g} / \mathrm{kW}$-hr. 
Table 12.25

Locomotives - Exhaust Emission Standards

\begin{tabular}{|c|c|c|c|c|c|c|c|c|c|}
\hline & $\begin{array}{l}\text { Duty- } \\
\text { cycle }^{\mathrm{b}}\end{array}$ & Tier & Year $^{\mathrm{c}}$ & $\begin{array}{c}\mathrm{HC}^{\mathrm{i}} \\
\text { (g/hp-hr) }\end{array}$ & $\begin{array}{c}\text { NOx } \\
\text { (g/bhp-hr) }\end{array}$ & $\begin{array}{c}\text { PM } \\
\text { (g/bhp-hr) }\end{array}$ & $\begin{array}{c}\text { CO } \\
\text { (g/bhp-hr) }\end{array}$ & $\begin{array}{c}\text { Smoke } \\
\text { (percentage) }^{\mathrm{m}}\end{array}$ & $\begin{array}{l}\text { Minimum useful life } \\
\text { (hours / years / miles) }\end{array}$ \\
\hline \multirow{11}{*}{ Federal $^{\mathrm{a}}$} & \multirow{6}{*}{$\begin{array}{l}\text { Line- } \\
\text { haul }\end{array}$} & Tier 0 & $\begin{array}{l}1973- \\
1992^{\mathrm{d}, \mathrm{e}}\end{array}$ & 1.0 & $9.5[\mathrm{ABT}]$ & $0.22[\mathrm{ABT}]$ & 5.0 & $30 / 40 / 50$ & $\begin{array}{c}(7.5 \times \mathrm{hp}) / 10 / \\
750,000^{\circ}\end{array}$ \\
\hline & & \multirow[t]{2}{*}{ Tier 1} & \multirow{2}{*}{$\begin{array}{l}1993- \\
2004^{\mathrm{d}, \mathrm{e}}\end{array}$} & \multirow[t]{2}{*}{0.55} & \multirow[t]{2}{*}{$7.4[\mathrm{ABT}]$} & \multirow[t]{2}{*}{$0.22[\mathrm{ABT}]$} & \multirow[t]{2}{*}{2.2} & \multirow[t]{2}{*}{$25 / 40 / 50$} & $\begin{array}{c}(7.5 \times \mathrm{hp}) / 10 / \\
750,000^{\circ}\end{array}$ \\
\hline & & & & & & & & & (7.5 x hp) / $10 /-$ \\
\hline & & Tier 2 & $\begin{array}{l}2005- \\
2011^{\mathrm{d}}\end{array}$ & 0.30 & $5.5[\mathrm{ABT}]$ & $0.10^{\mathrm{k}}[\mathrm{ABT}]$ & 1.5 & $20 / 40 / 50$ & (7.5 x hp) / $10 /$ - \\
\hline & & Tier 3 & $\begin{array}{l}2012- \\
2014^{f}\end{array}$ & 0.30 & $5.5[\mathrm{ABT}]$ & $0.10[\mathrm{ABT}]$ & 1.5 & $20 / 40 / 50$ & (7.5 x hp) / $10 /$ - \\
\hline & & Tier 4 & $2015+8$ & 0.14 & $1.3[\mathrm{ABT}]$ & $0.03[\mathrm{ABT}]$ & 1.5 & - & (7.5 x hp) / 10 / - \\
\hline & \multirow{5}{*}{ Switch } & Tier 0 & $\begin{array}{l}1973- \\
2001\end{array}$ & 2.10 & 11.8 [ABT] & $0.26[\mathrm{ABT}]$ & 8.0 & $30 / 40 / 50$ & $\begin{array}{l}(7.5 \times \mathrm{hp}) / 10 / \\
750,000^{\circ}\end{array}$ \\
\hline & & Tier 1 & $\begin{array}{l}2002- \\
2004^{\mathrm{h}}\end{array}$ & 1.20 & $11.0[\mathrm{ABT}]$ & $0.26[\mathrm{ABT}]$ & 2.5 & $25 / 40 / 50$ & (7.5 x hp) / $10 /$ - \\
\hline & & Tier 2 & $\begin{array}{l}2005- \\
2010^{\mathrm{h}}\end{array}$ & 0.60 & $8.1[\mathrm{ABT}]$ & $0.13^{1}[\mathrm{ABT}]$ & 2.4 & $20 / 40 / 50$ & (7.5 x hp) / $10 /-$ \\
\hline & & Tier 3 & $\begin{array}{l}2011- \\
2014\end{array}$ & 0.60 & $5.0[\mathrm{ABT}]$ & $0.10[\mathrm{ABT}]$ & 2.4 & $20 / 40 / 50$ & (7.5 x hp) / $10 /-$ \\
\hline & & Tier 4 & $2015+$ & $0.14^{\mathrm{j}}$ & $1.3^{\mathrm{j}}[\mathrm{ABT}]$ & $0.03[\mathrm{ABT}]$ & 2.4 & - & (7.5 x hp) / $10 /$ - \\
\hline
\end{tabular}

\section{Source:}

40 CFR 1033.101 = Emission Standards and Useful Life. (Additional resources: www.epa.gov/emission-standardsreference-guide/nonroad-engines-and-vehicles-emission-standards)

a These standards apply to locomotives that are propelled by engines with total rated horsepower (hp) of 750 kilowatts (kW) (1006 hp) or more, unless the owner chooses to have the equipment certified to meet the requirements of locomotives. This does not include vehicles propelled by engines with total rated horsepower of less than $750 \mathrm{~kW}$ (1006 hp); see the requirements in 40 Code of Federal Regulations (CFR) Parts 86, 89 and 1039. The test procedures specify chassis-based testing of locomotives. These test procedures include certification testing, production line testing, and in-use testing using the Federal Test Procedure (FTP) when the locomotive has reached between 50-70 percent of its useful life.

${ }^{\mathrm{b}}$ Line-haul locomotives are powered by an engine with a maximum rated power (or a combination of engines having a total rated power) greater than $2300 \mathrm{hp}$. Switch locomotives are powered by an engine with a maximum rated power (or a combination of engines having a total rated power) of $2300 \mathrm{hp}$ or less.

c The Tier 0 standards apply to locomotives manufactured after 1972 when they are manufactured or remanufactured. Note that interim standards may apply for Tier 0 or Tier 1 locomotives remanufactured in 2008 or 2009, or for Tier 2 locomotives manufactured or remanufactured in 2008-2012.

d Line-haul locomotives subject to the Tier 0 through Tier 2 emission standards must also meet switch standards of the same tier.

${ }^{\mathrm{e}}$ The Tier 0 standards apply for 1993-2001 locomotives not originally manufactured with a separate loop intake air cooling system.

${ }_{\mathrm{f}}$ Tier 3 line-haul locomotives must also meet Tier 2 switch standards.

g Manufacturers using credits may elect to meet a combined nitrogen oxides (NOx) plus hydrocarbon (HC) standard of 1.4 grams per brakehorsepower-hour (g/bhp-hr) instead of the otherwise applicable Tier 4 NOx and HC standards.

${ }^{\mathrm{h}}$ Tier 1 and Tier 2 switch locomotives must also meet line-haul standards of the same tier.

${ }^{i}$ The numerical emission standards for HC must be met based on the following types of hydrocarbon emissions for locomotives powered by the following fuels: (1) alcohol: total hydrocarbon equivalent (THCE) emissions for Tier 3 and earlier locomotives, and non-methane hydrocarbon equivalent (NMHCE) for Tier 4; (2) natural gas and liquefied petroleum gas: non-methane hydrocarbon (NMHC) emissions; and (3) diesel: total hydrocarbon (THC) emissions for Tier 3 and earlier locomotives, and NMHC for Tier 4. 


\section{Table 12.25 (continued) \\ Locomotives - Exhaust Emission Standards}

${ }^{\mathrm{j}}$ Manufacturers may elect to meet a combined NOx+HC standard of $1.4 \mathrm{~g} / \mathrm{bhp}-\mathrm{hr}$ instead of the otherwise applicable Tier 4 NOx and HC standards.

${ }^{\mathrm{k}}$ The line-haul particulate matter (PM) standard for newly remanufactured Tier 2 locomotives is $0.20 \mathrm{~g} / \mathrm{bhp}-\mathrm{hr}$ until January 1, 2013, except as specified in 40 CFR Part 1033.150(a).

${ }^{1}$ The switch PM standard for new Tier 2 locomotives is $0.24 \mathrm{~g} / \mathrm{bhp}$-hr until January 1, 2013, except as specified in 40 CFR Part 1033.150(a).

${ }^{\mathrm{m}}$ The smoke opacity standards apply only for locomotives certified to one or more PM standards or Family Emission Limits (FEL) greater than 0.05 g/bhp-hr. Percentages apply to smoke opacity at steady state/30-second peak/3-second peak, as measured continuously during testing.

${ }^{\mathrm{n}}$ Useful life and warranty period are expressed in megawatt-hours (MW-hr), years, or miles, whichever comes first. Manufacturers are required to certify to longer useful lives if their locomotives are designed to last longer between overhauls than the minimum useful life value.

${ }^{\circ}$ For locomotives originally manufactured before January 1, 2000, and not equipped with MW-hr meters. 
These standards apply to auxiliary and propulsion engines used by all types of recreational and commercial vessels, from small fishing boats to ocean-going ships.

Table 12.26

Marine Compression-Ignition (CI) Engines - Exhaust Emission Standards

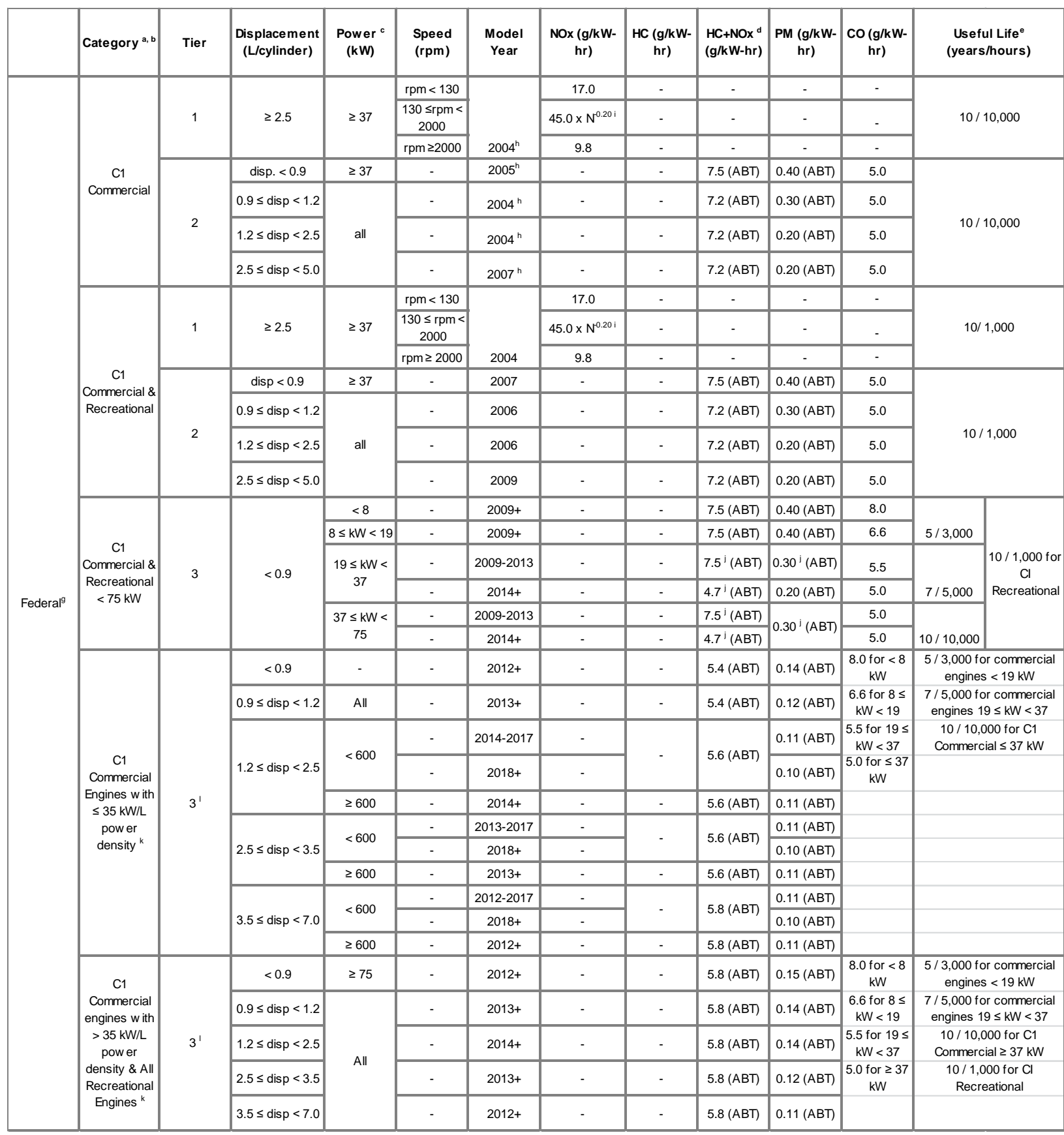

(Continued on next page) 
Table 12.26 (continued)

Marine Compression-Ignition (CI) Engines - Exhaust Emission Standards

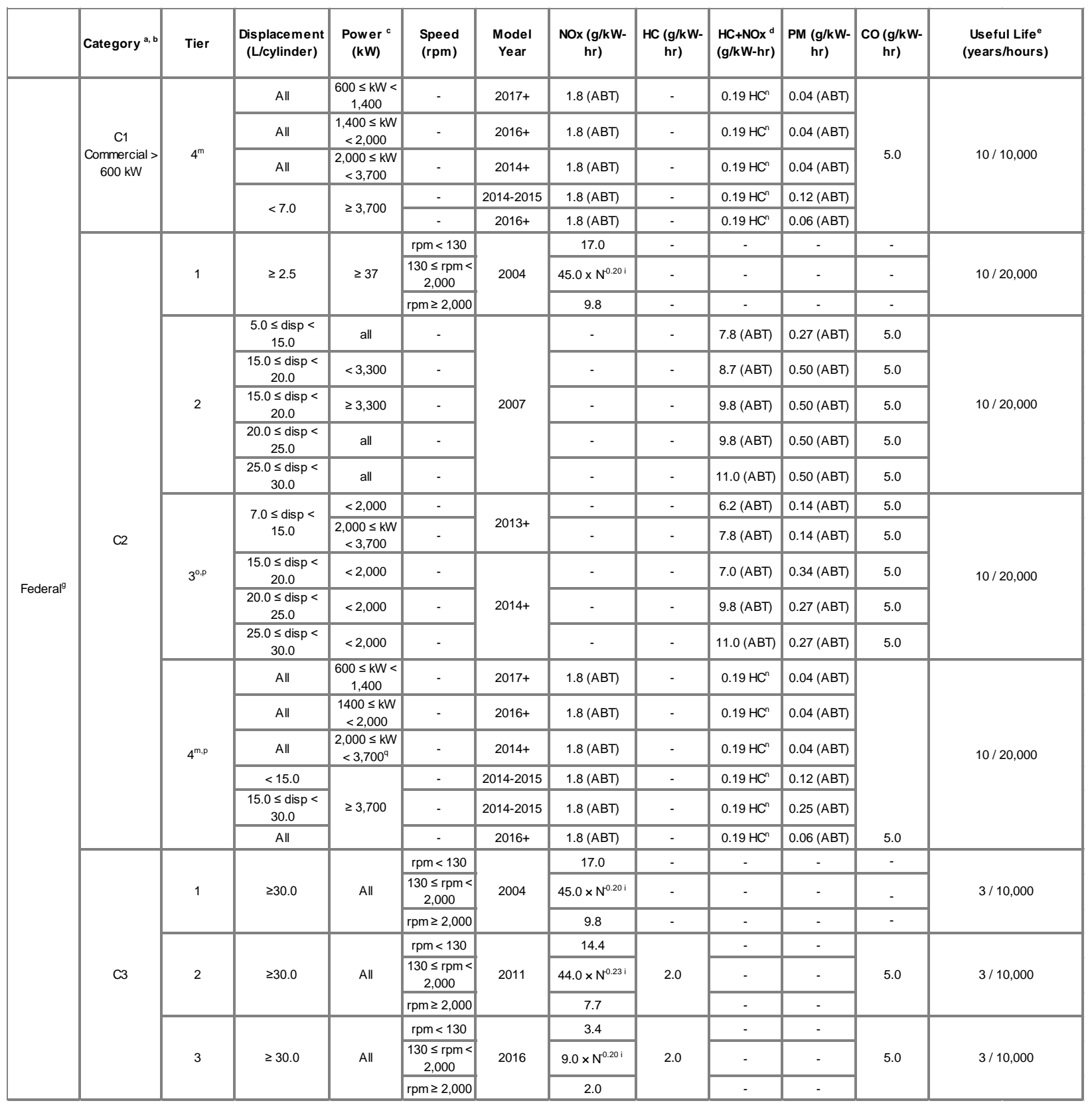

\section{Sources:}

40 CFR 89.104 = Tiers 1 and 2 useful life \& warranty period for marine CI engines less than $37 \mathrm{~kW}$ 40 CFR 89.112 = Tiers 1 and 2 emission standards for marine CI engines less than $37 \mathrm{~kW}$ 40 CFR 89 Subpart $\mathrm{E}=$ Tiers 1 and 2 test procedures for marine $\mathrm{CI}$ engines less than $37 \mathrm{~kW}$ 40 CFR 94.8 = Tiers 1 and 2 emission standards for C1 (both commercial \& recreational), C2 and C3 engines 40 CFR 94.9 = Tiers 1 and 2 useful life for C1 (both commercial \& recreational), C2 and C3 engines 40 CFR 94 Subpart B = Tiers 1 and 2 test procedures for C1 (both commercial \& recreational), C2 and C3 engines 40 CFR 1042.101 = Tiers 3 and 4 exhaust emission standards and useful life 


\section{Table 12.26 (continued) Marine Compression-Ignition (CI) Engines - Exhaust Emission Standards}

\section{Sources (continued):}

40 CFR 1042.107 = Tiers 3 and 4 evaporative emission standards engines using a volatile liquid fuel (e.g., methanol) 40 CFR $1042.120=$ Tiers 3 and 4 warranty period

40 CFR 1042 Subpart F = Tiers 3 and 4 test procedures (Additional resources: www.epa.gov/emission-standardsreference-guide/nonroad-engines-and-vehicles-emission-standards)

${ }^{a}$ For Tiers 1 and 2, Category 1 marine engines are greater than or equal to 37 kilowatts $(\mathrm{kW})$ and have a displacement less than 5.0 liters per cylinder (L/cylinder); Category 2 marine engines have a displacement greater than or equal to $5.0 \mathrm{~L} /$ cylinder and less than $30 \mathrm{~L} /$ cylinder; and Category 3 marine engines have a displacement greater than or equal to $30.0 \mathrm{~L} /$ cylinder. For Tiers 3 and 4, Category 1 represents engines up to $7 \mathrm{~L} /$ cylinder displacement; and Category 2 includes engines from 7 to $30 \mathrm{~L} /$ cylinder. The definition of Category 3 marine engines remains the same.

b Tiers 1 and 2 for marine engines less than $37 \mathrm{~kW}$ are subject to the same emission standards as for land-based engines. See Table 1 in 40 Code of Federal Regulations (CFR) Part 89.112 and 40 CFR Part 89.104.

${ }^{\mathrm{c}}$ For Tiers 1 and 2, this refers to the rated power; for Tiers 3 and 4, this refers to the maximum engine power.

d Total hydrocarbon (THC) plus nitrogen oxides (NOx) for Tier 2 standards.

e Useful life is expressed in hours or years, whichever comes first. For Tiers 3 and 4, a longer useful life in hours for an engine family must be specified if either:1) the engine is designed, advertised, or marketed to operate longer than the minimum useful life; or 2) the basic mechanical warranty is longer than the minimum useful life.

${ }^{\mathrm{f}}$ Warranty period is expressed in years and hours, whichever comes first.

${ }^{\mathrm{g}}$ For Tiers 3 and 4, there are no evaporative emission standards for diesel-fueled engines, or engines using other nonvolatile or nonliquid fuels (e.g., natural gas). If an engine uses a volatile liquid fuel, such as methanol, the engine's fuel system and the vessel in which the engine is installed must meet the evaporative emission requirements of 40 Code of Federal Regulations (CFR) Part 1045 that apply with respect to spark-ignition engines. Manufacturers subject to evaporative emission standards must meet the requirements of 40 CFR 1045.112 as described in 40 CFR 1060.1(a)(2).

${ }^{\mathrm{h}}$ Indicates the model years for which the specified standards start.

${ }^{\mathrm{i}} \mathrm{N}$ is the maximum test speed of the engine in revolutions per minute (rpm).

${ }^{\mathrm{j}}$ Manufacturers of Tier 3 engines greater than or equal to $19 \mathrm{~kW}$ and less than $75 \mathrm{~kW}$ with displacement below $0.9 \mathrm{~L} /$ cylinder may alternatively certify some or all of their engine families to a particulate matter (PM) emission standard of 0.20 grams per kilowatt-hour (g/kW-hr) and a NOx+HC emission standard fo $5.8 \mathrm{~g} / \mathrm{kW}$-hr for 2014 and later model years.

${ }^{k}$ The applicable Tier $2 \mathrm{NOx}+\mathrm{HC}$ standards continue to apply instead of the Tier 3 values for engines at or above $2000 \mathrm{~kW}$.

${ }^{1}$ These Tier 3 standards apply to Category 1 engines below $3700 \mathrm{~kW}$ except for recreational marine engines at or above $3700 \mathrm{~kW}$ (with any displacement), which must meet the Tier 3 standards specified for recreational marine engines with a displacement of 3.5 to $7.0 \mathrm{~L} /$ cylinder.

${ }^{m}$ The following provisions are optional: 1) Manufacturers may use NOx credits to certify Tier 4 engines to a NOX+HC emission standard of $1.9 \mathrm{~g} / \mathrm{kW}$-hr instead of the NOX and HC standards. See 40 CFR 1042.101(a)(8)(i) for more details. 2) For engines below $1000 \mathrm{~kW}$, manufacturers may delay complying with the Tier 4 standards until October 1, 2017. 3) For engines at or above $3700 \mathrm{~kW}$, manufacturers may delay complying with the Tier 4 standards until December 31, 2016.

${ }^{\mathrm{n}}$ The Tier 4 standard is for HC (not HC+NOx) in $\mathrm{g} / \mathrm{kW}$-hr.

${ }^{\circ}$ These Tier 3 standards apply to Category 2 engines below $3700 \mathrm{~kW}$; no Tier 3 standards apply for Category 2 engines at or above $3700 \mathrm{~kW}$, although there are Tier 4 standards that apply. 
Table 12.26 (continued)

Marine Compression-Ignition (CI) Engines - Exhaust Emission Standards

p An alternative set of Tier 3 and Tier 4 standards for PM, NOx, and HC are available for Category 2 engines at or above $1400 \mathrm{~kW}$, but must be applied to all of a manufacturer's engines in a given displacement category in model years 2012 through 2015.

\begin{tabular}{|c|c|c|c|c|c|}
\hline Tier & $\begin{array}{c}\text { Maximum } \\
\text { engine } \\
\text { power }\end{array}$ & $\begin{array}{l}\text { Model } \\
\text { year }\end{array}$ & $\begin{array}{c}\text { PM } \\
\text { (g/kW-hr) }\end{array}$ & $\begin{array}{c}\text { NOx } \\
\text { (g/kW-hr) }\end{array}$ & $\begin{array}{c}\mathrm{HC} \\
(\mathrm{g} / \mathrm{kW}-\mathrm{hr})\end{array}$ \\
\hline 3 & $\mathrm{~kW} \geq 1400$ & $2012-2014$ & 0.14 & \multicolumn{2}{|c|}{$7.8 \mathrm{NOx}+\mathrm{HC}$} \\
\hline 4 & $\begin{array}{c}1400 \leq \mathrm{kW}<3700 \\
\mathrm{~kW} \geq 3700\end{array}$ & $\begin{array}{l}2015 \\
2015\end{array}$ & $\begin{array}{l}0.04 \\
0.06\end{array}$ & $\begin{array}{l}1.8 \\
1.8\end{array}$ & $\begin{array}{l}0.19 \\
0.19\end{array}$ \\
\hline
\end{tabular}

q Interim Tier 4 PM standards apply for 2014 and 2015 model year Category 2 engines with per-cylinder displacement at or above 15.0 liters: $0.34 \mathrm{~g} / \mathrm{kW}$-hr for engines $2000=\mathrm{kW}<3000$, and $0.27 \mathrm{~g} / \mathrm{kW}$-hr for engines 3300 $=\mathrm{kW}<3700$. 
These standards apply to gasoline boats and personal watercraft, such as pleasure boats, jet-skis, outboard engines and sterndrive/inboard engines.

Table 12.27

Marine Spark-Ignition Engines and Vessels - Exhaust Emission Standards

\begin{tabular}{|c|c|c|c|c|c|c|c|c|}
\hline & & & \multirow[b]{2}{*}{$\begin{array}{c}\text { Model } \\
\text { year }\end{array}$} & \multicolumn{2}{|c|}{$\begin{array}{l}\mathrm{HC}+\mathrm{NOx}^{\mathrm{a}} \\
(\mathrm{g} / \mathrm{KW}-\mathrm{hr})\end{array}$} & \multicolumn{2}{|c|}{$\begin{array}{c}\mathrm{CO}^{\mathrm{c}} \\
\text { (g/KW-hr) }\end{array}$} & \multirow[b]{2}{*}{$\begin{array}{c}\begin{array}{c}\text { Useful life } \\
\text { (hours/years) }^{\mathrm{d}}\end{array} \\
\end{array}$} \\
\hline & \multicolumn{2}{|c|}{ Engine type } & & $\mathrm{P} \leq 4.3 \mathrm{~kW}^{\mathrm{b}}$ & $\mathrm{P}>4.3 \mathrm{~kW}^{\mathrm{b}}$ & $\begin{array}{c}\mathrm{P} \leq 4.3 \\
\mathrm{~kW}^{\mathrm{b}}\end{array}$ & $\begin{array}{c}\mathrm{P}>4.3 \\
\mathrm{~kW}^{\mathrm{b}}\end{array}$ & \\
\hline \multirow{14}{*}{ Federal $^{\mathrm{e}}$} & \multirow{10}{*}{\multicolumn{2}{|c|}{$\begin{array}{l}\text { Personal watercraft \& } \\
\text { outboard marine engines }\end{array}$}} & 1998 & $278 \mathrm{ABT}$ & $\begin{array}{c}(0.917 \times(151+ \\
\left.557 / \mathrm{P}^{0.9}+2.44\right) \\
{[\mathrm{ABT}]}\end{array}$ & -- & -- & \multirow{9}{*}{$350 / 5$} \\
\hline & & & 1999 & $253 \mathrm{ABT}$ & $\begin{array}{c}(0.833 \times(151+ \\
\left.557 / \mathrm{P}^{0.9}+2.89\right) \\
{[\mathrm{ABT}]}\end{array}$ & -- & -- & \\
\hline & & & 2000 & $228 \mathrm{ABT}$ & $\begin{array}{c}(0.750 \times(151+ \\
\left.557 / \mathrm{P}^{0.9}\right)+3.33 \\
{[\mathrm{ABT}]}\end{array}$ & -- & -- & \\
\hline & & & 2001 & $204 \mathrm{ABT}$ & $\begin{array}{c}(0.667 \times(151+ \\
\left.557 / \mathrm{P}^{0.9}\right)+3.78 \\
{[\mathrm{ABT}]}\end{array}$ & -- & -- & \\
\hline & & & 2002 & $179 \mathrm{ABT}$ & $\begin{array}{c}(0.583 \times(151+ \\
\left.557 / \mathrm{P}^{0.9}\right)+4.22 \\
{[\mathrm{ABT}]}\end{array}$ & -- & -- & \\
\hline & & & 2003 & $155 \mathrm{ABT}$ & $\begin{array}{c}(0.500 \times(151+ \\
\left.557 / \mathrm{P}^{0.9}\right)+4.67 \\
{[\mathrm{ABT}]}\end{array}$ & -- & -- & \\
\hline & & & 2004 & $130 \mathrm{ABT}$ & $\begin{array}{c}(0.417 \times(151+ \\
\left.557 / \mathrm{P}^{0.9}\right)+5.11 \\
{[\mathrm{ABT}]}\end{array}$ & -- & -- & \\
\hline & & & 2005 & $105 \mathrm{ABT}$ & $\begin{array}{c}(0.333 \times(151+ \\
\left.557 / \mathrm{P}^{0.9}\right)+5.56 \\
{[\mathrm{ABT}]}\end{array}$ & -- & -- & \\
\hline & & & $\begin{array}{l}2006- \\
2009\end{array}$ & $81 \mathrm{ABT}$ & $\begin{array}{c}(0.250 \times(151+ \\
\left.557 / \mathrm{P}^{0.9}\right)+6.00 \\
{[\mathrm{ABT}]}\end{array}$ & -- & -- & \\
\hline & & & $2010+\mathrm{t}$ & $30 \mathrm{ABT}$ & $\begin{array}{c}2.1+0.09 \mathrm{x} \\
(151+ \\
\left.557 / \mathrm{P}^{0.9}\right) \\
{[\mathrm{ABT}]} \\
\end{array}$ & $\begin{array}{c}500-5.0 \\
\quad x P\end{array}$ & 300 & $\begin{array}{c}\text { Personal } \\
\text { Watercraft: } 350 \\
\text { / } 5^{\mathrm{h}} \\
\text { Outboard: } 350 \\
/ 10^{\mathrm{h}} \\
\end{array}$ \\
\hline & \multirow{4}{*}{$\begin{array}{l}\text { Sterndrive/ } \\
\text { inboard } \\
\text { engines }\end{array}$} & $\begin{array}{c}\text { Conventional } \\
\text { engines }^{\mathrm{g}}\end{array}$ & $2010+$ & & $\begin{array}{l}.0 \\
\mathrm{BT}]\end{array}$ & $\begin{array}{r}7 \\
{[\mathrm{AF}} \\
\end{array}$ & & $480 / 10^{i}$ \\
\hline & & \multirow{3}{*}{$\begin{array}{l}\text { High- } \\
\text { performance } \\
\text { engines }\end{array}$} & & $\mathrm{P} \leq \mathrm{kW}^{\mathrm{b}}$ & $\mathrm{P}>485 \mathrm{~kW}^{\mathrm{b}}$ & \multirow{3}{*}{\multicolumn{2}{|c|}{350}} & $\begin{array}{l}\mathrm{P} \leq 485 \mathrm{~kW}: \\
150 / 3\end{array}$ \\
\hline & & & 2010 & 20.0 & 25.0 & & & $\begin{array}{l}\mathrm{P}>485 \mathrm{~kW}: \\
\quad 50 / 1\end{array}$ \\
\hline & & & $2011+$ & 16.0 & 22.0 & & & \\
\hline
\end{tabular}

\section{Sources:}

40 CFR 91.104 = Outboard and personal watercraft (PWC) exhaust emission standards (1998-2009)

40 CFR 91.105 = Outboard and PWC useful life (1998-2009)

40 CFR 1045.103 = Outboard and PWC exhaust emission standards (2010+)

40 CFR 1045.105 = Sterndrive/Inboard exhaust emission standards

40 CFR 1045.107 = Not-to-exceed exhaust emission standards (Additional resources: www.epa.gov/emissionstandards-reference-guide/nonroad-engines-and-vehicles-emission-standards)

a The numerical emission standards for hydrocarbons (HC) must be met based on the following types of HC emissions for engines powered by the following fuels: (1) total hydrocarbon equivalent for alcohol; (2) non-methane hydrocarbon for natural gas; and (3) total hydrocarbons for other fuels. 
Table 12.27 (continued) Marine Spark-Ignition Engines and Vessels - Exhaust Emission Standards

${ }^{\mathrm{b}} \mathrm{P}$ stands for the maximum engine power in kilowatts.

${ }^{\mathrm{c}}$ Manufacturers may generate or use emission credits for averaging, but not for banking or trading.

${ }^{\mathrm{d}}$ Useful life and warranty period are expressed hours or years of operation (unless otherwise indicated), whichever comes first.

e The test procedure for federal standards uses the International Organization for Standardization (ISO) 8178 E4 5-Mode Steady-State Test Cycle.

${ }^{\mathrm{f}}$ Also applies to model year (MY) 1997 engine families certified pursuant to 40 Code of Federal Regulations (CFR) 91.205.

g Not-to-exceed emission standards specified in 40 CFR 1045.107 also apply.

${ }^{\mathrm{h}}$ A longer useful life in terms of hours must be specified for the engine family if the average service life is longer than the minimum value as described in 40 CFR 1045.103(e)(3).

i The useful life may not be shorter than: (1) 150 hours of operation; (2) the recommended overhaul interval; or (3) the engine's mechanical warranty. A longer useful life must be specified in terms of hours if the average service life is longer than the minimum value as described in 40 CFR 1045.105(e)(3). 
These standards apply to land-based recreational vehicles, such as snowmobiles, dirt bikes, all-terrain vehicles and go-karts.

\section{Table 12.28}

Nonroad Recreational Engines and Vehicles - Exhaust Emission Standards

\begin{tabular}{|c|c|c|c|c|c|c|c|c|}
\hline & \multirow[b]{2}{*}{ Vehicle } & \multirow[b]{2}{*}{ Phase } & \multirow[b]{2}{*}{ Year } & \multirow{2}{*}{$\begin{array}{l}\mathrm{HC}^{\mathrm{a}} \\
\mathrm{g} / \mathrm{kW}-\mathrm{hr}\end{array}$} & \multirow{2}{*}{$\begin{array}{l}\mathrm{HC}+ \\
\mathrm{NOx} \\
\mathrm{g} / \mathrm{km}\end{array}$} & \multicolumn{2}{|c|}{$\mathrm{CO}$} & \multirow{2}{*}{$\begin{array}{l}\text { Minimum useful life } \\
\text { (hours/years/km) }^{\mathrm{b}}\end{array}$} \\
\hline & & & & & & g/kW-hr & $\mathrm{g} / \mathrm{km}$ & \\
\hline \multirow{6}{*}{ Federal } & \multirow{3}{*}{ Snowmobiles $^{c}$} & $1^{\mathrm{d}}$ & $2006+$ & $\begin{array}{c}100 \\
{[\mathrm{ABT}]}\end{array}$ & - & $\begin{array}{c}275 \\
{[\mathrm{ABT}]}\end{array}$ & - & \multirow{3}{*}{$400 / 5 / 8,000$} \\
\hline & & 2 & $\begin{array}{l}2010- \\
2011\end{array}$ & $\begin{array}{c}75 \\
{[\mathrm{ABT}]}\end{array}$ & - & $\begin{array}{c}275 \\
{[\mathrm{ABT}]}\end{array}$ & - & \\
\hline & & $3^{e}$ & $2012+$ & $\begin{array}{c}150^{\mathrm{f}} \\
{[\mathrm{ABT}]}\end{array}$ & - & $\begin{array}{c}400^{\mathrm{f}} \\
{[\mathrm{ABT}]}\end{array}$ & - & \\
\hline & \multirow{2}{*}{$\begin{array}{l}\text { Off-highway } \\
\text { motorcycles }^{g}\end{array}$} & \multirow{2}{*}{$1^{\mathrm{d}}$} & \multirow{2}{*}{$2006+$} & \multirow{2}{*}{-} & \multirow{2}{*}{$\begin{array}{l}2.0^{\mathrm{h}, \mathrm{i}} \\
{[\mathrm{ABT}]}\end{array}$} & \multirow[b]{2}{*}{-} & \multirow{2}{*}{$\begin{array}{c}25^{\mathrm{h}, \mathrm{i}} \\
{[\mathrm{ABT}]}\end{array}$} & $\begin{array}{c}>70 \text { cc } \\
\text { Displacement: - / } 5 \text { / } \\
10,000\end{array}$ \\
\hline & & & & & & & & $\begin{array}{c}\leq 70 \mathrm{cc} \\
\text { Displacement: - / } 5 \text { / } \\
5,000\end{array}$ \\
\hline & $\mathrm{ATVs}^{\mathrm{g}}$ & $1^{\mathrm{d}}$ & $2006+$ & - & $\begin{array}{l}1.5^{\mathrm{j}, \mathrm{k}} \\
{[\mathrm{ABT}]}\end{array}$ & - & $\begin{array}{c}35^{\mathrm{k}} \\
{[\mathrm{ABT}]}\end{array}$ & $\begin{array}{c}\geq 100 \mathrm{cc} \\
\text { Displacement: } 1000 \text { / } \\
5 \text { / 10,000 } \\
<100 \mathrm{cc} \\
\text { Displacement: } 500 \text { / } \\
\text { 5 / 5,000 }\end{array}$ \\
\hline
\end{tabular}

\section{Source:}

40 CFR 1051.101-115 = Emission standards (Additional resources: www.epa.gov/emission-standards-referenceguide/nonroad-engines-and-vehicles-emission-standards)

a The numerical emission standards for hydrocarbons (HC) must be met based on the following types of hydrocarbon emissions for recreational engines and vehicles powered by the following fuels: (1) non-methane hydrocarbons for natural gas; (2) total hydrocarbon equivalent for alcohol; and (3) total hydrocarbons for other fuels.

${ }^{\mathrm{b}}$ Useful life is expressed in hours, years, or kilometers, whichever comes first; warranty period is expressed in hours, months, or kilometers $(\mathrm{km})$, whichever comes first. Nonroad recreational engines and vehicles must meet emission standards over their full useful life. A longer useful life in terms of $\mathrm{km}$ and hours must be specified for the engine family if the average service life is longer than the minimum value as described in 40 Code of Federal Regulations (CFR) 1051 Subpart B.

${ }^{\mathrm{c}}$ Test procedures for snowmobiles use the equipment and procedures for spark-ignition engines in $40 \mathrm{CFR}$ Part 1065.

${ }^{\mathrm{d}}$ Phase 1 standards will be phased in: 50 percent by 2006, 100 percent by 2007.

e Litigation on the November 2002 final rule resulted in a court decision that requires EPA to clarify the evidence and analysis upon which the Phase 3 carbon monoxide (CO) and HC standards were based. EPA will address this in a future rulemaking.

${ }^{\mathrm{f}}$ These are the maximum allowable family emission limits (FEL). The HC and CO standards are defined by a functional relationship as described in 40 CFR 1051.103(a)(2).

${ }^{\mathrm{g}}$ For off-highway motorcycles and ATVs, chassis dynamometer emissions test procedures are specified in 40 CFR Part 86, Subpart F and engine dynamometer emissions test procedures are specified in 40 CFR Part 1065.

${ }^{\mathrm{h}}$ Maximum allowable FEL: 20.0 grams per kilometer (g/km) for HC plus nitrogen oxides (NOx) and $50 \mathrm{~g} / \mathrm{km}$ for CO. 
Table 12.28 (continued) Nonroad Recreational Engines and Vehicles - Exhaust Emission Standards

${ }^{\mathrm{i}}$ Manufacturers may certify off-highway motorcycles with engines that have total displacement of 70 cubic centimeters (cc) or less to an HC+NOx standard of 16.1 grams per kilowatt-hour (g/kW-hr) (with an FEL cap of 32.2 $\mathrm{g} / \mathrm{kW}$-hr) and a CO standard of $519 \mathrm{~g} / \mathrm{kW}-\mathrm{hr}$.

${ }^{\mathrm{j}}$ Maximum allowable FEL for HC+NOx is $20.0 \mathrm{~g} / \mathrm{km}$.

${ }^{\mathrm{k}}$ Manufacturers may certify all-terrain vehicles with engines that have total displacement of less than $100 \mathrm{cc}$ to an HC+NOx standard of $25.0 \mathrm{~g} / \mathrm{kW}$-hr (with an FEL cap of $40.0 \mathrm{~g} / \mathrm{kW}$-hr) and a CO standard of $500 \mathrm{~g} / \mathrm{kW}$-hr. 
The latest standards were established by the Environmental Protection Agency in conjunction with the Tier 3 emission standards.

Table 12.29

Gasoline Sulfur Standards

\begin{tabular}{|c|c|c|c|c|c|c|c|c|}
\hline & \multirow[b]{2}{*}{ Regulated entity } & \multicolumn{7}{|c|}{ Refinery average and per-gallon cap by year (ppm) } \\
\hline & & 2004 & 2005 & 2006 & 2007 & $2008-2016$ & $2017-2019$ & 2020 \\
\hline \multirow{4}{*}{ Federal } & $\begin{array}{c}\text { Large refiners / } \\
\text { importers }^{\mathrm{a}}\end{array}$ & $120^{\mathrm{b}} / 300^{\mathrm{c}}$ & $30 / 90^{\mathrm{b}} / 300$ & $30 / 80$ & $30 / 80$ & $30 / 80$ & $10 / 80$ & $10 / 80$ \\
\hline & $\begin{array}{c}\text { GPA } \\
\text { refiners } \mathrm{d} \text {, e }\end{array}$ & $150 / 300^{c}$ & $150 / 300$ & $150 / 300$ & $30 / 80$ & $30 / 80$ & $30 / 80$ & $10 / 80$ \\
\hline & $\begin{array}{c}\text { Small } \\
\text { refiners } \mathrm{f}, \mathrm{g}, \mathrm{h}\end{array}$ & $\mathrm{k}$ & $\mathrm{k}$ & $\mathrm{k}$ & $\mathrm{k}$ & $30 / 80$ & $30 / 80$ & $10 / 80$ \\
\hline & $\begin{array}{l}\text { Downstream } \\
\text { standards } i, j\end{array}$ & 378 & 326 & 95 & 95 & 95 & 95 & 95 \\
\hline
\end{tabular}

\section{Source:}

40 CFR 80 Subpart H (Additional resources: www.epa.gov/emission-standards-reference-guide/fuel-sulfurstandards)

a Standards effective January 1 at the refinery gate.

b No Refinery Average Standard applies in 2004; Corporate Average Standard applies in 2004 (120 ppm) and 2005 (90 ppm).

${ }^{\text {c }}$ Cap exceedances up to 50 ppm in 2004 must be made up in 2005.

${ }^{\mathrm{d}}$ Geographic Phase-in Area (GPA) refiners must also comply with the corporate average standards in 2004 and 2005 if less than 50\% of the refiner's gasoline is designated as GPA gasoline in a given compliance period.

e GPA refiners may receive an additional two years (i.e., through 2008) to comply with the 30 / 80 ppm gasoline sulfur standards in exchange for producing $95 \%$ of their highway diesel fuel at the 15 ppm sulfur standard by June 1 , 2006.

${ }^{\mathrm{f}}$ Small refiners may receive an additional two years (i.e., through 2009) to comply with the 30 / 80 ppm gasoline sulfur standards via a hardship demonstration.

g Small refiners may receive an additional three years (i.e., through 2010) to comply with the 30 / 80 ppm gasoline sulfur standards in exchange for producing $95 \%$ of their highway diesel fuel at the 15 ppm sulfur standard by June 1, 2006.

h Small refiners may receive a 20\% increase in their annual average and per-gallon cap standards in exchange for producing $95 \%$ of their highway, nonroad, locomotive, and marine diesel fuel at the 15 ppm sulfur standard by June 1, 2006.

${ }^{i}$ Downstream standards are effective February 1 at any downstream location other than at a retail outlet or wholesale purchaser-consumer (e.g., pipelines and terminals) and March 1 at any downstream location.

j Downstream standards for gasoline that is not blended with small refiner gasoline are shown. Refer to the Code of Federal Regulations (CFR) for the downstream standards that apply when a gasoline blend includes small refiner gasoline.

\begin{tabular}{c|c|c}
\hline $\begin{array}{c}\text { 1997-98 Refinery } \\
\text { baseline sulfur level } \\
\text { (ppm) }\end{array}$ & $\begin{array}{c}\text { Small refiner interim gasoline sulfur standards } \\
\text { (ppm) 2004-2007 }\end{array}$ \\
\hline 0 to 30 & Average & Cap \\
\hline 31 to 200 & 30 & 300 \\
\hline 201 to 400 & baseline level & 300 \\
\hline 401 to 600 & 200 & 1.5 x avg. standard \\
\hline 601 and above & $50 \%$ of baseline & 450 \\
\hline
\end{tabular}


Ultra-low sulfur diesel (ULSD) fuel is necessary for new advanced emission control technologies. It also reduces particulate matter in the existing fleet of nonroad engines and equipment.

Table 12.30

Highway, Nonroad, Locomotive, and Marine (NRLM) Diesel Fuel Sulfur Standards

\begin{tabular}{|c|c|c|c|c|c|c|c|c|c|c|c|}
\hline & & Covered & & & -gallon & ximum & Ifur leve & by yea & (ppm) & & \\
\hline & Regulated entity & fuel & $2006^{a}$ & $2007^{b}$ & 2008 & 2009 & $2010^{\mathrm{c}, \mathrm{d}}$ & 2011 & 2012 & 2013 & 2014 \\
\hline \multirow{9}{*}{ Federal } & \multirow{2}{*}{$\begin{array}{l}\text { Large refiners \& } \\
\text { importers }\end{array}$} & \multirow{2}{*}{ Highway } & \multicolumn{4}{|c|}{$80 \% 15$} & \multirow{3}{*}{\multicolumn{5}{|c|}{15}} \\
\hline & & & \multicolumn{4}{|c|}{$20 \% 500$} & & & & & \\
\hline & Small refiners & Highway & \multicolumn{4}{|c|}{500} & & & & & \\
\hline & \multirow{3}{*}{$\begin{array}{l}\text { Large refiners \& } \\
\text { importers }\end{array}$} & NR & - & 500 & 500 & 500 & 15 & 15 & 15 & 15 & 15 \\
\hline & & LM & - & 500 & 500 & 500 & 500 & 500 & 15 & 15 & 15 \\
\hline & & $\begin{array}{l}\text { NRLM } \\
\text { with } \\
\text { credits }^{\mathrm{e}}\end{array}$ & - & HS & HS & HS & 500 & 500 & 500 & 500 & 15 \\
\hline & Small refiners & NRLM $^{f}$ & - & HS & HS & HS & 500 & 500 & 500 & 500 & 15 \\
\hline & \multirow{2}{*}{$\begin{array}{c}\text { Transmix } \\
\text { processor \& in-use }\end{array}$} & $\mathrm{NR}^{\mathrm{e}}$ & - & HS & HS & HS & 500 & 500 & 500 & 500 & 15 \\
\hline & & $\mathrm{LM}^{\mathrm{e}}$ & - & HS & HS & HS & 500 & 500 & 500 & 500 & 500 \\
\hline
\end{tabular}

Source:

40 CFR 80 Subpart I (Additional resources: www.epa.gov/emission-standards-reference-guide/fuel-sulfurstandards)

a For highway diesel fuel, standards are effective June 1 for refiners/importers, September 1 for pipelines and terminals, and October 15 for retailers and wholesale purchaser-consumers. Anti-downgrading provisions effective October 16, 2006.

b For Nonroad, Locomotive, and Marine (NRLM) diesel fuel, standards are effective June 1 for refiners; downstream requirements apply for Northeast/Mid-Atlantic area only (August 1 for terminals, October 1 for retailers and wholesale purchaser-consumers, and December 1 for in-use).

c For highway diesel fuel, standards are effective June 1 for refiners/importers, October 1 for pipelines and terminals, and December 1 for retailers and wholesale purchaser-consumers.

${ }^{\mathrm{d}}$ For NRLM diesel fuel, standards are effective June 1 for refiners, August 1 for terminals, October 1 for retailers and wholesale purchaser-consumers, and December 1 for in-use.

e Excluding the Northeast and Alaska.

${ }^{\mathrm{f}}$ Excluding the Northeast, with approval in Alaska. 


\section{APPENDIX A}

SOURCES \& METHODOLOGIES 
A-2

TRANSPORTATION ENERGy DATA BOOK: EDITION 36-2017 


\section{SOURCES \& METHODOLOGIES}

This appendix contains documentation of the estimation procedures used by ORNL. The reader can examine the methodology behind the estimates and form an opinion as to their utility. The appendix is arranged by subject heading. Only tables which contain ORNL estimations are documented in Appendix A; all other tables have sources listed at the bottom of the table. Since abbreviations are used throughout the appendix, a list of abbreviations is also included.

\section{Contents of Appendix A}

1. List of Abbreviations Used in Appendix A .....................................................................

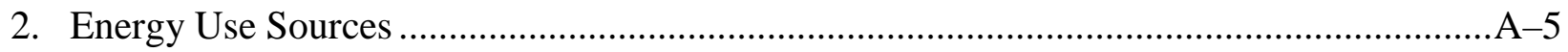

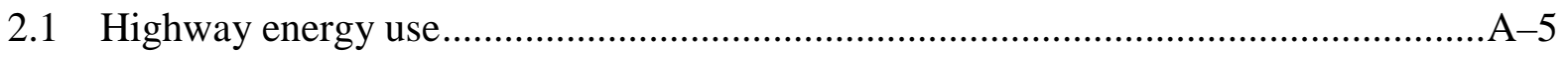

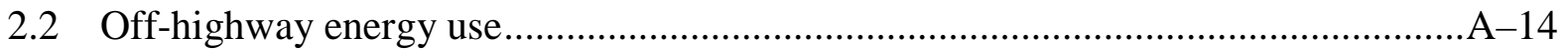

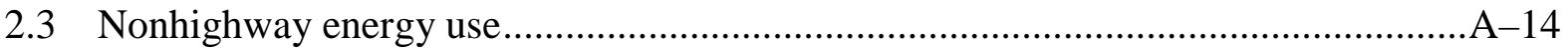

2.4 Calculation of Million Barrels per Day Crude Oil Equivalent ...............................A-24

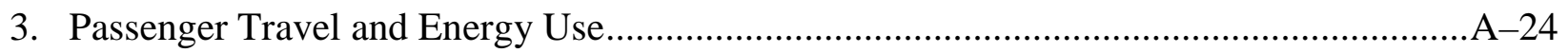

4. Highway Passenger Mode Energy Intensities................................................................28

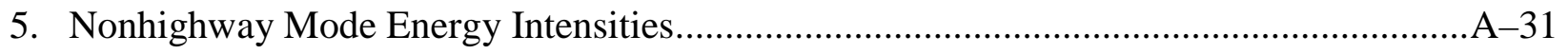

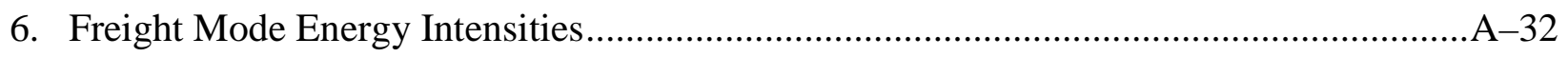

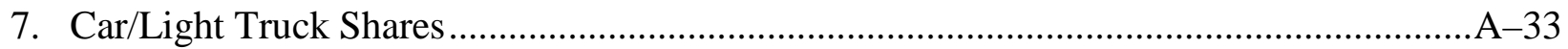




\section{LIST OF ABBREVIATIONS USED IN APPENDIX A}

\begin{tabular}{|c|c|}
\hline AAR & Association of American Railroads \\
\hline APTA & American Public Transportation Association \\
\hline Amtrak & National Railroad Passenger Corporation \\
\hline BTS & Bureau of Transportation Statistics \\
\hline Btu & British thermal unit \\
\hline CD & Compact Disc \\
\hline CNG & Compressed Natural Gas \\
\hline $\mathrm{CO}_{2}$ & Carbon Dioxide \\
\hline CPI & Consumer Price Index \\
\hline $\mathrm{CY}$ & Calendar Year \\
\hline DOE & Department of Energy \\
\hline DOT & Department of Transportation \\
\hline EIA & Energy Information Administration \\
\hline EPA & Environmental Protection Agency \\
\hline FAA & Federal Aviation Administration \\
\hline FHWA & Federal Highway Administration \\
\hline IRS & Internal Revenue Service \\
\hline gal & Gallons \\
\hline kWhr & Kilowatt hour \\
\hline $\mathrm{L}$ & Liter \\
\hline $\mathrm{lb}$ & Pound \\
\hline $\operatorname{lng}$ & Liquefied Natural Gas \\
\hline $\operatorname{lpg}$ & Liquefied Petroleum Gas \\
\hline mpg & Miles per Gallon \\
\hline NHTS & National Household Travel Survey \\
\hline NPTS & Nationwide Personal Transportation Survey \\
\hline NVPP & National Vehicle Population Profile \\
\hline ORNL & Oak Ridge National Laboratory \\
\hline RTECS & Residential Transportation Energy Consumption Survey \\
\hline SCF & Standard Cubic Feet \\
\hline TIUS & Truck Inventory and Use Survey \\
\hline VIUS & Vehicle Inventory and Use Survey \\
\hline vmt & vehicle-miles traveled \\
\hline
\end{tabular}




\section{ENERGY USE SOURCES}

\subsection{HIGHWAY ENERGY USE}

\subsubsection{Cars}

Fuel use in gallons (1970-2008) - DOT, FHWA, Highway Statistics 2008, Table VM-1 and annual editions back to 1996; DOT, FHWA, Highway Statistics Summary to 1995.

Fuel use in gallons (2009 - 2015) - See Section 7. Appendix A Car and Light Truck Shares.

Fuel type distribution for gallons - Fuel use was distributed among fuel types using the percentages shown in Table A.1. The FHWA discontinued gasohol data in 2005. Therefore, data from EIA, Alternatives to Traditional Transportation Fuels, 2006-2011, Table C1 were used.

Electricity use (2014) - U.S. Department of Energy, Energy Information Administration, Annual Energy Outlook 2016, July 2016, Table 37. Total light vehicle electricity usage was split between cars and light trucks using cumulative vehicle sales as a proxy.

Electricity use (2015) - Estimates derived using cumulative electric vehicle (EV) and plug-in hybrid vehicle (PHEV) sales as a proxy for vehicle population; sales-weighted vehicle efficiencies from the U.S. Department of Energy and U.S. Environmental Protection Agency's vehicle database on www.fueleconomy.gov; and annual miles traveled from varying PHEV utility factors and EV usage assumptions. A report documenting this methodology is forthcoming. 
Table A.1

Car Fuel Use and Fuel Type Shares for Calculation of Energy Use

\begin{tabular}{|c|c|c|c|c|c|c|}
\hline \multirow[b]{2}{*}{ Year } & \multirow{2}{*}{$\begin{array}{c}\text { Fuel use } \\
\text { (million gallons) }\end{array}$} & \multirow{2}{*}{$\begin{array}{c}\text { Source for } \\
\text { gasohol shares }\end{array}$} & \multirow{2}{*}{$\begin{array}{c}\text { Source for } \\
\text { gasoline/diesel shares }\end{array}$} & \multicolumn{3}{|c|}{ Shares by fuel type ${ }^{b}$} \\
\hline & & & & Gasoline & Gasohol & Diesel \\
\hline 1970 & 67,820 & & 1984 NVPP & $99.8 \%$ & $0.0 \%$ & $0.2 \%$ \\
\hline 1971 & 71,346 & & interpolated & $99.2 \%$ & $0.0 \%$ & $0.8 \%$ \\
\hline 1972 & 75,937 & & interpolated & $98.7 \%$ & $0.0 \%$ & $1.3 \%$ \\
\hline 1973 & 78,233 & & interpolated & $98.1 \%$ & $0.0 \%$ & $1.9 \%$ \\
\hline 1974 & 74,229 & & interpolated & $97.5 \%$ & $0.0 \%$ & $2.5 \%$ \\
\hline 1975 & 74,140 & & interpolated & $97.0 \%$ & $0.0 \%$ & $3.0 \%$ \\
\hline 1976 & 78,297 & & interpolated & $96.4 \%$ & $0.0 \%$ & $3.6 \%$ \\
\hline 1977 & 79,060 & & interpolated & $95.8 \%$ & $0.0 \%$ & $4.2 \%$ \\
\hline 1978 & 80,652 & & interpolated & $95.3 \%$ & $0.0 \%$ & $4.7 \%$ \\
\hline 1979 & 76,588 & & 1979 RTECS & $94.7 \%$ & $0.0 \%$ & $5.3 \%$ \\
\hline 1980 & 69,981 & FHWA, MF-33e & interpolated & $93.9 \%$ & $0.5 \%$ & $5.6 \%$ \\
\hline 1981 & 69,112 & FHWA, MF-33e & 1981 RTECS & $93.4 \%$ & $0.7 \%$ & $5.9 \%$ \\
\hline 1982 & 69,116 & FHWA, MF-33e & interpolated & $93.5 \%$ & $2.3 \%$ & $4.2 \%$ \\
\hline 1983 & 70,322 & FHWA, MF-33e & 1983 RTECS & $93.2 \%$ & $4.3 \%$ & $2.5 \%$ \\
\hline 1984 & 70,663 & FHWA, MF-33e & interpolated & $92.7 \%$ & $5.3 \%$ & $2.0 \%$ \\
\hline 1985 & 71,518 & FHWA, MF-33e & 1985 RTECS & $90.8 \%$ & $7.7 \%$ & $1.5 \%$ \\
\hline 1986 & 73,174 & FHWA, MF-33e & interpolated & $91.0 \%$ & $7.6 \%$ & $1.4 \%$ \\
\hline 1987 & 73,308 & FHWA, MF-33e & interpolated & $92.4 \%$ & $6.3 \%$ & $1.3 \%$ \\
\hline 1988 & 73,345 & FHWA, MF-33e & 1988 RTECS & $91.4 \%$ & $7.4 \%$ & $1.2 \%$ \\
\hline 1989 & 73,913 & FHWA, MF-33e & interpolated & $92.6 \%$ & $6.2 \%$ & $1.2 \%$ \\
\hline 1990 & 69,568 & FHWA, MF-33e & interpolated & $92.0 \%$ & $6.8 \%$ & $1.2 \%$ \\
\hline 1991 & 64,318 & FHWA, MF-33e & 1991 RTECS & $90.8 \%$ & $8.0 \%$ & $1.2 \%$ \\
\hline 1992 & 65,436 & FHWA, MF-33e & interpolated & $90.8 \%$ & $7.9 \%$ & $1.2 \%$ \\
\hline 1993 & 67,047 & FHWA, MF-33e & interpolated & $89.7 \%$ & $9.1 \%$ & $1.3 \%$ \\
\hline 1994 & 67,874 & FHWA, MF-33e & 1994 RTECS & $89.1 \%$ & $9.6 \%$ & $1.3 \%$ \\
\hline 1995 & 68,072 & FHWA, MF-33e & interpolated & $87.6 \%$ & $11.2 \%$ & $1.2 \%$ \\
\hline 1996 & 69,221 & FHWA, MF-33e & interpolated & $88.8 \%$ & $10.1 \%$ & $1.0 \%$ \\
\hline 1997 & 69,892 & FHWA, MF-33e & interpolated & $86.9 \%$ & $12.2 \%$ & $0.9 \%$ \\
\hline 1998 & 71,695 & FHWA, MF-33e & interpolated & $88.0 \%$ & $11.2 \%$ & $0.8 \%$ \\
\hline 1999 & 73,283 & FHWA, MF-33e & interpolated & $88.3 \%$ & $11.0 \%$ & $0.6 \%$ \\
\hline 2000 & 73,065 & FHWA, MF-33e & 2000 NVPP & $86.9 \%$ & $12.6 \%$ & $0.5 \%$ \\
\hline 2001 & 73,559 & FHWA, MF-33e & 2001 NVPP & $86.5 \%$ & $13.0 \%$ & $0.5 \%$ \\
\hline 2002 & 75,471 & FHWA, MF-33e & 2001 NVPP & $83.9 \%$ & $15.6 \%$ & $0.5 \%$ \\
\hline 2003 & 74,590 & FHWA, MF-33e & 2001 NVPP & $75.3 \%$ & $24.2 \%$ & $0.5 \%$ \\
\hline 2004 & 75,402 & FHWA, MF-33e & 2001 NVPP & $67.2 \%$ & $32.3 \%$ & $0.5 \%$ \\
\hline 2005 & 77,418 & FHWA, MF-33e & 2001 NVPP & $66.9 \%$ & $32.6 \%$ & $0.5 \%$ \\
\hline 2006 & 75,009 & EIA, C1 & 2001 NVPP & $78.2 \%$ & $21.3 \%$ & $0.5 \%$ \\
\hline 2007 & 74,377 & EIA, C1 & 2001 NVPP & $72.9 \%$ & $26.6 \%$ & $0.5 \%$ \\
\hline 2008 & 71,497 & EIA, C1 & 2001 NVPP & $61.8 \%$ & $37.7 \%$ & $0.5 \%$ \\
\hline 2009 & 66,587 & EIA, C1 & 2001 NVPP & $55.8 \%$ & $43.7 \%$ & $0.5 \%$ \\
\hline 2010 & 62,245 & EIA, C1 & 2001 NVPP & $49.5 \%$ & $50.0 \%$ & $0.5 \%$ \\
\hline 2011 & 59,646 & EIA, C1 & 2001 NVPP & $48.7 \%$ & $50.8 \%$ & $0.5 \%$ \\
\hline 2012 & 57,899 & EIA, C1 & 2001 NVPP & $48.7 \%$ & $50.8 \%$ & $0.5 \%$ \\
\hline 2013 & 57,290 & EIA, C1 & 2001 NVPP & $49.0 \%$ & $50.5 \%$ & $0.5 \%$ \\
\hline 2014 & 56,420 & EIA, C1 & 2001 NVPP & $50.1 \%$ & $49.4 \%$ & $0.5 \%$ \\
\hline \multirow[t]{2}{*}{2015} & 55,212 & EIA, C1 & 2001 NVPP & $50.0 \%$ & $49.5 \%$ & $0.5 \%$ \\
\hline & \multicolumn{2}{|c|}{ Heat content used for conversion to btu: } & & $\begin{array}{l}\text { 125,000 } \\
\text { btu/gallon }\end{array}$ & $\begin{array}{l}\text { 120,900 } \\
\text { btu/gallon }\end{array}$ & $\begin{array}{l}\text { 138,700 } \\
\text { btu/gallon }\end{array}$ \\
\hline
\end{tabular}

${ }^{\text {a }}$ Data are not continuous between 2008 and 2009 due to changes in source.

${ }^{\mathrm{b}}$ Percentages may not sum due to rounding. 


\subsubsection{Motorcycles}

DOT, FHWA, Highway Statistics 2015, Table VM-1, and annual editions. The FHWA made methodology changes for Highway Statistics 2009-10. At that time, they published historical data back to 2007 which do not match the previous data.

Table A.2

\section{Motorcycle Fuel Use}

\begin{tabular}{|c|c|c|c|c|}
\hline Year & $\begin{array}{c}\text { Fuel use } \\
\text { (thousand gallons) }\end{array}$ & Year & $\begin{array}{c}\text { Fuel use } \\
\text { (thousand gallons) }\end{array}$ & \\
\hline 1970 & 59,580 & 1993 & 198,120 & \\
\hline 1971 & 72,140 & 1994 & 204,800 & \\
\hline 1972 & 86,620 & 1995 & 198,262 & \\
\hline 1973 & 103,880 & 1996 & 195,940 & \\
\hline 1974 & 108,900 & 1997 & 201,620 & \\
\hline 1975 & 112,580 & 1998 & 205,660 & \\
\hline 1976 & 120,060 & 1999 & 211,680 & \\
\hline 1977 & 126,980 & 2000 & 209,380 & \\
\hline 1978 & 143,160 & 2001 & 192,780 & \\
\hline 1979 & 172,740 & 2002 & 191,040 & \\
\hline 1980 & 204,280 & 2003 & 190,780 & \\
\hline 1981 & 213,800 & 2004 & 202,447 & \\
\hline 1982 & 198,200 & 2005 & 189,495 & \\
\hline 1983 & 175,200 & 2006 & 221,030 & $\mathrm{a}$ \\
\hline 1984 & 175,680 & 2007 & 474,923 & \\
\hline 1985 & 181,720 & 2008 & 489,417 & \\
\hline 1986 & 187,940 & 2009 & 482,290 & \\
\hline 1987 & 190,120 & 2010 & 426,732 & \\
\hline 1988 & 200,480 & 2011 & 426,378 & \\
\hline 1989 & 207,420 & 2012 & 491,130 & \\
\hline 1990 & 191,140 & 2013 & 467,716 & \\
\hline 1991 & 183,560 & 2014 & 458,628 & \\
\hline 1992 & 191,140 & 2015 & 447,879 & \\
\hline \multicolumn{3}{|c|}{ Heat content used for conversion to btu: } & $125,000 \mathrm{btu} /$ gallon & \\
\hline
\end{tabular}

a Data are not continuous between 2006 and 2007 due to changes in estimation methodology. See source document for details. 


\subsubsection{Buses}

\section{Transit}

APTA, 2016 Public Transportation Fact Book, Washington, DC, 2016. Includes motorbus and trolley bus data. Data for 2015 were generated from the National Transit Database.

Table A.3

Transit Bus Fuel Use

\begin{tabular}{|c|c|c|c|c|c|c|c|c|}
\hline Year & $\begin{array}{c}\text { LNG } \\
\text { (million } \\
\text { gallons) }\end{array}$ & $\begin{array}{l}\text { LPG } \\
\text { (million } \\
\text { gallons) } \\
\end{array}$ & $\begin{array}{c}\text { CNG } \\
\text { (million } \\
\text { gallons) }\end{array}$ & $\begin{array}{l}\text { Gasoline } \\
\text { (million } \\
\text { gallons) }\end{array}$ & $\begin{array}{l}\text { Diesel } \\
\text { fuel } \\
\text { (million } \\
\text { gallons) }\end{array}$ & $\begin{array}{c}\text { Electricity } \\
\text { (thousand } \\
\text { kilowatt } \\
\text { hours) }\end{array}$ & $\begin{array}{l}\text { Biodiesel } \\
\text { (million } \\
\text { gallons) }\end{array}$ & $\begin{array}{l}\text { Methanol } \\
\text { (million } \\
\text { gallons) }\end{array}$ \\
\hline 1994 & 1.1 & 0.2 & 3.1 & 2.1 & 565.1 & 102.9 & $\mathrm{a}$ & 12.5 \\
\hline 1995 & 1.7 & 0.3 & 10.0 & 2.3 & 563.8 & 100.0 & $\mathrm{a}$ & 12.0 \\
\hline 1996 & 2.3 & 0.6 & 11.5 & 1.8 & 577.7 & 69.0 & $\mathrm{a}$ & 11.6 \\
\hline 1997 & 3.3 & 1.0 & 20.0 & 2.7 & 597.6 & 78.0 & $\mathrm{a}$ & 8.7 \\
\hline 1998 & 3.1 & 0.9 & 32.6 & 2.0 & 606.6 & 74.0 & $\mathrm{a}$ & 5.0 \\
\hline 1999 & 5.3 & 0.8 & 39.9 & 1.4 & 618.0 & 75.0 & a & 2.7 \\
\hline 2000 & 10.5 & 0.7 & 50.4 & 1.3 & 635.2 & 77.0 & $\mathrm{a}$ & 0.8 \\
\hline 2001 & 11.7 & 1.2 & 60.9 & 1.5 & 587.2 & 74.0 & a & 0.8 \\
\hline 2002 & 16.8 & 1.8 & 77.8 & 1.3 & 559.0 & 73.0 & $\mathrm{a}$ & 1.8 \\
\hline 2003 & 14.2 & 1.8 & 94.9 & 1.1 & 536.0 & 69.0 & $\mathrm{a}$ & 1.9 \\
\hline 2004 & 16.5 & 1.7 & 106.7 & 1.8 & 550.5 & 68.0 & a & 4.7 \\
\hline 2005 & 18.3 & 2.0 & 117.2 & 1.0 & 533.8 & 67.0 & a & 8.1 \\
\hline 2006 & 19.6 & 1.6 & 138.8 & 2.3 & 536.7 & 62.0 & 20.5 & 0.9 \\
\hline 2007 & 18.3 & a & 129.1 & 2.5 & 494.1 & 61.0 & 25.8 & 1.3 \\
\hline 2008 & 17.9 & a & 135.5 & 3.8 & 493.3 & 62.2 & 41.8 & 0.9 \\
\hline 2009 & 25.5 & a & 141.6 & 6.7 & 455.5 & 69.5 & 40.6 & 0.0 \\
\hline 2010 & 23.0 & a & 126.2 & 8.1 & 435.4 & 66.0 & 43.5 & 0.0 \\
\hline 2011 & 21.6 & a & 131.1 & 8.9 & 455.1 & 61.0 & 51.1 & 0.0 \\
\hline 2012 & 19.6 & a & 127.3 & 12.5 & 439.0 & 61.0 & 56.6 & 0.0 \\
\hline 2013 & 17.6 & 6.3 & 134.9 & 12.9 & 427.5 & 63.0 & 66.2 & 0.0 \\
\hline 2014 & 15.4 & 6.2 & 146.0 & 11.7 & 413.6 & 64.0 & 38.1 & 1.2 \\
\hline 2015 & 10.3 & 7.6 & 151.3 & 8.6 & 377.8 & 74.8 & 39.9 & 1.2 \\
\hline $\begin{array}{l}\text { Heat content used } \\
\text { for conversion } \\
\text { to btu: }\end{array}$ & $\begin{array}{l}84,800 \\
\text { btu/gallon }\end{array}$ & $\begin{array}{l}\text { 91,300 } \\
\text { btu/gallon }\end{array}$ & $\begin{array}{l}\text { 138,700 } \\
\text { btu/gallon }\end{array}$ & $\begin{array}{l}\text { 125,000 } \\
\text { btu/gallon }\end{array}$ & $\begin{array}{l}\text { 138,700 } \\
\text { btu/gallon }\end{array}$ & $\begin{array}{l}3,412 \\
\text { btu/kWhr }\end{array}$ & $\begin{array}{l}\text { 126,200 } \\
\text { btu/gallon }\end{array}$ & $\begin{array}{l}\text { 64,600 } \\
\text { btu/gallon }\end{array}$ \\
\hline
\end{tabular}

Note: CNG is reported in diesel-gallon equivalents.

\footnotetext{
${ }^{\text {a }}$ Data are not available.
} 


\section{Intercity and School}

Eno Transportation Foundation, Transportation in America, 2001, Nineteenth Edition, 2003, Washington, DC, pp. 20-23. School bus fuel was assumed to be $90 \%$ diesel fuel and $10 \%$ gasoline based on estimates from the National Association of State Directors of Pupil Transportation Services. Intercity bus fuel was assumed to be $100 \%$ diesel.

Table A.4

Intercity and School Bus Fuel Use

\begin{tabular}{|c|c|c|}
\hline Year & $\begin{array}{c}\text { Intercity } \\
\text { (million gallons) }\end{array}$ & $\begin{array}{c}\text { School } \\
\text { (million gallons) }\end{array}$ \\
\hline 1970 & 305.34 & 299.88 \\
\hline 1975 & 181.02 & 341.88 \\
\hline 1980 & 213.78 & 379.68 \\
\hline 1981 & 205.38 & 386.82 \\
\hline 1982 & 227.22 & 398.58 \\
\hline 1983 & 237.30 & 400.68 \\
\hline 1984 & 169.26 & 375.06 \\
\hline 1985 & 165.48 & 425.04 \\
\hline 1986 & 148.68 & 462.42 \\
\hline 1987 & 155.82 & 487.20 \\
\hline 1988 & 160.44 & 511.14 \\
\hline 1989 & 166.74 & 498.12 \\
\hline 1990 & 159.60 & 472.08 \\
\hline 1991 & 160.44 & 533.40 \\
\hline 1992 & 157.08 & 546.00 \\
\hline 1993 & 171.36 & 533.40 \\
\hline 1994 & 195.30 & 546.00 \\
\hline 1995 & 195.30 & 545.16 \\
\hline 1996 & 199.92 & 545.16 \\
\hline 1997 & 212.52 & 544.74 \\
\hline 1998 & 220.08 & 550.20 \\
\hline 1999 & 241.08 & 555.66 \\
\hline 2000 & 233.10 & 577.08 \\
\hline 2001 & $217.35^{*}$ & $538.08 *$ \\
\hline 2002 & $210.22 *$ & $520.44 *$ \\
\hline 2003 & $208.32 *$ & $515.72 *$ \\
\hline 2004 & $208.87^{*}$ & $517.09 *$ \\
\hline 2005 & $214.37 *$ & $530.70 *$ \\
\hline 2006 & $208.32 *$ & $515.72 *$ \\
\hline 2007 & $214.37 *$ & $530.70 *$ \\
\hline 2008 & $218.48 *$ & $540.89 *$ \\
\hline 2009 & $224.58 *$ & $556.00 *$ \\
\hline 2010 & $214.95 *$ & $532.15 *$ \\
\hline 2011 & $215.53 *$ & $533.58 *$ \\
\hline 2012 & 230.42 & $570.45^{*}$ \\
\hline 2013 & 236.76 & $586.14 *$ \\
\hline 2014 & 249.75 & $618.29 *$ \\
\hline 2015 & 253.35 & 627.22 \\
\hline Fuel type shares & $100 \%$ diesel & $\begin{array}{c}90 \% \text { diesel } \\
10 \% \text { gasoline }\end{array}$ \\
\hline $\begin{array}{l}\text { Heat content used for } \\
\text { conversion to btu: }\end{array}$ & $\begin{array}{c}\text { 138,700 } \\
\text { btu/gallon }\end{array}$ & $\begin{array}{l}138,700 \mathrm{btu} / \text { gallon } \\
125,000 \mathrm{btu} / \text { gallon }\end{array}$ \\
\hline
\end{tabular}

*Estimated using the rate of change of bus vehicle-miles traveled from FHWA Highway Statistics, Table VM-1 (recently revised). 


\subsubsection{Trucks}

\section{Light Trucks}

Fuel use in gallons (1970-2007) - DOT, FHWA, Highway Statistics 2008, Table VM-1 and annual editions back to 1996 and DOT, FHWA, Highway Statistics Summary to 1995.

Fuel use in gallons (2008 - 2014) - See Section 7. Appendix A Car and Light Truck Shares.

Fuel type distribution - Fuel use was distributed among fuel types using the percentages shown in Table A.1. The FHWA discontinued gasohol data in 2005. Therefore, data from EIA, Alternatives to Traditional Transportation Fuels, 2006-2011, Table C.1 were used.

Electricity (2015) - U.S. Department of Energy, Energy Information Administration, Annual Energy Outlook 2017, January 2017, Table 3.7. Total light vehicle electricity usage was split between cars and light trucks using cumulative vehicle sales as a proxy. 
Table A.5

Light Truck Fuel Use and Fuel Type Shares for Calculation of Energy Use

\begin{tabular}{|c|c|c|c|c|c|c|c|}
\hline \multirow[b]{2}{*}{ Year } & \multirow{2}{*}{$\begin{array}{l}\text { Fuel use } \\
\text { (million } \\
\text { gallons) }\end{array}$} & \multirow{2}{*}{$\begin{array}{c}\text { Source for } \\
\text { gasohol shares }\end{array}$} & \multirow{2}{*}{$\begin{array}{c}\text { Source for } \\
\text { gasoline/diesel } \\
\text { /lpg shares }\end{array}$} & \multicolumn{4}{|c|}{ Shares by fuel type } \\
\hline & & & & Gasoline & Gasohol & Diesel & Lpg \\
\hline 1970 & 12,313 & & 1977 TIUS & $97.6 \%$ & $0.0 \%$ & $1.6 \%$ & $0.8 \%$ \\
\hline 1975 & 19,081 & & 1977 TIUS & $97.6 \%$ & $0.0 \%$ & $1.6 \%$ & $0.8 \%$ \\
\hline 1976 & 20,828 & & 1977 TIUS & $97.6 \%$ & $0.0 \%$ & $1.6 \%$ & $0.8 \%$ \\
\hline 1977 & 22,383 & & 1977 TIUS & $97.6 \%$ & $0.0 \%$ & $1.6 \%$ & $0.8 \%$ \\
\hline 1978 & 24,162 & & Interpolated & $97.1 \%$ & $0.0 \%$ & $2.0 \%$ & $0.9 \%$ \\
\hline 1979 & 24,445 & & Interpolated & $96.7 \%$ & $0.0 \%$ & $2.4 \%$ & $1.0 \%$ \\
\hline 1980 & 23,796 & FHWA, MF-33e & Interpolated & $95.7 \%$ & $0.5 \%$ & $2.7 \%$ & $1.0 \%$ \\
\hline 1981 & 23,697 & FHWA, MF-33e & Interpolated & $95.1 \%$ & $0.7 \%$ & $3.1 \%$ & $1.1 \%$ \\
\hline 1982 & 22,702 & FHWA, MF-33e & 1982 TIUS & $93.0 \%$ & $2.3 \%$ & $3.5 \%$ & $1.2 \%$ \\
\hline 1983 & 23,945 & FHWA, MF-33e & Interpolated & $91.0 \%$ & $4.3 \%$ & $3.5 \%$ & $1.2 \%$ \\
\hline 1984 & 25,604 & FHWA, MF-33e & Interpolated & $90.0 \%$ & $5.3 \%$ & $3.5 \%$ & $1.2 \%$ \\
\hline 1985 & 27,363 & FHWA, MF-33e & Interpolated & $87.6 \%$ & $7.7 \%$ & $3.5 \%$ & $1.2 \%$ \\
\hline 1986 & 29,074 & FHWA, MF-33e & Interpolated & $87.7 \%$ & $7.6 \%$ & $3.5 \%$ & $1.2 \%$ \\
\hline 1987 & 30,598 & FHWA, MF-33e & 1987 TIUS & $89.0 \%$ & $6.3 \%$ & $3.5 \%$ & $1.2 \%$ \\
\hline 1988 & 32,653 & FHWA, MF-33e & Interpolated & $88.2 \%$ & $7.4 \%$ & $3.5 \%$ & $1.0 \%$ \\
\hline 1989 & 33,271 & FHWA, MF-33e & Interpolated & $89.5 \%$ & $6.2 \%$ & $3.4 \%$ & $0.8 \%$ \\
\hline 1990 & 35,611 & FHWA, MF-33e & Interpolated & $89.2 \%$ & $6.8 \%$ & $3.4 \%$ & $0.7 \%$ \\
\hline 1991 & 38,217 & FHWA, MF-33e & Interpolated & $88.1 \%$ & $8.0 \%$ & $3.3 \%$ & $0.5 \%$ \\
\hline 1992 & 40,929 & FHWA, MF-33e & 1992 TIUS & $88.5 \%$ & $7.9 \%$ & $3.3 \%$ & $0.3 \%$ \\
\hline 1993 & 42,851 & FHWA, MF-33e & Interpolated & $87.3 \%$ & $9.1 \%$ & $3.3 \%$ & $0.3 \%$ \\
\hline 1994 & 44,112 & FHWA, MF-33e & Interpolated & $86.8 \%$ & $9.6 \%$ & $3.3 \%$ & $0.3 \%$ \\
\hline 1995 & 45,605 & FHWA, MF-33e & Interpolated & $85.1 \%$ & $11.2 \%$ & $3.4 \%$ & $0.3 \%$ \\
\hline 1996 & 47,354 & FHWA, MF-33e & Interpolated & $86.2 \%$ & $10.1 \%$ & $3.4 \%$ & $0.3 \%$ \\
\hline 1997 & 49,388 & FHWA, MF-33e & 1997 VIUS & $84.2 \%$ & $12.2 \%$ & $3.4 \%$ & $0.2 \%$ \\
\hline 1998 & 50,462 & FHWA, MF-33e & Interpolated & $85.0 \%$ & $11.2 \%$ & $3.5 \%$ & $0.3 \%$ \\
\hline 1999 & 52,859 & FHWA, MF-33e & Interpolated & $84.9 \%$ & $11.0 \%$ & $3.6 \%$ & $0.4 \%$ \\
\hline 2000 & 52,939 & FHWA, MF-33e & Interpolated & $83.1 \%$ & $12.6 \%$ & $3.8 \%$ & $0.6 \%$ \\
\hline 2001 & 53,522 & FHWA, MF-33e & Interpolated & $82.4 \%$ & $13.0 \%$ & $3.9 \%$ & $0.7 \%$ \\
\hline 2002 & 55,220 & FHWA, MF-33e & 2002 VIUS & $79.6 \%$ & $15.6 \%$ & $4.0 \%$ & $0.8 \%$ \\
\hline 2003 & 60,758 & FHWA, MF-33e & 2002 VIUS & $71.0 \%$ & $24.2 \%$ & $4.0 \%$ & $0.8 \%$ \\
\hline 2004 & 63,417 & FHWA, MF-33e & 2002 VIUS & $62.9 \%$ & $32.3 \%$ & $4.0 \%$ & $0.8 \%$ \\
\hline 2005 & 58,869 & FHWA, MF-33e & 2002 VIUS & $62.6 \%$ & $32.6 \%$ & $4.0 \%$ & $0.8 \%$ \\
\hline 2006 & 60,685 & EIA, C1 & 2002 VIUS & $73.9 \%$ & $21.3 \%$ & $4.0 \%$ & $0.8 \%$ \\
\hline 2007 & 61,836 & EIA, C1 & 2002 VIUS & $68.6 \%$ & $26.6 \%$ & $4.0 \%$ & $0.8 \%$ \\
\hline 2008 & 61,199 & EIA, C1 & 2002 VIUS & $57.5 \%$ & $37.7 \%$ & $4.0 \%$ & $0.8 \%$ \\
\hline 2009 & 61,824 & EIA, C1 & 2002 VIUS & $51.5 \%$ & $43.7 \%$ & $4.0 \%$ & $0.8 \%$ \\
\hline 2010 & 64,687 & EIA, C1 & 2002 VIUS & $45.2 \%$ & $50.0 \%$ & $4.0 \%$ & $0.8 \%$ \\
\hline 2011 & 65,786 & EIA, C1 & 2002 VIUS & $44.4 \%$ & $50.8 \%$ & $4.0 \%$ & $0.8 \%$ \\
\hline 2012 & 66,395 & EIA, C1 & 2002 VIUS & $44.4 \%$ & $50.8 \%$ & $4.0 \%$ & $0.8 \%$ \\
\hline 2013 & 65,555 & EIA, C1 & 2002 VIUS & $44.7 \%$ & $50.5 \%$ & $4.0 \%$ & $0.8 \%$ \\
\hline 2014 & 69,012 & EIA, C1 & 2002 VIUS & $45.8 \%$ & $49.4 \%$ & $4.0 \%$ & $0.8 \%$ \\
\hline \multirow[t]{2}{*}{2015} & 67,730 & EIA, C1 & 2002 VIUS & $45.7 \%$ & $49.5 \%$ & $4.0 \%$ & $0.8 \%$ \\
\hline & & \multicolumn{2}{|c|}{ Heat content used for conversion to btu: } & $\begin{array}{l}\text { 125,000 } \\
\text { btu/gallon }\end{array}$ & $\begin{array}{l}\text { 120,900 } \\
\text { btu/gallon }\end{array}$ & $\begin{array}{l}\text { 138,700 } \\
\text { btu/gallon }\end{array}$ & $\begin{array}{l}90,800 \\
\text { btu/gallon }\end{array}$ \\
\hline
\end{tabular}

${ }^{\text {a }}$ Data are not continuous between 2008 and 2009 due to changes in source. 


\section{Medium/Heavy Trucks}

DOT, FHWA, Highway Statistics 2015, Table VM-1 and annual editions back to 1996 and DOT, FHWA, Highway Statistics Summary to 1995. The FHWA made methodology changes for Highway Statistics 2009. At that time, they published historical data back to 2007 which do not match the previous data. Total gallons for medium/heavy trucks are the sum of single-unit trucks and combination trucks.

Table A.6

Medium/Heavy Truck Fuel Use and Fuel Type Shares for Calculation of Energy Use

\begin{tabular}{|c|c|c|c|c|c|}
\hline \multirow[b]{2}{*}{ Year } & \multirow{2}{*}{$\begin{array}{c}\text { Fuel use } \\
\text { (million gallons) }\end{array}$} & \multirow{2}{*}{$\begin{array}{c}\text { Source for } \\
\text { fuel type shares }\end{array}$} & \multicolumn{3}{|c|}{ Shares by fuel type } \\
\hline & & & Gasoline & Diesel & Lpg \\
\hline 1970 & 11,316 & 1977 TIUS & $10.4 \%$ & $89.5 \%$ & $0.1 \%$ \\
\hline 1975 & 14,598 & 1977 TIUS & $10.4 \%$ & $89.5 \%$ & $0.1 \%$ \\
\hline 1980 & 19,960 & Interpolated & $27.9 \%$ & $71.4 \%$ & $0.6 \%$ \\
\hline 1981 & 20,376 & Interpolated & $33.8 \%$ & $65.4 \%$ & $0.8 \%$ \\
\hline 1982 & 20,386 & 1982 TIUS & $39.6 \%$ & $59.4 \%$ & $1.0 \%$ \\
\hline 1983 & 20,761 & Interpolated & $35.6 \%$ & $63.6 \%$ & $0.8 \%$ \\
\hline 1984 & 21,428 & Interpolated & $31.5 \%$ & $67.8 \%$ & $0.7 \%$ \\
\hline 1985 & 21,405 & Interpolated & $27.5 \%$ & $72.0 \%$ & $0.5 \%$ \\
\hline 1986 & 21,861 & Interpolated & $23.4 \%$ & $76.2 \%$ & $0.4 \%$ \\
\hline 1987 & 22,513 & 1987 TIUS & $19.4 \%$ & $80.4 \%$ & $0.2 \%$ \\
\hline 1988 & 22,925 & Interpolated & $18.8 \%$ & $81.0 \%$ & $0.3 \%$ \\
\hline 1989 & 23,512 & Interpolated & $18.1 \%$ & $81.6 \%$ & $0.3 \%$ \\
\hline 1990 & 24,490 & Interpolated & $17.5 \%$ & $82.1 \%$ & $0.4 \%$ \\
\hline 1991 & 24,981 & Interpolated & $16.8 \%$ & $82.7 \%$ & $0.4 \%$ \\
\hline 1992 & 25,453 & 1992 TIUS & $16.2 \%$ & $83.3 \%$ & $0.5 \%$ \\
\hline 1993 & 26,236 & Interpolated & $15.4 \%$ & $84.1 \%$ & $0.5 \%$ \\
\hline 1994 & 27,685 & Interpolated & $14.7 \%$ & $84.8 \%$ & $0.5 \%$ \\
\hline 1995 & 28,828 & Interpolated & $13.9 \%$ & $85.6 \%$ & $0.5 \%$ \\
\hline 1996 & 29,601 & Interpolated & $13.2 \%$ & $86.3 \%$ & $0.5 \%$ \\
\hline 1997 & 29,878 & 1997 VIUS & $12.4 \%$ & $87.1 \%$ & $0.5 \%$ \\
\hline 1998 & 30,841 & Interpolated & $12.1 \%$ & $87.4 \%$ & $0.5 \%$ \\
\hline 1999 & 33,909 & Interpolated & $11.8 \%$ & $87.6 \%$ & $0.5 \%$ \\
\hline 2000 & 35,229 & Interpolated & $11.6 \%$ & $87.9 \%$ & $0.5 \%$ \\
\hline 2001 & 35,179 & Interpolated & $11.3 \%$ & $88.1 \%$ & $0.5 \%$ \\
\hline 2002 & 36,800 & 2002 VIUS & $11.0 \%$ & $88.4 \%$ & $0.5 \%$ \\
\hline 2003 & 35,775 & 2002 VIUS & $11.0 \%$ & $88.4 \%$ & $0.5 \%$ \\
\hline 2004 & 33,150 & 2002 VIUS & $11.0 \%$ & $88.4 \%$ & $0.5 \%$ \\
\hline 2005 & 37,190 & 2002 VIUS & $11.0 \%$ & $88.4 \%$ & $0.5 \%$ \\
\hline 2006 & 37,959 & 2002 VIUS & $11.0 \%$ & $88.4 \%$ & $0.5 \%$ \\
\hline 2007 & 47,218 & 2002 VIUS & $11.0 \%$ & $88.4 \%$ & $0.5 \%$ \\
\hline 2008 & 47,705 & 2002 VIUS & $11.0 \%$ & $88.4 \%$ & $0.5 \%$ \\
\hline 2009 & 44,303 & 2002 VIUS & $11.0 \%$ & $88.4 \%$ & $0.5 \%$ \\
\hline 2010 & 45,024 & 2002 VIUS & $11.0 \%$ & $88.4 \%$ & $0.5 \%$ \\
\hline 2011 & 42,396 & 2002 VIUS & $11.0 \%$ & $88.4 \%$ & $0.5 \%$ \\
\hline 2012 & 42,351 & 2002 VIUS & $11.0 \%$ & $88.4 \%$ & $0.5 \%$ \\
\hline 2013 & 43,297 & 2002 VIUS & $11.0 \%$ & $88.4 \%$ & $0.5 \%$ \\
\hline 2014 & 44,012 & 2002 VIUS & $11.0 \%$ & $88.4 \%$ & $0.5 \%$ \\
\hline 2015 & 43,734 & 2002 VIUS & $11.0 \%$ & $88.4 \%$ & $0.5 \%$ \\
\hline Heat conte & or conversion to btu: & & $\begin{array}{l}\text { 125,000 } \\
\text { btu/gallon }\end{array}$ & $\begin{array}{l}\text { 138,700 } \\
\text { btu/gallon }\end{array}$ & $\begin{array}{l}90,800 \\
\text { btu/gallon }\end{array}$ \\
\hline
\end{tabular}

a Data are not continuous between 2006 and 2007 due to changes in methodology. See source for details. 
Shares of Class 3-6 and 7-8 energy use by fuel type were calculated from the 2002 Vehicle Inventory and Use Survey (VIUS) and applied to all years 1970-2015.

Table A.7

Share of Medium and Heavy Truck Energy Use

\begin{tabular}{lccc}
\hline & \multicolumn{2}{c}{ Share of energy use } & \\
\cline { 2 - 3 } Fuel type & Class 3-6 & Class 7-8 & Total \\
\hline Gasoline & $92 \%$ & $8 \%$ & $100 \%$ \\
Diesel & $14 \%$ & $86 \%$ & $100 \%$ \\
LPG & $99 \%$ & $1 \%$ & $100 \%$ \\
\hline
\end{tabular}




\subsection{OFF-HIGHWAY ENERGY USE}

U.S. Environmental Protection Agency, MOVES2014a model, results generated August 2017. Gallons of fuel by fuel type were produced for agricultural equipment, airport equipment, construction and mining equipment, industrial equipment, lawn and garden equipment, logging equipment, railroad maintenance equipment, and recreational equipment. Some nontransportation-related equipment, such as generators, chain saws, compressors, and pumps, were excluded from the data.

\subsection{NONHIGHWAY ENERGY USE}

\subsubsection{Air}

\section{General Aviation}

DOT, FAA, On-line General Aviation Activity and Air Taxi Activity Surveys: Annual Summary Report Calendar Year 2015, Table 5.1, and annual. 2011 Data: Aviation Forecasts, Tables 28 and 29, May 2013. (Additional resources:

www.faa.gov/data_research/aviation_data_statistics/general_aviation/CY2015/)

Table A.8

General Aviation Fuel Use

\begin{tabular}{|c|c|c|c|c|c|}
\hline Year & $\begin{array}{l}\text { Jet fuel } \\
\text { (million } \\
\text { gallons) }\end{array}$ & $\begin{array}{l}\text { Aviation gasoline } \\
\text { (million gallons) }\end{array}$ & Year & $\begin{array}{c}\text { Jet fuel } \\
\text { (million gallons) }\end{array}$ & $\begin{array}{l}\text { Aviation gasoline } \\
\text { (million gallons) }\end{array}$ \\
\hline 1970 & 208.0 & 551.0 & 1993 & 454.1 & 268.4 \\
\hline 1971 & 226.0 & 508.0 & 1994 & 470.8 & 264.1 \\
\hline 1972 & 245.0 & 584.0 & 1995 & 544.0 & 276.0 \\
\hline 1973 & 304.0 & 411.0 & 1996 & 567.5 & 286.5 \\
\hline 1974 & 357.0 & 443.0 & 1997 & 639.4 & 289.7 \\
\hline 1975 & 453.0 & 412.0 & 1998 & 814.6 & 311.4 \\
\hline 1976 & 495.0 & 432.0 & 1999 & 967.2 & 345.4 \\
\hline 1977 & 536.0 & 456.0 & 2000 & 998.1 & 336.3 \\
\hline 1978 & 763.0 & 518.0 & 2001 & 938.7 & 319.3 \\
\hline 1979 & 736.0 & 570.0 & 2002 & 815.5 & 261.4 \\
\hline 1980 & 766.0 & 520.0 & 2003 & 820.0 & 255.5 \\
\hline 1981 & 759.0 & 489.0 & 2004 & $1,075.2$ & 256.1 \\
\hline 1982 & 887.0 & 448.0 & 2005 & $1,507.4$ & 323.6 \\
\hline 1983 & 613.0 & 428.0 & 2006 & $1,636.3$ & 294.7 \\
\hline 1984 & 738.9 & 462.4 & 2007 & $1,516.3$ & 314.8 \\
\hline 1985 & 691.0 & 421.0 & 2008 & $1,688.6$ & 306.3 \\
\hline 1986 & 732.1 & 408.6 & 2009 & $1,350.6$ & 226.6 \\
\hline 1987 & 672.7 & 401.8 & 2010 & $1,451.5$ & 210.3 \\
\hline 1988 & 746.0 & 398.0 & 2011 & $1,490.7$ & 215.5 \\
\hline 1989 & 688.0 & 342.8 & 2012 & $1,492.1$ & 227.7 \\
\hline 1990 & 662.0 & 353.0 & 2013 & $1,353.6$ & 173.3 \\
\hline 1991 & 579.0 & 348.0 & 2014 & $1,454.1$ & 205.8 \\
\hline 1992 & 496.0 & 306.0 & 2015 & $1,384.4$ & 183.2 \\
\hline $\begin{array}{l}\text { Heat content used for } \\
\text { conversion to btu: }\end{array}$ & $\begin{array}{c}\text { 135,000 } \\
\text { btu/gallon }\end{array}$ & $\begin{array}{l}\text { 120,200 } \\
\text { btu/gallon }\end{array}$ & & $\begin{array}{l}\text { 135,000 } \\
\text { btu/gallon }\end{array}$ & $\begin{array}{c}\text { 120,200 } \\
\text { btu/gallon }\end{array}$ \\
\hline
\end{tabular}




\section{Domestic and International Air Carrier}

DOT, Bureau of Transportation Statistics, "Fuel Cost and Consumption Tables," www.transtats.bts.gov/fuel. The table below shows all international fuel use. Because the data for international include fuel purchased abroad, for the tables in Chapter 2, the international total was divided in half to estimate domestic fuel use for international flights.

Table A.9

Air Carrier Fuel Use

\begin{tabular}{|c|c|c|c|}
\hline Year & $\begin{array}{c}\text { Domestic } \\
\text { (thousand gallons) }\end{array}$ & $\begin{array}{c}\text { International } \\
\text { (thousand gallons) }\end{array}$ & $\begin{array}{c}\text { Total } \\
\text { (thousand gallons) }\end{array}$ \\
\hline 1970 & \multirow{3}{*}{\multicolumn{2}{|c|}{$\begin{array}{c}\text { Separate estimates for domestic } \\
\text { and international are not available } \\
\text { from 1970-1976. }\end{array}$}} & $10,085,000$ \\
\hline 1975 & & & $10,412,640$ \\
\hline 1976 & & & $10,400,040$ \\
\hline 1977 & $8,202,051$ & $1,708,376$ & $9,910,427$ \\
\hline 1978 & $8,446,117$ & $1,741,918$ & $10,188,035$ \\
\hline 1979 & $8,865,885$ & $1,828,435$ & $10,694,320$ \\
\hline 1980 & $8,519,233$ & $1,747,306$ & $10,266,539$ \\
\hline 1981 & $8,555,249$ & $2,032,520$ & $10,587,769$ \\
\hline 1982 & $8,432,465$ & $1,967,733$ & $10,400,198$ \\
\hline 1983 & $8,672,574$ & $1,998,289$ & $10,670,863$ \\
\hline 1984 & $9,625,958$ & $2,286,407$ & $11,912,365$ \\
\hline 1985 & $10,115,007$ & $2,487,929$ & $12,602,936$ \\
\hline 1986 & $11,137,331$ & $2,544,996$ & $13,682,327$ \\
\hline 1987 & $11,586,838$ & $2,893,617$ & $14,480,455$ \\
\hline 1988 & $11,917,904$ & $3,262,824$ & $15,180,728$ \\
\hline 1989 & $11,905,144$ & $3,557,294$ & $15,462,438$ \\
\hline 1990 & $12,429,305$ & $3,963,081$ & $16,392,386$ \\
\hline 1991 & $11,506,477$ & $3,939,666$ & $15,446,144$ \\
\hline 1992 & $11,762,852$ & $4,120,132$ & $15,882,983$ \\
\hline 1993 & $11,958,663$ & $4,113,321$ & $16,071,984$ \\
\hline 1994 & $12,475,549$ & $4,310,879$ & $16,786,428$ \\
\hline 1995 & $12,811,717$ & $4,511,418$ & $17,323,135$ \\
\hline 1996 & $13,187,305$ & $4,658,093$ & $17,845,398$ \\
\hline 1997 & $13,659,581$ & $4,964,181$ & $18,623,762$ \\
\hline 1998 & $13,876,971$ & $5,185,562$ & $19,062,533$ \\
\hline 1999 & $14,402,127$ & $5,250,492$ & $19,652,619$ \\
\hline 2000 & $14,844,592$ & $5,474,685$ & $20,319,277$ \\
\hline 2001 & $14,017,461$ & $5,237,487$ & $19,254,948$ \\
\hline 2002 & $12,848,329$ & $4,990,798$ & $17,839,127$ \\
\hline 2003 & $12,958,581$ & $4,836,356$ & $17,794,936$ \\
\hline 2004 & $13,622,603$ & $4,931,546$ & $18,554,149$ \\
\hline 2005 & $13,778,869$ & $5,520,889$ & $19,309,758$ \\
\hline 2006 & $13,694,437$ & $6,017,638$ & $19,712,075$ \\
\hline 2007 & $13,681,664$ & $6,204,502$ & $19,886,165$ \\
\hline 2008 & $12,666,911$ & $6,186,747$ & $18,853,658$ \\
\hline 2009 & $11,339,220$ & $5,721,298$ & $17,060,517$ \\
\hline 2010 & $11,256,900$ & $6,041,500$ & $17,288,400$ \\
\hline 2011 & $11,035,400$ & $6,522,600$ & $17,558,000$ \\
\hline 2012 & $10,439,700$ & $6,506,300$ & $16,946,000$ \\
\hline 2013 & $10,337,000$ & $6,487,300$ & $16,824,300$ \\
\hline 2014 & $10,458,600$ & $6,321,400$ & $16,780,000$ \\
\hline 2015 & $10,928,600$ & $6,420,600$ & $17,349,200$ \\
\hline Heat content used for & 135,000 & 135,000 & 135,000 \\
\hline conversion to btu: & btu/gallon & btu/gallon & btu/gallon \\
\hline
\end{tabular}




\subsubsection{Water}

\section{Freight}

Total - DOE, EIA, Petroleum and Other Liquids Database, August 2017. Adjusted sales of distillate and residual fuel oil for vessel bunkering. (This may include some amounts of bunker fuels used for recreational purposes.)

Table A.10

Diesel and Residual Fuel Oil for Vessel Bunkering

\begin{tabular}{|c|c|c|}
\hline Year & $\begin{array}{c}\text { Distillate fuel oil } \\
\text { (thousand gallons) }\end{array}$ & $\begin{array}{l}\text { Residual fuel oil } \\
\text { (thousand gallons) }\end{array}$ \\
\hline 1970 & 819,000 & $3,774,120$ \\
\hline 1975 & $1,097,880$ & $4,060,140$ \\
\hline 1980 & 717,376 & $7,454,242$ \\
\hline 1981 & $1,723,143$ & $7,922,512$ \\
\hline 1982 & $1,423,216$ & $6,408,818$ \\
\hline 1983 & $1,418,890$ & $5,724,115$ \\
\hline 1984 & $1,692,045$ & $5,688,931$ \\
\hline 1985 & $1,894,265$ & $5,269,733$ \\
\hline 1986 & $2,034,215$ & $5,690,250$ \\
\hline 1987 & $2,223,258$ & $5,869,154$ \\
\hline 1988 & $2,310,367$ & $6,025,511$ \\
\hline 1989 & $2,356,444$ & $6,621,100$ \\
\hline 1990 & $2,197,004$ & $6,248,095$ \\
\hline 1991 & $2,167,640$ & $6,786,055$ \\
\hline 1992 & $2,240,170$ & $7,199,078$ \\
\hline 1993 & $2,043,745$ & $6,269,882$ \\
\hline 1994 & $2,026,899$ & $5,944,383$ \\
\hline 1995 & $1,978,105$ & $6,431,238$ \\
\hline 1996 & $2,177,608$ & $5,804,977$ \\
\hline 1997 & $2,107,561$ & $4,789,861$ \\
\hline 1998 & $2,125,568$ & $4,640,153$ \\
\hline 1999 & $2,064,590$ & $5,598,630$ \\
\hline 2000 & $2,041,433$ & $6,192,294$ \\
\hline 2001 & $2,099,011$ & $4,345,284$ \\
\hline 2002 & $2,056,465$ & $4,783,956$ \\
\hline 2003 & $1,863,150$ & $3,801,425$ \\
\hline 2004 & $2,313,448$ & $4,886,978$ \\
\hline 2005 & 2,115,381 & $5,533,552$ \\
\hline 2006 & $2,206,690$ & $6,000,434$ \\
\hline 2007 & $2,158,930$ & $6,773,950$ \\
\hline 2008 & $1,980,729$ & $6,274,047$ \\
\hline 2009 & $2,138,690$ & $5,331,657$ \\
\hline 2010 & $2,427,051$ & $6,032,367$ \\
\hline 2011 & $2,651,859$ & $5,207,886$ \\
\hline 2012 & $1,842,107$ & $4,560,546$ \\
\hline 2013 & $1,655,258$ & $3,876,795$ \\
\hline 2014 & $1,626,527$ & $2,987,363$ \\
\hline 2015 & $2,415,253$ & $3,103,402$ \\
\hline $\begin{array}{l}\text { Heat content used for } \\
\text { conversion to btu: }\end{array}$ & $\begin{array}{c}\text { 138,700 } \\
\text { btu/gallon }\end{array}$ & $\begin{array}{c}\text { 149,700 } \\
\text { btu/gallon }\end{array}$ \\
\hline $\begin{array}{l}\text { Domestic share of total } \\
\text { fuel use }\end{array}$ & $77.5 \%$ & $9.3 \%$ \\
\hline
\end{tabular}




\section{Recreational Boating}

Fuel use by recreational boating comes from the EPA's MOVES2014a model.

Table A.11

Recreational Boating Fuel Use

\begin{tabular}{|c|c|c|}
\hline Year & $\begin{array}{l}\text { Diesel use } \\
\text { (gallons) }\end{array}$ & $\begin{array}{l}\text { Gasoline use } \\
\text { (gallons) }\end{array}$ \\
\hline 1970 & $39,589,953$ & $1,213,397,311$ \\
\hline 1975 & $77,294,680$ & $1,251,387,972$ \\
\hline 1976 & $84,835,632$ & $1,258,986,070$ \\
\hline 1977 & $92,376,573$ & $1,266,584,111$ \\
\hline 1978 & $99,917,523$ & $1,274,182,341$ \\
\hline 1979 & $107,458,470$ & $1,281,780,460$ \\
\hline 1980 & $114,999,421$ & $1,289,378,532$ \\
\hline 1981 & $122,540,357$ & $1,296,976,672$ \\
\hline 1982 & $130,081,302$ & $1,304,574,832$ \\
\hline 1983 & $137,622,248$ & $1,312,172,890$ \\
\hline 1984 & $145,163,202$ & $1,319,771,007$ \\
\hline 1985 & $152,704,140$ & $1,327,369,146$ \\
\hline 1986 & $160,245,074$ & $1,334,967,322$ \\
\hline 1987 & $167,786,030$ & $1,342,565,455$ \\
\hline 1988 & $175,326,970$ & $1,362,856,034$ \\
\hline 1989 & $182,867,916$ & 1,383,146,636 \\
\hline 1990 & $190,408,869$ & $1,403,437,194$ \\
\hline 1991 & $197,949,808$ & $1,429,688,292$ \\
\hline 1992 & $205,490,749$ & $1,455,939,504$ \\
\hline 1993 & $213,031,707$ & $1,482,190,597$ \\
\hline 1994 & $220,572,649$ & $1,539,794,180$ \\
\hline 1995 & $228,113,596$ & $1,597,269,921$ \\
\hline 1996 & $235,654,521$ & $1,654,446,069$ \\
\hline 1997 & $243,195,481$ & $1,657,737,628$ \\
\hline 1998 & $250,736,414$ & $1,659,056,085$ \\
\hline 1999 & $258,159,525$ & $1,657,198,161$ \\
\hline 2000 & $265,582,657$ & $1,652,906,973$ \\
\hline 2001 & $273,547,835$ & $1,655,303,922$ \\
\hline 2002 & $281,512,965$ & $1,653,583,696$ \\
\hline 2003 & $289,478,093$ & $1,648,070,959$ \\
\hline 2004 & $297,443,197$ & $1,639,713,127$ \\
\hline 2005 & $305,408,463$ & $1,629,873,278$ \\
\hline 2006 & $313,420,594$ & $1,619,603,593$ \\
\hline 2007 & $321,432,801$ & $1,609,567,873$ \\
\hline 2008 & $329,445,068$ & $1,599,830,522$ \\
\hline 2009 & $337,457,287$ & $1,590,749,216$ \\
\hline 2010 & $345,469,668$ & $1,578,405,558$ \\
\hline 2011 & $353,434,754$ & $1,566,937,275$ \\
\hline 2012 & $361,399,927$ & $1,557,381,573$ \\
\hline 2013 & $369,365,038$ & $1,550,075,141$ \\
\hline 2014 & $377,330,139$ & $1,544,411,821$ \\
\hline 2015 & $385,295,170$ & $1,540,441,668$ \\
\hline $\begin{array}{l}\text { Heat content used for } \\
\text { conversion to btu: }\end{array}$ & $\begin{array}{c}138,700 \\
\text { btu/gallon }\end{array}$ & $\begin{array}{c}\text { 125,000 } \\
\text { btu/gallon }\end{array}$ \\
\hline
\end{tabular}




\subsubsection{Pipeline}

The sum of natural gas, crude petroleum and petroleum product, and coal slurry and water.

\section{Natural Gas}

The amount of natural gas used to transport natural gas was defined as "pipeline fuel" as reported in DOE, EIA, Natural Gas Annual 2015, Table 1. Cubic feet were converted to Btu using 1,031 $\mathrm{Btu} / \mathrm{ft} 3$. Electricity use was estimated using the following procedure as reported on p. 5-110 of J. N. Hooker et al., End Use Energy Consumption DataBase: Transportation Sector. The energy consumption of a natural gas pipeline was taken to be the energy content of the fuel used to drive the pumps. Some $94 \%$ of the installed pumping horsepower was supplied by natural gas. The remaining $6 \%$ of the horsepower was generated more efficiently, mostly by electric motors. The energy consumed by natural gas pipeline pumps that were electrically powered was not known. In order to estimate the electricity consumed, the Btu of natural gas pipeline fuel consumed was multiplied by a factor of 0.015 .

\section{Crude Petroleum and Petroleum Product}

J. N. Hooker, Oil Pipeline Energy Consumption and Efficiency, ORNL-5697, ORNL, Oak Ridge, TN, 1981. (Data held constant; Latest available data.)

\section{Coal Slurry and Water}

W. F. Banks, Systems, Science and Software, Energy Consumption in the Pipeline Industry, LaJolla, CA, October 1977. (Data held constant; Latest available data.) 
Table A.12

Pipeline Fuel Use

\begin{tabular}{|c|c|c|c|}
\hline Year & $\begin{array}{c}\text { Natural gas } \\
\text { (million cubic } \\
\text { feet) }\end{array}$ & $\begin{array}{l}\text { Estimated natural } \\
\text { gas pipeline } \\
\text { electricity use } \\
\text { (million kWhr) }\end{array}$ & $\begin{array}{l}\text { Electricity } \\
\text { constant } \\
\text { (trillion btu) }\end{array}$ \\
\hline 1970 & 722,166 & $3,272.9$ & 70.0 \\
\hline 1975 & 582,963 & $2,642.0$ & 70.0 \\
\hline 1976 & 548,323 & $2,485.0$ & 70.0 \\
\hline 1977 & 532,669 & $2,414.1$ & 70.0 \\
\hline 1978 & 530,451 & $2,404.0$ & 70.0 \\
\hline 1979 & 600,964 & 2,723.6 & 70.0 \\
\hline 1980 & 634,622 & $2,876.1$ & 70.0 \\
\hline 1981 & 642,325 & $2,911.0$ & 70.0 \\
\hline 1982 & 596,411 & $2,703.0$ & 70.0 \\
\hline 1983 & 490,042 & $2,220.9$ & 70.0 \\
\hline 1984 & 528,754 & 2,396.3 & 70.0 \\
\hline 1985 & 503,766 & 2,283.1 & 70.0 \\
\hline 1986 & 485,041 & 2,198.2 & 70.0 \\
\hline 1987 & 519,170 & $2,352.9$ & 70.0 \\
\hline 1988 & 613,912 & $2,782.3$ & 70.0 \\
\hline 1989 & 629,308 & $2,852.0$ & 70.0 \\
\hline 1990 & 659,816 & $2,990.3$ & 70.0 \\
\hline 1991 & 601,305 & $2,725.1$ & 70.0 \\
\hline 1992 & 587,710 & 2,663.5 & 70.0 \\
\hline 1993 & 624,308 & $2,829.4$ & 70.0 \\
\hline 1994 & 685,362 & $3,106.1$ & 70.0 \\
\hline 1995 & 700,335 & 3,173.9 & 70.0 \\
\hline 1996 & 711,446 & $3,224.3$ & 70.0 \\
\hline 1997 & 751,470 & $3,405.7$ & 70.0 \\
\hline 1998 & 635,477 & $2,880.0$ & 70.0 \\
\hline 1999 & 645,319 & 2,924.6 & 70.0 \\
\hline 2000 & 642,210 & $2,910.5$ & 70.0 \\
\hline 2001 & 624,964 & $2,832.3$ & 70.0 \\
\hline 2002 & 666,920 & $3,022.5$ & 70.0 \\
\hline 2003 & 591,492 & $2,680.7$ & 70.0 \\
\hline 2004 & 566,187 & $2,566.0$ & 70.0 \\
\hline 2005 & 584,026 & $2,646.8$ & 70.0 \\
\hline 2006 & 584,213 & $2,647.7$ & 70.0 \\
\hline 2007 & 621,364 & $2,816.0$ & 70.0 \\
\hline 2008 & 647,956 & 2,936.6 & 70.0 \\
\hline 2009 & 670,174 & $3,037.2$ & 70.0 \\
\hline 2010 & 674,124 & $3,055.1$ & 70.0 \\
\hline 2011 & 687,784 & $3,117.0$ & 70.0 \\
\hline 2012 & 730,790 & $3,312.0$ & 70.0 \\
\hline 2013 & 833,061 & $3,775.4$ & 70.0 \\
\hline 2014 & 700,150 & 3,173.1 & 70.0 \\
\hline 2015 & 666,214 & $3,019.3$ & 70.0 \\
\hline $\begin{array}{l}\text { Heat content used for } \\
\text { conversion to btu: }\end{array}$ & $\begin{array}{c}1,031 \\
\text { btu/cubic foot }\end{array}$ & $\begin{array}{c}\text { 3,412 } \\
\text { Btu/kWhr }\end{array}$ & \\
\hline
\end{tabular}

Note: Formula for estimating electricity use for natural gas pipelines is:

Natural gas use (in million cubic ft) $\times 1,031$ btu/cubic ft $\times 0.015 \times 29.305 \times 10-5 \mathrm{kWhr} / \mathrm{btu}$. 


\subsubsection{Rail}

\section{Freight}

AAR, Railroad Facts, 2016 Edition, Washington, DC, 2016.

Table A.13

Class I Freight Railroad Fuel Use

\begin{tabular}{|c|c|}
\hline Year & $\begin{array}{c}\text { Diesel fuel } \\
\text { (thousand gallons) }\end{array}$ \\
\hline 1970 & $3,807,663$ \\
\hline 1971 & $3,822,907$ \\
\hline 1972 & 3,996,985 \\
\hline 1973 & $4,160,730$ \\
\hline 1974 & $4,175,375$ \\
\hline 1975 & $3,736,484$ \\
\hline 1976 & $3,895,542$ \\
\hline 1977 & $3,985,069$ \\
\hline 1978 & $3,968,007$ \\
\hline 1979 & $4,072,187$ \\
\hline 1980 & $3,955,996$ \\
\hline 1981 & $3,756,439$ \\
\hline 1982 & $3,178,116$ \\
\hline 1983 & $3,137,295$ \\
\hline 1984 & $3,388,173$ \\
\hline 1985 & $3,144,190$ \\
\hline 1986 & $3,039,069$ \\
\hline 1987 & $3,102,227$ \\
\hline 1988 & $3,182,267$ \\
\hline 1989 & $3,190,815$ \\
\hline 1990 & $3,134,446$ \\
\hline 1991 & $2,925,970$ \\
\hline 1992 & $3,022,108$ \\
\hline 1993 & $3,111,981$ \\
\hline 1994 & $3,355,802$ \\
\hline 1995 & $3,503,096$ \\
\hline 1996 & $3,600,649$ \\
\hline 1997 & $3,602,793$ \\
\hline 1998 & $3,619,341$ \\
\hline 1999 & $3,749,428$ \\
\hline 2000 & $3,720,107$ \\
\hline 2001 & 3,729,985 \\
\hline 2002 & $3,751,413$ \\
\hline 2003 & $3,849,229$ \\
\hline 2004 & $4,082,236$ \\
\hline 2005 & $4,119,879$ \\
\hline 2006 & $4,214,459$ \\
\hline 2007 & $4,087,405$ \\
\hline 2008 & $3,911,178$ \\
\hline 2009 & $3,220,059$ \\
\hline 2010 & $3,519,021$ \\
\hline 2011 & $3,710,485$ \\
\hline 2012 & $3,634,025$ \\
\hline 2013 & $3,712,582$ \\
\hline 2014 & $3,897,113$ \\
\hline 2015 & $3,723,491$ \\
\hline \multirow{2}{*}{$\begin{array}{l}\text { Heat content used for } \\
\text { conversion to btu: }\end{array}$} & 138,700 \\
\hline & Btu/gallon \\
\hline
\end{tabular}




\section{Passenger}

Commuter - APTA, 2016 Public Transportation Fact Book, Washington, DC, 2016. Data for 2015 are directly from the U.S. Department of Transportation, Federal Transit Administration, National Transit Database.

Table A.14

Commuter Rail Fuel Use

\begin{tabular}{|c|c|c|}
\hline Year & $\begin{array}{c}\text { Diesel } \\
\text { (thousand gallons) }\end{array}$ & $\begin{array}{c}\text { Electricity } \\
\text { (million kWhr) }\end{array}$ \\
\hline 1984 & 58,320 & 901 \\
\hline 1985 & 55,372 & 1,043 \\
\hline 1986 & 54,608 & 1,170 \\
\hline 1987 & 51,594 & 1,155 \\
\hline 1988 & 53,054 & 1,195 \\
\hline 1989 & 52,516 & 1,293 \\
\hline 1990 & 52,681 & 1,226 \\
\hline 1991 & 54,315 & 1,239 \\
\hline 1992 & 54,951 & 1,124 \\
\hline 1993 & 59,766 & 1,196 \\
\hline 1994 & 61,900 & 1,244 \\
\hline 1995 & 63,064 & 1,253 \\
\hline 1996 & 61,888 & 1,255 \\
\hline 1997 & 63,195 & 1,270 \\
\hline 1998 & 69,200 & 1,299 \\
\hline 1999 & 73,005 & 1,322 \\
\hline 2000 & 70,818 & 1,370 \\
\hline 2001 & 72,204 & 1,354 \\
\hline 2002 & 72,847 & 1,334 \\
\hline 2003 & 72,264 & 1,383 \\
\hline 2004 & 71,999 & 1,449 \\
\hline 2005 & 76,714 & 1,484 \\
\hline 2006 & 78,600 & 1,478 \\
\hline 2007 & 80,700 & 1,763 \\
\hline 2008 & 83,500 & 1,718 \\
\hline 2009 & 95,000 & 1,780 \\
\hline 2010 & 93,200 & 1,797 \\
\hline 2011 & 93,900 & 1,813 \\
\hline 2012 & 92,800 & 1,808 \\
\hline 2013 & 98,700 & 1,816 \\
\hline 2014 & 93,900 & 1,809 \\
\hline 2015 & 95,728 & 1,792 \\
\hline \multirow{2}{*}{$\begin{array}{l}\text { Heat content used for } \\
\text { conversion to btu: }\end{array}$} & 138,700 & 3,412 \\
\hline & Btu/gallon & Btu/kWhr \\
\hline
\end{tabular}


Transit - APTA, 2016 Public Transportation Fact Book, Washington, DC, 2016. Includes light rail and heavy rail. Data for 2015 are directly from the U.S. Department of Transportation, Federal Transit Administration, National Transit Database.

Table A.15

Transit Rail Fuel Use

\begin{tabular}{|c|c|c|c|}
\hline \multirow[b]{2}{*}{ Year } & \multicolumn{3}{|c|}{ Electricity (million kWhr) } \\
\hline & Light rail & Heavy rail & Total \\
\hline 1970 & & & 2,561 \\
\hline 1975 & \multirow{3}{*}{\multicolumn{2}{|c|}{$\begin{array}{l}\text { Light rail and heavy rail data are } \\
\text { not available separately from }\end{array}$}} & 2,646 \\
\hline 1976 & & & 2,576 \\
\hline 1977 & & & 2,303 \\
\hline 1978 & \multicolumn{2}{|c|}{1970 to 1985.} & 2,223 \\
\hline 1979 & & & 2,473 \\
\hline 1980 & & & 2,446 \\
\hline 1981 & & & 2,655 \\
\hline 1982 & & & 2,722 \\
\hline 1983 & & & 2,930 \\
\hline 1984 & & & 3,092 \\
\hline 1985 & & & 2,928 \\
\hline 1986 & 173 & 3,066 & 3,239 \\
\hline 1987 & 191 & 3,219 & 3,410 \\
\hline 1988 & 243 & 3,256 & 3,499 \\
\hline 1989 & 242 & 3,286 & 3,528 \\
\hline 1990 & 239 & 3,284 & 3,523 \\
\hline 1991 & 274 & 3,248 & 3,522 \\
\hline 1992 & 297 & 3,193 & 3,490 \\
\hline 1993 & 281 & 3,287 & 3,568 \\
\hline 1994 & 282 & 3,431 & 3,713 \\
\hline 1995 & 288 & 3,401 & 3,689 \\
\hline 1996 & 321 & 3,322 & 3,643 \\
\hline 1997 & 363 & 3,253 & 3,616 \\
\hline 1998 & 382 & 3,280 & 3,662 \\
\hline 1999 & 416 & 3,385 & 3,801 \\
\hline 2000 & 563 & 3,549 & 4,112 \\
\hline 2001 & 587 & 3,646 & 4,233 \\
\hline 2002 & 510 & 3,683 & 4,193 \\
\hline 2003 & 507 & 3,632 & 4,138 \\
\hline 2004 & 553 & 3,684 & 4,237 \\
\hline 2005 & 571 & 3,769 & 4,339 \\
\hline 2006 & 634 & 3,709 & 4,343 \\
\hline 2007 & 687 & 3,817 & 4,505 \\
\hline 2008 & 721 & 3,898 & 4,619 \\
\hline 2009 & 738 & 3,866 & 4,624 \\
\hline 2010 & 749 & 3,780 & 4,529 \\
\hline 2011 & 789 & 3,854 & 4,643 \\
\hline 2012 & 806 & 3,795 & 4,601 \\
\hline 2013 & 882 & 3,856 & 4,738 \\
\hline 2014 & 985 & 3,812 & 4,797 \\
\hline 2015 & 898 & 3,816 & 4,713 \\
\hline t content used for & 3,412 & 3,412 & 3,412 \\
\hline conversion to btu: & Btu/kWhr & Btu/kWhr & Btu/kWhr \\
\hline
\end{tabular}


Intercity - Personal communication with Amtrak, Washington, DC, 2016.

Table A.16

Intercity Rail Fuel Use

\begin{tabular}{ccc}
\hline Year & $\begin{array}{c}\text { Diesel fuel } \\
\text { (thousand } \\
\text { gallons) }\end{array}$ & $\begin{array}{c}\text { Electricity } \\
\text { (thousand kWhr) }\end{array}$ \\
\hline 1994 & 73,516 & 308,948 \\
1995 & 72,371 & 335,818 \\
1996 & 71,226 & 362,689 \\
1997 & 75,656 & 389,559 \\
1998 & 75,999 & 416,429 \\
1999 & 79,173 & 443,300 \\
2000 & 94,968 & 470,170 \\
2001 & 96,846 & 455,703 \\
2002 & 84,432 & 518,306 \\
2003 & 74,621 & 536,950 \\
2004 & 68,605 & 550,695 \\
2005 & 65,477 & 531,377 \\
2006 & 62,463 & 548,856 \\
2007 & 61,824 & 577,864 \\
2008 & 63,428 & 582,022 \\
2009 & 61,704 & 564,968 \\
2010 & 63,474 & 558,662 \\
2011 & 63,450 & 555,425 \\
2012 & 63,058 & 549,201 \\
2013 & 66,036 & 525,127 \\
2014 & 65,711 & 515,332 \\
2015 & 62,468 & 504,017 \\
\hline Heat content used for & 138,700 & 3,412 \\
conversion to Btu & Btu/gallon & Btu/kWhr \\
\hline
\end{tabular}

\subsection{CALCULATION OF MILLION BARRELS PER DAY CRUDE OIL EQUIVALENT}

One gallon of gasoline, diesel fuel, or lpg is estimated to be the equivalent of one gallon of crude oil. Petroleum used for electricity was calculated using the following formula:

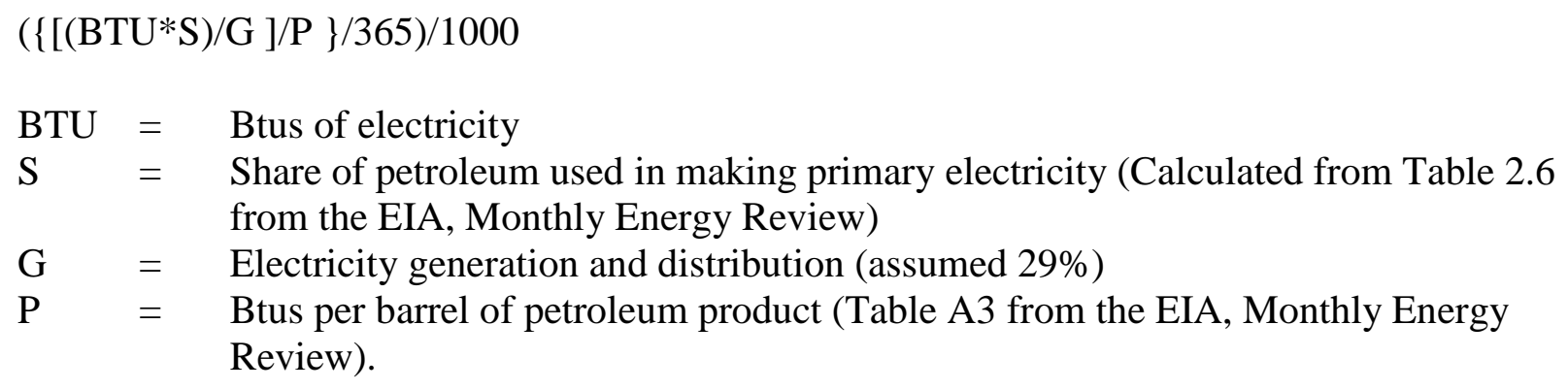




\section{PASSENGER TRAVEL AND ENERGY USE}

\subsection{CARS}

Number of vehicles - DOT, FHWA, Highway Statistics 2014, Table MV-1 and annual editions back to 2009.

Vehicle-miles - See Appendix A, Section 7. Car and Light Truck Shares.

Passenger-miles - Vehicle-miles multiplied by an average load factor.

Load factor - 2009 NHTS shows car load factor as 1.55 persons per vehicle.

Energy intensities -

Btu per vehicle-mile - Car energy use divided by vehicle-miles.

Btu per passenger-mile - Car energy use divided by passenger-miles.

Energy use - See Section 2. Energy Use Sources. Data series shown in Table 2.9.

\subsection{LIGHT TRUCKS}

Number of vehicles - DOT, FHWA, Highway Statistics 2014, Table MV-9 and annual editions back to 2009. Columns for pickups, vans, sport utility vehicles, and other light trucks. Data were multiplied by the shares of light trucks which are for personal use (Table A.17) which were derived by ORNL from the 2002 VIUS Micro Data File on CD.

Vehicle-miles - See Appendix A, Section 7. Car and Light Truck Shares. Data were multiplied by the shares of vehicle miles which are for personal use (Table A.17) which were derived by ORNL from the 2002 VIUS Micro Data File on CD.

Passenger-miles - Vehicle-miles multiplied by an average load factor.

Load factor - 2009 NHTS shows personal light truck load factor as 1.84 persons per vehicle.

Energy intensities -

Btu per vehicle-mile - Personal light truck energy use divided by personal light truck vehicle-miles.

Btu per passenger-mile - Personal light truck energy use divided by personal light truck passenger-miles.

Energy use - See Section 2. Energy Use Sources (light trucks, medium/heavy trucks). Data by truck type were multiplied by the shares of truck fuel use which are for personal use (Table A.17) which were derived by ORNL from the 2002 VIUS Micro Data File on CD. 
Table A.17

Share of Trucks, Truck Travel, and Fuel Use for Personal Travel

\begin{tabular}{ll}
\hline Personal trucks & \\
$85.6 \%$ & 2-axle, 4-tire trucks \\
$26.9 \%$ & Other single-unit and combination trucks \\
$\begin{array}{l}\text { Personal truck travel } \\
80.9 \%\end{array}$ & 2-axle, 4-tire trucks \\
$13.1 \%$ & Other single-unit and combination trucks \\
$\begin{array}{ll}\text { Personal truck fuel use } \\
78.0 \%\end{array}$ & 2-axle, 4-tire trucks \\
$6.0 \%$ & Other single-unit and combination trucks \\
\hline
\end{tabular}

Note: Since these shares come from the 2002 VIUS, they may underestimate the amount of personal trucks, truck travel, and energy use for 2015.

\subsection{MOTORCYCLES}

Number of vehicles, vehicle-miles - DOT, FHWA, Highway Statistics 2015, Table VM-1.

Passenger-miles - Vehicle-miles multiplied by an average load factor.

Load factor - 2009 NHTS shows motorcycle load factor as 1.16 persons per vehicle.

Energy intensities -

Btu per vehicle-mile - Motorcycle energy use divided by vehicle-miles.

Btu per passenger-mile - Motorcycle energy use divided by passenger-miles.

Energy use - See Section 2. Energy Use Sources. Data series shown in Table 2.9.

\subsection{DEMAND RESPONSE}

Number of vehicles, vehicle-miles, passenger-miles - APTA, 2016 Public Transportation Fact Book, Washington, DC, 2016.

Load factor - Passenger-miles divided by vehicle-miles.

Energy intensities -

Btu per vehicle-mile - Energy use divided by vehicle-miles.

Btu per passenger-mile - Energy use divided by passenger-miles.

Energy use - APTA, 2016 Public Transportation Fact Book, Washington, DC, 2016.

Note that all Demand Response data are for 2014. 


\subsection{BUSES}

\subsubsection{Transit}

Number of vehicles, vehicle-miles, passenger-miles - APTA, 2016 Public Transportation Fact Book, Washington, DC, 2016. Data series shown on Table 5.18. Data for 2015 are directly from the U.S. Department of Transportation, Federal Transit Administration, National Transit Database.

Load factor - Passenger-miles divided by vehicle-miles.

Energy intensities -

Btu per vehicle-mile - Transit bus energy use divided by transit bus vehicle-miles.

Btu per passenger-mile - Transit bus energy use divided by transit bus passenger-miles.

Energy use - See Section 2. Energy Use Sources. Data series shown in Table 5.18.

\subsubsection{Intercity}

Energy use - See Section 2. Energy Use Sources. Because the data past 2000 are not available, the rate of change in bus VMT from FHWA, Highway Statistics 2015, was used to estimate the change in energy use.

\subsubsection{School}

Number of vehicles - DOT, FHWA, Highway Statistics 2015, Table MV-10.

Energy use - See Section 2. Energy Use Sources. Because the data past 2000 are not available, the rate of change in bus VMT from FHWA, Highway Statistics 2015, was used to estimate the change in energy use.

\subsection{AIR}

\subsubsection{Certificated Air Carriers}

Aircraft-miles, passenger-miles - DOT, BTS, U.S. Air Traffic Statistics Through December 2016, www.transtats.bts.gov, Washington, DC.

Load factor - Passenger-miles divided by aircraft-miles.

Energy intensities -

Btu per passenger-mile - Certificated air carrier energy use divided by passenger-miles.

Energy use - See Section 2. Energy Use Sources. All of domestic fuel use and half of international fuel use was considered to be domestic use.

Note: These data differ from the data in Table 9.2 because that table contains data on ALL domestic AND international air carrier energy use and passenger-miles.

\subsubsection{General Aviation}

Number of vehicles - DOT, FAA, General Aviation and Air Taxi Activity Surveys - CY 2015. 2011 Data: Aviation Forecasts, Tables 28 and 29, May 2013. Data series shown in Table 9.3. 
Energy intensities -

Btu per passenger-mile - General aviation energy use divided by passenger-miles.

Energy use - See Section 2. Energy Use Sources. Data series shown in Table 9.3.

\subsection{RECREATIONAL BOATING}

Number of vehicles and energy use - U.S. EPA's MOVES2014a model.

\subsection{RAIL}

\subsubsection{Intercity}

Number of vehicles, vehicle-miles, passenger-miles - AAR, Railroad Facts, 2016 Edition, Washington, DC, 2016.

Load factor - Passenger-miles divided by vehicle-miles.

Energy Intensities -

Btu per vehicle-mile - Intercity rail energy use divided by vehicle-miles.

Btu per passenger-mile - Intercity rail energy use divided by passenger-miles.

Energy use - See Section 2. Energy Use Sources. Data series shown in Table 9.10.

\subsubsection{Transit}

Number of vehicles, vehicle-miles, passenger-miles - APTA, 2016 Public Transportation Fact Book, Washington, DC, 2016. Sum of light and heavy rail transit. Data series shown on Table 9.12. Data for 2015 are directly from the U.S. Department of Transportation, Federal Transit Administration, National Transit Database.

Load factor - Passenger-miles divided by vehicle-miles.

Energy intensities -

Btu per vehicle-mile - Light and heavy transit rail energy use divided by vehicle-miles. Btu per passenger-mile - Light and heavy transit rail energy use divided by passengermiles.

Energy use - See Section 2. Energy Use Sources. Data series shown in Table 9.12.

\subsubsection{Commuter}

Number of vehicles, vehicle-miles, passenger-miles - APTA, 2016 Public Transportation Fact Book, Washington, DC, 2016. Data series shown on Table 9.11. Data for 2015 are directly from the U.S. Department of Transportation, Federal Transit Administration, National Transit Database.

Load factor - Passenger-miles divided by vehicle-miles.

Energy intensities -

Btu per vehicle-mile - Commuter rail energy use divided by vehicle-miles.

Btu per passenger-mile - Commuter rail energy use divided by passenger-miles.

Energy use - See Section 2. Energy Use Sources. Data series shown in Table 9.11. 


\section{HIGHWAY PASSENGER MODE ENERGY INTENSITIES}

\subsection{CARS}

Btu per vehicle-mile - Car energy use divided by car vehicle miles of travel.

Energy use - See Section 2. Energy Use Sources. Data series shown in Table 2.9.

Vehicle-miles - 1970-2008: DOT, FHWA, Highway Statistics 2009, Table VM-1 and annual editions back to 1996 and DOT, FHWA, Highway Statistics Summary to 1995. Data series shown in Table 4.1.

2009-2015: See Appendix A, Section 7. Car and Light Truck Shares.

Btu per passenger-mile - Car energy use divided by car passenger-miles.

Energy use - See Section 2. Energy Use Sources. Data series shown in Table 2.9.

Passenger miles - Vehicle miles multiplied by an average load factor.

Vehicle-miles - 1970-2008: DOT, FHWA, Highway Statistics 2009, Table VM-1 and annual editions back to 1996 and DOT, FHWA, Highway Statistics Summary to 1995. Data series shown in Table 4.1.

2009-2015: See Appendix A, Section 7. Car and Light Truck Shares.

Load factor - NPTS 1969, 1977, 1983/84, 1990, and 1995; NHTS 2001 and 2009. Data series shown in Table A.18. 
Table A.18

Car Load Factor used to Calculate Passenger-Miles

\begin{tabular}{|c|c|c|}
\hline Year & Source & Load Factor \\
\hline 1970 & 1969 NPTS & 1.90 \\
\hline 1971 & Interpolated & 1.90 \\
\hline 1972 & Interpolated & 1.90 \\
\hline 1973 & Interpolated & 1.90 \\
\hline 1974 & Interpolated & 1.90 \\
\hline 1975 & Interpolated & 1.90 \\
\hline 1976 & Interpolated & 1.90 \\
\hline 1977 & 1977 NPTS & 1.90 \\
\hline 1978 & Interpolated & 1.88 \\
\hline 1979 & Interpolated & 1.87 \\
\hline 1980 & Interpolated & 1.85 \\
\hline 1981 & Interpolated & 1.83 \\
\hline 1982 & Interpolated & 1.82 \\
\hline 1983 & 1983/84 NPTS & 1.80 \\
\hline 1984 & Interpolated & 1.77 \\
\hline 1985 & Interpolated & 1.74 \\
\hline 1986 & Interpolated & 1.71 \\
\hline 1987 & Interpolated & 1.69 \\
\hline 1988 & Interpolated & 1.66 \\
\hline 1989 & Interpolated & 1.63 \\
\hline 1990 & 1990 NPTS & 1.60 \\
\hline 1991 & Interpolated & 1.60 \\
\hline 1992 & Interpolated & 1.60 \\
\hline 1993 & Interpolated & 1.60 \\
\hline 1994 & Interpolated & 1.60 \\
\hline 1995 & 1995 NPTS & 1.60 \\
\hline 1996 & Interpolated & 1.60 \\
\hline 1997 & Interpolated & 1.59 \\
\hline 1998 & Interpolated & 1.59 \\
\hline 1999 & Interpolated & 1.58 \\
\hline 2000 & Interpolated & 1.58 \\
\hline 2001 & 2001 NHTS & 1.57 \\
\hline 2002 & 2001 NHTS & 1.57 \\
\hline 2003 & 2001 NHTS & 1.57 \\
\hline 2004 & 2001 NHTS & 1.57 \\
\hline 2005 & 2001 NHTS & 1.57 \\
\hline 2006 & 2001 NHTS & 1.57 \\
\hline 2007 & 2001 NHTS & 1.57 \\
\hline 2008 & 2009 NHTS & 1.55 \\
\hline 2009 & 2009 NHTS & 1.55 \\
\hline 2010 & 2009 NHTS & 1.55 \\
\hline 2011 & 2009 NHTS & 1.55 \\
\hline 2012 & 2009 NHTS & 1.55 \\
\hline 2013 & 2009 NHTS & 1.55 \\
\hline 2014 & 2009 NHTS & 1.55 \\
\hline 2015 & 2009 NHTS & 1.55 \\
\hline
\end{tabular}




\subsection{LIGHT TRUCKS}

Btu per vehicle-mile - Light truck energy use divided by light truck vehicle miles of travel. Energy use - See Section 2. Energy Use Sources. Data series shown in Table 2.9. Vehicle-miles - 1970-2008: DOT, FHWA, Highway Statistics 2008, Table VM-1 and annual editions back to 1996 and DOT, FHWA, Highway Statistics Summary to 1995. Data series shown in Table 4.2. 2009-2015: See Appendix A, Section 7. Car and Light Truck Shares.

\subsection{Buses}

\subsubsection{Transit}

Btu per vehicle-mile - Transit bus energy use divided by transit bus vehicle-miles.

Energy use - See Section 2. Energy Use Sources. Data series shown in Table 7.9.

Vehicle-miles - APTA, 2016 Public Transportation Fact Book, Washington, DC, 2016. Data series shown on Table 7.9. Data for 2015 are directly from the U.S. Department of Transportation, Federal Transit Administration, National Transit Database.

Btu per passenger-mile - Transit bus energy use divided by transit bus passenger-miles. Energy use - See Section 2. Energy Use Sources. Data series shown in Table 7.9. Passenger-miles - APTA, 2016 Public Transportation Fact Book, Washington, DC, 2016. Data series shown on Table 7.9. Data for 2015 are directly from the U.S. Department of Transportation, Federal Transit Administration, National Transit Database.

\subsubsection{Intercity}

Btu per passenger-mile - Data are not available.

Energy use - See Section 2. Energy Use Sources. Because the data past 2000 are not available, the rate of change in bus VMT from FHWA, Highway Statistics 2015, was used to estimate the change in energy use.

Passenger-miles - Data are not available. 


\section{NONHIGHWAY MODE ENERGY INTENSITIES}

\subsection{AIR}

\subsubsection{Certificated Air Carriers}

Btu per passenger-mile - Certificated air carrier energy use divided by passenger-miles. Energy use - See Section 2. Energy Use Sources. All of domestic fuel use and half of international fuel use was considered to be domestic use.

Passenger-miles - DOT, BTS, Air Carrier Traffic Statistics, Washington, DC, www.transtats.bts.gov. Pre-1994 data are from various editions of the FAA Statistical Handbook of Aviation (no longer published). Scheduled service passenger-miles of domestic air carriers and half of international air carriers were used to coincide with fuel use.

Note: These data differ from the data in Table 9.2 because that table contains data on ALL domestic AND international air carrier energy use and passenger-miles.

\subsubsection{General Aviation}

Btu per passenger-mile - Data are not available.

Energy use - See Section 2. Energy Use Sources. Data series shown in Table 9.3.

Passenger-miles - Data are not available.

\subsection{RAIL}

\subsubsection{Intercity}

Btu per passenger-mile - Intercity rail energy use divided by passenger-miles.

Energy use - See Section 2. Energy Use Sources. Data series shown in Table 9.10.

Passenger-miles - AAR, Railroad Facts, 2016 Edition, and previous annual editions.

\subsubsection{Transit}

Btu per passenger-mile - Transit rail energy use divided by passenger-miles.

Energy use - See Section 2. Energy Use Sources. Data series shown in Table 9.12. Passenger-miles - APTA, 2016 Public Transportation Fact Book, Washington, DC, 2016. Data series shown on Table 9.12. Data for 2015 are directly from the U.S. Department of Transportation, Federal Transit Administration, National Transit Database. 


\subsubsection{Commuter}

Btu per passenger-mile - Commuter rail energy use divided by passenger-miles.

Energy use - See Section 2. Energy Use Sources. Data series shown in Table 9.11. Passenger-miles - APTA, 2016 Public Transportation Fact Book, Washington, DC, 2016. Data series shown on Table 9.11. Data for 2015 are directly from the U.S. Department of Transportation, Federal Transit Administration, National Transit Database. 


\section{FREIGHT MODE ENERGY INTENSITIES}

\subsection{TRUCK}

Btu per vehicle-mile - Heavy single-unit and combination truck energy use divided by vehicle miles

Energy use - See Section 2. Energy Use Sources (medium/heavy trucks).

Vehicle-miles - DOT, FHWA, Highway Statistics 2015, Table VM-1 and annual editions back to 1996 and DOT, FHWA, Highway Statistics Summary to 1995. Data series is the total of vehicle travel data on Tables 5.1 and 5.2.

\subsection{RAIL}

Btu per freight car-mile - Class I rail energy use divided by freight car-miles.

Energy use - See Section 2. Energy Use Sources. Data series shown in Table 9.8.

Freight car miles - AAR, Railroad Facts, 2016 Edition, Washington, DC, 2016. Data series shown in Table 9.8.

Btu per ton-mile - Class I rail energy use divided by ton-miles.

Energy use - See Section 2. Energy Use Sources. Data series shown in Table 9.8.

Ton-miles - AAR, Railroad Facts, 2016 Edition, Washington, DC, 2016. Data series shown in Table 9.8.

\subsection{WATER}

Btu per ton-mile - Domestic waterborne commerce energy use on taxable waterways divided by ton-miles on taxable waterways.

Energy use - Modeled by Chrisman A. Dager, University of Tennessee, Knoxville, using Waterborne Commerce Statistics Center detail records and annual IRS reports on the Inland Waterway Trust Fund tax on diesel fuel used on the inland waterway.

Note: These data are not available for 2015.

Ton-miles - Based on detailed records from the U.S. Department of the Army, Army Corps of Engineers, Waterborne Commerce Statistics Center. Includes only ton-miles on taxable waterways. 


\section{CAR AND LIGHT TRUCK SHARES}

In 2011, the Federal Highway Administration (FHWA) changed the methodology for producing the data on the VM-1 Table in the annual Highway Statistics publication. Historically, VM-1 included individual categories for passenger cars and 2-axle, 4-tire trucks. VM-1 included the vehicle miles of travel (VMT), registrations, fuel use, and fuel economy of passenger cars and 2axle, 4-tire trucks. After the methodology change, the categories of light vehicles on VM-1 changed to Light-Duty Vehicles with Short wheelbase (less than or equal to 121 inches) and Light-Duty Vehicles with Long Wheelbase (over 121 inches). As some passenger cars have long wheelbases and some 2-axle, 4-tire trucks have short wheelbases, the categories of cars and 2axle, 4-tire trucks are no longer available. Despite these changes, there are many transportation analysts who require information on cars and 2-axle, 4-tire trucks. Thus, a new methodology to estimate the data in these categories was developed for years 2009 through 2015.

\subsection{CARS}

Registrations - DOT, FHWA, Highway Statistics 2015, Table MV-1 and annual editions back to 2009.

\section{Vehicle travel -}

Total for all light vehicles - DOT, FHWA, Highway Statistics 2015, Table VM-1 and annual editions back to 2009; sum of light-duty short wheelbase and light-duty long wheelbase VMT.

Cars - Using historical shares of passenger cars/2-axle, 4-tire trucks from the Highway Statistics, the percent of light vehicle travel attributable to cars was estimated for 20092015, keeping in mind the economic conditions present in those years and the general trend in total light vehicle VMT. The estimated share was applied to total VMT as shown in Table A.19.

Table A.19

Estimated Car VMT

\begin{tabular}{cccc}
\hline Year & $\begin{array}{c}\text { Total Light Vehicle } \\
\text { VMT (billions) }\end{array}$ & $\begin{array}{c}\text { Share Attributable to } \\
\text { Cars }\end{array}$ & $\begin{array}{c}\text { Total Car } \\
\text { VMT (billions) }\end{array}$ \\
\hline 2009 & $2,633.3$ & $59.5 \%$ & $1,566.8$ \\
2010 & $2,648.5$ & $56.5 \%$ & $1,496.4$ \\
2011 & $2,650.5$ & $55.0 \%$ & $1,457.8$ \\
2012 & $2,664.1$ & $54.0 \%$ & $1,438.6$ \\
2013 & $2,677.8$ & $54.0 \%$ & $1,446.0$ \\
2014 & $2,710.6$ & $53.0 \%$ & $1,436.6$ \\
2015 & $2,779.7$ & $52.0 \%$ & $1,445.4$ \\
\hline
\end{tabular}

Miles per Vehicle - Vehicle travel divided by registrations.

Fuel Use - Vehicle travel divided by fuel economy.

Fuel Economy - DOE, EIA, Annual Energy Outlook 2017, June 2017 and annual editions back to 2012. 


\subsection{2-AXLE, 4-TIRE TRUCKS}

Registrations - DOT, FHWA, Highway Statistics 2015, Table MV-1 and annual editions back to 2009.

Vehicle travel -

Total for all light vehicles - DOT, FHWA, Highway Statistics 2015, Table VM-1 and annual editions back to 2009; sum of light-duty short wheelbase and light-duty long wheelbase VMT.

2-axle, 4-tire truck VMT - Using historical shares of passenger cars/2-axle, 4-tire trucks from the Highway Statistics, the percent of light vehicle travel attributable to cars was estimated for 2009-2015, keeping in mind the economic conditions present in those years and the general trend in total light vehicle VMT. The estimated share was applied to total VMT as shown in Table A.20.

Table A.20

Estimated 2-axle, 4-tire Truck VMT

\begin{tabular}{cccc}
\hline Year & $\begin{array}{c}\text { Total Light Vehicle } \\
\text { VMT (billions) }\end{array}$ & $\begin{array}{c}\text { Share Attributable to } \\
\text { 2-axle, 4-tire Trucks }\end{array}$ & $\begin{array}{c}\text { Total 2-axle, 4-tire } \\
\text { Truck VMT (billions) }\end{array}$ \\
\hline 2009 & $2,633.2$ & $40.5 \%$ & $1,066.5$ \\
2010 & $2,648.5$ & $43.5 \%$ & $1,152.1$ \\
2011 & $2,650.5$ & $45.0 \%$ & $1,192.7$ \\
2012 & $2,664.1$ & $46.0 \%$ & $1,225.5$ \\
2013 & $2,677.8$ & $46.0 \%$ & $1,231.8$ \\
2014 & $2,710.6$ & $47.0 \%$ & $1,274.0$ \\
2015 & $2,779.7$ & $48.0 \%$ & $1,334.3$ \\
\hline
\end{tabular}

Miles per Vehicle - Vehicle travel divided by registrations.

Fuel Use - Vehicle travel divided by fuel economy.

Fuel Economy - DOE, EIA, Annual Energy Outlook 2017, June 2017 and annual editions back to 2012. 
A-36

TRANSPORTATION ENERGY DATA BOOK: EDITION 36-2017 
APPENDIX B

\section{CONVERSIONS}


B-2

TRANSPORTATION ENERGY DATA BOOK: EDITION 36-2017 


\section{CONVERSIONS}

\section{A Note about Heating Values}

The heat content of a fuel is the quantity of energy released by burning a unit amount of that fuel. However, this value is not absolute and can vary according to several factors. For example, empirical formulae for determining the heating value of liquid fuels depend on the fuels' American Petroleum Institute (API) gravity. The API gravity varies depending on the percent by weight of the chemical constituents and impurities in the fuel, both of which are affected by the combination of raw materials used to produce the fuel and by the type of manufacturing process. Temperature and climatic conditions are also factors.

Because of these variations, the heating values in Table B.4 may differ from values in other publications. The figures in this report are representative or average values, not absolute ones. The gross (higher) heating values used here agree with those used by the Energy Information Administration (EIA).

Heating values fall into two categories, usually referred to as "higher" (or gross) and "lower" (or net). If the products of fuel combustion are cooled back to the initial fuel-air or fueloxidizer mixture temperature and the water formed during combustion is condensed, the energy released by the process is the higher (gross) heating value. If the products of combustion are cooled to the initial fuel-air temperature, but the water is considered to remain as a vapor, the energy released by the process is the lower (net) heating value. Usually the difference between the gross and net heating values for fuels used in transportation is around 5 to 8 percent; however, it is important to be consistent in their use.

The Transportation Energy Data Book has always used gross heating values for fuel conversion. 
Table B.1

Hydrogen Heat Content

\begin{tabular}{cc}
\hline \multicolumn{2}{c}{1 kilogram hydrogen $=$} \\
\hline Higher heating value & Lower heating value \\
$134,200 \mathrm{Btu}$ & $113,400 \mathrm{Btu}$ \\
$39.3 \mathrm{kWhr}$ & $33.2 \mathrm{kWhr}$ \\
$141,600 \mathrm{~kJ}$ & $119,600 \mathrm{~kJ}$ \\
$33,800 \mathrm{kCal}$ & $28,560 \mathrm{kCal}$ \\
\hline
\end{tabular}

Table B.2

Hydrogen Conversions

\begin{tabular}{|c|c|c|c|c|c|c|}
\hline & \multicolumn{2}{|c|}{ Weight } & \multicolumn{2}{|c|}{ Gas } & \multicolumn{2}{|c|}{ Liquid } \\
\hline & $\begin{array}{l}\text { Pounds } \\
\text { (lb) }\end{array}$ & $\begin{array}{c}\text { Kilograms } \\
(\mathrm{kg})\end{array}$ & $\begin{array}{c}\text { Standard } \\
\text { cubic feet } \\
(\mathrm{SCF}) \\
\end{array}$ & $\begin{array}{c}\text { Normal } \\
\text { cubic meter } \\
\left(\mathrm{Nm}^{3}\right)\end{array}$ & $\begin{array}{c}\text { Gallons } \\
\text { (gal) }\end{array}$ & $\begin{array}{c}\text { Liters } \\
\text { (L) }\end{array}$ \\
\hline $1 \mathrm{lb}$ & 1.0 & 0.4536 & 192.00 & 5.047 & 1.6928 & 6.408 \\
\hline $1 \mathrm{~kg}$ & 2.205 & 1.0 & 423.3 & 11.126 & 3.733 & 14.128 \\
\hline 1 SCF gas & 0.005209 & 0.002363 & 1.0 & 0.02628 & 0.00882 & 0.0339 \\
\hline $1 \mathrm{Nm}^{3}$ gas & 0.19815 & 0.08988 & 38.04 & 1.0 & 0.3355 & 1.2699 \\
\hline 1 gal liquid & 0.5906 & 0.2679 & 113.41 & 2.981 & 1.0 & 3.785 \\
\hline $1 \mathrm{~L}$ liquid & 0.15604 & 0.07078 & 29.99 & 0.77881 & 0.2642 & 1.0 \\
\hline
\end{tabular}

Table B.3

Pressure Conversions

\begin{tabular}{lccc}
\hline & Bar & Atmosphere & lb/in ${ }^{2}$ (or psi) \\
\hline Bar & 1.0 & 0.987 & 14.5 \\
Atmosphere & 1.013 & 1.0 & 14.696 \\
lb/in ${ }^{2}$ or psi) & 0.0689 & 0.0680 & 1.0 \\
\hline
\end{tabular}


Table B.4

Heat Content for Various Fuels

\begin{tabular}{|c|c|}
\hline Conventional gasoline & $125,000 \mathrm{Btu} /$ gal (gross) $=115,400 \mathrm{Btu} / \mathrm{gal}$ (net) \\
\hline E10 & $120,900 \mathrm{Btu} /$ gal $($ gross $)=112,400 \mathrm{Btu} / \mathrm{gal}$ (net) \\
\hline E15 & $119,000 \mathrm{Btu} /$ gal $($ gross $)=109,400 \mathrm{Btu} /$ gal (net) \\
\hline Hydrogen & 134,200 Btu/kg (gross) = 113,400 Btu/kg (net) \\
\hline Low-sulfur diesel & $138,700 \mathrm{Btu} /$ gal $($ gross $)=128,700 \mathrm{Btu} / \mathrm{gal}$ (net) \\
\hline Biodiesel & $126,200 \mathrm{Btu} /$ gal $($ gross $)=117,100 \mathrm{Btu} /$ gal (net) \\
\hline Methanol & 64,600 Btu/gal (gross) = 56,600 Btu/gal (net) \\
\hline Ethanol & 84,600 Btu/gal (gross) = 75,700 Btu/gal (net) \\
\hline E85 & 90,700 Btu/gal (gross) = 81,600 Btu/gal (net) \\
\hline Aviation gasoline & $120,200 \mathrm{Btu} /$ gal $($ gross) $=112,000 \mathrm{Btu} / \mathrm{gal}$ (net) \\
\hline Liquefied petroleum gas (LPG) & 91,300 Btu/gal (gross) = 83,500 Btu/gal (net) \\
\hline Butane & 103,000 Btu/gal (gross) = 93,000 Btu/gal (net) \\
\hline Jet fuel (naphtha) & $127,500 \mathrm{Btu} /$ gal $($ gross) $=118,700 \mathrm{Btu} /$ gal $($ net) \\
\hline Jet fuel (kerosene) & $135,000 \mathrm{Btu} /$ gal $($ gross $)=128,100 \mathrm{Btu} /$ gal (net) \\
\hline Lubricants & $144,400 \mathrm{Btu} /$ gal (gross) $=130,900 \mathrm{Btu} / \mathrm{gal}$ (net) \\
\hline Waxes & $131,800 \mathrm{Btu} /$ gal $($ gross $)=120,200 \mathrm{Btu} /$ gal (net) \\
\hline Asphalt and road oil & $158,000 \mathrm{Btu} /$ gal $($ gross $)=157,700 \mathrm{Btu} /$ gal $($ net) \\
\hline Liquefied natural gas (LNG) & 23,700 Btu/lb (gross) = 20,900 Btu/lb (net) \\
\hline Compressed natural gas (CNG) & 22,500 Btu/lb (gross) = 20,200 Btu/lb (net) \\
\hline Crude petroleum & $138,100 \mathrm{Btu} /$ gal $($ gross) $=131,800 \mathrm{Btu} /$ gal (net) \\
\hline \multicolumn{2}{|l|}{ Fuel Oils } \\
\hline Residual & $149,700 \mathrm{Btu} /$ gal (gross) $=138,400 \mathrm{Btu} / \mathrm{gal}$ (net) \\
\hline Distillate & $138,700 \mathrm{Btu} /$ gal $($ gross) $=131,800 \mathrm{Btu} /$ gal (net) \\
\hline \multicolumn{2}{|l|}{ Coal } \\
\hline Production average & $19.880 \times 10^{6} \mathrm{Btu} / \mathrm{short}$ ton \\
\hline Consumption average & $19.499 \times 10^{6} \mathrm{Btu} / \mathrm{short}$ ton \\
\hline
\end{tabular}

Note: Heat content values are approximate. Data are rounded to the nearest hundred. 
Table B.5

Fuel Equivalents

1 million bbl crude oil/day

1 billion bbl crude oil/year

1 quadrillion Btu/year

1 billion short tons coal/year

1 billion metric tons coal/year

1 trillion $\mathrm{ft}^{3}$ natural gas/year

1 petajoule/year
$=0.365$ billion bbl crude oil/year

$=2.089$ quadrillion Btu/year

$=107.110 \mathrm{million}$ short tons coal $/$ year

$=97.170 \mathrm{million}$ metric tons coal/year

$=2.016$ trillion $\mathrm{ft}^{3}$ natural gas/year

$=2,203$ petajoules/year

$=2.740$ million bbl crude oil/day

$=5.722$ quadrillion Btu/year

$=293.451 \mathrm{million}$ short tons coal $/$ year

$=266.219$ million metric tons coal/year

$=5.523$ trillion $\mathrm{ft}^{3}$ natural gas/year

$=6,037$ petajoules/year

$=8.000$ million gasoline gallon equivalents

$=0.479$ million bbl crude oil/day

$=174.764 \mathrm{million} \mathrm{bbl}$ crude oil/year

$=51.285$ million short tons coal/year

$=46.525 \mathrm{million}$ metric tons coal $/$ year

$=965.251$ billion $\mathrm{ft}^{3}$ natural gas/year

$=1,055$ petajoules/year

$=0.907 \mathrm{billion}$ metric tons coal/year

$=9.336$ million bbl crude oil/day

$=3.408$ billion bbl crude oil/year

$=19.499$ quadrillion Btu/year

$=18.821$ trillion $\mathrm{ft}^{3}$ natural gas/year

$=20,572$ petajoules/year

$=1.102$ billion short tons coal/year

$=8.470 \mathrm{million}$ bbl crude oil $/$ day

$=3.091 \mathrm{billion} \mathrm{bbl}$ crude oil/year

$=17.689$ quadrillion btu/year

$=17.075$ trillion $\mathrm{ft}^{3}$ natural gas/year

$=18,662$ petajoules/year

$=0.496$ million bbl crude oil/day

$=0.181$ billion bbl crude oil/year

$=1.036$ quadrillion Btu/year

$=53.131 \mathrm{million}$ short tons coal/year

$=48.200 \mathrm{million}$ metric tons coal $/$ year

$=1,093$ petajoules/year

$=453.844 \mathrm{bbl}$ crude oil/day

$=165.653$ thousand bbl crude oil/year

$=0.948$ trillion Btu/year

$=48.661$ thousand short tons coal/year

$=44.100$ thousand metric tons coal/year

$=0.915$ billion $\mathrm{ft}^{3}$ natural gas/year 
Table B.6

Energy Unit Conversions

\begin{tabular}{|c|c|c|c|}
\hline $1 \mathrm{Btu}$ & $\begin{array}{l}=778.2 \mathrm{ft}-\mathrm{lb} \\
=107.6 \mathrm{~kg}-\mathrm{m} \\
=1055 \mathrm{~J} \\
=39.30 \times 10^{-5} \mathrm{hp}-\mathrm{h} \\
=39.85 \times 10^{-5} \text { metric hp-h } \\
=29.31 \times 10^{-5} \mathrm{kWhr}\end{array}$ & $1 \mathrm{kWhr}$ & $\begin{array}{l}=3412 \mathrm{Btu}^{\mathrm{a}} \\
=2.655 \times 10^{6} \mathrm{ft}-\mathrm{lb} \\
=3.671 \times 10^{5} \mathrm{~kg}-\mathrm{m} \\
=3.600 \times 10^{6} \mathrm{~J} \\
=1.341 \mathrm{hp}-\mathrm{h} \\
=1.360 \text { metric hp-h }\end{array}$ \\
\hline 1 kg-m & $\begin{array}{l}=92.95 \times 10^{-4} \text { Btu } \\
=7.233 \mathrm{ft}-\mathrm{lb} \\
=9.806 \mathrm{~J} \\
=36.53 \times 10^{-7} \mathrm{hp}-\mathrm{h} \\
=37.04 \times 10^{-7} \text { metric hp-h } \\
=27.24 \times 10^{-7} \mathrm{kWhr}\end{array}$ & 1 Joule & $\begin{array}{l}=94.78 \times 10^{-5} \mathrm{Btu} \\
=0.7376 \mathrm{ft}-\mathrm{lb} \\
=0.1020 \mathrm{~kg}-\mathrm{m} \\
=37.25 \times 10^{-8} \mathrm{hp}-\mathrm{h} \\
=37.77 \times 10^{-8} \text { metric hp-h } \\
=27.78 \times 10^{-8} \mathrm{kWhr}\end{array}$ \\
\hline 1 hp-h & $\begin{array}{l}=2544 \mathrm{Btu} \\
=1.98 \times 10^{6} \mathrm{ft}-\mathrm{lb} \\
=2.738 \times 10^{6} \mathrm{kgm} \\
=2.685 \times 10^{6} \mathrm{~J} \\
=1.014 \text { metric hp-h } \\
=0.7475 \mathrm{kWhr}\end{array}$ & 1 metric hp-h & $\begin{array}{l}=2510 \mathrm{Btu} \\
=1.953 \times 10^{6} \mathrm{ft}-\mathrm{lb} \\
=27.00 \times 10^{4} \mathrm{~kg}-\mathrm{m} \\
=2.648 \times 10^{6} \mathrm{~J} \\
=0.9863 \mathrm{hp}-\mathrm{h} \\
=0.7355 \mathrm{kWhr}\end{array}$ \\
\hline
\end{tabular}

${ }^{a}$ This figure does not take into account the fact that electricity generation and distribution efficiency is approximately 33\%. If generation and distribution efficiency are taken into account, $1 \mathrm{kWhr}=10,339 \mathrm{Btu}$.

Table B.7

International Energy Conversions

\begin{tabular}{|c|c|c|c|c|c|}
\hline To: & Petajoules & $\begin{array}{c}\begin{array}{c}\text { Giga- } \\
\text { calories }\end{array} \\
\end{array}$ & $\begin{array}{c}\text { Million } \\
\text { metric tons of } \\
\text { oil equivalent }\end{array}$ & $\begin{array}{c}\text { Million } \\
\text { Btu }\end{array}$ & $\begin{array}{c}\begin{array}{c}\text { Gigawatt- } \\
\text { hours }\end{array} \\
\end{array}$ \\
\hline From: & multiply by: & & & & \\
\hline Petajoules & 1 & $238.8 \times 10^{3}$ & $2.388 \times 10^{-2}$ & $947.8 \times 10^{3}$ & 277.8 \\
\hline Gigacalories & $4.1868 \times 10^{-6}$ & 1 & $10^{-7}$ & 3.968 & $1.163 \times 10^{-3}$ \\
\hline $\begin{array}{r}\text { Million metric tons } \\
\text { of oil equivalent }\end{array}$ & 41.868 & $10^{7}$ & 1 & $3.968 \times 10^{7}$ & 11,630 \\
\hline Million Btu & $1.0551 \times 10^{-6}$ & 0.252 & $2.52 \times 10^{-8}$ & 1 & $2.931 \times 10^{-4}$ \\
\hline Gigawatthours & $3.6 \times 10^{-3}$ & 860 & $8.6 \times 10^{-5}$ & 3412 & 1 \\
\hline
\end{tabular}


Table B.8

Distance and Velocity Conversions

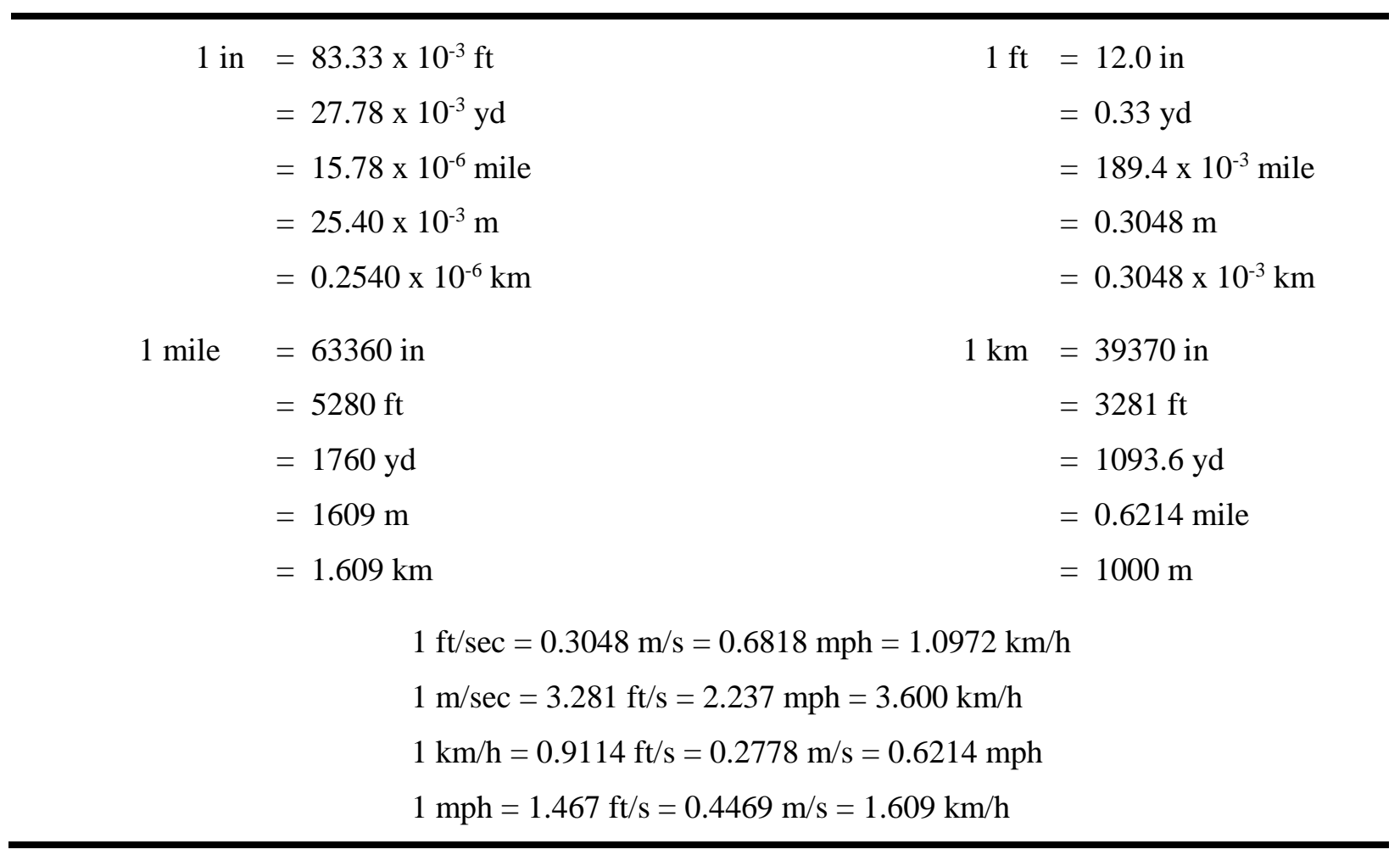

Table B.9

Alternative Measures of Greenhouse Gases

1 pound methane, measured in carbon units $\left(\mathrm{CH}_{4}\right)$

1 pound carbon dioxide, measured in carbon units $\left(\mathrm{CO}_{2}-\mathrm{C}\right)$

1 pound carbon monoxide, measured in carbon units (CO-C)

1 pound nitrous oxide, measured in nitrogen units $\left(\mathrm{N}_{2} \mathrm{O}-\mathrm{N}\right)$
1.333 pounds methane, measured at full

$=$ molecular weight $\left(\mathrm{CH}_{4}\right)$

3.6667 pounds carbon dioxide, measured at

$=$ full molecular weight $\left(\mathrm{CO}_{2}\right)$

2.333 pounds carbon monoxide, measured at

$=$ full molecular weight (CO)

= $\quad 1.571$ pounds nitrous oxide, measured at full molecular weight $\left(\mathrm{N}_{2} \mathrm{O}\right)$ 
Table B.10

Volume and Flow Rate Conversions ${ }^{\mathrm{a}}$

\begin{tabular}{|c|c|c|c|}
\hline \multirow[t]{6}{*}{1 U.S. gal } & $=231 \mathrm{in}^{3}$ & \multirow[t]{6}{*}{1 liter } & $=61.02 \mathrm{in}^{3}$ \\
\hline & $=0.1337 \mathrm{ft}^{3}$ & & $=3.531 \times 10^{-2} \mathrm{ft}^{3}$ \\
\hline & $=3.785$ liters & & $=0.2624$ U.S. gal \\
\hline & $=0.8321$ imperial gal & & $=0.2200$ imperial gal \\
\hline & $=0.0238 \mathrm{bbl}$ & & $=6.29 \times 10^{-3} \mathrm{bbl}$ \\
\hline & $=0.003785 \mathrm{~m}^{3}$ & & $=0.001 \mathrm{~m}^{3}$ \\
\hline \multicolumn{4}{|c|}{ A U.S. gallon of gasoline weighs 6.2 pounds } \\
\hline \multirow[t]{6}{*}{1 imperial gal } & $=277.4 \mathrm{in}^{3}$ & $1 \mathrm{bbl}$ & $=9702 \mathrm{in}^{3}$ \\
\hline & $=0.1606 \mathrm{ft}^{3}$ & & $=5.615 \mathrm{ft}^{3}$ \\
\hline & $=4.545$ liters & & $=158.97$ liters \\
\hline & $=1.201$ U.S.gal & & $=42$ U.S. gal \\
\hline & $=0.0286 \mathrm{bbl}$ & & $=34.97$ imperial gal \\
\hline & $=0.004546 \mathrm{~m}^{3}$ & & $=0.15897 \mathrm{~m}^{3}$ \\
\hline \multirow[t]{4}{*}{1 U.S. gal/hr } & $=3.209 \mathrm{ft}^{3} /$ day & & $=1171 \mathrm{ft}^{3} /$ year \\
\hline & $=90.84$ liter/day & & $=33157$ liter/year \\
\hline & $=19.97$ imperial gal $/$ day & & = $7289 \mathrm{imperial} \mathrm{gal} /$ year \\
\hline & $=0.5712 \mathrm{bbl} / \mathrm{day}$ & & $=207.92 \mathrm{bbl} /$ year \\
\hline \multicolumn{4}{|c|}{ For Imperial gallons, multiply above values by 1.201} \\
\hline \multirow[t]{4}{*}{1 liter/hr } & $=0.8474 \mathrm{ft}^{3} / \mathrm{day}$ & & $=309.3 \mathrm{ft}^{3} /$ year \\
\hline & $=6.298$ U.S. gal/day & & $=2299$ U.S. gal/year \\
\hline & $=5.28 \mathrm{imperial} \mathrm{gal} / \mathrm{day}$ & & = $1927 \mathrm{imperial} \mathrm{gal} /$ year \\
\hline & $=0.1510 \mathrm{bbl} / \mathrm{day}$ & & $=55.10 \mathrm{bbl} /$ year \\
\hline \multirow[t]{4}{*}{$1 \mathrm{bbl} / \mathrm{hr}$} & $=137.8 \mathrm{ft}^{3} /$ year & & $=49187 \mathrm{ft}^{3}$ year \\
\hline & $=1008$ U.S. gal/day & & $=3.679 \times 10^{5}$ U.S. gal/year \\
\hline & = 839.3 imperial gal/day & & $=3.063 \times 10^{5}$ imperial gal $/$ year \\
\hline & $=3815$ liter/day & & $=1.393 \times 10^{6}$ liter $/$ day \\
\hline
\end{tabular}

${ }^{\mathrm{a}}$ The conversions for flow rates are identical to those for volume measures, if the time units are identical. 
Table B.11

Power Conversions

\begin{tabular}{|c|c|c|c|c|c|c|}
\hline \multirow[b]{2}{*}{ FROM } & \multicolumn{6}{|c|}{ TO } \\
\hline & Horsepower & Kilowatts & $\begin{array}{c}\text { Metric } \\
\text { horsepower }\end{array}$ & Ft-lb per sec & $\begin{array}{c}\text { Kilocalories } \\
\text { per sec }\end{array}$ & Btu per sec \\
\hline Horsepower & 1 & 0.7457 & 1.014 & 550 & 0.1781 & 0.7068 \\
\hline Kilowatts & 1.341 & 1 & 1.360 & 737.6 & 0.239 & 0.9478 \\
\hline $\begin{array}{l}\text { Metric } \\
\text { horsepower }\end{array}$ & 0.9863 & 0.7355 & 1 & 542.5 & 0.1757 & 0.6971 \\
\hline Ft-lb per sec & $1.36 \times 10^{-3}$ & $1.356 \times 10^{-3}$ & $1.84 \times 10^{-3}$ & 1 & $0.3238 \times 10^{-3}$ & $1.285 \times 10^{-3}$ \\
\hline $\begin{array}{l}\text { Kilocalories } \\
\text { per sec }\end{array}$ & 5.615 & 4.184 & 5.692 & 3088 & 1 & 3.968 \\
\hline Btu per sec & 1.415 & 1.055 & 1.434 & 778.2 & 0.2520 & 1 \\
\hline
\end{tabular}

Table B.12

Mass Conversions

\begin{tabular}{llllll}
\hline & \multicolumn{5}{c}{ TO } \\
\cline { 2 - 6 } FROM & Pound & Kilogram & Short ton & Long ton & Metric ton \\
\hline Pound & 1 & 0.4536 & $5.0 \times 10^{-4}$ & $4.4643 \times 10^{-4}$ & $4.5362 \times 10^{-4}$ \\
Kilogram & 2.205 & 1 & $1.1023 \times 10^{-3}$ & $9.8425 \times 10^{-4}$ & $1.0 \times 10^{-3}$ \\
Short ton & 2,000 & 907.2 & 1 & 0.8929 & 0.9072 \\
Long ton & 2,240 & 1,106 & 1.12 & 1 & 1.016 \\
Metric ton & 2,205 & 1,000 & 1.102 & 0.9842 & 1 \\
\hline
\end{tabular}


Table B.13

Fuel Efficiency Conversions

\begin{tabular}{|c|c|c|c|c|c|}
\hline MPG & Miles/liter & Kilometers/L & $\begin{array}{c}\text { L/100 } \\
\text { kilometers }\end{array}$ & $\begin{array}{l}\text { Grams of } \\
\mathrm{CO}_{2} \\
\text { per mile }\end{array}$ & $\begin{array}{c}\text { Pounds of } \mathrm{CO}_{2} \\
\text { per mile }\end{array}$ \\
\hline 10 & 2.64 & 4.25 & 23.52 & 877.80 & 1.94 \\
\hline 15 & 3.96 & 6.38 & 15.68 & 585.20 & 1.29 \\
\hline 20 & 5.28 & 8.50 & 11.76 & 438.90 & 0.97 \\
\hline 25 & 6.60 & 10.63 & 9.41 & 351.12 & 0.78 \\
\hline 30 & 7.92 & 12.75 & 7.84 & 292.60 & 0.65 \\
\hline 35 & 9.25 & 14.88 & 6.72 & 250.80 & 0.55 \\
\hline 40 & 10.57 & 17.00 & 5.88 & 219.45 & 0.49 \\
\hline 45 & 11.89 & 19.13 & 5.23 & 195.07 & 0.43 \\
\hline 50 & 13.21 & 21.25 & 4.70 & 175.56 & 0.39 \\
\hline 55 & 14.53 & 23.38 & 4.28 & 159.60 & 0.35 \\
\hline 60 & 15.85 & 25.51 & 3.92 & 146.30 & 0.32 \\
\hline 65 & 17.17 & 27.63 & 3.62 & 135.05 & 0.30 \\
\hline 70 & 18.49 & 29.76 & 3.36 & 125.40 & 0.28 \\
\hline 75 & 19.81 & 31.88 & 3.14 & 117.04 & 0.26 \\
\hline 80 & 21.13 & 34.01 & 2.94 & 109.73 & 0.24 \\
\hline 85 & 22.45 & 36.13 & 2.77 & 103.27 & 0.23 \\
\hline 90 & 23.77 & 38.26 & 2.61 & 97.53 & 0.22 \\
\hline 95 & 25.09 & 40.38 & 2.48 & 92.40 & 0.20 \\
\hline 100 & 26.42 & 42.51 & 2.35 & 87.78 & 0.19 \\
\hline 105 & 27.74 & 44.64 & 2.24 & 83.60 & 0.18 \\
\hline 110 & 29.06 & 46.76 & 2.14 & 79.80 & 0.18 \\
\hline 115 & 30.38 & 48.89 & 2.05 & 76.33 & 0.17 \\
\hline 120 & 31.70 & 51.01 & 1.96 & 73.15 & 0.16 \\
\hline 125 & 33.02 & 53.14 & 1.88 & 70.22 & 0.16 \\
\hline 130 & 34.34 & 55.26 & 1.81 & 67.52 & 0.15 \\
\hline 135 & 35.66 & 57.39 & 1.74 & 65.02 & 0.14 \\
\hline 140 & 36.98 & 59.51 & 1.68 & 62.70 & 0.14 \\
\hline 145 & 38.30 & 61.64 & 1.62 & 60.54 & 0.13 \\
\hline 150 & 39.62 & 63.76 & 1.57 & 58.52 & 0.13 \\
\hline Formula & MPG/3.785 & MPG/[3.785/1.609] & 235.24/MPG & 8,778/MPG & 19.4/MPG \\
\hline
\end{tabular}

${ }^{\text {a }}$ For gasoline-fueled vehicles. 
Table B.14

SI Prefixes and Their Values

\begin{tabular}{llll}
\hline & Value & Prefix & Symbol \\
\hline One million million millionth & $10^{-18}$ & atto & $\mathrm{a}$ \\
One thousand million millionth & $10^{-15}$ & femto & $\mathrm{f}$ \\
One million millionth & $10^{-12}$ & pico & $\mathrm{p}$ \\
One thousand millionth & $10^{-9}$ & nano & $\mu$ \\
One millionth & $10^{-6}$ & micro & $\mathrm{m}$ \\
One thousandth & $10^{-3}$ & milli & $\mathrm{c}$ \\
One hundredth & $10^{-2}$ & centi & \\
One tenth & $10^{-1}$ & deci & \\
One & $10^{0}$ & & \\
Ten & $10^{1}$ & deca & $\mathrm{k}$ \\
One hundred & $10^{2}$ & hecto & $\mathrm{M}$ \\
One thousand & $10^{3}$ & kilo & $\mathrm{G}$ \\
One million & $10^{6}$ & mega & $\mathrm{T}$ \\
One billion $^{\mathrm{a}}$ & $10^{9}$ & giga & $\mathrm{P}$ \\
One trillion $^{\mathrm{a}}$ & $10^{12}$ & tera & $\mathrm{E}$ \\
One quadrillion $^{\mathrm{a}}$ & $10^{15}$ & peta & \\
One quintillion $^{\mathrm{a}}$ & $10^{18}$ & exa & \\
\hline
\end{tabular}

${ }^{\text {a }}$ Care should be exercised in the use of this nomenclature, especially in foreign correspondence, as it is either unknown or carries a different value in other countries. A "billion," for example, signifies a value of $10^{12}$ in most other countries.

Table B.15

Metric Units and Abbreviations

\begin{tabular}{lll}
\hline \multicolumn{1}{c}{ Quantity } & \multicolumn{1}{c}{ Unit name } & \multicolumn{1}{c}{ Symbol } \\
\hline & & $\mathrm{J}$ \\
Energy & Joule & $\mathrm{J} / \mathrm{kg}$ \\
Specific energy & Joule/kilogram & $\mathrm{J} /(\mathrm{kg} \bullet \mathrm{km})$ \\
Specific energy consumption & Joule/kilogram•kilometer & $\mathrm{J} / \mathrm{km}$ \\
Energy consumption & Joule/kilometer & $\mathrm{km} / \mathrm{kJ}$ \\
Energy economy & kilometer/kilojoule & $\mathrm{kW}$ \\
Power & kilowatt & $\mathrm{W} / \mathrm{kg}$ \\
Specific power & Watt/kilogram & $\mathrm{W} / \mathrm{m}^{3}$ \\
Power density & Watt/meter ${ }^{3}$ & $\mathrm{~km} / \mathrm{h}$ \\
Speed & kilometer/hour & $\mathrm{m} / \mathrm{s}^{2}$ \\
Acceleration & meter/second ${ }^{2}$ & $\mathrm{~km}$ \\
Range (distance) & kilometer & $\mathrm{kg}$ \\
Weight & kilogram & $\mathrm{N} \bullet \mathrm{m}$ \\
Torque & Newton•meter & $\mathrm{m} 3$ \\
Volume & meter & $\mathrm{kg}$ \\
Mass; payload & kilogram & $\mathrm{m}$ \\
Length; width & meter & $\mathrm{kg} / \mathrm{J}$ \\
Brake specific fuel consumption & kilogram/Joule & $\mathrm{L} / 100 \mathrm{~km}$ \\
Fuel economy (heat engine) & Liters/100 km & \\
\hline
\end{tabular}


Table B.16

Carbon Coefficients

(Million metric tons carbon per quadrillion Btu)

\begin{tabular}{llc}
\hline Energy Source & Fuel Type & Carbon Coefficients \\
\hline Coal & & \\
& Anthractie & 28.28 \\
& Bituminous & 25.45 \\
& Subbituminous & 26.51 \\
& Lignite & 26.65 \\
& Coke & 31.12 \\
Natural gas & Coal (All types) & 26.00 \\
& Natural Gas & 14.47 \\
& Flared natural gas & 14.92 \\
& Propane & 17.20 \\
Butane & 17.71 \\
& Butane/Propane Mix & 17.46 \\
& Gasoline & 19.45 \\
& Diesel fuel & 19.95 \\
& Jet Fuel & 19.34 \\
Aviation Gas & 18.87 \\
& Kerosene & 19.72 \\
& Residual Heating Fuel & 21.49 \\
& Petroleum coke & 27.85 \\
Asphalt and Road Oil & 20.62 \\
Lubricants & 20.24 \\
& Petrochemical Feedstocks & 19.37 \\
& Special Naphthas (solvents) & 19.85 \\
Waxes & 19.81 \\
Other petroleum \& miscellaneous & 19.81 \\
\hline
\end{tabular}

Note: Additional information:

www.eia.gov/environment/emissions/co2_vol_mass.cfm 


\section{Conversion of Constant Dollar Values}

Many types of information in this data book are expressed in dollars. Generally, constant dollars are used-that is, dollars of a fixed value for a specific year, such as 2010 dollars. Converting current dollars to constant dollars, or converting constant dollars for one year to constant dollars for another year, requires conversion factors (Table B.17 and Table B.18). Table B.17 shows conversion factors for the Consumer Price Index inflation factors. Table B.18 shows conversion factors using the Gross National Product Implicit Price Deflator. 
Table B.17

Consumer Price Inflation (CPI) Index

\begin{tabular}{|c|c|c|c|c|c|c|c|c|c|c|}
\hline From: & 1970 & 1971 & 1972 & 1973 & 1974 & 1975 & 1976 & 1977 & 1978 & 1979 \\
\hline 1970 & 1.000 & 1.044 & 1.077 & 1.144 & 1.271 & 1.387 & 1.466 & 1.562 & 1.680 & 1.871 \\
\hline 1971 & 0.958 & 1.000 & 1.032 & 1.096 & 1.217 & 1.328 & 1.405 & 1.496 & 1.610 & 1.793 \\
\hline 1972 & 0.928 & 0.969 & 1.000 & 1.062 & 1.179 & 1.287 & 1.361 & 1.450 & 1.560 & 1.737 \\
\hline 1973 & 0.874 & 0.912 & 0.941 & 1.000 & 1.110 & 1.212 & 1.282 & 1.365 & 1.468 & 1.635 \\
\hline 1974 & 0.787 & 0.822 & 0.848 & 0.901 & 1.000 & 1.091 & 1.154 & 1.229 & 1.323 & 1.473 \\
\hline 1975 & 0.721 & 0.753 & 0.777 & 0.825 & 0.916 & 1.000 & 1.058 & 1.126 & 1.212 & $1.34 \mathrm{~S}$ \\
\hline 1976 & 0.682 & 0.712 & 0.735 & 0.780 & 0.866 & 0.946 & 1.000 & 1.065 & 1.146 & $1.27 €$ \\
\hline 1977 & 0.640 & 0.668 & 0.690 & 0.733 & 0.814 & 0.888 & 0.939 & 1.000 & 1.076 & 1.198 \\
\hline 1978 & 0.595 & 0.621 & 0.641 & 0.681 & 0.756 & 0.825 & 0.873 & 0.929 & 1.000 & 1.113 \\
\hline 1979 & 0.534 & 0.558 & 0.576 & 0.612 & 0.679 & 0.741 & 0.784 & 0.835 & 0.898 & 1.000 \\
\hline 1980 & 0.471 & 0.492 & 0.507 & 0.539 & 0.598 & 0.653 & 0.691 & 0.735 & 0.791 & 0.881 \\
\hline 1981 & 0.427 & 0.446 & 0.460 & 0.488 & 0.542 & 0.592 & 0.626 & 0.667 & 0.717 & $0.79 \mathrm{~s}$ \\
\hline 1982 & 0.402 & 0.420 & 0.433 & 0.460 & 0.511 & 0.558 & 0.590 & 0.628 & 0.676 & 0.752 \\
\hline 1983 & 0.390 & 0.407 & 0.420 & 0.446 & 0.495 & 0.540 & 0.571 & 0.608 & 0.655 & $0.72 \mathrm{~S}$ \\
\hline 1984 & 0.373 & 0.390 & 0.402 & 0.427 & 0.474 & 0.518 & 0.548 & 0.583 & 0.628 & $0.69 \mathrm{~s}$ \\
\hline 1985 & 0.361 & 0.376 & 0.388 & 0.413 & 0.458 & 0.500 & 0.529 & 0.563 & 0.606 & 0.675 \\
\hline 1986 & 0.354 & 0.370 & 0.381 & 0.405 & 0.450 & 0.491 & 0.519 & 0.553 & 0.595 & 0.662 \\
\hline 1987 & 0.342 & 0.357 & 0.368 & 0.391 & 0.434 & 0.474 & 0.501 & 0.533 & 0.574 & 0.639 \\
\hline 1988 & 0.328 & 0.342 & 0.353 & 0.375 & 0.417 & 0.455 & 0.481 & 0.512 & 0.551 & 0.614 \\
\hline 1989 & 0.313 & 0.327 & 0.337 & 0.358 & 0.398 & 0.434 & 0.459 & 0.489 & 0.526 & 0.585 \\
\hline 1990 & 0.297 & 0.310 & 0.320 & 0.340 & 0.377 & 0.412 & 0.435 & 0.464 & 0.499 & 0.555 \\
\hline 1991 & 0.285 & 0.297 & 0.307 & 0.326 & 0.362 & 0.395 & 0.418 & 0.445 & 0.479 & 0.533 \\
\hline 1992 & 0.277 & 0.289 & 0.298 & 0.316 & 0.351 & 0.383 & 0.406 & 0.432 & 0.465 & 0.517 \\
\hline 1993 & 0.269 & 0.280 & 0.289 & 0.307 & 0.341 & 0.372 & 0.394 & 0.419 & 0.451 & 0.502 \\
\hline 1994 & 0.262 & 0.273 & 0.282 & 0.300 & 0.333 & 0.363 & 0.384 & 0.409 & 0.440 & 0.490 \\
\hline 1995 & 0.255 & 0.266 & 0.274 & 0.291 & 0.323 & 0.353 & 0.373 & 0.398 & 0.428 & $0.47 €$ \\
\hline 1996 & 0.247 & 0.258 & 0.266 & 0.283 & 0.314 & 0.343 & 0.363 & 0.386 & 0.416 & 0.463 \\
\hline 1997 & 0.242 & 0.252 & 0.260 & 0.277 & 0.307 & 0.335 & 0.355 & 0.378 & 0.406 & 0.452 \\
\hline 1998 & 0.238 & 0.248 & 0.256 & 0.272 & 0.302 & 0.330 & 0.349 & 0.372 & 0.400 & 0.445 \\
\hline 1999 & 0.233 & 0.243 & 0.251 & 0.267 & 0.296 & 0.323 & 0.342 & 0.364 & 0.391 & 0.436 \\
\hline 2000 & 0.225 & 0.235 & 0.243 & 0.258 & 0.286 & 0.312 & 0.330 & 0.352 & 0.379 & 0.422 \\
\hline 2001 & 0.219 & 0.229 & 0.236 & 0.251 & 0.278 & 0.304 & 0.321 & 0.342 & 0.368 & 0.410 \\
\hline 2002 & 0.216 & 0.225 & 0.232 & 0.247 & 0.274 & 0.299 & 0.316 & 0.337 & 0.362 & 0.404 \\
\hline 2003 & 0.211 & 0.220 & 0.227 & 0.241 & 0.268 & 0.292 & 0.309 & 0.329 & 0.354 & 0.395 \\
\hline 2004 & 0.205 & 0.214 & 0.221 & 0.235 & 0.261 & 0.285 & 0.301 & 0.321 & 0.345 & 0.384 \\
\hline 2005 & 0.199 & 0.207 & 0.214 & 0.227 & 0.252 & 0.275 & 0.291 & 0.310 & 0.334 & 0.372 \\
\hline 2006 & 0.192 & 0.201 & 0.207 & 0.220 & 0.245 & 0.267 & 0.282 & 0.301 & 0.323 & 0.360 \\
\hline 2007 & 0.187 & 0.195 & 0.202 & 0.214 & 0.238 & 0.259 & 0.274 & 0.292 & 0.314 & 0.350 \\
\hline 2008 & 0.180 & 0.188 & 0.194 & 0.206 & 0.229 & 0.250 & 0.264 & 0.281 & 0.303 & 0.337 \\
\hline 2009 & 0.181 & 0.189 & 0.195 & 0.207 & 0.230 & 0.251 & 0.265 & 0.282 & 0.304 & 0.338 \\
\hline 2010 & 0.178 & 0.186 & 0.192 & 0.204 & 0.226 & 0.247 & 0.261 & 0.278 & 0.299 & 0.333 \\
\hline 2011 & 0.172 & 0.180 & 0.186 & 0.197 & 0.219 & 0.239 & 0.253 & 0.269 & 0.290 & 0.323 \\
\hline 2012 & 0.169 & 0.176 & 0.182 & 0.193 & 0.215 & 0.234 & 0.248 & 0.264 & 0.284 & $0.31 €$ \\
\hline 2013 & 0.167 & 0.174 & 0.179 & 0.191 & 0.212 & 0.231 & 0.244 & 0.260 & 0.280 & 0.312 \\
\hline 2014 & 0.164 & 0.171 & 0.177 & 0.188 & 0.208 & 0.227 & 0.240 & 0.256 & 0.275 & 0.307 \\
\hline 2015 & 0.164 & 0.171 & 0.176 & 0.187 & 0.208 & 0.227 & 0.240 & 0.256 & 0.275 & $0.30 €$ \\
\hline
\end{tabular}


Table B.17

Consumer Price Inflation (CPI) Index (Continued)

\begin{tabular}{|c|c|c|c|c|c|c|c|c|c|c|}
\hline From: & 1980 & 1981 & 1982 & 1983 & 1984 & 1985 & 1986 & 1987 & 1988 & 1989 \\
\hline 1970 & 2.124 & 2.343 & 2.487 & 2.567 & 2.678 & 2.773 & 2.825 & 2.928 & 3.049 & 3.196 \\
\hline 1971 & 2.035 & 2.244 & 2.383 & 2.459 & 2.565 & 2.657 & 2.706 & 2.805 & 2.921 & 3.062 \\
\hline 1972 & 1.971 & 2.175 & 2.309 & 2.383 & 2.486 & 2.574 & 2.622 & 2.718 & 2.830 & 2.967 \\
\hline 1973 & 1.856 & 2.047 & 2.173 & 2.243 & 2.340 & 2.423 & 2.468 & 2.559 & 2.664 & 2.793 \\
\hline 1974 & 1.671 & 1.844 & 1.957 & 2.020 & 2.108 & 2.183 & 2.223 & 2.304 & 2.400 & 2.515 \\
\hline 1975 & 1.532 & 1.690 & 1.794 & 1.851 & 1.931 & 2.000 & 2.037 & 2.112 & 2.199 & 2.305 \\
\hline 1976 & 1.448 & 1.598 & 1.696 & 1.750 & 1.826 & 1.891 & 1.926 & 1.996 & 2.079 & 2.179 \\
\hline 1977 & 1.360 & 1.500 & 1.592 & 1.644 & 1.715 & 1.776 & 1.809 & 1.875 & 1.952 & 2.046 \\
\hline 1978 & 1.264 & 1.394 & 1.480 & 1.528 & 1.594 & 1.650 & 1.681 & 1.742 & 1.814 & 1.902 \\
\hline 1979 & 1.135 & 1.252 & 1.329 & 1.372 & 1.431 & 1.482 & 1.510 & 1.565 & 1.629 & 1.708 \\
\hline 1980 & 1.000 & 1.103 & 1.171 & 1.209 & 1.261 & 1.306 & 1.330 & 1.379 & 1.436 & 1.505 \\
\hline 1981 & 0.906 & 1.000 & 1.062 & 1.096 & 1.143 & 1.184 & 1.206 & 1.250 & 1.301 & 1.364 \\
\hline 1982 & 0.854 & 0.942 & 1.000 & 1.032 & 1.077 & 1.115 & 1.136 & 1.177 & 1.226 & 1.285 \\
\hline 1983 & 0.827 & 0.913 & 0.969 & 1.000 & 1.043 & 1.080 & 1.100 & 1.141 & 1.188 & 1.245 \\
\hline 1984 & 0.793 & 0.875 & 0.929 & 0.959 & 1.000 & 1.036 & 1.055 & 1.093 & 1.139 & 1.193 \\
\hline 1985 & 0.766 & 0.845 & 0.897 & 0.926 & 0.966 & 1.000 & 1.019 & 1.056 & 1.099 & 1.152 \\
\hline 1986 & 0.752 & 0.829 & 0.880 & 0.909 & 0.948 & 0.982 & 1.000 & 1.036 & 1.079 & 1.131 \\
\hline 1987 & 0.725 & 0.800 & 0.849 & 0.877 & 0.915 & 0.947 & 0.965 & 1.000 & 1.041 & 1.092 \\
\hline 1988 & 0.697 & 0.768 & 0.816 & 0.842 & 0.878 & 0.910 & 0.926 & 0.960 & 1.000 & 1.048 \\
\hline 1989 & 0.665 & 0.733 & 0.778 & 0.803 & 0.838 & 0.868 & 0.884 & 0.916 & 0.954 & 1.000 \\
\hline 1990 & 0.630 & 0.695 & 0.738 & 0.762 & 0.795 & 0.823 & 0.839 & 0.869 & 0.905 & 0.949 \\
\hline 1991 & 0.605 & 0.667 & 0.709 & 0.731 & 0.763 & 0.790 & 0.805 & 0.834 & 0.869 & 0.910 \\
\hline 1992 & 0.587 & 0.648 & 0.688 & 0.710 & 0.741 & 0.767 & 0.781 & 0.810 & 0.843 & 0.884 \\
\hline 1993 & 0.570 & 0.629 & 0.668 & 0.689 & 0.719 & 0.745 & 0.758 & 0.786 & 0.819 & 0.858 \\
\hline 1994 & 0.556 & 0.613 & 0.651 & 0.672 & 0.701 & 0.726 & 0.740 & 0.767 & 0.798 & 0.837 \\
\hline 1995 & 0.541 & 0.596 & 0.633 & 0.654 & 0.682 & 0.706 & 0.719 & 0.745 & 0.776 & 0.814 \\
\hline 1996 & 0.525 & 0.579 & 0.615 & 0.635 & 0.662 & 0.686 & 0.699 & 0.724 & 0.754 & 0.790 \\
\hline 1997 & 0.513 & 0.566 & 0.601 & 0.621 & 0.647 & 0.670 & 0.683 & 0.708 & 0.737 & 0.773 \\
\hline 1998 & 0.506 & 0.558 & 0.592 & 0.611 & 0.637 & 0.660 & 0.672 & 0.697 & 0.726 & 0.761 \\
\hline 1999 & 0.495 & 0.546 & 0.579 & 0.598 & 0.624 & 0.646 & 0.658 & 0.682 & 0.710 & 0.744 \\
\hline 2000 & 0.479 & 0.528 & 0.560 & 0.578 & 0.603 & 0.625 & 0.636 & 0.660 & 0.687 & 0.720 \\
\hline 2001 & 0.465 & 0.513 & 0.545 & 0.562 & 0.587 & 0.608 & 0.619 & 0.641 & 0.668 & 0.700 \\
\hline 2002 & 0.458 & 0.505 & 0.536 & 0.554 & 0.578 & 0.598 & 0.609 & 0.631 & 0.658 & 0.689 \\
\hline 2003 & 0.448 & 0.494 & 0.524 & 0.541 & 0.565 & 0.585 & 0.596 & 0.617 & 0.643 & 0.674 \\
\hline 2004 & 0.436 & 0.481 & 0.511 & 0.527 & 0.550 & 0.570 & 0.580 & 0.601 & 0.626 & 0.656 \\
\hline 2005 & 0.422 & 0.465 & 0.494 & 0.510 & 0.532 & 0.551 & 0.561 & 0.582 & 0.606 & 0.635 \\
\hline 2006 & 0.409 & 0.451 & 0.479 & 0.494 & 0.515 & 0.534 & 0.544 & 0.563 & 0.587 & 0.615 \\
\hline 2007 & 0.397 & 0.438 & 0.465 & 0.480 & 0.501 & 0.519 & 0.529 & 0.548 & 0.571 & 0.598 \\
\hline 2008 & 0.383 & 0.422 & 0.448 & 0.463 & 0.483 & 0.500 & 0.509 & 0.528 & 0.549 & 0.576 \\
\hline 2009 & 0.384 & 0.424 & 0.450 & 0.464 & 0.484 & 0.502 & 0.511 & 0.530 & 0.551 & 0.578 \\
\hline 2010 & 0.378 & 0.417 & 0.443 & 0.457 & 0.476 & 0.493 & 0.503 & 0.521 & 0.543 & 0.569 \\
\hline 2011 & 0.366 & 0.404 & 0.429 & 0.443 & 0.462 & 0.478 & 0.487 & 0.505 & 0.526 & 0.551 \\
\hline 2012 & 0.359 & 0.396 & 0.420 & 0.434 & 0.453 & 0.469 & 0.477 & 0.495 & 0.515 & 0.540 \\
\hline 2013 & 0.354 & 0.390 & 0.414 & 0.428 & 0.446 & 0.462 & 0.470 & 0.488 & 0.508 & 0.532 \\
\hline 2014 & 0.348 & 0.384 & 0.408 & 0.421 & 0.439 & 0.455 & 0.463 & 0.480 & 0.500 & 0.524 \\
\hline 2015 & 0.348 & 0.384 & 0.407 & 0.420 & 0.438 & 0.454 & 0.462 & 0.479 & 0.499 & 0.523 \\
\hline
\end{tabular}


Table B.17

Consumer Price Inflation (CPI) Index (Continued)

\begin{tabular}{|c|c|c|c|c|c|c|c|c|c|c|}
\hline From: & 1990 & 1991 & 1992 & 1993 & 1994 & 1995 & 1996 & 1997 & 1998 & 1999 \\
\hline 1970 & 3.369 & 3.510 & 3.616 & 3.724 & 3.820 & 3.928 & 4.044 & 4.137 & 4.201 & 4.294 \\
\hline 1971 & 3.227 & 3.363 & 3.464 & 3.568 & 3.659 & 3.763 & 3.874 & 3.963 & 4.025 & 4.114 \\
\hline 1972 & 3.127 & 3.258 & 3.356 & 3.457 & 3.545 & 3.646 & 3.754 & 3.840 & 3.900 & 3.986 \\
\hline 1973 & 2.944 & 3.068 & 3.160 & 3.255 & 3.338 & 3.432 & 3.534 & 3.615 & 3.671 & 3.752 \\
\hline 1974 & 2.651 & 2.763 & 2.846 & 2.931 & 3.006 & 3.091 & 3.183 & 3.256 & 3.306 & 3.379 \\
\hline 1975 & 2.429 & 2.532 & 2.608 & 2.686 & 2.755 & 2.833 & 2.916 & 2.983 & 3.030 & 3.097 \\
\hline 1976 & 2.297 & 2.394 & 2.466 & 2.540 & 2.605 & 2.678 & 2.757 & 2.821 & 2.865 & 2.928 \\
\hline 1977 & 2.157 & 2.248 & 2.315 & 2.384 & 2.446 & 2.515 & 2.589 & 2.649 & 2.690 & 2.749 \\
\hline 1978 & 2.005 & 2.089 & 2.152 & 2.216 & 2.273 & 2.337 & 2.406 & 2.462 & 2.500 & 2.555 \\
\hline 1979 & 1.800 & 1.876 & 1.933 & 1.990 & 2.041 & 2.099 & 2.161 & 2.211 & 2.245 & 2.295 \\
\hline 1980 & 1.586 & 1.653 & 1.703 & 1.754 & 1.799 & 1.850 & 1.904 & 1.948 & 1.978 & 2.022 \\
\hline 1981 & 1.438 & 1.498 & 1.543 & 1.590 & 1.630 & 1.677 & 1.726 & 1.766 & 1.793 & 1.833 \\
\hline 1982 & 1.354 & 1.411 & 1.454 & 1.497 & 1.536 & 1.579 & 1.626 & 1.663 & 1.689 & 1.726 \\
\hline 1983 & 1.312 & 1.367 & 1.409 & 1.451 & 1.488 & 1.530 & 1.575 & 1.611 & 1.637 & 1.673 \\
\hline 1984 & 1.258 & 1.311 & 1.350 & 1.391 & 1.426 & 1.467 & 1.510 & 1.545 & 1.569 & 1.603 \\
\hline 1985 & 1.215 & 1.266 & 1.304 & 1.343 & 1.377 & 1.416 & 1.458 & 1.492 & 1.515 & 1.548 \\
\hline 1986 & 1.193 & 1.243 & 1.280 & 1.318 & 1.352 & 1.391 & 1.432 & 1.464 & 1.487 & 1.520 \\
\hline 1987 & 1.151 & 1.199 & 1.235 & 1.272 & 1.305 & 1.342 & 1.381 & 1.413 & 1.435 & 1.467 \\
\hline 1988 & 1.105 & 1.151 & 1.186 & 1.221 & 1.253 & 1.288 & 1.326 & 1.357 & 1.378 & 1.408 \\
\hline 1989 & 1.054 & 1.098 & 1.131 & 1.165 & 1.195 & 1.229 & 1.265 & 1.294 & 1.315 & 1.344 \\
\hline 1990 & 1.000 & 1.042 & 1.073 & 1.106 & 1.134 & 1.166 & 1.200 & 1.228 & 1.247 & 1.275 \\
\hline 1991 & 0.960 & 1.000 & 1.030 & 1.061 & 1.088 & 1.119 & 1.152 & 1.178 & 1.197 & 1.223 \\
\hline 1992 & 0.932 & 0.971 & 1.000 & 1.030 & 1.056 & 1.086 & 1.118 & 1.144 & 1.162 & 1.187 \\
\hline 1993 & 0.904 & 0.943 & 0.971 & 1.000 & 1.026 & 1.055 & 1.086 & 1.111 & 1.128 & 1.153 \\
\hline 1994 & 0.882 & 0.919 & 0.947 & 0.975 & 1.000 & 1.028 & 1.059 & 1.083 & 1.100 & 1.124 \\
\hline 1995 & 0.858 & 0.894 & 0.921 & 0.948 & 0.972 & 1.000 & 1.030 & 1.053 & 1.070 & 1.093 \\
\hline 1996 & 0.833 & 0.868 & 0.894 & 0.921 & 0.945 & 0.971 & 1.000 & 1.023 & 1.039 & 1.062 \\
\hline 1997 & 0.814 & 0.849 & 0.874 & 0.900 & 0.923 & 0.950 & 0.978 & 1.000 & 1.016 & 1.038 \\
\hline 1998 & 0.802 & 0.836 & 0.861 & 0.887 & 0.909 & 0.935 & 0.963 & 0.985 & 1.000 & 1.022 \\
\hline 1999 & 0.785 & 0.818 & 0.842 & 0.867 & 0.890 & 0.915 & 0.942 & 0.963 & 0.978 & 1.000 \\
\hline 2000 & 0.759 & 0.791 & 0.815 & 0.839 & 0.861 & 0.885 & 0.911 & 0.932 & 0.947 & 0.967 \\
\hline 2001 & 0.738 & 0.769 & 0.792 & 0.816 & 0.837 & 0.861 & 0.886 & 0.906 & 0.920 & 0.941 \\
\hline 2002 & 0.727 & 0.757 & 0.780 & 0.803 & 0.824 & 0.847 & 0.872 & 0.892 & 0.906 & 0.926 \\
\hline 2003 & 0.710 & 0.740 & 0.763 & 0.785 & 0.805 & 0.828 & 0.853 & 0.872 & 0.886 & 0.905 \\
\hline 2004 & 0.692 & 0.721 & 0.743 & 0.765 & 0.785 & 0.807 & 0.831 & 0.850 & 0.863 & 0.882 \\
\hline 2005 & 0.669 & 0.697 & 0.718 & 0.740 & 0.759 & 0.780 & 0.803 & 0.822 & 0.835 & 0.853 \\
\hline 2006 & 0.648 & 0.676 & 0.696 & 0.717 & 0.735 & 0.756 & 0.778 & 0.796 & 0.809 & 0.826 \\
\hline 2007 & 0.630 & 0.657 & 0.677 & 0.697 & 0.715 & 0.735 & 0.757 & 0.774 & 0.786 & 0.804 \\
\hline 2008 & 0.607 & 0.633 & 0.652 & 0.671 & 0.688 & 0.708 & 0.729 & 0.745 & 0.757 & 0.774 \\
\hline 2009 & 0.609 & 0.635 & 0.654 & 0.674 & 0.691 & 0.710 & 0.731 & 0.748 & 0.760 & 0.777 \\
\hline 2010 & 0.599 & 0.625 & 0.643 & 0.663 & 0.680 & 0.699 & 0.720 & 0.736 & 0.748 & 0.764 \\
\hline 2011 & 0.581 & 0.605 & 0.624 & 0.642 & 0.659 & 0.678 & 0.698 & 0.714 & 0.725 & 0.741 \\
\hline 2012 & 0.569 & 0.593 & 0.611 & 0.629 & 0.645 & 0.664 & 0.683 & 0.699 & 0.710 & 0.726 \\
\hline 2013 & 0.561 & 0.585 & 0.602 & 0.620 & 0.636 & 0.654 & 0.674 & 0.689 & 0.700 & 0.715 \\
\hline 2014 & 0.552 & 0.575 & 0.593 & 0.610 & 0.626 & 0.644 & 0.663 & 0.678 & 0.689 & 0.704 \\
\hline 2015 & 0.551 & 0.575 & 0.592 & 0.610 & 0.625 & 0.643 & 0.662 & 0.677 & 0.688 & 0.703 \\
\hline
\end{tabular}


Table B.17

Consumer Price Inflation (CPI) Index (Continued)

\begin{tabular}{|c|c|c|c|c|c|c|c|c|c|c|}
\hline From: & 2000 & 2001 & 2002 & 2003 & 2004 & 2005 & 2006 & 2007 & 2008 & 2009 \\
\hline 1970 & 4.438 & 4.564 & 4.637 & 4.742 & 4.869 & 5.034 & 5.196 & 5.344 & 5.549 & 5.529 \\
\hline 1971 & 4.252 & 4.373 & 4.442 & 4.543 & 4.664 & 4.822 & 4.978 & 5.120 & 5.316 & 5.297 \\
\hline 1972 & 4.120 & 4.237 & 4.304 & 4.402 & 4.519 & 4.672 & 4.823 & 4.960 & 5.151 & 5.132 \\
\hline 1973 & 3.878 & 3.989 & 4.052 & 4.144 & 4.255 & 4.399 & 4.541 & 4.670 & 4.849 & 4.832 \\
\hline 1974 & 3.493 & 3.592 & 3.649 & 3.732 & 3.832 & 3.961 & 4.089 & 4.206 & 4.367 & 4.352 \\
\hline 1975 & 3.201 & 3.292 & 3.344 & 3.420 & 3.511 & 3.630 & 3.747 & 3.854 & 4.002 & 3.988 \\
\hline 1976 & 3.026 & 3.112 & 3.162 & 3.234 & 3.320 & 3.432 & 3.543 & 3.644 & 3.784 & 3.770 \\
\hline 1977 & 2.842 & 2.922 & 2.969 & 3.036 & 3.117 & 3.223 & 3.327 & 3.421 & 3.553 & 3.540 \\
\hline 1978 & 2.641 & 2.716 & 2.759 & 2.822 & 2.897 & 2.995 & 3.092 & 3.180 & 3.302 & 3.290 \\
\hline 1979 & 2.372 & 2.439 & 2.478 & 2.534 & 2.602 & 2.690 & 2.777 & 2.856 & 2.966 & 2.955 \\
\hline 1980 & 2.090 & 2.149 & 2.183 & 2.233 & 2.292 & 2.370 & 2.447 & 2.516 & 2.613 & 2.604 \\
\hline 1981 & 1.894 & 1.948 & 1.979 & 2.024 & 2.078 & 2.149 & 2.218 & 2.281 & 2.369 & 2.360 \\
\hline 1982 & 1.784 & 1.835 & 1.864 & 1.907 & 1.958 & 2.024 & 2.089 & 2.149 & 2.231 & 2.223 \\
\hline 1983 & 1.729 & 1.778 & 1.806 & 1.847 & 1.897 & 1.961 & 2.024 & 2.082 & 2.162 & 2.154 \\
\hline 1984 & 1.657 & 1.705 & 1.731 & 1.771 & 1.818 & 1.880 & 1.940 & 1.996 & 2.072 & 2.065 \\
\hline 1985 & 1.600 & 1.646 & 1.672 & 1.710 & 1.756 & 1.815 & 1.874 & 1.927 & 2.001 & 1.994 \\
\hline 1986 & 1.571 & 1.616 & 1.641 & 1.679 & 1.724 & 1.782 & 1.839 & 1.892 & 1.964 & 1.957 \\
\hline 1987 & 1.516 & 1.559 & 1.584 & 1.620 & 1.663 & 1.719 & 1.775 & 1.825 & 1.895 & 1.889 \\
\hline 1988 & 1.456 & 1.497 & 1.521 & 1.555 & 1.597 & 1.651 & 1.704 & 1.753 & 1.820 & 1.813 \\
\hline 1989 & 1.389 & 1.428 & 1.451 & 1.484 & 1.523 & 1.575 & 1.626 & 1.672 & 1.736 & 1.730 \\
\hline 1990 & 1.318 & 1.355 & 1.376 & 1.408 & 1.445 & 1.494 & 1.542 & 1.586 & 1.647 & 1.641 \\
\hline 1991 & 1.264 & 1.300 & 1.321 & 1.351 & 1.387 & 1.434 & 1.480 & 1.522 & 1.581 & 1.575 \\
\hline 1992 & 1.227 & 1.262 & 1.282 & 1.311 & 1.346 & 1.392 & 1.437 & 1.478 & 1.535 & 1.529 \\
\hline 1993 & 1.192 & 1.226 & 1.245 & 1.273 & 1.307 & 1.352 & 1.395 & 1.435 & 1.490 & 1.485 \\
\hline 1994 & 1.162 & 1.195 & 1.214 & 1.242 & 1.275 & 1.318 & 1.360 & 1.399 & 1.453 & 1.448 \\
\hline 1995 & 1.130 & 1.162 & 1.180 & 1.207 & 1.240 & 1.281 & 1.323 & 1.360 & 1.413 & 1.408 \\
\hline 1996 & 1.098 & 1.129 & 1.147 & 1.173 & 1.204 & 1.245 & 1.285 & 1.321 & 1.372 & 1.367 \\
\hline 1997 & 1.073 & 1.103 & 1.121 & 1.146 & 1.177 & 1.217 & 1.256 & 1.292 & 1.341 & 1.337 \\
\hline 1998 & 1.056 & 1.087 & 1.104 & 1.129 & 1.159 & 1.198 & 1.237 & 1.272 & 1.321 & 1.316 \\
\hline 1999 & 1.034 & 1.063 & 1.080 & 1.104 & 1.134 & 1.172 & 1.210 & 1.245 & 1.292 & 1.288 \\
\hline 2000 & 1.000 & 1.028 & 1.045 & 1.069 & 1.097 & 1.134 & 1.171 & 1.204 & 1.250 & 1.246 \\
\hline 2001 & 0.972 & 1.000 & 1.016 & 1.039 & 1.067 & 1.103 & 1.138 & 1.171 & 1.216 & 1.211 \\
\hline 2002 & 0.957 & 0.984 & 1.000 & 1.023 & 1.050 & 1.086 & 1.121 & 1.153 & 1.197 & 1.193 \\
\hline 2003 & 0.936 & 0.963 & 0.978 & 1.000 & 1.027 & 1.061 & 1.096 & 1.127 & 1.170 & 1.166 \\
\hline 2004 & 0.912 & 0.938 & 0.952 & 0.974 & 1.000 & 1.034 & 1.067 & 1.098 & 1.140 & 1.136 \\
\hline 2005 & 0.882 & 0.907 & 0.921 & 0.942 & 0.967 & 1.000 & 1.032 & 1.062 & 1.102 & 1.098 \\
\hline 2006 & 0.854 & 0.878 & 0.892 & 0.913 & 0.937 & 0.969 & 1.000 & 1.028 & 1.068 & 1.064 \\
\hline 2007 & 0.831 & 0.854 & 0.868 & 0.887 & 0.911 & 0.942 & 0.972 & 1.000 & 1.038 & 1.035 \\
\hline 2008 & 0.800 & 0.823 & 0.836 & 0.855 & 0.877 & 0.907 & 0.936 & 0.963 & 1.000 & 0.996 \\
\hline 2009 & 0.803 & 0.825 & 0.839 & 0.858 & 0.881 & 0.910 & 0.940 & 0.966 & 1.004 & 1.000 \\
\hline 2010 & 0.790 & 0.812 & 0.825 & 0.844 & 0.866 & 0.896 & 0.925 & 0.951 & 0.987 & 0.984 \\
\hline 2011 & 0.766 & 0.787 & 0.800 & 0.818 & 0.840 & 0.868 & 0.896 & 0.922 & 0.957 & 0.954 \\
\hline 2012 & 0.750 & 0.771 & 0.784 & 0.801 & 0.823 & 0.851 & 0.878 & 0.903 & 0.938 & 0.934 \\
\hline 2013 & 0.739 & 0.760 & 0.772 & 0.790 & 0.811 & 0.838 & 0.865 & 0.890 & 0.924 & 0.921 \\
\hline 2014 & 0.727 & 0.748 & 0.760 & 0.777 & 0.798 & 0.825 & 0.852 & 0.876 & 0.909 & 0.906 \\
\hline 2015 & 0.727 & 0.747 & 0.759 & 0.776 & 0.797 & 0.824 & 0.851 & 0.875 & 0.908 & 0.905 \\
\hline
\end{tabular}


Table B.17

Consumer Price Inflation (CPI) Index (Continued)

\begin{tabular}{|c|c|c|c|c|c|c|}
\hline From: & 2010 & 2011 & 2012 & 2013 & 2014 & 2015 \\
\hline 1970 & 5.620 & 5.797 & 5.917 & 6.004 & 6.101 & 6.109 \\
\hline 1971 & 5.384 & 5.554 & 5.669 & 5.752 & 5.845 & 5.852 \\
\hline 1972 & 5.217 & 5.381 & 5.493 & 5.573 & 5.664 & 5.670 \\
\hline 1973 & 4.911 & 5.066 & 5.171 & 5.247 & 5.332 & 5.338 \\
\hline 1974 & 4.423 & 4.563 & 4.657 & 4.725 & 4.802 & 4.808 \\
\hline 1975 & 4.053 & 4.181 & 4.268 & 4.330 & 4.400 & 4.406 \\
\hline 1976 & 3.832 & 3.953 & 4.035 & 4.094 & 4.161 & 4.166 \\
\hline 1977 & 3.598 & 3.712 & 3.789 & 3.844 & 3.907 & 3.911 \\
\hline 1978 & 3.344 & 3.450 & 3.521 & 3.573 & 3.631 & 3.635 \\
\hline 1979 & 3.004 & 3.098 & 3.162 & 3.209 & 3.261 & 3.265 \\
\hline 1980 & 2.646 & 2.730 & 2.786 & 2.827 & 2.873 & 2.876 \\
\hline 1981 & 2.399 & 2.475 & 2.526 & 2.563 & 2.604 & 2.607 \\
\hline 1982 & 2.260 & 2.331 & 2.379 & 2.414 & 2.453 & 2.456 \\
\hline 1983 & 2.189 & 2.258 & 2.305 & 2.339 & 2.377 & 2.380 \\
\hline 1984 & 2.099 & 2.165 & 2.210 & 2.242 & 2.278 & 2.281 \\
\hline 1985 & 2.027 & 2.091 & 2.134 & 2.165 & 2.200 & 2.203 \\
\hline 1986 & 1.990 & 2.052 & 2.095 & 2.126 & 2.160 & 2.163 \\
\hline 1987 & 1.920 & 1.980 & 2.021 & 2.051 & 2.084 & 2.086 \\
\hline 1988 & 1.843 & 1.901 & 1.941 & 1.969 & 2.001 & 2.004 \\
\hline 1989 & 1.759 & 1.814 & 1.852 & 1.879 & 1.909 & 1.911 \\
\hline 1990 & 1.668 & 1.721 & 1.757 & 1.782 & 1.811 & 1.813 \\
\hline 1991 & 1.601 & 1.652 & 1.686 & 1.710 & 1.738 & 1.740 \\
\hline 1992 & 1.554 & 1.603 & 1.636 & 1.660 & 1.687 & 1.689 \\
\hline 1993 & 1.509 & 1.557 & 1.589 & 1.612 & 1.638 & 1.640 \\
\hline 1994 & 1.471 & 1.518 & 1.549 & 1.572 & 1.597 & 1.599 \\
\hline 1995 & 1.431 & 1.476 & 1.507 & 1.529 & 1.553 & 1.555 \\
\hline 1996 & 1.390 & 1.434 & 1.463 & 1.485 & 1.509 & 1.511 \\
\hline 1997 & 1.359 & 1.401 & 1.430 & 1.451 & 1.475 & 1.477 \\
\hline 1998 & 1.338 & 1.380 & 1.409 & 1.429 & 1.452 & 1.454 \\
\hline 1999 & 1.309 & 1.350 & 1.378 & 1.398 & 1.421 & 1.423 \\
\hline 2000 & 1.266 & 1.306 & 1.333 & 1.353 & 1.375 & 1.376 \\
\hline 2001 & 1.231 & 1.270 & 1.296 & 1.315 & 1.337 & 1.338 \\
\hline 2002 & 1.212 & 1.250 & 1.276 & 1.295 & 1.316 & 1.317 \\
\hline 2003 & 1.185 & 1.222 & 1.248 & 1.266 & 1.287 & 1.288 \\
\hline 2004 & 1.154 & 1.191 & 1.215 & 1.233 & 1.253 & 1.255 \\
\hline 2005 & 1.117 & 1.152 & 1.176 & 1.193 & 1.212 & 1.214 \\
\hline 2006 & 1.082 & 1.116 & 1.139 & 1.156 & 1.174 & 1.176 \\
\hline 2007 & 1.052 & 1.085 & 1.107 & 1.124 & 1.142 & 1.143 \\
\hline 2008 & 1.013 & 1.045 & 1.066 & 1.082 & 1.100 & 1.101 \\
\hline 2009 & 1.016 & 1.048 & 1.070 & 1.086 & 1.103 & 1.105 \\
\hline 2010 & 1.000 & 1.032 & 1.053 & 1.068 & 1.086 & 1.087 \\
\hline 2011 & 0.969 & 1.000 & 1.021 & 1.036 & 1.052 & 1.054 \\
\hline 2012 & 0.950 & 0.980 & 1.000 & 1.015 & 1.031 & 1.032 \\
\hline 2013 & 0.936 & 0.966 & 0.986 & 1.000 & 1.016 & 1.017 \\
\hline 2014 & 0.921 & 0.950 & 0.970 & 0.984 & 1.000 & 1.001 \\
\hline 2015 & 0.920 & 0.949 & 0.969 & 0.983 & 0.999 & 1.000 \\
\hline
\end{tabular}

Source:

U.S. Bureau of Labor Statistics. 
Table B.18

Gross National Product Implicit Price Deflator

\begin{tabular}{|c|c|c|c|c|c|c|c|c|c|c|}
\hline From: & 1970 & 1971 & 1972 & 1973 & 1974 & 1975 & 1976 & 1977 & 1978 & 1979 \\
\hline 1970 & 1.000 & 1.051 & 1.097 & 1.156 & 1.260 & 1.377 & 1.453 & 1.543 & 1.651 & 1.788 \\
\hline 1971 & 0.952 & 1.000 & 1.043 & 1.100 & 1.199 & 1.310 & 1.382 & 1.468 & 1.571 & 1.701 \\
\hline 1972 & 0.912 & 0.958 & 1.000 & 1.055 & 1.149 & 1.256 & 1.325 & 1.407 & 1.506 & 1.630 \\
\hline 1973 & 0.865 & 0.909 & 0.948 & 1.000 & 1.090 & 1.191 & 1.256 & 1.334 & 1.428 & 1.546 \\
\hline 1974 & 0.794 & 0.834 & 0.870 & 0.918 & 1.000 & 1.093 & 1.153 & 1.224 & 1.311 & 1.419 \\
\hline 1975 & 0.726 & 0.763 & 0.796 & 0.840 & 0.915 & 1.000 & 1.055 & 1.121 & 1.199 & 1.298 \\
\hline 1976 & 0.688 & 0.723 & 0.755 & 0.796 & 0.867 & 0.948 & 1.000 & 1.062 & 1.137 & 1.231 \\
\hline 1977 & 0.648 & 0.681 & 0.711 & 0.749 & 0.817 & 0.892 & 0.942 & 1.000 & 1.070 & 1.159 \\
\hline 1978 & 0.606 & 0.636 & 0.664 & 0.700 & 0.763 & 0.834 & 0.880 & 0.934 & 1.000 & 1.083 \\
\hline 1979 & 0.559 & 0.588 & 0.613 & 0.647 & 0.705 & 0.770 & 0.813 & 0.863 & 0.924 & 1.000 \\
\hline 1980 & 0.513 & 0.539 & 0.563 & 0.593 & 0.647 & 0.707 & 0.745 & 0.792 & 0.847 & 0.917 \\
\hline 1981 & 0.469 & 0.493 & 0.515 & 0.543 & 0.591 & 0.646 & 0.682 & 0.724 & 0.775 & 0.839 \\
\hline 1982 & 0.442 & 0.464 & 0.485 & 0.511 & 0.557 & 0.608 & 0.642 & 0.682 & 0.730 & 0.790 \\
\hline 1983 & 0.425 & 0.447 & 0.466 & 0.492 & 0.536 & 0.585 & 0.617 & 0.656 & 0.702 & 0.760 \\
\hline 1984 & 0.411 & 0.431 & 0.450 & 0.475 & 0.517 & 0.565 & 0.596 & 0.633 & 0.678 & 0.734 \\
\hline 1985 & 0.398 & 0.418 & 0.436 & 0.460 & 0.501 & 0.548 & 0.578 & 0.614 & 0.657 & 0.711 \\
\hline 1986 & 0.390 & 0.410 & 0.428 & 0.451 & 0.491 & 0.537 & 0.566 & 0.602 & 0.644 & 0.697 \\
\hline 1987 & 0.380 & 0.399 & 0.417 & 0.440 & 0.479 & 0.523 & 0.552 & 0.586 & 0.628 & 0.679 \\
\hline 1988 & 0.367 & 0.386 & 0.403 & 0.425 & 0.463 & 0.506 & 0.533 & 0.567 & 0.606 & 0.656 \\
\hline 1989 & 0.353 & 0.371 & 0.388 & 0.409 & 0.445 & 0.487 & 0.513 & 0.545 & 0.584 & 0.632 \\
\hline 1990 & 0.341 & 0.358 & 0.374 & 0.394 & 0.429 & 0.469 & 0.495 & 0.526 & 0.563 & 0.609 \\
\hline 1991 & 0.330 & 0.346 & 0.362 & 0.381 & 0.415 & 0.454 & 0.479 & 0.509 & 0.544 & 0.589 \\
\hline 1992 & 0.322 & 0.339 & 0.353 & 0.373 & 0.406 & 0.444 & 0.468 & 0.497 & 0.532 & 0.576 \\
\hline 1993 & 0.315 & 0.331 & 0.345 & 0.364 & 0.397 & 0.434 & 0.457 & 0.486 & 0.520 & 0.563 \\
\hline 1994 & 0.308 & 0.324 & 0.338 & 0.357 & 0.389 & 0.425 & 0.448 & 0.476 & 0.509 & 0.551 \\
\hline 1995 & 0.302 & 0.317 & 0.331 & 0.349 & 0.381 & 0.416 & 0.439 & 0.466 & 0.499 & 0.540 \\
\hline 1996 & 0.297 & 0.312 & 0.325 & 0.343 & 0.374 & 0.408 & 0.431 & 0.458 & 0.490 & 0.530 \\
\hline 1997 & 0.292 & 0.306 & 0.320 & 0.337 & 0.367 & 0.401 & 0.424 & 0.450 & 0.481 & 0.521 \\
\hline 1998 & 0.288 & 0.303 & 0.316 & 0.334 & 0.363 & 0.397 & 0.419 & 0.445 & 0.476 & 0.516 \\
\hline 1999 & 0.284 & 0.299 & 0.312 & 0.329 & 0.358 & 0.392 & 0.413 & 0.439 & 0.470 & 0.508 \\
\hline 2000 & 0.278 & 0.292 & 0.305 & 0.322 & 0.350 & 0.383 & 0.404 & 0.429 & 0.459 & 0.497 \\
\hline 2001 & 0.272 & 0.286 & 0.298 & 0.314 & 0.343 & 0.374 & 0.395 & 0.419 & 0.449 & 0.486 \\
\hline 2002 & 0.268 & 0.281 & 0.294 & 0.310 & 0.337 & 0.369 & 0.389 & 0.413 & 0.442 & 0.479 \\
\hline 2003 & 0.262 & 0.276 & 0.288 & 0.303 & 0.331 & 0.361 & 0.381 & 0.405 & 0.433 & 0.469 \\
\hline 2004 & 0.255 & 0.268 & 0.280 & 0.295 & 0.322 & 0.352 & 0.371 & 0.394 & 0.422 & 0.457 \\
\hline 2005 & 0.248 & 0.260 & 0.271 & 0.286 & 0.312 & 0.341 & 0.360 & 0.382 & 0.409 & 0.442 \\
\hline 2006 & 0.240 & 0.252 & 0.263 & 0.278 & 0.303 & 0.331 & 0.349 & 0.370 & 0.397 & 0.429 \\
\hline 2007 & 0.234 & 0.246 & 0.256 & 0.270 & 0.295 & 0.322 & 0.340 & 0.361 & 0.386 & 0.418 \\
\hline 2008 & 0.229 & 0.241 & 0.252 & 0.265 & 0.289 & 0.316 & 0.333 & 0.354 & 0.379 & 0.410 \\
\hline 2009 & 0.228 & 0.239 & 0.250 & 0.263 & 0.287 & 0.313 & 0.331 & 0.351 & 0.376 & 0.407 \\
\hline 2010 & 0.225 & 0.236 & 0.246 & 0.260 & 0.283 & 0.309 & 0.326 & 0.347 & 0.371 & 0.402 \\
\hline 2011 & 0.220 & 0.232 & 0.242 & 0.255 & 0.278 & 0.303 & 0.320 & 0.340 & 0.364 & 0.394 \\
\hline 2012 & 0.217 & 0.228 & 0.237 & 0.250 & 0.273 & 0.298 & 0.315 & 0.334 & 0.358 & 0.387 \\
\hline 2013 & 0.213 & 0.224 & 0.234 & 0.247 & 0.269 & 0.294 & 0.310 & 0.329 & 0.352 & 0.381 \\
\hline 2014 & 0.210 & 0.220 & 0.230 & 0.243 & 0.264 & 0.289 & 0.305 & 0.324 & 0.346 & 0.375 \\
\hline 2015 & 0.207 & 0.217 & 0.226 & 0.239 & 0.260 & 0.284 & 0.300 & 0.319 & 0.341 & 0.369 \\
\hline 2016 & 0.204 & 0.214 & 0.224 & 0.236 & 0.257 & 0.281 & 0.296 & 0.315 & 0.337 & 0.365 \\
\hline
\end{tabular}


Table B.18

Gross National Product Implicit Price Deflator (Continued)

\begin{tabular}{|c|c|c|c|c|c|c|c|c|c|c|}
\hline From: & 1980 & 1981 & 1982 & 1983 & 1984 & 1985 & 1986 & 1987 & 1988 & 1989 \\
\hline 1970 & 1.949 & 2.131 & 2.263 & 2.353 & 2.436 & 2.514 & 2.565 & 2.631 & 2.723 & 2.830 \\
\hline 1971 & 1.854 & 2.028 & 2.153 & 2.239 & 2.318 & 2.392 & 2.441 & 2.504 & 2.591 & 2.693 \\
\hline 1972 & 1.777 & 1.943 & 2.064 & 2.145 & 2.222 & 2.293 & 2.339 & 2.399 & 2.484 & 2.580 \\
\hline 1973 & 1.685 & 1.843 & 1.957 & 2.034 & 2.107 & 2.174 & 2.218 & 2.275 & 2.355 & 2.447 \\
\hline 1974 & 1.547 & 1.691 & 1.796 & 1.867 & 1.933 & 1.995 & 2.036 & 2.088 & 2.161 & 2.246 \\
\hline 1975 & 1.415 & 1.548 & 1.644 & 1.709 & 1.769 & 1.826 & 1.863 & 1.911 & 1.978 & 2.055 \\
\hline 1976 & 1.341 & 1.467 & 1.558 & 1.619 & 1.677 & 1.731 & 1.766 & 1.811 & 1.875 & 1.948 \\
\hline 1977 & 1.263 & 1.381 & 1.467 & 1.525 & 1.579 & 1.629 & 1.662 & 1.705 & 1.765 & 1.834 \\
\hline 1978 & 1.180 & 1.290 & 1.370 & 1.425 & 1.475 & 1.522 & 1.553 & 1.593 & 1.649 & 1.714 \\
\hline 1979 & 1.090 & 1.192 & 1.266 & 1.316 & 1.363 & 1.406 & 1.435 & 1.472 & 1.523 & 1.583 \\
\hline 1980 & 1.000 & 1.093 & 1.161 & 1.207 & 1.250 & 1.290 & 1.316 & 1.350 & 1.398 & 1.452 \\
\hline 1981 & 0.915 & 1.000 & 1.062 & 1.104 & 1.143 & 1.180 & 1.204 & 1.235 & 1.278 & 1.328 \\
\hline 1982 & 0.861 & 0.942 & 1.000 & 1.040 & 1.076 & 1.111 & 1.133 & 1.163 & 1.203 & 1.250 \\
\hline 1983 & 0.828 & 0.906 & 0.962 & 1.000 & 1.035 & 1.069 & 1.090 & 1.118 & 1.158 & 1.203 \\
\hline 1984 & 0.800 & 0.875 & 0.929 & 0.966 & 1.000 & 1.032 & 1.053 & 1.080 & 1.118 & 1.162 \\
\hline 1985 & 0.775 & 0.848 & 0.900 & 0.936 & 0.969 & 1.000 & 1.020 & 1.047 & 1.083 & 1.126 \\
\hline 1986 & 0.760 & 0.831 & 0.882 & 0.917 & 0.950 & 0.980 & 1.000 & 1.026 & 1.062 & 1.103 \\
\hline 1987 & 0.741 & 0.810 & 0.860 & 0.894 & 0.926 & 0.956 & 0.975 & 1.000 & 1.035 & 1.075 \\
\hline 1988 & 0.716 & 0.782 & 0.831 & 0.864 & 0.895 & 0.923 & 0.942 & 0.966 & 1.000 & 1.039 \\
\hline 1989 & 0.689 & 0.753 & 0.800 & 0.831 & 0.861 & 0.888 & 0.906 & 0.930 & 0.962 & 1.000 \\
\hline 1990 & 0.664 & 0.726 & 0.771 & 0.802 & 0.830 & 0.857 & 0.874 & 0.897 & 0.928 & 0.964 \\
\hline 1991 & 0.643 & 0.703 & 0.746 & 0.776 & 0.803 & 0.829 & 0.846 & 0.867 & 0.898 & 0.933 \\
\hline 1992 & 0.628 & 0.687 & 0.730 & 0.758 & 0.785 & 0.810 & 0.827 & 0.848 & 0.878 & 0.912 \\
\hline 1993 & 0.614 & 0.671 & 0.713 & 0.741 & 0.767 & 0.792 & 0.808 & 0.828 & 0.858 & 0.891 \\
\hline 1994 & 0.601 & 0.657 & 0.698 & 0.725 & 0.751 & 0.775 & 0.791 & 0.811 & 0.840 & 0.872 \\
\hline 1995 & 0.588 & 0.644 & 0.683 & 0.710 & 0.736 & 0.759 & 0.775 & 0.795 & 0.822 & 0.855 \\
\hline 1996 & 0.578 & 0.632 & 0.671 & 0.698 & 0.722 & 0.746 & 0.761 & 0.780 & 0.808 & 0.839 \\
\hline 1997 & 0.568 & 0.621 & 0.660 & 0.686 & 0.710 & 0.733 & 0.748 & 0.767 & 0.794 & 0.825 \\
\hline 1998 & 0.562 & 0.615 & 0.653 & 0.679 & 0.703 & 0.725 & 0.740 & 0.759 & 0.786 & 0.816 \\
\hline 1999 & 0.554 & 0.606 & 0.644 & 0.669 & 0.693 & 0.715 & 0.729 & 0.748 & 0.774 & 0.805 \\
\hline 2000 & 0.542 & 0.592 & 0.629 & 0.654 & 0.677 & 0.699 & 0.713 & 0.732 & 0.757 & 0.787 \\
\hline 2001 & 0.530 & 0.579 & 0.615 & 0.639 & 0.662 & 0.683 & 0.697 & 0.715 & 0.740 & 0.769 \\
\hline 2002 & 0.522 & 0.570 & 0.606 & 0.630 & 0.652 & 0.673 & 0.687 & 0.704 & 0.729 & 0.757 \\
\hline 2003 & 0.511 & 0.559 & 0.594 & 0.617 & 0.639 & 0.660 & 0.673 & 0.691 & 0.715 & 0.743 \\
\hline 2004 & 0.498 & 0.544 & 0.578 & 0.601 & 0.622 & 0.642 & 0.655 & 0.672 & 0.696 & 0.723 \\
\hline 2005 & 0.482 & 0.527 & 0.560 & 0.582 & 0.603 & 0.622 & 0.635 & 0.651 & 0.674 & 0.700 \\
\hline 2006 & 0.468 & 0.512 & 0.543 & 0.565 & 0.585 & 0.604 & 0.616 & 0.632 & 0.654 & 0.679 \\
\hline 2007 & 0.456 & 0.498 & 0.529 & 0.550 & 0.570 & 0.588 & 0.600 & 0.615 & 0.637 & 0.662 \\
\hline 2008 & 0.447 & 0.489 & 0.519 & 0.540 & 0.559 & 0.577 & 0.588 & 0.604 & 0.625 & 0.649 \\
\hline 2009 & 0.444 & 0.485 & 0.515 & 0.536 & 0.555 & 0.572 & 0.584 & 0.599 & 0.620 & 0.644 \\
\hline 2010 & 0.438 & 0.479 & 0.508 & 0.529 & 0.547 & 0.565 & 0.576 & 0.591 & 0.612 & 0.636 \\
\hline 2011 & 0.429 & 0.469 & 0.499 & 0.518 & 0.537 & 0.554 & 0.565 & 0.580 & 0.600 & 0.623 \\
\hline 2012 & 0.422 & 0.461 & 0.490 & 0.509 & 0.527 & 0.544 & 0.555 & 0.570 & 0.590 & 0.613 \\
\hline 2013 & 0.416 & 0.455 & 0.483 & 0.502 & 0.520 & 0.536 & 0.547 & 0.561 & 0.581 & 0.604 \\
\hline 2014 & 0.409 & 0.447 & 0.475 & 0.493 & 0.511 & 0.527 & 0.538 & 0.552 & 0.571 & 0.593 \\
\hline 2015 & 0.402 & 0.440 & 0.467 & 0.486 & 0.503 & 0.519 & 0.530 & 0.543 & 0.562 & 0.584 \\
\hline 2016 & 0.397 & 0.435 & 0.461 & 0.480 & 0.497 & 0.513 & 0.523 & 0.537 & 0.555 & 0.577 \\
\hline
\end{tabular}


Table B.18

Gross National Product Implicit Price Deflator (Continued)

\begin{tabular}{|c|c|c|c|c|c|c|c|c|c|c|}
\hline From: & 1990 & 1991 & 1992 & 1993 & 1994 & 1995 & 1996 & 1997 & 1998 & 1999 \\
\hline 1970 & 2.935 & 3.033 & 3.102 & 3.176 & 3.243 & 3.311 & 3.372 & 3.429 & 3.467 & 3.517 \\
\hline 1971 & 2.793 & 2.886 & 2.952 & 3.022 & 3.086 & 3.151 & 3.209 & 3.263 & 3.299 & 3.346 \\
\hline 1972 & 2.676 & 2.766 & 2.829 & 2.896 & 2.958 & 3.020 & 3.075 & 3.128 & 3.162 & 3.207 \\
\hline 1973 & 2.538 & 2.623 & 2.683 & 2.746 & 2.805 & 2.863 & 2.916 & 2.966 & 2.998 & 3.041 \\
\hline 1974 & 2.329 & 2.407 & 2.462 & 2.520 & 2.574 & 2.628 & 2.676 & 2.722 & 2.751 & 2.791 \\
\hline 1975 & 2.131 & 2.203 & 2.253 & 2.307 & 2.356 & 2.405 & 2.449 & 2.491 & 2.518 & 2.554 \\
\hline 1976 & 2.020 & 2.088 & 2.135 & 2.186 & 2.233 & 2.279 & 2.321 & 2.361 & 2.387 & 2.421 \\
\hline 1977 & 1.902 & 1.966 & 2.011 & 2.058 & 2.102 & 2.146 & 2.185 & 2.223 & 2.247 & 2.279 \\
\hline 1978 & 1.777 & 1.837 & 1.879 & 1.923 & 1.964 & 2.005 & 2.042 & 2.077 & 2.099 & 2.130 \\
\hline 1979 & 1.642 & 1.697 & 1.735 & 1.776 & 1.814 & 1.852 & 1.886 & 1.918 & 1.939 & 1.967 \\
\hline 1980 & 1.506 & 1.556 & 1.592 & 1.630 & 1.664 & 1.699 & 1.730 & 1.760 & 1.779 & 1.805 \\
\hline 1981 & 1.377 & 1.423 & 1.456 & 1.490 & 1.522 & 1.554 & 1.582 & 1.609 & 1.627 & 1.650 \\
\hline 1982 & 1.297 & 1.340 & 1.371 & 1.403 & 1.433 & 1.463 & 1.490 & 1.516 & 1.532 & 1.554 \\
\hline 1983 & 1.247 & 1.289 & 1.319 & 1.350 & 1.379 & 1.408 & 1.433 & 1.458 & 1.474 & 1.495 \\
\hline 1984 & 1.205 & 1.245 & 1.273 & 1.304 & 1.331 & 1.359 & 1.384 & 1.408 & 1.423 & 1.444 \\
\hline 1985 & 1.167 & 1.206 & 1.234 & 1.263 & 1.290 & 1.317 & 1.341 & 1.364 & 1.379 & 1.399 \\
\hline 1986 & 1.144 & 1.182 & 1.209 & 1.238 & 1.264 & 1.291 & 1.315 & 1.337 & 1.352 & 1.371 \\
\hline 1987 & 1.115 & 1.153 & 1.179 & 1.207 & 1.233 & 1.259 & 1.282 & 1.303 & 1.318 & 1.337 \\
\hline 1988 & 1.078 & 1.114 & 1.139 & 1.166 & 1.191 & 1.216 & 1.238 & 1.259 & 1.273 & 1.291 \\
\hline 1989 & 1.037 & 1.072 & 1.096 & 1.122 & 1.146 & 1.170 & 1.192 & 1.212 & 1.225 & 1.243 \\
\hline 1990 & 1.000 & 1.033 & 1.057 & 1.082 & 1.105 & 1.128 & 1.149 & 1.169 & 1.181 & 1.198 \\
\hline 1991 & 0.968 & 1.000 & 1.023 & 1.047 & 1.069 & 1.092 & 1.112 & 1.131 & 1.143 & 1.159 \\
\hline 1992 & 0.946 & 0.978 & 1.000 & 1.024 & 1.046 & 1.067 & 1.087 & 1.106 & 1.118 & 1.134 \\
\hline 1993 & 0.924 & 0.955 & 0.977 & 1.000 & 1.021 & 1.043 & 1.062 & 1.080 & 1.092 & 1.107 \\
\hline 1994 & 0.905 & 0.935 & 0.956 & 0.979 & 1.000 & 1.021 & 1.040 & 1.057 & 1.069 & 1.084 \\
\hline 1995 & 0.886 & 0.916 & 0.937 & 0.959 & 0.979 & 1.000 & 1.018 & 1.036 & 1.047 & 1.062 \\
\hline 1996 & 0.870 & 0.899 & 0.920 & 0.942 & 0.962 & 0.982 & 1.000 & 1.017 & 1.028 & 1.043 \\
\hline 1997 & 0.856 & 0.884 & 0.905 & 0.926 & 0.946 & 0.966 & 0.983 & 1.000 & 1.011 & 1.025 \\
\hline 1998 & 0.847 & 0.875 & 0.895 & 0.916 & 0.936 & 0.955 & 0.973 & 0.989 & 1.000 & 1.014 \\
\hline 1999 & 0.835 & 0.862 & 0.882 & 0.903 & 0.922 & 0.942 & 0.959 & 0.975 & 0.986 & 1.000 \\
\hline 2000 & 0.816 & 0.843 & 0.863 & 0.883 & 0.902 & 0.921 & 0.938 & 0.954 & 0.964 & 0.978 \\
\hline 2001 & 0.798 & 0.824 & 0.843 & 0.863 & 0.882 & 0.900 & 0.917 & 0.932 & 0.942 & 0.956 \\
\hline 2002 & 0.786 & 0.812 & 0.830 & 0.850 & 0.868 & 0.886 & 0.903 & 0.918 & 0.928 & 0.941 \\
\hline 2003 & 0.770 & 0.796 & 0.814 & 0.834 & 0.851 & 0.869 & 0.885 & 0.900 & 0.910 & 0.923 \\
\hline 2004 & 0.750 & 0.775 & 0.792 & 0.811 & 0.829 & 0.846 & 0.861 & 0.876 & 0.886 & 0.898 \\
\hline 2005 & 0.726 & 0.751 & 0.768 & 0.786 & 0.803 & 0.820 & 0.835 & 0.849 & 0.858 & 0.870 \\
\hline 2006 & 0.705 & 0.728 & 0.745 & 0.763 & 0.779 & 0.795 & 0.810 & 0.824 & 0.832 & 0.844 \\
\hline 2007 & 0.686 & 0.709 & 0.726 & 0.743 & 0.759 & 0.775 & 0.789 & 0.802 & 0.811 & 0.823 \\
\hline 2008 & 0.673 & 0.696 & 0.712 & 0.729 & 0.744 & 0.760 & 0.773 & 0.787 & 0.795 & 0.807 \\
\hline 2009 & 0.668 & 0.690 & 0.706 & 0.723 & 0.738 & 0.754 & 0.768 & 0.781 & 0.789 & 0.800 \\
\hline 2010 & 0.659 & 0.681 & 0.697 & 0.713 & 0.729 & 0.744 & 0.758 & 0.770 & 0.779 & 0.790 \\
\hline 2011 & 0.647 & 0.668 & 0.683 & 0.700 & 0.715 & 0.729 & 0.743 & 0.756 & 0.764 & 0.775 \\
\hline 2012 & 0.635 & 0.657 & 0.672 & 0.688 & 0.702 & 0.717 & 0.730 & 0.743 & 0.751 & 0.761 \\
\hline 2013 & 0.626 & 0.647 & 0.662 & 0.677 & 0.692 & 0.706 & 0.719 & 0.732 & 0.740 & 0.750 \\
\hline 2014 & 0.616 & 0.636 & 0.651 & 0.666 & 0.680 & 0.695 & 0.707 & 0.719 & 0.727 & 0.738 \\
\hline 2015 & 0.606 & 0.626 & 0.641 & 0.656 & 0.670 & 0.684 & 0.696 & 0.708 & 0.716 & 0.727 \\
\hline 2016 & 0.598 & 0.618 & 0.633 & 0.648 & 0.661 & 0.675 & 0.688 & 0.699 & 0.707 & 0.718 \\
\hline
\end{tabular}


Table B.18

Gross National Product Implicit Price Deflator (Continued)

\begin{tabular}{|c|c|c|c|c|c|c|c|c|c|c|}
\hline From: & 2000 & 2001 & 2002 & 2003 & 2004 & 2005 & 2006 & 2007 & 2008 & 2009 \\
\hline 1970 & 3.596 & 3.679 & 3.735 & 3.810 & 3.915 & 4.040 & 4.164 & 4.275 & 4.359 & 4.393 \\
\hline 1971 & 3.422 & 3.501 & 3.555 & 3.626 & 3.725 & 3.845 & 3.963 & 4.068 & 4.148 & 4.180 \\
\hline 1972 & 3.280 & 3.355 & 3.407 & 3.475 & 3.570 & 3.685 & 3.798 & 3.899 & 3.976 & 4.006 \\
\hline 1973 & 3.110 & 3.181 & 3.230 & 3.295 & 3.385 & 3.494 & 3.601 & 3.697 & 3.770 & 3.799 \\
\hline 1974 & 2.854 & 2.920 & 2.965 & 3.024 & 3.107 & 3.206 & 3.305 & 3.393 & 3.460 & 3.487 \\
\hline 1975 & 2.612 & 2.672 & 2.713 & 2.767 & 2.843 & 2.934 & 3.025 & 3.105 & 3.166 & 3.191 \\
\hline 1976 & 2.476 & 2.532 & 2.571 & 2.623 & 2.695 & 2.781 & 2.867 & 2.943 & 3.001 & 3.024 \\
\hline 1977 & 2.331 & 2.384 & 2.421 & 2.470 & 2.537 & 2.619 & 2.699 & 2.771 & 2.825 & 2.847 \\
\hline 1978 & 2.178 & 2.228 & 2.262 & 2.307 & 2.371 & 2.447 & 2.522 & 2.589 & 2.640 & 2.660 \\
\hline 1979 & 2.012 & 2.058 & 2.090 & 2.131 & 2.190 & 2.260 & 2.330 & 2.392 & 2.439 & 2.457 \\
\hline 1980 & 1.846 & 1.888 & 1.917 & 1.955 & 2.009 & 2.073 & 2.137 & 2.194 & 2.237 & 2.254 \\
\hline 1981 & 1.688 & 1.726 & 1.753 & 1.788 & 1.837 & 1.896 & 1.954 & 2.006 & 2.046 & 2.062 \\
\hline 1982 & 1.589 & 1.626 & 1.651 & 1.684 & 1.730 & 1.785 & 1.840 & 1.889 & 1.926 & 1.941 \\
\hline 1983 & 1.529 & 1.564 & 1.588 & 1.620 & 1.664 & 1.717 & 1.770 & 1.817 & 1.853 & 1.867 \\
\hline 1984 & 1.476 & 1.510 & 1.533 & 1.564 & 1.607 & 1.659 & 1.710 & 1.755 & 1.789 & 1.803 \\
\hline 1985 & 1.431 & 1.463 & 1.486 & 1.516 & 1.557 & 1.607 & 1.657 & 1.701 & 1.734 & 1.747 \\
\hline 1986 & 1.402 & 1.434 & 1.456 & 1.485 & 1.526 & 1.575 & 1.624 & 1.667 & 1.700 & 1.713 \\
\hline 1987 & 1.367 & 1.398 & 1.420 & 1.448 & 1.488 & 1.536 & 1.583 & 1.625 & 1.657 & 1.670 \\
\hline 1988 & 1.321 & 1.351 & 1.372 & 1.399 & 1.437 & 1.484 & 1.529 & 1.570 & 1.601 & 1.613 \\
\hline 1989 & 1.271 & 1.300 & 1.320 & 1.347 & 1.383 & 1.428 & 1.472 & 1.511 & 1.541 & 1.553 \\
\hline 1990 & 1.225 & 1.254 & 1.273 & 1.298 & 1.334 & 1.377 & 1.419 & 1.457 & 1.485 & 1.497 \\
\hline 1991 & 1.186 & 1.213 & 1.232 & 1.256 & 1.291 & 1.332 & 1.373 & 1.410 & 1.437 & 1.448 \\
\hline 1992 & 1.159 & 1.186 & 1.204 & 1.228 & 1.262 & 1.302 & 1.343 & 1.378 & 1.405 & 1.416 \\
\hline 1993 & 1.132 & 1.158 & 1.176 & 1.200 & 1.233 & 1.272 & 1.311 & 1.346 & 1.373 & 1.383 \\
\hline 1994 & 1.109 & 1.134 & 1.152 & 1.175 & 1.207 & 1.246 & 1.284 & 1.318 & 1.344 & 1.355 \\
\hline 1995 & 1.086 & 1.111 & 1.128 & 1.151 & 1.182 & 1.220 & 1.258 & 1.291 & 1.317 & 1.327 \\
\hline 1996 & 1.067 & 1.091 & 1.108 & 1.130 & 1.161 & 1.198 & 1.235 & 1.268 & 1.293 & 1.303 \\
\hline 1997 & 1.049 & 1.073 & 1.089 & 1.111 & 1.141 & 1.178 & 1.214 & 1.247 & 1.271 & 1.281 \\
\hline 1998 & 1.037 & 1.061 & 1.077 & 1.099 & 1.129 & 1.165 & 1.201 & 1.233 & 1.257 & 1.267 \\
\hline 1999 & 1.023 & 1.046 & 1.062 & 1.083 & 1.113 & 1.149 & 1.184 & 1.216 & 1.240 & 1.249 \\
\hline 2000 & 1.000 & 1.023 & 1.039 & 1.059 & 1.088 & 1.123 & 1.158 & 1.189 & 1.212 & 1.222 \\
\hline 2001 & 0.978 & 1.000 & 1.015 & 1.036 & 1.064 & 1.098 & 1.132 & 1.162 & 1.185 & 1.194 \\
\hline 2002 & 0.963 & 0.985 & 1.000 & 1.020 & 1.048 & 1.082 & 1.115 & 1.145 & 1.167 & 1.176 \\
\hline 2003 & 0.944 & 0.966 & 0.980 & 1.000 & 1.027 & 1.060 & 1.093 & 1.122 & 1.144 & 1.153 \\
\hline 2004 & 0.919 & 0.940 & 0.954 & 0.973 & 1.000 & 1.032 & 1.064 & 1.092 & 1.114 & 1.122 \\
\hline 2005 & 0.890 & 0.911 & 0.925 & 0.943 & 0.969 & 1.000 & 1.031 & 1.058 & 1.079 & 1.087 \\
\hline 2006 & 0.864 & 0.883 & 0.897 & 0.915 & 0.940 & 0.970 & 1.000 & 1.027 & 1.047 & 1.055 \\
\hline 2007 & 0.841 & 0.861 & 0.874 & 0.891 & 0.916 & 0.945 & 0.974 & 1.000 & 1.020 & 1.028 \\
\hline 2008 & 0.825 & 0.844 & 0.857 & 0.874 & 0.898 & 0.927 & 0.955 & 0.981 & 1.000 & 1.008 \\
\hline 2009 & 0.819 & 0.837 & 0.850 & 0.867 & 0.891 & 0.920 & 0.948 & 0.973 & 0.992 & 1.000 \\
\hline 2010 & 0.808 & 0.826 & 0.839 & 0.856 & 0.879 & 0.908 & 0.936 & 0.960 & 0.979 & 0.987 \\
\hline 2011 & 0.792 & 0.810 & 0.823 & 0.839 & 0.862 & 0.890 & 0.917 & 0.942 & 0.960 & 0.968 \\
\hline 2012 & 0.779 & 0.797 & 0.809 & 0.825 & 0.848 & 0.875 & 0.902 & 0.926 & 0.944 & 0.951 \\
\hline 2013 & 0.767 & 0.785 & 0.797 & 0.813 & 0.835 & 0.862 & 0.888 & 0.912 & 0.930 & 0.937 \\
\hline 2014 & 0.755 & 0.772 & 0.784 & 0.800 & 0.822 & 0.848 & 0.874 & 0.898 & 0.915 & 0.922 \\
\hline 2015 & 0.744 & 0.761 & 0.772 & 0.788 & 0.809 & 0.835 & 0.861 & 0.884 & 0.901 & 0.908 \\
\hline 2016 & 0.734 & 0.751 & 0.762 & 0.778 & 0.799 & 0.825 & 0.850 & 0.873 & 0.890 & 0.897 \\
\hline
\end{tabular}


Table B.18

Gross National Product Implicit Price Deflator (Continued)

\begin{tabular}{|c|c|c|c|c|c|c|c|}
\hline From: & 2010 & 2011 & 2012 & 2013 & 2014 & 2015 & 2016 \\
\hline 1970 & 4.451 & 4.539 & 4.618 & 4.688 & 4.767 & 4.841 & 4.903 \\
\hline 1971 & 4.236 & 4.319 & 4.395 & 4.461 & 4.536 & 4.607 & 4.666 \\
\hline 1972 & 4.059 & 4.140 & 4.212 & 4.275 & 4.348 & 4.415 & 4.472 \\
\hline 1973 & 3.849 & 3.925 & 3.994 & 4.054 & 4.123 & 4.187 & 4.241 \\
\hline 1974 & 3.533 & 3.602 & 3.665 & 3.720 & 3.784 & 3.842 & 3.892 \\
\hline 1975 & 3.233 & 3.297 & 3.354 & 3.405 & 3.463 & 3.516 & 3.562 \\
\hline 1976 & 3.064 & 3.125 & 3.179 & 3.227 & 3.282 & 3.333 & 3.376 \\
\hline 1977 & 2.885 & 2.942 & 2.993 & 3.038 & 3.090 & 3.138 & 3.179 \\
\hline 1978 & 2.696 & 2.749 & 2.797 & 2.839 & 2.887 & 2.932 & 2.970 \\
\hline 1979 & 2.490 & 2.539 & 2.583 & 2.622 & 2.667 & 2.708 & 2.743 \\
\hline 1980 & 2.284 & 2.329 & 2.370 & 2.406 & 2.447 & 2.485 & 2.517 \\
\hline 1981 & 2.089 & 2.130 & 2.167 & 2.200 & 2.237 & 2.272 & 2.301 \\
\hline 1982 & 1.967 & 2.006 & 2.041 & 2.072 & 2.107 & 2.139 & 2.167 \\
\hline 1983 & 1.892 & 1.929 & 1.963 & 1.993 & 2.027 & 2.058 & 2.085 \\
\hline 1984 & 1.827 & 1.863 & 1.896 & 1.924 & 1.957 & 1.987 & 2.013 \\
\hline 1985 & 1.771 & 1.806 & 1.837 & 1.865 & 1.896 & 1.926 & 1.951 \\
\hline 1986 & 1.735 & 1.770 & 1.801 & 1.828 & 1.859 & 1.888 & 1.912 \\
\hline 1987 & 1.692 & 1.725 & 1.755 & 1.782 & 1.812 & 1.840 & 1.864 \\
\hline 1988 & 1.634 & 1.667 & 1.696 & 1.721 & 1.751 & 1.778 & 1.801 \\
\hline 1989 & 1.573 & 1.604 & 1.632 & 1.657 & 1.685 & 1.711 & 1.733 \\
\hline 1990 & 1.517 & 1.547 & 1.574 & 1.597 & 1.625 & 1.650 & 1.671 \\
\hline 1991 & 1.468 & 1.497 & 1.523 & 1.546 & 1.572 & 1.596 & 1.617 \\
\hline 1992 & 1.435 & 1.463 & 1.489 & 1.511 & 1.537 & 1.561 & 1.581 \\
\hline 1993 & 1.402 & 1.429 & 1.454 & 1.476 & 1.501 & 1.525 & 1.544 \\
\hline 1994 & 1.372 & 1.400 & 1.424 & 1.445 & 1.470 & 1.493 & 1.512 \\
\hline 1995 & 1.344 & 1.371 & 1.395 & 1.416 & 1.440 & 1.462 & 1.481 \\
\hline 1996 & 1.320 & 1.346 & 1.370 & 1.390 & 1.414 & 1.436 & 1.454 \\
\hline 1997 & 1.298 & 1.324 & 1.347 & 1.367 & 1.390 & 1.412 & 1.430 \\
\hline 1998 & 1.284 & 1.309 & 1.332 & 1.352 & 1.375 & 1.397 & 1.415 \\
\hline 1999 & 1.266 & 1.291 & 1.313 & 1.333 & 1.354 & 1.375 & 1.393 \\
\hline 2000 & 1.238 & 1.262 & 1.284 & 1.303 & 1.324 & 1.345 & 1.362 \\
\hline 2001 & 1.210 & 1.234 & 1.255 & 1.274 & 1.295 & 1.315 & 1.332 \\
\hline 2002 & 1.192 & 1.215 & 1.236 & 1.255 & 1.275 & 1.295 & 1.312 \\
\hline 2003 & 1.168 & 1.191 & 1.212 & 1.230 & 1.250 & 1.270 & 1.286 \\
\hline 2004 & 1.137 & 1.160 & 1.180 & 1.198 & 1.217 & 1.236 & 1.252 \\
\hline 2005 & 1.102 & 1.123 & 1.143 & 1.160 & 1.179 & 1.197 & 1.213 \\
\hline 2006 & 1.069 & 1.090 & 1.109 & 1.126 & 1.144 & 1.161 & 1.176 \\
\hline 2007 & 1.041 & 1.062 & 1.080 & 1.097 & 1.114 & 1.131 & 1.146 \\
\hline 2008 & 1.021 & 1.041 & 1.059 & 1.075 & 1.092 & 1.109 & 1.124 \\
\hline 2009 & 1.013 & 1.033 & 1.051 & 1.067 & 1.084 & 1.101 & 1.115 \\
\hline 2010 & 1.000 & 1.020 & 1.038 & 1.053 & 1.070 & 1.086 & 1.100 \\
\hline 2011 & 0.981 & 1.000 & 1.017 & 1.033 & 1.048 & 1.064 & 1.078 \\
\hline 2012 & 0.964 & 0.983 & 1.000 & 1.015 & 1.030 & 1.045 & 1.059 \\
\hline 2013 & 0.949 & 0.968 & 0.985 & 1.000 & 1.015 & 1.029 & 1.042 \\
\hline 2014 & 0.935 & 0.954 & 0.971 & 0.986 & 1.000 & 1.010 & 1.024 \\
\hline 2015 & 0.920 & 0.940 & 0.957 & 0.972 & 0.990 & 1.000 & 1.013 \\
\hline 2016 & 0.909 & 0.928 & 0.945 & 0.960 & 0.977 & 0.987 & 1.000 \\
\hline
\end{tabular}

Source:

U.S. Department of Commerce, Bureau of Economic Analysis, Survey of Current Business, Washington, DC, monthly. 


\section{APPENDIX C}

MAPS 
C-2

TRANSPORTATION ENERGY DATA BOOK: EDITION 36-2017 
Table C.1

Census Regions and Divisions

\begin{tabular}{|c|c|c|c|}
\hline \multicolumn{4}{|c|}{ Northeast Region } \\
\hline \multicolumn{2}{|c|}{ Mid-Atlantic division } & \multicolumn{2}{|c|}{ New England division } \\
\hline $\begin{array}{l}\text { New Jersey } \\
\text { New York }\end{array}$ & Pennsylvania & $\begin{array}{l}\text { Connecticut } \\
\text { Maine } \\
\text { Massachusetts }\end{array}$ & $\begin{array}{l}\text { New Hampshire } \\
\text { Rhode Island } \\
\text { Vermont }\end{array}$ \\
\hline \multicolumn{4}{|c|}{ South Region } \\
\hline $\begin{array}{l}\text { West South Central } \\
\text { division }\end{array}$ & $\begin{array}{l}\text { East South Central } \\
\text { division }\end{array}$ & \multicolumn{2}{|c|}{$\begin{array}{l}\text { South Atlantic } \\
\text { division }\end{array}$} \\
\hline $\begin{array}{l}\text { Arkansas } \\
\text { Louisiana } \\
\text { Oklahoma } \\
\text { Texas }\end{array}$ & $\begin{array}{l}\text { Alabama } \\
\text { Kentucky } \\
\text { Mississippi } \\
\text { Tennessee }\end{array}$ & $\begin{array}{l}\text { Delaware } \\
\text { Florida } \\
\text { Georgia } \\
\text { Maryland } \\
\text { North Carolina }\end{array}$ & $\begin{array}{l}\text { South Carolina } \\
\text { Virginia } \\
\text { Washington, DC } \\
\text { West Virginia }\end{array}$ \\
\hline \multicolumn{4}{|c|}{ West Region } \\
\hline \multicolumn{2}{|c|}{ Pacific division } & \multicolumn{2}{|c|}{ Mountain division } \\
\hline $\begin{array}{l}\text { Alaska } \\
\text { California } \\
\text { Hawaii }\end{array}$ & $\begin{array}{l}\text { Oregon } \\
\text { Washington }\end{array}$ & $\begin{array}{l}\text { Arizona } \\
\text { Colorado } \\
\text { Idaho } \\
\text { Montana }\end{array}$ & $\begin{array}{l}\text { Nevada } \\
\text { New Mexico } \\
\text { Utah } \\
\text { Wyoming }\end{array}$ \\
\hline \multicolumn{4}{|c|}{ Midwest Region } \\
\hline \multicolumn{2}{|c|}{ West North Central division } & \multicolumn{2}{|c|}{ East North Central division } \\
\hline $\begin{array}{l}\text { Iowa } \\
\text { Kansas } \\
\text { Minnesota } \\
\text { Missouri }\end{array}$ & $\begin{array}{l}\text { Nebraska } \\
\text { North Dakota } \\
\text { South Dakota }\end{array}$ & $\begin{array}{l}\text { Illinois } \\
\text { Indiana } \\
\text { Michigan }\end{array}$ & $\begin{array}{l}\text { Ohio } \\
\text { Wisconsin }\end{array}$ \\
\hline
\end{tabular}

Source:

U.S. Census Bureau. 
Figure C.1. Census Regions and Divisions

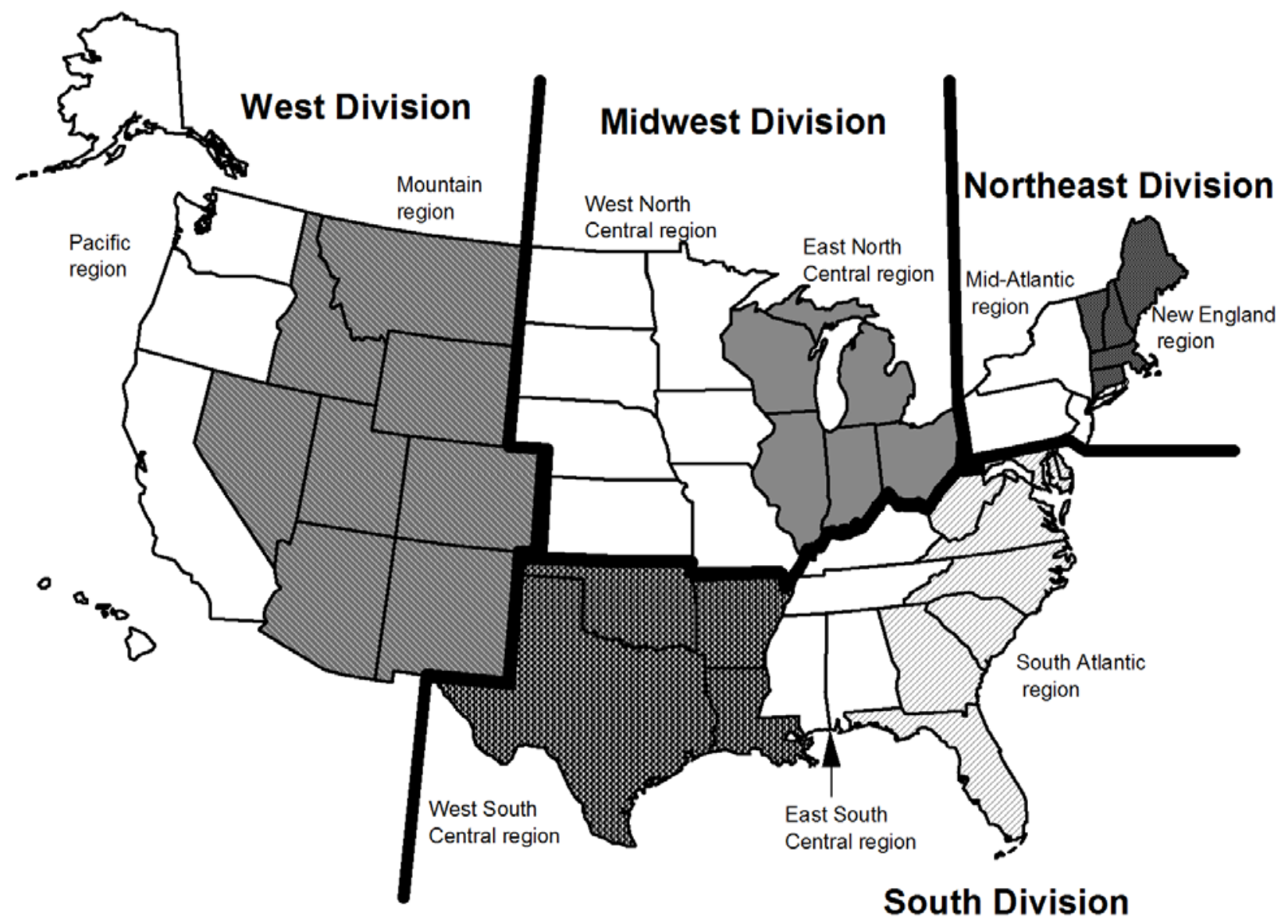

Source:

See Table C.1. 
Table C.2

Petroleum Administration for Defense Districts (PADD)

\begin{tabular}{|l|l|l|}
\hline District & Subdistrict & States \\
\hline $\begin{array}{l}\text { PAD District 1 } \\
\text { East Coast }\end{array}$ & $\begin{array}{l}\text { Subdistrict 1A } \\
\text { New England }\end{array}$ & $\begin{array}{l}\text { Connecticut, Maine, Massachusetts, New } \\
\text { Hampshire, Rhode Island, Vermont }\end{array}$ \\
\hline $\begin{array}{l}\text { Subdistrict 1B } \\
\text { Central Atlantic }\end{array}$ & $\begin{array}{l}\text { Delaware, District of Columbia, Maryland, New } \\
\text { Jersey, New York, Pennsylvania }\end{array}$ \\
\hline $\begin{array}{l}\text { MAD District 2 } \\
\text { Midwest }\end{array}$ & Lower Atlantic & $\begin{array}{l}\text { Florida, Georgia, North Carolina, South Carolina, } \\
\text { Virginia, West Virginia }\end{array}$ \\
\hline $\begin{array}{l}\text { PAD District 3 } \\
\text { Gulf Coast }\end{array}$ & $\begin{array}{l}\text { Illinois, Indiana, Iowa, Kansas, Kentucky, } \\
\text { Michigan, Minnesota, Missouri, Nebraska, North } \\
\text { Dakota, South Dakota, Ohio, Oklahoma, } \\
\text { Tennessee, Wisconsin }\end{array}$ \\
\hline $\begin{array}{l}\text { PAD District 4 } \\
\text { Rocky Mountains }\end{array}$ & & $\begin{array}{l}\text { Alabama, Arkansas, Louisiana, Mississippi, New } \\
\text { Mexico, Texas }\end{array}$ \\
\hline $\begin{array}{l}\text { PAD District 5 } \\
\text { West Coast }\end{array}$ & Colorado Idaho, Montana, Utah, Wyoming \\
\hline
\end{tabular}

\section{Source:}

Energy Information Administration web site:

http://www.eia.gov/tools/glossary/index.cfm?id=P\#PADD_def 
Figure C.2. Petroleum Administration for Defense Districts

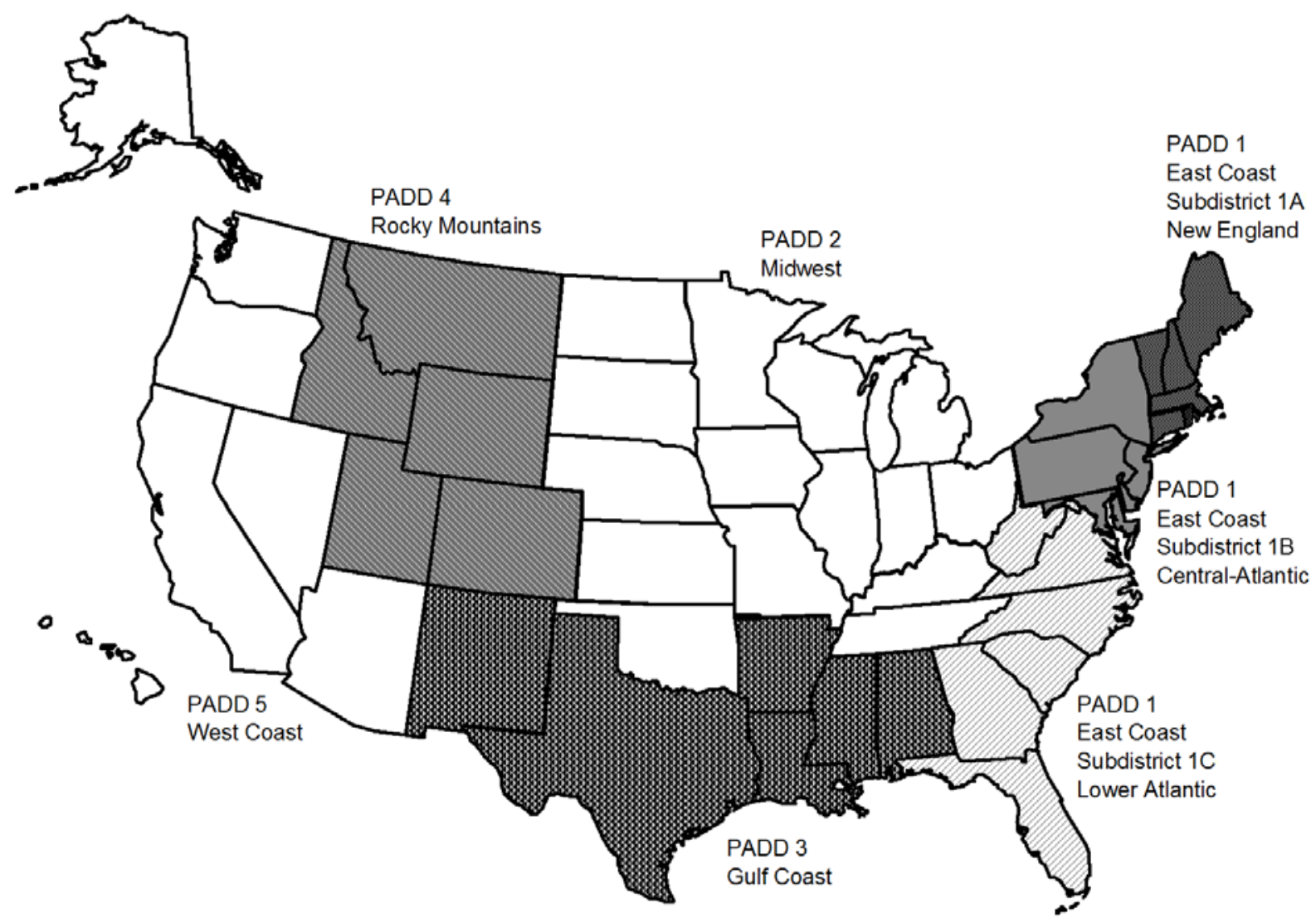

Source:

See Table C.2. 
Table C.3. Counties Where Reformulated Gasoline is Sold

\begin{tabular}{|c|c|c|}
\hline \multicolumn{3}{|c|}{ Reformulated Gasoline (RFG) used in entire county } \\
\hline \multicolumn{3}{|c|}{ California } \\
\hline Fresno County & Orange County & Stanislaus County \\
\hline Kings County & Sacramento County & Tulare County \\
\hline Los Angeles County & San Diego County & Ventura County \\
\hline Madera County & San Joaquin & Yolo County \\
\hline Merced County & & \\
\hline \multicolumn{3}{|c|}{ Connecticut } \\
\hline Fairfield County & Windham County & \\
\hline \multicolumn{3}{|c|}{ Delaware } \\
\hline New Castle County & Kent County & Sussex County \\
\hline \multicolumn{3}{|c|}{ Illinois } \\
\hline Cook County & Lake County & Monroe County \\
\hline Du Page County & McHenry County & St. Clair County \\
\hline Jersey County & Madison County & Will County \\
\hline Kane County & & \\
\hline \multicolumn{3}{|c|}{ Indiana } \\
\hline Lake County & Porter County & \\
\hline \multicolumn{3}{|c|}{ Kentucky } \\
\hline Boone County & Jefferson County & Kenton County \\
\hline Campbell County & & \\
\hline \multicolumn{3}{|c|}{ Maine } \\
\hline Androscoggin County & Knox County & Sagadahoc County \\
\hline Cumberland County & Lincoln County & York County \\
\hline Kennebec County & & \\
\hline \multicolumn{3}{|c|}{ Maryland } \\
\hline Anne Arundel County & Cecil County & Montgomery County \\
\hline Baltimore County & Frederick County & Prince George's County \\
\hline Calvert County & Harford County & The City of Baltimore \\
\hline Carroll County & Howard County & Queen Anne's County \\
\hline Charles County & Kent County & \\
\hline \multicolumn{3}{|c|}{ Massachusetts } \\
\hline Barnstable County & Franklin County & Norfolk County \\
\hline Berkshire County & Hampden County & Plymouth County \\
\hline Bristol County & Hampshire County & Suffolk County \\
\hline Dukes County & Middlesex County & Worcester County \\
\hline Essex County & Nantucket County & \\
\hline \multicolumn{3}{|c|}{ Missouri } \\
\hline Franklin County & St. Louis County & St. Charles County \\
\hline Jefferson County & St Louis (city) & \\
\hline \multicolumn{3}{|c|}{ New Hampshire } \\
\hline Hillsborough County & Merrimack County & Strafford County \\
\hline Rockingham County & & \\
\hline \multicolumn{3}{|c|}{ New Jersey } \\
\hline Bergen County & Gloucester County & Ocean County \\
\hline Burlington County & Hudson County & Passaic County \\
\hline Camden County & Hunterdon County & Salem County \\
\hline Cumberland County & Mercer County & Somerset County \\
\hline Atlantic County & Middlesex County & Sussex County \\
\hline Cape May County & Monmouth County & Union County \\
\hline Essex County & Morris County & Warren County \\
\hline \multicolumn{3}{|c|}{ New York } \\
\hline Bronx County & New York County & Richmond County \\
\hline Dutchess County & Orange County & Rockland County \\
\hline Kings County & Putnam & Suffolk County \\
\hline Nassau County & Queens County & Westchester County \\
\hline
\end{tabular}


Table C.3. Counties Where Reformulated Gasoline is Sold (continued)

\begin{tabular}{|c|c|c|}
\hline \multicolumn{3}{|c|}{ RFG used in entire county (continued) } \\
\hline \multicolumn{3}{|c|}{ Pennsylvania } \\
\hline Bucks County & Delaware County & Philadelphia County \\
\hline Chester County & Montgomery County & \\
\hline \multicolumn{3}{|c|}{ Rhode Island } \\
\hline Bristol County & Newport County & Washington County \\
\hline Kent County & Providence County & \\
\hline \multicolumn{3}{|c|}{ Texas } \\
\hline Brazoria County & Denton County & Liberty County \\
\hline Chambers County & Fort Bend County & Montgomery County \\
\hline Collin County & Galveston County & Tarrant County \\
\hline Dallas County & Harris County & Waller County \\
\hline \multicolumn{3}{|c|}{ Virginia } \\
\hline Alexandria & Hanover County & Poquoson \\
\hline Arlington County & Henrico County & Portsmouth \\
\hline Charles City County & Hopewell & Prince William County \\
\hline Chesapeake & James City County & Richmond \\
\hline Chesterfield County & Loudoun County & Stafford County \\
\hline Colonial Heights & Manassas & Suffolk \\
\hline Fairfax & Manassas Park & Virginia Beach \\
\hline Fairfax County & Newport News & Williamsburg \\
\hline Falls Church & Norfolk & York County \\
\hline Hampton & & \\
\hline \multicolumn{3}{|c|}{ Wisconsin } \\
\hline Kenosha County & Ozaukee County & Washington County \\
\hline Milwaukee County & Racine County & Waukesha County \\
\hline \multicolumn{3}{|c|}{ Partial RFG Counties } \\
\hline \multicolumn{3}{|c|}{ California } \\
\hline El Dorado County & Riverside County & Solano County \\
\hline Kern County & San Bernardino County & Sutter County \\
\hline Placer County & & \\
\hline \multicolumn{3}{|c|}{ Connecticut } \\
\hline Hartford County & Middlesex County & New London County \\
\hline Litchfield County & New Haven County & Tolland County \\
\hline \multicolumn{3}{|c|}{ Illinois } \\
\hline Grundy County & Kendall County & \\
\hline \multicolumn{3}{|c|}{ Kentucky } \\
\hline Bullitt County & Oldham County & \\
\hline \multicolumn{3}{|c|}{ New York } \\
\hline Essex Count & & \\
\hline
\end{tabular}

Note: RFG is also sold in the District of Columbia. Reformulated gasoline is a motor gasoline specially formulated to achieve significant reductions in vehicle emissions of ozone-forming and toxic air pollutants. The Clean Air Act of 1990 mandates reformulated gasoline use in areas with ozone-air pollution problems, but some of these counties opted-in to the RFG program.

\section{Source:}

U.S. Environmental Protection Agency, RFG Areas, accessed September 15, 2017, http://www.epa.gov/gasolinestandards/reformulated-gasoline 
Figure C.3. Counties Where Reformulated Gasoline is Sold

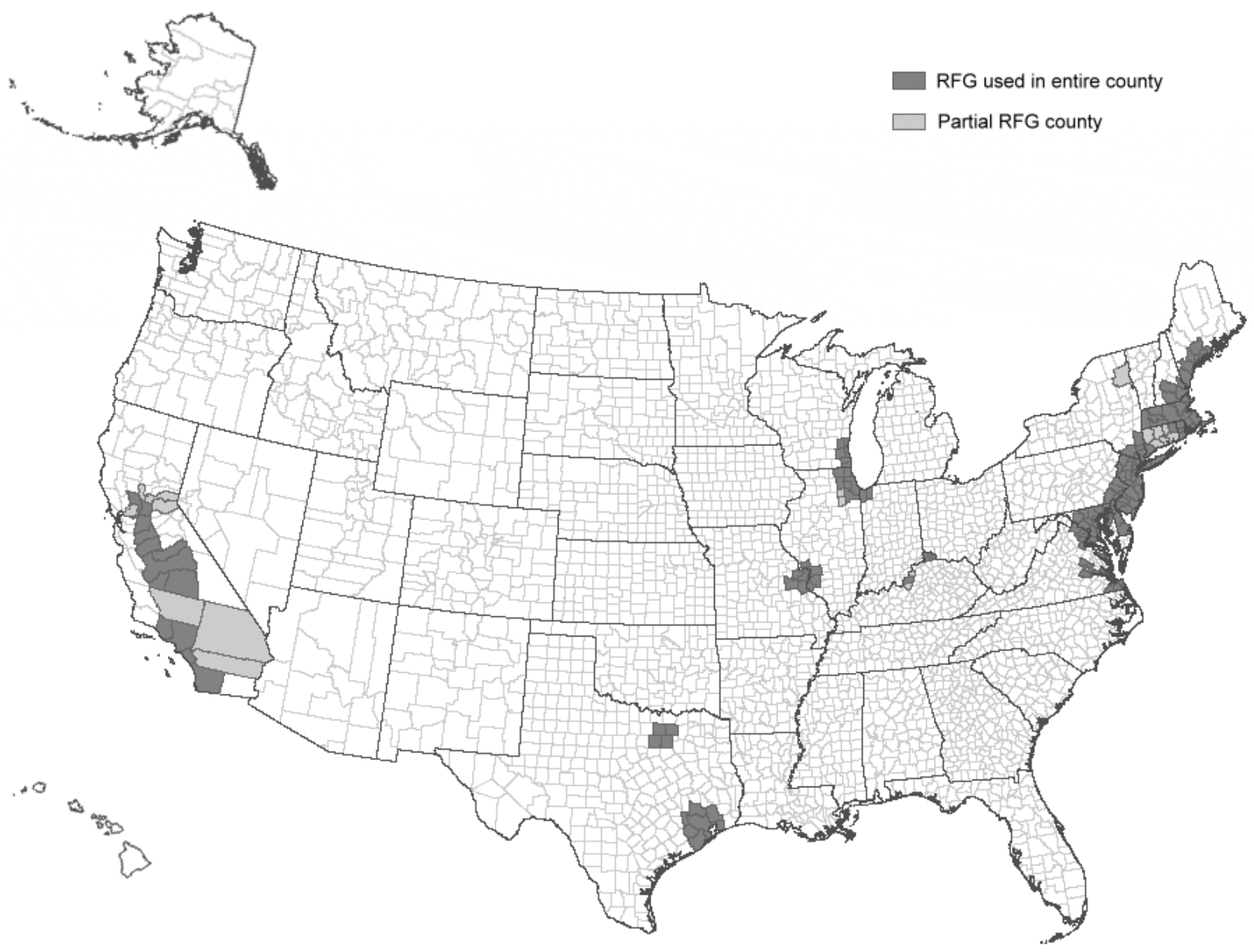

Note: Reformulated gasoline (RFG) is a motor gasoline specially formulated to achieve significant reductions in vehicle emissions of ozone-forming and toxic air pollutants. The Clean Air Act of 1990 mandates reformulated gasoline use in areas with ozone-air pollution problems, but some of these counties opted-in to the RFG program.

\section{Source:}

See Table C.3. 
C-10

TRANSPORTATION ENERGY DATA BOOK: EDITION 36-2017 


\section{GLOSSARY}

Acceleration power - Often measured in kilowatts. Pulse power obtainable from a battery used to accelerate a vehicle. This is based on a constant current pulse for 30 seconds at no less than $2 / 3$ of the maximum open-circuit-voltage, at $80 \%$ depth-of-discharge relative to the battery's rated capacity and at $20^{\circ} \mathrm{C}$ ambient temperature.

Age - The amount of time a person or thing has existed.

Air Carrier - The commercial system of air transportation consisting of certificated air carriers, air taxis (including commuters), supplemental air carriers, commercial operators of large aircraft, and air travel clubs.

Certificated route air carrier: An air carrier holding a Certificate of Public Convenience and Necessity issued by the Department of Transportation to conduct scheduled interstate services. Nonscheduled or charter operations may also be conducted by these carriers. These carriers operate large aircraft (30 seats or more, or a maximum payload capacity of 7,500 pounds or more) in accordance with Federal Aviation Regulation part 121.

Domestic air operator: Commercial air transportation within and between the 50 States and the District of Columbia. Includes operations of certificated route air carriers, Pan American, local service, helicopter, intra-Alaska, intra-Hawaii, all-cargo carriers and other carriers. Also included are transborder operations conducted on the domestic route segments of U.S. air carriers. Domestic operators are classified based on their operating revenue as follows:

Majors - over $\$ 1$ billion

Nationals - $\$ 100$ million to $\$ 1$ billion

Large Regionals - \$20 million to \$99 million

Medium Regionals - Less than $\$ 20$ million

International air operator: Commercial air transportation outside the territory of the United States, including operations between the U.S. and foreign countries and between the U.S. and its territories and possessions.

Supplemental air carrier: A class of air carriers which hold certificates authorizing them to perform passenger and cargo charter services supplementing the scheduled service of the certificated route air carriers. Supplemental air carriers are often referred to as nonscheduled air carriers or "nonskeds."

Alcohol - The family name of a group of organic chemical compounds composed of carbon, hydrogen, and oxygen. The molecules in the series vary in chain length and are composed of a hydrocarbon plus a hydroxyl group. Alcohol includes methanol and ethanol. 
Alternative fuel - For transportation applications, includes the following: methanol; denatured ethanol, and other alcohols; fuel mixtures containing 85 percent or more by volume of methanol, denatured ethanol, and other alcohols with gasoline or other fuels; natural gas; liquefied petroleum gas (propane); hydrogen; coal-derived liquid fuels; fuels (other than alcohol) derived from biological materials (biofuels such as soy diesel fuel); and electricity (including electricity from solar energy). The term "alternative fuel" does not include alcohol or other blended portions of primarily petroleum-based fuels used as oxygenates or extenders, i.e. MTBE, ETBE, other ethers, and the 10-percent ethanol portion of gasohol.

Amtrak - See Rail.

Anthropogenic - Human made. Usually used in the context of emissions that are produced as the result of human activities.

Aviation - See General aviation.

Aviation gasoline - All special grades of gasoline for use in aviation reciprocating engines, as given in the American Society for Testing and Materials (ASTM) Specification D 910. Includes all refinery products within the gasoline range that are to be marketed straight or in blends as aviation gasoline without further processing (any refinery operation except mechanical blending). Also included are finished components in the gasoline range which will be used for blending or compounding into aviation gasoline.

Barges - Shallow, non-self-propelled vessels used to carry bulk commodities on the rivers and the Great Lakes.

Battery efficiency - Measured in percentage. Net DC energy delivered on discharge, as a percentage of the total DC energy required to restore the initial state-of-charge. The efficiency value must include energy losses resulting from self-discharge, cell equalization, thermal loss compensation, and all battery-specific auxiliary equipment.

Btu - British thermal unit. The amount of energy required to raise the temperature of 1 pound of water 1 degree Fahrenheit at or near 39.2 degrees Fahrenheit. An average Btu content of fuel is the heat value per quantity of fuel as determined from tests of fuel samples.

Bunker - A storage tank.

Bunker fuels - Fuel supplied to ships and aircraft, both domestic and foreign, consisting primarily of residual and distillate fuel oil for ships and kerosene-based jet fuel for aircraft.

Bus -A mode of transit service characterized by roadway vehicles powered by diesel, gasoline, battery, or alternative fuel engines contained within the vehicle.

Intercity bus: A standard size bus equipped with front doors only, high backed seats, luggage compartments separate from the passenger compartment and usually with restroom facilities, for high-speed long distance service. 
Motor bus: Rubber-tired, self-propelled, manually-steered bus with fuel supply on board the vehicle. Motor bus types include intercity, school, and transit.

School and other nonrevenue bus: Bus services for which passengers are not directly charged for transportation, either on a per passenger or per vehicle basis.

Transit bus: A bus designed for frequent stop service with front and center doors, normally with a rear-mounted diesel engine, low-back seating, and without luggage storage compartments or restroom facilities.

Trolley coach: Rubber-tired electric transit vehicle, manually-steered, propelled by a motor drawing current, normally through overhead wires, from a central power source not on board the vehicle.

Calendar year - The period of time between January 1 and December 31 of any given year.

Captive imports - Products produced overseas specifically for domestic manufacturers.

Car size classifications - Size classifications of cars are established by the Environmental Protection Agency (EPA) as follows:

Minicompact - less than 85 cubic feet of passenger and luggage volume.

Subcompact - between 85 to 99 cubic feet of passenger and luggage volume.

Compact - between 100 to 109 cubic feet of passenger and luggage volume.

Midsize - between 110 to 119 cubic feet of passenger and luggage volume.

Large - 120 cubic feet or more of passenger and luggage volume.

Two seater - cars designed primarily to seat only two adults.

Small station wagon - less than 130 cubic feet of passenger and luggage volume.

Mid-size station wagon - between 130 to 159 cubic feet of passenger and luggage volume.

Large station wagon - 160 or more cubic feet of passenger and luggage volume.

Carbon dioxide $\left(\mathbf{C O}_{2}\right)$ - A colorless, odorless, non-poisonous gas that is a normal part of the ambient air. Carbon dioxide is a product of fossil fuel combustion.

Carbon monoxide (CO) - A colorless, odorless, highly toxic gas that is a by-product of incomplete fossil fuel combustion. Carbon monoxide, one of the major air pollutants, can be harmful in small amounts if breathed over a certain period of time.

Car-mile (railroad) - A single railroad car moved a distance of one mile.

Cargo ton-mile - See Ton-mile.

Certificated route air carriers - See Air carriers.

Class I freight railroad - See Rail.

Coal slurry - Finely crushed coal mixed with sufficient water to form a fluid. 
Combination trucks - Consist of a power unit (a truck tractor) and one or more trailing units (a semi-trailer or trailer). The most frequently used combination is popularly referred to as a "tractor-semitrailer" or "tractor trailer."

Commercial sector - An energy-consuming sector that consists of service-providing facilities of: businesses; Federal, State, and local governments; and other private and public organizations, such as religious, social or fraternal groups. Includes institutional living quarters.

Commuter rail - A mode of transit service (also called metropolitan rail, regional rail, or suburban rail) characterized by an electric or diesel propelled railway for urban passenger train service consisting of local short distance travel operating between a central city and adjacent suburbs.

Compact car - See car size classifications.

Compression ignition - The form of ignition that initiates combustion in a diesel engine. The rapid compression of air within the cylinders generates the heat required to ignite the fuel as it is injected.

Constant dollars - A time series of monetary figures is expressed in constant dollars when the effect of change over time in the purchasing power of the dollar has been removed. Usually the data are expressed in terms of dollars of a selected year or the average of a set of years.

Consumer Price Index (CPI) - A measure of the average change over time in the prices paid by urban consumers for a market basket of consumer goods and services.

Continuous discharge capacity - Measured as percent of rated energy capacity. Energy delivered in a constant power discharge required by an electric vehicle for hill climbing and/or high-speed cruise, specified as the percent of its rated energy capacity delivered in a one hour constant-power discharge.

Conventional Refueling Station - An establishment for refueling motor vehicles with traditional transportation fuels, such as gasoline and diesel fuel.

Corporate Average Fuel Economy (CAFE) Standards - CAFE standards were originally established by Congress for new cars, and later for light trucks, in Title V of the Motor Vehicle Information and Cost Savings Act (15 U.S.C.1901, et seq.) with subsequent amendments. Under CAFE, car manufacturers are required by law to produce vehicle fleets with a composite sales-weighted fuel economy which cannot be lower than the CAFE standards in a given year, or for every vehicle which does not meet the standard, a fine of $\$ 5.00$ is paid for every one-tenth of a mpg below the standard.

Criteria pollutant - A pollutant determined to be hazardous to human health and regulated under EPA's National Ambient Air Quality Standards. The 1970 amendments to the Clean Air Act require EPA to describe the health and welfare impacts of a pollutant as the "criteria" for inclusion in the regulatory regime. 
Crude oil - A mixture of hydrocarbons that exists in the liquid phase in natural underground reservoirs and remains liquid at atmospheric pressure after passing through surface separating facilities. Crude oil production is measured at the wellhead and includes lease condensate.

Crude oil imports - The volume of crude oil imported into the 50 States and the District of Columbia, including imports from U.S. territories, but excluding imports of crude oil into the Hawaiian Foreign Trade Zone.

Curb weight - The weight of a vehicle including all standard equipment, spare tire and wheel, all fluids and lubricants to capacity, full tank of fuel, and the weight of major optional accessories normally found on the vehicle.

Current dollars - Represents dollars current at the time designated or at the time of the transaction. In most contexts, the same meaning would be conveyed by the use of the term "dollars." See also constant dollars.

Demand Response - A transit mode that includes passenger cars, vans, and small buses operating in response to calls from passengers to the transit operator who dispatches the vehicles. The vehicles do not operate over a fixed route on a fixed schedule. Can also be known as paratransit or dial-a-ride.

Diesel fuel - See Distillate fuel oil.

Disposable personal income - See Income.

Distillate fuel oil - The lighter fuel oils distilled off during the refining process. Included are products known as ASTM grades numbers 1 and 2 heating oils, diesel fuels, and number 4 fuel oil. The major uses of distillate fuel oils include heating, fuel for on-and off-highway diesel engines, and railroad diesel fuel.

Domestic air operator - See Air carrier.

Domestic water transportation - See Internal water transportation.

E85 - 85\% ethanol and 15\% gasoline.

E95 - 95\% ethanol and 5\% gasoline.

Electric utilities sector - Consists of privately and publicly owned establishments which generate electricity primarily for resale.

Emission standards - Limits or ranges established for pollution levels emitted by vehicles as well as stationary sources. The first standards were established under the 1963 Clean Air Act.

End-use sector - See Sector.

Energy capacity - Often measured in kilowatt hours. The energy delivered by the battery up to termination of discharge specified by the battery manufacturer. 
Energy efficiency - In reference to transportation, the inverse of energy intensiveness: the ratio of outputs from a process to the energy inputs; for example, miles traveled per gallon of fuel (mpg).

Energy intensity - In reference to transportation, the ratio of energy inputs to a process to the useful outputs from that process; for example, gallons of fuel per passenger-mile or Btu per ton-mile.

Ethanol ( $\left.\mathbf{C}_{2} \mathrm{H}_{5} \mathrm{OH}\right)$ - Otherwise known as ethyl alcohol, alcohol, or grain-spirit. A clear, colorless, flammable oxygenated hydrocarbon with a boiling point of 78.5 degrees Celsius in the anhydrous state. In transportation, ethanol is used as a vehicle fuel by itself (E100 $100 \%$ ethanol by volume), blended with gasoline (E85 - 85\% ethanol by volume), or as a gasoline octane enhancer and oxygenate (10\% by volume).

Excise tax - Paid when purchases are made on a specific good, such as gasoline. Excise taxes are often included in the price of the product. There are also excise taxes on activities, such as highway usage by trucks.

Ferry boat - A transit mode comprising vessels carrying passengers and in some cases vehicles over a body of water, and that are generally steam or diesel-powered.

Fixed operating cost - See Operating cost.

Fleet vehicles -

Private fleet vehicles: Ideally, a vehicle could be classified as a member of a fleet if it is:

a) operated in mass by a corporation or institution,

b) operated under unified control, or

c) used for non-personal activities.

However, the definition of a fleet is not consistent throughout the fleet industry. Some companies make a distinction between cars that were bought in bulk rather than singularly, or whether they are operated in bulk, as well as the minimum number of vehicles that constitute a fleet (i.e. 4 or 10).

Government fleet vehicles: Includes vehicles owned by all Federal, state, county, city, and metro units of government, including toll road operations.

Foreign freight - Movements between the United States and foreign countries and between Puerto Rico, the Virgin Islands, and foreign countries. Trade between U.S. territories and possessions (e.g. American Samoa, Guam, North Mariana Islands and U.S. Outlying Islands) and foreign countries is excluded. Traffic to or from the Panama Canal Zone is included, but traffic with U.S. origin and U.S. destination traveling through the Panama Canal is not.

Gas Guzzler Tax - Originates from the 1978 Energy Tax Act (Public Law 95-618). A new car purchaser is required to pay the tax if the car purchased has a combined city/highway fuel economy rating that is below the standard for that year. For model years 1986 and later, the standard is $22.5 \mathrm{mpg}$. 
Gasohol - A mixture of $10 \%$ anhydrous ethanol and $90 \%$ gasoline by volume; $7.5 \%$ anhydrous ethanol and $92.5 \%$ gasoline by volume; or 5.5\% anhydrous ethanol and $94.5 \%$ gasoline by volume. There are other fuels that contain methanol and gasoline, but these fuels are not referred to as gasohol.

\section{Gasoline - See Motor gasoline.}

General aviation - That portion of civil aviation which encompasses all facets of aviation except air carriers. It includes any air taxis, commuter air carriers, and air travel clubs which do not hold Certificates of Public Convenience and Necessity.

Global warming potential (GWP) - An index used to compare the relative radiative forcing of different gases without directly calculating the changes in atmospheric concentrations. GWPs are calculated as the ratio of the radiative forcing that would result from the emission of one kilogram of a greenhouse gas to that from the emission of one kilogram of carbon dioxide over a fixed period of time, such as 100 years.

Greenhouse gases - Those gases, such as water vapor, carbon dioxide, nitrous oxide, methane, hydrofluorocarbons (HFCs), perfluorocarbons (PFCs) and sulfur hexafluoride, that are transparent to solar (short-wave) radiation but opaque to long-wave (infrared) radiation, thus preventing long-wave radiant energy from leaving Earth's atmosphere. The net effect is a trapping of absorbed radiation and a tendency to warm the planet's surface.

Gross National Product - A measure of monetary value of the goods and services becoming available to the nation from economic activity. Total value at market prices of all goods and services produced by the nation's economy. Calculated quarterly by the Department of Commerce, the Gross National Product is the broadest available measure of the level of economic activity.

Gross vehicle weight (gvw) - The weight of the empty truck plus the maximum anticipated load weight, including passengers, fluids, and cargo.

Gross vehicle weight rating (gvwr) - The gross vehicle weight which is assigned to each new truck by the manufacturer. This rating may be different for trucks of the same model because of certain features, such as heavy-duty suspension. Passenger cars are not assigned gross vehicle weight ratings.

Heavy-heavy truck - See Truck size classifications.

Heavy rail - A mode of transit service (also called metro, subway, rapid transit, or rapid rail) operating on an electric railway with the capacity for a heavy volume of traffic. Characterized by high speed and rapid acceleration of passenger rail cars.

Household - Consists of all persons who occupy a housing unit, including the related family members and all unrelated persons, if any, who share the housing unit.

Housing unit - A house, apartment, a group of rooms, or a single room occupied or intended for occupancy as separate living quarters. Separate living quarters are those in which the 
occupants do not live and eat with any other persons in the structure and which have either (1) direct access from the outside of the building or through a common hallway intended to be used by the occupants of another unit or by the general public, or (2) complete kitchen facilities for the exclusive use of the occupants. The occupants may be a single family, one person living alone, two or more families living together, or any other group of related or unrelated persons who share living arrangements.

Hybrid-electric vehicles - Combines the benefits of gasoline engines and electric motors and can be configured to obtain different objectives, such as improved fuel economy, increased power, or additional auxiliary power for electronic devices and power tools.

Hydrocarbon (HC) - A compound that contains only hydrogen and carbon. The simplest and lightest forms of hydrocarbon are gaseous. With greater molecular weights they are liquid, while the heaviest are solids.

Income -

Disposable personal income: Personal income less personal tax and non-tax payments.

National income: The aggregate earnings of labor and property which arise in the current production of goods and services by the nation's economy.

Personal income: The current income received by persons from all sources, net of contributions for social insurance.

Industrial sector - Construction, manufacturing, agricultural and mining establishments.

Inertia weight - The curb weight of a vehicle plus 300 pounds.

Intercity bus - See Bus.

Intermodal - Transportation activities involving more than one mode of transportation, including transportation connections and coordination of various modes.

Internal water transportation - Includes all local (intraport) traffic and traffic between ports or landings wherein the entire movement takes place on inland waterways. Also termed internal are movements involving carriage on both inland waterways and the water of the Great Lakes, and inland movements that cross short stretches of open water that link inland systems.

International air operator - See Air carrier.

International freight - See Foreign freight.

Jet fuel - Includes both naphtha-type and kerosene-type fuels meeting standards for use in aircraft turbine engines. Although most jet fuel is used in aircraft, some is used for other purposes such as generating electricity in gas turbines.

Kerosene-type jet fuel: A quality kerosene product with an average gravity of 40.7 degrees API and $10 \%$ to $90 \%$ distillation temperatures of 217 to 261 degrees Celsius. Used 
primarily as fuel for commercial turbojet and turboprop aircraft engines. It is a relatively low freezing point distillate of the kerosene type.

Naphtha-type jet fuel: A fuel in the heavy naphtha boiling range with an average gravity of 52.8 degrees API and $10 \%$ to $90 \%$ distillation temperatures of 117 to 233 degrees Celsius used for turbojet and turboprop aircraft engines, primarily by the military. Excludes ramjet and petroleum.

Kerosene - A petroleum distillate in the 300 to 500 degrees Fahrenheit boiling range and generally having a flash point higher than 100 degrees Fahrenheit by the American Society of Testing and Material (ASTM) Method D56, a gravity range from 40 to 46 degrees API, and a burning point in the range of 150 to 175 degrees Fahrenheit. It is a clean-burning product suitable for use as an illuminant when burned in wick lamps. Includes grades of kerosene called range oil having properties similar to Number 1 fuel oil, but with a gravity of about 43 degrees API and an end point of 625 degrees Fahrenheit. Used in space heaters, cooking stoves, and water heaters.

Kerosene-type jet fuel - See Jet fuel.

Large car - See Car size classifications.

Lease Condensate - A liquid recovered from natural gas at the well or at small gas/oil separators in the field. Consists primarily of pentanes and heavier hydrocarbons (also called field condensate).

Light duty vehicles - Cars and light trucks combined.

Light truck - Unless otherwise noted, light trucks are defined in this publication as two-axle, four-tire trucks. The U.S. Bureau of Census classifies all trucks with a gross vehicle weight less than 10,000 pounds as light trucks (See Truck size classifications).

Light-heavy truck - See Truck size classifications.

Light rail - Mode of transit service (also called streetcar, tramway or trolley) operating passenger rail cars singly (or in short, usually two-car or three-car trains) on fixed rails in right-ofway that is often separated from other traffic for part or much of the way.

Liquefied petroleum gas (lpg) - Consists of propane and butane and is usually derived from natural gas. In locations where there is no natural gas and the gasoline consumption is low, naphtha is converted to lpg by catalytic reforming.

Load factor - Total passenger miles divided by total vehicle miles.

Low emission vehicle - Any vehicle certified to the low emission standards which are set by the Federal government and/or the state of California.

M85 - 85\% methanol and 15\% gasoline.

M100 - 100\% methanol. 
Medium truck - See Truck size classifications.

Methanol ( $\left.\mathrm{CH}_{3} \mathbf{O H}\right)$ - A colorless highly toxic liquid with essentially no odor and very little taste. It is the simplest alcohol and boils at 64.7 degrees Celsius. In transportation, methanol is used as a vehicle fuel by itself (M100), or blended with gasoline (M85).

Midsize car - See Car size classifications.

Minicompact car - See Car size classifications.

Model year - In this publication, model year is referring to the "sales" model year, the period from October 1 to the next September 31.

Motor bus - See Bus.

Motor gasoline - A mixture of volatile hydrocarbons suitable for operation of an internal combustion engine whose major components are hydrocarbons with boiling points ranging from 78 to 217 degrees Celsius and whose source is distillation of petroleum and cracking, polymerization, and other chemical reactions by which the naturally occurring petroleum hydrocarbons are converted into those that have superior fuel properties.

Regular gasoline: Gasoline having an antiknock index, i.e., octane rating, greater than or equal to 85 and less than 88 . Note: Octane requirements may vary by altitude.

Midgrade gasoline: Gasoline having an antiknock index, i.e., octane rating, greater than or equal to 88 and less than or equal to 90 . Note: Octane requirements may vary by altitude.

Premium gasoline: Gasoline having an antiknock index, i.e., octane rating, greater than 90. Note: Octane requirements may vary by altitude.

Reformulated gasoline: Finished motor gasoline formulated for use in motor vehicles, the composition and properties of which meet the requirements of the reformulated gasoline regulations promulgated by the U.S. Environmental Protection Agency under Section 211(k) of the Clean Air Act. For more details on this clean fuel program see http://www.epa.gov/otaq/fuels/gasolinefuels/rfg/index.htm. Note: This category includes oxygenated fuels program reformulated gasoline (OPRG) but excludes reformulated gasoline blendstock for oxygenate blending (RBOB).

MTBE - Methyl Tertiary Butyl Ether-a colorless, flammable, liquid oxygenated hydrocarbon containing 18.15 percent oxygen.

Naphtha-type jet fuel - See Jet fuel.

National income - See Income.

Nationwide Personal Transportation Survey (NPTS) - A nationwide survey of households that provides information on the characteristics and personal travel patterns of the U.S. 
population. Surveys were conducted in 1969, 1977, 1983, 1990, and 1995 by the U.S. Bureau of Census for the U.S. Department of Transportation.

Natural gas - A mixture of hydrocarbon compounds and small quantities of various nonhydrocarbons existing in the gaseous phase or in solution with crude oil in natural underground reservoirs at reservoir conditions.

Natural gas, dry: Natural gas which remains after: 1) the liquefiable hydrocarbon portion has been removed from the gas stream; and 2) any volumes of nonhydrocarbon gases have been removed where they occur in sufficient quantity to render the gas unmarketable. Dry natural gas is also known as consumer-grade natural gas. The parameters for measurement are cubic feet at 60 degrees Fahrenheit and 14.73 pounds per square inch absolute.

Natural gas, wet: The volume of natural gas remaining after removal of lease condensate in lease and/or field separation facilities, if any, and after exclusion of nonhydrocarbon gases where they occur in sufficient quantity to render the gas unmarketable. Natural gas liquids may be recovered from volumes of natural gas, wet after lease separation, at natural gas processing plants.

Natural gas plant liquids: Natural gas liquids recovered from natural gas in processing plants and from natural gas field facilities and fractionators. Products obtained include ethane, propane, normal butane, isobutane, pentanes plus, and other products from natural gas processing plants.

Nitrogen oxides $\left(\mathbf{N O}_{\mathbf{x}}\right)$ - A product of combustion of fossil fuels whose production increases with the temperature of the process. It can become an air pollutant if concentrations are excessive.

Nonattainment area - Any area that does not meet the national primary or secondary ambient air quality standard established by the Environmental Protection Agency for designated pollutants, such as carbon monoxide and ozone.

Oil Stocks - Oil stocks include crude oil (including strategic reserves), unfinished oils, natural gas plant liquids, and refined petroleum products.

\section{Operating cost -}

Fixed operating cost: In reference to passenger car operating cost, refers to those expenditures that are independent of the amount of use of the car, such as insurance costs, fees for license and registration, depreciation and finance charges.

Variable operating cost: In reference to passenger car operating cost, expenditures which are dependent on the amount of use of the car, such as the cost of gas and oil, tires, and other maintenance.

Organization for Economic Cooperation and Development (OECD) - Consists of Australia, Austria, Belgium, Canada, Chile, Czech Republic, Denmark, Estonia, Finland, France, Germany, Greece, Hungary, Iceland, Ireland, Israel, Italy, Japan, Luxembourg, Mexico, Netherlands, New Zealand, Norway, Poland, Portugal, Slovak Republic, Slovenia, South Korea, Spain, Sweden, Switzerland, Turkey, United Kingdom, and United States. Total 
OECD includes the United States Territories (Guam, Puerto Rico, and the U.S. Virgin Islands). Total OECD excludes data for Czech Republic, Hungary, Mexico, Poland, and South Korea which are not yet available.

OECD Europe: Consists of Austria, Belgium, Czech Republic, Denmark, Estonia, Finland, France, Germany, Greece, Hungary, Iceland, Ireland, Italy, Luxembourg, Netherlands, Norway, Poland, Portugal, Slovak Republic, Slovenia, Spain, Sweden, Switzerland, Turkey, and United Kingdom. OECD Europe excludes data for Czech Republic, Hungary, and Poland which are not yet available.

OECD Pacific: Consists of Australia, Japan, South Korea, and New Zealand.

Organization for Petroleum Exporting Countries (OPEC) - Includes Algeria, Angola, Ecuador, Iran, Iraq, Kuwait, Libya, Nigeria, Qatar, Saudi Arabia, United Arab Emirates, and Venezuela.

Arab OPEC - Consists of Algeria, Bahrain, Egypt, Iraq, Kuwait, Libya, Qatar, Saudi Arabia, Syria, Tunisia, and the United Arab Emirates.

Other single-unit truck - See Single-unit truck.

Oxygenate - A substance which, when added to gasoline, increases the amount of oxygen in that gasoline blend. Includes fuel ethanol, methanol, and methyl tertiary butyl ether (MTBE).

Paratransit - Mode of transit service (also called demand response or dial-a-ride) characterized by the use of passenger cars, vans or small buses operating in response to calls from passengers or their agents to the transit operator, who then dispatches a vehicle to pick up the passengers and transport them to their destinations.

Particulates - Carbon particles formed by partial oxidation and reduction of the hydrocarbon fuel. Also included are trace quantities of metal oxides and nitrides, originating from engine wear, component degradation, and inorganic fuel additives. In the transportation sector, particulates are emitted mainly from diesel engines.

Passenger-miles traveled (PMT) - One person traveling the distance of one mile. Total passenger-miles traveled, thus, give the total mileage traveled by all persons.

Passenger rail - See Rail, "Amtrak" and "Transit Railroad".

Persian Gulf countries - Consists of Bahrain, Iran, Iraq, Kuwait, Qatar, Saudi Arabia, and the United Emirates.

Personal Consumption Expenditures (PCE) - As used in the national accounts, the market value of purchases of goods and services by individuals and nonprofit institutions and the value of food, clothing, housing, and financial services received by them as income in kind. It includes the rental value of owner-occupied houses but excludes purchases of dwellings, which are classified as capital goods (investment).

Personal income - See Income. 
Petroleum - A generic term applied to oil and oil products in all forms, such as crude oil, lease condensate, unfinished oil, refined petroleum products, natural gas plant liquids, and nonhydrocarbon compounds blended into finished petroleum products.

Petroleum consumption: A calculated demand for petroleum products obtained by summing domestic production, imports of crude petroleum and natural gas liquids, imports of petroleum products, and the primary stocks at the beginning of the period and then subtracting the exports and the primary stocks at the end of the period.

Petroleum exports: Shipments of petroleum products from the 50 States and the District of Columbia to foreign countries, Puerto Rico, the Virgin Islands, and other U.S. possessions and territories.

Petroleum imports: All imports of crude petroleum, natural gas liquids, and petroleum products from foreign countries and receipts from Guam, Puerto Rico, the Virgin Islands, and the Hawaiian Trade Zone. The commodities included are crude oil, unfinished oils, plant condensate, and refined petroleum products.

Petroleum inventories: The amounts of crude oil, unfinished oil, petroleum products, and natural gas liquids held at refineries, at natural gas processing plants, in pipelines, at bulk terminals operated by refining and pipeline companies, and at independent bulk terminals. Crude oil held in storage on leases is also included; these stocks are known as primary stocks. Secondary stocks-those held by jobbers dealers, service station operators, and consumers-are excluded. Prior to 1975, stock held at independent bulk terminals were classified as secondary stocks.

Petroleum products supplied: For each petroleum product, the amount supplied is calculated by summing production, crude oil burned directly, imports, and net withdrawals from primary stocks and subtracting exports.

Plug-in hybrid-electric vehicles (PHEVs) - Hybrid-electric vehicles with high capacity batteries that can be charged by plugging them into an electrical outlet or charging station. There are two basic PHEV configurations:

Parallel or Blended PHEV: Both the engine and electric motor are mechanically connected to the wheels, and both propel the vehicle under most driving conditions. Electric-only operation usually occurs only at low speeds.

Series PHEVs, also called Extended Range Electric Vehicles (EREVs): Only the electric motor turns the wheels; the gasoline engine is only used to generate electricity. Series PHEVs can run solely on electricity until the battery needs to be recharged. The gasoline engine will then generate the electricity needed to power the electric motor. For shorter trips, these vehicles might use no gasoline at all.

Processing Gain - The amount by which the total volume of refinery output is greater than the volume of input for given period of time. The processing gain arises when crude oil and 
other hydrocarbons are processed into products that are, on average, less dense than the input.

Processing Loss - The amount by which the total volume of refinery output is less than the volume of input for given period of time. The processing loss arises when crude oil and other hydrocarbons are processed into products that are, on average, denser than the input.

Proved Reserves of Crude Oil - The estimated quantities of all liquids defined as crude oil, which geological and engineering data demonstrate with reasonable certainty to be recoverable in future years from known reservoirs under existing economic and operating conditions.

Quad - Quadrillion, 10 ${ }^{15}$. In this publication, a Quad refers to Quadrillion Btu.

\section{Rail -}

Amtrak (American Railroad Tracks): Operated by the National Railroad Passenger Corporation of Washington, DC. This rail system was created by President Nixon in 1970, and was given the responsibility for the operation of intercity, as distinct from suburban, passenger trains between points designated by the Secretary of Transportation.

Class I freight railroad: Defined by the Interstate Commerce Commission each year based on annual operating revenue. A railroad is dropped from the Class I list if it fails to meet the annual earnings threshold for three consecutive years.

Commuter railroad: Those portions of mainline railroad (not electric railway) transportation operations which encompass urban passenger train service for local travel between a central city and adjacent suburbs. Commuter railroad service-using both locomotive-hauled and self-propelled railroad passenger cars-is characterized by multitrip tickets, specific station-to-station fares, and usually only one or two stations in the central business district. Also known as suburban railroad.

Transit railroad: Includes "heavy" and "light" transit rail. Heavy transit rail is characterized by exclusive rights-of-way, multi-car trains, high speed rapid acceleration, sophisticated signaling, and high platform loading. Also known as subway, elevated railway, or metropolitan railway (metro). Light transit rail may be on exclusive or shared rights-of-way, high or low platform loading, multi-car trains or single cars, automated or manually operated. In generic usage, light rail includes streetcars, trolley cars, and tramways.

Refiner sales price - Sales from the refinery made directly to ultimate consumers, including bulk consumers (such as agriculture, industry, and electric utilities) and residential and commercial consumers.

Reformulated gasoline (RFG) - See Motor gasoline.

RFG area - An ozone nonattainment area designated by the Environmental Protection Agency which requires the use of reformulated gasoline.

Residential sector - An energy consuming sector that consists of living quarters for private households. Excludes institutional living quarters. 
Residential Transportation Energy Consumption Survey (RTECS) - This survey was designed by the Energy Information Administration of the Department of Energy to provide information on how energy is used by households for personal vehicles. It has been conducted five times since 1979, the most recent being 1991.

Residual fuel oil - The heavier oils that remain after the distillate fuel oils and lighter hydrocarbons are boiled off in refinery operations. Included are products know as ASTM grade numbers 5 and 6 oil, heavy diesel oil, Navy Special Fuel Oil, Bunker C oil, and acid sludge and pitch used as refinery fuels. Residual fuel oil is used for the production of electric power, for heating, and for various industrial purposes.

Rural - Usually refers to areas with population less than 5,000.

Sales period - October 1 of the previous year to September 30 of the given year. Approximately the same as a model year.

Sales-weighted miles per gallon (mpg) - Calculation of a composite vehicle fuel economy based on the distribution of vehicle sales.

Scrappage rate - As applied to motor vehicles, it is usually expressed as the percentage of vehicles of a certain type in a given age class that are retired from use (lacking registration) in a given year.

School and other nonrevenue bus - See Bus.

Sector - A group of major energy-consuming components of U.S. society developed to measure and analyze energy use. The sectors most commonly referred to are: residential, commercial, industrial, transportation, and electric power.

Single-unit truck - Includes two-axle, four-tire trucks and other single-unit trucks.

Two-axle, four-tire truck: A motor vehicle consisting primarily of a single motorized device with two axles and four tires.

Other single-unit truck: A motor vehicle consisting primarily of a single motorized device with more than two axles or more than four tires.

Spark ignition engine - An internal combustion engine in which the charge is ignited electrically (e.g., with a spark plug).

Special fuels - Consist primarily of diesel fuel with small amount of liquefied petroleum gas, as defined by the Federal Highway Administration.

Specific acceleration power - Measured in watts per kilogram. Acceleration power divided by the battery system weight. Weight must include the total battery system.

Specific energy - Measured in watt hours per kilogram. The rated energy capacity of the battery divided by the total battery system weight. 
Subcompact car - See Car size classifications.

Supplemental air carrier - See Air carrier.

Survival rate - As applied to motor vehicles, it is usually expressed as the percentage of vehicles of a certain type in a given age class that will be in use at the end of a given year.

Tax incentives - In general, a means of employing the tax code to stimulate investment in or development of a socially desirable economic objective without direct expenditure from the budget of a given unit of government. Such incentives can take the form of tax exemptions or credits.

Test weight - The weight setting at which a vehicle is tested on a dynamometer by the U.S. Environmental Protection Agency (EPA). This weight is determined by the EPA using the inertia weight of the vehicle.

Ton-mile - The movement of one ton of freight the distance of one mile. Ton-miles are computed by multiplying the weight in tons of each shipment transported by the distance hauled.

\section{Transmission types -}

A3 - Automatic three speed

A4 - Automatic four speed

A5 - Automatic five speed

L4 - Automatic lockup four speed

M5 - Manual five speed

Transit bus - See Bus.

Transit railroad - See Rail.

Transportation sector - Consists of both private and public passenger and freight transportation, as well as government transportation, including military operations.

Truck Inventory and Use Survey (TIUS) - Survey designed to collect data on the characteristics and operational use of the nation's truck population. It is conducted every five years by the U.S. Bureau of the Census. Surveys were conducted in 1963, 1967, 1972, 1977, 1982, 1987, and 1992. For the 1997 survey, it was renamed the Vehicle Inventory and Use Survey in anticipation of including additional vehicle types. However, no additional vehicle types were added to the 1997 survey.

Trolleybus - Mode of transit service (also called transit coach) using vehicles propelled by a motor drawing current from overhead wires via connecting poles called a trolley pole, from a central power source not onboard the vehicle.

Truck size classifications - U.S. Bureau of the Census has categorized trucks by gross vehicle weight (gvw) as follows:

Light - Less than 10,000 pounds gvw (Also see Light Truck.) 
Medium - 10,001 to 20,000 pounds gvw

Light-heavy - 20,001 to 26,000 pounds gvw

Heavy-heavy - 26,001 pounds gvw or more.

Two-axle, four-tire truck - See Single-unit truck.

Two seater car - See Car size classifications.

Ultra-low emission vehicle - Any vehicle certified to the ultra-low emission standards which are set by the Federal government and/or the state of California.

Urban - Usually refers to areas with population of 5,000 or greater.

Vanpool: A ridesharing prearrangement using vans or small buses providing round-trip transportation between the participant's prearranged boarding points and a common and regular destination.

Variable operating cost - See Operating cost.

Vehicle Inventory and Use Survey - Last conducted in 2002. See Truck Inventory and Use Survey.

Vehicle-miles traveled (vmt) - One vehicle traveling the distance of one mile. Total vehicle miles, thus, is the total mileage traveled by all vehicles.

Volatile organic compounds (VOCs) - Organic compounds that participate in atmospheric photochemical reactions.

\section{Waterborne Commerce -}

Coastwise: Domestic traffic receiving a carriage over the ocean, or the Gulf of Mexico. Traffic between Great Lakes ports and seacoast ports, when having a carriage over the ocean, is also termed Coastwise.

Domestic: Includes coastwise, lakewise, and internal waterborne movements.

Foreign: Waterborne import, export, and in-transit traffic between the United States, Puerto Rico and the Virgin Islands and any foreign country.

Internal: Vessel movements (origin and destination) which take place solely on inland waterways. An inland waterway is one geographically located within the boundaries of the contiguous 48 states or within the boundaries of the State of Alaska.

Lakewise: Waterborne traffic between the United States ports on the Great Lakes System. The Great Lakes System is treated as a separate waterway system rather than as a part of the inland waterway system. In comparing historical data for the Great Lakes System, one should note that prior to calendar year 1990, marine products, sand and gravel being moved 
from the Great Lakes to Great Lake destinations were classified as local traffic. From 1990on, these activities are classified as lakewise traffic.

Well-to-wheel - A life cycle analysis used in transportation to consider the entire energy cycle for a given mode, rather than just tailpipe emissions. The analysis starts at the primary energy source and ends with the turning wheels of the vehicle.

Zero-emission vehicle - Any vehicle certified to the zero emission standards which are set by the Federal government and/or the state of California. These standards apply to the vehicle emissions only. 